\title{
ORCHESTRATION: THE MOVEMENT AND VOCAL BEHAVIOR OF FREE-RANGING NORWEGIAN KILLER WHALES (ORCINUS ORCA)
}

By

\author{
Ari Daniel Shapiro \\ M.Phil., University of St. Andrews, 2002 \\ B.S., Boston College, 2001
}

Submitted in partial fulfillment of the requirements for the degree of

Doctor of Philosophy

at the

\section{MASSACHUSETTS INSTITUTE OF TECHNOLOGY}

and the

\section{WOODS HOLE OCEANOGRAPHIC INSTITUTION}

June 2008

(C) 2008 Ari D. Shapiro

All rights reserved.

The author hereby grants to MIT and WHOI permission to reproduce and to distribute publicly paper and electronic copies of this thesis document in whole or in part in any medium now known or hereafter created.

Signature of Author

Joint Program in Oceanography/Applied Ocean Science and Engineering
Massachusetts Institute of Technology
and Woods Hole Oceanographic Institution
May 2008

Certified by

Peter Lloyd Tyack

Thesis Supervisor

Accepted by

Edward DeLong Chair, Joint Committee for the Biology Department Woods Hole Oceanographic Institution 


\title{
ORCHESTRATION: THE MOVEMENT AND VOCAL BEHAVIOR OF FREE-RANGING NORWEGIAN KILLER WHALES (ORCINUS ORCA)
}

\author{
By \\ Ari Daniel Shapiro \\ Submitted in partial fulfillment of the requirements for the degree of \\ Doctor of Philosophy at the Massachusetts Institute of Technology \\ and the Woods Hole Oceanographic Institution
}

\begin{abstract}
Studying the social and cultural transmission of behavior among animals helps to identify patterns of interaction and information content flowing between individuals. Killer whales are likely to acquire traits culturally based on their population-specific feeding behaviors and group-distinctive vocal repertoires. I used digital tags to explore the contributions of individual Norwegian killer whales to group carousel feeding and the relationships between vocal and non-vocal activity.

Periods of tail slapping to incapacitate herring during feeding were characterized by elevated movement variability, heightened vocal activity and call types containing additional orientation cues. Tail slaps produced by tagged animals were identified using a rapid pitch change and occurred primarily within $20 \mathrm{~m}$ of the surface. Two simultaneously tagged animals maneuvered similarly when tail slapping within 60 s of one another, indicating that the position and composition of the herring ball influenced their behavior.
\end{abstract}

Two types of behavioral sequence preceding the tight circling of carousel feeding were apparent. First, the animals engaged in periods of directional swimming. They were silent in 2 of 3 instances, suggesting they may have located other foraging groups by eavesdropping. Second, tagged animals made broad horizontal loops as they dove in a manner consistent with corralling. All 4 of these occasions were accompanied by vocal activity, indicating that this and tail slapping may benefit from social communication. No significant relationship between the call types and the actual movement measurements was found.

Killer whale vocalizations traditionally have been classified into discrete call types. Using human speech processing techniques, I considered that calls are alternatively comprised of shared segments that can be recombined to form the stereotyped and variable repertoire. In a classification experiment, the characterization of calls using the whole call, a set of unshared segments, or a set of shared segments yielded equivalent performance. The shared segments required less information to parse the same vocalizations, suggesting a more parsimonious system of representation.

This closer examination of the movements and vocalizations of Norwegian killer 
whales, combined with future work on ontogeny and transmission, will inform our understanding of whether and how culture plays a role in achieving population-specific behaviors in this species. 


\section{ACKNOWLEDGEMENTS}

A dissertation is a journey requiring the effort and energy of many people - mentors, colleagues, friends and family. I will start by acknowledging the immense amount of inspiration, insight and joy that my advisor, Peter Tyack, poured into both me and this document. He recharged my intellectual and psychological voltage each time that we met, offering up creative analytical directions, steering and then refining and then finessing the trajectory of my dissertation, and infusing such genuine enthusiasm and passion into each of our conversations. Peter revised numerous drafts of each chapter, making suggestions and additions that immeasurably improved their content and approach. He has been an ideal mentor to me, guiding me through the political intricacies of organizing field work and teaching me about the insights gained by honoring the novelties and nuances of animals behaving in the wild. He helped me take the idea of studying killer whales in northern Norway and turn it into the reality of this dissertation. Peter maintains a high standard of professional and personal excellence that I admire. I respect him greatly as both a scientist and a human being and have benefited from witnessing how he blends these two aspects so naturally.

The other members of my committee have provided crucial guidance on numerous components of my dissertation. Andy Solow and I met during my Summer Student Fellowship at WHOI in 2000 and it was then that we established the tradition of meeting nearly once each week. We have discussed the design and execution of statistical analyses, how to navigate the issues of authorship and collaboration in science, and the politics and humor in the world around us. Andy encourages and teaches in a style that is at once engaging and exciting. Stephanie Seneff thought resourcefully and productively about how to connect up the realm of human speech recognition with killer whale communication. The syntax work presented here particularly benefited from her thoughtful consideration, but she offered important comments on numerous other aspects of my dissertation as well. I thank her for finding me a home in the Stata Center at MIT and allowing countless undergraduates to use the computers outside of her office to help process the vocal data. Nearly all of the data analyzed here were fetched because of Patrick's heroic tagging efforts. He tagged when everyone else thought the waves and weather dictated otherwise. Patrick's strong contributions in the field were complemented by his helpful and guiding comments on my dissertation chapters.

The field teams in Norway in November 2005 and 2006 offered a boundless supply of energy, effort and enthusiasm to gather the data for this dissertation. Tiu Similä beautifully cultivated positive relationships between the scientists, fisherfolk, whale watch operators and local community members. Her natural ability to build alliances greatly benefited our field efforts. She was a tremendous intellectual resource as well, offering guidance about how and where to find the whales and making observations about their behavior. Our skipper, Geoff Magee, is an incredibly skilled boat driver who balanced maneuvering the vessel at a respectable distance from the animals with approaching close 
enough to allow the science to happen. His sense of humor and song graced the project, providing levity, perspective and wisdom. I thank the field team for all of their work and for keeping their spirits up even when the whales seemed like a distant promise: Mads Christoffersen, Mike deRoos, Andy Foote, Ari Friedlaender, Petter Helgevold-Kvadsheim, Jesper Jensen, Pamela Kiekebosch, Sanna Kuningas, Michi Main, Patrick Miller, Sari Oksanen, Alice Pope, Filipa Samarra, Outi Tervo and Hajime Yoshino. There are two other field crews that I would like to acknowledge for teaching me about the fruits of collaboration. The first was the group with whom I studied narwhals on Baffin Island, Canada during the summer of 2004 and included Rune Dietz, Jack Orr, Mehdi Bakhtiari, Sila Akikuluk, Sandie Black, Moe Keenainak, Martin Nweeia, Hans-Christian Schmidt, Seemee Tunraq and Keith Yip. I worked with the second team off of southeast Alaska where we tagged transient killer whales. I don't remember ordering such a delightful collection of memories - just desiring them. Thanks to Volker Deecke, Jim and Gayle Eastwood, Mike deRoos, Michi Main, Patrick Miller and Filipa Samarra.

I greatly appreciate the efforts of Mark Johnson and Tom Hurst to teach me about the tags, help me prepare for my field work and troubleshoot problems that I encountered in the field. I relied on the generous instruction and counsel of numerous friends and colleagues associated with Peter's lab including Ann Allen, Kira Barton, Alex Bocconcelli, Stacy DeRuiter, Amanda Hansen, Peter Madsen, Amanda Searby, Alex Shorter, Stephanie Watwood and Walter Zimmer. Thirteen industrious undergraduate students contributed hundreds of hours towards sorting spectrograms, tracing contours, double-checking call type assignments, untangling the morasses of overlapping calls and then uncrossing their eyes. I am so grateful for the hard work of Marlene Brito, Deirdre Connolly, Ivan Dimitrov, Sara Kim, Gary Matthias, Rebecca McGowan, Diane Rak, Maitagorri Schade, Levi Schmidt, Katie Smyth, Katie Stanchak, Clara Stefanov-Wagner and Jia Xin Wu. Ghinwa Chouetier and Scott Cyphers helped me to decipher the SLS computers at the Stata Center. Robert Gross, my friend and calculus professor from Boston College, offered patient, eloquent and clear instruction on how to fit Legendre polynomials to the contour traces.

The WHOI Academic Programs Office has provided financial and psychological guidance since I was a summer student. Special thanks to Marsha Gomes, Julia Westwater, Shona Vitelli, Valerie Caron, Jim Price, Jim Yoder, John Farrington and Ronni Schwartz. Without an actual office at MIT, Lindgren Library became a second home for me. Joe Hankins and Chris Sherratt generously greeted and welcomed me at the library each day, offering me filtered water, refrigerator space, cookies, use of the telephone, crossword puzzles and special deliveries to my desk of books about Túngara frogs that I had placed on reserve. I greatly appreciate the efforts of Lonny Lippsett, Matt Villano and Oceanus magazine to feature a story on the work that we were doing in Norway and I am grateful to Matt Barton, Jack Cook, Fritz Heide, E. Paul Oberlander, Tim Silva and the rest of the Graphics Department for their beautiful artistic and audiovisual efforts to render the content of my dissertation more understandable. 
I must thank a variety of funding sources. The Ocean Life Institute at WHOI and the National Geographic Society financed the two seasons of field work in Norway. My stipend was paid for 3 years by the National Defense Science and Engineering Graduate Fellowship, a grant I was especially pleased with since I felt like I was using money that would have been spent on fighter jets and turning it into chocolate chip cookies. My remaining time in graduate school was financed by a National Science Foundation Graduate Fellowship, the Academic Programs Office at WHOI and Dennis McLaughlin at MIT. Thanks to the MIT UROP office and the Ocean Life Institute for their fiduciary support of several of the undergraduate students mentioned above.

My path towards the Ph.D. has been illuminated and enriched by numerous friendships. I thank many for their warm companionship and rich laughter: Kate Buckman, Kumar Chandran, Andrea Christoforou, Evan and Emily Dvorin, Nicole Estvanik, Lauren Freeman, Rachel Gold, Leia Grossman, Joanna Gyory, Elke Hodson, Annette Hynes, Elana Levites-Agababa, Paul and Laura London, Christian Karaba, Ari Mueller, Eli Pristoop, Sarah Reynolds, Andrea Schaffer, Jerome Sherman, Elesheva and Meira Soloff, Elanor Starmer, Samantha Sutton, Mark Stephenson, Yuki Takahashi, Zak Vassar and Pat and Emily Venanzi. My friends at Atlantic Public Media and the Cape and Islands NPR station, Sam Broun, Viki Merrick, Jay Allison, Steve Young and Syd Lewis, have mentored and believed in me, a gift that I cherish. Stacy DeRuiter and I have been labmates since we began the Joint Program and she has been a confidante, a study buddy, a helper and most importantly - a friend. I am grateful to Mrs. Nora Doerder for exciting me about biology at Charles F. Brush High School and motivating me to follow that enthusiasm, to Dr. Don Griffin for introducing me to the simultaneous serious and spontaneous nature of field work when I helped him study Canadian beavers in Concord, and to Peter Slater and Vincent Janik for teaching me about independent research and being a scientist.

I thank a few friends in particular. Desireé Plata ignites the world with her passion for people and compassion for our planet - I admire her natural ability to actively appreciate those around her. Kristen Grauman is one of the most thoughtful listeners and laughers I've ever met and I treasure our many, many conversations in which we've talked about our futures and I've confided some of my dearest thoughts and feelings. Haley Naik is an amazing blend of adventure, joy and advice - she provides dermatological insights in one breath and thoughts on pop fashion in the next. I so treasure her friendship and the fun we've had. Over the last 4 years I have lived with four very special people: Anne Thompson, Kjetil and Selina Vage, and Stephanie Waterman. They've supported and celebrated me when I needed it the most, and we've gone through a lot of life together. We began as roommates, grew to become dear friends, and now they feel like family. When I return to 33 Dimick Street, it feels like I'm coming home. 
Through it all, my family has been a source of constant support. Mom and Dad have given me perhaps the greatest gift of all - they have loved me for who I am since the day that I arrived on this planet. That has meant the world to me. Tammy and Kristie are not only sisters to me but best friends as well. Our visits each and every week are like honey. They teach me perspective, patience and so many lessons, including that if you listen, the world sings back to you.

And finally, I'd like to dedicate my dissertation to one very special woman who helped to make me who I am in a significant and beautiful way. That woman is Nana, my grandmother, who passed away earlier this year at the age of 90 . She taught me that love and laughter make life worth living. I love you so much, Nana. 


\section{TABLE OF Contents}

SIGNATURE PAGE

ABSTRACT $\ldots \ldots \ldots$

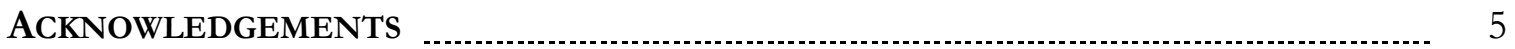

TABLE OF CONTENTS

LIST OF FIGURES

LIST OF TABLES

CHAPTER 1: GENERAL INTRODUCTION _.......................................................... 25

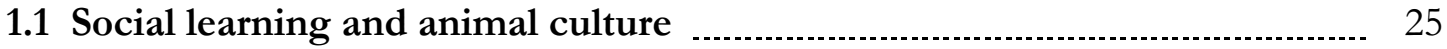

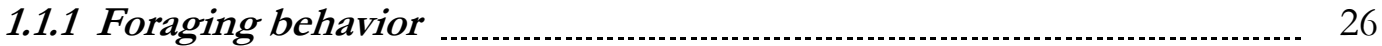

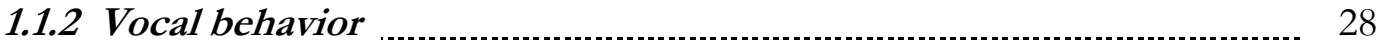

1.2 Killer whales as candidates for using culture ....................................... 29

1.3 Introduction to dissertation research ................................................ 33

1.3.1 Field procedure: Daily sailing routine and tagging procedure ........... 37

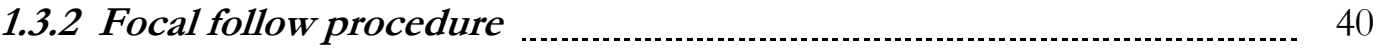

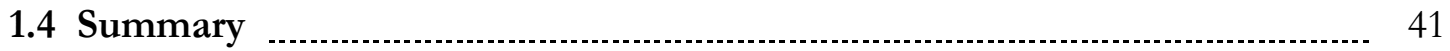

CHAPTER 2: INDIVIDUAL BEHAVIORS OF CAROUSEL FEEDING

NORWEGIAN KILLER WHALES

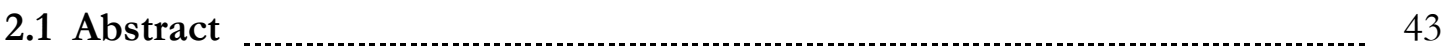

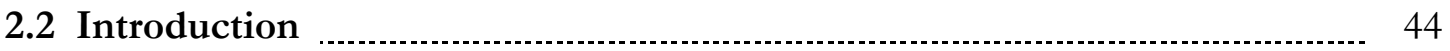

2.3 General materials and methods ..................................................... 50

2.4 Analysis 1: General movement and depth features ................................. 52

2.4.1 Methods

2.4.1.1 Identifying tail slaps and tail slapping periods .................................... 52

2.4.1.2 Derived movement measurements ..................................................... 53

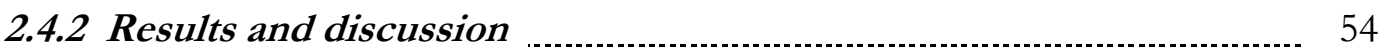

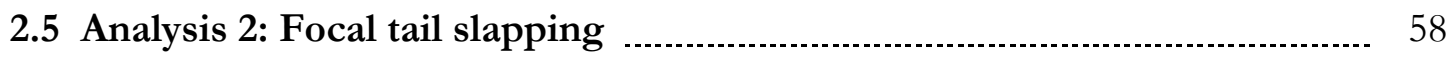

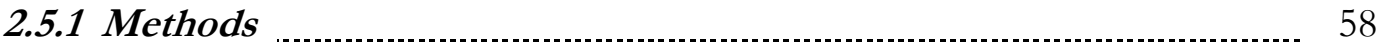

2.5.2 Results and discussion .................................................... 59

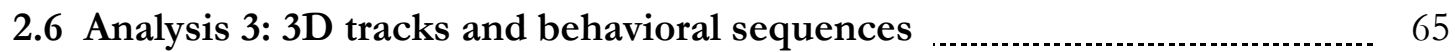

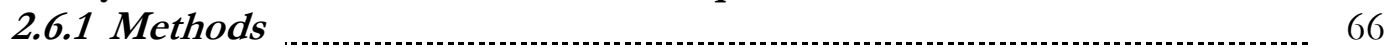

2.6.2 Results and discussion $\ldots$

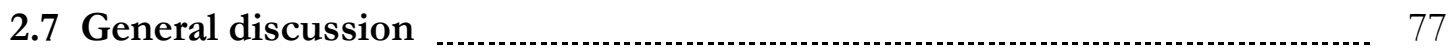

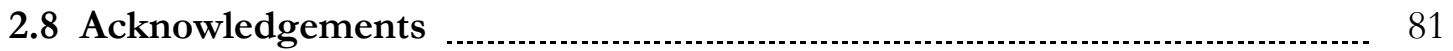


Chapter 3: Everything but the Cetacean Synch: An Example-Driven Discussion OF BEHAVIORAL ASSOCIATION IN MARINE MAMMALS . ...................... 83

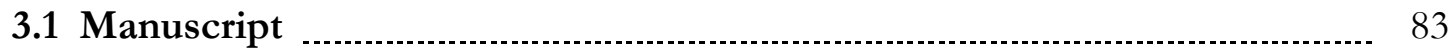

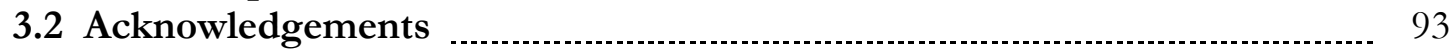

CHAPTER 4: RELATIONSHIP BETWEEN MOVEMENT AND VOCAL BEHAVIOR OF FREE-RANGING NORWEGIAN KILLER WHALES ………........................... 95

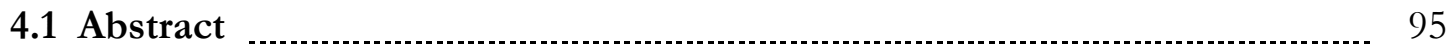

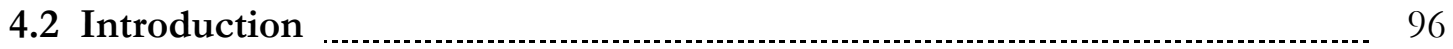

4.3 General materials and methods …..................................................... 101

4.4 Analysis 1: State-dependent models …................................................. 103

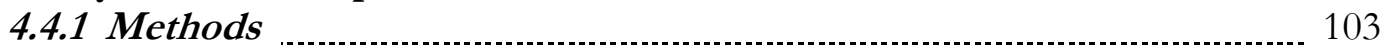

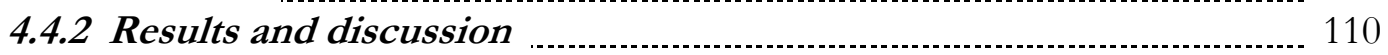

4.5 Analysis 2: Incorporating the movement sensors …................................. 120

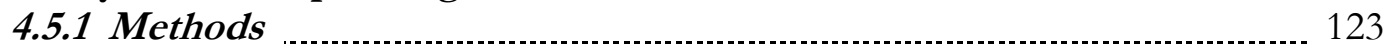

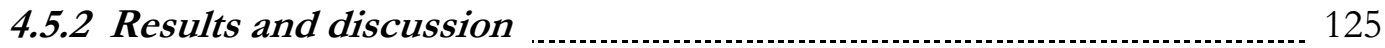

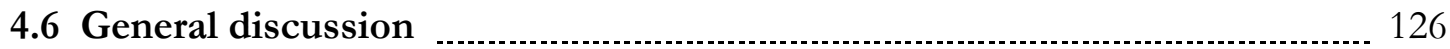

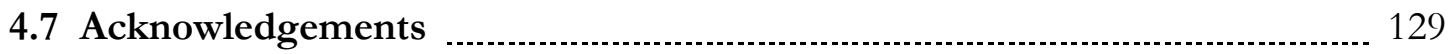

Chapter 5: Phonemic Segment Characterization of Norwegian Killer

WHALE CALL TYPES

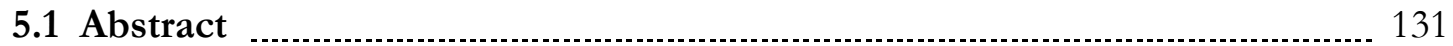

5.2 Introduction

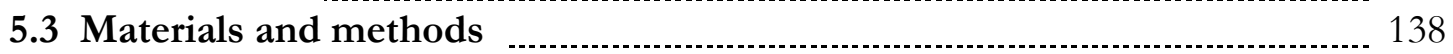

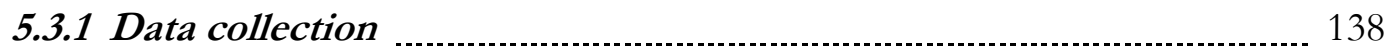

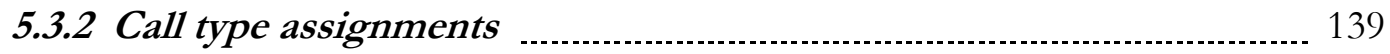

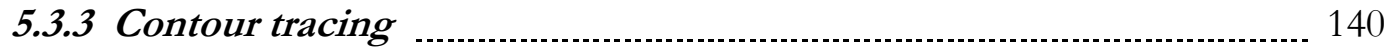

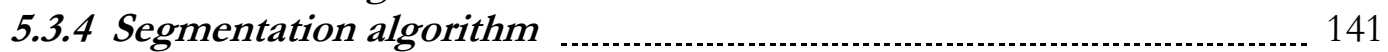

5.3.5 Call classification experiments

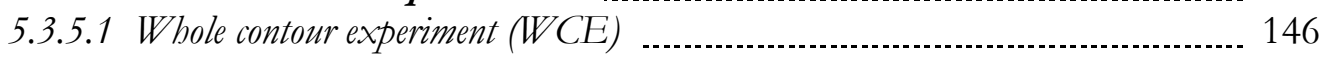

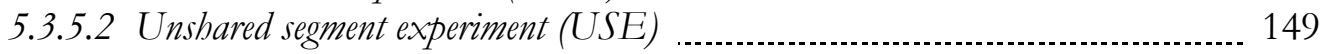

5.3.5.3 Shared segment experiment (SSE) ...................................................... 150

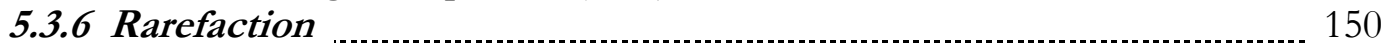

5.3.7 Variable Norwegian killer whale calls and stereotyped Pacific

Northwest resident and transient killer whale calls .......................... 151

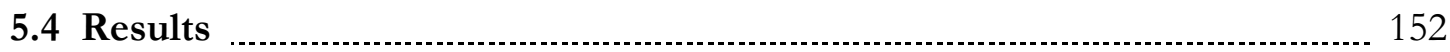

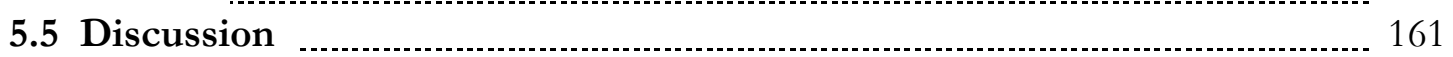

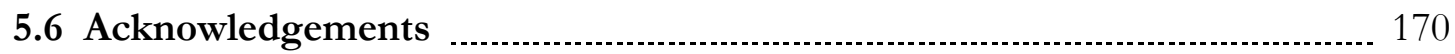

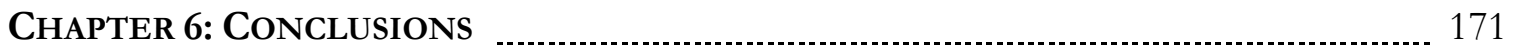

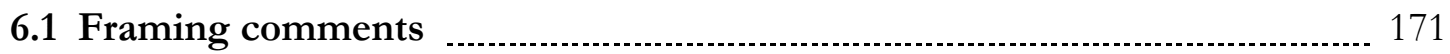

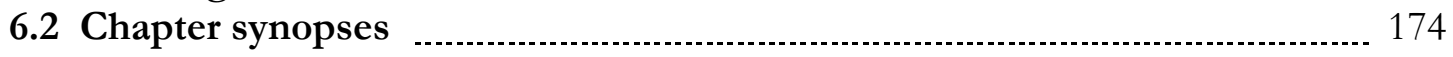

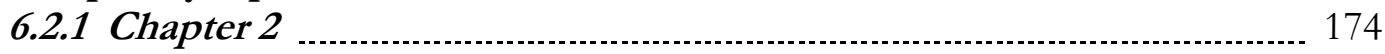


6.2.2 Chapter 3

6.2.3 Chapter 4

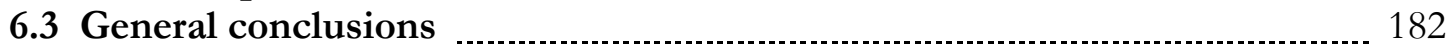

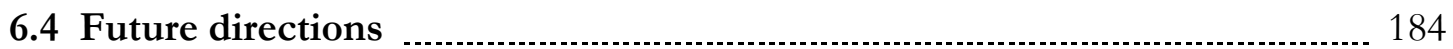

CHAPTER 7: REFERENCES

APPENdix 1: AdDitional Movement Plots _.............................................. 197

A1.1 Pitch, depth and Az variation profiles centered on focal tail slaps .......... 197

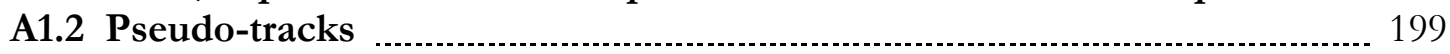

A1.3 Movement data summary plots _...................................................... 213

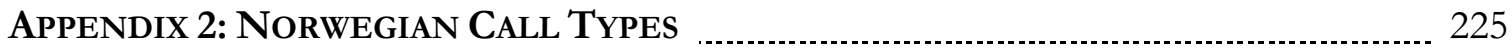

Appendix 3: Norwegian Stereotyped Call Contours _................................. 243

APPENDiX 4: PRELIMINARY EVIDENCE FOR SIgNATURE VOCALIZATIONS AMONG

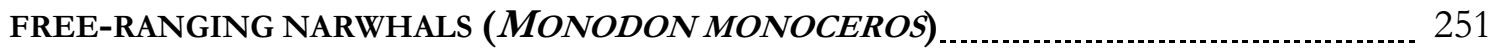

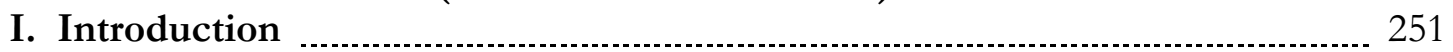

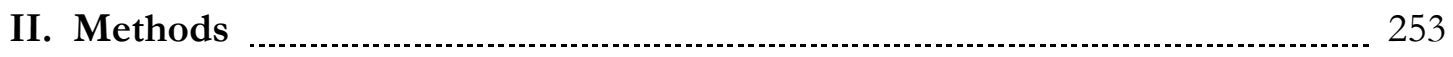

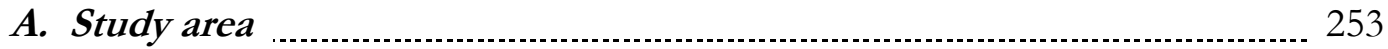

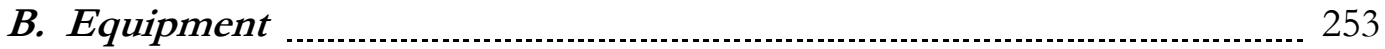

C. Capturing and tagging protocol ……......................................... 253

D. Vocalization extraction

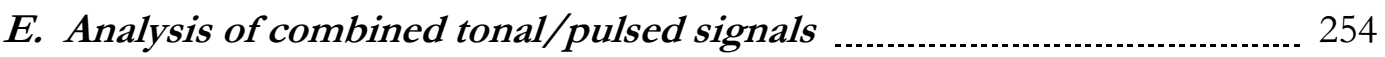

F. Whistle extraction and analysis ........................................................ 254

1. Nonparametric comparison ................................................................... 255

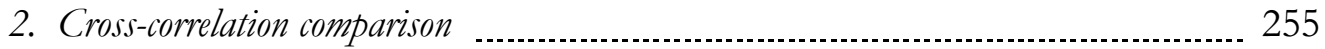

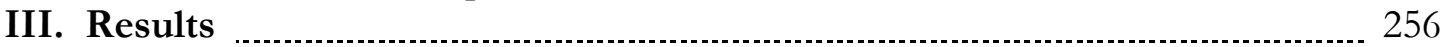

IV. Discussion and conclusions _................................................... 258

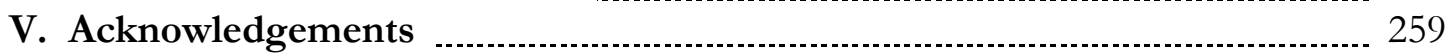

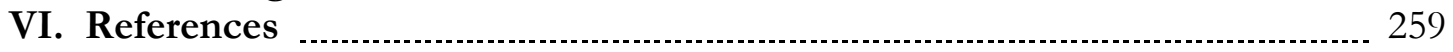

APPENDIX 5: TESTING FOR ORIENTATION RESPONSES OF INDIVIDUAL SPERM WHALES TO A VARYING SONAR EXPOSURE LEVEL _.......................................... 263

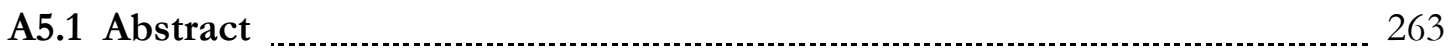

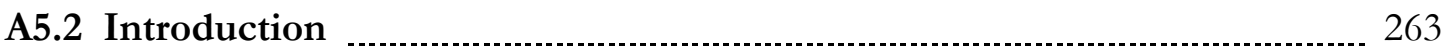

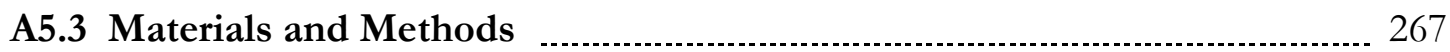




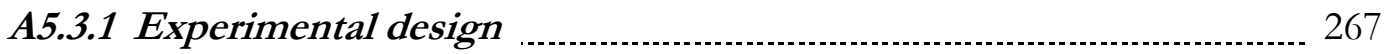

A5.3.1.1 Field protocol ...................................................................... 267

A5.3.1.2 Received sound exposure level (RL) calculations _................................ 269

A5.3.1.3 Animal and vessel paths ..................................................... 271

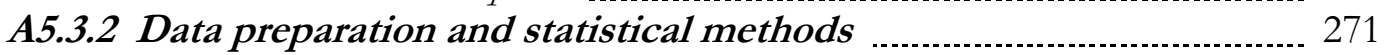

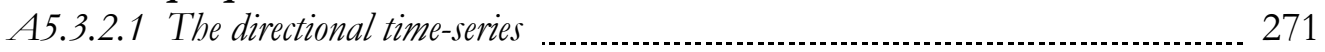

A5.3.2.2 Assumptions ...................................................................... 273

A5.3.2.3 Circular regression on residual deviations from path trend ......................... 274

A5.3.2.4 Testing significance ....................................................................... 276

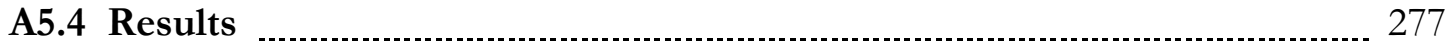

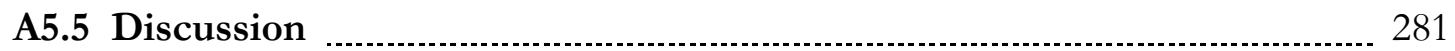

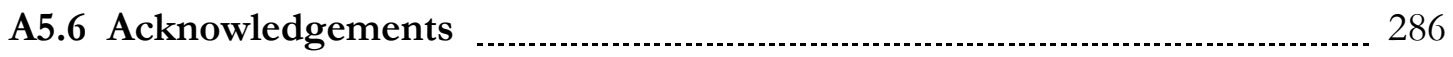

A5.7 References

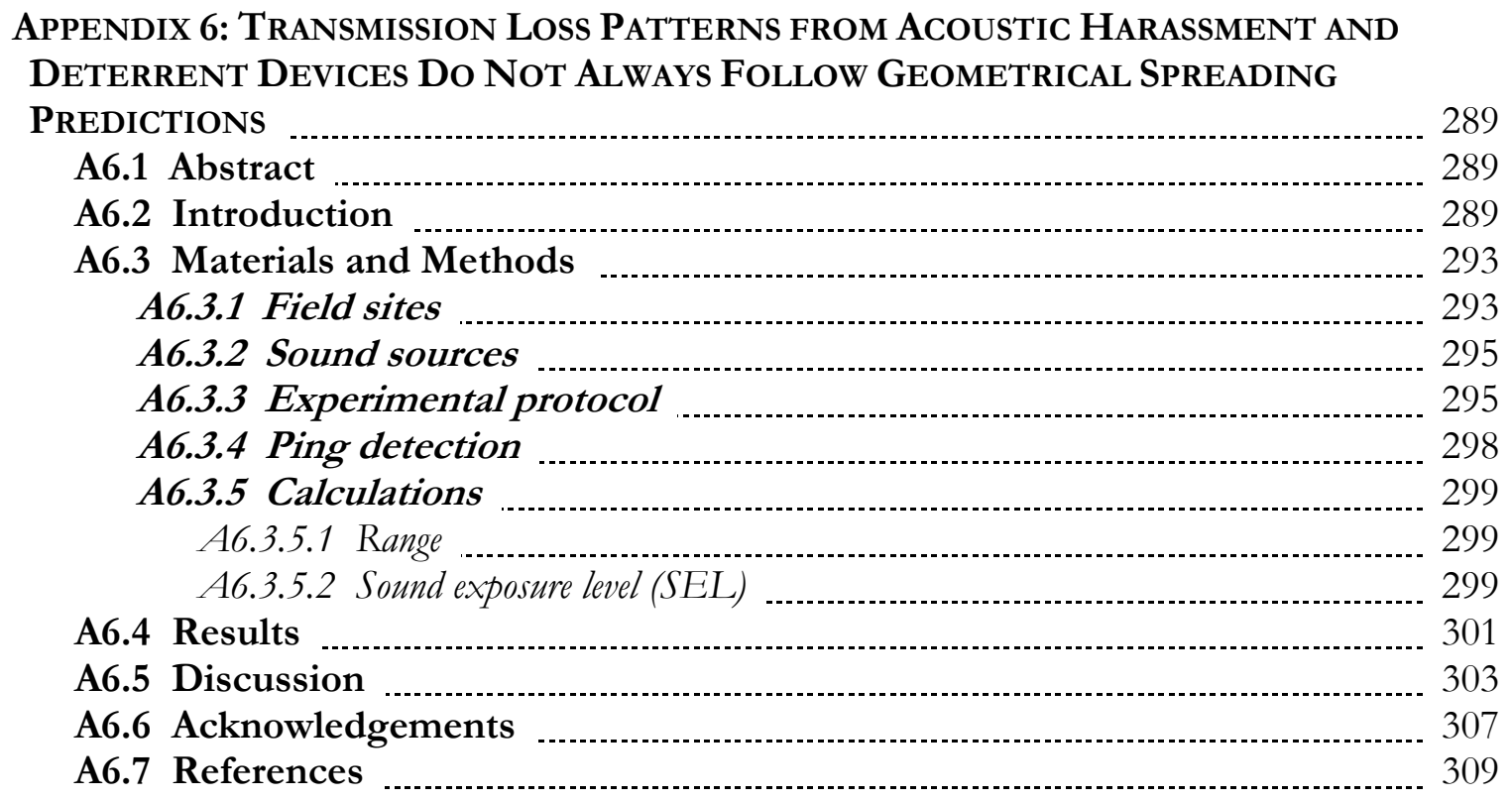




\section{LIST OF FIGURES}

Figure 1.1. Map of field site. Top: Scandinavia with box corresponding to the zoomed in plot on the bottom. Most of the tagouts were conducted in Tysfjord though a small number were completed in Vestfjord.

Figure 1.2. DTAG on killer whale from the 2006 field season. Photograph courtesy of Cathy Harlow.

Figure 2.1. Left: cumulative plot of depth for all periods. Percentage of total time spent in different depth bins during tail slapping (center, 5.4h) and not tail slapping (right, 26.7h) periods. Both panels are bounded by the same axis limits.

Figure 2.2. Top: Histograms of maximum depth for each dive exceeding $10 \mathrm{~m}$ for tail slapping periods (left) and not tail slapping periods (right). Bottom: Histograms of interdive intervals between all dives exceeding $10 \mathrm{~m}$ for tail slapping periods (left) and not tail slapping periods (right). Each horizontal pair of panels is bounded by the same axis limits.

Figure 2.3. Standard deviation of 5 movement measures (see text for details) for the 9 animals exhibiting both tail slapping (TS, black bars) and not tail slapping (NTS, white bars) periods. The panels in the upper and middle left contain circular datasets, requiring the calculation of the circular standard deviation instead of the conventional standard deviation used for the remaining panels. The first four characters of the whale identification labels have been dropped for legibility. The lengths of the time periods of TS and NTS periods are available in Table 2.1.

Figure 2.4. Pitch values associated with the 2 s flanking 10 randomly selected focal (left) and non-focal (right) tail slaps across all whales. Note the consistent directional change in pitch associated with the focal tail slaps compared to the flatter, more level pitch data associated with the non-focal tail slaps.

Figure 2.5. Histograms of pitch, roll and heading measurements (in degrees) taken during focal tail slap events (left, $N=89$ ) and non-focal tail slap events (right, $N=981$ ). Axis bounds are identical for each pair of focal and non-focal measurements. Focal tail slaps were centered on the zero crossing of the pitch measurement (see text for justification).

Figure 2.6. Top two trios of panels: Pitch (left), depth (center) and Az variation (right) profiles centered on focal tail slaps (gray dotted line) produced by male oo05_322b (top triplet) and female oo06_327s (middle triplet). Each line color corresponds to a unique focal tail slap and is consistent across each horizontal triptych of plots. Individual oo05_322b initiated many of his tail slaps by surfacing and diving to $10-20 \mathrm{~m}$ whereas oo06_327s remained at a more constant depth for nearly all of her tail slaps. Fluking intensity increased during the approach or lunge phase $2-3$ s preceding the tail slap (dotted line bracket) and then once the tail slap was executed (solid line bracket). Bottom panel: Four pairs of depth profiles surrounding tail slaps produced by group members oo05_322a (dotted line) and oo05_322b (solid line) within 60s of one another (turquoise: $3 \mathrm{~s}$, green: $12 \mathrm{~s}$, red: $39 \mathrm{~s}$, blue: $54 \mathrm{~s}$ ).

Figure 2.7. Box and whisker plots depicting the depths of focal tail slaps (top) and the change in depth from 5 s preceding the tail slap to the moment of the tail slap (bottom; 
positive values: descents; negative values: ascents) for all 9 whales. The horizontal lines of each box correspond to the lower quartile, median and upper quartile values of each dataset. Whiskers show the extent of the data and outliers are indicated $(+)$. The sample size for each of these bars is given in the penultimate column of Table 2.1.

Figure 2.8. Simultaneous dive profiles of two carousel feeding whales oo05_322a and oo05_322b, zoomed into tail slapping activity. Colored circles indicate focal tail slaps (see legend).

Figure 2.9. Top panel: Histogram of linearity index (LI) gathered once every second from all whales. Bottom panel: Histogram of LI values at moments of focal tail slaps $(N=$ 82). A normal distribution was fit to the bottom histogram and is plotted in both panels as the dark line $(\mu=0.59 ; \sigma=0.09)$. The LI threshold of 0.76 is plotted in red in the top panel and was computed to contain $95 \%$ of the data in the lower panel. This threshold split the data into low $(<0.76)$ and high $(>0.76)$ linearity.

Figure 2.10. Partial pseudo-track (non-geo-referenced inertial path) of male oo05_320b (1.3h segment). Horizontal movement is plotted as relative distance and the beginning and ending of this segment are shown ( $\square$ and $\odot$, respectively). In the left panel, depth is colorized, the filled gray circles represent tail slaps produced by a non-tagged individual, and the red circles indicate tail slaps produced by the tagged, focal animal. In the right panel, the red sections of the track correspond to periods of low linearity (thresholded using the focal tail slap data, see text and Figure 2.9). This path reveals movement characterized by high linearity and directional swimming leading into movement characterized by low linearity.

Figure 2.11. Movement data summary plots for male oo05_320b. The linearity index (LI) is plotted as a thin continuous black line in the two uppermost panels and ranges from 0 (no displacement) to 1 (straight path). The LI plots two rough states: a low linearity (high circuitous) state when the animals were tail slapping and a high linearity (low circuitous) state when few tail slaps were observed (see Figure 2.9). Dive duration and maximum dive depth are indicated with the magenta squares and red circles in the left and right panels, respectively. The small gray squares and open black circles on the LI curve indicate non-focal and focal tail slaps, respectively. The two tail slapping (TS) periods are indicated by the black horizontal bars at the top of the plot. The low LI episodes are shown by the blue horizontal bars. The two small triangles at the bottom of the plots mark the time interval plotted in Figure 2.10. Beginning in the second row and reading left to right, the remaining panels plot the change in pitch (degrees), depth $(\mathrm{m})$, change in roll (degrees), vertical velocity $(\mathrm{m} / \mathrm{s})$, residual heading (degrees) and variation in the $z$-axis of the accelerometer (a proxy for fluking energy, relative units). See text for computation details. The change in pitch, change in roll, residual heading and Az variation all increased during TS periods. Time is reported locally and runs identically along the $\mathrm{x}$-axis of each panel.

Figure 2.12. Partial pseudo-track of female oo05_322a (1.6h segment). Key as in Figure 2.10. The right panel shows that the high circuitousness sections (red) accompanied by tail slapping were interspersed with a high linearity section (gray) that contained vertical excursions and broad horizontal looping but lacked frequent tail slapping. ............... 74

Figure 2.13. Movement data summary plots for female oo05_322a. See Figure 2.11 for key. The TS period was flanked by two NTS periods. The two small triangles at the 
Figure 3.1. Pairs of dive profiles from simultaneously tagged Norwegian orcas belonging to the same social group. Top: Two animals traveling at opposite ends of a coherent group, 17 November 2005. Bottom: Two killer whales carousel feeding, 18 November 2005.

Figure 3.2. Half-weight index (HWI) as a function of the time interval considered to be a synchronous surfacing for each pairing of animals in Figure 3.1.

Figure 4.1. Depth records of whales with calling activity overlaid. During NTS episodes, the purple bars indicate the non-calling periods that precede and pair with the green bars that overlie the bouts of calling activity. Sections without a horizontal bar belong to NTS periods that are not part of such a pairing. Orange bars span TS episodes. For records characterized entirely by NTS, calls are plotted in yellow. For records characterized by both NTS and TS periods, LFC calls are plotted in black and L/HFC calls are plotted in maroon. (It was only for these records that this distinction was relevant for the analysis.) This coloration scheme was not applied to oo06_314a or oo06_317s because their records contained only 1 or 0 L/HFC calls, respectively.

Figure 4.2. Mean depth (left) and standard deviation of the vertical velocity (right) plotted for bouts without calling activity versus successive bouts with calling activity during NTS periods for 7 whales. The 1:1 line is plotted in each figure. With the exception of a single mean depth value for oo05_322b, all measurements were smaller during a calling bout than in the non-vocal time period preceding it.

Figure 4.3. Horizontal tracks of two tagged killer whales with the linearity index (LI) plotted in color. Thin sections of the track correspond to an absence of calling behavior. Each call has been plotted by thickening the track. In both plots, the dotted square boxes indicate the portions of the track associated with tail slapping and concomitant vocal activity. In panel (a), this carousel feeding period is preceded by putative corralling (dotted circle) that contains high levels of calling. In panel (b), however, the preceding period of directional travel was quiet vocally.

Figure 4.4. These plots present the percentage contribution of each call type to the calls produced during NTS (black bars) and TS (white bars) periods.

Figure 4.5. Call type sequences for oo05_316a (top) and oo05_322b (bottom). The legends to the right provide the call type labels and the letters above the square plots indicate the NTS $(\mathrm{N})$ and TS (T) documents. Notice that sometimes call type onset and offset occur at the document boundaries (e.g., N8, bottom plot) whereas other times they do not (e.g., N10.3, top plot).

Figure 5.1. Examples of call types that motivated this study. The three spectrogram panels on the left contain the same primary call base labeled N72. N72.2 contains a short additional utterance at the end of the call and N72.3 contains two such additional utterances. The four spectrogram panels on the right can be assembled from top to bottom by sequentially adding both low and high frequency components to the N16.1 base. 
Figure 5.2. Abrupt, non-continuous spectral shifts (left: N9.2) or intervals of silence (right: N72.3) in the contour were considered boundaries defining segment edges (marked here with black dotted lines).

Figure 5.3. Call types whose LFCs were segmented according to the algorithm described in the text. Segmentation divided the LFCs of these calls into two (N16.1, N32) or three (N91) segments.

Figure 5.4. Contour trace of N32 call (see Figure 5.3 for sample spectrogram) in black (without the noisy onset) with $4^{\text {th }}$-order Legendre polynomial fit of entire trace superimposed in red. The segmentation algorithm first located the time point of maximum deviation between the actual contour and fitted polynomial (blue dotted line). Splitting the contour into two at this location and then scanning forwards and backwards, the time point that minimized the deviation in fit between the two new segments (heavy black lines, 0.05 s to either side of the split) and their individual polynomial fits (magenta lines) was used as the location of the actual segmentation split of the contour (green dotted line).

Figure 5.5. RMS error difference between contour traces and polynomial fits for the three calls on which the segmentation algorithm was run (top panels) and for all other low and high frequency components (bottom panels). Each of the top distributions was significantly different from each of the bottom distributions (Mann-Whitney $\mathrm{U}$ test, $P<$ $0.001)$.

Figure 5.6. Schematic illustration of polynomial and labeling treatment of contours for each of the three experiments described in the text. The top row depicts the original traces for the low frequency components of 3 different call types: N12.1, a continuous and descending vocalization; N16.1, a continuous call subjected to the segmentation algorithm due to its rapid internal frequency change marked by the thin dotted line; N72.2, a call containing two segments separated by a brief period of silence. The second row plots the $4^{\text {th }}$-order Legendre polynomial fits used in the first whole contour experiment (WCE) in orange. Each contour was considered continuous and silent intervals were interpolated over (indicated by the thick dotted line connecting the two N72.2 segments). The contours were labeled by adding zeros until two places to the right of the decimal point were filled. The unshared segment experiment (USE) is shown in the third row. Here, the segments of each call were honored with a polynomial representation (N16.1 and N72.2 were divided into two segments using the segmentation algorithm and silent interval, respectively) but were labeled distinctly. The hundredths place in the label was used to count each successive segment for a particular call type. The final row demonstrates treatment according to the shared segment experiment (SSE). The segmentation decisions and polynomial fits were the same as in the USE but the labeling allowed call types to share syllables. For example, the second segments of both N16.1 and N72.2 were collapsed into syllable category 4. See the text for a description of the syllable collapse. Segments that appeared only in a single call retained their USE label (e.g., 12.11).

Figure 5.7. Top: Number of contours of each call type used in the 3 experiments described in the text. Bottom: Cumulative plot of the number of call types according to the number of calls associated with each type.

Figure 5.8. Histograms of error rates for each experiment after 100 runs. Call contours 
were categorized to type based on the entire call type (upper left), unshared segments (bottom row) or a combination of shared and call-specific segments (upper right). The unshared segments were scored strictly (i.e., each segment within a call type had to match to itself for the call to succeed, lower left) and more leniently (i.e., a call could also succeed if each of its composite segments matched to segments belonging to the same phonemic classes, lower right).

Figure 5.9. Rarefaction curves plotting the number of call types (top left) and shared syllables (top right) as a function of the number of whales considered. Bottom: Normalized number of shared syllables plotted against normalized number of call types and viewed for all data (left) and zoomed region (right, magnified to box depicted on left).

Figure 5.10. Sampling of matches of alternative calls (dark black traces) with Norwegian stereotyped call syllable matches (gray traces). The alternative calls included Norwegian variable (first column), Pacific Northwest resident (second column) and transient call segments (third column) that performed at threshold percentages (see Table 5.3) of 90\%, $25 \%$ and $25 \%$, respectively. The title of each sub-panel lists the number of the syllable match and whether the match was a LFC or HFC. The LFC or HFC label also refers to the component of the Pacific Northwest calls and the titles in these sub-panels conclude with the call type of the resident or transient call. The number following the hyphen in the transient call label refers to the segment number.

Figure 5.11. a: Syllables found in at least two call types. Note different axis scales. b: Color code for syllable traces of different call type origins (legend locations here match trace plot locations). The digits before the decimal point and the first digit after the decimal point refer to call type (e.g., N8, N76.2, etc.). The second digit after the decimal point corresponds to the segment number in the call. (See Figure 6 for further elaboration.) Certain numbers are repeated in this figure but this ambiguity is resolved when differentiating between the LFC and HFC indicated in the titles of the sub-panels of the figure.

Figure A4.1. Narwhal shown with Crittercam (contained within the dashed ellipse) and DTAG (contained within the dashed rectangle) attached immediately before release. Photograph courtesy of Rune Dietz.

Figure A4.2. Sample spectrograms (larger, top plots) and waveforms (smaller, bottom plots) of a combined tonal/pulsed signal produced by individual (a) mm224 and (b) $\mathrm{mm} 226$ with a FFT size and frame length of 512 points, $50 \%$ window overlap, and a maximum frequency displayed of $48 \mathrm{kHz}$. The low frequency energy associated with most of the pulses is likely due to the resonance of the air sacs involved in sound production or transmission. The solid arrows in both spectrograms indicate the synchronous FM tonal component produced by the tagged animal.

Figure A4.3. Spectrogram composite of all four whistles of mm224 (a-d) and 14 of the 17 whistles of $\mathrm{mm} 226$ ( $\mathrm{f}-\mathrm{s}$ ) with a FFT size and frame length of 512 points, $50 \%$ window overlap, and a maximum frequency displayed of $20 \mathrm{kHz}$. The remaining 3 whistles of $\mathrm{mm} 226$ resembled those displayed here but were excluded for graphical convenience. The waveforms displayed in subplots e and $t$ are of the same whistles used to generate subplots $\mathrm{d}$ and s, respectively. 
Figure A4.4. Digitized traces of the fundamental frequency contours of the whistles displayed in Fig. 3. Each trace is shown with 100 equally spaced points that have been normalized on a horizontal time axis from 0 to 1 . Again, panels a-d correspond to the whistles produced by $\mathrm{mm} 224$ and $\mathrm{f}-\mathrm{s}$ to those by $\mathrm{mm} 226$. Note the difference in the frequency ranges for the two individuals.

Figure A4.5. Illustration of temporal and spectral features extracted from a traced whistle produced by mm224 (subplot $\mathrm{d}$ in Figs. 3 and 4). The initial and ending frequencies are indicated by filled circles $(\bullet)$ while the other spectral components are marked with horizontal dashed lines. Note that the mean frequency is closer to the minimum frequency because $\sim 60 \%$ of the whistle's frequency content lies below $3700 \mathrm{~Hz}$. ...... 255

Figure A4.6. Illustration of points used for cross-correlation comparison of whistles (see text for the equation). In (a), contour A (darker, from mm226: subplot $\mathrm{i}$ in Figs. 3 and 4) and B (lighter, from mm224: subplot $d$ in Figs. 3 and 4) are depicted normalized in time with their original frequency content. In (b), contour B has been shifted along the frequency axis to minimize the frequency difference between the two contours. All 100 points along the contours were used to compute Eq. (1)..

Figure A4.7. Approximate depths where combined tonal/pulsed signals (triangles, $\triangle$ ) and whistles (circles, O) were produced adjacent to a frequency histogram of depth bins (bars) for mm224 (a) and mm226 (b). The frequency plotted on the abscissa is expressed as a fraction of the total amount of time spent at all depths. The maximum depths achieved for mm224 and mm226 during the DTAG deployments were roughly 125 and $210 \mathrm{~m}$, respectively. 256

Figure A4.8. Visual representations of temporal features of combined tonal/pulsed signals. Normalized pulse number (top two panels) and pulse repetition rate (pulses per second, bottom two panels) as a function of normalized duration. Note the clear differences in general morphology of these plots between the two individuals.

Figure A5.1. The adjusted animal paths are displayed as the heavy, dark black lines. Observer-sighted locations (black + ) with \pm 0.1 reticule error ovals (gray) are indicated. All sightings ( 0 ) were placed within (or on) their associated error ellipses to minimize the manipulation required to adjust the remaining positions. (Some of the error ellipses are too small to be visible relative to the sighting circles.) The beginning of the whale track is indicated $(\bullet)$ and the first sighting position are marked $(\square)$.

Figure A5.2. The 3D track of each sperm whale (thin black line) is displayed with the surface track of the vessel (thick gray line). The starting points of the whale and vessel paths are indicated $(\bullet$ and $\circ$, respectively).

Figure A5.3. Scenarios illustrating the computation of the directional time-series. Scenario 1, Animal facing away from the vessel: The vector directed from the vessel to the animal (V) is parallel to the vector of the animal's heading (W). In other words, $\left|v_{\theta}-\omega_{\theta}\right|=0$ and $v_{\varphi}=\omega_{\varphi}$, which means $\cos ^{-1}(\mathbf{V} \cdot \mathbf{W})=0$. Scenario 2, Animal broadside to the vessel: Oriented broadside, the value of $\omega_{\varphi}$ is always zero. In addition, because $\left|v_{\theta}-\omega_{\theta}\right|=\pi / 2$, $\cos ^{-1}(\mathbf{V} \cdot \mathbf{W})=\pi / 2$. Scenario 3, Animal facing towards the vessel: $\mathbf{V}$ and $\mathbf{W}$ are antiparallel. Now, $\left|v_{\theta}-\omega_{\theta}\right|=\pi$ and $v_{\varphi}=-\omega_{\varphi}, \operatorname{soc}^{-1}(\mathbf{V} \cdot \mathbf{W})=\pi$.

Figure A5.4. Received sound exposure level data plotted as energy flux density (dB re $\left.1 \mu \mathrm{Pa}^{2} \mathrm{~s}\right)$ as a function of time for 2001-2003. 
Figure A5.5. Directional time-series data plots. The ordinate ranges from 0 (away from) to $\pi$ (towards) as illustrated in Figure A5.3. Black horizontal lines at the top of the plots correspond to the intervals during which the sonar source was active. The low frequency trend of the vessel's path and the high frequency jitter of the animal's movements are simultaneously visible in these plots.

Figure A5.6. Orientation of whale with respect to the source vessel plotted as $|\theta|$ vs. sonar $\mathrm{RL}(\bullet)$ and noise RL $(\bullet)$. The ordinate range is the same as in Figure A5.4. 281

Figure A5.7. (a) Proposed experimental design for controlled exposure experiments involving ramp up. Once the general heading trend of the study animal (thick, dark gray line) can be determined, the vessel can either close its distance with the whale by passing in front from broadside in quadrant I or move behind from broadside in quadrant III as the whale swims away from the path of the vessel (thick, dotted black lines). The source can either be ramped up $(\uparrow)$ or maintained at a constant level $\left(^{-}\right)$. Each exposure period therefore allows four possible combinations of vessel movement and source level trend. (b) A sequence was determined using a semi-randomized order; one sample realization is listed here. This design covers the full RL space and all bearing angles from the vessel to the animal (after folding from $\theta$ to $|\theta|$ ) and balances vessel approaches with retreats and increasing with constant levels. See text for further discussion.

Figure A6.1. Maps of study locations.

Figure A6.2. Waveforms (left), spectra (center) and spectrograms (right) for each of the sound sources. The SaveWave signal was an example taken from the larger repertoire of signals (see Table A6.1). Sweep duration, start and end frequencies and number of repetitions changed randomly from signal to signal.

Figure A6.3. Source energy level (at one meter distance) of the Airmar and Aquamark pingers recorded in various directions. The levels of the CF (constant frequency) and sweep ping are denoted uniquely ( + and $\circ$, respectively). The orientation scenarios 1-6 of the pingers and receivers are illustrated graphically beneath the plots. The pinger (black and white oval) was recorded from the direction indicated by the origin of the arrow. The first pinger was recorded from its north pole, the middle four from the equator at four different pinger orientations and the final image from the south pole.

Figure A6.4. Received sound exposure level as a function of range. Slopes obeying cylindrical and spherical spreading laws and absorption are shown by the dotted and solid lines, respectively.

Figure A6.5. Received sound exposure level from a Lofitech AHD source as a function of range for a recording using a hydrophone that continuously approached a stationary pinger. Imagining an animal moving along a trackline similar to the one here, a steadily reliable decrease with increasing range would not occur since the levels fluctuate dramatically. See text for further elaboration. 


\section{LIST OF TABLES}

Table 1.1. Tagged killer whale subjects and durations analyzed in this dissertation.

Table 2.1. Tagged animals, sex and age class if known, maximum depth, tagout duration, the relative amounts of time spent tail slapping and not tail slapping, and the number of tail slaps produced by tagged (focal) and non-tagged (non-focal) individuals. The presence of a subsequent controlled sonar exposure to the data period analyzed here is indicated by an asterisk in the tagout duration column. Animal id corresponds to the

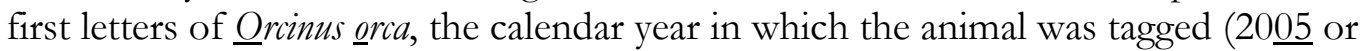
2006), the julian day of the tagout (e.g., $\underline{316}$ ) and the order of tagging (first from a platform: $\underline{a}$ or $\underline{s}$; second from a platform: $\underline{b})$. The sums of the columns were calculated from the actual data and not the rounded data displayed here.

Table 2.2. Behavioral sequence narrations for all animals based on track, LI and diving data. The LI threshold of 0.76 divided the records into low LI (tight circling generally accompanied by focal and non-focal tail slaps) and high LI (directional swimming and broad looping behavior) periods.

Table 2.3. Time intervals for low and high LI periods of at least 2 minutes in duration, excluding the first and last 4 minutes because of the smoothing window of a filter (see text).

Table 3.1. Tagging and analysis details. Tagout durations proceed in the same order as the listing of animal identification labels. The last two rows marked by asterisks contain inter-surfacing time interval threshold between individuals and the depth threshold used to determine whether animals surfaced together.

Table 4.1. Counts of call types in different recordings. Yellow cells indicate call types that were documented in 3 or fewer recordings.

Table 4.2. Call type component counts, durations, mean frequencies.

Table 4.3. Results of the rotation test to assess pairing of L/HFC or LFC calls during TS versus NTS periods. The first and second columns provide the identification of the whale and the total number of calls used in the test. The next two columns list the percentage of L/HFC call production during TS and NTS periods, respectively. The odds ratio and the significance of the rotation test are offered in the last two columns.

Table 4.4. Results of Markov chain test to compare call type frequency distributions during NTS versus TS periods. A significant $P$-value indicates that, once the serial dependence was considered by modeling the sequence using a Markov chain, the counts of call types differed between NTS and TS episodes. Call types are italicized if they were lowered during NTS or elevated during TS periods and are in bold if there were elevated during NTS or lowered during TS periods (the predicted outcomes). L/HFC calls are underlined and calls that do not fit into either LFC or L/HFC are listed parenthetically. Call type N15 was characterized by a short, fairly flat contour but spanned a wide spectral range and N84 contained a HFC only.

Table 4.5. Results of MANOVA test for the 12 whales and 3 sets of movement measures: 
the running standard deviation $(\sigma)$, running mean $(\mu)$ and difference in means $(\mathrm{d} \mu)$. Significant $P$-values are marked in bold.

Table 5.1. Tabulation of call and type counts from each recording used for visual sorting and the subsequent classification experiments. The number of spectrograms used to do the sorting is listed in the second column. The next set of 4 columns contains the call type counts determined by each of the observers. Agreement between me and at least two of the observers on a category defined it as a call type and the number of types determined from each recording is listed in the next column. The numbers of stereotyped and variable calls actually used in the syntax analysis are included in the last two columns of the table. Some calls were not used because they were too faint. The last row sums these counts over all the recordings to yield the total amount of data analyzed.

Table 5.2. Counts of call types and contour traces affected by segmentation (see sections 5.3.3 and 5.3.4 in Materials \& Methods).

Table 5.3. Performance of alternative calls when evaluated against self-sorted Norwegian stereotyped call data. The first column in the table lists the self-sorted threshold that the alternative call needed to exceed to qualify as a match (see Section 5.3.7). For each alternative category heading (i.e., Norwegian variable calls and Pacific Northwest resident and transient stereotyped calls), the first and second columns contain the percent and count of calls, respectively, that matched one of the Norwegian stereotyped syllables at the performance threshold.

Table 5.4. Counts of all call types (first row), call types containing both a LFC and HFC (second row), a LFC alone (third row) and a HFC alone (fourth row) that were comprised of call-specific syllables only, a mixture of shared and call-specific syllables, and shared syllables only.

Table A4.1. Summary statistics of the acoustic features of combined tonal/pulsed signals.

Table A4.2. Summary statistics of the acoustic features of whistles.

Table A4.3. Cross-correlation comparison of whistles between the same and different individuals. These data were computed in arbitrary units with higher values indicating a greater difference between the contours being compared. The intra-individual comparisons are italicized.

Table A5.1. Descriptive measurements made for each experiment. The italicized sonar ping frequency in 2001 was near the Nyquist frequency of $16 \mathrm{kHz}$. Because it was not possible to measure the RLs of these pings reliably, they were excluded from the analysis.

Table A5.2. Bandwidth, duration and level (energy flux density or sound exposure level (SEL), sound pressure level RMS (SPL $\mathrm{RMS}$ ), and sound pressure level peak-peak $\left(\mathrm{SPL}_{\mathrm{p}-\mathrm{p}}\right)$ ) of sonar frequencies used in all years. The frequency of each ping increased from $f_{0}-1 / 2$ $b$ to $f_{0}+1 / 2 b$ where $f_{0}$ corresponds to the mean frequency given in Table A5.1 and $b$ to the bandwidth listed here. The ranges listed here defined the middle $90 \%$ of the data values. 
Table A6.1. Specifications of sound sources described in this study.

Table A6.2. Equipment used at each field site with corresponding amplification and filtering details. Abbreviations: B\&K = Brüel and Kjær (Danish hydrophone company), DAT $=$ Digital Audio Tape Recorder, HP = high pass filter; LP = low pass filter, $\mathrm{DAB}=$ Data Acquisition Board. SSs: Saltö, Sweden, spring, KSf: Kosterhamn, Sweden, fall, SSf: Saltö, Sweden, fall, JDf: Jammerland, Denmark, fall. All hydrophones were calibrated in the laboratory before

fieldwork.

Table A6.3. Recording duration and number of signals analyzed for each sound source and field site. See Table A6.1 for abbreviations. 


\section{Chapter 1. General INTRODUCTION}

\subsection{Social learning and animal culture}

The social intelligence hypothesis proposes that social environments have placed a premium on learning and the cognitive and behavioral adaptability associated therein, which may have led to the development of intelligence more generally. Whiten \& Byrne (1988) asserted that observational learning may have become more prevalent as social intelligence became increasingly developed. Individuals belonging to social species must interact with other dynamic agents to form relationships with predictable patterns of affiliative, manipulative or aggressive behaviors (Byrne \& Whiten, 1988; Whiten \& Byrne, 1997). Social transmission of behavior can occur vertically between parents and offspring, horizontally between members of the same generation and obliquely between individuals of different generations (Cavalli-Sforza \& Feldman, 1981). Originally documented with regard to primates, evidence now indicates that numerous species of fishes exhibit behaviors consistent with social intelligence (see Bshary et al., 2002 for a review) and it is reasonable to suppose that other taxa are similarly capable. Social learning may be influenced by both preexisting social dynamics (Coussi-Korbel \& Fragaszy, 1995) and the cognitive capacity determining the information content that can be transmitted successfully (Byrne et al., 2004). Because learners must approach others closely to observe and model their behaviors, relationships between kin or bonded animals often provide the gregariousness and tolerance that afford the necessary proximity (Coussi-Korbel \& Fragaszy, 1995; van Schaik et al., 1999). A theoretical continuum of instruction ranges from drawing attention to and indirectly encouraging interaction with certain features of the environment to the active instruction and shaping of behavior more directly (Caro \& Hauser, 1992; Boesch \& 
Tomasello, 1998).

Social learning is fundamental to cultural transmission, which refers to the dissemination of behaviors via imprinting, conditioning, imitation, facilitation, teaching and local or stimulus enhancement (Zentall, 2006). Culture and cultural transmission are terms first used by social anthropologists for human beings exclusively. When, in the 1970s, biological anthropologists first introduced evidence to support the idea of animal culture (e.g., Kummer, 1971), it seemed paradoxical and even heretical. Culture, they argued, was evident when communities of animals were distinguishable based on a characteristic set of behaviors where genetic (i.e., heritable) and ecological or environmental explanations were unsupported (see de Waal, 1999; Boesch, 2003). The notion of animal culture has come to help frame arguments both about how animals learn and transmit information, underscoring the ways in which organisms and the cultures they adopt shape one another, and about conservation (Laiolo \& Jovani, 2006). As a process, cultural transmission of a trait commences when a new behavior called an innovation is introduced and subsequently diffuses through all or part of the population as increasing numbers of individuals learn the behavior from one another (Laland \& Hoppitt, 2003). I next turn to research that has addressed the social and cultural transmission of behavior in the wild and in captivity. Then, I address killer whales (Orcinus orca) as an ideal species for exploring foraging traditions and population-specific vocal dialects, which both show evidence for being acquired via social learning.

\subsubsection{Foraging behavior}

The earliest work on animal culture and social transmission focused primarily on the 
tool use and foraging specializations of highly social non-human primates. One of the earliest examples documented the spreading of sweet potato washing behavior developed originally by an 18-month-old Japanese macaque (Macaca fuscata) to other members of her social group (Imanishi, 1957). The slow rate of spreading of this behavior, however, was thought by some to be inconsistent with cultural diffusion as a transmission mechanism (reviewed in Whiten, 2000). Recently, Whiten et al. (1999) collated 39 separate behaviors that characterized certain chimpanzee (Pan troglodytes) communities but not others across seven field sites (Whiten et al., 1999). Many of these behaviors related to population-specific foraging activities. The authors asserted that these behaviors were culturally transmitted but recent work has suggested that the discrediting of ecological explanations may have been premature. Humle \& Matsuzawa (2002) demonstrated that the differences in ant-dipping foraging techniques by different chimpanzee populations - the example emphasized by Whiten et al. (1999) - could be explained by the aggressiveness of the ant species rather than by cultural inheritance. The same criticism of failing to take environmental or physical features into account may be levied against the assertion of culture in orangutans (Pongo pygmaeus) by van Schaik et al. (2003) who used a similar compilation and comparison approach to the Whiten et al. (1999) study.

Stronger evidence for foraging culture has emerged from research conducted on chimpanzees in captivity. The general experimental paradigm has involved seeding a different feeding-related behavioral sequence that achieves the same objective in two populations and observing whether and how these behaviors propagate within the communities. This approach is modeled after the observation that wild chimpanzee populations often display unique sequences of behavior to achieve an identical goal (Whiten 
\& Boesch, 2001; Whiten, 2005). Although the collective results of these experiments have been ambiguous (e.g., Horner et al., 2006; Hopper et al., 2007), a more general point is that species that lend themselves to experimental manipulation with appropriate genetic and environmental controls can provide more compelling evidence for animal culture (Laland \& Hoppitt, 2003). Within the foraging domain, for example, feeding preferences among captive Norwegian rats were transmitted culturally (Rattus norvegicus, Galef \& Allen, 1995). Other examples include the social transmission of migration routes among wild French grunts (Haemulon flavolineatum, Helfman \& Schultz, 1984), mating site preferences among wild bluehead wrasse (Thalassoma bifasciatum, Warner, 1988) and affiliative and competitive behaviors among captive cowbirds (Molothrus ater, White et al., 2007). These species tend to be more tractable for experimental manipulation, observation and interpolation whereas the lack of experimental controls has caused the ape work to remain largely inconclusive.

\subsubsection{Vocal behavior}

Another suite of evidence for social learning and animal culture concerns vocal behavior. Vocal production learning occurs when an animal modifies its acoustic signals due to experience with other individuals to render these signals either more or less similar to the model that it hears (Janik \& Slater, 1997; Janik \& Slater, 2000). It is a social process that can lead to the transmission of an acoustic repertoire between signalers and receivers. Songbirds are an excellent illustration of this phenomenon as they generally learn species- and often population-specific song through an iterated procedure of listening and vocalizing (Slater, 1986). Similarly, in the case of male humpback whales (Megaptera novaeangliae) whose song converges and evolves simultaneously among all individuals within a population (Payne et al., 
1984), intra- and intersexual selection on vocal behavior may be relevant if females compare males based on song quality and fidelity as research suggests (Tyack, 1999).

Tyack \& Sayigh (1997: 230) reported, "Vocal learning may provide a mechanism whereby the vocal repertoire can develop to match the particular social system experienced by an individual." Compared with vocal learning, vocal culture underscores the stable social bonds that allow information to be transmitted between conspecifics and across generations. A focus on vocal culture emphasizes the ways in which the social relationships allow learning to occur. Vocal culture can cause acoustic badges of membership to emerge among certain animal groupings. For example, the different contact calls of newly formed groups of captive male budgerigars (Melopsittacus undulatus) converged on the same dominant call, which then experienced synchronous changes (Farabaugh et al., 1994). Called conformity bias, this phenomenon plays a role more generally in integrating individuals into social groups (see Boesch et al., 1994; Sapolsky \& Share, 2004; Whiten et al., 2005 for additional examples). In addition, though other explanations including genetics have not yet been excluded, Rendell \& Whitehead (2003) proposed that coda variation among sperm whales (Physeter macrocephalus) may be best explained by cultural transmission that follows association patterns among social units spread over an entire ocean basin.

\subsection{Killer whales as candidates for using culture}

To summarize the earlier sections, primates offer suggestive but inconclusive evidence for animal culture. Data from more tractable species including fishes and rats have been of better quality but of somewhat limited scope. The cultural aspects of vocal learning exemplify the ways in which social bonds influence and allow the transmission of vocal 
behavior. An understanding of the consequences of cultural transmission for social behavior requires a broad taxonomic comparison of animals in the wild. It is important to ascertain whether, why and how these phenomena are deployed by a variety of species in their natural habitats. If genetic inheritance can be discredited, group-distinctive behaviors provide a promising starting point for investigating cultural traits since frequent opportunities for social behavioral interaction and transmission are available between group members.

A lively debate persists over the presence and nature of culture among cetaceans more generally (see the review by Rendell \& Whitehead, 2001 and the ensuing commentary). Killer whales in particular are excellent candidates for investigating the areas of animal culture and social learning. They are characterized by stable social groups, populationspecific foraging strategies and vocal repertoires that are likely to be transmitted through learning. Their feeding behaviors include hunting fishes, cephalopods, sea turtles, sea birds, mustelids, pinnipeds and cetaceans (e.g., Martinez \& Klinghammer, 1970; Christensen, 1978; Smith et al., 1981; Hoelzel, 1991; Jefferson et al., 1991; Baird et al., 1992; Similä \& Ugarte, 1993; Matkin \& Saulitis, 1994; Baird \& Dill, 1995; Baird \& Dill, 1996; Fertl et al., 1996; Similä et al., 1996; Ford et al., 1998; Baird \& Whitehead, 2000; Ford \& Ellis, 2006; Simon et al., 2006). The diversity of hunting strategies parallels the diversity of prey on which they feed. Though sufficient ecological differences in habitat and food sources exist between many of these populations to explain the variety of foraging strategies, the adaptability and flexibility of this species are evident.

Two sympatric populations in the Pacific Northwest have partitioned their niche according to a diet consisting exclusively of salmon or marine mammals (Ford et al., 1998; Saulitis et al., 2000). Another example of social transmission of foraging behavior concerns 
Guinet \& Bouvier's (1995) report of adult killer whales in the Crozet Archipelago teaching their calves how to beach themselves and capture pinniped prey. This observation has not been subjected to proper experimental scrutiny, however, and remains speculative. Longitudinal data collection across multiple calves and juveniles is required to demonstrate teaching in this population. The absence of both regional and global genetic variation, the former likely due to matrilineal group structure and the latter suggestive of an earlier bottleneck event (Hoelzel et al., 2002), suggests that the variability in foraging behaviors may have been more likely to arise from learning and/or ecological differences.

Compared to the fission-fusion societies of bottlenose dolphins described above, killer whale social groupings are remarkably stable, generating a set of long-lasting relationships between a set of animals that interact reliably with known conspecifics. This feature of killer whale social behavior, combined with an aptitude for learning, create conditions in which cultural transmission could occur. Among fish-eating resident orcas, the matriline is the fundamental social unit from which neither male nor female offspring emigrate even after achieving sexual maturity (Bigg et al., 1990). (Unless otherwise stated, the research discussed here comes from the large body of work conducted on the population and vocal dynamics governing the killer whale communities of the Pacific Northwest.) Matrilines that associate over half of the time (based on visual observations at the surface) are considered to belong to the same pod (Bigg et al., 1990) and matrilines and pods sharing elements of their acoustic repertoire are referred to as clans (Ford, 1991; Yurk et al., 2002). Finally, clans that associate and interact regularly are considered part of the same community even if their association measures less than half (Bigg et al., 1990). This stable, long-lasting and gregarious social context allows these animals to observe and learn from one another, a 
scenario supported by their vocal behavior.

The members of each matriline produce a set of specific call types, which serve as group-distinctive vocal signatures (Ford, 1991; Miller \& Bain, 2000). Resident pods possess unique vocal repertoires of stereotyped pulsed calls with primary energy between 1 and 6 $\mathrm{kHz}$ (Ford, 1989). These calls contain both a high-frequency component (HFC between 2 and $12 \mathrm{kHz}$, Hoelzel \& Osborne, 1986) beamed forward from the melon and a less directional low frequency component (LFC between 80 and $2400 \mathrm{~Hz}$, Ford, 1987). The relative energy of these two components may cue conspecifics into the orientation of the signaler (Miller, 2002). Discrete, stereotyped calls constitute the majority of vocalizations in most behavioral contexts (Ford, 1989). Hitherto, the individual call type has largely been regarded as an arbitrary and interchangeable unit without any specific behavioral significance. Certain call types may be more common in certain contexts (see Deecke et al., 2005; Van Opzeeland et al., 2005) but in general, it has been assumed that the full vocal repertoire is used by social groups to differentiate one another. If this is the sole function, however, it is not clear why so many call types are required. It is possible that the functions of these call types may depend on a behavioral, social or interactive context that we have not yet been able to discern adequately.

Although no definitive study has been conducted demonstrating vocal learning in killer whales, several separate observations support both this conclusion (Bowles et al., 1988; Foote et al., 2006; Nousek et al., 2006; Riesch et al., 2006) and that cultural transmission may be the mechanism underlying development and acquisition of the vocal repertoire. Ford (1991) suggested that when pods become too large to afford all members adequate access to resources, they splinter into two or more smaller pods and acoustic dialects begin to diverge 
slowly through cultural drift whereby changes arise from small copying errors in vocal imitation and transmission. Over time, it is believed that this gradual divergence has led to the development of unique repertoires between pods and changes in the relative numbers of call types within pods. A comparison of two stereotyped calls over 12-13 years revealed that different call types have undergone structural modifications at different rates, likely arising from cultural drift (Deecke et al., 2000).

Until recently, analysis of the social and vocal behavior of free-ranging killer whales was conducted at the group level because it was not possible to monitor individual animals continuously in time once they left the surface. Recent advances in recording technology including towed beamforming arrays (Miller \& Tyack, 1998) and digital archival tags (Johnson \& Tyack, 2003) are allowing more detailed investigations of individual behavior. For example, Ford (1989) described that calls of the same type tended to follow one another but it was unclear whether a single individual produced them in a series or multiple individuals were exchanging these calls. Using a towed array to isolate the calling behavior of a single individual within a group, Miller et al. (2004c) ascertained that this matched counter-calling behavior was due to vocal exchanges between individuals. In demonstrating fine-scale vocal interactions, this study suggested that the contextual and vocal learning of stereotyped calls may be reinforced by matched counter-calling, which could play a role in coordinating group travel or maintaining group cohesion.

\subsection{Introduction to dissertation research}

The observational support for social learning and cultural transmission in killer whales is certainly suggestive, though it has not been demonstrated explicitly. The diverse 
array of feeding strategies speaks to the behavioral versatility and environmental adaptability of the species, features that tend to be associated with learning. This dissertation was motivated by an interest in understanding how the behavior of individual killer whales contributed to these group foraging behaviors. Their stable family groupings provide a network of reliable and regular interactions through which behavior could propagate culturally, a point reinforced by the pod-specific vocal repertoires. My dissertation also sought to explore both the relationships between the vocal and non-vocal activity patterns of these animals and the possible components of their vocalizations that might be learned and subsequently concatenated to form the call types of the repertoire.

This study benefited from selecting as its study species the killer whales that inhabit the fjords of northern Norway (Figure 1.1) in the wintertime as they follow spring-spawning herring (Clupea harengus) stocks (Similä et al., 1996). My research took advantage of a few long-term studies of the Norwegian killer whales, including a catalog of almost 600 identified animals, records of pod composition and detailed descriptions of carousel feeding, a group foraging behavior involving corralling and incapacitating herring. In particular, groups of Norwegian (and Icelandic as documented by Simon et al., 2005) killer whales corral herring by circling the fish to trigger their coalescing, ultimately lunging towards and tail-slapping the prey ball to incapacitate the fish before feeding (Christensen, 1978; Similä \& Ugarte, 1993; Simon et al., 2005). The primary objectives of my dissertation were to examine the movement behaviors and association patterns of individual free-ranging Norwegian killer whales, whether their movement and vocal behavior related to one another during both foraging and non-foraging contexts, and whether call subunits were assembled syntactically into discrete calls and call sequences. In Chapter 2, I investigated how individual animals 


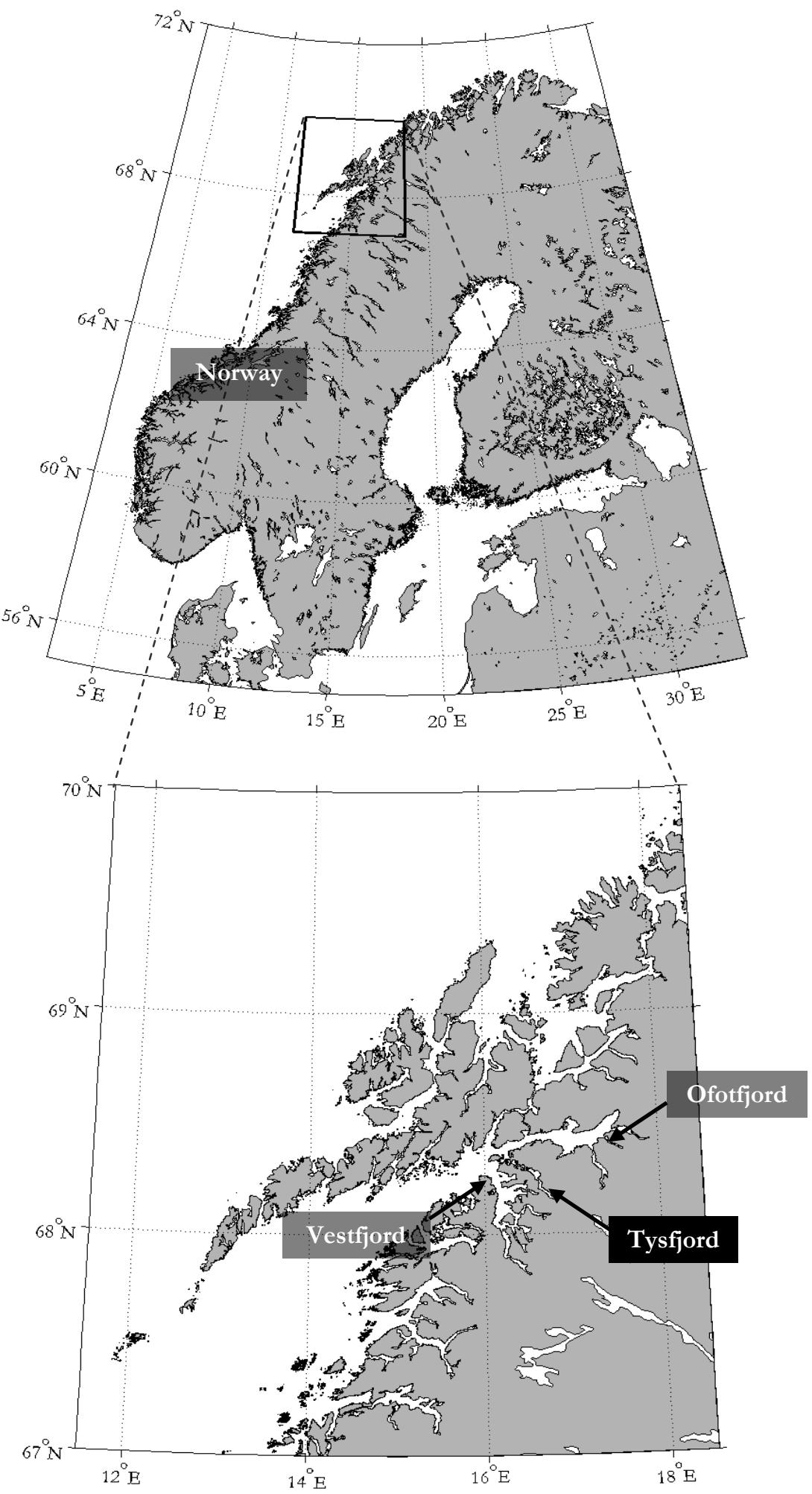

Figure 1.1. Map of field site. Top: Scandinavia with box corresponding to the zoomed in plot on the bottom. 
Most of the tagouts were conducted in Tysfjord though a small number were completed in Vestfjord. contributed within their group to execute their foraging sequences, building as detailed a spatial and temporal picture as possible of the carousel feeding behavior. Chapter 3 considered the different association patterns of simultaneously tagged killer whales during traveling versus carousel feeding periods.

The killer whales of northern Norway produce repertoires of 3-16 call types (Strager, 1993; 1995). Call subtypes corresponded to calls or call components whose duration or repetition rate was altered significantly or to calls in which at least one component was added or removed. Strager (1995) additionally described compound calls, which either contained two or more calls that were also produced individually as discrete calls or included a component that was produced within multiple discrete call types. These call components underwent significant duration and repetition rate modifications when combined in tandem with other calls.

The behavioral context associated with the acoustic activity of an animal is important when assessing the social, communicative, foraging or other functions of a vocal repertoire. Only one call type showed different rates of production between the two behavioral categories of seiner and carousel feeding (Van Opzeeland et al., 2005). Otherwise, call types have not been associated with particular behaviors. I examined the potential relationships between the movement and vocal behaviors of these animals in Chapter 4, which are valuable for understanding the function of vocal behavior and whether and why animals deploy different signals from their repertoire in different contexts. Finally, in Chapter 5, I used techniques adapted from human speech processing to test whether stereotyped Norwegian calls could be represented by a set of flexibly arranged and smaller phonemic 
segments, exploring the possibility of vocal syntax in these animals. I offered my conclusions in Chapter 6.

Appendices 1 - 3 include additional data plots that are referenced by the dissertation data chapters. Appendices $4-6$ contain either published or submitted first author manuscripts that pertain to different subjects than the primary narrative arc of culture in killer whales. Appendix 4 is a manuscript on the signature content of two vocalization classes, combined tonal/pulsed signals and whistles, produced by two adult male narwhals (Monodon monoceros) tagged off the shores of Admiralty Inlet on Baffin Island, Canada. It was published in the Journal of the Acoustical Society of America in September 2006. In Appendix 5, I explored whether sperm whales tagged in the Ligurian Sea displayed any consistent angular response relative to a source vessel producing mid-frequency sonar pings as a function of sound exposure level. This document was co-authored by Peter Tyack and Andrew Solow and we are revising it based on reviewer comments before resubmitting to Marine Mammal Science for publication. Appendix 6 reports on the high variability of sound exposure levels as a function of range for several kinds of acoustic harassment and deterrent devices used to discourage marine mammals from feeding on stock caught by fisheries. It explored the consequences of an animal attempting to minimize its exposure upon encountering a complicated 3D field of levels. Originating as a class project for a summer bioacoustics course that I attended in Tjarnö, Sweden in 2005, I have submitted this manuscript with 8 co-authors to Marine Mammal Science.

\subsubsection{Field procedure: Daily sailing routine and tagging procedure}

To collect the continuous movement and acoustic data required, digital archival tags 
(DTAGs, Johnson \& Tyack, 2003) were attached to individual free-ranging killer whales

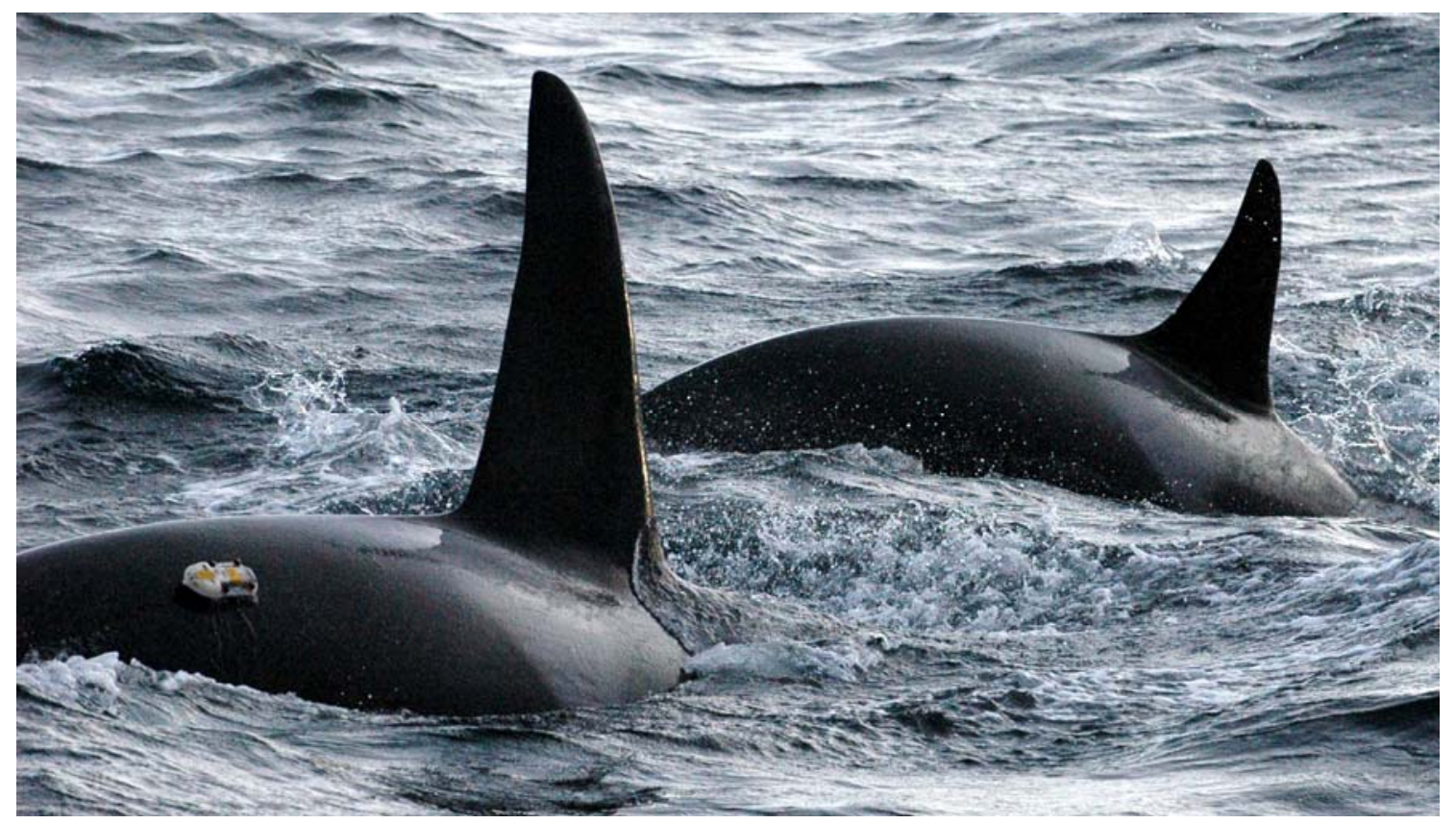

Figure 1.2. DTAG on killer whale from the 2006 field season. Photograph courtesy of Cathy Harlow.

(Figure 1.2). All field work was conducted during November 2005 and 2006. Tagged animals were visually tracked and their range, bearing and behavior were recorded for subsequent ground truthing with the tag data and for monitoring the behavioral context of both the tagged animal's group and other neighboring groups. Two vessels were used in both years: a $12 \mathrm{~m}$ sailboat Iolaire observation platform and a small RHIB from which we deployed and recovered the tags. In 2006, we benefited from an additional set of platforms: the research vessels Sverdrup and Nokken and two RHIBs. The Iolaire or Nokken followed the first tagged animal (defined as the focal) and its group, towing the hydrophone array at a relatively constant range of $\sim 100 \mathrm{~m}$ from the animals until the tag released, allowing reasonably close visual inspection without excessive maneuvering near the animals. Focal follows were necessary for both tag recovery purposes and behavioral tracking of the tagged 
animal's group. Once the tags released and were recovered, data were offloaded ashore and the tag was recharged and sterilized for subsequent use. The effort was considered complete once all of the tags were recovered and there was no longer sufficient daylight to attempt further tagging. Because it was difficult to follow animals in bad weather against the wind and the waves, tagging was not attempted when the weather forecast was poor and aborted if conditions grew too severe or dangerous while at sea.

Across both years, we achieved 15 tag carries ranging between 1.2 and 5.5 hours combined with visual tracking and frequent photo-identification including three pairs of simultaneous tag deployments, two of which were on animals belonging to the same social group. Endeavoring to tag more than one whale in a group was a high science priority because additional recorders allowed an examination of association and synchrony of group members during carousel feeding and other behaviors. This data collection scenario would help reveal whether individuals engage in same or different activities during a particular behavioral episode, providing preliminary observations of what might be considered coordination or role playing. Animals were tagged during both foraging and non-foraging contexts. Three tag carries in 2006 occurred during a behavioral response stuy of the effects of sonar. Only the pre-exposure periods from these recordings were considered here, reducing the dataset to 14 tag carries lasting between 0.6 and 4.7 hours (Table 1.1). In total, 32.2 hours of movement data and 33.3 hours of vocal data were analyzed. (A low battery contributed to sensor calibration difficulties for one of the deployments, causing the discrepancy in the durations of the movement and vocal data.) The hydrophone array data were not analyzed here. 


\begin{tabular}{c|c|c} 
animal id & duration (h) & notes \\
\hline \hline oo05_316a & 2.7 & $\begin{array}{c}\text { Synchronous tagout of two animals } \\
\text { in different social groups }\end{array}$ \\
\hline oo05_320a & 4.7 & $\begin{array}{c}\text { Synchronous tagout of two animals } \\
\text { in the same social group }\end{array}$ \\
\hline oo05_320b & 2.2 & $\begin{array}{c}\text { Synchronous tagout of two animals } \\
\text { in the same social group }\end{array}$ \\
\hline oo05_321a & 4.2 & \\
\hline oo05_321b & 1.7 & \\
\hline oo05_322a & 3.6 & \\
\hline oo05_322b & 3.1 & \\
\hline oo05_324a & 2.2 & Unable to calibrate all of the movement sensors \\
oo06_314a & 2.2 & because of low battery during deployment \\
\hline oo06_314s & 2.1 & \\
\hline oo06_317s & 0.6 & \\
\hline oo06_324s & 1.2 &
\end{tabular}

Table 1.1. Tagged killer whale subjects and durations analyzed in this dissertation.

\subsubsection{Focal follow procedure}

The overall goal of the focal follow was to provide information on group behavior as context for the movement and acoustic data streams. At regular intervals once every two minutes, data were recorded on the dynamics of the focal group. This information was useful as a cross reference to identify the time periods when the animals were foraging and paralleled the behavioral state information acquired in earlier studies. In particular, milling index, group formation, group size and the presence of gulls interacting with the water's surface were noted. Changes in the milling index, direction of travel and group composition were recorded opportunistically, generally at intervals lasting longer than two minutes. One of the three simultaneous tag deployments involved two animals from different social groups. In this instance, once the tag released from the first focal individual, behavioral observations ended with its group and began with the group containing the second focal 
animal. In the other two instances of simultaneous tagging, the two animals belonged to the same social group on which behavioral observations were recorded for the duration of the two tag deployments.

\subsection{Summary}

Killer whale populations around the world are characterized by distinctive foraging behaviors and vocal repertoires of call types that are likely socially learned through cultural transmission. In my dissertation, I use digital archival tag data to explore the movement and carousel feeding behaviors of free-ranging individual Norwegian killer whales and the detailed vocal behavior and syntax of their groups. I conclude by discussing how these

results impact future work, which should continue to probe the nexus of social behavior and learning. 


\section{Chapter 2. INdividual BeHAVIORS OF CAROUSEl FeEding NORWEGIAN KILLER WHALES}

\subsection{Abstract}

Among marine mammals, group foraging that facilitates consumption of evasive or large prey has generally remained unexplored from the perspective of the individual predators. Norwegian killer whales (Orcinus orca) herd herring from depth into balls at the surface, incapacitate them with tail slaps and feed on the stunned fish one by one in a behavior called carousel feeding. I explored carousel feeding at the level of the individual killer whale by analyzing data of digital archival tags that recorded each animal's depth, orientation and acoustic environment. Bouts of carousel feeding were defined based on acoustic evidence of tail slaps. Measures of changes in orientation and depth, fluking energy and variation in heading all showed distinct increases during carousel feeding compared to other time periods. Using a rapid change in pitch angle to determine which tail slaps were produced by the tagged animal, I found that most tail slaps occurred at shallow depths within $20 \mathrm{~m}$ of the surface. Two synchronously tagged animals revealed similar dive profiles when tail slapping within 60s of one another, suggesting that the location and geometry of the herring ball was influencing their tail slapping movements. A linearity index measurement was used to split the dataset into periods of high and low circuitousness; two behavior patterns preceding carousel feeding were evident. In the first, killer whales initiated tail slapping behavior after a period of directional swimming, suggesting an absence of corralling at depth. Similar to observations reported by earlier studies, the second sequence involved killer whales engaging in broad looping movements consistent with corralling before commencing their tail slapping activity. Together, these results indicate that carousel 
feeding is fluid and opportunistic. Individual animals maneuver within the group to collect and herd herring either already at the surface or originating at depth and they maintain the geometry of the fish ball as long as possible during feeding.

\subsection{Introduction}

The extent to which animals forage on their own or in groups depends on the distribution of food resources, the ease and relative payoffs of locating, grazing, or hunting food independently or collectively, and the nature and dynamics of the social interactions of the population. Multiple individuals of a species may aggregate at a plentiful food patch without interacting or they may engage with one another through competition or cooperation. Competition arises when animals exploit or monopolize a food source at the expense of others. Cooperative foraging, the focus of this chapter involves individuals that work together to locate, incapacitate, handle, and/or feed on prey. Kin selection rewards cooperation among related individuals by enhancing the inclusive fitness of the participants (e.g., lions (Panthera leo), Packer et al., 1990). Cooperation can also arise under reciprocal altruism, the serial exchange of beneficial behavior between related or non-related individuals (e.g., vampire bats (Desmodus rotundus), Wilkinson, 1984; dolphins, Connor \& Norris, 1982). In other cases of social foraging, the nutritional benefit:cost ratio of feeding together must exceed that associated with resource competition and independent hunting (Macdonald, 1983; Nudds, 1978; Clark, 1986; Packer \& Ruttan, 1988). Modeling suggests that cooperative foraging behaviors are likely to persist when hunting success, defined in terms of either the number or size of the prey being sought, improves in groups versus when alone, a pattern borne out by wild Taï chimpanzees (Pan troglodytes: Boesch, 1994), captive 
black-headed gulls (Larus ridibundus: Götmark et al., 1986) and mammal-eating transient killer whales (Orinus orca: Baird \& Dill, 1996).

Cooperative activity can enhance foraging efforts by 1) making the search phase for food more efficient, 2) allowing animals to capture prey that would be difficult to secure alone, and 3) introducing a division of labor in which individuals specialize repeatedly on different tasks within the group (though specialization can be inefficient if animals are unable to behave flexibly). I will consider each of these three points in turn. First, foraging in groups often arises to optimize the search, especially when food patches are evanescent and large but challenging to locate, rendering them difficult to monopolize via territoriality (Dittus, 1984; Elgar, 1986; Brown et al., 1991; Wilkinson, 1992; Cocroft, 2005). Second, the simultaneous (yet possibly dispersed) pursuit of prey allows predators to develop countermeasures against some defenses of their quarry (e.g., Creel \& Creel, 1995). Multiple pairs of spinner dolphins (Stenella longirostris) near Hawai'i, for example, overcame the evasive and dynamic schooling tendencies of their micronekton prey by echolocating to track the horizontal and vertical excursions of these fishes, shrimps and squid and herding them into a more accommodating three dimensional (3D) geometry for capture (Benoit-Bird \& Au, 2003). One of the critical features of this study involved the ability to map the distribution of both dolphins and prey simultaneously. The dolphins clearly demonstrated well coordinated behavior. Although the patterns of prey response and the structured formation of these dolphin pairs were taken as evidence for cooperative foraging, the methods were unable to track individuals through time or detect the fine-scale movements of either predator or prey. To demonstrate cooperation, the following additional observations would have been required: each animal involved was participating in the effort either consistently or 
via staggered turn taking, all individuals received an opportunity to eat and the dolphin group responded globally to alteration in 3D prey structure.

Third, under certain circumstances, cooperative foraging allows role specialization in which individual animals each repeatedly conduct a particular task within the collective effort. Stander (1992) distinguished between Namibian lioness "wings" that initiated hunts by stalking and circling their prey and lioness "centers" that captured the prey once it was driven towards them. Similarly, among two bottlenose dolphin (Tursiops truncatus) groups in the Cedar Keys, Florida, one animal repeatedly assumed the role of the "driver" that steered the fish towards 2-5 closely spaced "barrier" animals (Gazda et al., 2005). In another example, once groups of Dusky dolphins (Lagenorbynchus obscurus) corralled southern anchovies (Engraulis anchoita) into a ball at the surface, individual animals took turns to break rank and swim through the ball to eat a mouthful of fish before rejoining the group corralling effort (Würsig \& Würsig, 1980; Würsig, 1986). Larger groups fed more of the time and for longer periods than smaller groups. Finally, female humpback whales (Megaptera novaeangliae) in Alaska formed a tight spatial configuration during bubble net feeding on euphausiids and vertical lunge-feeding on herring (Clupea harengus) in a manner suggestive of a division of labor (D’Vincent et al., 1985). A call was produced that preceded feeding sequences in which a ring of whales assumed individually-consistent positions and ascended to enclose and trap their prey against the air-water interface. Role specialization can be demonstrated when different functional behaviors and spatial configurations are executed consistently by the same animals within a group.

Killer whales display a striking array of population-specific feeding strategies, some of which show evidence for collective foraging and food provisioning (e.g., Smith et al., 
1981; Hoelzel, 1991; Baird \& Dill, 1995; Ford \& Ellis, 2006). Transient killer whales in British Columbia, for example, often form groups of three animals to fetch the most amount of meat for their combined effort (Baird \& Dill, 1996; Baird \& Whitehead, 2000). This optimal group size allows them to locate and hunt their marine mammal prey without being easily detected. Fish-eating resident killer whales travel in much larger groups, however, and do not share food or depend on one another to catch prey that is easily hunted and handled individually (see Baird et al., 1992; Baird \& Whitehead, 2000). Historically, the behavioral states that have captured these dynamics (e.g., feeding, traveling, group size and composition) have been defined based on surface observations of killer whale group activity (Bain, 1986; Morton et al., 1986). This group follow approach (see Mann, 2000; Whitehead, 2004), however, has not considered underwater or individual activity. To understand the patterns of group foraging in $3 \mathrm{D}$, it is crucial to examine the continuous behavior of the individual killer whales within these groups. This will both provide information on how actively each animal participates in the feeding effort and verify that all group members are indeed afforded the chance to eat (except for younger animals that feed by suckling milk), as long as feeding corresponds with a noticeable 3D orientation signal.

Norwegian killer whales partake in a group foraging sequence called carousel feeding in which animals feed on schools of herring, apparently herding the fish from depth, corralling them into a tight ball against the surface of the water and tail slapping the edge of the ball to incapacitate the fish and eat them one at a time (Christensen, 1978; Similä \& Ugarte, 1993). Earlier work has suggested, but not demonstrated rigorously, that these animals are interacting cooperatively. It is likely that such a group strategy boosts the benefit:cost ratio for the individuals participating by improving the net caloric intake and 
managing an otherwise evasive prey species. This behavior could be motivated by kin selection (since they live in family groups) or reciprocal altruism (since group composition is stable). Diving up to 160-180 $\mathrm{m}$ to locate herring patches, different groups of killer whales converge first to herd a large swath of herring away from the total aggregation and then fragment it into smaller, more manageable schools as they drive the fish to shoal (Nøttestad \& Similä, 2001; Nøttestad et al., 2002). The sonar technology used by these earlier studies to describe the corralling behavior at depth documented the generic movements of all animals within a group simultaneously without tracking individual whales continuously through time.

Once the herring have been gathered at the surface, the orcas further corral them into a small, compact and circular ball by swimming around the fish, flashing their white ventral sides and releasing bubbles (Similä \& Ugarte, 1993; Nøttestad \& Axelsen, 1999). They capitalize on some of the same herring responses that the lunge-feeding humpback whales exploit (D’Vincent et al., 1985; Sharpe \& Dill, 1997). Individual killer whales sometimes lunge into the school, presumably to steer the fish since no feeding was observed at this time (Domenici et al., 2000). The animals tail slap the edge of the ball and stun herring likely through cavitation or direct contact, some of which are then consumed (Simon et al., 2005). Killer whale tail slaps can exceed herring escape velocities and accelerations (Domenici et al., 2000; Domenici, 2001). The absence of direct feeding by engulfing mouthfuls of fish suggests an alternative strategy to the Dusky dolphins mentioned earlier. Here, the killer whales maintain the geometry and density of the ball, perhaps to prevent herring from escaping laterally or vertically. Differences in the response behavior of the prey, the compactness of the fish ball and the size of the predators may contribute to the distinct feeding strategies pursued by these two cetacean populations. Carousel feeding at 
the surface both pushes the herring against an air-water barrier and decreases the vertical distance that the orcas must traverse to take a breath. Carousel feeding allows the killer whales to successfully manage the herring's deep vertical migration, schooling behavior and enhanced maneuverability.

Much of the previous research has explored the overall carousel feeding sequence from the level of the group. Although the work of Domenici et al. (2000) quantified movement features of individuals, they averaged the observed behaviors over all group members because they were unable to track the same animals continuously once they moved out of view on the video recording. Observing the behavior of an individual participating in cooperative foraging has been more straightforward for terrestrial than marine animals but this kind of approach is essential for exploring individual- versus group-specific behaviors and the possibilities of role playing and turn taking (i.e., changing subsets of killer whales corralling and tail slapping the fish over the course of a single carousel feeding episode). Here, I analyze and present unbiased and continuous data gathered from digital archival movement and audio tags deployed on free-ranging Norwegian killer whales. I explore the behaviors of individual killer whales operating within their larger foraging groups. In addition, I describe the detailed spatial and temporal patterns of the horizontal and vertical movements of carousel feeding animals and examine the consequent implications of individual variability and role playing. After outlining the general methods in the next section, I will present 3 analyses. The first broadly characterizes the differences in the values and variability of the movements and depth of the animals between periods involving tail slapping and periods that do not. Second, I examine tail slaps produced by the tagged animal more closely, developing an approach to distinguish them from those 
produced by other group members and exploring the diving patterns associated with this movement signature. The third analysis considers these two sets of results together with a linearity index of movement to explore the behavioral sequences preceding successful carousel feeding episodes.

\subsection{General materials and methods}

I conducted field work in Tysfjord and Vestfjord, Norway, in the fjord system just south of the Lofoten islands $\left(\sim 68^{\circ} 15^{\prime} \mathrm{N}, \sim 16^{\circ} \mathrm{E}\right)$. These fjords receive a massive influx of spring-spawning herring in the winter time, attracting foraging killer whales (Similä et al., 1996). Digital archival tags (DTAGs: Johnson \& Tyack, 2003) were attached from a rigid hull inflatable boat with a $7 \mathrm{~m}$ hand pole to 8 orcas in November 2005 and to 7 orcas in November 2006 for between 1.2 and 5.5 hours each. Tag deployment durations were deliberately short because of limited daylight and difficult nighttime tracking conditions. These tags sampled sound at $96 \mathrm{kHz}$ and movement at $50 \mathrm{~Hz}$ via a tri-axial accelerometer, a tri-axial magnetometer and a pressure sensor for depth. Although some of the animals in the 2006 dataset were introduced to sonar after a quiet pre-exposure session, only time periods before these exposures were analyzed here. In addition, the movement record of one animal (oo06_324s) could not be calibrated due to a low battery. These constraints reduced the dataset to 13 animals and 32.3 tag recording hours in all. Table 2.1 lists the 13 animals that were tagged, tagout durations, sex and age class when known, maximum dive depth and data related to tail slapping behavior and measurements. Tagged killer whales were tracked using the tag's VHF beacon. The behavioral state of the group was monitored visually from aboard the sailboat Iolaire or the research vessel Sverdrup. Tags were 


\begin{tabular}{|c|c|c|c|c|c|c|c|c|}
\hline animal id & date & sex/age class & $\begin{array}{l}\text { maximum } \\
\text { depth }(\mathrm{m})\end{array}$ & $\begin{array}{c}\text { tagout } \\
\text { duration }(\mathrm{h}) \\
\end{array}$ & $\begin{array}{l}\text { time spent tail } \\
\text { slapping (h) }\end{array}$ & $\begin{array}{l}\text { time spent not } \\
\text { tail slapping (h) }\end{array}$ & $\begin{array}{c}\text { \# focal } \\
\text { tail slaps }\end{array}$ & $\begin{array}{c}\text { \# non-focal } \\
\text { tail slaps }\end{array}$ \\
\hline "0o05_316a & 12 Nov 2005 & & 49 & 2.7 & & 2.7 & & \\
\hline oo05_320a & 16 Nov 2005 & $q$, adult? & 43 & 4.7 & & 4.7 & & \\
\hline oo05_321a & 17 Nov 2005 & & 106 & 4.2 & 0.3 & 3.9 & 1 & 40 \\
\hline oo05_321b & 17 Nov 2005 & O , adult & 51 & 1.7 & & 1.7 & & \\
\hline oo05_322a & 18 Nov 2005 & O , adult & 123 & 3.6 & 0.8 & 2.8 & 5 & 281 \\
\hline oo05_322b & 18 Nov 2005 & $\hat{\sigma}$, adult & 47 & 3.1 & 0.9 & 2.2 & 30 & 199 \\
\hline oo05_324a & 20 Nov 2005 & & 92 & 2.2 & & 2.2 & & \\
\hline oo06_313s & 9 Nov 2006 & q & 93 & 2.2 & 0.2 & 2.0 & 3 & 5 \\
\hline oo06_314a & 10 Nov 2006 & $\hat{o}$, adult & 67 & 2.1 & 0.3 & 1.8 & 8 & 25 \\
\hline 0o06_314s & 10 Nov 2006 & $\hat{o}$, sub-adult & 140 & 2.1 & 0.6 & 1.5 & 4 & 62 \\
\hline 0o06_317s & 13 Nov 2006 & $\hat{\sigma}$, adult & 38 & $0.6^{*}$ & 0.3 & 0.3 & 11 & 11 \\
\hline 0o06_327s & 23 Nov 2006 & O, adult & 45 & $0.9^{*}$ & 0.5 & 0.4 & 12 & 227 \\
\hline total & & & & 32.3 & 5.0 & 27.3 & 89 & 981 \\
\hline
\end{tabular}

Table 2.1. Tagged animals, sex and age class if known, maximum depth, tagout duration, the relative amounts of time spent tail slapping and not tail slapping, and the number of tail slaps produced by tagged (focal) and non-tagged (non-focal) individuals. The presence of a subsequent controlled sonar exposure to

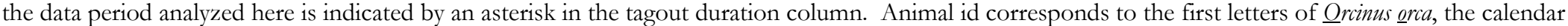

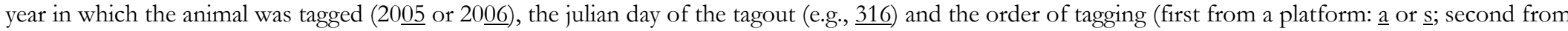
a platform: $\underline{b})$. The sums of the columns were calculated from the actual data and not the rounded data displayed here. 
programmed to release from the animal at a pre-determined time, floating to the surface where they were then located using the VHF signal. Data were offloaded in the field and saved to CD. All movement data were subsequently calibrated to convert from the tag frame to the pitch $\left(-90^{\circ}=\right.$ pitched vertically downwards; $+90^{\circ}=$ pitched vertically upwards), roll $\left(0^{\circ}=\right.$ dorsal side up; $180^{\circ}=$ ventral side up) and heading (compass bearing) of the whale frame (PRH: Johnson \& Tyack, 2003; Miller et al., 2004b). The acoustic records were audited manually by listening and logging each acoustic event.

\subsection{Analysis 1: General movement and depth features}

Compared with periods of travel or resting, killer whales that are carousel feeding must maneuver and reorient continuously to maintain the herring ball (Similä \& Ugarte, 1993; Domenici et al., 2000). In this analysis, a suite of movement measurements and their variability were broadly compared between periods of tail slapping (TS), which served as an initial proxy for feeding activity, and periods of not tail slapping (NTS).

\subsubsection{Methods}

\subsubsection{Identifying tail slaps and tail slapping periods}

A tail slap produced a characteristic acoustic signature (Simon et al., 2005; Van Opzeeland et al., 2005) that could be detected when listening through the tag sound recordings. TS periods were defined as the 2 -min window flanking any set of at least 3 tail slaps occurring within 2-min of one another. These thresholds were chosen to decrease the chance of including faint or brief sounds produced by other percussive sources that resembled tail slapping. To ignore bouts of tail slapping involving a group composed 
entirely of non-tagged animals that were detected acoustically at a distance, TS periods were additionally confined to bouts in which the tagged or focal animal produced at least a single tail slap. (These focal tail slaps were accompanied by a concomitant pitch change signature. See Analysis 2 for more detail.) The remaining sections of the record were considered to be NTS periods. Tail slap clustering within each bout was assessed by computing the Greenwood statistic on the temporal spacings between tail slap events (Greenwood, 1946; Stephens, 1986) and comparing it to 1000 datasets generated under the null hypothesis of a uniform distribution.

\subsubsection{Derived movement measurements}

To explore the variability of the animals' movements, I computed the circular standard deviation of the derivative of both the pointing angle and roll measures and the conventional standard deviation of the vertical velocity (depth derivative) once per second. The pointing angle collapsed the pitch and heading measurements into a single variable that described the 3D orientation of the whale's longitudinal axis (Miller et al., 2004a). I also computed the residual heading of the animal by first low-pass filtering (to remove high frequency fluking activity using a cut-off frequency of half of $0.43 \mathrm{~Hz}$; see Sato et al., 2007

for the computation of the mean stroke cycle frequency of killer whales) and then high-pass filtering (to remove slow maneuvering or trend of the animal over a 30s window) the calibrated heading data. The fluking intensity of the animal was computed by band-pass filtering the $z$-axis of the accelerometer using frequencies of $0.3-0.5 \mathrm{~s}$, squaring the result and then taking the running average (see Miller et al., 2004b; Hooker et al., 2005). Because the measurements associated with focal tail slaps were considered explicitly by Analysis 2 (see 
below), the data contained in the time immediately spanning each focal tail slap were removed before comparing the TS and NTS episodes. Inter-dive intervals (IDIs), the lengths of time spent at or near the surface between dives, were calculated for all dives exceeding $10 \mathrm{~m}$ in depth.

\subsubsection{Results and discussion}

Nine of the 13 whales exhibited both TS and NTS periods; the remainder contained only NTS episodes. None of the maximum dive depths in Table 2.1 exceeded the 160-180 m depths observed by Nøttestad et al. (2002), suggesting the herring were located at shallower depths here. The killer whales restricted $98 \%$ and $72 \%$ of their overall diving to within the upper $50 \mathrm{~m}$ and $20 \mathrm{~m}$, respectively (Figure 2.1). More specifically, they tended to stay near the surface regardless of their activity state, though the deepest dives occurred during and often just prior to tail slapping (TS) periods (Figure 2.1, Appendix 1). Despite animals spending comparatively less time in the depth bin closest to the surface during TS than NTS periods, TS episodes were characterized by a greater proportion of time spent occupying the depth bins between 5 and $25 \mathrm{~m}$. The frequency of occupying deeper depths decreased monotonically for TS periods and non-monotonically for NTS periods. A greater number of dives between 10 and 20 m occurred during TS compared to NTS periods (Figure 2.2, top; $49 \%$ vs. $21 \%$ ). This tendency of TS periods to contain shallower dives was likely due to the whales interacting with the herring ball close to the surface.

Short inter-dive intervals were observed more frequently and deep excursions occurred more quickly during TS compared with NTS periods (Figure 2.2). During TS periods, $73 \%$ of inter-dive intervals (IDIs) lasted less than 1 minute, which was considerably 


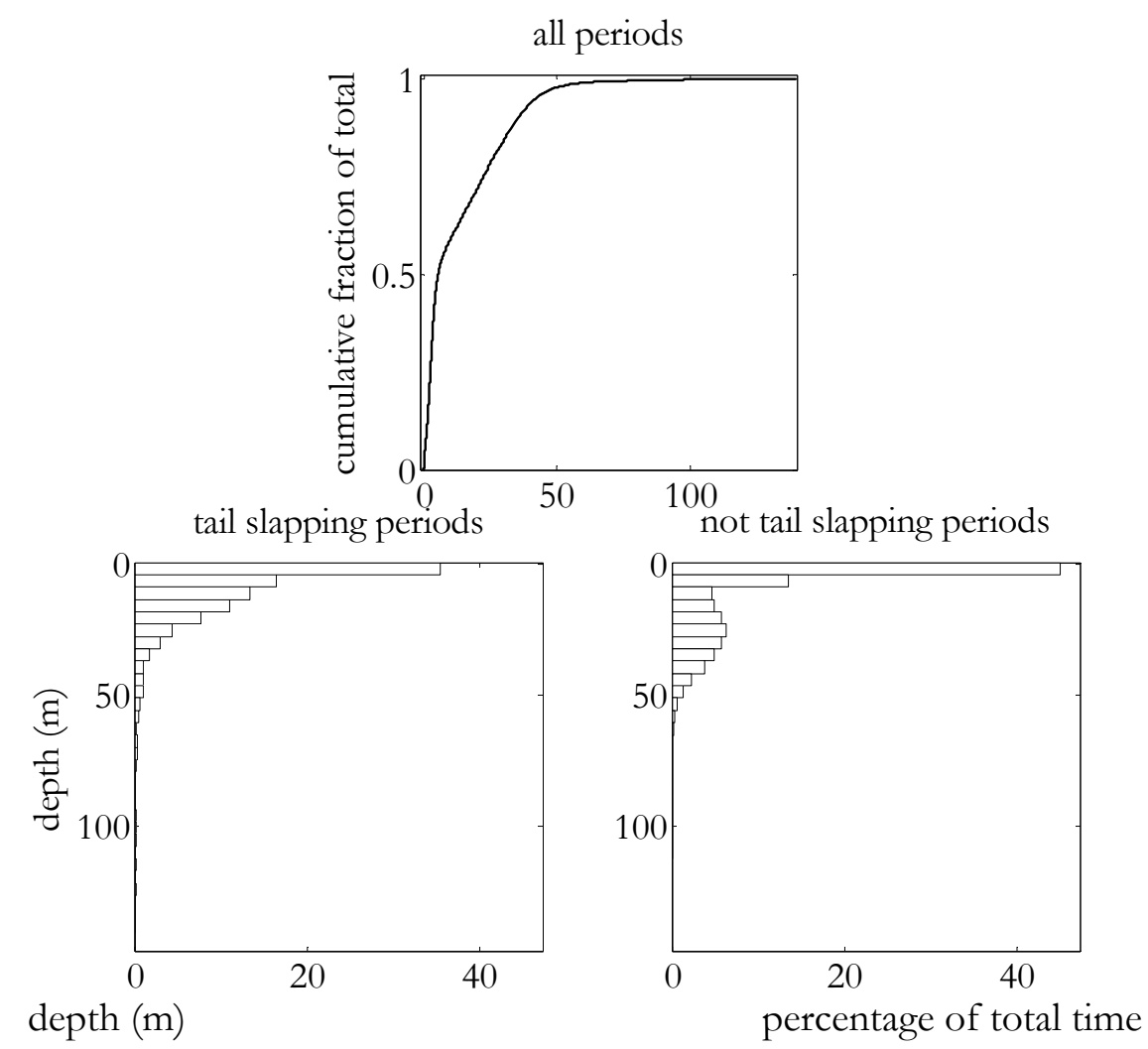

Figure 2.1. Left: cumulative plot of depth for all periods. Percentage of total time spent in different depth bins during tail slapping (center, 5.4h) and not tail slapping (right, 26.7h) periods. Both panels are bounded by the same axis limits.

higher than the value of $19 \%$ observed during NTS periods (Figure 2.2, bottom). There were 43 instances in which IDIs exceeded 4 minutes during NTS periods compared to none during TS periods. The variability of the depth did not reveal any consistent differences across animals between TS and NTS periods (Figure 2.3). The vertical velocity, change in pointing angle, change in roll movement and fluking intensity measures, however, were more variable during TS than NTS periods for these animals except for the vertical velocity of a single whale (Figure 2.3). Because the time surrounding each focal tail slap was withheld to calculate the variability of the movement measures, Figure 2.3 indicates that the animals were moving more variably during TS segments even when they were not actually producing tail 

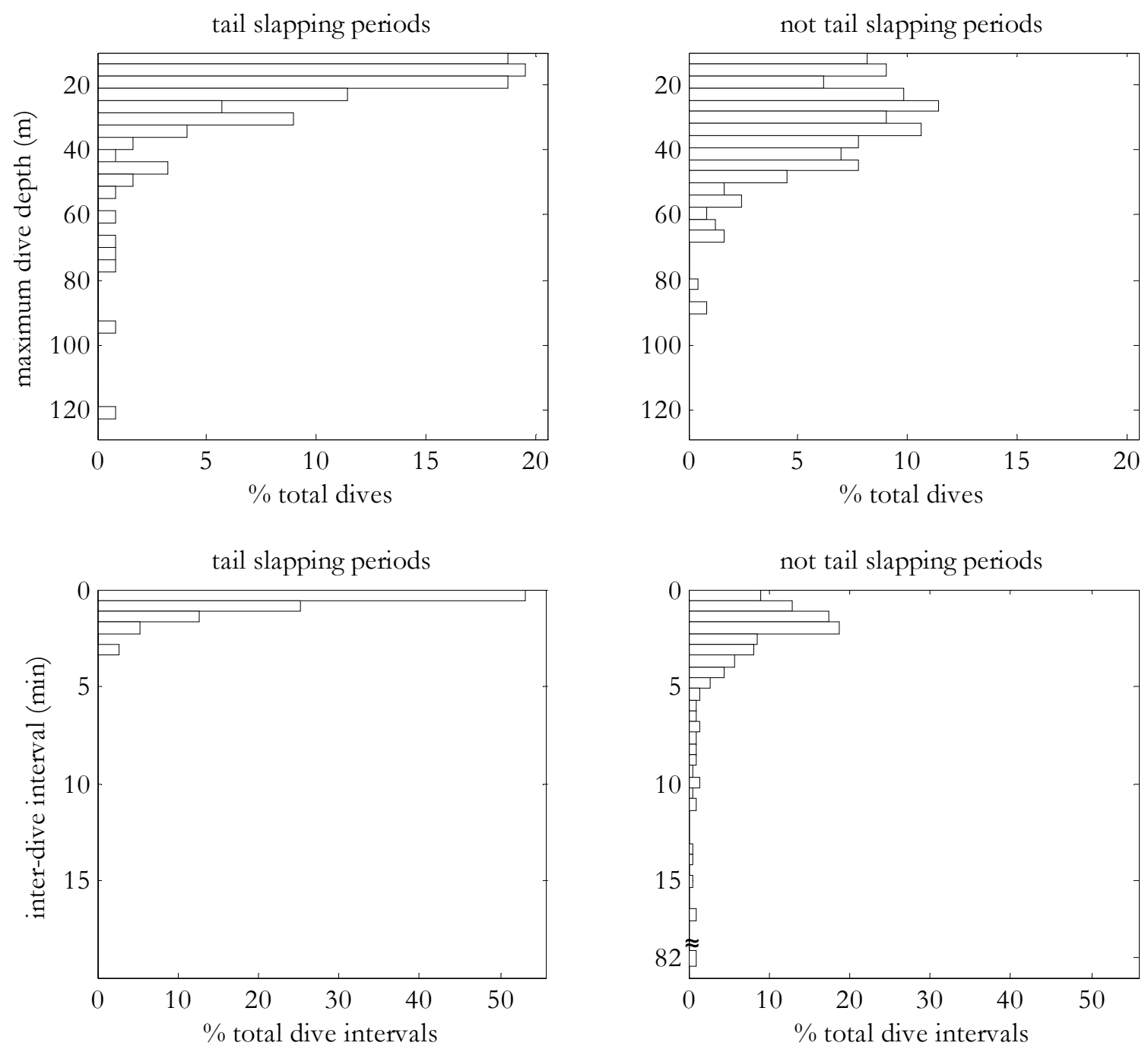

Figure 2.2. Top: Histograms of maximum depth for each dive exceeding $10 \mathrm{~m}$ for tail slapping periods (left) and not tail slapping periods (right). Bottom: Histograms of inter-dive intervals between all dives exceeding $10 \mathrm{~m}$ for tail slapping periods (left) and not tail slapping periods (right). Each horizontal pair of panels is bounded by the same axis limits.

slaps. Given relatively constant running mean values of the movement measures, this increased variability may have elevated the aerobic demands of the animals, requiring more frequent trips to the surface to breathe, briefer excursions to depth and a consequent need to keep the herring ball near the surface. (Of course, feeding on the herring ball at the surface 

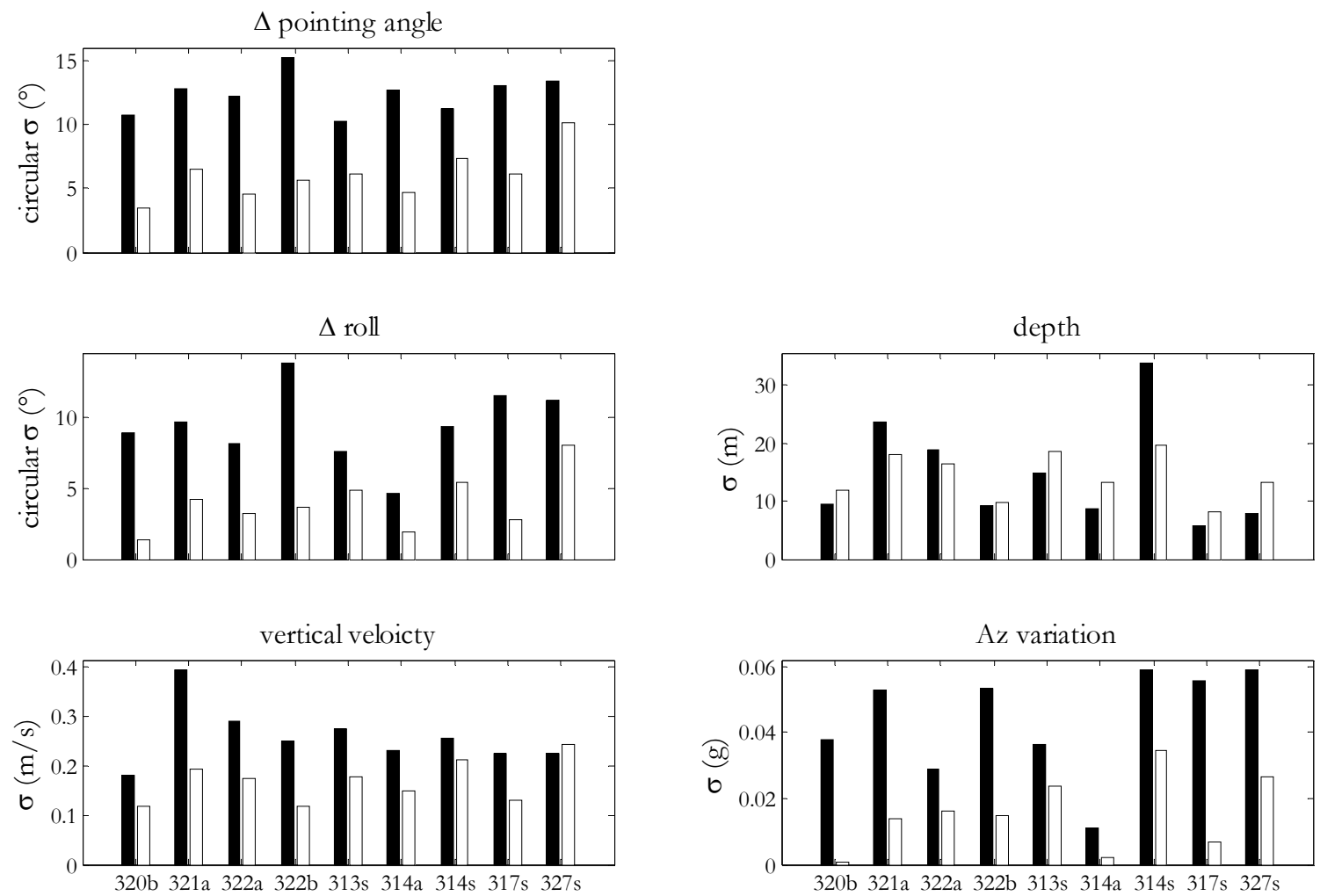

Figure 2.3. Standard deviation of 5 movement measures (see text for details) for the 9 animals exhibiting both tail slapping (TS, black bars) and not tail slapping (NTS, white bars) periods. The panels in the upper and middle left contain circular datasets, requiring the calculation of the circular standard deviation instead of the conventional standard deviation used for the remaining panels. The first four characters of the whale identification labels have been dropped for legibility. The lengths of the time periods of TS and NTS periods are available in Table 2.1.

also allowed the whales to trap the fish against the air-water interface.)

Six of the nine whales contained at least one TS period with tail slaps that were significantly more clustered than expected if they were distributed uniformly (Greenwood statistic). This suggests two different temporal regimes for tail slapping behavior: one in which tail slaps clustered in time (7 of 18 TS periods, $1: P<0.05 ; 2: P<0.01 ; 4: P<0.001$ ) and another in which tail slaps were produced more uniformly in time (11 of 18 TS periods). Group tail slap rates were higher than previously reported, varying between 0.7 and 9.6 per 
minute during carousel feeding. Tail slap rates from individual tagged whales ranged between 0.2 and 0.7 per minute.

\subsection{Analysis 2: Focal tail slapping}

The tail slap is a distinctive feature of carousel feeding and it offered a salient acoustic and movement signature pairing when produced by the tagged, focal animal. Here, I quantify the more detailed movements surrounding and characterizing focal tail slaps.

\subsubsection{Methods}

Tail slapping individuals undergo a concomitant pitch change as they move through a partial vertical turn resulting from the momentum of the slap (Similä \& Ugarte, 1993; Domenici et al., 2000; see printed and supplementary video material from Simon et al., 2005). The subset of tail slaps meeting an individual-specific, minimum instantaneous change in pitch threshold were assigned to the tagged animal (i.e., considered to be focal tail slaps). Figure 2.4 contrasts the consistent change in pitch associated with 10 randomly selected focal tail slaps with the more constant pitch values of 10 randomly selected nonfocal tail slaps. Often, focal tail slaps were preceded by elevated flow noise, presumably due to water moving faster over the tag as the animal fluked into position. Just prior to the tail slap, this flow noise went quiet. These acoustic features were used to help classify focal tail slaps as well. The time stamps of the focal tail slaps were centered on the zero crossing of the pitch signal because this cue could be reliably and consistently measured. Alignment at the onset of the percussive acoustic signal would have been more variable because its occurrence depended on the relative position, concentration and incapacitation of the fish. 


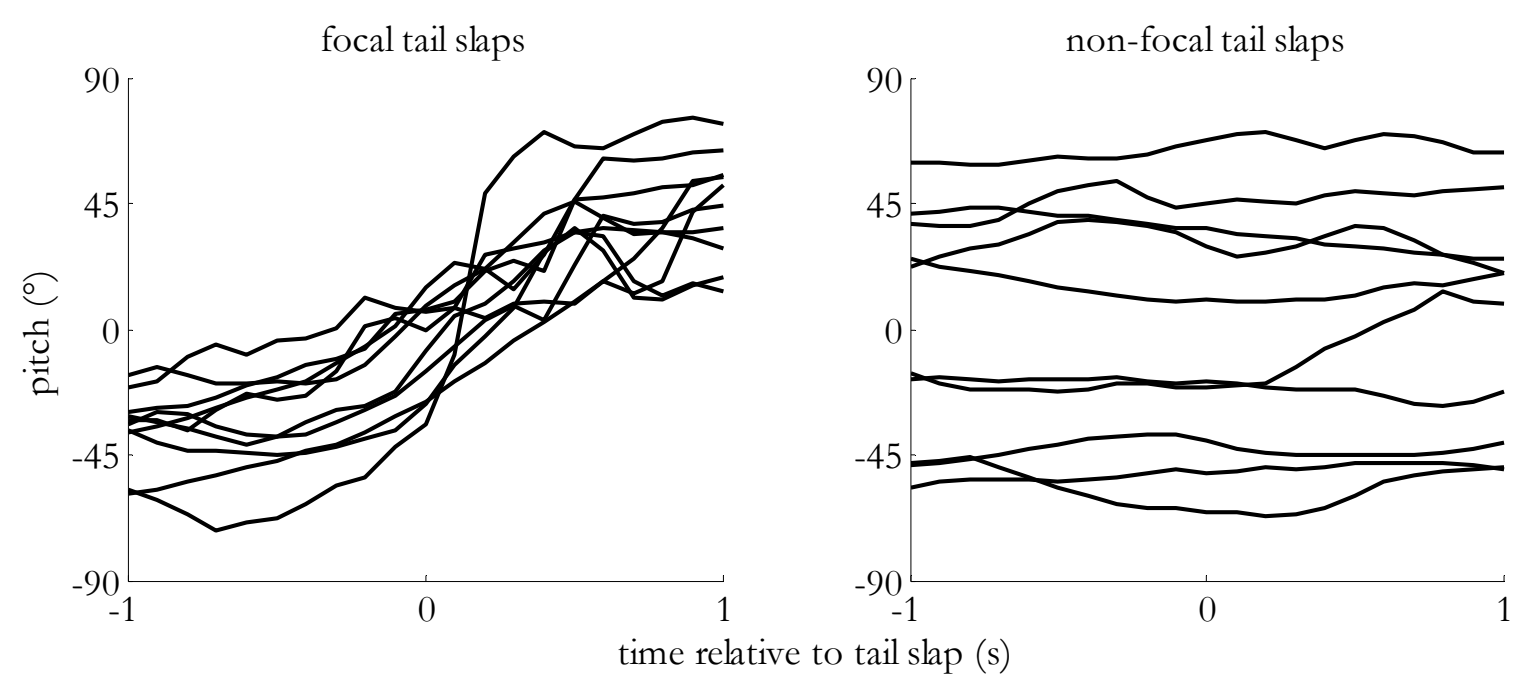

Figure 2.4. Pitch values associated with the 2 s flanking 10 randomly selected focal (left) and non-focal (right) tail slaps across all whales. Note the consistent directional change in pitch associated with the focal tail slaps compared to the flatter, more level pitch data associated with the non-focal tail slaps.

The relative proportion of focal to non-focal tail slaps was quite variable (Table 2.1).

\subsubsection{Results and discussion}

Focal tail slaps were generally louder with a higher signal-to-noise ratio than nonfocal tail slaps. They were also characterized by an instantaneous change in pitch caused by the animals spinning from a negative pitch angle (i.e., pointing downwards) to an opposite and approximately equal positive pitch angle (i.e., pointing upwards, Figure 2.4). This led to a high kurtosis for the distribution of pitch angles sampled at the times of focal tail slaps with most values inevitably stacking close to $0^{\circ}$ (Figure 2.5a). The distribution of pitch angles during non-focal tail slaps was more evenly distributed with an overall preference for generally level orientation (Figure 2.5b). This trend was reversed for the roll data (Figure $2.5 \mathrm{c}-\mathrm{d})$. Most non-focal tail slaps were characterized by a rather level roll $\left(\sim 0^{\circ}\right.$, or dorsal side up) but all orientations were observed. During focal tail slaps, however, the animals 
(a)

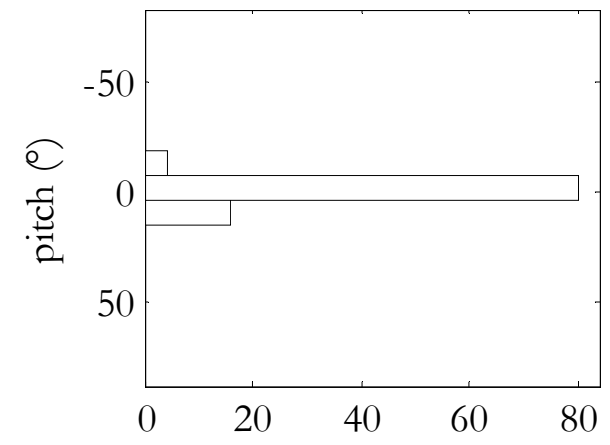

(c)

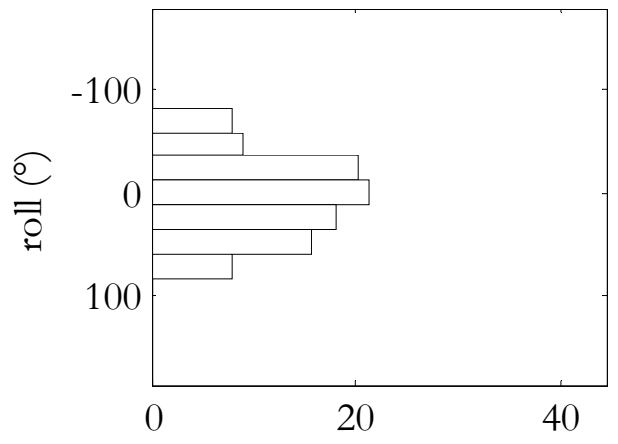

(e)

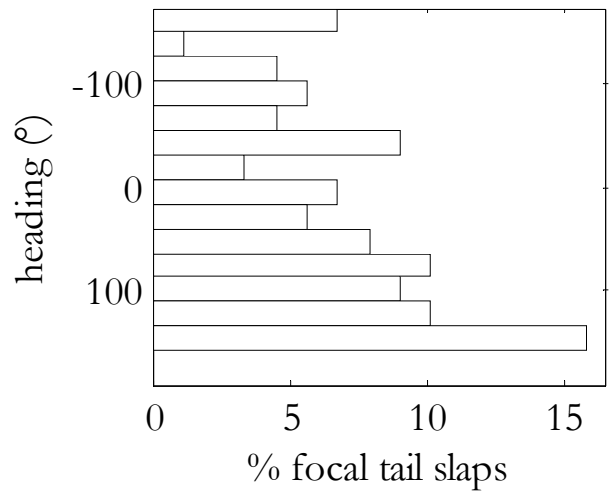

(b)

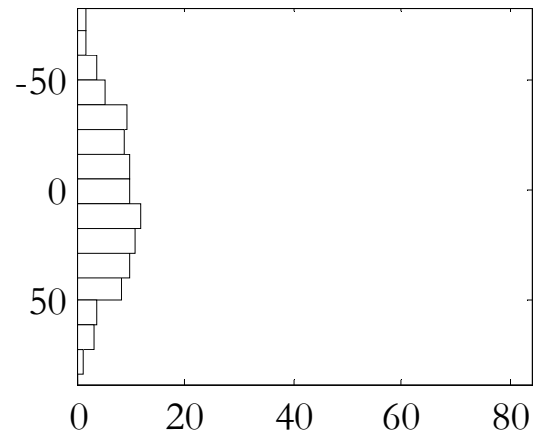

(d)

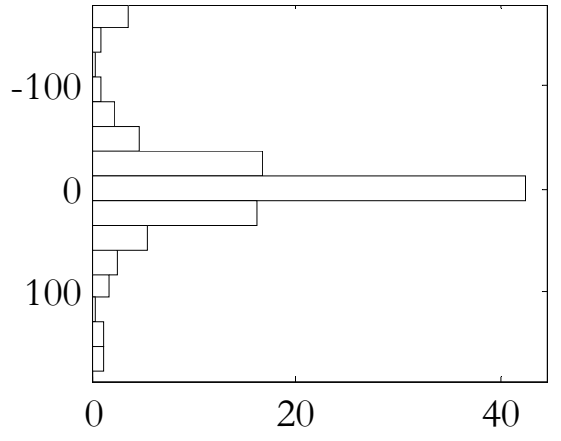

(f)

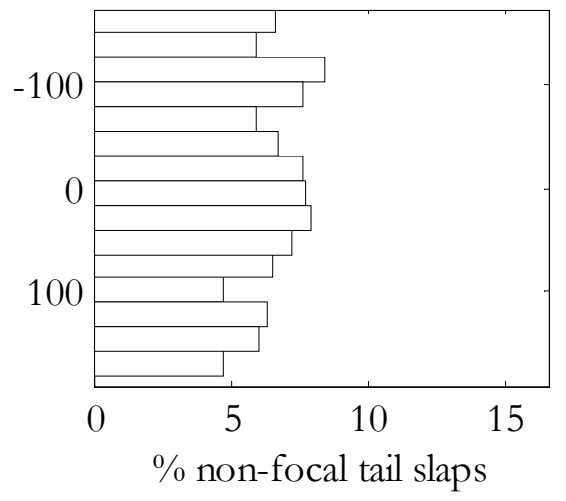

Figure 2.5. Histograms of pitch, roll and heading measurements (in degrees) taken during focal tail slap events (left, $N=89$ ) and non-focal tail slap events (right, $N=981$ ). Axis bounds are identical for each pair of focal and non-focal measurements. Focal tail slaps were centered on the zero crossing of the pitch measurement (see text for justification).

were rolled between $\pm 90^{\circ}$ with only a slight preference for orientations closer to $0^{\circ}$.

Although the orcas rotated their bodies somewhat as they tail slapped, they appeared to be 
constrained within a particular range of orientations. For example, no inverted tail slaps (ventral side up) were detected, suggesting that the animals were not approaching from the underside of the ball. This preferred set of roll orientations may reflect a geometry that delivers sufficient tail slapping force to incapacitate the herring that might have themselves adopted a particular suite of favored orientations as well. No differences between the heading of the animals during focal and non-focal tail slaps were observed (Figure 2.5e-f). There was only one occasion in which a tail slap that was accompanied by a substantial change in heading was preceded by elevated flow noise and fluking energy. It was not included here as a focal tail slap. This tail slap may have reflected an alternative movement strategy to tail slap fish by rotating in a horizontal plane but apparently was deployed much less frequently than the tail slaps produced by changing pitch.

The top two sets of triple plots in Figure 2.6 plot the pitch, depth and fluking intensity profiles for an adult male, oo05_322b (top row), and an adult female, oo06_327s (middle row), during their focal tail slaps (see Appendix 1 for similar figures for the remaining animals). The left column depicts the pitch change accompanying tail slaps produced by the tagged animal. The center column reveals differences in the diving profiles associated with tail slapping. The male generally started at the surface before diving to 10$20 \mathrm{~m}$ to initiate a tail slap whereas the female maintained a more constant depth level, often closer to the surface, before and during her tail slaps. The column to the far right indicates a general tendency of two surges in fluking intensity, the first likely corresponding to the animal fluking into position and/or lunging (dotted line bracket, see Introduction and Domenici et al., 2000 for a further description) and the second to the tail slapping event itself (solid line bracket). The relative amounts of fluking energy invested in the lunge and 

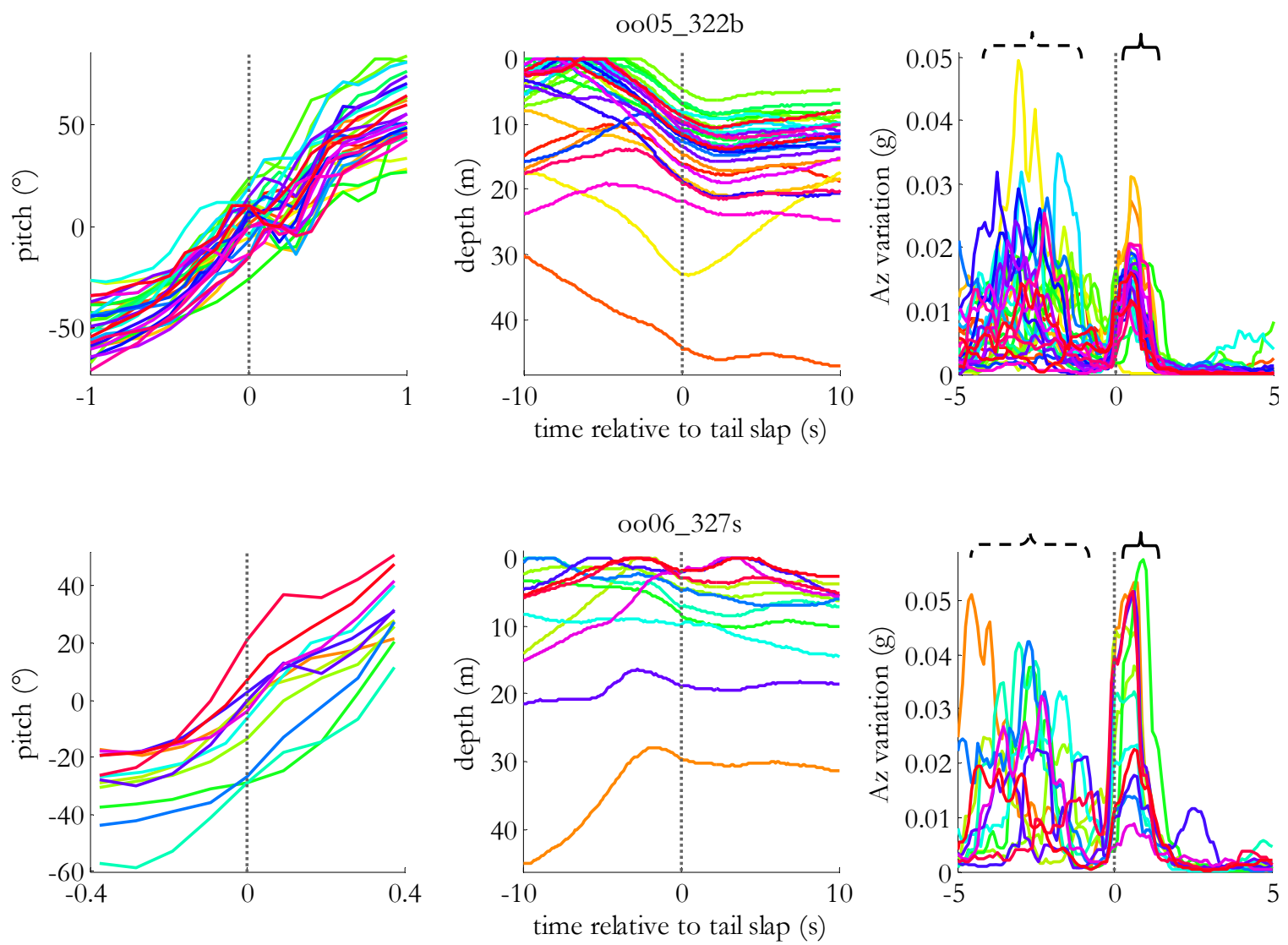

selected oo05_322a \& oo05_322b

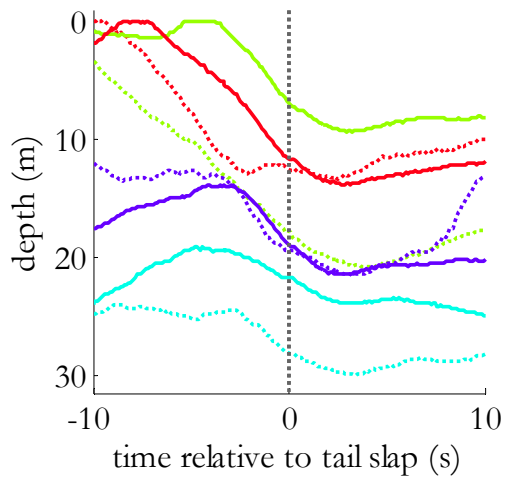

Figure 2.6. Top two trios of panels: Pitch (left), depth (center) and Az variation (right) profiles centered on focal tail slaps (gray dotted line) produced by male oo05_322b (top triplet) and female oo06_327s (middle triplet). Each line color corresponds to a unique focal tail slap and is consistent across each horizontal triptych of plots. Individual oo05_322b initiated many of his tail slaps by surfacing and diving to $10-20 \mathrm{~m}$ whereas oo06_327s remained at a more constant depth for nearly all of her tail slaps. Fluking intensity increased during the approach or lunge phase 2-3s preceding the tail slap (dotted line bracket) and then once the tail slap was executed (solid line bracket). Bottom panel: Four pairs of depth profiles surrounding tail slaps produced by group members oo05_322a (dotted line) and oo05_322b (solid line) within 60s of one another (turquoise: 3s, green: 12 s, red: 39 s, blue: 54 s). 
tail slap phases varied between animals due to tag placement but were generally similar within individuals.

On one occasion, two carousel feeding animals, an adult female (oo05_322a) and an adult male (oo05_322b), were tagged simultaneously. No regular patterns were observed in the pitch, roll and heading of these two animals to indicate that they were synchronizing or staggering their movement behaviors reliably (data not shown here). The bottom panel in Figure 2.6 compares the depth profiles associated with four pairs of their tail slaps in the same ball of herring occurring within 60s of one another towards the end of a feeding episode (the remaining pair was separated by $83 \mathrm{~s}$ ). Each pairing showed a similar profile in terms of both the change in depth 5 s preceding the tail slap to the moment of the tail slap (difference ranged from 0.5 to $3.5 \mathrm{~m}$ with $\bar{x}=1.6 \mathrm{~m}$ ) and the actual depth of the tail slaps (difference ranged from 0.4 to $10.9 \mathrm{~m}$ with $\bar{x}=4.7 \mathrm{~m}$ ). Given the male's tendency to surface before diving to 5-20 $\mathrm{m}$ to tail slap (Figure 2.6, middle panel of top row), this matching of the deeper, more level trajectories was especially striking. This strong overlap therefore suggests that the location and geometry of the herring ball were primarily influencing the details of tail slapping movements. The difference in profiles between this male and the other adult female (oo06_327s, middle set of panels, Figure 2.6) that maintained a more level depth profile surrounding her tail slaps, then, were less likely to have resulted from individually-stereotyped behaviors.

The depths of focal tail slaps and the changes in depth associated with 5 s before to the instant of the tail slap are plotted in Figure 2.7 for the 9 whales that produced their own tail slaps. In light of the earlier discussion, the differences observed here were most likely determined by the depth of the herring ball. Female oo05_322a produced only 5 tail slaps 
(a)

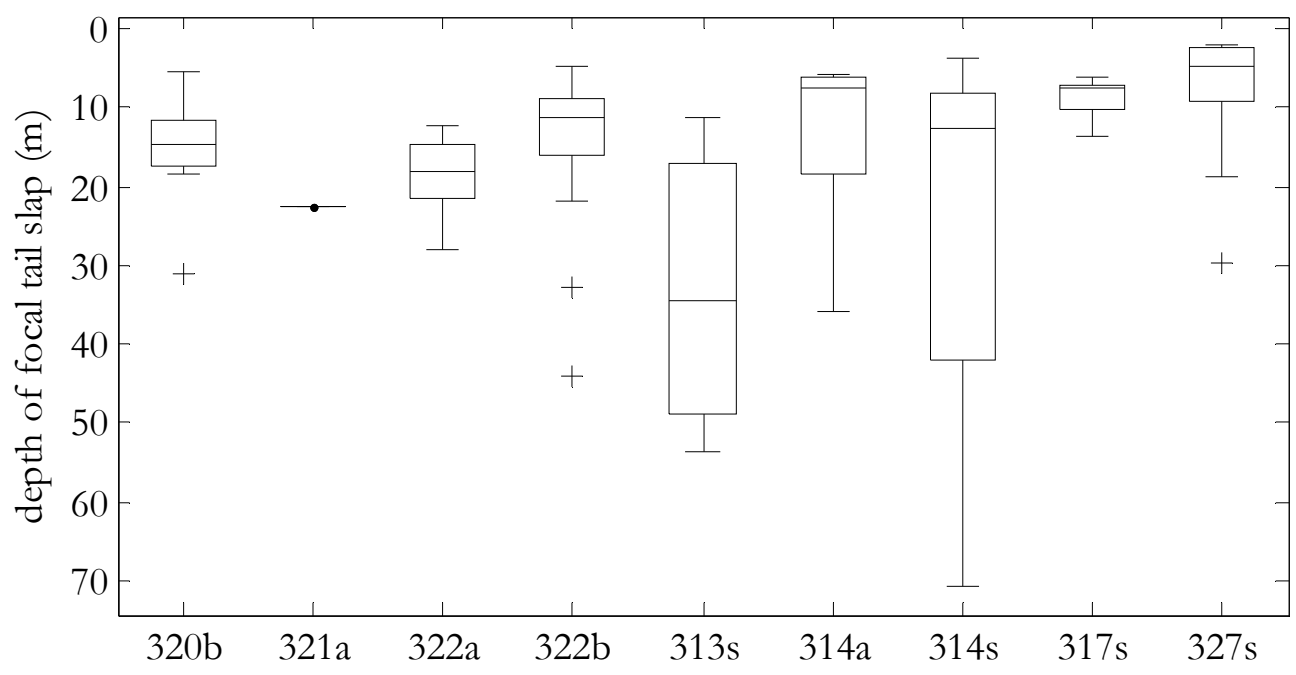

(b)

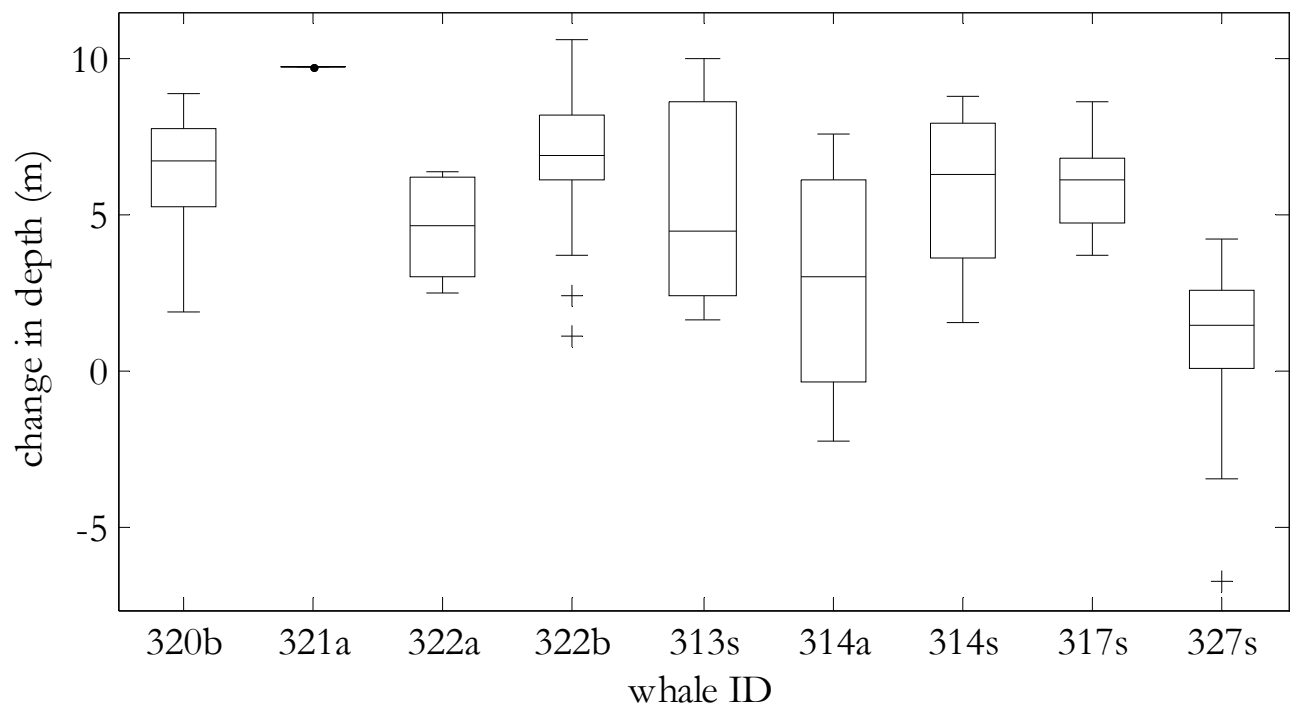

Figure 2.7. Box and whisker plots depicting the depths of focal tail slaps (top) and the change in depth from 5 s preceding the tail slap to the moment of the tail slap (bottom; positive values: descents; negative values: ascents) for all 9 whales. The horizontal lines of each box correspond to the lower quartile, median and upper quartile values of each dataset. Whiskers show the extent of the data and outliers are indicated $(+)$. The sample size for each of these bars is given in the penultimate column of Table 2.1.

over a much shorter time period at the very end of the feeding sequence whereas the simultaneously tagged male oo05_322b produced 30 tail slaps throughout the carousel 


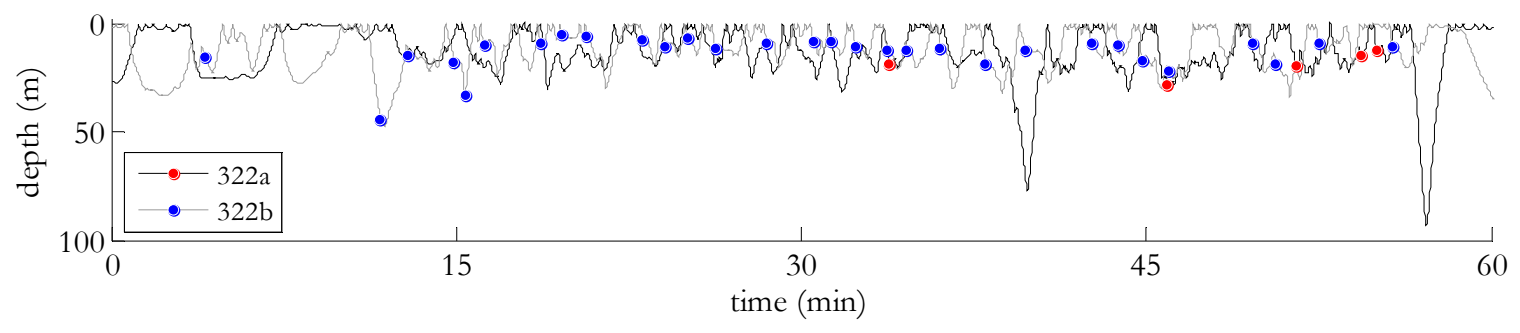

Figure 2.8. Simultaneous dive profiles of two carousel feeding whales oo05_322a and oo05_322b, zoomed into tail slapping activity. Colored circles indicate focal tail slaps (see legend).

feeding episode. Figure 2.8 shows the diving sequences and timing of the focal tail slaps of these two simultaneously tagged animals. Both whales worked the vertical dimension actively, even as they tail slapped, but did so non-synchronously, presumably to preserve the coherence of the prey ball (see Chapter 3). It is possible that killer whales consumed the fish that were incapacitated as a result of their own tail slaps (see Similä \& Ugarte, 1993; Domenici et al., 2000). If that were true, the female in the carousel here could have consumed six times fewer fish than the male.

\subsection{Analysis 3: 3D tracks and behavioral sequences}

This section considers the behavioral sequences that lead to successful foraging episodes. Carousel feeding has been described as essentially a two-stage process involving corralling herring from depth and feeding. More specifically, corralling has been associated previously with animals first driving herring to the surface, which required successively shallower dives, and then herding the fish into a compact ball (Similä \& Ugarte, 1993; Nøttestad \& Similä, 2001; Nøttestad et al., 2002). Feeding consists of circling about this condensed herring ball at the surface and occasionally breaking to tail slap and feed (Similä \& Ugarte, 1993; Domenici et al., 2000; Simon et al., 2005). The index of 2D travel 
introduced in this section relied explicitly on the movement data to divide the dataset into periods of low and high circling. Using the data streams from all three analyses, I examined the carousels in light of the corralling and feeding behaviors previously described.

\subsubsection{Methods}

The PRH data allowed the calculation of a pseudo-track, a non-geo-referenced inertial track of the animal's 2D movement path that assumed a constant swimming speed and current velocity (see Johnson \& Tyack, 2003; Zimmer et al., 2005). The third dimension of depth was measured directly with the pressure sensor. The pseudo-track was not useful for calculating absolute distances traveled since geo-referencing based on both imperfect visual sightings and the assumption of constant velocity would have introduced large errors. It did allow relative measurements to be made, however, such as track tortuosity. I sought a path-based metric of tortuosity and used a linearity index (LI) that was calculated and smoothed over a sliding 30s window by dividing the pseudo-distance between the beginning and ending of this section of the path (the crow's flight) by the pseudo-path covered by the whale (see Wilson et al., 2007). While the entire pseudo-track accumulated error over the full recording, the LI measurement did so only over the length of the short smoothing window selected to capture path variation on a tight time scale. For this measure, 1 corresponded to a straight path (i.e., the crow's path was identical to the whale's path and roughly expected when the animal was swimming directionally) and 0 corresponded to absolute circuitousness (i.e., no displacement since the beginning and ending points were the same). The LI was smoothed again with a running average filter and the final measure excluded the first and last several minutes of the record to accommodate the lag of the filter. 
Dive duration, maximum depth and IDI were examined in relation to the LI as well.

\subsubsection{Results and discussion}

The TS versus NTS distinction established in Analysis 1 relied on acoustic evidence of tail slaps to divide the dataset. In this analysis, I examined whether the LI, which derived directly from the movement data, justified some kind of division as well. Figure 2.9a plots a histogram of the linear index (LI) sampled once every second across all animals. All of the LI data were greater than 0.37 and $84 \%$ were larger than 0.80 . The LI values corresponding to 82 of the 89 focal tail slaps (the remaining 7 tail slaps occurred within the 4 minute lag at the beginning and ending of each record created by the smoothing window) are accumulated in Figure 2.9b. A normal distribution was fit to these data $(\mu=0.59, \sigma=0.09)$ and plotted in both panels of Figure 2.9. It is evident that focal tail slaps occurred during segments of the record that were characterized by lower linearity (i.e., higher circuitousness). I used this second distribution to motivate dividing the data into low and high LI segments of at least 2 minutes in duration using a threshold of 0.76 , the LI value exceeding $95 \%$ of the focal tail slap data. Compared with a value exceeding all of the focal tail slap data, 0.76 was more likely to classify intermediate measurements near the threshold as high LI. There was a slight distinction between dividing the data into TS and NTS episodes (Analysis 1) and into low and high LI periods (here). In particular, the LI division included periods of high circuitousness that may not have resulted in successful focal tail slaps (e.g., see first low LI episode in Figure 2.12). TS episodes were generally slightly longer than low LI periods, suggesting that the tight circling movements of the focal animal (low LI) lasted longer than the time span in which group members were producing audible tail slaps (TS periods). 
(a) all tags

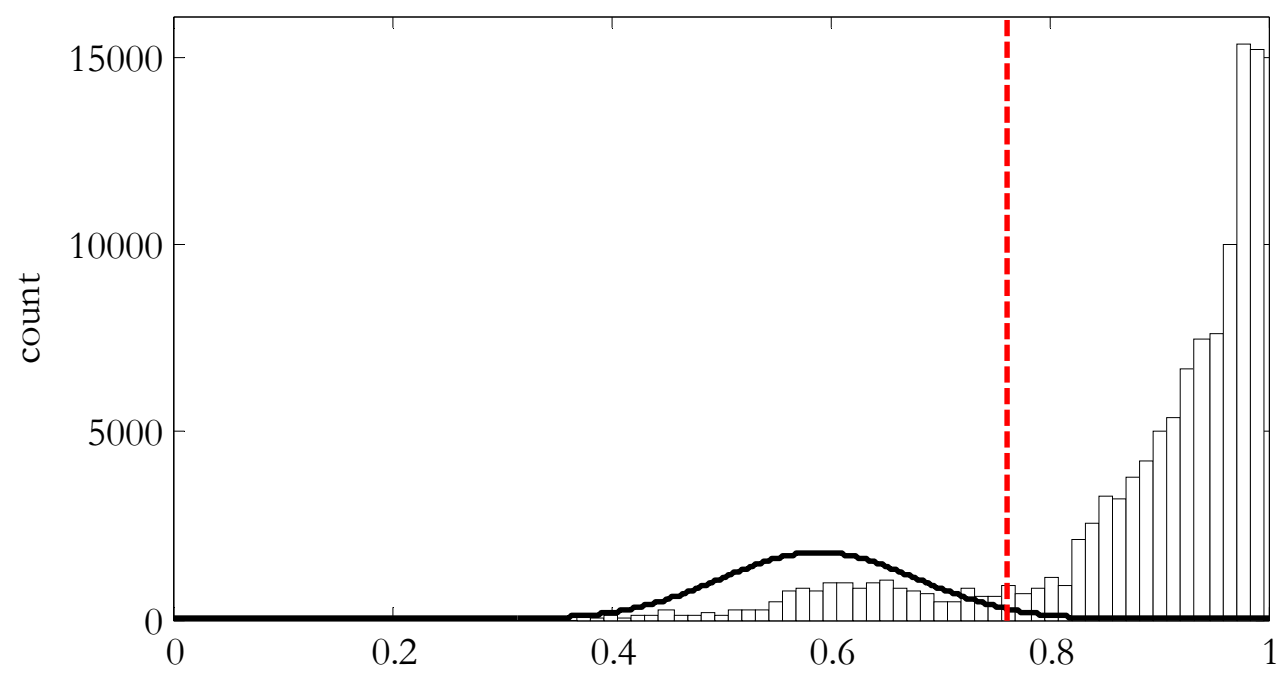

high circuitousness

high linearity

(b) focal tail slaps

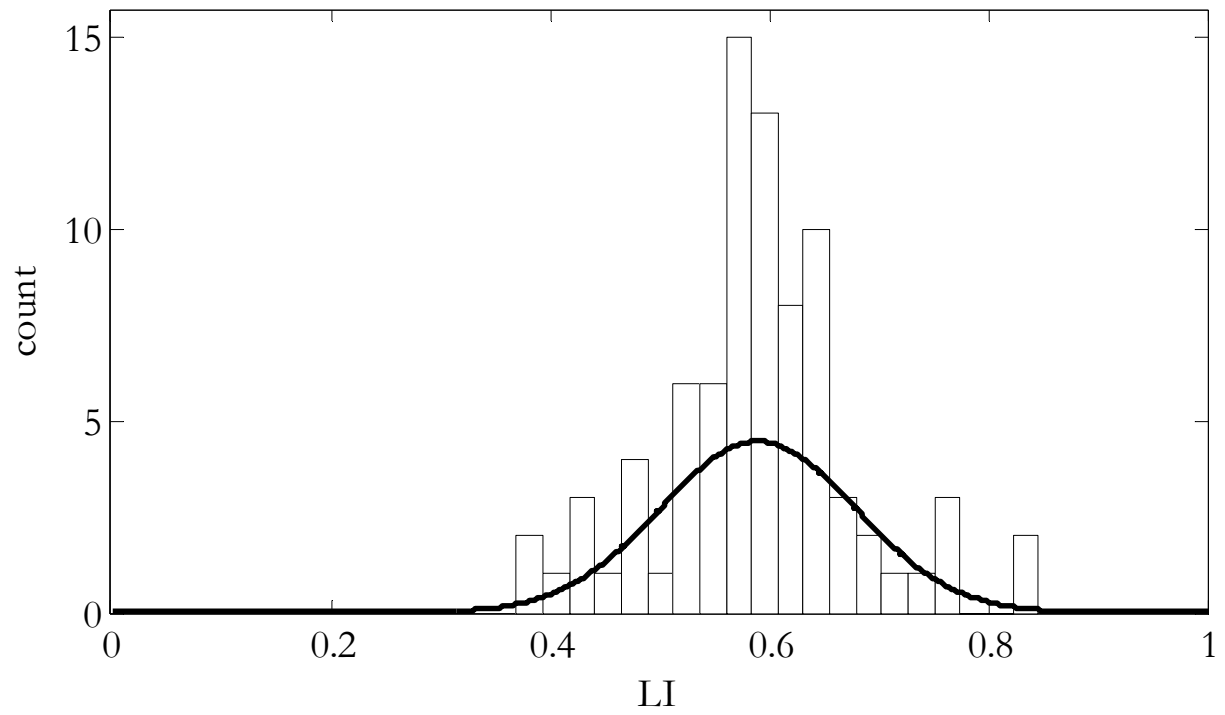

Figure 2.9. Top panel: Histogram of linearity index (LI) gathered once every second from all whales. Bottom panel: Histogram of LI values at moments of focal tail slaps $(N=82)$. A normal distribution was fit to the bottom histogram and is plotted in both panels as the dark line $(\mu=0.59 ; \sigma=0.09)$. The LI threshold of 0.76 is plotted in red in the top panel and was computed to contain $95 \%$ of the data in the lower panel. This threshold split the data into low $(<0.76)$ and high $(>0.76)$ linearity.

Table 2.2 provides behavioral sequence narrations for all 13 animals based on pseudo-track, LI and direct and derived movement measurements. The derived movement 


\begin{tabular}{|c|c|}
\hline Tag & Behavioral sequence narration \\
\hline oo05_316a & $\begin{array}{l}\text { The deepest dive of } 49 \mathrm{~m} \text { was followed by several dives that became progressively shallower. No } \\
\text { looping in the track or tail slap activity was observed. Only high LI activity was evident. }\end{array}$ \\
\hline oo05_320a & $\begin{array}{l}\text { Periods of directional movement alternated with broad looping behavior for the duration of this } \\
\text { recording. The first period of broad looping occurred at the very beginning of the record with a } \\
\text { concomitant shoaling of maximum dive depth. Only a single non-focal tail slap was observed } \\
\text { during the entire recording. High LI activity characterized the entire tagout. }\end{array}$ \\
\hline oo05_320b & $\begin{array}{l}\text { Two periods of low LI and tail slapping at the very beginning and ending of this record were } \\
\text { separated by a long episode of more directional movement. No obvious shoaling of maximum } \\
\text { dive depth was apparent. }\end{array}$ \\
\hline oo05_321a & $\begin{array}{l}\text { The first period of this record was highly circuitous and contained tail slapping activity. This was } \\
\text { followed by an episode of high LI containing two periods of directional movement separated by } \\
\text { a section of broad looping. The deepest dive occurred at the beginning of the record and the } \\
\text { maximum dive depths became shallower with time. No focal tail slapping was observed after the } \\
\text { initial high LI episode. }\end{array}$ \\
\hline oo05_321b & $\begin{array}{l}\text { This animal was tagged as it traced out broad loops, corresponding to the same looping behavior } \\
\text { of oo05_321a. The remainder of the track was characterized by travel in a consistent direction. } \\
\text { Dive durations and depths were fairly uniform for the entire tagout. The LI gradually } \\
\text { approached } 1 \text {. }\end{array}$ \\
\hline oo05_322a & $\begin{array}{l}\text { A long period of initial directional movement was followed by an episode of circling initiated by } \\
\text { the deepest dive of the record. The maximum dive depth became progressively shallower. The } \\
\text { low LI period involved tight circling behavior and a handful of focal tail slaps. This was followed } \\
\text { by broad looping and the record ended with a return to high LI directional swimming. }\end{array}$ \\
\hline oo05_322b & $\begin{array}{l}\text { An initial episode of broad looping transitioned into a tail slapping and low LI phase with } \\
\text { numerous focal tail slaps. The deepest dive of the record occurred as the tail slapping was getting } \\
\text { underway. The remainder of the tagout was characterized by consistently directed swimming } \\
\text { interspersed with occasional broad loops. }\end{array}$ \\
\hline oo05_324a & $\begin{array}{l}\text { This whale swam consistently towards the west with right angle changes of direction. No tail } \\
\text { slaps or suggestive diving patterns were observed. }\end{array}$ \\
\hline oo06_313s & $\begin{array}{l}\text { A long period of initial directional swimming and an occasional small loop involving an early } \\
\text { deep dive was followed by a brief period of broad looping before a quick episode of low LI and } \\
\text { focal tail slapping. The record ended with the animal returning to high LI movement. }\end{array}$ \\
\hline oo06_314a & $\begin{array}{l}\text { This animal moved directionally for the majority of the record until beginning a low LI episode at } \\
\text { the very end accompanied by focal tail slapping. }\end{array}$ \\
\hline oo06_314s & $\begin{array}{l}\text { An initial period of high LI ended with a sharp } 180^{\circ} \text { turn and deep dive, after which tail slapping } \\
\text { accompanied a low LI. A short episode of subsequent broad looping was followed by another } \\
\text { longer period of directional travel. One focal tail slap was produced during the first high LI } \\
\text { segment. }\end{array}$ \\
\hline 0006_317s & $\begin{array}{l}\text { An initial episode of tail slapping and low LI transitioned into a high LI phase characterized by a } \\
\text { single deep dive and broader looping behavior. Directional swimming occurred subsequently. }\end{array}$ \\
\hline 0o06_327s & $\begin{array}{l}\text { Although nearly the entire record of this animal was characterized by low LI, periods of high and } \\
\text { moderate circling were both present. The looping at the beginning was accompanied by the } \\
\text { deepest dives compared to later in the sequence. }\end{array}$ \\
\hline
\end{tabular}

Table 2.2. Behavioral sequence narrations for all animals based on track, LI and diving data. The LI threshold of 0.76 divided the records into low LI (tight circling generally accompanied by focal and non-focal tail slaps) and high LI (directional swimming and broad looping behavior) periods.

measures tended to vary considerably during low LI periods and moderately or minimally during high LI periods. Table 2.3 lists the time intervals corresponding to periods of low 


\begin{tabular}{|c|c|c|c|c|}
\hline Tag & LI & Time interval (minutes) & Time interval (local time) & mean LI \\
\hline oo05_316a & high & $4-158$ & $12: 09-14: 42$ & 0.95 \\
\hline oo05_320a & high & $4-278$ & $8: 49-13: 23$ & 0.91 \\
\hline \multirow{5}{*}{ oo05_320b } & low & $4-15$ & $12: 42-12: 53$ & 0.62 \\
\hline & high & $15-86$ & $12: 53-14: 05$ & 0.97 \\
\hline & low & $86-102$ & $14: 05-14: 20$ & 0.68 \\
\hline & high & $102-107$ & $14: 20-14: 25$ & 0.80 \\
\hline & low & $107-128$ & $14: 25-14: 46$ & 0.63 \\
\hline \multirow{2}{*}{ oo05_321a } & low & $4-15$ & $10: 45-10: 56$ & 0.71 \\
\hline & high & $15-245$ & $10: 56-14: 45$ & 0.94 \\
\hline oo05_321b & high & $4-96$ & $11: 52-13: 25$ & 0.92 \\
\hline \multirow{5}{*}{ oo05_322a } & high & $4-66$ & $10: 14-11: 16$ & 0.94 \\
\hline & low & $66-72$ & $11: 16-11: 22$ & 0.70 \\
\hline & high & $72-126$ & $11: 22-12: 16$ & 0.89 \\
\hline & low & $126-166$ & $12: 16-12: 56$ & 0.66 \\
\hline & high & $166-211$ & $12: 56-13: 41$ & 0.91 \\
\hline \multirow{8}{*}{ oo05_322b } & low & $4-9$ & $11: 31-11: 36$ & 0.65 \\
\hline & high & $9-27$ & $11: 36-11: 54$ & 0.87 \\
\hline & low & $27-30$ & $11: 54-11: 56$ & 0.74 \\
\hline & high & $30-35$ & $11: 56-12: 01$ & 0.81 \\
\hline & low & $35-39$ & $12: 01-12: 05$ & 0.73 \\
\hline & high & $39-42$ & $12: 05-12: 07$ & 0.79 \\
\hline & low & $42-90$ & $12: 07-12: 56$ & 0.59 \\
\hline & high & $90-179$ & $12: 56-14: 25$ & 0.94 \\
\hline oo05_324a & high & $4-126$ & $13: 39-15: 41$ & 0.98 \\
\hline \multirow{3}{*}{ oo06_313s } & high & $4-95$ & $13: 42-15: 13$ & 0.92 \\
\hline & low & $95-99$ & $15: 13-15: 17$ & 0.73 \\
\hline & high & $99-128$ & $15: 17-15: 45$ & 0.96 \\
\hline \multirow{2}{*}{ oo06_314a } & high & $4-113$ & $10: 37-12: 27$ & 0.96 \\
\hline & low & $113-121$ & $12: 27-12: 34$ & 0.65 \\
\hline \multirow{5}{*}{ oo06_314s } & high & $4-43$ & $12: 00-12: 39$ & 0.91 \\
\hline & low & $43-45$ & $12: 39-12: 41$ & 0.74 \\
\hline & high & $45-48$ & $12: 41-12: 44$ & 0.83 \\
\hline & low & $48-64$ & $12: 44-13: 00$ & 0.63 \\
\hline & high & $64-120$ & $13: 00-13: 56$ & 0.91 \\
\hline \multirow{2}{*}{ oo06_317s } & low & $4-17$ & $14: 37-14: 50$ & 0.59 \\
\hline & high & $17-34$ & $14: 50-15: 06$ & 0.87 \\
\hline oo06_327s & low & $4-50$ & $13: 47-14: 32$ & 0.58 \\
\hline
\end{tabular}

Table 2.3. Time intervals for low and high LI periods of at least 2 minutes in duration, excluding the first and last 4 minutes because of the smoothing window of a filter (see text).

and high LI and their mean LI values.

Figures $2.10-2.13$ visually summarize the two general types of behavioral sequences of the animals observed preceding and during carousel feeding (see Appendix 1 for the full tracks of all 13 animals). The first involved a period of directional swimming followed by 

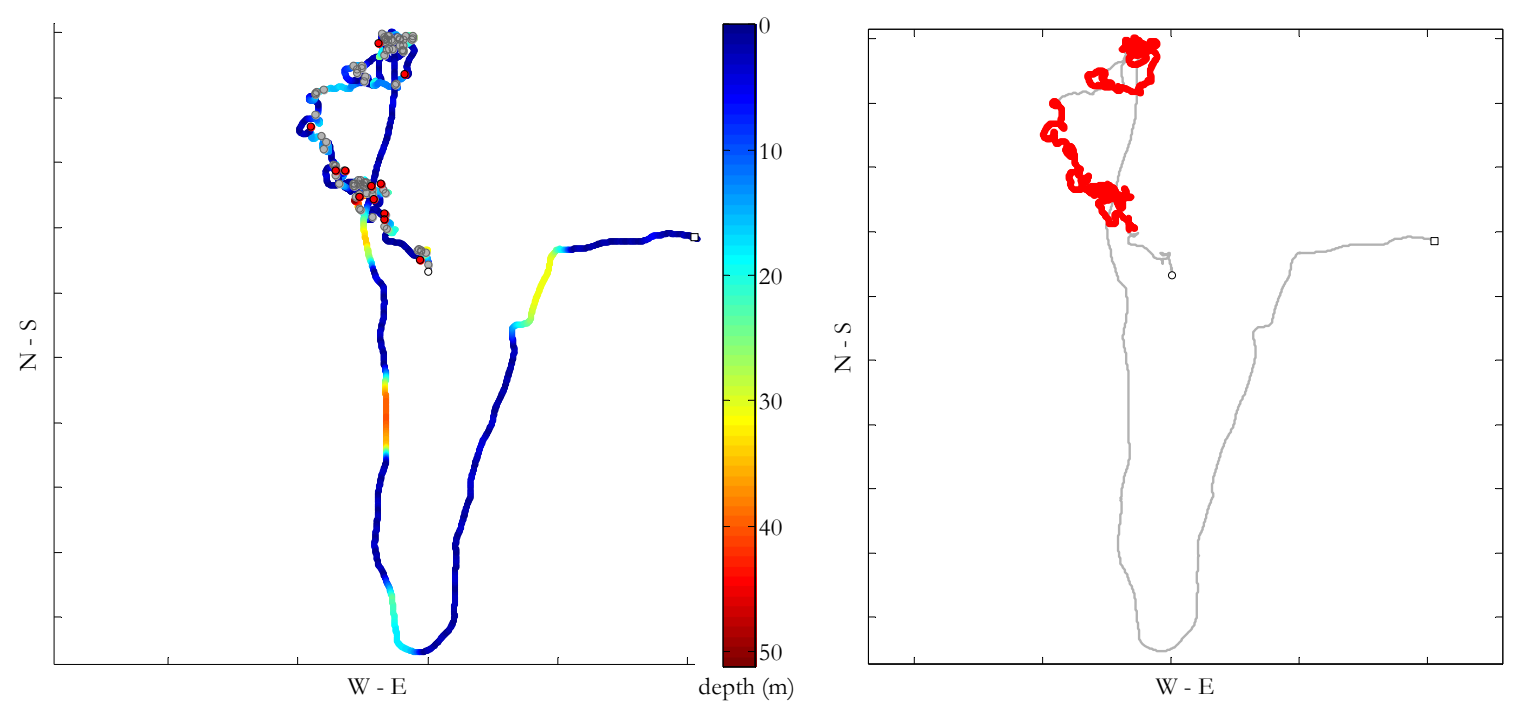

Figure 2.10. Partial pseudo-track (non-geo-referenced inertial path) of male oo05_320b (1.3h segment). Horizontal movement is plotted as relative distance and the beginning and ending of this segment are shown ( $\square$ and $\odot$, respectively). In the left panel, depth is colorized, the filled gray circles represent tail slaps produced by a non-tagged individual, and the red circles indicate tail slaps produced by the tagged, focal animal. In the right panel, the red sections of the track correspond to periods of low linearity (thresholded using the focal tail slap data, see text and Figure 2.9). This path reveals movement characterized by high linearity and directional swimming leading into movement characterized by low linearity.

heavy tail slapping activity. The track segment in Figure 2.10 depicts an adult male (oo05_320b) that initially swam southwards until making a sharp hairpin turn to the north. This period of movement was characterized by high LI (gray in the right panel) and an absence of focal tail slaps. Subsequently, the male began a period of tight circling and his path became highly circuitous (red in the right panel). The elevated rates of focal and nonfocal tail slap production were indicative of feeding behavior (Similä \& Ugarte, 1993; Domenici et al., 2000; Simon et al., 2005).

The LI, dive profile and derived movement measurements for this male are plotted in Figure 2.11 (see Appendix 1 to view these plots for the remaining animals). The LI oscillated between lower values during TS periods and a higher value during the NTS period. The derived movement measurements all exhibited increased activity and variability during 
oo05_320b
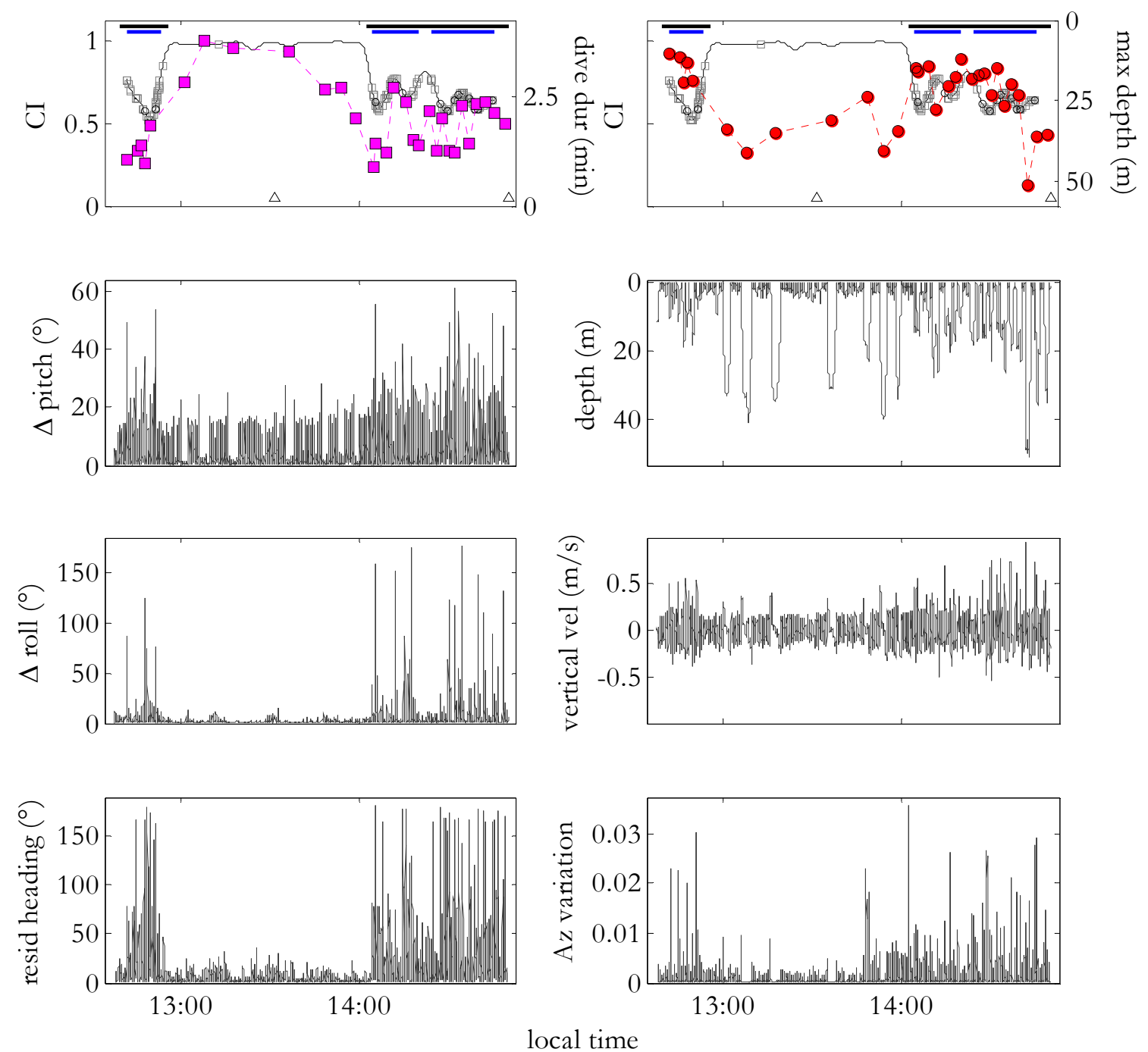

Figure 2.11. Movement data summary plots for male oo05_320b. The linearity index (LI) is plotted as a thin continuous black line in the two uppermost panels and ranges from 0 (no displacement) to 1 (straight path).

The LI plots two rough states: a low linearity (high circuitous) state when the animals were tail slapping and a high linearity (low circuitous) state when few tail slaps were observed (see Figure 2.9). Dive duration and maximum dive depth are indicated with the magenta squares and red circles in the left and right panels, respectively. The small gray squares and open black circles on the LI curve indicate non-focal and focal tail slaps, respectively. The two tail slapping (TS) periods are indicated by the black horizontal bars at the top of the plot. The low LI episodes are shown by the blue horizontal bars. The two small triangles at the bottom of the plots mark the time interval plotted in Figure 2.10. Beginning in the second row and reading left to right, the remaining panels plot the change in pitch (degrees), depth $(\mathrm{m})$, change in roll (degrees), vertical velocity $(\mathrm{m} / \mathrm{s})$, residual heading (degrees) and variation in the z-axis of the accelerometer (a proxy for fluking energy, relative units). See text for computation details. The change in pitch, change in roll, residual heading and Az variation all increased during TS periods. Time is reported locally and runs identically along the $\mathrm{x}$-axis of each panel. 
TS periods compared to NTS periods (see Figure 2.3 as well). The percussive tail slapping sounds, echolocation clicks and numerous pulsed calls make feeding an acoustically active time (Similä \& Ugarte, 1993; Van Opzeeland et al., 2005; Simon et al., 2007), which may attract nearby groups of killer whales to a ball of herring that has already been brought to the surface. This may explain the sudden change in direction by the male here since focal tail slapping activity ensued suddenly after roughly 18 minutes of directional swimming following the turn. The possible strategy of feeding on a previously corralled herring school may offer an opportunity for these animals to forego herding the fish at depth and participate only in the feeding endgame. Indeed, visual observations confirmed that this male and his group joined another group that was already carousel feeding. Two other animals displayed a similar pattern in which a highly circuitous path coupled with tail slapping activity was preceded by a low LI period. In one instance (oo06_314a), the direction of movement remained consistent but in the other (oo06_314s), a rapid change in direction preceding the tail slapping activity similar to oo05_320b was observed. No additional visual observations of the presence or behavior of nearby groups were available for these other two whales.

When carried through effectively, the second type of foraging sequence involved broad horizontal looping followed by tail slapping and feeding. The path of the female oo05_322a (Figure 2.12) exemplified this behavioral routine as it transitioned from high to low LI (after an initial low LI episode). In contrast to oo05_320b, the high LI period contained numerous broad loops accompanied by moderate to deep dives to $20-40 \mathrm{~m}$. The absence of tail slapping activity suggested that no feeding was taking place. The diving depths gradually shoaled after the deepest dive to $123 \mathrm{~m}$ in this record. This use of 3D 

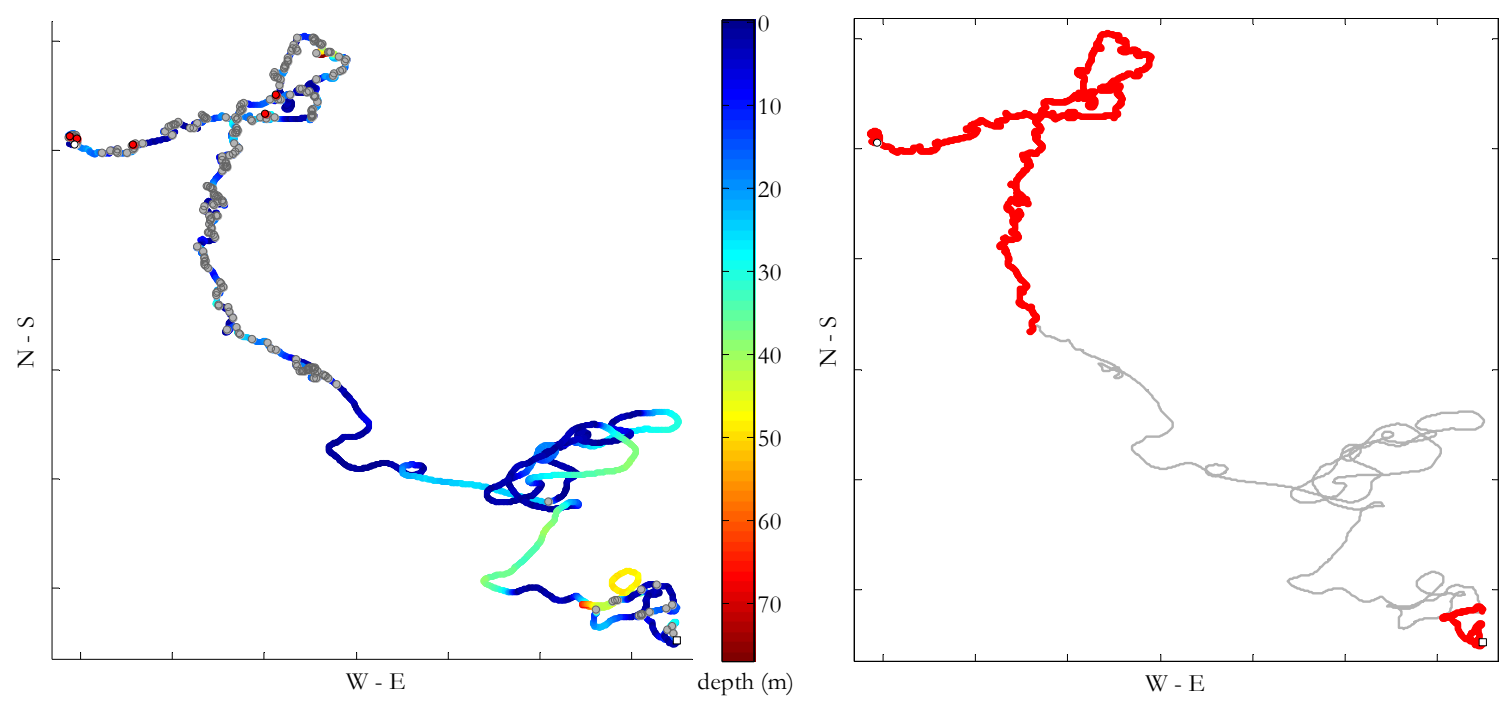

Figure 2.12. Partial pseudo-track of female oo05_322a (1.6h segment). Key as in Figure 2.10. The right panel shows that the high circuitousness sections (red) accompanied by tail slapping were interspersed with a high linearity section (gray) that contained vertical excursions and broad horizontal looping but lacked frequent tail slapping.

space was consistent with the corralling behaviors previously described (Similä \& Ugarte, 1993; Nøttestad \& Similä, 2001; Nøttestad et al., 2002). In particular, this animal may have traveled to depth to locate herring, brought them to the surface after a set of repeated and increasingly shallow dives, and contained them by broadly looping about the circumference.

The subsequent tighter, low LI loops were again accompanied by numerous instances of non-focal and focal tail slapping (Figure 2.12). The derived movement measurements registered increased activity levels during the low LI phase (Figure 2.13). The other period at $\sim 11: 30$ exhibiting elevated activity corresponded to another section of tight circling and numerous non-focal tail slaps displayed as the short first span of red in Figure 2.12. Three other whales contained similar sequences of transitions from broad to tight horizontal looping (oo05_322b, oo06_313s, oo06_327s), which may have reflected that the herring ball required restructuring by the group before feeding could progress. The two 

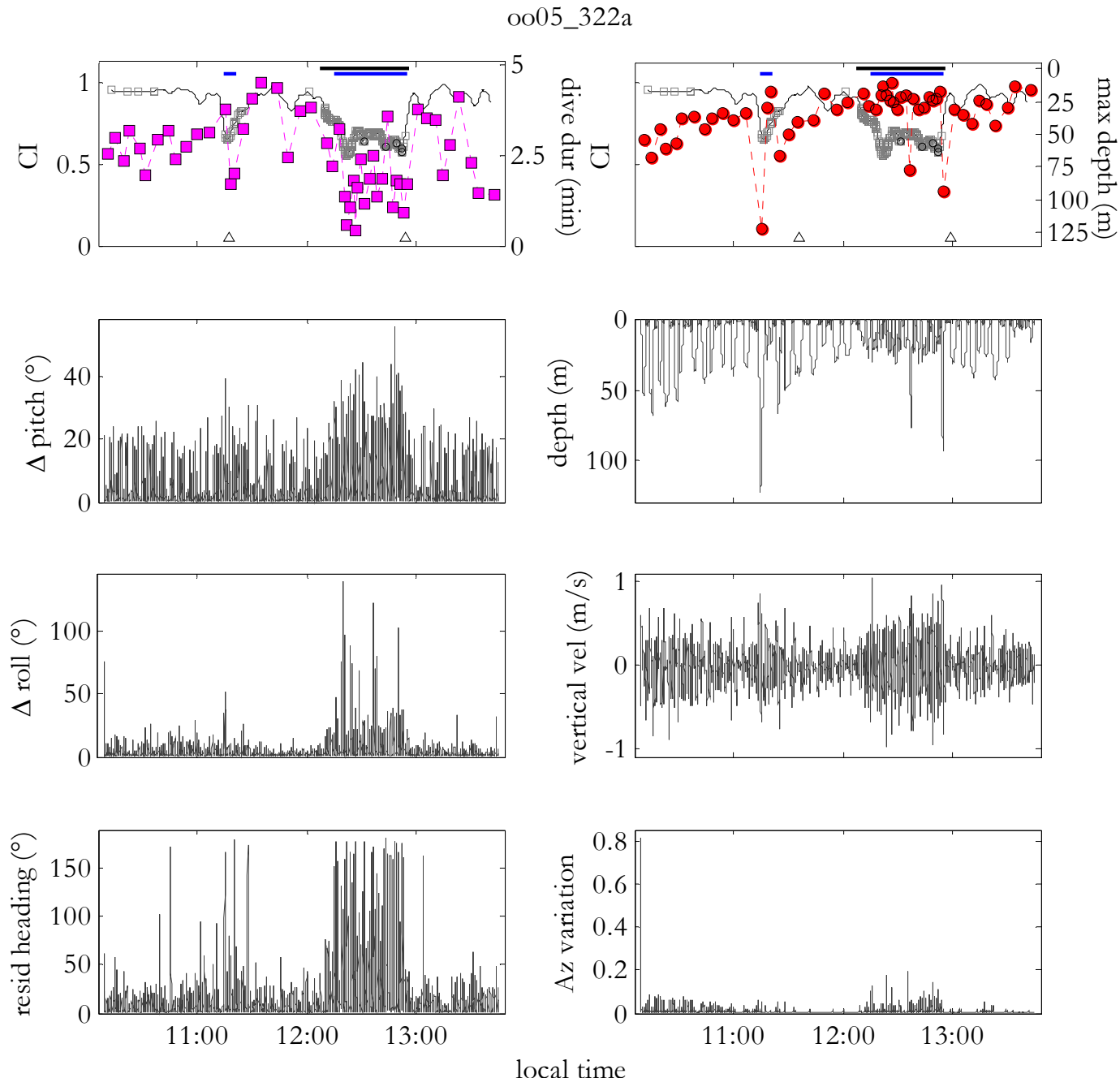

Figure 2.13. Movement data summary plots for female oo05_322a. See Figure 2.11 for key. The TS period was flanked by two NTS periods. The two small triangles at the bottom of the top two sub-panels mark the time interval plotted in Figure 2.12.

animals that were tagged simultaneously, the adult female oo05_322a and the adult male oo05_322b, traced out similar paths of low LI leading to high LI (see Appendix 1), suggesting that closely coordinated and sequenced movements may be essential for group members to contain and feed on herring.

In addition to oo05_322a, 7 other animals displayed this broad looping behavior 
during which only occasional focal tail slaps were produced (i.e., oo05_320a, oo05_321a, oo05_321b, oo06_317s), the putative corralling occurred after successful tail slapping and feeding had been replaced by directional swimming. The episodes of putative corralling displayed by 3 other animals (oo05_321a,oo06_313s, oo06_314s) contained non-focal tail slaps, suggesting either that they were produced by a more distant non-focal group or that the animals may have taken turns between corralling, in which group members including the focal animal preserved the integrity of the herring ball, and tail slapping. This kind of coordinated turn taking would prevent the animals from feeding all at once, a scenario that could lead to the dispersal of the fish ball. To test for turn taking, sufficient numbers of animals need to be tagged simultaneously in future studies to capture all of the behaviors that may be co-occurring and examine the numbers and patterns of animals participating in each task.

In 7 cases, broad horizontal looping did not always lead to tail slapping and feeding. Four animals interspersed periods of directional swimming with periods of putative corralling (e.g., oo05_320a, oo05_321a, oo05_321b,oo06_317s). Anti-predator responses of herring have been documented (Nøttestad \& Axelsen, 1999) and it is probable that herring take advantage of momentary breaks in orca group formation to disperse laterally and dive, rendering their geometry and vertical position more challenging for tail slapping. In addition, certain fish densities and quantities may be insufficient to merit further shoaling, leading the orcas to abort their efforts prematurely (Nøttestad et al., 2002). A subset of the 7 whales remained closer to the surface after a successful tail slapping episode, suggesting that they may have been trying to re-corral the ball upon which the group had been feeding. The data presented here reveal that putative corralling efforts may go unrewarded rather 
regularly. This would make the strategy of feeding upon herring that require minimal corralling (since they have already been gathered close to the surface by the focal or nonfocal group) all the more advantageous.

\subsection{General discussion}

The data presented here provide a detailed portrait of the movement behavior of individual Norwegian killer whales as they forage in groups on herring. Previous work described carousel feeding episodes consisting of a deeper, gradually shoaling corralling phase followed by a shallower feeding phase (Similä \& Ugarte, 1993; Nøttestad \& Similä, 2001; Nøttestad et al., 2002). Using a threshold calculated from the distribution of linearity index (LI) values taken at the moments of focal tail slaps (Figure 2.9), the recordings were examined with these phases in mind. High LI periods mapped onto segments of track characterized by either directional swimming or broad horizontal looping. Both involved moderate to deep vertical excursions and occasional non-focal tail slaps. Directional swimming and the broad looping seemed to fit the putative behavioral categories of "travel" and "corralling," respectively. Low LI segments involved tight circling and contained numerous instances of focal tail slapping that likely indicated feeding activity.

Low LI periods were by definition associated with more variable 3D movement features. With the exception of a single measurement for one individual, the vertical velocity, fluking energy and changes in pointing angle and roll were highly variable (Figures $2.3,2.11 \& 2.13)$. This is consistent with the frequent changes in orientation of these animals as they feed that have been observed (Similä \& Ugarte, 1993; Domenici et al., 2000; Simon et al., 2005). To produce effective tail slaps and to compensate for the dynamic 
responses of both conspecifics and the herring ball, the killer whales must vary and make constant adjustments to their 3D orientation and position. Additional data from whales engaged across multiple carousel feeding events are required to explore how the animals balance the individual need to feed with the group need to preserve the coherence of the herring ball.

A subset of the tagged whales displayed a carousel sequence consistent with the observations of earlier research. In particular, the broad horizontal looping, deeper vertical excursions and high LI that defined putative corralling in the tag data gave way to the tight circling, abundant focal and non-focal tail slaps and low LI of feeding (Figures 2.12 \& 2.13). Data from other whales, however, suggested that it was not uncommon for putative corralling to be required minimally or not at all as animals began feeding on herring already at the surface (Figures $2.10 \& 2.11$ ). The herring follow diurnal vertical migration patterns, remaining at depth during the daytime to avoid visual predators and traveling to the surface at night to feed. This made it doubtful that the fish first located at the surface by the killer whales had shoaled on their own. Rather, it was more likely that they had escaped from an earlier corralling effort organized by the focal or non-focal group moments earlier.

The order and timing of these behaviors and movements varied and likely depended on the circumstances of the actual feeding event. For example, tail slapping did not always follow putative corralling. No cues in the movement data consistently anticipated transitions from one behavior to the next. Rather, these stages may be coordinated vocally, a topic that will be considered in Chapter 4. Carousel feeding demands a significant energetic input as evidenced by the elevated fluking energy and more variable movement measures during feeding periods (Figures 2.3, $2.11 \& 2.13$ ). The data presented here have indicated that the 
cost:benefit ratio may be even higher since corralling efforts do not always lead to actual feeding episodes. Furthermore, it is likely that carouseling should yield a larger energetic gain for killer whale groups that locate herring that have already been corralled or that are closer to the surface due to bathymetry or fish behavior since less effort is required to initiate the feeding. The extent to which turn taking or general behavioral state synchrony and different tail slap timing and energetic patterns are preferred may depend less on the killer whales and more on the immediate demands imposed by the movements of a particular fish school. This seems especially likely given that otherwise distinct diving profiles produced by the two synchronously tagged animals became more similar when preceding their closely spaced tail slaps (Figure 2.5, see earlier discussion).

Carousel feeding by Norwegian killer whales shares features common to group foraging behaviors documented in other animal species. First, their prey source is abundant: a vast amount of herring biomass entered the fjords each winter (Similä et al., 1996). Secondly, the herring possess the advantages of greater maneuverability and acceleration compared to the killer whales (Domenici et al., 2000; Domenici, 2001). These two characteristics of the prey field make carousel feeding an especially productive strategy for the orcas since as a collective, they can exploit a food source that is otherwise difficult to capture. The benefits of group foraging have likely contributed at least partially to the development of the highly social nature of these animals, offsetting the benefits of a more solitary lifestyle.

Together, these results point out that carousel feeding is dynamic and opportunistic, requiring animals to secure herring at depth or closer to the surface, herd fish schools that may or may not have been already corralled by another group, and ensure the spatial 
integrity of the ball for as long as feeding is taking place. The animals worked frequently to corral fish (both unsuccessfully and successfully) into a preferred configuration, presumably organizing their behaviors to avoid interfering with one another when taking advantage of tail slapping and feeding opportunities . Although the putative corralling behavior was not evident from visual observation alone because it either occurred at depth or resembled feeding at the surface, the tag movement data helped to distinguish this behavior. The common movement and focal tail slapping patterns observed across animals during putative feeding periods implied that the tagged whales all participated in the carousel and received an opportunity to eat.

To ascertain whether carousel feeding is truly cooperative, however, more work is required to demonstrate that all of the animals in a group contribute consistently to the foraging effort and that each individual is eating. This study strongly motivates future tagging studies of Norwegian killer whales combined with simultaneous sonar or video observations of herring to characterize the dynamics between predator and prey. Longer, synchronous tag deployments would allow comparisons of individual performance across multiple carousels to gain insights into the likelihood of role playing and behavioral stereotypy. Synchronous tagging on multiple animals combined with prey mapping would allow an investigation of how killer whales both distribute themselves in 3D space to contain the herring ball and respond as a group once the herring manage an escape and the ball dissipates. It would allow further exploration of whether turn taking or synchrony of group members is occurring. A comparative tagging study of bubble net feeding humpback whales and carousel feeding killer whales could offer insights into the elements of convergence between these two animal systems that forage in groups on herring. It would highlight both 
the unique strategies deployed by each species and the constraints imposed on the collective by certain biological and physical aspects of the feeding paradigm.

\subsection{Acknowledgements}

Peter Tyack, Andy Solow and Patrick Miller provided substantive and iterative assistance with the development of the analysis and exposition of this chapter. In addition, Patrick Miller gave me his Matlab code for computing the fluking intensity and he verified the calibrated PRH files for all of the whales, considering both the raw movement data and field observations to inform his perspective. Sanna Kuningas offered her field notes to help reconstruct the movement and behavior of these animals. Special thanks to Peter Tyack and the MIT Student Assistance Fund for supporting the presentation of this work at the $30^{\text {th }}$ International Ethological Congress in Halifax, Canada in August 2007. 


\section{Chapter 3. Everything but the Cetacean SynCh: An Example Driven Discussion of BeHavioral Association in Marine MAMMALS}

\subsection{Manuscript}

A relationship can be defined as a sequence of interactions between a pair of individuals that recognize one another (Hinde, 1976; 1979). They are characterized by the character and patterning (i.e., the timing and rates) of these interactions and rely on the participation of both partners (Hinde, 1976). It has become common in animal behavior to define an association index as a helpful tool that uses the frequency of specific interactions and behaviors to indicate the strength of a social relationship. Indeed, animals that interact rarely do not generally have much of a relationship but those that interact more frequently are predicted to share a strong relationship.

In the marine mammal literature, association is defined operationally as the sighting of two or more individuals at the same time and in the same place. Field work and logistical restrictions aside, association parameters are ideally grounded in the biology of the study animal and the behaviors that are used to maintain social relationships. Nevertheless, the spatial and temporal scales of these association patterns vary dramatically depending on the species, the behavioral context and the research study itself. The differences in the quantification of the spatial and temporal extent of marine mammal groups illustrate how observer-biased definitions can lead to highly variable interpretations of the same social association data.

Ballance (1990), for example, assigned bottlenose dolphins (Tursiops truncatus) to the same social group if they were sighted simultaneously from the research vessel, allowing 
separation distances to range from a couple of body lengths to a few kilometers. In studies of this same species in Shark Bay, Australia, however, a 10m chain rule was adopted to define a group as all individuals within 10m of one another (Smolker et al., 1992; 1993; Mann, 2000). Both approaches reported that despite allowing the possibility of groups to contain animals separated by large distances, associating individuals generally surfaced only within a couple of meters of each other. Relevant spatial scales should depend on the sensory modality and sensitivity of the organism being studied. Bottlenose dolphin whistles propagate for much shorter distances (i.e., kilometers: Janik, 2000) than the calls of certain species of baleen whales (i.e., hundreds of kilometers), for instance, which dramatically influences the range over which social coordination can operate. Visual and tactile cues function across even tighter spatial distances.

Various time restrictions have been applied to determine grouping patterns in marine mammals as well. Some associations have been scored by the concurrent appearance of multiple surfacing individuals within single or adjacent photographic frames (e.g., bottlenose dolphins: Ballance, 1990; killer whales (Orinus orca): Bigg et al., 1990). This imposes a narrow time window on the order of a couple of seconds in which animals must synchronize their breathing to be considered associated. (Photographs grouped more broadly according to encounter do not provide sufficient data to describe or infer diving synchrony information.) As Würsig (1978) discussed, instances of non-synchronous surfacing may occur for both associated and unassociated individuals. Other experimental paradigms have relaxed these time constraints, assigning all dolphin individuals passing by an observational lookout in a single day to the same group (Würsig \& Würsig, 1977; Würsig, 1978).

Timing often depends on the behavioral context as well, however. In certain 
contexts, animals may function well as a group with more synchronized patterns of movement and breathing. Synchrony can confer certain advantages including predator avoidance and detection (Pulliam, 1973), information storage, and smarter decision-making (Parrish \& Edelstein-Keshet, 1999). In particular, synchronous surfacing to breathe was thought to decrease the probability of predation upon certain air-breathing fishes (Kramer \& Graham, 1976; Chapman \& Chapman, 1994). In contrast, groups of wild ostriches (Struthio camelus) lessened their predation risk by desynchronizing their vigilance behavior (i.e., time spent with head up, Bertram, 1980). Individual vigilance decreased as a function of group size and allowed the animals to use information gathered from the environment to improve their safety.

But synchrony can also reflect a competitive agenda. The function of synchronizing vocal output, for example, ranges from social affiliation at one extreme (e.g., vocal convergence in pair bonding budgerigars (Melopsittacus undulatus), Hile et al., 2000) to competition for reproductive access at the other (e.g., vocal contests in black-capped chickadees (Poecile atricapillus), Mennill \& Ratcliffe, 2004). Coordinated behaviors may also require individuals sharing social relationships to act in ways that are highly nonsynchronized in space or time. Indeed, Whitehead (1996) documented that the diving behavior of groups of sperm whales (Physeter macrocephalus) near the Galápagos Islands were more asynchronous when calves were present compared to groups without calves, suggesting coordination of alloparental care of calves at the surface. Desynchronizing dives increased the amount of time that at least one adult was near the calf.

Whitehead (1996) and Whitehead \& Weilgart (1991) pointed out that sperm whales tended to have two basic behavioral contexts: socializing while at the surface and foraging at 
depth. Other factors influencing behavioral context may be social, including affiliative or aggressive behaviors (e.g., roving males during the breeding season), biological (e.g., changes in the depth of the prey layer or to allow the metabolism of lactic acid accumulated from deep diving), or physical, including oceanographic properties like water temperature.

Indeed, sperm whales off the Galápagos tended to aggregate at the surface in the afternoons, perhaps in part to take advantage of shallower waters warmed by the sun (Whitehead \& Weilgart, 1991).

Synchrony among marine mammals, especially related to breathing at the surface, has been considered an indicator of social relationship (Fripp et al., 2005) and alliance membership (Connor et al., 2006). Social association has also been defined in terms of nearest neighbor identification. Association indexes that employ surfacing behaviors (see Mann, 2000 for a review) are biased in 2D since animals spend most of their time below the surface, are free to orient in any direction underwater, and may converge or spread apart at depth. Here, we illustrate the differences between and implications of social association among free-ranging Norwegian killer whales.

Digital archival tags (DTAGs) that contained hydrophones for sampling sound and an accelerometer, magnetometer and pressure sensor for sampling 3D movement were attached to each animal (Johnson \& Tyack, 2003). A VHF beacon allowed tracking of the tagged animal for the duration of the attachment. After retrieving the released tag, the timestamped movement data were calibrated to the whale's frame of reference (Johnson \& Tyack, 2003). See Table 3.1 for the tagging details associated with the experiment. In particular, tags were deployed simultaneously on two pairs of killer whales each belonging to the same social group (i.e., 4 animals in all). The movement data for the concurrently tagged 


\begin{tabular}{c|c} 
Number of animals & 2 pairs \\
\hline Date and animal id & 17 Nov 2005: \\
& $\begin{array}{c}\text { oo05_321a \& oo05_321b; } \\
\text { 18 Nov 2005: } \\
\text { oo05_322a \& oo05_322b }\end{array}$ \\
\hline Study site & Tysfjord, Norway \\
\hline Sttachment mechanism & carbon fiber hand pole \\
\hline Tagout duration $(\mathrm{h})$ & $4.2 ; 2.1 ; 3.6 ; 3.1$ \\
\hline Additional visual data & 50 \\
\hline Time interval threshold ${ }^{*}$ & annotated rough \\
\hline Depth threshold $(\mathrm{m})^{*}$ & behavioral state of group \\
\hline
\end{tabular}

Table 3.1. Tagging and analysis details. Tagout durations proceed in the same order as the listing of animal identification labels. The last two rows marked by asterisks contain inter-surfacing time interval threshold between individuals and the depth threshold used to determine whether animals surfaced together.

animals were aligned as closely as possible using the tag time stamps that were each set to GPS time before every deployment. The half-weight index (HWI: Cairns \& Schwager, 1987; Bräger et al., 1994) was used to compute coefficients of association between the pairs or trios of whales during the time ranges depicted in Figure 3.1. In the equation:

$$
\mathrm{HWI}=2 \mathrm{~N} /\left(n_{1}+n_{2}\right),
$$

$2 \mathrm{~N}$ is the total number of joint sightings counted once for every appearance of the two individuals together, $n_{1}$ is the total number of sightings for one individual and $n_{2}$ is the total for the other individual. The surfacings of two individuals were considered coincident if they occurred within a set time interval and a new surfacing was only counted after the animals had each dived beyond a set depth threshold. These thresholds are listed in Table 3.1.

Norwegian killer whales travel in matrilineal groups and forage cooperatively on herring in a group behavior called carousel feeding (Similä \& Ugarte, 1993; Similä, 1997; Domenici et al., 2000; Simon et al., 2005). In a series of coordinated maneuvers, they corral the fish into a ball at the surface, tail slap to incapacitate the fish and eat them one by one. 
a: killer whale double tagout: traveling

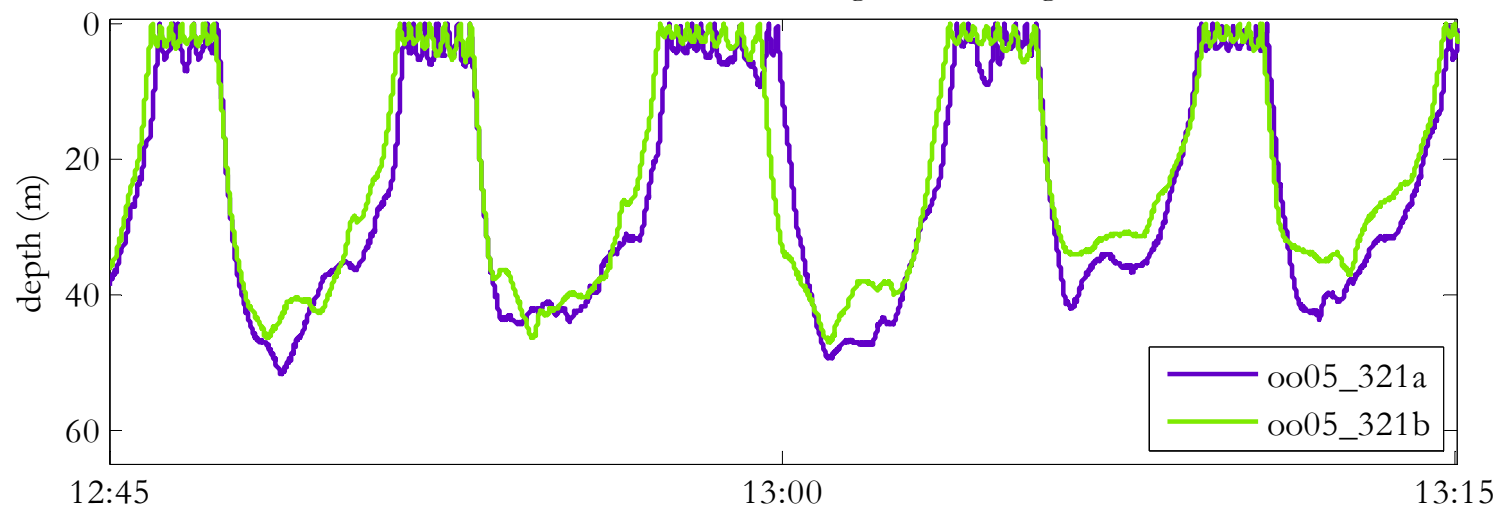

b: killer whale double tagout: carousel feeding

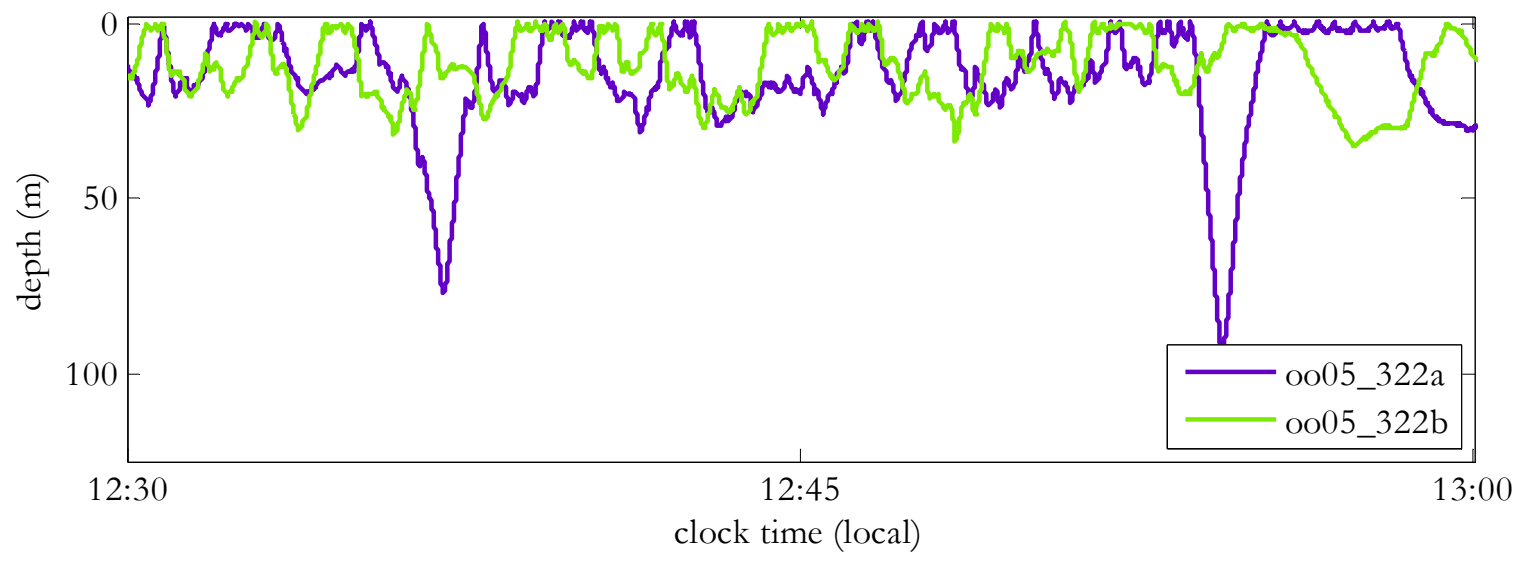

Figure 3.1. Pairs of dive profiles from simultaneously tagged Norwegian orcas belonging to the same social group. Top: Two animals traveling at opposite ends of a coherent group, 17 November 2005. Bottom: Two killer whales carousel feeding, 18 November 2005.

The sequence of traveling and foraging behaviors is sufficiently well defined in killer whales to allow us to ask whether the degree of synchrony or coordination displayed by a group of related animals can be viewed as a stable indicator of their social relationship or whether synchrony may vary as a function of the requirements of different behavioral states. The dive profiles of two pairs of killer whales tagged within two social matrilineal groups were compared. On 17 November 2005, a small juvenile whale (oo05_321a) and an adult female (oo05_321b) were tagged in a group of $\sim 16$ killer whales. At the end of the follow, all of the 
animals in the group were traveling together steadily to the northwest. The tagged individuals swam at opposite ends of this group for a portion of the time that they were simultaneously tagged (102 minutes). Figure 3.1a plots the depth profiles of these two animals during 30 minutes of this traveling period $(\mathrm{HWI}=0.19)$. These animals surfaced and dove in a fairly synchronized fashion. The deep portion of their dives tended to diverge more (i.e., oo05_321b tended to dive shallower than oo05_321a) than their more overlapping descents and ascents. Although their surfacing and diving synchrony might suggest close social association, they were never each other's nearest neighbor when observed at the surface. Therefore, the use of the nearest neighbor metric of association would have missed the 3D coordination and interactions of these groups. Nearest neighbor data may be useful, however, when discerning the relative importance of individual relationships among more fluid group structures such as those found in bottlenose dolphin communities.

On 18 November 2005, an adult female (oo05_322a) and adult male (oo05_322b) were tagged in a group of $\sim 15$ carousel feeding animals. The tags recorded data simultaneously for 140 minutes; 30 minutes of their overlapping dive profiles are presented in Figure 3.1b $(\mathrm{HWI}=0.11)$. In contrast to oo05_321 a and oo05_321b, the foraging efforts of oo05_322a and oo05_322b did not result in consistently synchronized diving, even as they used tail slaps to feed on the herring ball. We therefore observed highly synchronous diving behavior by the two animals that were traveling steadily (Figure 3.1a), which differed from the asynchronous diving by the two individuals that were coordinating with their group to carousel feed (Figure 3.1b). The HWI was higher for the two traveling animals than the two carousel feeding whales. The shallower depth and shorter time interval thresholds used 
for the four killer whales contributed to their low HWI scores.

While the differences in synchrony and HWI of the two pairs of tagged killer whales may have been a function of the individual differences in the strength of social relationship among the tagged whales, differences in the behavioral state (i.e., traveling versus carousel feeding) could just as likely be the primary influence. To maintain contact and cohesion during travel, it is likely that synchronized vertical movement through the water column was useful to the group since vocal activity was largely absent. The similar breathing frequencies of oo05_321a and oo05_321b at the surface (assuming that every depth zero-crossing corresponds to a single breath) also implies comparable oxygen demands due to diving to similar depths.

The movement and behavioral demands of carousel feeding on a group are different than traveling: the animals must continuously and dynamically preserve the integrity of a ball of herring while individual killer whales tail slap and consume fish from its periphery. The dive records in Figure 3.1b reveal certain portions that were out of phase with each other and fewer portions that were in phase. If members of a group were to dive synchronously all of the time, the fish might be able to take advantage of the absence of whales at a particular depth to escape. Instead, the orcas appear to work different depths to their advantage, trapping the herring against the surface and preventing their lateral dispersal by taking turns at depth. Breathing requires an orca to position itself at the surface because if all group members took a breath synchronously, the fish would have a greater opportunity to escape.

It is important to exercise caution when making inferences about the social implications of behavioral synchrony. For example, one might assume that individuals 
oo05_321a and oo05_321b maintain a closer social bond than oo05_322a and oo05_322b based on a higher HWI and the extent to which they synchronize their diving. It is worth considering the possibility that the behavioral regime and context may be the principal factor affecting the synchrony of diving patterns of these three whale species. Evidence of synchrony may indicate individual or group relationship but an absence of synchrony does not demand an absence of social relationship: it could suggest that a different kind of behavioral coordination is present. Indeed, HWI alone reflects a $2 \mathrm{D}$ temporal relationship that does not necessarily depict the extent to which two animals may be coordinating their behaviors spatially and associating in 3D.

Figure 3.2 plots HWI as a function of the time interval considered to constitute a synchronous surfacing between animals. Naturally, as the time interval grew and additional surfacings separated by longer periods of time were considered synchronous, the HWI increased incrementally. The magnitude of the change in this step size depended on the total number of dives considered in the calculation and the spread of the inter-surfacing intervals. Killer whales oo05_322a and oo05_322b, for example, surfaced numerous times with a wide range of inter-surfacing intervals and therefore displayed short step lengths in Figure 3.2. This figure shows that HWI in this dataset is sensitive to the time interval selected and that certain diving regimes may be more tolerant to flexibility in selecting this interval. It is useful to examine such a plot to ascertain the stability of the HWI value.

The behavior-dependent diving synchrony or asynchrony for the species described here demonstrated that social bonds can be expressed in a variety of ways. Animals that cluster at the surface may associate affiliatively in 3D or they may disband underwater and 


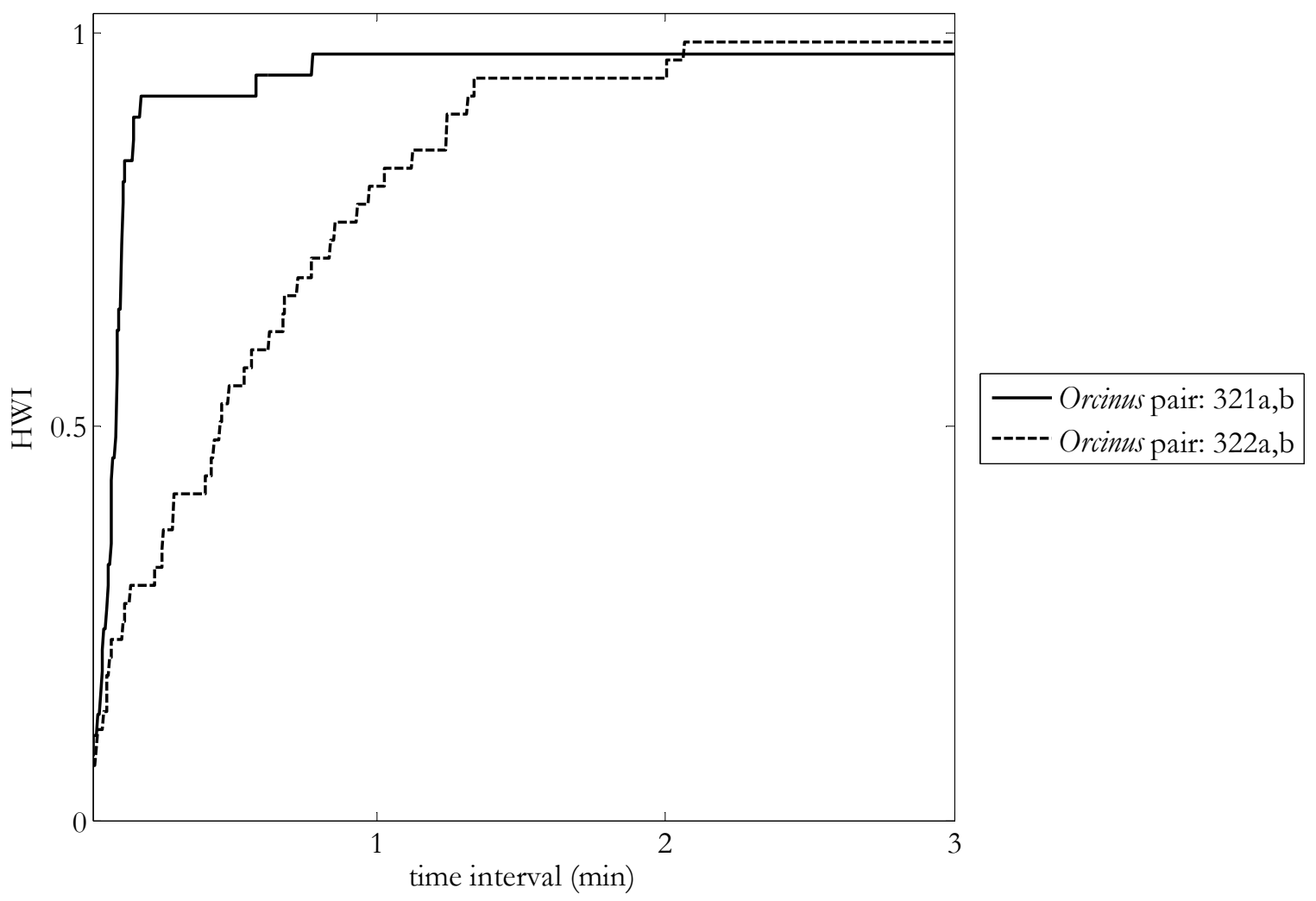

Figure 3.2. Half-weight index (HWI) as a function of the time interval considered to be a synchronous surfacing for each pairing of animals in Figure 3.1.

function either independently or cooperatively. Animals that do not cluster at the surface may still coordinate their independent behavior through vocal communication or turn taking (e.g., Whitehead, 1996). Visual association indexes may have made the best of incomplete observations of marine mammals but we are no longer limited to these opportunistic sightings of surfacing individuals. New electronic data collection tools like the DTAG are yielding movement and behavioral data that permit direct measurement of vocal communication and continuous social association indexes. These metrics are more relevant to the animals, rendering realistic aspects of their socially complex behaviors accessible to study. 
Tagging multiple animals will permit an understanding of whether certain behavioral contexts better predict synchrony of movement. Tagging studies can be combined more with traditional photographic methods of computing association indexes, which would help determine whether these two techniques can function independently. It is likely, however, that the two will inform one another as the photography can complement the tag data, offering nearest neighbor information and helping subsequently to synchronize the timing of the multiple tag records. In addition, it might be possible to connect certain surfacing behaviors with corresponding 3D association patterns and vice versa. When designing these studies, it is important to consider 1) the social and behavioral implications of the presence or absence of synchrony, association and coordination; 2) the possibility that behavioral coordination may or may not lead to synchronization; and 3) the ways in which individual or groups of marine mammals engage in simultaneous behavior either as a result of encountering and reacting to a common stimulus or to achieve a shared goal. These elements will all help to provide information on the strength of social relationships between individuals within a population.

\subsection{Acknowledgements}

We are grateful to Tiu Similä, Geoff Magee, Mads Christoffersen, Mike deRoos, Andy Foote, Petter Helgevold-Kvadsheim, Jesper Jensen, Sanna Kuningas, Michi Main, Patrick Miller, Sari Oksanen, Filipa Samarra and Outi Tervo who helped to collect the killer whale data. P. Miller was supported by a fellowship from the Royal Society. We thank Mark Johnson, Tom Hurst, Alex Bocconcelli and Alex Shorter for providing tag and logistical support. This work was funded by the Ocean Life Institute of the Woods Hole 
Oceanographic Institution, the National Geographic Society, WWF Sweden and Discovery Initiatives. 


\section{Chapter 4. RELATIONSHIP BETWEen MOVEMENT AND Vocal BEHAVIOR of FreE-RANGiNg NorWEgian KILlER WHALES}

\subsection{Abstract}

Though the rates of killer whale pulsed calls fluctuate according to behavior state, the individual call types are generally considered to be functionally interchangeable. I test this assertion using individual movement data and group calling sequences recorded with digital archival tags attached to free-ranging Norwegian killer whales. Twelve animals were tagged and seven of these engaged in carousel feeding, a vocally active time when herring were trapped at the surface, tail slapped and eaten. On 4 occasions, carousel feeding was preceded by a vocally active period of putative corralling involving broad looping presumably to locate herring at depth. In contrast, in 2 of the 3 instances in which carouseling was anticipated by directional travel, the animals were silent, suggesting that they may have eavesdropped to locate conspecific groups that were already feeding on herring at the surface. The recordings were then divided into two general behavioral states: tail slapping (TS) periods that coincided with carousel feeding activity and periods with no tail slapping (NTS). I predicted that killer whales depended on orientation cues more during carousel feeding than other behaviors. The relative differences in level between the low and high frequency components of pulsed calls may provide such an orientation cue of the signaler to the receiver. My prediction was confirmed using a rotation test that preserved the serial dependence of the original data: more calls characterized by both components than the low component alone were produced during TS than NTS episodes in 5 of the 7 whales. These results were consistent with the top three call types contributing to the significant differences in the rates of call type production between TS and NTS periods in all but one of 
the whales. Collectively, these results are consistent with the premise that Norwegian killer whales use their vocalizations to provide information to conspecifics about their $3 \mathrm{D}$ position and orientation as they corral and feed in groups. No significant relationship was found between call type and the actual measurements from the movement sensors. Additional data collected across multiple days and carousels are required to explore the possibility of more detailed relationships between movement and vocal behavior.

\subsection{Introduction}

A classical ethological approach suggested that much of animal communication involves a linked system of inherited motor patterns that generate signals with specific functions coupled with sensory mechanisms in the receiver to detect, classify and respond appropriately to the signal. Following this approach, many ethologists attempt to link single vocal categories to specific behavioral contexts of the signaler and specific behavioral responses of conspecifics within earshot. Some marine mammal studies report strong links between signaling, context and response. In southeast Alaska, groups of humpback whales (Megaptera novaeangliae) produce a series of cries that crescendo to one especially loud cry that immediately anticipates their coordinated surfacing and lunge feeding on herring (Clupea harengus) (D’Vincent et al., 1985; Cerchio \& Dahlheim, 2001). It is possible that the humpback whales use these cries to synchronize the endgame of their foraging sequence. Icelandic killer whales (Orcinus orca) likely herd herring using a loud I36 pulsed call (Simon et al., 2006). The fundamental frequency of this call resembles the resonant frequency of the herring swim bladder and the frequency of maximum hearing sensitivity in herring. Thus, whereas the humpbacks appeared to use their cries to coordinate the behavior of 
conspecifics, the killer whales seemed to exploit the physical properties of their call to handle their prey more effectively.

Because of the traditional difficulties associated with acquiring continuous behavioral observations of individual marine mammals, the functional links between their behaviors and many of their vocalizations were, until recently, generally not as apparent. One common analytical approach involved dividing the behavioral sequence into a series of states and determining whether these states were characterized by a reliable subset of vocal classes (e.g., Atlantic spotted dolphins (Stenella frontalis) and bottlenose dolphins (Tursiops truncatus), Herzing, 1996). Links between the function and context of vocal behavior have improved substantially using digital archival tags that simultaneously record movement and acoustic data. Northeast Pacific blue whales (Balaenoptera musculus), for example, produced singular D calls frequently and singular B calls occasionally during daytime foraging dives, which suggested a function relating to feeding (Oleson et al., 2007a; 2007b). Blue whale song, however, was produced at dawn and dusk exclusively by males, implying a reproductive purpose.

Extensive evidence of vocal production learning among marine mammals raises questions about the interpretation of communication in these species. During the 1960s, ethologists recognized that oscine songbirds do not inherit the motor patterns that generate their songs, but rather need to hear songs to produce the typical songs of their population. These birds have evolved neural mechanisms that enable more flexibility in vocal development in which communication signals produced by males can be influenced by both what the male hears and feedback from females.

As has been demonstrated for many reproductive advertisement displays, sexual 
selection can lead to the elaboration of complex displays. One form of complexity of bird song involves song repertoires where one male may sing many different songs. Females of some species prefer males with larger song repertoires and in these species selection favors males with the capacity to remember and produce many songs. Early reports of song repertoires assumed that songs were interchangeable and functionally equivalent (see Krebs \& Kroodsma, 1980; McGregor, 1991). Subsequent investigation of communication between males, however, uncovered that males may select specific songs from their repertoires to modulate aggressive or territorial interactions. If a territorial male matches the song of a neighbor, this is likely to escalate the fight On the other hand, if a male responds to a neighbor with a different song, but one that these two birds shared in common (male song sparrows (Melospiza melodia), Beecher et al., 1996), this "repertoire matching" can help to prevent the interaction from escalating into a fight.

Like songbirds, killer whales have been reported to imitate sounds (Bowles et al., 1988; Foote et al., 2006) and members of each killer whale group produce a repertoire of shared calls. Most research on these stereotyped calls has largely concluded that the different calls in the repertoire are contextually and functionally equivalent. Ford (1989) found no evidence that different call types were produced reliably according to behavioral state by resident fish-eating killer whales in the Pacific Northwest. The overall rate of vocalization of killer whales may vary by behavioral context. Vocalization rates tended to climb during periods of high arousal (Bain, 1986), whereas calling activity generally fell to low levels or zero during periods of resting (Hoelzel \& Osborne, 1986; Ford, 1989). Transient killer whales must additionally remain silent as they forage to prevent alerting their marine mammal prey whose hearing is sensitive in the frequency range of pulsed calls 
(Deecke et al., 2002). During the other behaviors of surface activity, slow travel and milling after a kill, no difference in call type usage by the transients was observed (Deecke et al., 2005).

There is some evidence for differential usage of call types in different contexts. Some call types may be used more frequency during intra-pod meetings (Miller \& Bain, 2000). Van Opzeeland et al. (2005) explored the vocal behavior of Norwegian killer whales during two types of foraging. Seiner feeding involved orcas feeding on herring discarded from fishing boats. Carousel feeding, by contrast, referred to the efforts of a group of whales that corralled herring from depth, trapped them in a tight ball against the surface and tail slapped the edge of the ball to stun the fish before eating them one by one (Christensen, 1978; Similä \& Ugarte, 1993). N21 was the only call type that showed statistically-significant differential usage as it was produced more frequently during seiner than carousel feeding (Van Opzeeland et al., 2005). The sample sizes were somewhat unbalanced, however, since only 2 seiner feeding events were observed relative to 16 carousel feeding episodes. Another potential confounding factor of this analysis was the difference in call repertoires between pods. Each Norwegian killer whale pod vocalizes 3 to 16 call types (Strager, 1995). These repertoires can overlap but it is possible that the groups might produce shared call types in different contexts or proportions.

Killer whale pulsed calls are often characterized by two simultaneously-produced, yet independently-modulated components: a low frequency component (LFC) occurring between $80 \mathrm{~Hz}$ and $2.4 \mathrm{kHz}$ (Ford, 1987) and a high frequency component (HFC) ranging from 2 to $12 \mathrm{kHz}$ (Hoelzel \& Osborne, 1986). These components differ in terms of their directionality as well. The HFC is beamed forward from the melon of the animal whereas 
the LFC is less directional (Miller, 2002). When Miller (2002) recorded directly in front of a vocalizing animal in the wild, the calls contained roughly equal levels of LFC and HFC energy. When recording from behind the animal, however, the HFC was considerably softer or absent altogether. Combined with other spectral characteristics (Miller et al., 2007), the relative proportion of energy between the LFC and HFC may allow the signaler to provide conspecifics with orientation cues. In addition, the peak energy ratio between the first and second harmonics of the LFC was significantly greater for calls vocalized by adult female versus adult male killer whales (Miller et al., 2007). These somewhat subtle details of pulsed calls may constitute salient elements to which conspecifics attend and might be related to the use of certain call types over others in different behavioral contexts.

Much of the earlier research has supported a null hypothesis that killer whale call types are interchangeably produced independent of behavioral context. Analyses incorporating the categories of call types being produced and more detailed representations of the movements of the animals, however, may help reveal the relationships between vocal and non-vocal behavior. Using data gathered from digital archival tags, this chapter explores the possible relationships between the individual movements and group vocal behavior of free-ranging Norwegian killer whales. I specifically query whether call types are interchangeable at both coarse and fine time and categorization scales.

Killer whale call types do not appear to be generated as a string of independent events. Often, for example, call types tend to be repeated in series (Ford, 1989; Miller et al., 2004c). One of the primary methodological difficulties is dealing with the serial dependence of these sequences, or the tendency for the occurrence of a call type to depend on the preceding call type. Because serial dependence renders invalid standard statistical tests that 
treat each call as an independent event, I instead took advantage of non-parametric rotation tests and parametric bootstraps using fitted Markov chain models

At first, this analysis parallels earlier studies by dividing the dataset into behavioral states. The tail slapping and not tail slapping states were defined based on the acoustic and movement signatures of tail slaps identified in Chapter 2. It considers broad divisions of calls according to spectral characteristics as well as a more specific treatment of the individual call types. Secondly, I take advantage of the detailed movement sensor data to test whether call type can serve as a reliable predictor of movement features and/or whether particular movement data regimes can anticipate certain call types dependably.

\subsection{General materials and methods}

Field work was conducted aboard a sailing vessel and rigid hull inflatable boat in November 2005 and 2006 in Tysfjord and Vestfjord in northern Norway. Digital archival tags were attached to 14 free-ranging killer whales using a handheld $7 \mathrm{~m}$ carbon-fiber pole (Johnson \& Tyack, 2003). These tags recorded movement data at $50 \mathrm{~Hz}$ and sound data at $96 \mathrm{kHz}$, released from the animal after a designated period of time and were collected for data offload and subsequent redeployment. All sensor and audio files were burned to CD. The sensor data were calibrated and converted to the whale's frame of reference (pitch: $-90^{\circ}$ $=$ pointing downwards and $+90^{\circ}=$ pointing upwards; roll: $0^{\circ}=$ dorsal side up and $180^{\circ}=$ ventral side up; heading: compass bearing). The pressure sensor provided depth measurements. Several measurements were derived from the calibrated movement data including fluking intensity (calculated as the mean square of the filtered z-axis of the accelerometer: Miller et al., 2004b; Hooker et al., 2005; Chapter 2) and derivatives of roll, 
depth (i.e., vertical velocity) and pointing angle (a variable that combined the pitch and heading data to compute the 3D orientation of the whale's longitudinal axis: Miller et al., 2004a). A linearity index (LI), a path-based measure of the inverse tortuosity of the horizontal pseudo-track, was calculated according to the method described in Chapter 2. Briefly, a window was slid along the record and for each section of track, the shortest distance between the beginning and ending points was divided by the length of the full path traversed by the whale (see Wilson et al., 2007). This ratio was concerned with the relative difference in scale between these two quantities and not the actual distance measures, which were not known. The values ranged from 0 , or absolute circuitousness, to 1 , or straight line travel.

The audio records were scanned manually and all acoustic events (i.e., tail slaps, stereotyped and variable pulsed calls, echolocation clicks, etc.) were documented. Three naïve observers and I sorted the pulsed calls into different categories by visual inspection of the spectrograms. Call types were labeled according to earlier catalogues (Strager, 1993; Van Opzeeland et al., 2005) or given new numbers if they had not been described previously. (See Chapter 5 for a more detailed description of assigning calls to type.) Table 4.1 lists which call types occurred in each recording and Table 4.2 offers the duration and mean frequency data for all call types measured by the contour tracing described in Chapter 5. Calling bouts were defined as periods of time containing at least 10 calls occurring within 5 minutes of one another. The movement record of one of the whales(oo06_324s) could not be calibrated and the audio record of another one of the whales (oo05_321b) did not contain any vocal activity. Because the analysis here required both the movement and vocal data streams, these two tagouts were jettisoned from the analysis, leaving 12 whales and 
recordings that totaled 30.6 hours of data.

\subsection{Analysis 1: State-dependent models}

Previous research has relied on observations of surface activity to segment killer whale activity into general behavior states (Bain, 1986; Hoelzel \& Osborne, 1986; Ford, 1989; Deecke et al., 2005). State-dependent models assume an ability to define most or all of the important phases of a particular time course, which would correspond here to the behavioral states of the tagged animal. Paralleling earlier studies, then, this section examines whether there is any evidence for state-dependent usage of calls. I first examined whether the presence or absence of calling activity is associated with particular movement features and then with two specific feeding-related behavioral sequences. Next, I tested for differences in call type usage based on the functional benefit of cueing orientation during carousel feeding.

\subsubsection{Methods}

Two behavioral states were identified based on the occurrence of tail slaps in the audio record (see Chapter 2 for complete details). Tail slapping (TS) periods contained at least 3 tail slaps that were produced within 2 minutes of one another. To exclude episodes of tail slapping that were conducted entirely by a non-focal group, TS periods included at least one tail slap that was produced by the tagged focal animal. All other segments of the record were considered not tail slapping (NTS) periods. Because tail slapping occurred during carousel feeding, TS episodes were considered representative of foraging behavior. NTS periods, however, likely encompassed a wide array of behaviors that excluded 
Table 4.1. Occurrence of call types in different recordings (continued onto next page). Yellow cells indicate call types that were documented in 3 or fewer recordings.

\begin{tabular}{|c|c|c|c|c|c|c|c|c|c|c|c|c|c|c|}
\hline call type & $316 \mathrm{a}$ & $320 \mathrm{a}$ & $320 \mathrm{~b}$ & $321 \mathrm{a}$ & $321 \mathrm{~b}$ & $322 \mathrm{a}$ & $322 \mathrm{~b}$ & $324 \mathrm{a}$ & $313 \mathrm{~s}$ & $314 a$ & $314 \mathrm{~s}$ & $317 \mathrm{~s}$ & $324 s$ & $327 \mathrm{~s}$ \\
\hline 8 & 2 & 9 & 37 & 12 & & 28 & 23 & & 16 & & 4 & & & 59 \\
\hline 9.2 & & & & 1 & & & & & 27 & & & & & \\
\hline 10 & & 27 & 30 & 2 & & 99 & 41 & & 5 & 3 & 3 & & 2 & 1 \\
\hline 10.3 & & & & & & & 1 & & & & 10 & & & \\
\hline 12.1 & 337 & 449 & 125 & 1 & & & & 32 & & 3 & & & & \\
\hline 15 & & & 7 & 4 & & 9 & 17 & 1 & 2 & & 1 & & & 7 \\
\hline 16.1 & 81 & 193 & 98 & 9 & & 1 & 1 & 2 & 36 & 2 & 11 & & & \\
\hline 21 & 62 & 116 & 43 & & & & & 1 & & & & & & \\
\hline 23.2 & & & & & & 87 & 129 & & & & 2 & & & \\
\hline 23.3 & & & & 4 & & & & & 2 & & 4 & & & 10 \\
\hline 26.2 & & & & 12 & & & & 1 & 68 & & 1 & & & 2 \\
\hline 32 & 229 & 247 & 42 & & & & & & & & & & & \\
\hline 32.2 & 3 & 2 & 4 & & & & & & & & & & & \\
\hline 45 & 46 & 64 & 35 & 2 & & 11 & 7 & 13 & 4 & & 4 & & 1 & 1 \\
\hline 64 & 137 & 150 & 27 & & & & 3 & 2 & 19 & & & & & 2 \\
\hline 65 & & & & & & 11 & 14 & & & & & & & \\
\hline 66 & 245 & 99 & 29 & & & & & 16 & 1 & & & & & \\
\hline 66.2 & 2 & 1 & 2 & & & & & & & & & & & \\
\hline 67 & 189 & 70 & 18 & & & 3 & & 1 & 13 & 2 & & & & \\
\hline 68 & & & & & & 5 & 16 & & & & & & & \\
\hline 69 & 43 & 63 & 8 & & & & & & 2 & & 3 & & & \\
\hline 69.2 & 2 & & & & & & & & & & & & & \\
\hline 71 & & & & & & 3 & 5 & & & & & & & \\
\hline 72 & & 1 & 20 & 2 & & 325 & 246 & & & 2 & 64 & 2 & & 17 \\
\hline 72.2 & & 3 & 37 & 9 & & 301 & 242 & & & 153 & 242 & 3 & 1 & 132 \\
\hline 72.3 & & 1 & 6 & & & 71 & 37 & & & 14 & 65 & & & 40 \\
\hline 73 & & 3 & 37 & & & 377 & 221 & 55 & 3 & 116 & 282 & 12 & 4 & 103 \\
\hline 73.3 & & & & & & & & & & 4 & & & & \\
\hline 74 & & & & & & & & 9 & & & 1 & & & \\
\hline 75 & & & & & & & 11 & & & & & & & \\
\hline 76 & 96 & 44 & 2 & & & 1 & & 3 & & & 1 & & & \\
\hline 76.2 & & & & & & & & & & & & & & 43 \\
\hline 77 & & & 9 & & & 88 & 67 & 24 & & 168 & 36 & 10 & 8 & 72 \\
\hline 77.2 & & & 6 & & & 8 & 10 & 1 & & & & 1 & 1 & 7 \\
\hline 77.3 & & & & & & & & & 22 & & & & & \\
\hline 78 & & & & & & 103 & 120 & & & & & & & \\
\hline 79 & & & 28 & & & & & & & & & & & \\
\hline 80 & & & & & & 95 & 150 & & 9 & & 4 & & & 5 \\
\hline 81 & & 2 & & 76 & & 15 & 128 & & 2 & 1 & 2 & & & 3 \\
\hline 81.3 & & & & & & 15 & 1 & & & & & & & \\
\hline 82 & & & & & & 9 & & & 10 & & 4 & & & \\
\hline 83 & 1 & 1 & 1 & 3 & & & & & & & & & & 19 \\
\hline
\end{tabular}




\begin{tabular}{|c|c|c|c|c|c|c|c|c|c|c|c|c|c|c|}
\hline call type & $316 a$ & $320 a$ & $320 b$ & $321 a$ & $321 b$ & $322 a$ & $322 b$ & $324 a$ & $313 \mathrm{~s}$ & $314 a$ & $314 s$ & $317 \mathrm{~s}$ & $324 \mathrm{~s}$ & $327 \mathrm{~s}$ \\
\hline 84 & & & & 19 & & 11 & 13 & & & & & & & 24 \\
\hline 85 & & 2 & & 11 & & 42 & 29 & & 12 & & 8 & & 3 & 14 \\
\hline 86 & & & 1 & & & 7 & 7 & & & & & & & \\
\hline 87 & 1 & 13 & 5 & 10 & & 4 & & & 3 & & 3 & & & \\
\hline 88 & & 13 & 1 & & & & & & & & & & & \\
\hline 89 & & 1 & 29 & 1 & & 3 & 3 & & 19 & & 3 & & & \\
\hline 90 & & & & 12 & & & & & & & & & & \\
\hline 91 & 153 & 51 & 14 & & & & & & & & & & & \\
\hline 91.2 & 2 & 1 & 4 & & & & & & & & & & & \\
\hline 91.3 & 1 & & & & & & & & & & 1 & & & \\
\hline 93 & & & & 5 & & 6 & & & & & & & & \\
\hline 94 & & & 7 & & & 3 & & & & & 1 & & & \\
\hline 95 & & & 14 & & & 1 & & & & & 11 & & 1 & \\
\hline 96 & & & & & & & & & 9 & & & & & \\
\hline 98 & & & & & & 4 & & & & & & & & \\
\hline 99 & & & & & & 2 & & & & & & & & \\
\hline 100 & & & & & & & & & & & & & & 9 \\
\hline 101 & & & & & & 6 & & & & 1 & & & & \\
\hline 102 & & & 1 & & & 3 & 1 & & & 2 & & & & 1 \\
\hline 103 & & & & & & & & & & & & 6 & & \\
\hline
\end{tabular}

successful carousel feeding.

In Chapter 2, I identified two primary behavioral sequences preceding the tail slapping and tight circling that indicated carousel feeding. Both of these sequences were classified as NTS periods. In the first sequence, carousel feeding was anticipated by vertical excursions and broad horizontal looping, a set of behaviors that strongly suggested that the animals were corralling and shoaling herring from depth (Nøttestad \& Similä, 2001; Nøttestad et al., 2002). In the second sequence, feeding occurred after a period of directional travel that involved minimal or no looping. These two sequences will be referred to as putative corralling and putative traveling, respectively. I examined the presence or absence of stereotyped calling associated with each of these behaviors.

For the next analysis, call types were divided into two general categories: those 
Table 4.2. Call type component counts, durations, mean frequencies (continued onto next two pages).

\begin{tabular}{|c|c|c|c|c|c|c|c|}
\hline \multirow[b]{2}{*}{ call type } & \multirow[b]{2}{*}{ LFC or HFC } & \multirow[b]{2}{*}{ Component number } & \multirow[b]{2}{*}{$\boldsymbol{N}$} & \multicolumn{2}{|c|}{ duration (s) } & \multicolumn{2}{|c|}{ mean frequency $(\mathrm{Hz})$} \\
\hline & & & & $\mu$ & $\sigma$ & $\mu$ & $\sigma$ \\
\hline 8 & $\mathrm{LFC}$ & 1 & 76 & 0.94 & 0.32 & 1592.27 & 315.26 \\
\hline 8 & $\mathrm{HFC}$ & 1 & 67 & 0.95 & 0.27 & 7530.44 & 820.30 \\
\hline 9.2 & LFC & 1 & 19 & 0.71 & 0.22 & 343.60 & 37.13 \\
\hline 9.2 & LFC & 2 & 19 & 0.81 & 0.17 & 2311.35 & 133.29 \\
\hline 10 & LFC & 1 & 72 & 0.62 & 0.16 & 696.39 & 247.58 \\
\hline 10 & $\mathrm{HFC}$ & 1 & 52 & 0.05 & 0.03 & 4910.92 & 406.57 \\
\hline 10 & $\mathrm{HFC}$ & 2 & 52 & 0.69 & 0.19 & 8579.36 & 1541.60 \\
\hline 10.3 & LFC & 1 & 7 & 0.72 & 0.06 & 571.15 & 75.52 \\
\hline 12.1 & LFC & 1 & 387 & 1.55 & 0.34 & 1617.06 & 292.76 \\
\hline 15 & LFC & 1 & 8 & 0.16 & 0.07 & 2040.73 & 1170.76 \\
\hline 15 & $\mathrm{HFC}$ & 1 & 1 & 0.08 & & 4770.55 & \\
\hline 16.1 & LFC & 1 & 109 & 0.69 & 0.28 & 1113.11 & 158.66 \\
\hline 16.1 & LFC & 2 & 109 & 0.24 & 0.13 & 2190.94 & 304.52 \\
\hline 16.1 & $\mathrm{HFC}$ & 1 & 99 & 0.11 & 0.08 & 5447.29 & 619.48 \\
\hline 16.1 & $\mathrm{HFC}$ & 2 & 99 & 1.03 & 0.37 & 7639.61 & 671.67 \\
\hline 21 & LFC & 1 & 104 & 1.16 & 0.23 & 970.70 & 80.12 \\
\hline 21 & $\mathrm{HFC}$ & 1 & 82 & 0.68 & 0.33 & 6912.27 & 479.28 \\
\hline 23.2 & LFC & 1 & 62 & 0.16 & 0.06 & 1919.91 & 196.84 \\
\hline 23.2 & LFC & 2 & 62 & 1.09 & 0.16 & 694.55 & 50.71 \\
\hline 23.3 & LFC & 1 & 15 & 0.30 & 0.09 & 2091.71 & 152.86 \\
\hline 23.3 & $\mathrm{HFC}$ & 1 & 8 & 0.34 & 0.08 & 8627.46 & 604.28 \\
\hline 26.2 & $\mathrm{LFC}$ & 1 & 69 & 1.11 & 0.25 & 1040.48 & 127.51 \\
\hline 32 & LFC & 1 & 226 & 0.45 & 0.08 & 2280.51 & 228.25 \\
\hline 32 & LFC & 2 & 226 & 0.70 & 0.12 & 805.70 & 69.70 \\
\hline 32.2 & $\mathrm{LFC}$ & 1 & 7 & 0.47 & 0.11 & 2011.22 & 80.46 \\
\hline 32.2 & LFC & 2 & 7 & 0.36 & 0.11 & 879.36 & 51.55 \\
\hline 32.2 & $\mathrm{HFC}$ & 1 & 5 & 0.66 & 0.11 & 8739.74 & 260.58 \\
\hline 45 & LFC & 1 & 61 & 0.09 & 0.05 & 1533.52 & 550.59 \\
\hline 64 & LFC & 1 & 161 & 0.92 & 0.27 & 639.56 & 132.99 \\
\hline 64 & $\mathrm{HFC}$ & 1 & 4 & 0.18 & 0.05 & 5492.33 & 107.90 \\
\hline 64 & $\mathrm{HFC}$ & 2 & 4 & 0.74 & 0.30 & 6284.77 & 155.52 \\
\hline 65 & $\mathrm{LFC}$ & 1 & 8 & 0.37 & 0.06 & 1209.53 & 48.52 \\
\hline 65 & $\mathrm{HFC}$ & 1 & 6 & 0.50 & 0.08 & 5442.28 & 122.40 \\
\hline 66 & LFC & 1 & 187 & 0.55 & 0.09 & 1326.60 & 85.47 \\
\hline 66 & $\mathrm{HFC}$ & 1 & 175 & 0.52 & 0.09 & 7780.92 & 249.63 \\
\hline 66.2 & LFC & 1 & 5 & 0.92 & 0.12 & 1157.29 & 35.37 \\
\hline 66.2 & $\mathrm{HFC}$ & 1 & 4 & 0.73 & 0.09 & 7706.28 & 346.20 \\
\hline 67 & $\mathrm{LFC}$ & 1 & 113 & 0.86 & 0.16 & 251.12 & 20.01 \\
\hline 68 & LFC & 1 & 1 & 0.35 & & 156.82 & \\
\hline 69 & $\mathrm{LFC}$ & 1 & 66 & 0.85 & 0.12 & 964.08 & 100.58 \\
\hline 69 & $\mathrm{HFC}$ & 1 & 65 & 0.25 & 0.15 & 5105.79 & 495.75 \\
\hline 69 & $\mathrm{HFC}$ & 2 & 65 & 0.90 & 0.12 & 6498.46 & 393.75 \\
\hline 69.2 & $\mathrm{LFC}$ & 1 & 2 & 0.50 & 0.01 & 795.43 & 3.99 \\
\hline 69.2 & $\mathrm{HFC}$ & 1 & 2 & 0.33 & 0.08 & 5414.35 & 47.34 \\
\hline 69.2 & $\mathrm{HFC}$ & 2 & 2 & 0.57 & 0.03 & 5883.39 & 76.44 \\
\hline 71 & LFC & 1 & 5 & 0.65 & 0.09 & 1918.95 & 346.32 \\
\hline 72 & LFC & 1 & 136 & 0.94 & 0.30 & 362.77 & 154.04 \\
\hline 72.2 & $\mathrm{LFC}$ & 1 & 329 & 1.12 & 0.23 & 425.41 & 129.24 \\
\hline 72.2 & LFC & 2 & 329 & 0.07 & 0.05 & 1639.42 & 515.22 \\
\hline 72.3 & $\mathrm{LFC}$ & 1 & 87 & 1.12 & 0.12 & 500.81 & 90.52 \\
\hline 72.3 & LFC & 2 & 87 & 0.04 & 0.01 & 1589.93 & 441.65 \\
\hline 72.3 & LFC & 3 & 87 & 0.04 & 0.02 & 2586.41 & 590.15 \\
\hline 73 & LFC & 1 & 538 & 0.60 & 0.14 & 1143.64 & 185.51 \\
\hline 73.3 & LFC & 1 & 2 & 0.16 & 0.06 & 711.57 & 39.34 \\
\hline 73.3 & LFC & 2 & 2 & 0.03 & 0.00 & 1730.90 & 62.52 \\
\hline 74 & LFC & 1 & 7 & 1.22 & 0.22 & 871.31 & 37.14 \\
\hline 75 & $\mathrm{LFC}$ & 1 & 1 & 0.54 & & 437.18 & \\
\hline 75 & $\mathrm{HFC}$ & 1 & 1 & 0.06 & & 4380.48 & \\
\hline 75 & $\mathrm{HFC}$ & 2 & 1 & 0.51 & & 6437.04 & \\
\hline
\end{tabular}




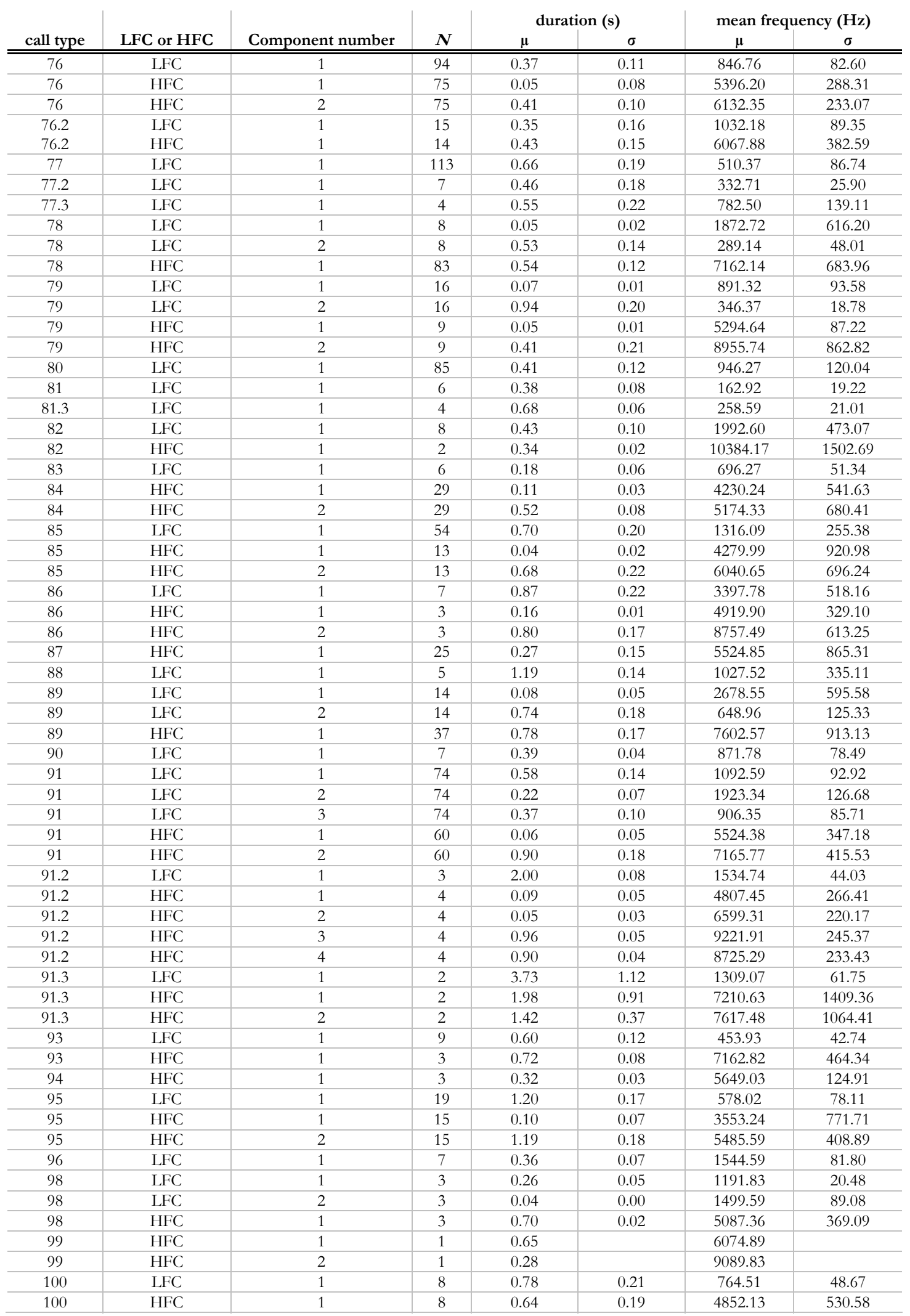




\begin{tabular}{|c|c|c|c|c|c|c|c|}
\hline \multirow[b]{2}{*}{ call type } & \multirow[b]{2}{*}{ LFC or HFC } & \multirow[b]{2}{*}{ Component number } & \multirow[b]{2}{*}{$\boldsymbol{N}$} & \multicolumn{2}{|c|}{ duration (s) } & \multicolumn{2}{|c|}{ mean frequency $(\mathrm{Hz})$} \\
\hline & & & & $\mu$ & $\sigma$ & $\mu$ & $\sigma$ \\
\hline 101 & $\mathrm{LFC}$ & 1 & 1 & 0.06 & & 847.54 & \\
\hline 101 & $\mathrm{LFC}$ & 2 & 1 & 0.18 & & 737.87 & \\
\hline 101 & LFC & 3 & 1 & 0.04 & & 1521.14 & \\
\hline 101 & $\mathrm{HFC}$ & 1 & 4 & 0.05 & 0.01 & 5007.29 & 340.19 \\
\hline 101 & $\mathrm{HFC}$ & 2 & 4 & 0.28 & 0.04 & 8786.62 & 1612.26 \\
\hline 102 & LFC & 1 & 5 & 0.07 & 0.01 & 1637.03 & 930.29 \\
\hline 102 & $\mathrm{LFC}$ & 2 & 5 & 0.34 & 0.10 & 1864.25 & 332.24 \\
\hline 102 & $\mathrm{HFC}$ & 1 & 4 & 0.06 & 0.02 & 5269.08 & 321.51 \\
\hline 102 & $\mathrm{HFC}$ & 2 & 4 & 0.33 & 0.13 & 9713.54 & 1695.25 \\
\hline 103 & LFC & 1 & 5 & 0.89 & 0.10 & 690.90 & 83.04 \\
\hline
\end{tabular}

containing only a low frequency component (LFC) and those containing both low and high frequency components together (L/HFC). Because orientation cues (see Introduction) may have been especially important as animals were carousel feeding, I expected this foraging behavior to be characterized by more L/HFC than LFC calls if they function as orientation cues. To test this prediction, I compared the kinds of call types produced during NTS versus TS periods. Of the 9 tag recordings that contained vocal activity during NTS and TS periods (the remaining 3 (i.e., oo05_316a, oo05_320a, oo05_324a) were characterized exclusively by NTS activity), only 7 were considered here. The other two (oo06_314a and oo06_317s) included almost all LFC calls with only 0 or 1 L/HFC calls.

An odds ratio was calculated from the $2 \times 2$ contingency table containing the actual tallies of LFC and L/HFC calls during NTS and TS periods. One concern with this dataset was its serial dependence. In particular, LFC calls tended to follow LFC calls and L/HFC calls tended to follow L/HFC calls significantly more often than expected by chance (as assessed by a chi-square test). To test for significance of the odds ratio and accommodate the inherent serial dependence of the data, a rotation test was conducted that rotated the calling sequence relative to the NTS and TS divisions. The odds ratio was calculated for each of 1000 such rotations and this distribution was compared to the actual value to 
determine a $P$-value.

In addition, a pooled result was tabulated across all of the whales except oo05_322b. The tag records of individuals oo05_322a and oo05_322b were not independent because they were tagged simultaneously. Animal oo05_322a was retained for the pooled analysis because of the greater power afforded by the longer recording duration (3.6 hours instead of 3.1 hours). A single $2 \times 2$ contingency table was formed by summing the data from the 6 whales. The rotation test was conducted as above except the results of each rotation were summed across the 6 whales. The odds ratio was computed for the 1000 rotations and this distribution was compared to the actual pooled odds ratio value to determine the $P$-value.

The next analysis examined differential call use more closely by exploring whether call types were produced at different rates in different behavioral contexts. A $2 \times N$ contingency table was constructed to tally the number of each of the $N$ call types that was produced during the NTS versus TS behavior states. A parametric bootstrap using a fitted Markov chain model was employed to gain more power than a rotation test would have allowed given the large number of call types. The stationary distribution was derived from the set of call type transition probability estimates. Rather than conditioning on the first call type in the actual sequences, a set of 1000 randomized realizations of the calling sequence were generated using the stationary distribution to determine the first call type for each sequence. The chi-square statistic was re-computed for each realization. Brown (1974) formulated a formal sequential approach that operated under the assumption of independence to identify sources of significance in two-way contingency tables. Because the data here were not independent, I used this test informally to determine the 3 call types that contributed primarily to a significant chi-square value. The procedure involved locating the 
cell in the contingency table with the largest deviation between the observed value and the expected value given by:

$$
E_{i j}^{*}=\left(r_{i}-a_{i j}\right)\left(c_{j}-a_{i j}\right) /\left(N-r_{i}-c_{j}+a_{i j}\right)
$$

where $r_{i}$ is the $i^{\text {th }}$ row sum, $c_{j}$ is the $j^{\text {th }}$ column sum and $a_{i j}$ is the observed value for cell $(i, j)$. After replacing the entry of this cell with $E_{i j}^{*}$, the process was repeated. Three such iterations yielded the three call types that contributed principally to a significant chi-square score (Brown, 1974).

\subsubsection{Results and discussion}

There were some general patterns that related the vocal activity and movement behavior of the animals. Focusing initially on NTS periods, movement measurements during bouts of calling activity were compared to preceding bouts that lacked calling activity. There was at least one such transition during a NTS period from a bout without calling activity to a bout with calling activity in the recordings of 7 animals (i.e., oo05_316a, oo05_320a,oo05_322a, oo05_322b, oo05_324a, oo06_313s and oo06_314a). Figure 4.1 plots the timing of stereotyped pulsed calls on the depth records for all of the whales whose records were analyzed for this chapter. For male oo06_314a, for example, the period without calling spanned $0-71$ minutes (purple horizontal bar) and the subsequent bout of calling activity occurred between 71 and 79 minutes (green horizontal bar). The remainder of the record was excluded from this particular analysis because the next phase of calling activity was paired with tail slapping (orange horizontal bar). Of the movement measures compared before and during the calling bouts for the 7 animals, calling activity bouts 
Figure 4.1. Depth records of whales with calling activity overlaid. During NTS episodes, the purple bars indicate the non-calling periods that precede and pair with the green bars that overlie the bouts of calling activity. Sections without a horizontal bar belong to NTS periods that are not part of such a pairing. Orange bars span TS episodes. For records characterized entirely by NTS, calls are plotted in yellow. For records characterized by both NTS and TS periods, LFC calls are plotted in black and L/HFC calls are plotted in maroon. (It was only for these records that this distinction was relevant for the analysis.) This coloration scheme was not applied to oo06_314a or oo06_317s because their records contained only 1 or 0 L/HFC calls, respectively.
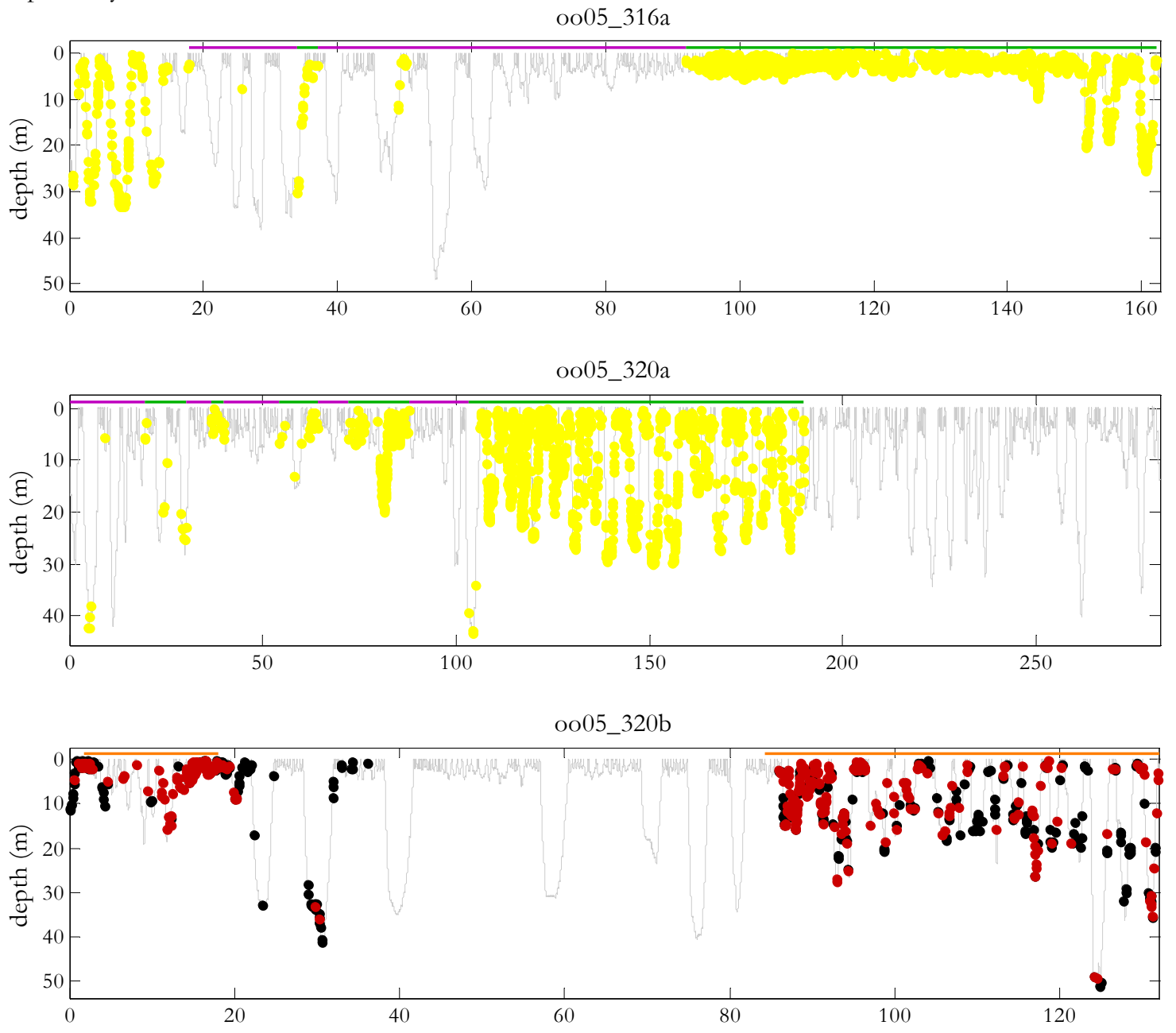

oo05_321a

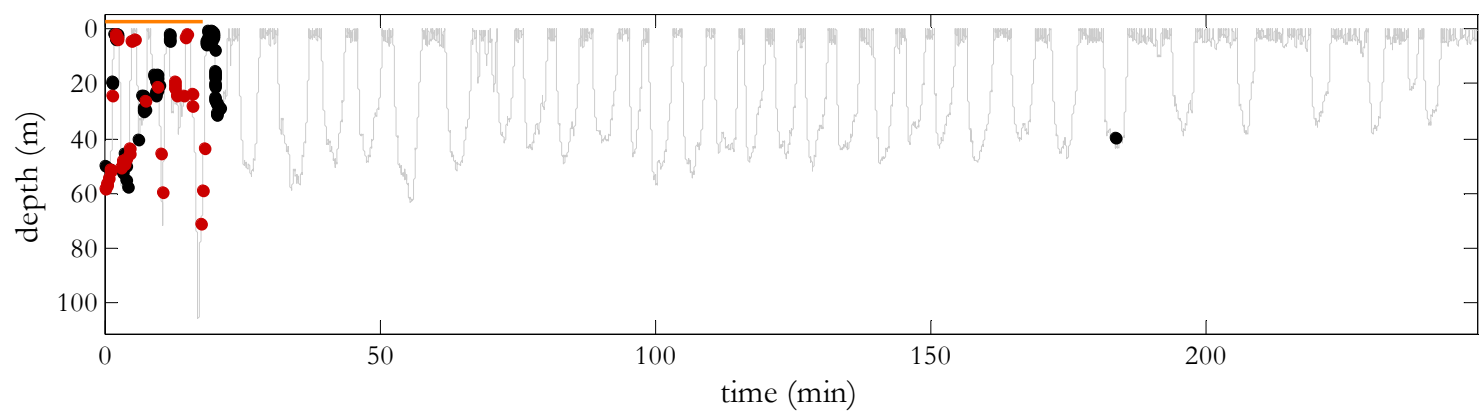


oo05_322a
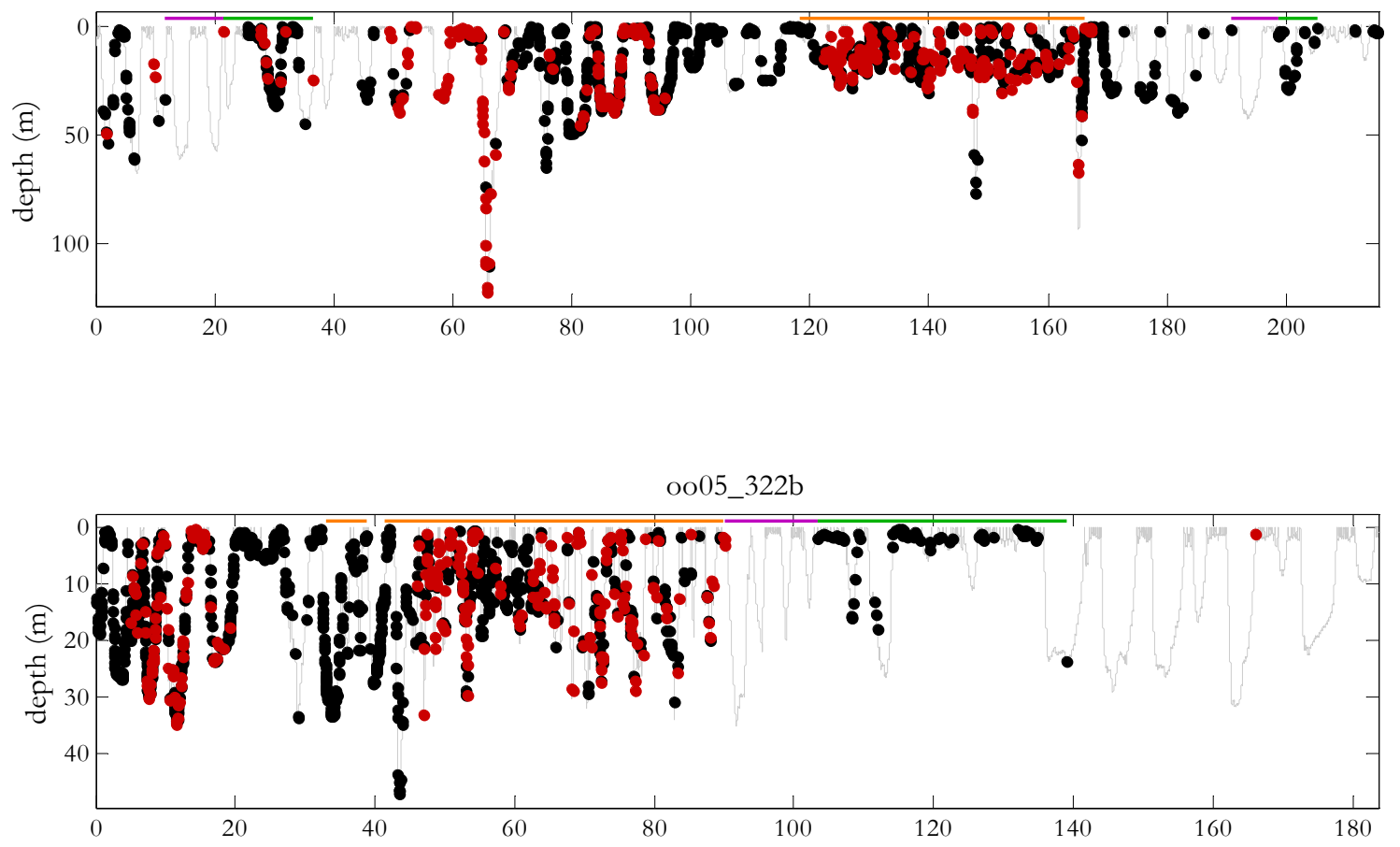

oo05_324a

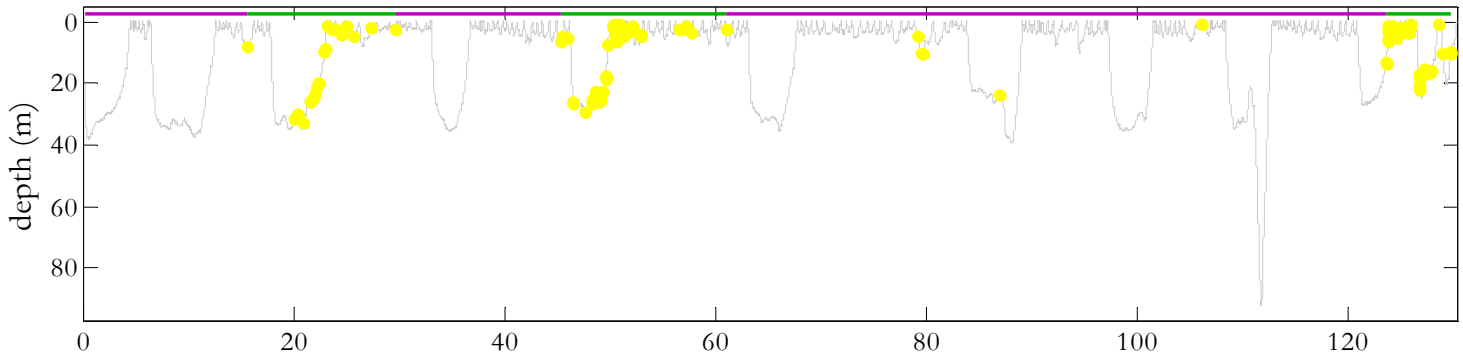

oo06_313s

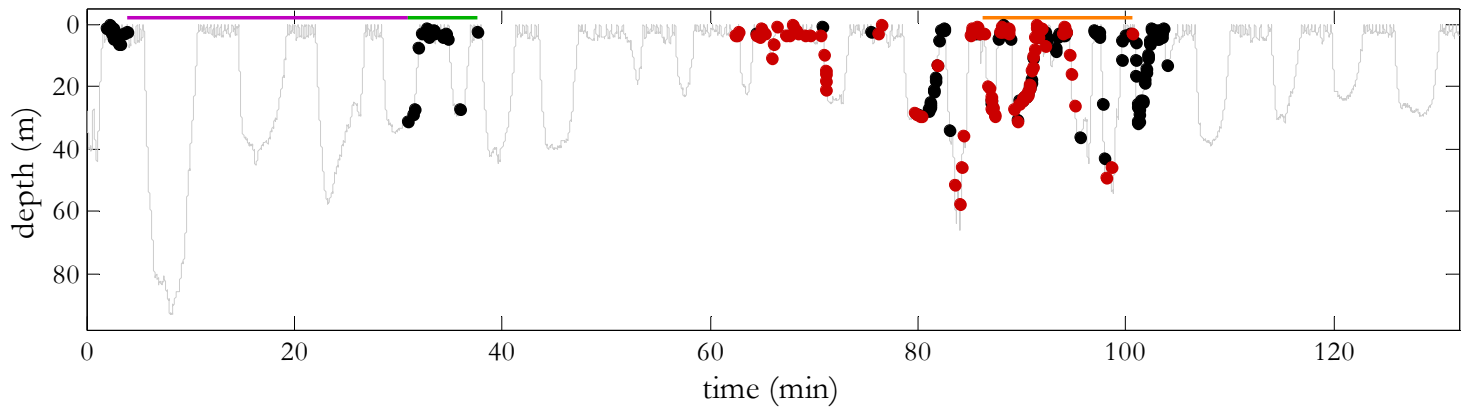


oo06_314a
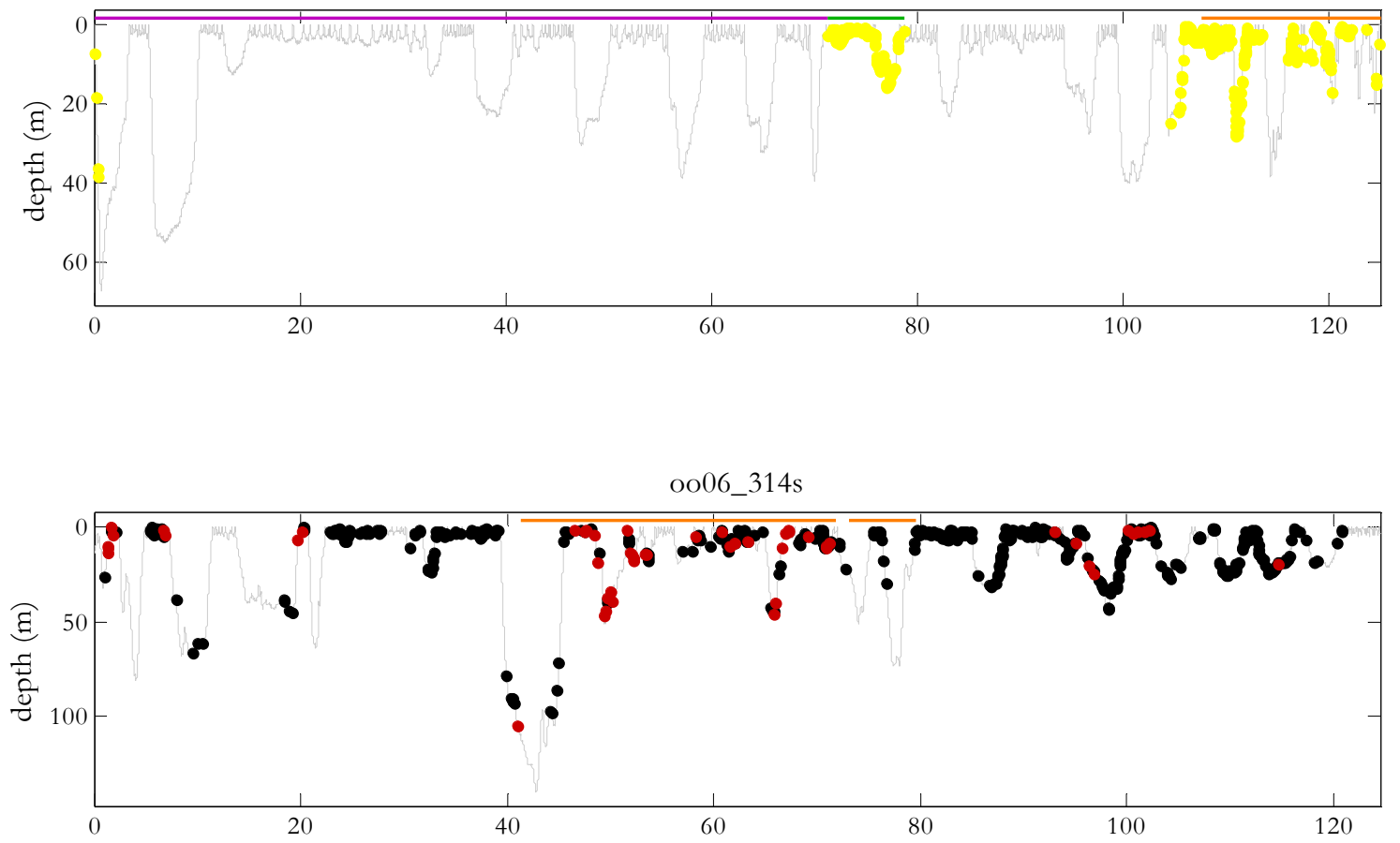

oo06_317s
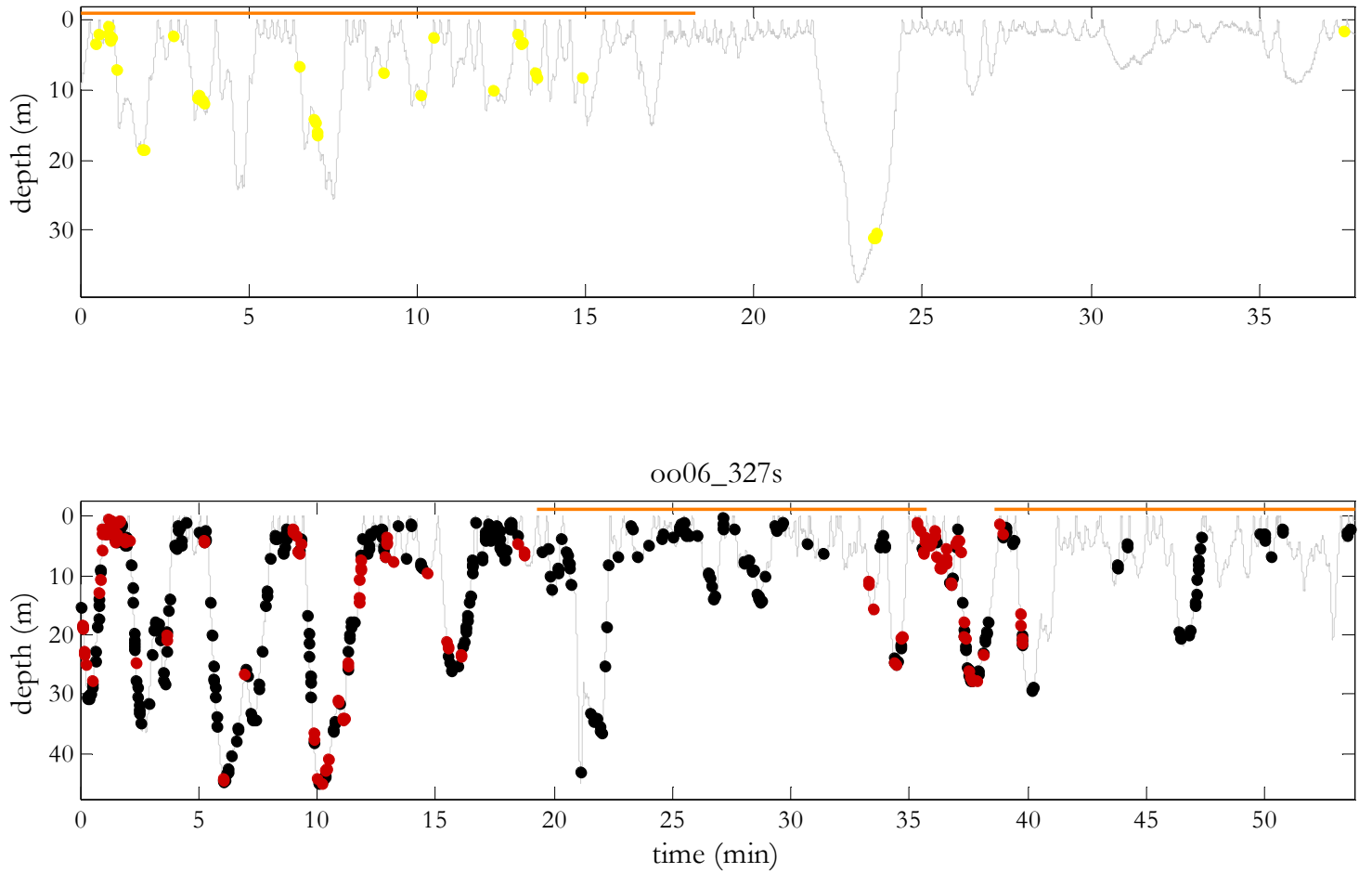

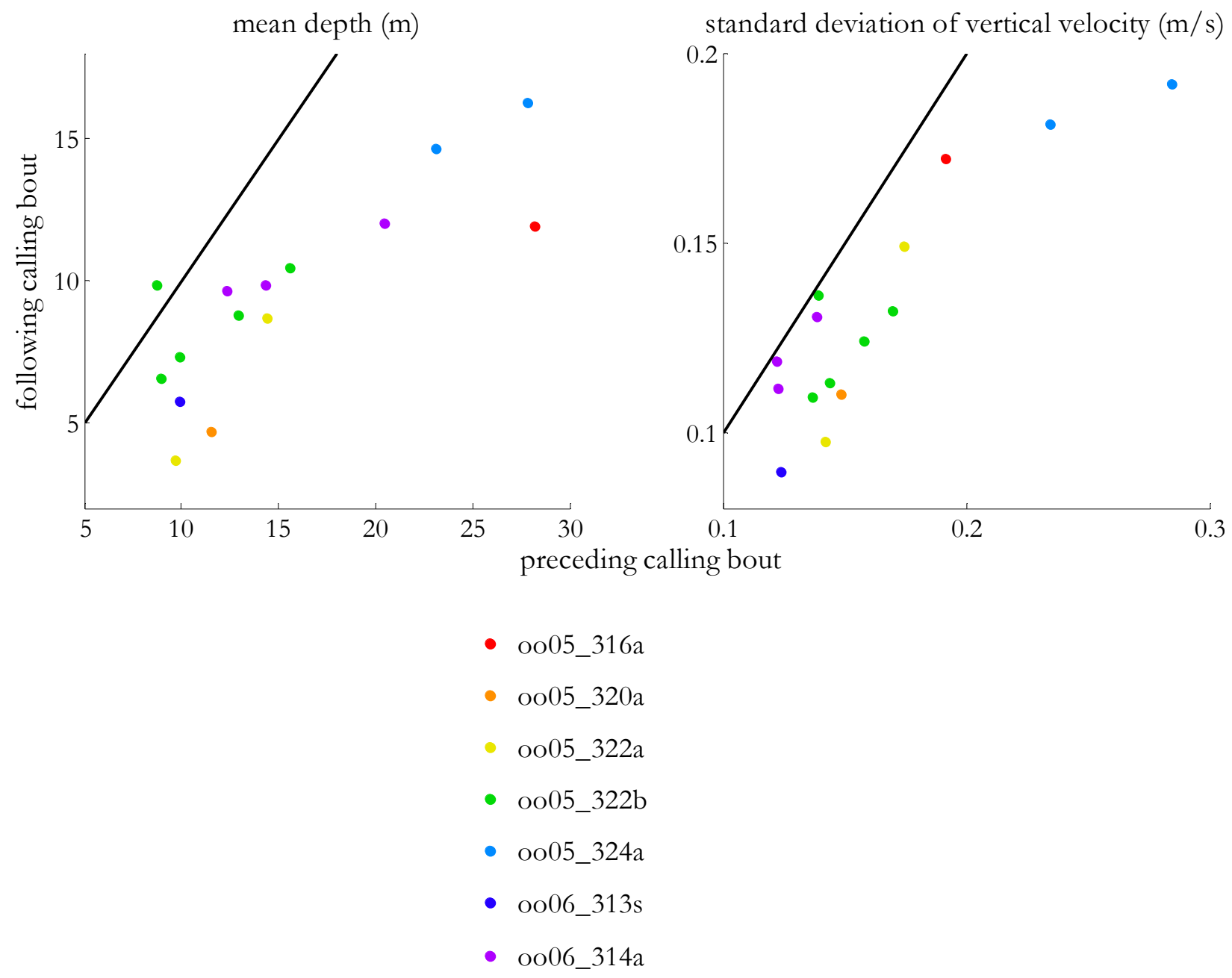

Figure 4.2. Mean depth (left) and standard deviation of the vertical velocity (right) plotted for bouts without calling activity versus successive bouts with calling activity during NTS periods for 7 whales. The 1:1 line is plotted in each figure. With the exception of a single mean depth value for oo05_322b, all measurements were smaller during a calling bout than in the non-vocal time period preceding it.

consistently occurred at shallower depths (except on one occasion by a single whale) and were characterized by less variable vertical velocities (Figure 4.2). It is reasonable that animals occupying shallower depths during calling periods would traverse less vertical distance, leading to smaller variation in their vertical velocities. This pattern is also evident in Figure 4.1. No further trends were evident for the standard deviation of the change in pointing angle, change in roll, or fluking intensity (data now shown). 
(a) $0005 \_322 a$

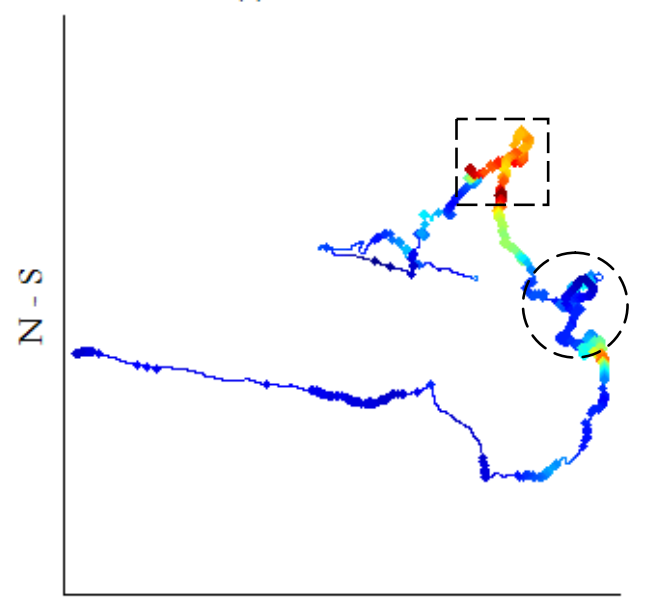

LI

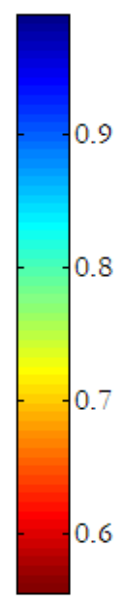

(b) $0005 \_320 \mathrm{~b}$

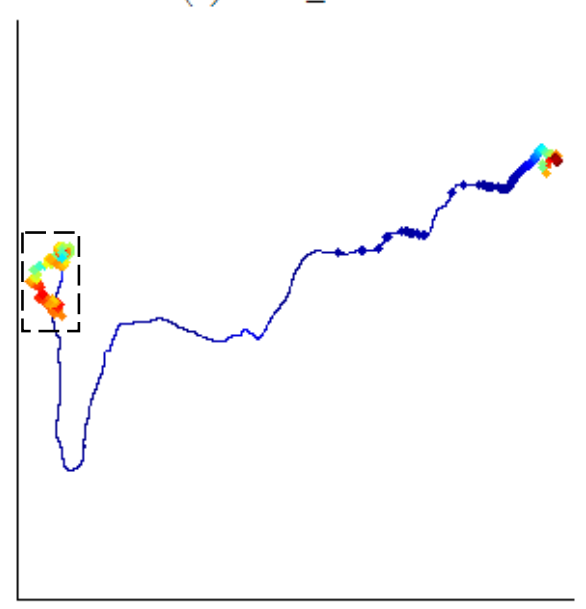

LI

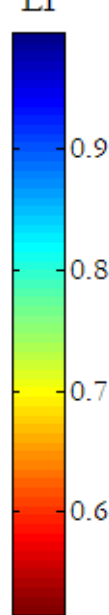

Figure 4.3. Horizontal tracks of two tagged killer whales with the linearity index (LI) plotted in color. Thin sections of the track correspond to an absence of calling behavior. Each call has been plotted by thickening the track. In both plots, the dotted square boxes indicate the portions of the track associated with tail slapping and concomitant vocal activity. In panel (a), this carousel feeding period is preceded by putative corralling (dotted circle) that contains high levels of calling. In panel (b), however, the preceding period of directional travel was quiet vocally.

Once these different movement and vocal behaviors during NTS periods were identified, their relationship with trends during the TS episodes were explored. Tail slapping activity was consistently matched with heightened vocal activity. In Chapter 2, two primary behavioral sequences were found to anticipate carousel feeding: one that involved a preceding period of putative corralling and another that involved putative travel. In all 4 of the sequences in which putative corralling anticipated tail slapping, calling activity was observed during both of these behaviors (Figure 4.3a). In 2 of the 3 instances in which directional travel (but no putative corralling) preceded the tail slapping, calling activity was absent during travel but began suddenly once the tail slapping commenced (Figure 4.3b). The animals were vocally active during feeding regardless of the prior behavior, suggesting that they were communicating with one another. For the four animals whose feeding was preceded by putative corralling, their vocal activity similarly suggested some kind of acoustic 
communication or elevated arousal level. The recording from female oo05_322a contained substantial vocal activity during the earlier directional travel periods as well (Figure 4.3a). The absence of vocal activity in the 2 cases of travel indicates that they did not need to communicate acoustically during this time and/or they were relying on passive listening to locate distant groups of animals that were feeding noisily. In the case depicted in Figure 4.3b, visual observations confirmed that the tagged animal and his group did approach another group of killer whales that was already engaged in carousel feeding.

The vocal behavior was then considered more closely by separating it into the L/HFC calls that feature additional orientation cues (Miller, 2002) and the less directional LFC calls. As described above, I predicted that the group carousel feeding activity associated with TS periods might have benefited from orientation signaling and therefore may have been characterized by more L/HFC than LFC calls. The results from this analysis are presented in Table 4.3. L/HFC calls occurred more during TS periods and LFC calls more during NTS periods than expected by chance for 5 of the 7 whales that were tested (one of these whales, oo05_322b, showed borderline significance). The pooled analysis in which the results of 6 whales were considered at once was highly significant $(P<0.001)$. This finding supports the notion that Norwegian killer whales may use the L/HFC calls to provide an orientation cue to group members during carousel feeding. Table 4.3 reveals that the proportion of LFC calls during NTS periods was consistently higher than during TS periods. For the whales displaying significant results, the proportion of L/HFC calls during TS periods was always larger than the proportion during NTS periods even if the actual counts of L/HFC calls were almost always smaller than LFC calls in both TS and NTS periods. Individuals oo05_322a and oo05_322b were tagged simultaneously. Results 


\begin{tabular}{c|c|c|c|c|c} 
whale id & $\boldsymbol{N}$ & L/HFC: TS & L/HFC: NTS & odds ratio & $\boldsymbol{P}$-value \\
\hline \hline Oo05_320b & 663 & 56.0 & 18.9 & 5.39 & 0.007 \\
Oo05_321a & 162 & 46.2 & 1.5 & 39.34 & 0.002 \\
Oo05_322a & 1728 & 31.4 & 13.0 & 3.04 & 0.013 \\
Oo05_322b & 1513 & 25.4 & 10.1 & 3.00 & 0.109 \\
Oo06_313s & 279 & 46.8 & 29.0 & 2.14 & 0.215 \\
Oo06_314s & 766 & 21.9 & 3.4 & 7.91 & 0.012 \\
oo06_327s & 540 & 15.7 & 29.2 & 0.46 & 0.838
\end{tabular}

Table 4.3. Results of the rotation test to assess pairing of L/HFC or LFC calls during TS versus NTS periods. The first and second columns provide the identification of the whale and the total number of calls used in the test. The next two columns list the percentage of L/HFC call production during TS and NTS periods, respectively. The odds ratio and the significance of the rotation test are offered in the last two columns.

\begin{tabular}{|c|c|c|}
\hline whale id & $P$-value & call types acting as sources of significance \\
\hline oo05_320b & $<0.001$ & N12.1, N64, N45 \\
\hline oo05_321a & $<0.001$ & $\underline{\mathrm{N} 8}, \underline{\mathrm{N} 16.1}, \underline{\mathrm{N} 85}$ \\
\hline oo05_322a & $<0.001$ & $\underline{\mathrm{N} 85}, \underline{\mathrm{N} 8}, \underline{\mathrm{N} 65}$ \\
\hline oo05_322b & $<0.001$ & $\underline{\mathrm{N} 10}, \mathrm{~N} 81, \underline{\mathrm{N} 85}$ \\
\hline oo06_313s & 0.074 & $\underline{\mathbf{N 8 9}}, N 77.3, \underline{\mathbf{N 8 2}}$ \\
\hline oo06_314s & $<0.001$ & N73, $\underline{\mathbf{N 8 5}}, N 72.2$ \\
\hline 0o06_327s & 0.002 & (N15), (N84), N72.3 \\
\hline
\end{tabular}

Table 4.4. Results of Markov chain test to compare call type frequency distributions during NTS versus TS periods. A significant $P$-value indicates that, once the serial dependence was considered by modeling the sequence using a Markov chain, the counts of call types differed between NTS and TS episodes. Call types are italicized if they were lowered during NTS or elevated during TS periods and are in bold if there were elevated during NTS or lowered during TS periods (the predicted outcomes). L/HFC calls are underlined and calls that do not fit into either LFC or L/HFC are listed parenthetically. Call type N15 was characterized by a short, fairly flat contour but spanned a wide spectral range and N84 contained a HFC only.

for both of these animals are reported in Table 4.3 though they are not independent. The especially large $P$-value for 0o06_327s may have resulted from the conservative division of NTS and TS periods used here. The tag record of this whale contained either putative corralling (considered NTS) or tail slapping episodes. Both of these behaviors may have benefited from a higher incidence of L/HFC calls, causing the lack of significance.

The next analysis explored whether different call types were used in the two different NTS and TS behavioral states. When a Markov chain was used to model the serial dependence of the call type sequence, all of the 7 whales demonstrated significant results (Table 4.4; one animal, oo06_313s, showed borderline significance). This indicated that the 
Figure 4.4. These plots present the percentage contribution of each call type to the calls produced during NTS (black bars) and TS (white bars) periods.
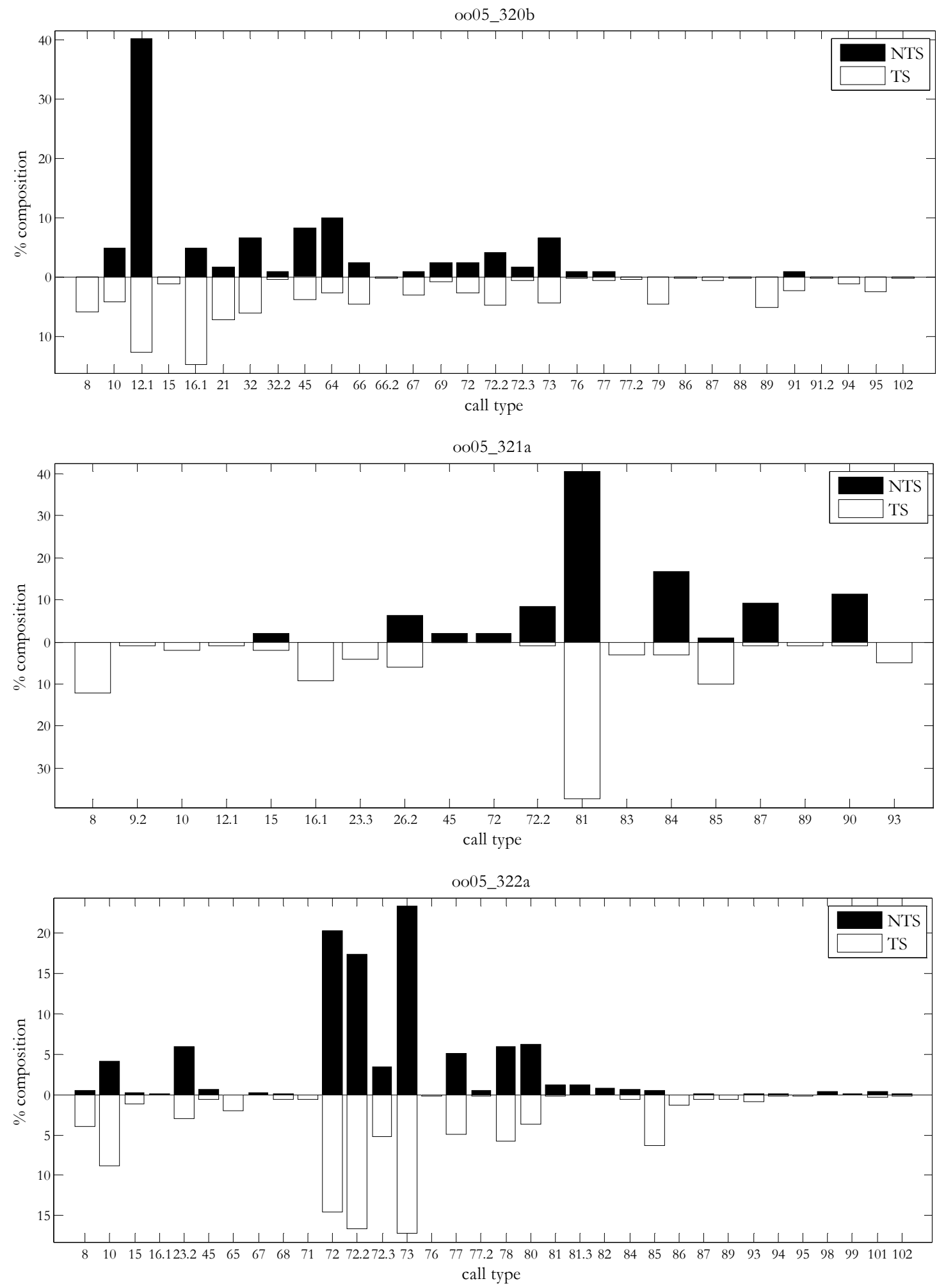

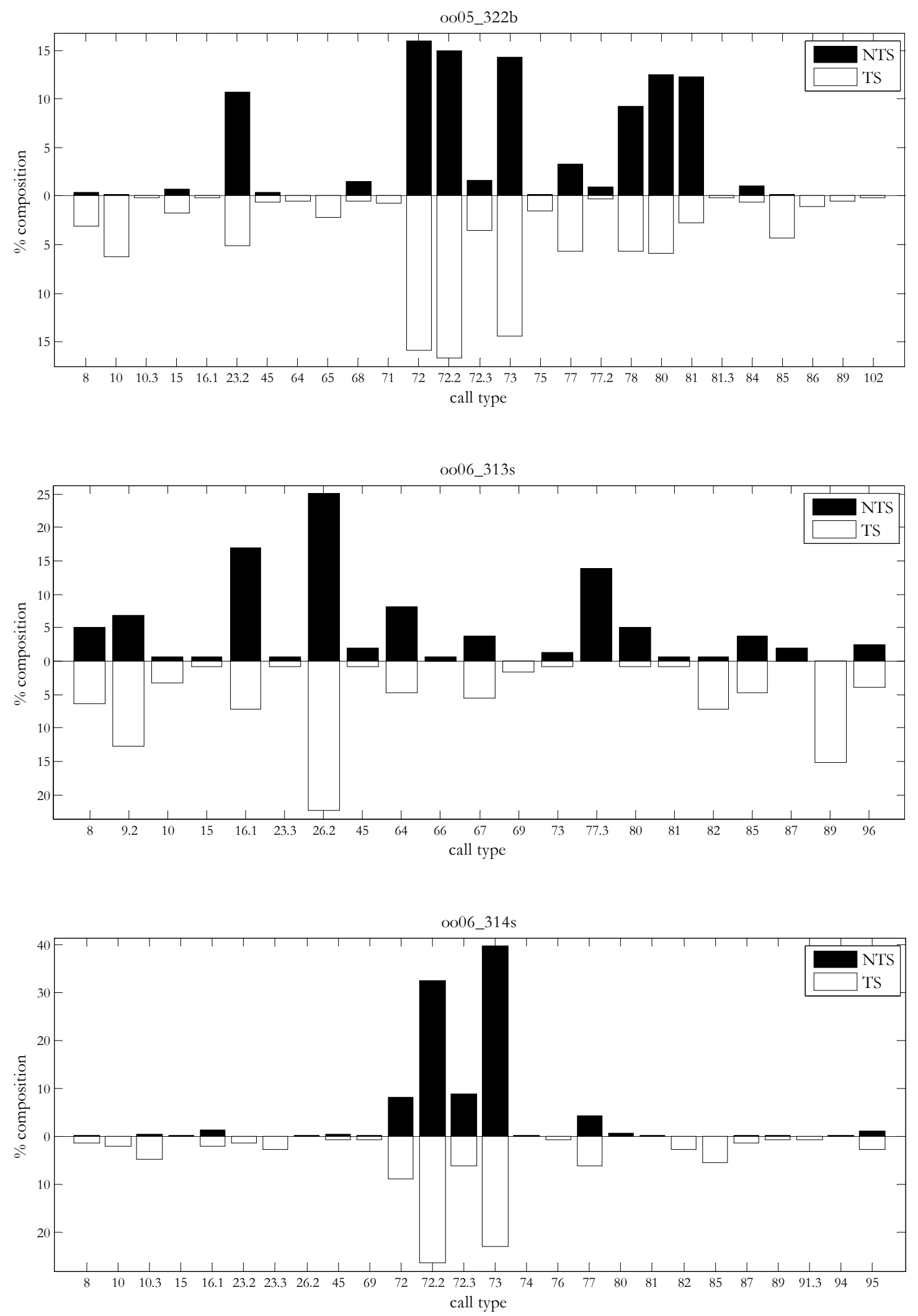


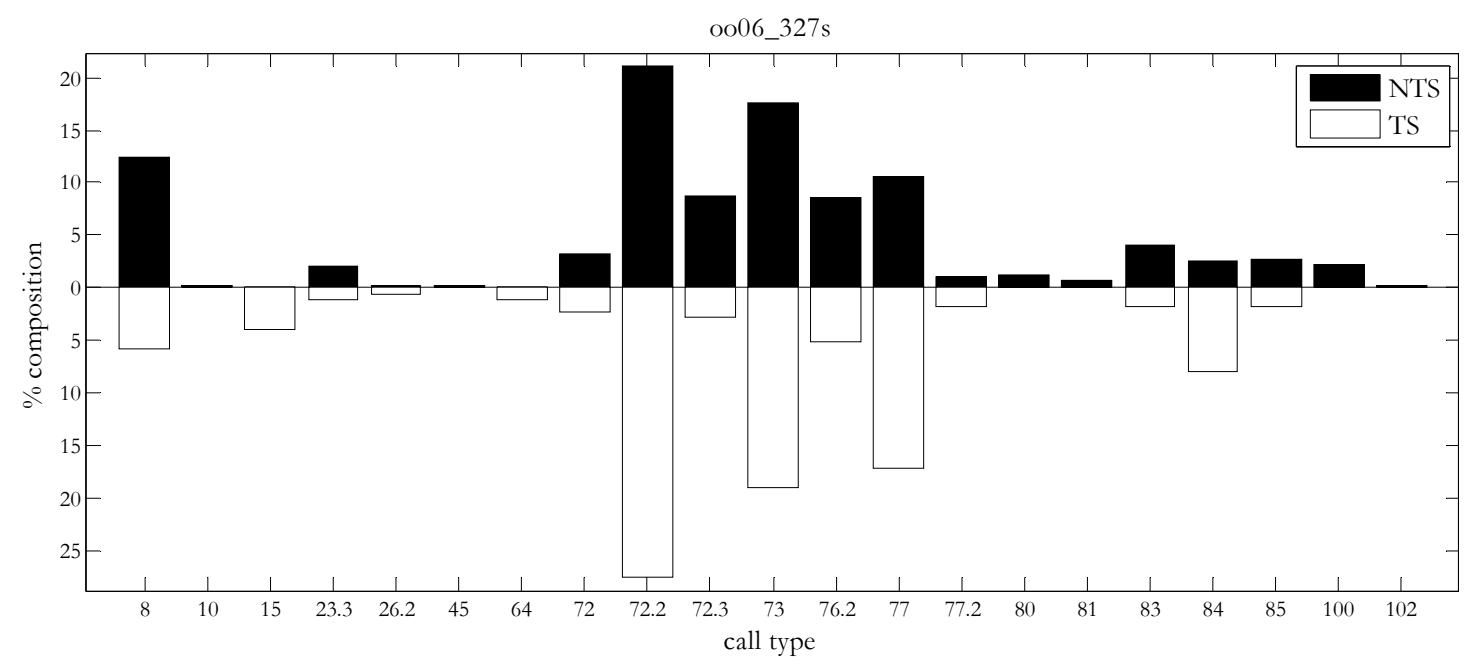

distribution of call type rates was significantly different between the NTS and TS periods.

Figure 4.4 plots the percent contribution of each call type during NTS and TS periods for 7 whales displaying both behavioral states. Then, the call types contributing to this significant difference were studied more closely (see last two columns of Table 4.4). In six whales, all three of these call types were consistent with the pattern observed above: LFC call types were more abundant during NTS periods (or less abundant during TS periods) and L/HFC call types were more abundant during TS periods (or less abundant during NTS periods). For the remaining whale (i.e., oo06_327s), one of the top three call types followed this pattern, one covered a wide spectral range (N15) and the third contained a HFC only (N84). These results were consistent with the orientation cueing prediction and support the possibility that the killer whales produced subsets of call types at different rates depending on the behavioral context.

\subsection{Analysis 2: Incorporating the movement sensors}

Sometimes the onset or ending of calling activity corresponded rather closely to the 


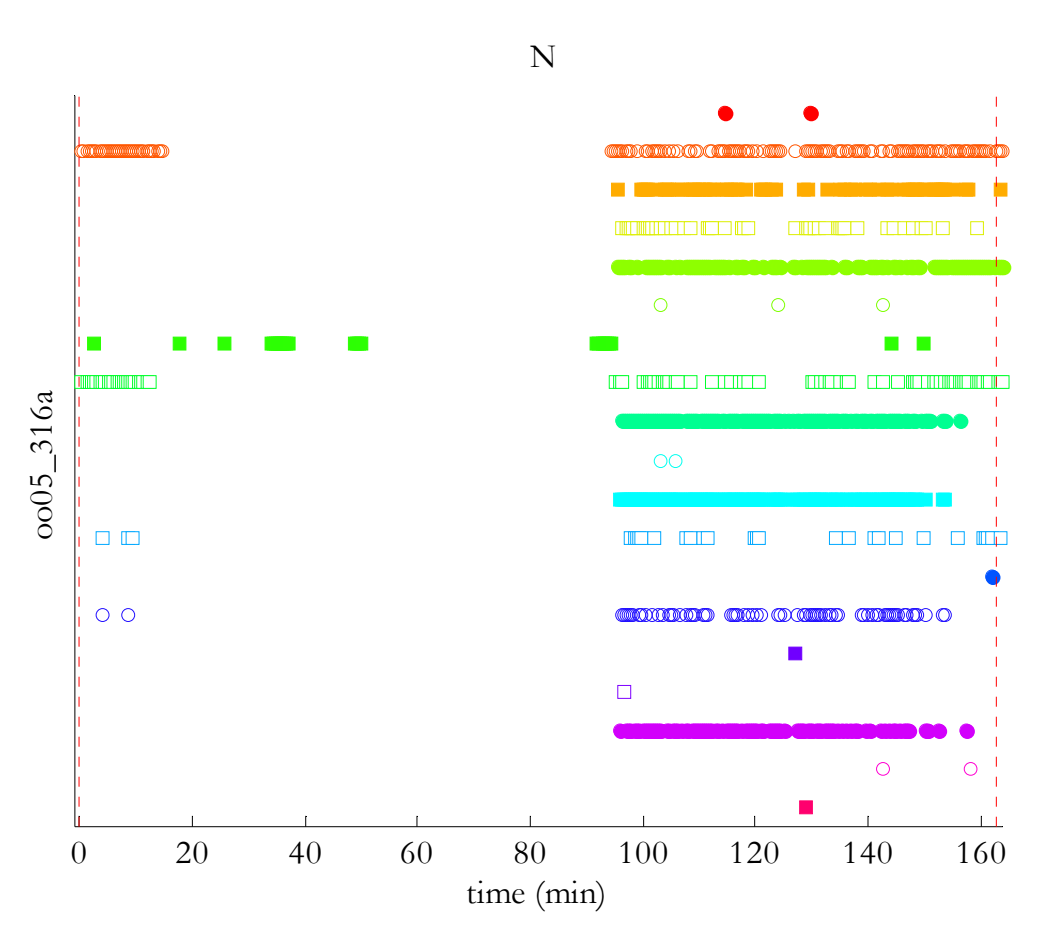

- N8

N10

- N10.3

N15

- N16.1

N23.2

- N45

- N64

- N65

N68

- N71

$\square$ N72

- N72.2

N72.3

- N73

$\square$ N75

- N77

- N77.2

- N78

- N80

- N81

$\mathrm{N} 81.3$

- N84

N85

- N86

N89

- N102

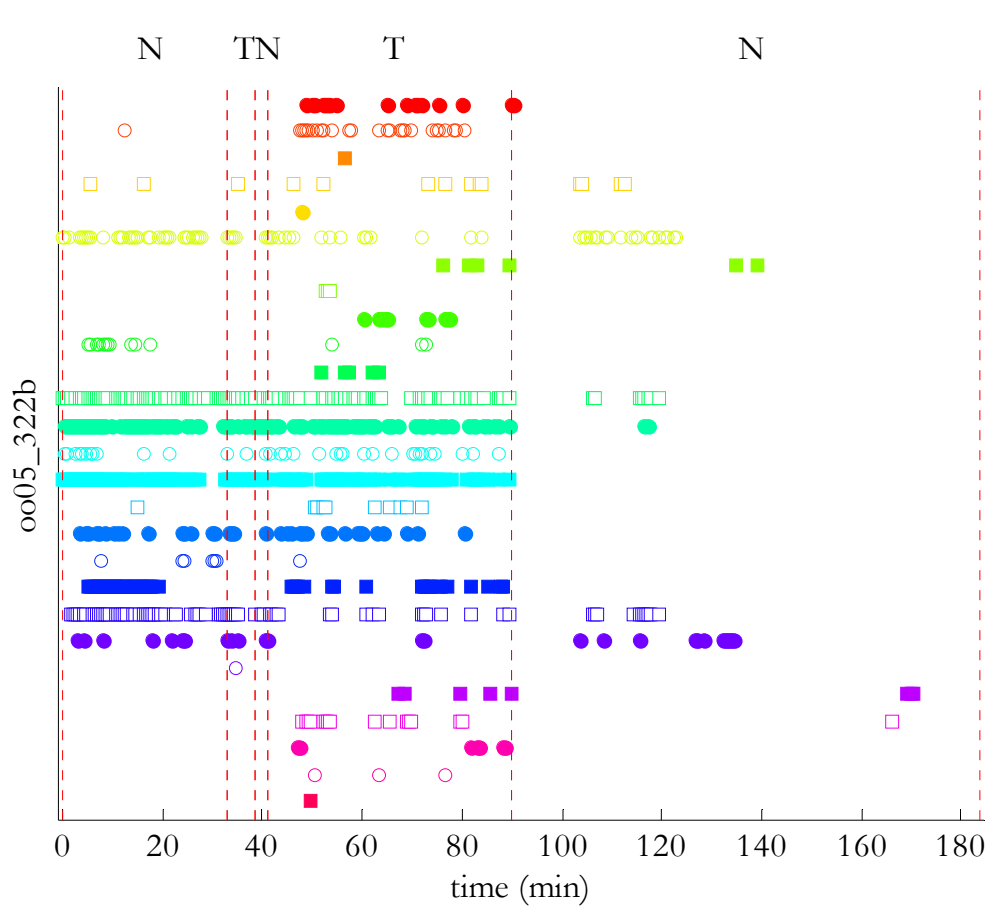

- N8

N12.1

- N16.1

N21

- N32

N32.2

- $\mathrm{N} 45$

$\square$ N64

- N66

N66.2

- N67

- N69

- N69.2

N76

- $\mathrm{N} 83$

N87

- N91

N91.2

- N91.3

Figure 4.5. Call type sequences for oo05_316a (top) and oo05_322b (bottom). The legends to the right provide the call type labels and the letters above the square plots indicate the NTS (N) and TS (T) documents. Notice that sometimes call type onset and offset occur at the document boundaries (e.g., N8, bottom plot) whereas other times they do not (e.g., N10.3, top plot). 
boundaries of the NTS and TS periods while other times there appeared to be little obvious connection (Figure 4.5). Individual oo05_316a, for example, only displayed NTS behavior. Its calling activity showed distinct patterning with most call types beginning around 95 minutes (Figure 4.5, top). Other call types occurred earlier and call type N45 was produced throughout the recording. Certain call types (e.g., N8, N65, N71, N86) clearly clustered in the primary TS section of the oo05_322b recording (Figure 4.5, bottom). Others (e.g., N68, N72.3, N73, N77, N78) spanned from the first NTS section (containing the putative corralling) through the primary TS section. A few of the call types (e.g., N23.2, N72, N80, N81) continued to be produced in the final NTS section.

Although it is possible that the calls types may have been produced interchangeably, the calling sequence patterns described in Section 4.4 and Figure 4.4 suggest otherwise. Previous approaches have studied the functions of killer whale call types by testing in which behavioral states they occur. These states have been assumed to be wholly representative of what an animal can do. This traditional approach of using a state-dependent model (i.e., partitioning the record into NTS and TS periods) may not be powerful enough to capture the rich dynamics of vocal behavior. I therefore explored what could be gained by taking advantage of the densely sampled sensor data and relating it to each call type. In the first analysis, I considered the question of whether different call types triggered particular movement responses in the tagged animal. In the second analysis, this perspective was reversed by investigating whether certain movement regimes were characterized by and could predict different sets of call types. 


\subsubsection{Methods}

In this section, a vocal sequence was considered to be a marked point process, or a time series of calling events that was each defined according to a call type. Five continuous movement features were incorporated as multivariate data streams: depth, pointing angle, roll, vertical velocity and fluking intensity (as defined above). The pointing angle and roll quantities were measured in radians. A running mean and standard deviation were computed over these movement data using a window length of 30s. Each vocalization could be associated with the coincident values of these measurements.

A multivariate analysis of variance (MANOVA) was conducted first, which tested whether the mean vectors of the movement data associated with each call type were significantly different. The running means and standard deviations of the movement data as well as the differences in the mean values before and after each call type were considered. This approach relied on computing the Wilks lambda criterion:

$$
\Lambda=\frac{|\mathbf{E}|}{|\mathbf{H}+\mathbf{E}|}
$$

where $\mathbf{E}$ and $\mathbf{H}$ are the error and hypothesis sum-of-squares and cross-products (SSCP) matrices, respectively (see Huberty \& Olejnik, 2006 for further elaboration, derivation and discussion). The F test statistic was then calculated as:

$$
F=\frac{1-\Lambda}{\Lambda} \frac{\mathrm{df}_{e}-p+1}{p}
$$

where $p$ was the number of outcome variables (i.e., 5) and $\mathrm{df}_{e}$ denoted the error degrees of freedom, which was computed as the difference between the number of data points and $p$. A rotation test was conducted to assess significance while preserving the serial dependence 
of the calling data. The timing of the calls was rotated relative to the movement data and the mean vectors were recomputed. This rotation procedure was performed 100 times and the observed value of the $F$ statistic was compared to the distribution of values produced by rotation to determine a $P$-value.

The second analysis conducted here involved a multinomial logistic regression in which the dependence of the distribution of call type on the multivariate movement measurements was modeled. The regression was of the form:

$$
P(Y=s \mid \mathbf{x})=\frac{e^{g_{j}(\mathbf{x})}}{\sum_{i=1}^{n} e^{g_{k}(\mathbf{x})}}
$$

where $g_{j}(\mathbf{x})=\sum_{k=0}^{5} \beta_{j k} x_{k}$ was a linear predictor involves a constant term (since $\left.x_{0} \equiv 1\right)$ and 5 covariates that corresponded to each of the movement measurements (Hosmer \& Lemeshow, 2000). The left-hand side of equation (4.4) denotes the probability that a calling event, $Y$, was of a particular type, $s$, given a particular combination of movement features, $\mathbf{x}$. There were a total of $n$ call type categories. This multinomial logistic regression model was fit to the dataset through maximum log likelihood by maximizing the likelihood function:

$$
L(\boldsymbol{\beta})=\sum_{i=1}^{n}\left(\sum_{j=0}^{5} y_{j i} g_{j}\left(\mathbf{x}_{i}\right)-\ln \sum_{j=0}^{5} e^{g_{j}\left(\mathbf{x}_{i}\right)}\right) .
$$

To preserve the serial dependence of the calling behavior when assessing significance, the call type sequence was again rotated relative to the movement data for each whale and the same maximum log likelihood value $L$ was computed. 


\begin{tabular}{c|c|c|c} 
whale id & $\boldsymbol{\sigma}$ & $\boldsymbol{\mu}$ & $\mathbf{d} \boldsymbol{\mu}$ \\
\hline \hline oo05_316a & 0.06 & 0.16 & $\mathbf{0 . 0 1}$ \\
o005_320a & 0.18 & $\mathbf{0 . 0 3}$ & 0.66 \\
o005_320b & 0.54 & 0.27 & 1.00 \\
oo05_321a & 0.56 & 0.57 & 0.61 \\
o005_322a & 0.16 & 0.14 & 0.48 \\
o005_322b & 0.10 & 0.21 & 0.09 \\
oo05_324a & 0.21 & 0.84 & 0.40 \\
oo06_313s & 0.11 & 0.54 & $\mathbf{0 . 0 5}$ \\
o006_314a & 0.07 & $\mathbf{0 . 0 4}$ & 0.12 \\
oo06_314s & $<\mathbf{0 . 0 1}$ & $<\mathbf{0 . 0 1}$ & $<\mathbf{0 . 0 1}$ \\
oo06_317s & 0.37 & 0.10 & 0.27 \\
oo06_327s & 0.26 & 0.76 & 0.10
\end{tabular}

Table 4.5. Results of MANOVA test for the 12 whales and 3 sets of movement measures: the running standard deviation $(\sigma)$, running mean $(\mu)$ and difference in means $(\mathrm{d} \mu)$. Significant $P$-values are marked in bold.

\subsubsection{Results and discussion}

The results of the MANOVA test are presented in Table 4.5. The call types of only one recording, oo06_314s, were significantly associated with different mean vectors of the running standard deviation of the movement features. The results were significant for 3 of the 12 recordings for both the running mean and the change in mean value. Thus, of 36 whale-movement stream combinations, only 7 were significant $(19 \%$ of the total, Table 4.5$)$. This small quantity of significant results supported the claim that the call types were interchangeable. This conclusion was reinforced by the lack of significance of the multinomial logistic regression, the second analysis described above, for all whales and movement streams. In addition, no significant findings emerged when the data were inspected with multinomial regressions using time as the regressor instead of movement. Multidimensional scaling and principal components analysis, techniques that reduced the dimensionality of the dataset for more straightforward handling and visualization purposes, did not yield any evident patterns either. Therefore, no convincing evidence was found to support the hypothesis that different call types produced by the group were associated with 
particular movement signatures of the tagged individual or vice versa.

\subsection{General Discussion}

The results reflected certain connections between the vocal and movement data at a very broad level. Across 7 animals during NTS periods, bouts of calls were produced closer to the surface and during spans of less variable vertical velocity compared with silent periods occurring immediately prior (Figures $4.1 \& 4.2$ ). This behavior resembled sperm whale codas that tend to be produced near the surface for what is likely a social communication purpose. TS episodes were dependably associated with high rates of calling activity, as were 4 out of 4 putative corralling episodes preceding instances of carousel feeding. On 2 of 3 occasions when putative travel anticipated carousel feeding, the absence of calling behavior during the travel portion gave way to intense vocal activity during the carousel.

These putative travel periods were generally silent and occasionally punctuated by a sharp turn resulting in a path that converged on a carousel feeding opportunity (see Chapter 2; Figure 4.3). This suggests that the animals may have remained quiet to rely on passive listening to locate other carousel feeding groups whose calling and tail slapping would have rendered their position and activity evident, a scenario confirmed with visual observations on at least one occasion. More data are required to assess the likelihood of this hypothesis since one of the traveling periods did contain vocal activity. This could provide a structured opportunity to investigate whether animals choose to vocalize based on the composition of the tail slapping group, which they could likely determine by listening to its vocal repertoire. Putative corralling and carousel feeding were reliably associated with heightened calling behavior (Figure 4.3). It is possible that these activities produced an elevated arousal level 
that released more vocal behavior. This relationship has been observed in other populations of killer whales (e.g., transients, Deecke et al., 2005). An alternative explanation for this observation provides that calling is somehow necessary for the proper execution of these foraging-related behaviors.

The next set of results supported this second conclusion since L/HFC call types, vocalizations containing both the low and high frequency components necessary to provide the orientation cue hypothesized by Miller (2002), were more abundant during TS versus NTS periods. The reverse was true for LFC call types, which were more common during NTS episodes. This conclusion was consistent both when calls were divided broadly into the two classes of LFC and L/HFC types (Table 4.3) and when the rates of individual call types were considered separately (Table 4.4; Figure 4.4). Corralling and carouseling involved multiple animals gathering, positioning and condensing a ball of herring to allow successful tail slapping and feeding. Killer whales involved in this incessant maneuvering would benefit from knowing the location, orientation and direction of movement of other group members. The higher incidence of L/HFC call types during TS episodes was consistent with the prediction that the animals could have relied on the orientation cues embedded within these calls to manage the ball of herring more effectively. Because L/HFC calls tend to be louder (Miller, 2006), it is also possible that this change in usage was due to louder call production intended to attract other groups or resulting from the excitement of the animals.

This first set of results suggested differential call type production according to behavioral state. Bouts of calling behavior often commenced and terminated rather suddenly (Figure 4.5). There were likely transitions occurring between behavioral states more refined than the coarse TS/NTS distinction made earlier. The detailed movement data 
streams were considered more closely to explore whether they could serve as reliable predictors of call types or vice versa. Only 7 of the 36 whale-movement stream combinations produced a significant difference between the means of the movement vectors when they were separated according to call type (Table 4.5). No significant results arose when call type was regressed onto the multivariate movement features or when several other techniques were attempted. The second set of analyses did not support the notion that call types could be associated reliably with distinctive sets of movement features. I find it likely that either the detailed movement data were not effectively or wholly characterizing relevant behaviors or the calls had been sorted into call type categories that may not have mattered to the animals.

Indeed, the findings presented in this chapter suggest that Norwegian killer whale call types are not entirely interchangeable as they may function to inform conspecifics about the $3 \mathrm{D}$ orientation and position of the signaler. Carousel feeding in particular requires multiple animals to corral and maintain the spatial integrity of a ball of herring for successful foraging to occur. During this dynamic enterprise, killer whales would certainly profit from orientation cues. In addition, Ford (1989) proposed that the group-specific vocal repertoires of killer whales may function as a badge for group identity but did not explain why more than a single call type was necessary to achieve this function. It is possible that large repertoires allow animals to distinguish association patterns and levels of interaction more finely. For example, killer whales that share $50 \%$ of their repertoire are likely to encounter one another more often than those sharing 25\%. The extent of repertoire overlap presented in Table 4.1 could therefore be used to formulate a hierarchy of degrees of social association between the recorded groups. This overlap could result from vocal convergence (see Mitani 
\& Gros-Louis, 1998; Smolker \& Pepper, 1999; Hile et al., 2000) or repertoire matching (Beecher et al., 1996; see Introduction).

One element missing from the findings presented here concerns the behavioral consistency of individual whales over time courses that extend beyond a few hours. Data collection efforts in the future should therefore focus on gathering recordings of the same animals across multiple carousels that ideally include multiple instances of both the traveling and corralling sequences anticipating the feeding. Additional data are also necessary to verify the absence of a relationship between the fine movement and vocal data analyzed here in Section 4.5. If the same result continues to emerge, then either the killer whale pulsed calls really are context-independent or we lack sufficient information about other social or environmental cues that may be triggering certain bouts of calling activity. Playback experiments of carousel feeding calling sequences and tail slaps to groups of traveling killer whales could test whether these animals do eavesdrop on and exploit conspecifics that have already corralled and brought a ball of herring to the surface. Additional research effort is likely to be rewarded with the discovery of more links between the vocal and non-vocal behavior of free-ranging killer whales, testing explicitly whether their call types are interchangeable and functionally equivalent.

\subsection{Acknowledgements}

Special thanks to Andy Solow and Peter Tyack for facilitating the analysis of this chapter. Diane Rak, Katie Stanchak and Jia Xin Wu helped to sort the spectrograms into different call types. They received financial support from the Ocean Life Institute at the Woods Hole Oceanographic Institution. 


\section{Chapter 5. Phonemic Segment Characterization of Norwegian KILLER \\ Whale Call Types}

\subsection{Abstract}

Killer whale vocal production has traditionally been categorized by human observers into a set of discrete call types. These call types often contain internal spectral shifts, silent gaps and synchronously produced low and high frequency components. The fundamental units used to build signals into a repertoire and the syntactic rules associated with that assembly are essential to understand animal vocal communication systems. Inspired by human speech processing techniques and algorithms, this chapter tested whether call types could be represented by a set of flexibly arranged and smaller phonemic segments. In particular, I evaluated whether segmented characterizations of stereotyped Norwegian killer whale calls yielded automated classification results of contour traces that paralleled a classification scheme using whole call type designations. Representations of calls in their entirety or as sets of either distinct or shared syllables did achieve similar performance. Calls composed of shared segments may provide a more parsimonious approach to parsing the vocal stream since there were fewer segments than call types, nearly $75 \%$ of all call types contained at least one shared syllable, and some syntactic patterns were evident. Such a system could flexibly generate the killer whale vocal repertoire as a subset of all possible combinations of segments.

\subsection{Introduction}

An important question in animal behavior and communication concerns how the 
brain archives and generates a sequence of acoustic signals, which has implications for individual and interactive vocal performance (see Lee et al., 2004). Chunking, the process by which serial information is segmented during memory formation to facilitate subsequent recall (Simon, 1974; Terrace, 1987), can help explain certain perceptual and production features of sequences generated from a vocal repertoire. More elaborate repertoires can arise when a smaller inventory of chunked signals are reshuffled to generate a vast array of new vocal combinations (e.g., Dobson \& Lemon, 1979). Zebra finches (Taeniopygia guttata), for example, copy serial strings of sung syllables and intervening periods of silence from a variety of adult tutors and then reorder these chunks to produce their own song (Williams \& Staples, 1992). Similarly, nightingales (Luscinia megarbynchos) acquire song by extracting and rearranging coherent packages of 3-5 song types from longer model sequences (Hultsch \& Todt, 1989). Individual birds of both species tended to segment their vocal sequences at different breakpoints and likely exploit the chunking of songs to memorize a larger array of vocal components. Chunking helps to organize vocal information by breaking received sequences into smaller elements, which can then be used to build and produce identical or novel sequences.

Syntax may be broadly defined as the set of rules that inform how smaller communication tokens are organized into larger phrases (Snowdon, 1982; Hailman \& Ficken, 1986). Likely candidates for these tokens include discrete, intact segments that are found within different sequences. Syntax constrains the ordering of such subunits within the set of all possible combinations and sequences and its analysis can help identify the chunks that compose a repertoire. Certain features help define the boundaries of these chunks. In birdsong, for example, syllables are generally defined as continuous and discrete elements 
separated by silence (Lemon \& Chatfield, 1971; Eales, 1985; Eens et al., 1989). Indeed, zebra finches whose songs were experimentally interrupted usually stopped singing between song syllables, suggesting that the syllable was a meaningful unit at least in terms of basic production (Cynx, 1990). In human speech, positions of maximum spectral transition are important for consonant and vowel perception (Furui, 1986). Similarly, Yurk (2005) used abrupt and discontinuous spectral shifts to define boundaries between syllables in killer whale (Orcinus orca) vocalizations.

Marler (1977) distinguished phonological syntax, in which elements from the repertoire are rearranged in specific ways to generate new vocalizations (the focus of this chapter), from lexical syntax, in which the meaning of a newly combined vocalization is derived from both the order and the constituent meanings of its components. Birdsong lasting on the order of seconds tends to be built from individual notes whose speciesspecific phonological arrangement is required to evoke an appropriate behavioral response (e.g., European starlings (Sturnus vulgaris): Böhner \& Todt, 1996; white-crowned sparrows (Zonotrichia leucophrys): Soha \& Marler, 2001). Humpback whale (Megaptera novaeangliae) song lasts on the order of minutes and is constructed phonologically from themes that consist of repeated phrases (Payne \& McVay, 1971). These songs change over time as themes are added and lost and phrases are modified (Payne et al., 1984; Noad et al., 2000). Bird and humpback whale song repertoires are both constituted from a set of flexibly arranged, smaller and more basic units of vocal production. The temporal and spectral properties of these tokens differ but their role as the building blocks of a syntactically-organized repertoire is the same.

Vocal production characterized by phonological syntax has been observed in social 
species with a fixed repertoire of sounds. Sequences of discrete calls are well suited for syntactic analysis since the onset and ending of vocalizations are usually evident and stereotypy can facilitate categorization of individual calls (Slater, 1973; 1983). Killer whales produce such a set of stereotyped pulsed calls (see Ford, 1987; Strager, 1993; Filatova et al., 2004) and they live in stable family groups, interact vocally in a variety of contexts (Ford, 1989; Deecke et al., 2005; Van Opzeeland et al., 2005; Simon et al., 2007) and show evidence for acoustic mimicry (see Bowles et al., 1988; Foote et al., 2006). Call types have been classified similarly by human observers sorting calls aurally and spectrographically and by neural networks relying on temporal and spectral features of a trace of the fundamental frequency (Deecke et al., 1999; Deecke \& Janik, 2006). A portion of the repertoire has been labeled variable, a miscellaneous class of vocal behavior containing the calls that have not sorted neatly into one of the stereotyped categories.

Here, I take advantage of techniques that have been developed in the field of human speech recognition to explore phonological syntax in Norwegian killer whales. Two of the hallmarks of human language include an ability to generate a vast array of words from a set of a few dozen phonemic units and to use recursive or hierarchical procedures to form appropriate combinations of these signals (Nowak et al., 2000; Hauser et al., 2002). Early attempts at using words to drive speech recognizers gave way to phonemic representations that improved performance on large vocabularies considerably (see Lee et al., 1989). Traditionally, killer whale call types have been regarded as the fundamental units of vocal production. Fashioned after the approaches developed in the arena of human speech research, orca vocalizations were decomposed in this study to test whether a simpler set of phonemic segments may define chunks that can be assembled to form the repertoire. Unlike 
human speech processing tasks where we know when the computer has conducted an accurate and efficient parsing, I assess the traditional and phonemic models of killer whale vocalizations by comparing their call type classification efficiency. In this chapter, the terms segment and syllable are used interchangeably.

A few additional features of killer whale calls motivate their decomposition into smaller segments to explore the possibility of phonological syntax. First, the pulsed calls can contain synchronously produced low and high frequency components (LFCs and HFCs, respectively, Hoelzel \& Osborne, 1986; Miller \& Bain, 2000) that are presumably generated by two independent sources located in close proximity to one another (see Miller et al., 2007 for a discussion). These components are spectrographically distinct entities: the LFC is characterized by a fundamental frequency between $80-2400 \mathrm{~Hz}$ (Ford, 1987) whereas the HFC extends between 2 and $12 \mathrm{kHz}$ (Hoelzel \& Osborne, 1986). The proposed independent control required to produce these two components would allow the animals to increase their repertoire dramatically simply by varying the LFC and HFC pairings.

Second, Norwegian killer whales produce compound calls, concatenations of multiple discrete calls that are produced elsewhere in the record either individually or within other compound calls (Strager, 1993; 1995). This combinatorial and syntactic rearrangement of entire call types to generate compound vocal patterns suggests that these animals may take advantage of a similar mechanism to fashion the call types themselves from a set of even smaller subunits. The call type could even be considered a compound call itself if its subunits were also produced elsewhere on their own. The approach presented here offers a simple framework for understanding killer whale vocal behavior by arranging production into segments that are flexibly combined to form call types, which can be variably ordered 
themselves to generate the compound calling sequences. Third, subtype designations have been used to distinguish "structurally unique variants" of a call type based on 1) consistent temporal or spectral differences of particular features or 2) the presence or absence of certain segments (Ford, 1991; Strager, 1995). These segments may serve as the phonological tokens that are syntactically rearranged to generate call types. Indeed, Yurk (2005) extracted syllables from the calls of resident and transient killer whales living in British Columbia, categorized them by eye (using their "gestalt" to aid the differentiation) and found that human classifiers agreed with these divisions. Different arrangements of these phonemes formed the call types from these animals.

Figure 5.1 presents a set of motivating spectrograms for this work. Each column contains a series of calls that are successively built upon one another. In the first column, the first call is N72, a gradual frequency modulated upsweep. The next two calls in the vertical series, N72.2 and N72.3, contain the same upsweep followed by one or two very short higher frequency segments, respectively. These segments are separated by brief (< 0.1s) spans of silence. The foundation of the call series in the second column of Figure 5.1 is N16.1, a continuous call type characterized by both a LFC and HFC. Each successive LFC in the vertical series accumulates an additional constant frequency or slightly frequency modulated segment. The HFC segments are added gradually with N16.1 and N81 containing one segment and N91.2 and N91.3 containing two segments. In addition to sharing structural similarity, call types N16.1 and N91 occasionally occupied the same final position in the compound call series beginning with the calling sequence N66 $\rightarrow$ N67. The examples presented in Figure 5.1 are certainly consistent with the notion that a set of syllables can be flexibly arranged and sequenced to generate a large repertoire of call types. 

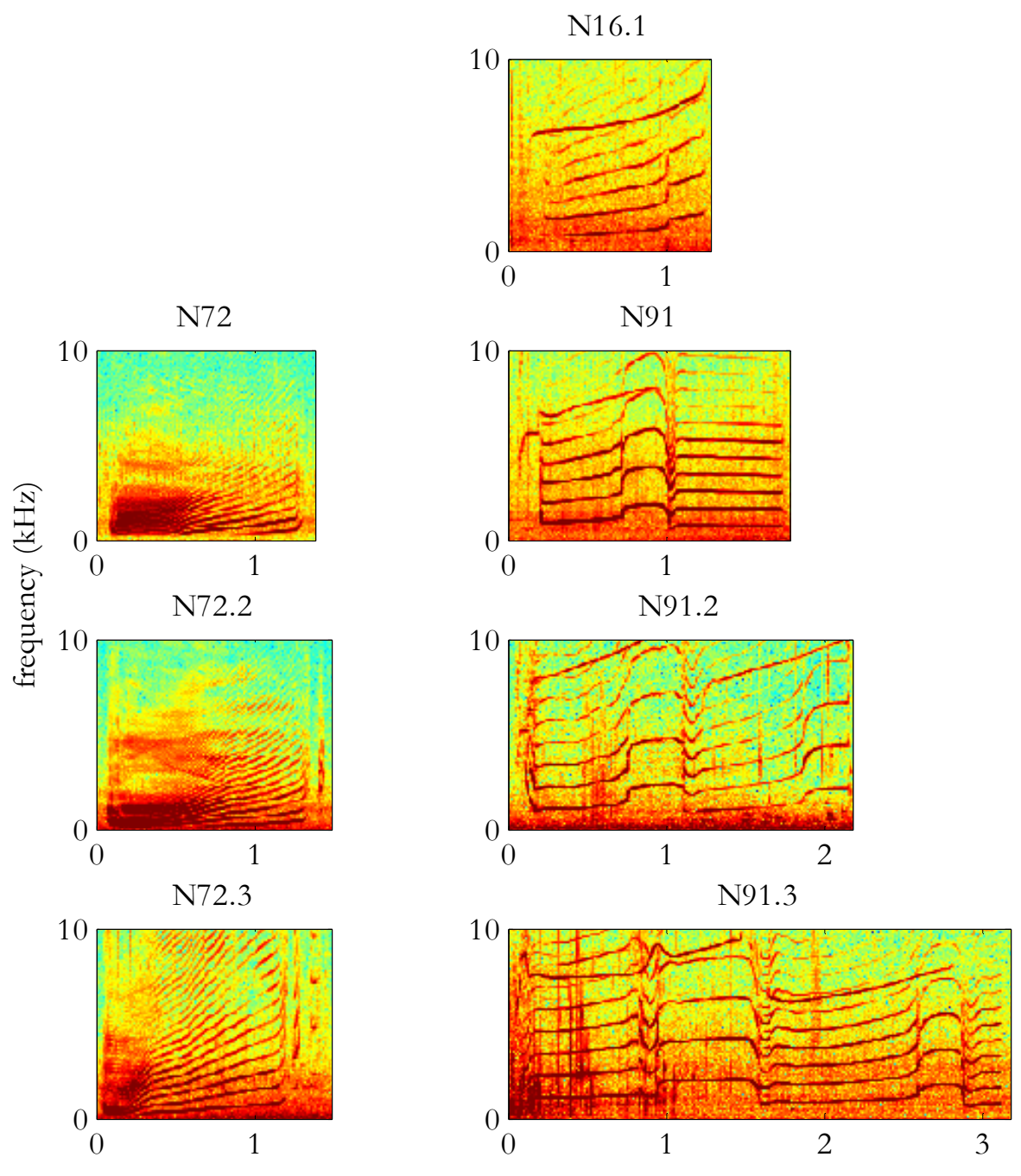

time (s)

Figure 5.1. Examples of call types that motivated this study. The three spectrogram panels on the left contain the same primary call base labeled N72. N72.2 contains a short additional utterance at the end of the call and N72.3 contains two such additional utterances. The four spectrogram panels on the right can be assembled from top to bottom by sequentially adding both low and high frequency components to the N16.1 base.

Here, leveraging techniques developed for human speech recognition, I confirm the earlier observation of shared syllables by Yurk (2005) and extend it further, evaluating formally whether phonemic divisions of Norwegian killer whale stereotyped calls allow automated classification to call type with an efficiency that parallels a classification scheme 
based on whole call type designations. This chapter presents evidence that the performance of these two approaches is comparable. The characterization of pulsed calls with a set of shared syllables may be superior to the whole call type approach, however, because the phoneme model is simpler, requiring less information and computation to represent the dataset. I also test the possibility that certain variable calls may be built from the same phonological segments constituting the stereotyped calls. Finally, inspired by the small yet universal set of phonemes that are sub-sampled to form each human language (see Schultz \& Waibel, 2001; Zhu et al., 2005), I quantify how completely the phonemic inventory derived from Norwegian stereotyped calls characterizes the repertoire of resident and transient killer whales from the Pacific Northwest.

\subsection{Materials and methods}

\subsubsection{Data collection}

Field work was conducted in November 2005 and 2006 in the northern Norwegian fjords of Tysfjord and Vestfjord near the Lofoten islands $\left(\sim 68^{\circ} 15^{\prime} \mathrm{N}, \sim 16^{\circ} \mathrm{E}\right)$. Fourteen free-ranging killer whales were outfitted with digital archival tags that sampled audio at 96 $\mathrm{kHz}$ and individual movement at $50 \mathrm{~Hz}$, storing all data to flash memory (Johnson \& Tyack, 2003). The animals were approached in a rigid hull inflatable boat and the tags were attached using a $7 \mathrm{~m}$ carbon fiber hand pole. A VHF beacon was used to track the tagged animal from an observation platform (the sailboat Iolaire or the Norwegian research vessel Sverdrup) and recover the tag once it released after a pre-programmed period of time. The data were offloaded using an infrared interface, checked for errors, backed up onto CD and then cleared from memory to allow re-deployment of the tag. A total of 31.8 hours of 
recordings were made from 13 animals (one of the recordings did not contain any vocalizations). In addition, calls produced by resident and transient killer whales in the Pacific Northwest were recorded with towed hydrophone arrays and single hydrophones, respectively (see Miller \& Tyack, 1998; Deecke et al., 2005 for data collection and processing details) and were kindly provided for the analysis here.

\subsubsection{Call type assignments}

Initially, all recordings were audited manually to listen to and flag every vocalization. Calls of either sufficient signal-to-noise ratio (SNR, generally $10 \mathrm{~dB}$ ) or spectrographic visibility were used for classification purposes. Categorization of calls to type by visual inspection of spectrograms was used here since earlier studies using this approach demonstrated high inter-observer reliability scores and compared favorably to automated approaches involving neural networks (Bain, 1986; Ford, 1991; Deecke et al., 1999; Yurk et

al., 2002; Deecke \& Janik, 2006). Three observers and I each sorted spectrograms of calls from each recording into our own sets of categories. Call types were derived for each recording from spectrogram sets that were identically classified by me and at least two of the observers (see Table 5.1 for counts).

Because calls from different tag recordings assigned to the same type were found by visual inspection to be more similar than calls assigned to different types, call types were subsequently compared across whales to determine global classes, matching them whenever possible to pre-existing call type labels (Strager, 1993; Van Opzeeland et al., 2005). Strager (1993) labeled the first 34 categories with the initial "N" for "Norwegian" followed by a whole number to indicate the call type (i.e., N1 to N34). Subtypes were designated by a 


\begin{tabular}{|c|c|c|c|c|c|c|c|c|}
\hline \multirow[b]{2}{*}{ animal id } & \multirow{2}{*}{$\begin{array}{l}\text { \# spectrograms } \\
\text { used for sorting }\end{array}$} & \multicolumn{4}{|c|}{$\begin{array}{l}\text { \# types determined } \\
\text { by observer: }\end{array}$} & \multirow{2}{*}{$\begin{array}{l}\text { \# types used } \\
\text { in analysis }\end{array}$} & \multirow{2}{*}{$\begin{array}{l}\text { \# stereotyped calls } \\
\text { used in analysis sorted } \\
\text { into these types }\end{array}$} & \multirow{2}{*}{$\begin{array}{l}\text { \# variable calls } \\
\text { used in analysis }\end{array}$} \\
\hline & & 1 & 2 & 3 & 4 & & & \\
\hline coo05_316a & 489 & 11 & 29 & 21 & 19 & 12 & 814 & 39 \\
\hline oo05_320a & 218 & 14 & 20 & 20 & 18 & 15 & 594 & 46 \\
\hline oo05_320b & 109 & 14 & 27 & 17 & 19 & 21 & 268 & 96 \\
\hline oo05_321a & 63 & 12 & 17 & 14 & 17 & 11 & 48 & 11 \\
\hline oo05_322a & 591 & 26 & 35 & 39 & 34 & 17 & 572 & 54 \\
\hline oo05_322b & 120 & 20 & 50 & 48 & 34 & 14 & 341 & 23 \\
\hline oo05_324a & 41 & 4 & 5 & 6 & 5 & 7 & 70 & 12 \\
\hline 0006_313s & 113 & 16 & 19 & 14 & 17 & 13 & 139 & 38 \\
\hline oo06_314a & 126 & 6 & 11 & 9 & 14 & 7 & 196 & 13 \\
\hline 0o06_314s & 150 & 7 & 18 & 13 & 16 & 16 & 220 & 78 \\
\hline 0006_317s & 35 & 3 & 8 & 8 & 7 & 4 & 13 & 23 \\
\hline 0006_324s & 18 & 3 & 6 & 4 & 3 & 4 & 5 & 3 \\
\hline 0006_327s & 258 & 19 & 38 & 35 & 24 & 11 & 250 & 51 \\
\hline total & 2331 & & & & & & 3530 & 487 \\
\hline
\end{tabular}

Table 5.1. Tabulation of call and type counts from each recording used for visual sorting and the subsequent classification experiments. The number of spectrograms used to do the sorting is listed in the second column. The next set of 4 columns contains the call type counts determined by each of the observers. Agreement between me and at least two of the observers on a category defined it as a call type and the number of types determined from each recording is listed in the next column. The numbers of stereotyped and variable calls actually used in the syntax analysis are included in the last two columns of the table. Some calls were not used because they were too faint. The last row sums these counts over all the recordings to yield the total amount of data analyzed.

number in the tenths place (e.g., N23.2). Additional call types from N35 to N63 were added subsequently by Van Opzeeland et al. (2005). New categories identified here were assigned new numbers (N64 to N103); spectrograms of the call types used can be found in Appendix

2. For this analysis, I assume that the initial call type assignments made here were correct. Single instances of a call that could not be classified into a class were considered variable calls $(11.6 \%$ of the Norwegian calls considered in this study). The calls from the resident and transient killer whales in the Pacific Northwest had already been sorted to type.

\subsubsection{Contour tracing}

Contour tracing was restricted to killer whale calls with evident onset and offset (ensuring representation by a complete trace compared with calls that faded in or out in the 
spectrogram) and whose contour was entirely visible (i.e., free of vocal activity or surfacing noises that overlapped and obscured the call). A pitch tracking algorithm developed for human telephone speech that relies on the harmonic structure of a vocal signal (Wang \& Seneff, 2000; Wang, 2001) was used to trace the fundamental frequency of both the low and high frequency components (LFC and HFC, respectively) of killer whale calls when present. Three separate parameter settings were used to optimize traces for LFCs below $600 \mathrm{~Hz}$, LFCs between $400-4000 \mathrm{~Hz}$ and HFCs between 4-12 kHz (Wang \& Shapiro, In prep). Using a customized Matlab interface, all pitch contours were checked manually against the original spectrograms and if necessary, portions were smoothed via linear interpolation, corrected for pitch doubling or halving errors and re-traced using the mouse. Each contour was further prepared by manually removing any sharp and noisy onset or offset transients that could not be reliably traced, rejecting LFCs and HFCs that were too faint to discern completely, standardizing the number of segments (see below) according to call type and thresholding the allowable proportion of non-tonal (and therefore non-traceable) contour according to call type segment.

Research on birdsong, killer whale calls and human speech have established syllable boundaries at intervening periods of silence and abrupt spectral shifts (see Introduction). Killer whale calls were divided into segments and traced non-continuously based on these rules (Figure 5.2). The contour traces of all calls used here are inventoried in Appendix 3. The LFC and HFC of a call were often divided into segments at different time points.

\subsubsection{Segmentation algorithm}

Several calls contained quick yet spectrally continuous frequency changes (i.e., the 

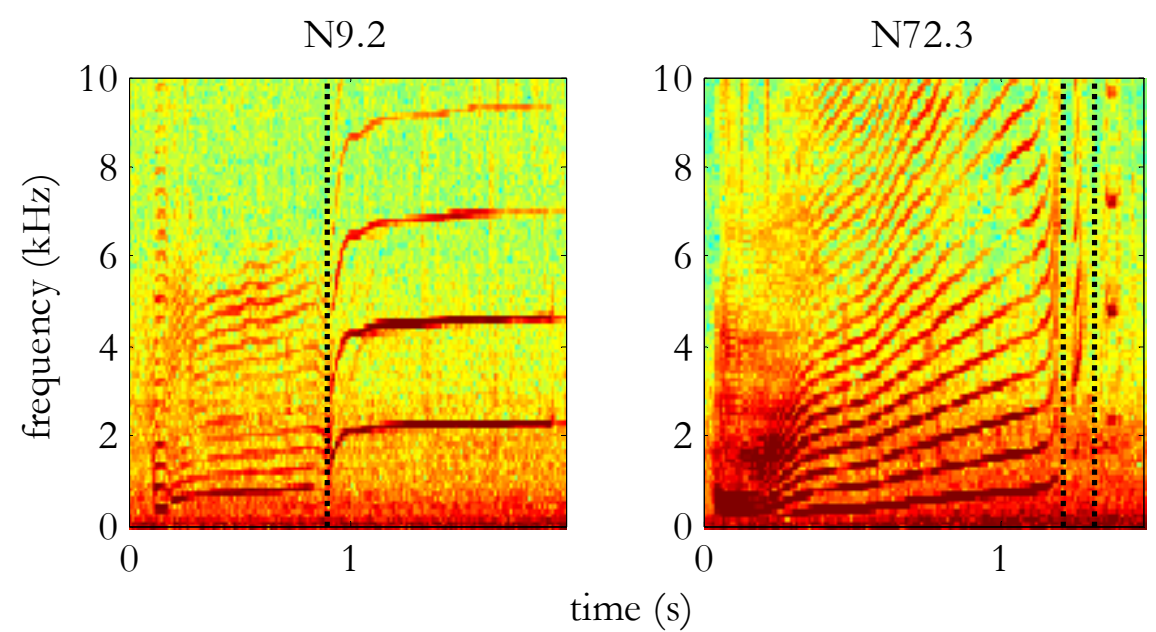

Figure 5.2. Abrupt, non-continuous spectral shifts (left: N9.2) or intervals of silence (right: N72.3) in the contour were considered boundaries defining segment edges (marked here with black dotted lines).
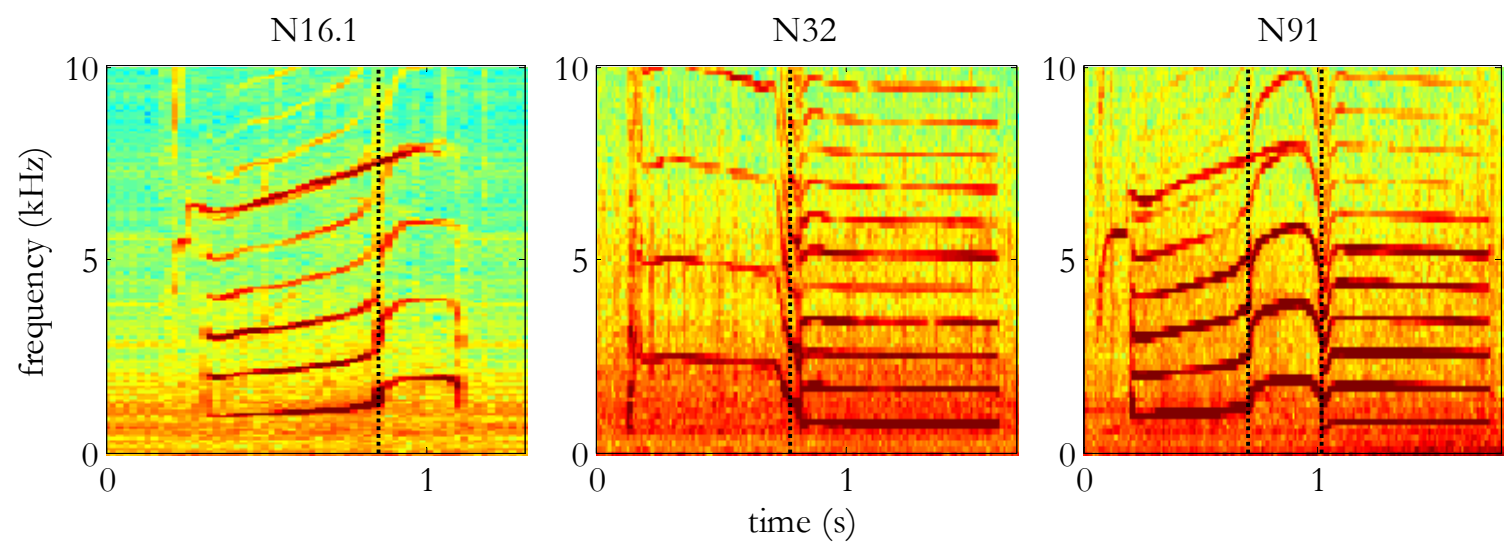

Figure 5.3. Call types whose LFCs were segmented according to the algorithm described in the text. Segmentation divided the LFCs of these calls into two (N16.1, N32) or three (N91) segments.

LFCs of N16.1, N32, N91, Figure 5.3). These transitions were also used to separate the calls into segments but it was less obvious where the divisions should be made. A segmentation algorithm was developed to divide the contours with these breakpoints consistently into a certain number of segments according to call type (Figure 5.4). First, a Legendre polynomial curve of $4^{\text {th }}$-order was fit to the contour (Aburdene \& Dorband, 1996). Although any polynomial family would have performed similarly, the class of Legendre polynomials was 


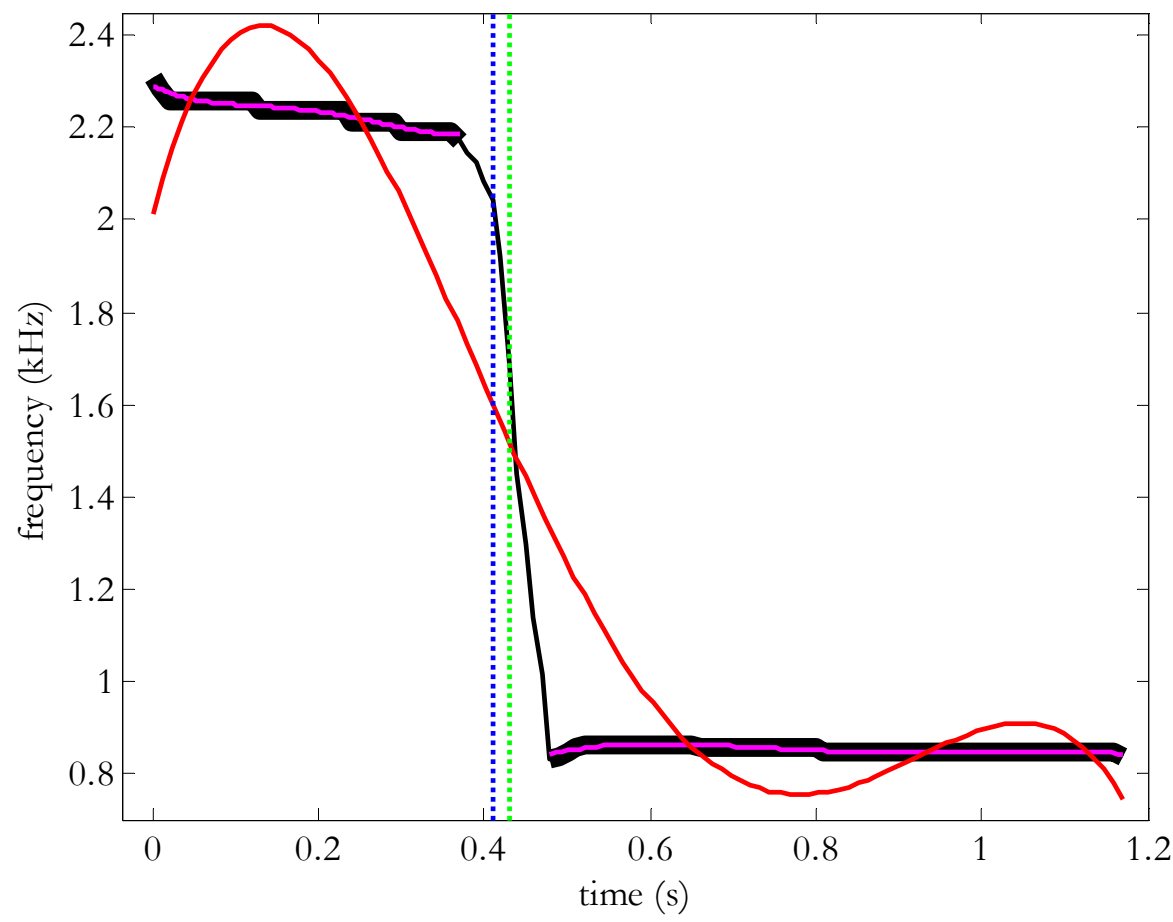

Figure 5.4. Contour trace of N32 call (see Figure 5.3 for sample spectrogram) in black (without the noisy onset) with $4^{\text {th }}$-order Legendre polynomial fit of entire trace superimposed in red. The segmentation algorithm first located the time point of maximum deviation between the actual contour and fitted polynomial (blue dotted line). Splitting the contour into two at this location and then scanning forwards and backwards, the time point that minimized the deviation in fit between the two new segments (heavy black lines, $0.05 \mathrm{~s}$ to either side of the split) and their individual polynomial fits (magenta lines) was used as the location of the actual segmentation split of the contour (green dotted line).

selected because of its precedence in human speech research (i.e., used to characterize the tones of Mandarin Chinese: Chen \& Wang, 1990; Wang \& Seneff, 1998; Wang, 2001).

For a contour being split into two (e.g., N16.1, N32), the time point of the maximum difference between the contour and the polynomial was computed. Using this as an initial breakpoint, two new $4^{\text {th }}$-order Legendre polynomials were fit to the contour traces preceding and following it. (This polynomial order was selected because of precedence in the literature (Chen \& Wang, 1990; Wang \& Seneff, 1998; Wang, 2001). See Discussion for further 
elaboration on the tradeoffs of this decision.) The breakpoint itself was exempted from tracing by excluding $0.05 \mathrm{~s}$ to either side of it, the approximate duration of this transition. The algorithm then slid this breakpoint forwards and backwards by increments of 0.1 s (i.e., the sampling interval of the pitch tracking algorithm) until the deviation between the two resulting contour segments and two new polynomial fits was minimized. This minimum was taken as the segmentation point. For a contour being split into three (i.e., N91), both the maximum and second highest difference between the whole contour and the fitted polynomial were determined. Fixing one, the second was shifted forwards and backwards until the deviation between the three resulting segments and polynomial fits was again minimized. The second was then fixed at this point and the original was shifted forwards and backwards until a new minimum between the traces and fits was located. This procedure was iterated until the deviation between the three contour segments and the three new polynomial fits was globally minimized.

A final manual review of all automated segmentation decisions was conducted to discard the calls containing erroneous divisions (22\% of the total) from all 3 experiments (see next section), generally resulting from discontinuities elsewhere in the pitch track or an overly gradual change in the trace between the segments. For the remaining $78 \%$, the two or three segments were separated from one another by non-traced transition regions of $0.1 \mathrm{~s}$. RMS values were calculated between the difference in the trace and curve fit of every contour before the segmentation algorithm was imposed. This term provided a measurement of the extent of frequency modulation, since the Legendre polynomial fit highly modulated contour shapes with numerous inflection points more poorly, leading to a greater RMS value. I expected that contours selected for segmentation with this algorithm 

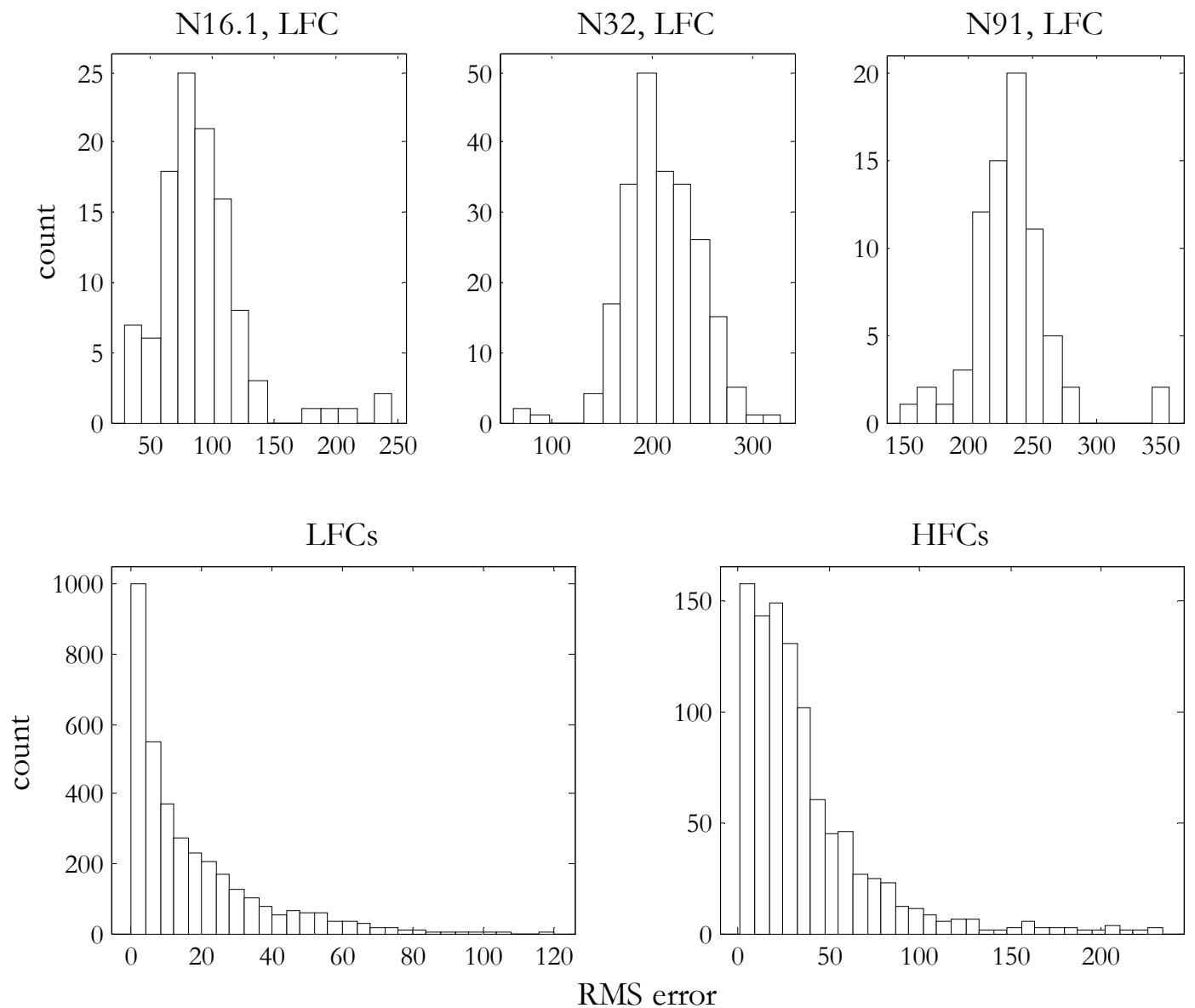

Figure 5.5. RMS error difference between contour traces and polynomial fits for the three calls on which the segmentation algorithm was run (top panels) and for all other low and high frequency components (bottom panels). Each of the top distributions was significantly different from each of the bottom distributions (MannWhitney $\mathrm{U}$ test, $P<0.001)$.

would be characterized by greater frequency modulation than the remaining contours. To verify and justify this claim, a Mann-Whitney $\mathrm{U}$ test was conducted to compare the distribution of RMS values of the three segmented call types to those of the non-segmented call types. The distributions were significantly different $(P<0.01)$ and are plotted in Figure 5.5 for purposes of graphical comparison. 


\subsubsection{Call classification experiments}

Phonemic classification schemes have yielded better and more efficient results in human speech recognition tasks than larger whole word divisions (see Introduction). In particular, mixed Gaussian models have achieved robust performance when characterizing the differences between phonemes in human speech (e.g., Bonafonte et al., 1996; Schultz \& Waibel, 2001). Based on these observations, a set of experiments was designed to evaluate whether describing the killer whale repertoire using syllables categorized calls at least as efficiently as more traditional whole call type divisions. This whole call type approach was the standard against which the other models of representation were compared. Equivalent or improved efficiency of these alternatives was needed to justify their ability to characterize the repertoire in a manner consistent with earlier studies and to parse this repertoire more parsimoniously using less information. The experiments employed here involved summarizing call type or segment features using mixed Gaussian models. Only call types with at least 10 exemplars were considered; this reduced the total number of call types from 62 to 31 . Figure 5.6 summarizes these three experiments graphically.

\subsubsection{Whole contour experiment (WCE)}

This experiment operated under the classic view that considered the entire call type to be the basic unit of killer whale vocal production. A $4^{\text {th }}$-order Legendre polynomial was fit to the entire LFC and/or HFC, linearly interpolating between segments separated by silent intervals. This interpolation permitted the entire call to be represented continuously, similar to how a human sorting spectrograms might consider the vocal and silent pieces together when evaluating whole calls. Six parameters that characterized the properties of 


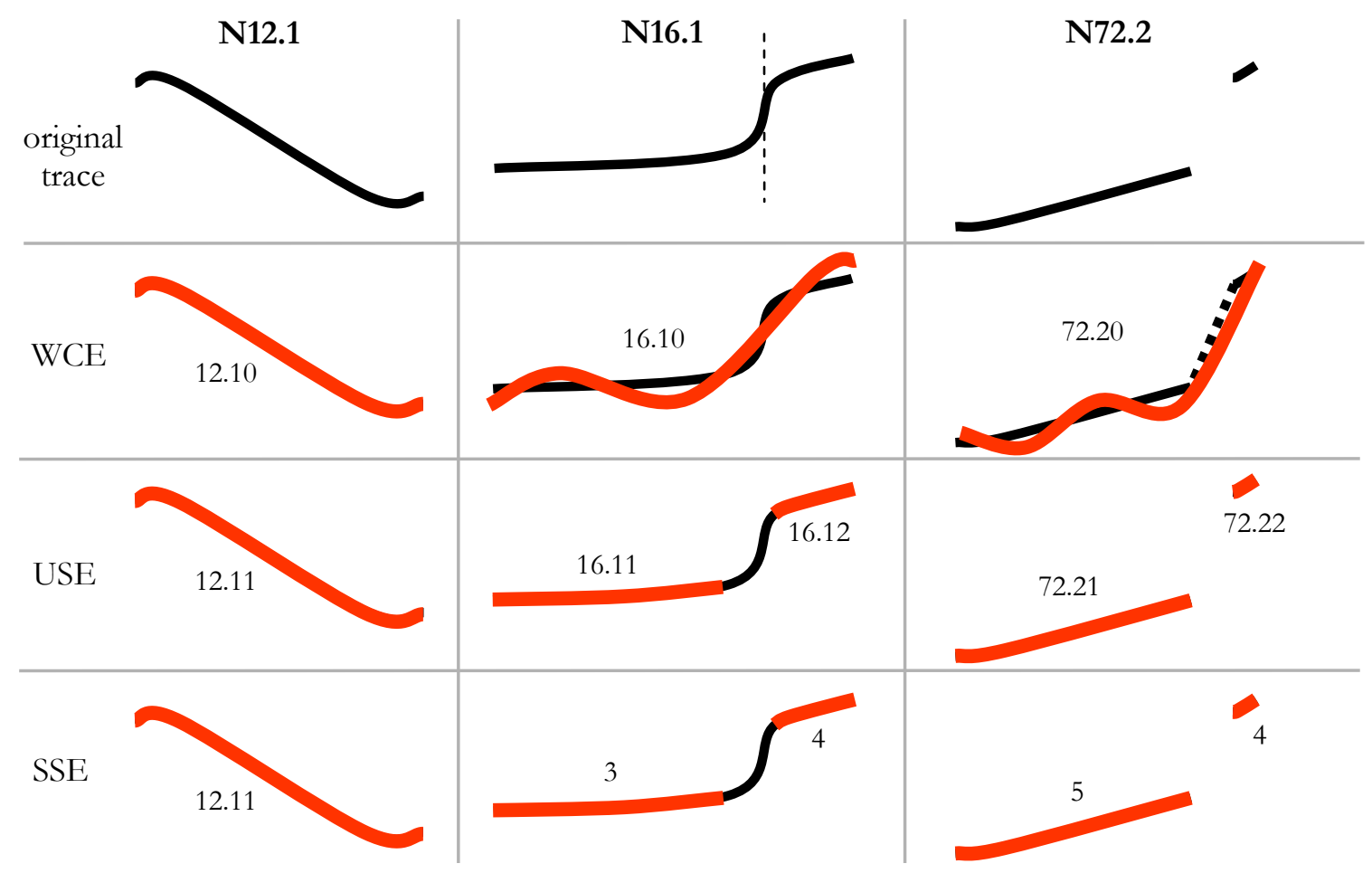

Figure 5.6. Schematic illustration of polynomial and labeling treatment of contours for each of the three experiments described in the text. The top row depicts the original traces for the low frequency components of 3 different call types: N12.1, a continuous and descending vocalization; N16.1, a continuous call subjected to the segmentation algorithm due to its rapid internal frequency change marked by the thin dotted line; N72.2, a call containing two segments separated by a brief period of silence. The second row plots the $4^{\text {th }}$-order Legendre polynomial fits used in the first whole contour experiment (WCE) in orange. Each contour was considered continuous and silent intervals were interpolated over (indicated by the thick dotted line connecting the two N72.2 segments). The contours were labeled by adding zeros until two places to the right of the decimal point were filled. The unshared segment experiment (USE) is shown in the third row. Here, the segments of each call were honored with a polynomial representation (N16.1 and N72.2 were divided into two segments using the segmentation algorithm and silent interval, respectively) but were labeled distinctly. The hundredths place in the label was used to count each successive segment for a particular call type. The final row demonstrates treatment according to the shared segment experiment (SSE). The segmentation decisions and polynomial fits were the same as in the USE but the labeling allowed call types to share syllables. For example, the second segments of both N16.1 and N72.2 were collapsed into syllable category 4. See the text for a description of the syllable collapse. Segments that appeared only in a single call retained their USE label (e.g., 12.11).

each contour were calculated: (1) the duration provided temporal information, $(2-5)$ the first 4 Legendre coefficients represented the basic spectral shape and (6) the RMS error between the polynomial curve and the actual trace captured the extent of frequency modulation. Deriving 6 features from the fundamental frequency alone represented a radical 
oversimplification of the spectrogram since this consolidation lacked information about the energy, harmonics and other details of the original signal. In human speech, for example, the intelligible properties of vowels are contained in the harmonics. Such information in the killer whale calls might have been lost using the curve fits employed here but the goal was to provide as simple a rendering as possible in this first treatment of the dataset.

For each call type, a training set was formed from a randomly selected $90 \%$ of its contour traces. This $90-10$ split allowed the training set to be formed from the majority of the dataset while leaving a sufficiently large test set to assess categorization efficiency (see Heuber et al., 2007 for discussion about the stability of such leave-one-out approaches; Elisseeff \& Pontil, 2002 for a human speech application). A multi-normal distribution was then defined for each call type by computing the mean vector and covariance matrix of the 6 features of its corresponding training set. All of the remaining $10 \%$ of the calls, the test set, were subsequently assigned to a call type by selecting the class whose training set data yielded the highest multi-normal probability density. The calls assigned to the training and test sets were preserved across the three experiments.

A test contour containing a LFC alone was compared against the LFC of all contours that had a LFC only or a LFC and HFC. A test contour containing a HFC alone was compared against the HFC of all contours that had a HFC only or a LFC and HFC. Finally, a test contour containing a LFC and HFC was compared against only those contours containing both as well. In this last case, the multi-normal probability density of the LFC evaluation was added to that of the HFC evaluation and the call type yielding the maximum sum constituted the matching class. This density summation was considered a mixed Gaussian model. In general, for this and all experiments, classification was aided by only 
considering as possible matches those call types that were characterized by the same component assignments (just described) and number of segments (see USE below) as the test contour. All three experiments were assessed based on how often an incorrect call type was chosen for each member of the test set. Results were reported as error rates \pm the standard error (s.e.), which was computed as:

$$
\text { s.e. }=\sqrt{\frac{p(1-p)}{N}}
$$

where $p$ was the error rate and $N$ was the sample size of the test set. The standard error calculation allowed comparison of the scoring between the three experiments to provide an indication of improved, weakened or equivalent performance.

\subsubsection{Unshared segment experiment (USE)}

This experiment operated under the alternative view that call types were composed of different segments but did not allow multiple calls to share the same segment. Every segment type $(N=62)$ was therefore considered individually and formed its own Gaussian

model. For calls with multiple segments, the multi-normal probability density was computed for each segment and summed; the maximum sum determined the matching class. In a second scoring for this experiment, performance was also rewarded for a selected call type match that contained a combination of the same phonemic classes (see SSE) as the correct call type (i.e., confusions with segments from other calls but with the same phonemic label were forgiven). The intervening $0.1 \mathrm{~s}$ spans between segments determined by the segmentation algorithm were ignored by the USE and SSE. 


\subsubsection{Shared segment experiment (SSE)}

This experiment operated under the alternative view that calls were composed of different segments that may have been shared across type. These segments were collapsed into phonemic classes by consolidating syllables that confused with one another when separated from their call type of origin and sorted, resulting in 26 syllables. The mean vector and covariance matrix were computed for each shared and call-specific phonemic class to generate the Gaussian models. Although each call type was defined by a unique combination of syllables, certain LFC or HFC segment sequences were occasionally shared across types (see Results and Discussion). Certain call types had exemplars that contained both a LFC and HFC but had entries in the test set that contained traces of the LFC or HFC alone because the SNR of the other component was too low for accurate pitch tracking. Because of the inability to resolve the confusion in favor of a correct assignment arising from these overlapping syllable combinations, these test set entries were considered to match incorrectly during the evaluation for this experiment.

\subsubsection{Rarefaction}

As each of the 13 recordings was added to the analysis, the rate at which new syllables accumulated was examined as a function of the rate at which new call types were contributed to the repertoire. The mean number of call types (or syllables), s, observed in a sample of $m$ individuals was given by:

$$
s(m)=K-\sum_{j=1}^{K}\left(\begin{array}{c}
M-L_{j} \\
m
\end{array}\right) /\left(\begin{array}{l}
M \\
m
\end{array}\right)
$$

where $K$ was the total number of call types (or syllables), $M$ was the total number of 
individuals and $L_{j}$ was the number of individuals in which call type $j$ occurred (analogous to certain formulations of biodiversity discovery curves: see Solow \& Smith, 1991). For the data here, $K=31$ call types or $K=26$ syllables and $M=14$ animals.

\subsubsection{Variable Norwegian killer whale calls and stereotyped Pacific Northwest resident and transient killer whale calls}

Variable Norwegian calls and stereotyped calls from resident and transient animals in the Pacific Northwest were evaluated identically and will be referred to collectively as "alternative calls." These calls were separated into segments based on intervening periods of silence (described in section 5.3.3). The segmentation algorithm was applied to all variable call traces with an RMS value exceeding $99 \%$ of the RMS values of stereotyped LFC and HFC contours that were not processed with the segmentation algorithm (bottom two histograms in Figure 5.5). Many of these contours did not contain an obvious breakpoint for segmentation (due to substantial frequency modulation, for example). The resulting divisions were inspected visually and only reasonable segmentation decisions were retained ( $24 \%$ of the total). The other contours were included as single, non-segmented traces. Each of the 26 Norwegian stereotyped phonemic classes was defined by a set of self-sorted density values resulting from correct pairings of training and test set members (see description of these syllable pairings and the collapse technique in the SSE section above). Each alternative call segment was characterized by the same 6 measures presented in section 5.3.5 above and classified to the Norwegian stereotyped phonemic class that returned the largest multi-normal probability density. Assessment was conducted by tabulating the proportion of self-sorted density values of this matching class that was exceeded by the 
density value of the alternative call. If this density value of the alternative call were greater than $25 \%$ of the self-sorted values, for example, this would be considered a match at the $25 \%$ self-sorted threshold.

\subsection{Results}

Of the 3696 calls traced, 3530 belonged to 31 call types (16 with LFC only, 3 with HFC only and 12 with both LFC and HFC) containing at least 10 exemplars. By excluding some $5.3 \%$ of the dataset belonging to call types with 9 or fewer samples, certain syllables may have been missed but an insufficient number of traces would have been available to summarize these classes quantitatively. In addition to tallying call type counts, Figure 5.7 accumulates a count of the stereotyped call type categories used in this analysis according to the number of calls that were assigned to each type. The 31 call types provided the basis of comparison for the whole contour experiment (WCE) whose treatment of the dataset paralleled traditional whole spectrogram sorting. Table 5.2 tallies the number of call types and contour traces that were divided into segments based on intervening periods of silence and quick spectral shifts. The unshared segment experiment (USE) assumed that each call type was composed of a set of distinct segments that were unshared across type. A total of 62 different segments (39 LFC segments and 23 HFC segments) was considered by the USE. The shared segment experiment (SSE) allowed call types to share syllables, reducing this number to 26 different syllables (19 LFC syllables and 7 HFC syllables). Naturally, LFC syllables tended to occupy a lower frequency range than HFC syllables. The syllables also varied in shape and duration and were labeled numerically in the order that they were identified. 

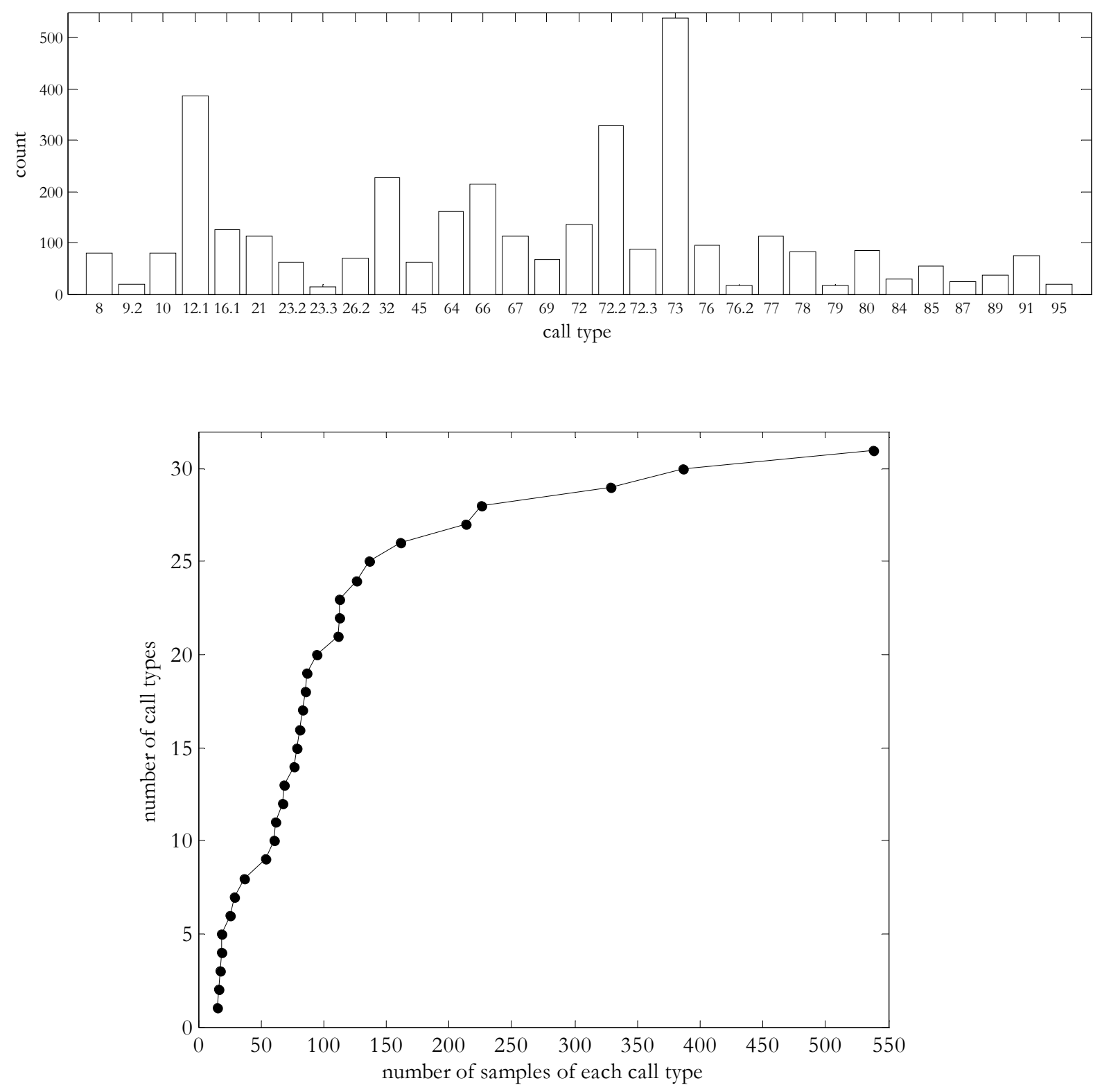

Figure 5.7. Top: Number of contours of each call type used in the 3 experiments described in the text. Bottom: Cumulative plot of the number of call types according to the number of calls associated with each type.

The categorization efficiency of all three experiments was equivalent (error rates \pm standard error for WCE: $0.079 \pm 0.014$; USE first scoring: $0.093 \pm 0.015$; USE second scoring: $0.091 \pm 0.015$; SSE: $0.071 \pm 0.014)$. The distributions of the error rates formed from 100 runs of these experiments overlapped (Figure 5.8). In other words, representing 


\begin{tabular}{c|c|c|c} 
component & number of segments & number of call types & number of contour traces \\
\hline \hline \multirow{2}{*}{ LFC } & 2 & 7 & 775 \\
\cline { 2 - 4 } & 3 & 2 & 161 \\
\hline \multirow{2}{*}{ HFC } & 2 & 8 & 309
\end{tabular}

Table 5.2. Counts of call types and contour traces affected by segmentation (see sections 5.3.3 and 5.3.4 in Materials \& Methods).

\begin{tabular}{c|c|r|r|r|r|r}
$\begin{array}{c}\text { self-sorted } \\
\text { threshold (\%) }\end{array}$ & \multicolumn{2}{|c|}{$\begin{array}{c}\text { Norwegian } \\
\text { variable calls }\end{array}$} & \multicolumn{2}{c|}{$\begin{array}{c}\text { resident } \\
\text { stereotyped calls }\end{array}$} & \multicolumn{2}{c}{$\begin{array}{c}\text { transient } \\
\text { stereotyped calls }\end{array}$} \\
\hline \hline 5 & 72.4 & 489 & 30.7 & 59 & 56.0 & 116 \\
\hline 10 & 53.5 & 361 & 20.3 & 39 & 39.6 & 82 \\
\hline 25 & 26.2 & 177 & 3.6 & 7 & 11.6 & 24 \\
\hline 50 & 10.8 & 73 & 0 & 0 & 2.9 & 6 \\
\hline 75 & 4.0 & 27 & 0 & 0 & 0.0 & 0 \\
\hline 90 & 1.9 & 13 & 0 & 0 & 0.0 & 0 \\
\hline 95 & 1.1 & 7 & 0 & 0 & 0.0 & 0
\end{tabular}

Table 5.3. Performance of alternative calls when evaluated against self-sorted Norwegian stereotyped call data. The first column in the table lists the self-sorted threshold that the alternative call needed to exceed to qualify as a match (see Section 5.3.7). For each alternative category heading (i.e., Norwegian variable calls and Pacific Northwest resident and transient stereotyped calls), the first and second columns contain the percent and count of calls, respectively, that matched one of the Norwegian stereotyped syllables at the performance threshold.

stereotyped calling behavior in terms of whole calls, unshared syllables and shared syllables all provided equally strong categorization results. The classification of whole call types by human judges was the standard against which these efficiencies were scored. The discovery curves in Figure 5.9 revealed that the numbers of both call types and syllables apparently saturated after the first 7 or 8 animals were considered (top panels). The eventual rate at which syllables accumulated was lower than that of call types.

Results of the Norwegian variable calls ( $N=576$ calls; 675 segments) and Pacific Northwest resident ( $N=192$ calls and segments since each call only had a single segment) and transient ( $N=162$ calls; 207 segments) stereotyped calls against the self-sorted Norwegian stereotyped calls are presented in Table 5.3. The values in this table correspond to the percentage (or number) of alternative calls whose probability density score associated 

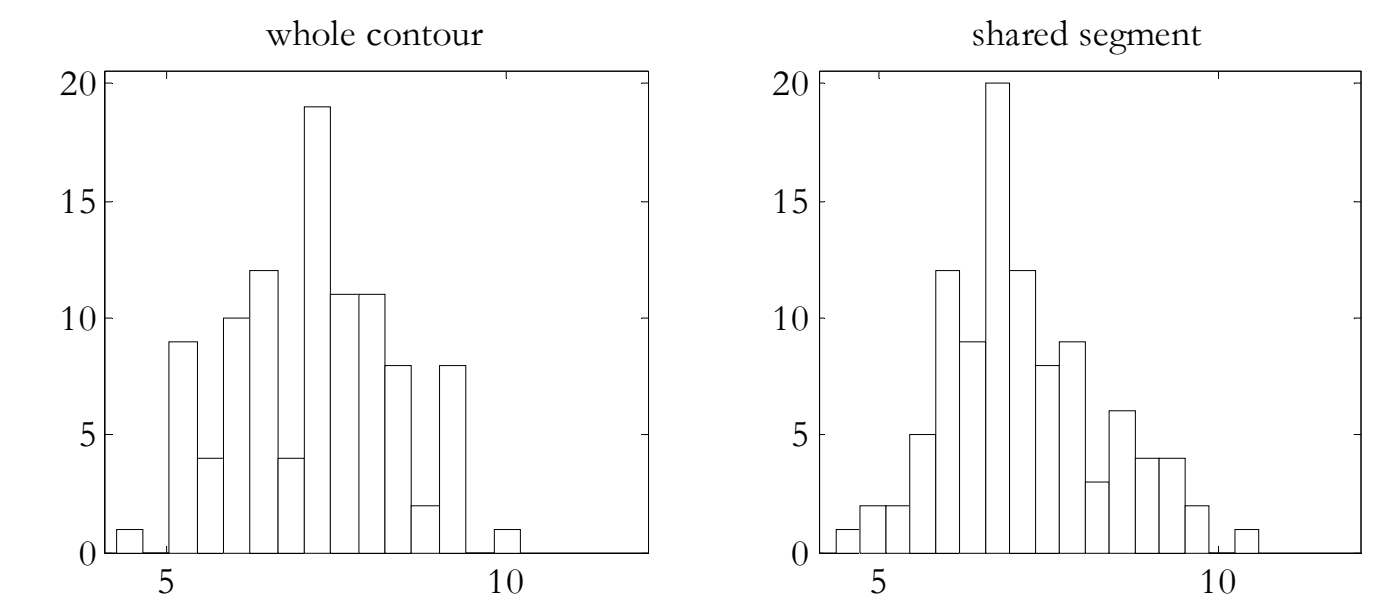

节

unshared segment, first scoring
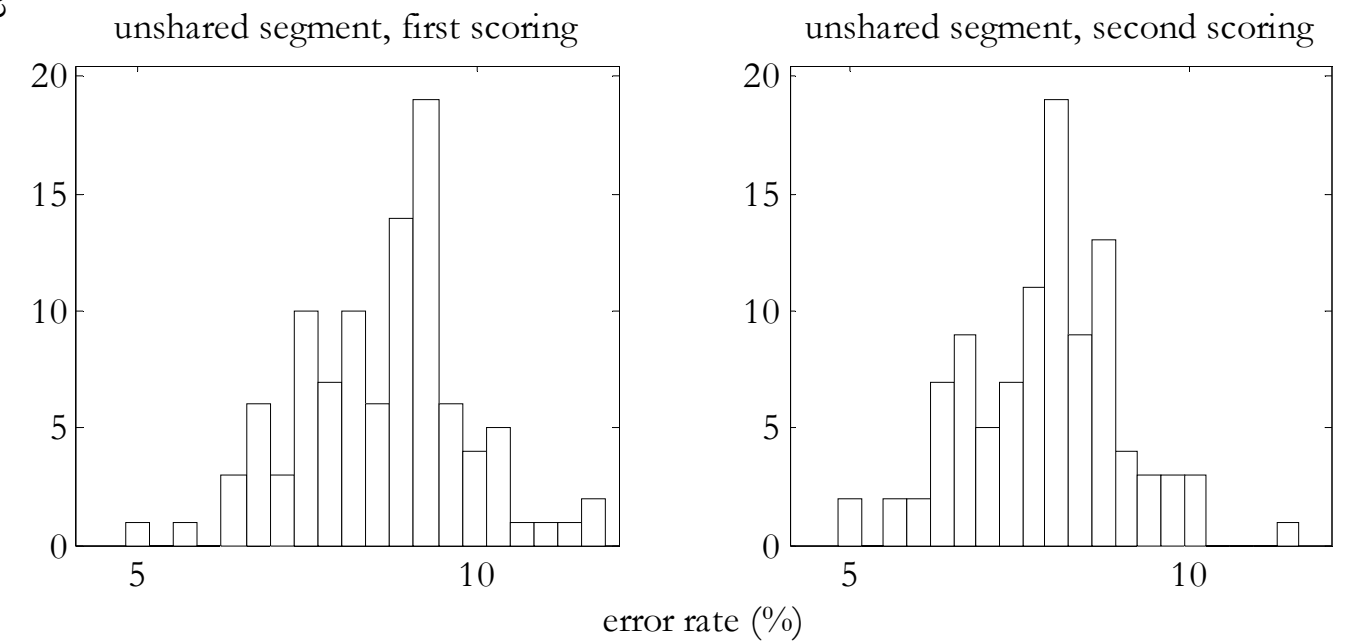

Figure 5.8. Histograms of error rates for each experiment after 100 runs. Call contours were categorized to type based on the entire call type (upper left), unshared segments (bottom row) or a combination of shared and call-specific segments (upper right). The unshared segments were scored strictly (i.e., each segment within a call type had to match to itself for the call to succeed, lower left) and more leniently (i.e., a call could also succeed if each of its composite segments matched to segments belonging to the same phonemic classes, lower right).

with its best Norwegian syllable match exceeded a threshold calculated from a density distribution of this Norwegian syllable when it correctly matched to its own model. The variable calls outperformed both sets of Pacific Northwest calls and the transient calls showed better performance than resident calls. At the $25 \%$ self-sorted threshold (see Section 5.3 .7 ), 84\%, $43 \%$ and $96 \%$ of the variable, resident and transient segments, 

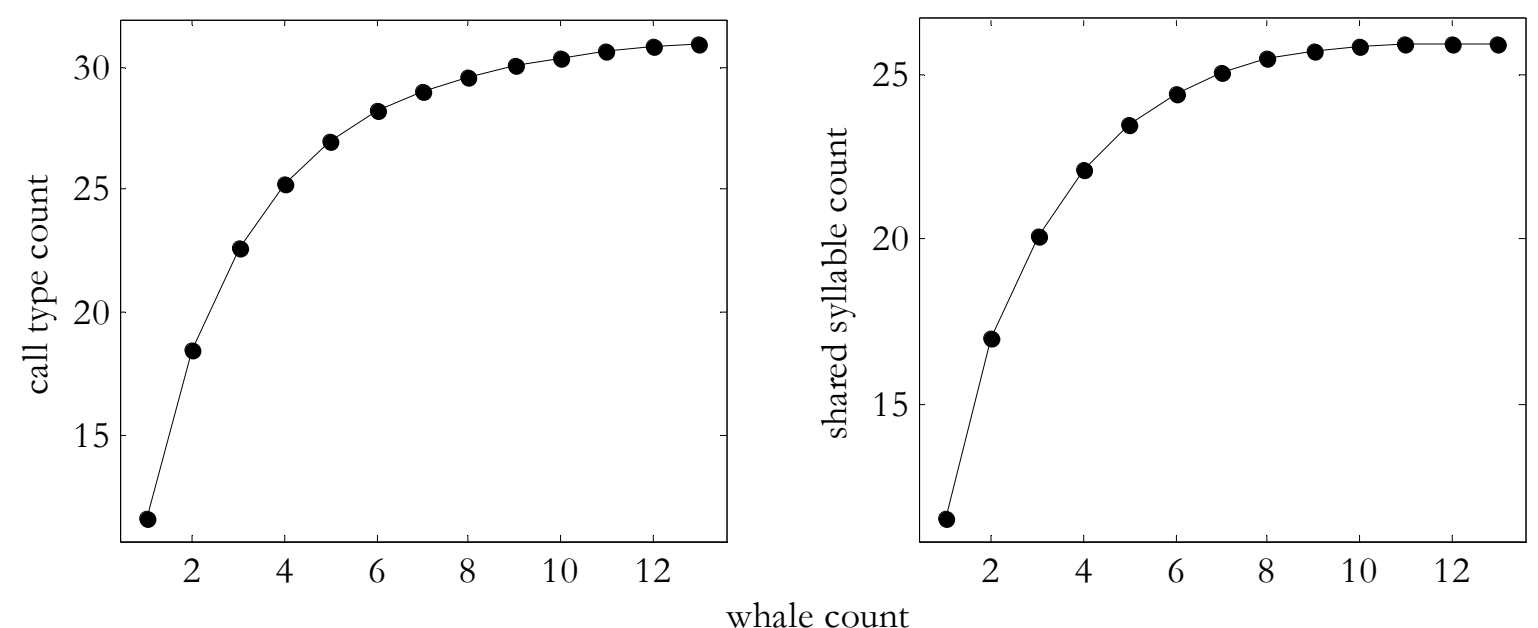

whale count

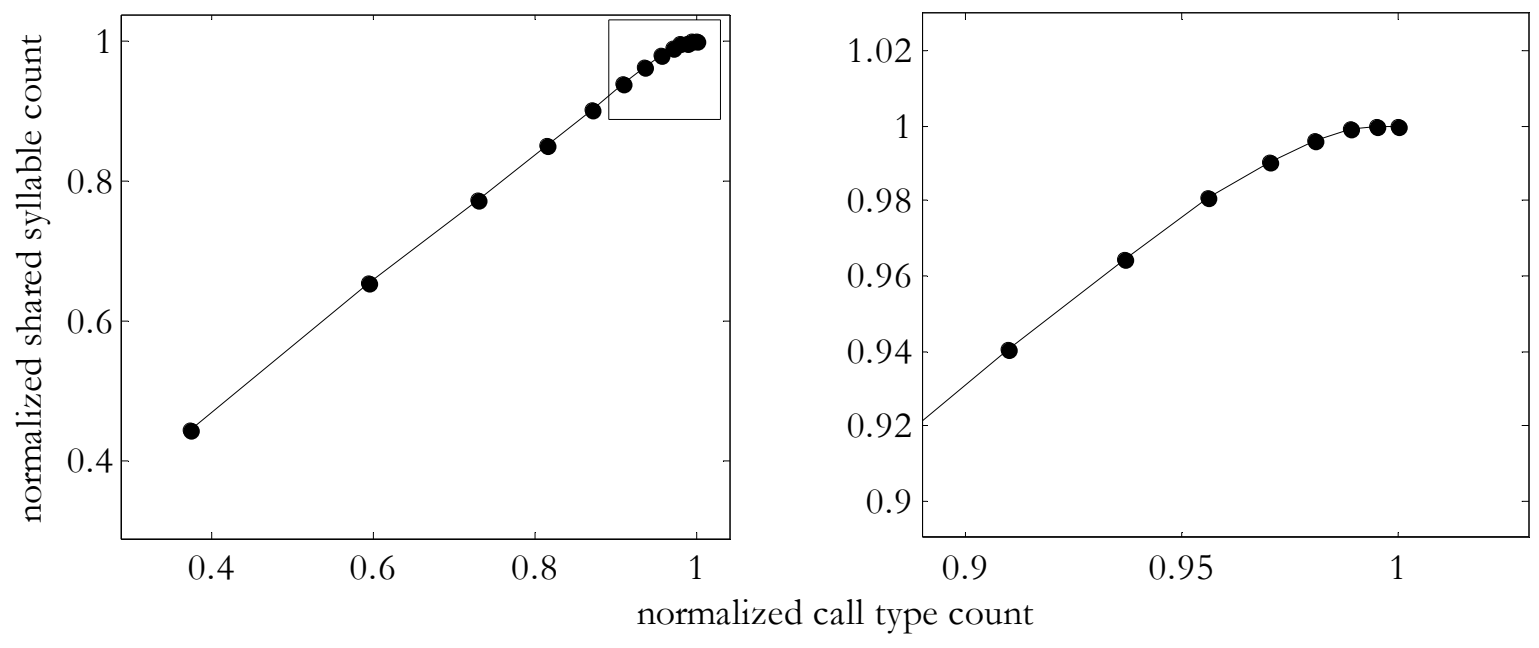

Figure 5.9. Rarefaction curves plotting the number of call types (top left) and shared syllables (top right) as a function of the number of whales considered. Bottom: Normalized number of shared syllables plotted against normalized number of call types and viewed for all data (left) and zoomed region (right, magnified to box depicted on left).

respectively, matched with one of the shared syllables from the stereotyped Norwegian repertoire. Figure 5.10 plots a sampling of the best matches for each alternative call category superimposed upon its corresponding syllable class.

After condensing the number of syllables required to represent the vocal repertoire, I examined how many call types drew on this shared pool and what their patterns of 

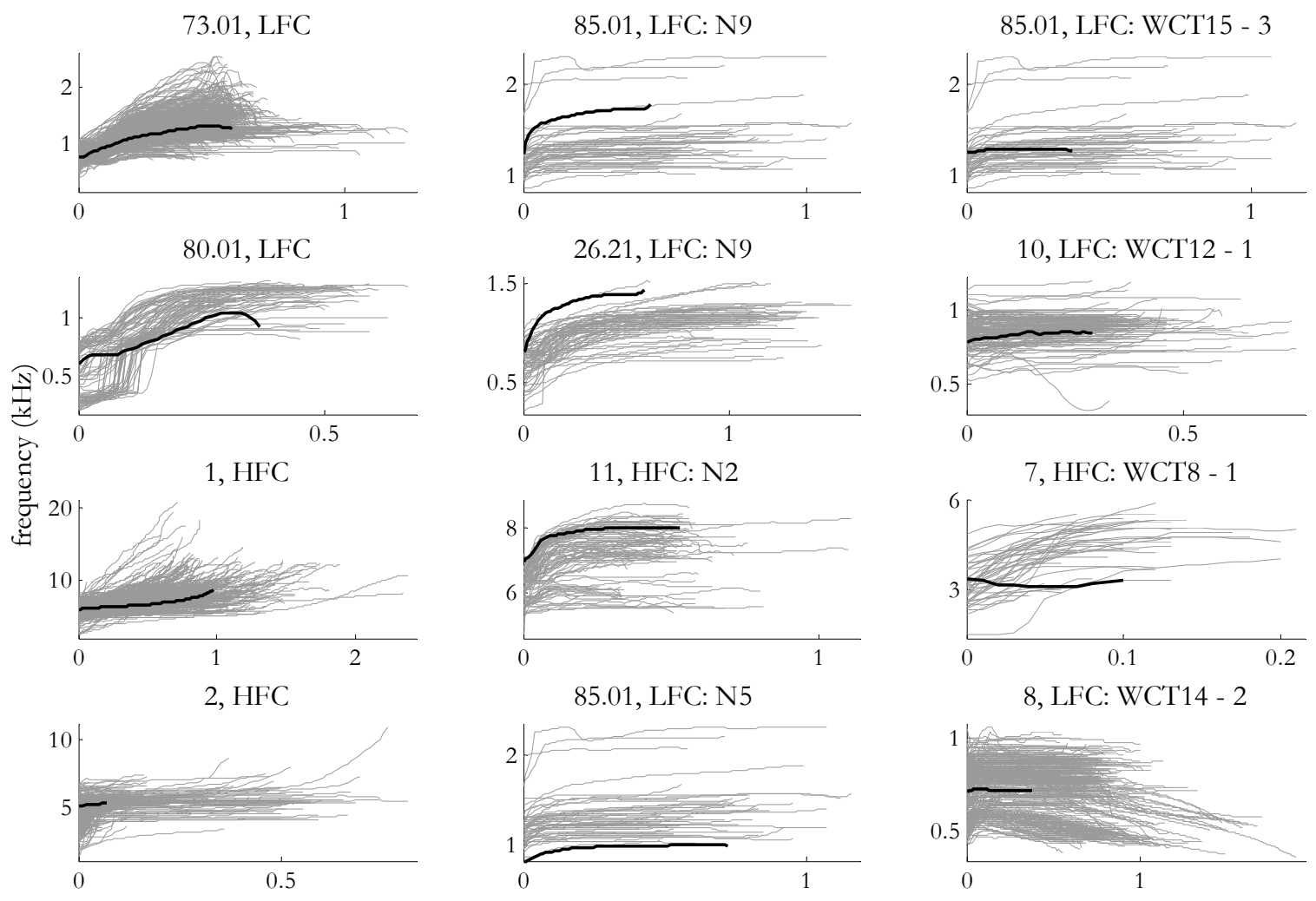

10, LFC: WCT12 - 1

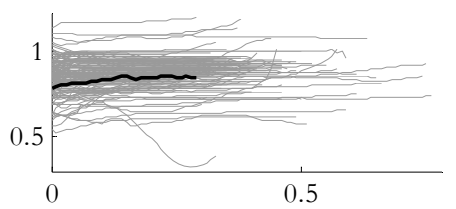

11, HFC: N2

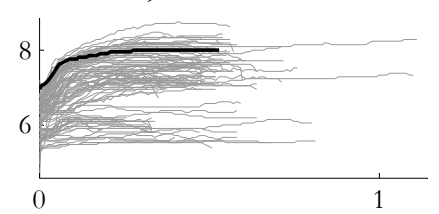

7, HFC: WCT8 - 1

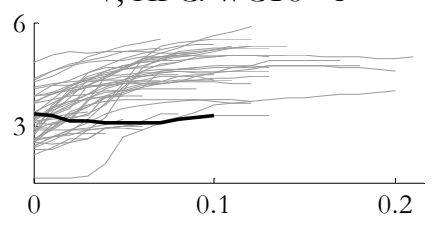

85.01, LFC: N5

8, LFC: WCT14 - 2
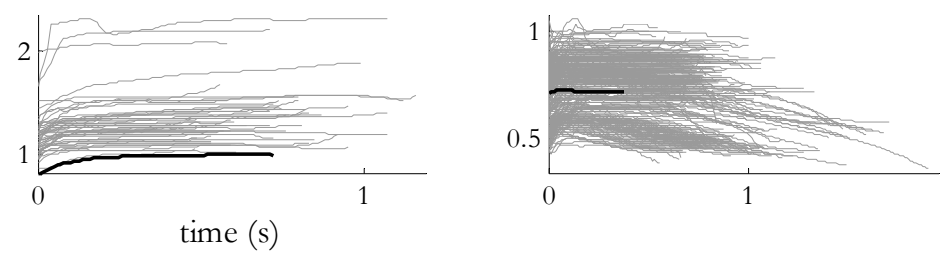

Figure 5.10. Sampling of matches of alternative calls (dark black traces) with Norwegian stereotyped call syllable matches (gray traces). The alternative calls included Norwegian variable (first column), Pacific Northwest resident (second column) and transient call segments (third column) that performed at threshold percentages (see Table 5.3) of $90 \%, 25 \%$ and $25 \%$, respectively. The title of each sub-panel lists the number of the syllable match and whether the match was a LFC or HFC. The LFC or HFC label also refers to the component of the Pacific Northwest calls and the titles in these sub-panels conclude with the call type of the resident or transient call. The number following the hyphen in the transient call label refers to the segment number.

occurrence were. Fifteen syllables appeared in only one call type. The remaining 7 LFC and 4 HFC syllables were formed from collapsing the segments from at least two different call types (Figure 5.11). Table 5.4 counts the number of call types (altogether and separated based on presence or absence of LFC and/or HFC) composed entirely of shared syllables, a mixture of shared and call-specific syllables and entirely of call-specific syllables. All but one of the shared LFC syllables constituted the entire LFC in at least one call type. Three of the 
syllable $1, \mathrm{HFC}$

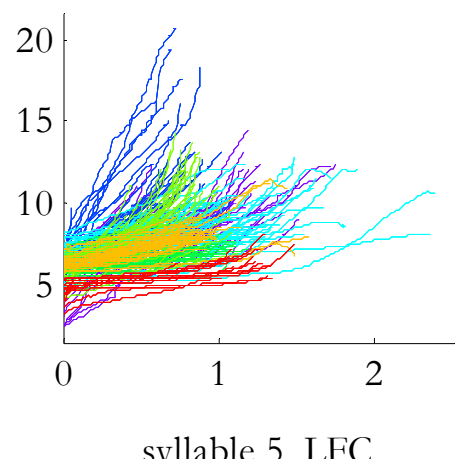

syllable 5, LFC

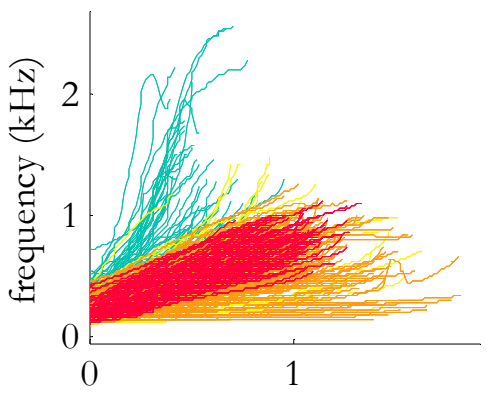

syllable 9, LFC

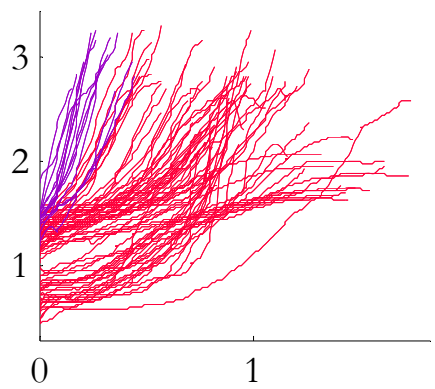

syllable 2, HFC
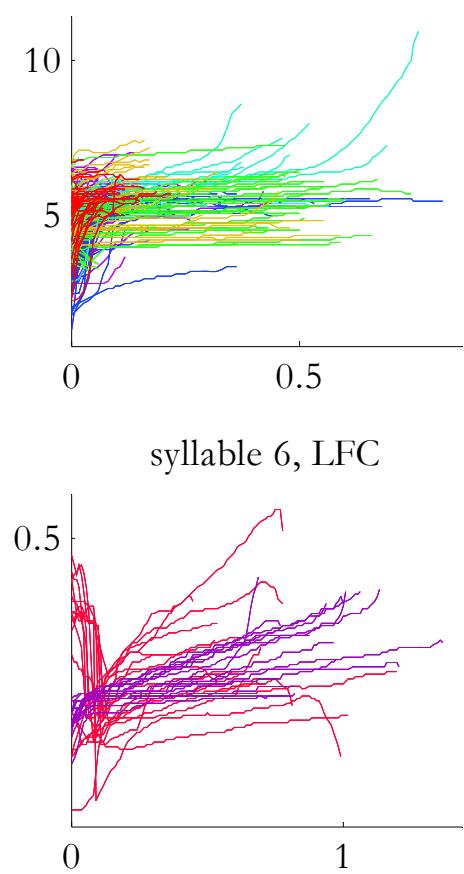

syllable 10 , LFC

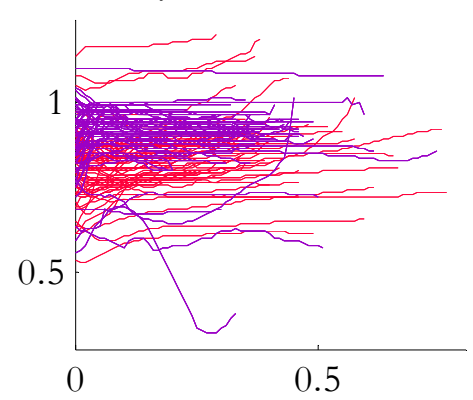

syllable 3, LFC
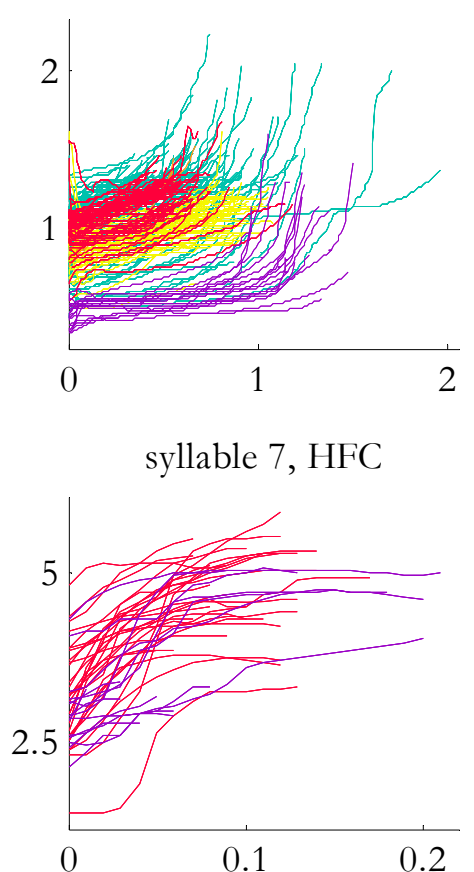

syllable $11, \mathrm{HFC}$

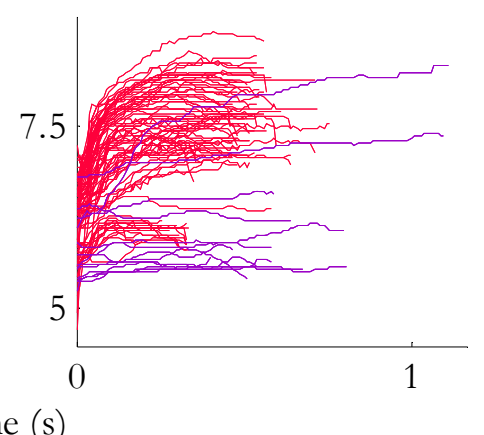

syllable 4, LFC
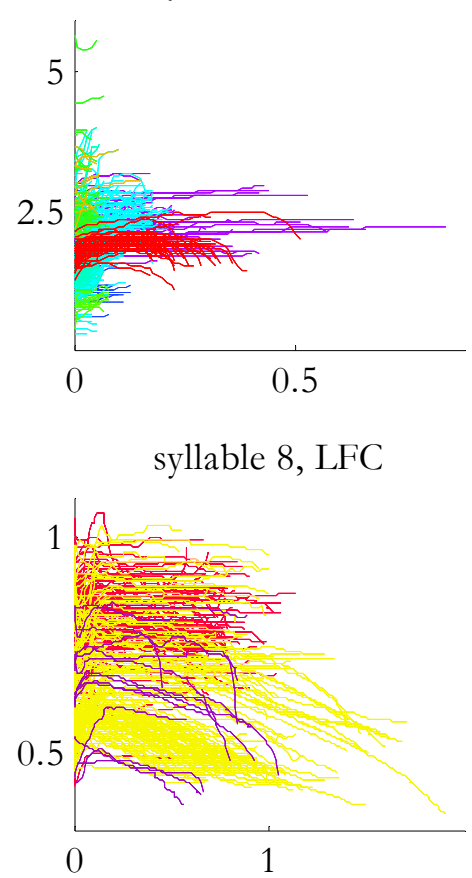

Figure 5.11a. 

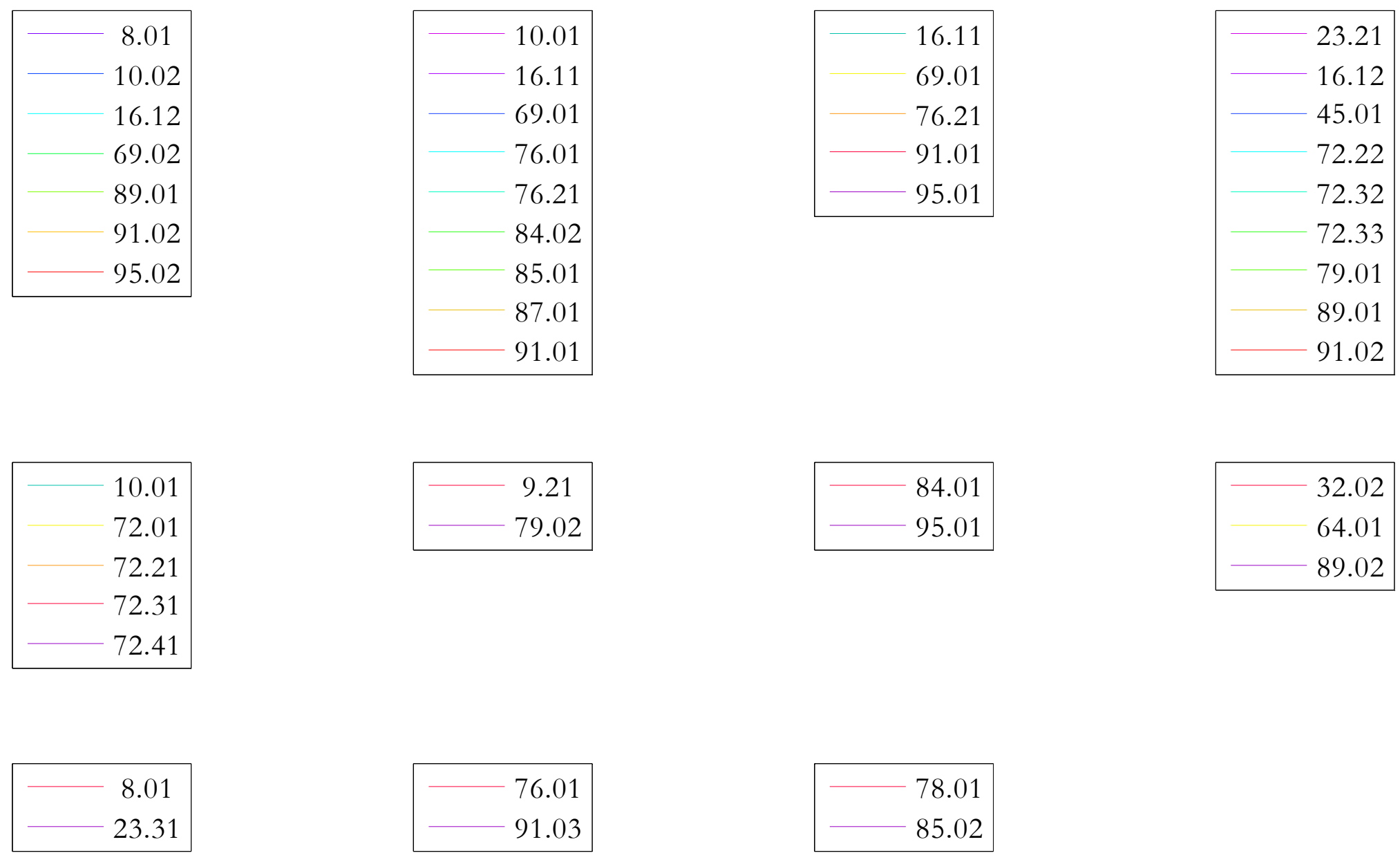

Figure 5.11b. a: Syllables found in at least two call types. Note different axis scales. b: Color code for syllable traces of different call type origins (legend locations here match trace plot locations). The digits before the decimal point and the first digit after the decimal point refer to call type (e.g., N8, N76.2, etc.). The second digit after the decimal point corresponds to the segment number in the call. (See Figure 5.6 for further elaboration.) Certain numbers are repeated in this figure but this ambiguity is resolved when differentiating between the LFC and HFC indicated in the titles of the sub-panels of the figure. 


\begin{tabular}{c|c|c|c} 
& call-specific syllables & syllable mixture & shared syllables \\
\hline \hline all call types & 8 & 5 & 18 \\
\hline LFC \& HFC & 2 & 2 & 8 \\
\hline LFC & 6 & 3 & 7 \\
\hline HFC & 0 & 0 & 3
\end{tabular}

Table 5.4. Counts of all call types (first row), call types containing both a LFC and HFC (second row), a LFC alone (third row) and a HFC alone (fourth row) that were comprised of call-specific syllables only, a mixture of shared and call-specific syllables, and shared syllables only.

seven LFC syllables arose in multiple call types at the same position in a series (i.e., first or last) and two appeared in multiple call types at different positions in a series.

Now I will describe three syllables in greater detail that occurred commonly and at a reliable location in a sequence. Syllable 7 was produced in the first position of a two-syllable sequence in two call types and never in the last position. Of the 13 HFCs constructed from at least one shared syllable, 12 contained syllable 1, syllable 2 or both (see Figure 5.11 for plots of these syllables). In fact, 4 call types had HFCs built from a syllable $2 \rightarrow 1$ sequence. The corresponding LFCs for these call types contained syllables that were all shared with at least one other call. Syllable 1 occurred second in the only other instance in which it was paired with another syllable that was not syllable 2 . Syllable 2 occurred first in two of the three other instances in which it was paired with a second syllable that was not syllable 1.

The patterns of certain call types seemed to be most parsimoniously explained by a set of shared and flexibly ordered syllables. Several sets of call types could be described by the simple addition of extra syllables to a common base segment or set of segments in the manner described in the Introduction. For example, call type N72 (Figure 5.1) was comprised of a long and slowly ascending initial segment (syllable 5). Call types N72.2 and N72.3 contained syllable 5 followed by one or two rapid higher frequency segments at the end (a single or double instance of syllable 4, respectively). These three subtypes can be 
generated easily by variably arranging or repeating two LFC syllables. Another example involves call type N16.1, which was built from two LFC syllables and two HFC syllables (Figure 5.1). N91 was identically composed except for an additional LFC syllable at the end (Figure 5.1). In fact, the LFCs of N91.2 and N91.3 (Figure 5.1, not used in the analysis here because they were recorded fewer than 10 times each) could be synthesized by appending another one or two LFC syllables, respectively, to the end of an N91.

\subsection{Discussion}

Taking advantage of human speech processing methods, the pitch tracking algorithm, segmentation algorithm, and single and mixed Gaussian models were successfully adapted and applied here to Norwegian killer whale vocalizations. Human speech can be broken into words or phonemes and though each division is meaningful from a production perspective, the phonemes afford improved speech recognition algorithms on large vocabularies in particular (see Lee et al., 1989) and are relevant for our perception of consonants and vowels (Furui, 1986). Analogously, though the call type has been viewed historically as the basic unit of killer whale stereotyped vocal production, I used a classification test to explore whether the vocal repertoire could be parsed and represented just as efficiently using smaller phonological units. Compared to the approach of categorizing whole call types, a few pieces of evidence from this study support the notion that a syllabic inventory could provide a set of simpler shared components that the killer whales use to assemble at least some of their calls. In particular, 1) equivalent classification efficiencies were achieved when the sorting was based on either the non-segmented whole call type or the syllabic divisions, 2) nearly $75 \%$ of all stereotyped calls contained at least one 
syllable shared across calls, and 3) the set of stereotyped syllables provided reasonable matches for many of the variable calls.

First, the results indicated that temporal and spectral representations of calls in their entirety (by the whole contour experiment (WCE)) or as sets of either distinct (by the unshared segment experiment (USE)) or shared (by the shared segment experiment (SSE)) syllables achieved similar performance when classifying these calls to type as judged by human observers (Figure 5.8). All three experiments provided equivalently robust means of characterizing stereotyped calling behavior. Compared to the WCE and USE, the SSE relied on a reduced amount of information to perform the classification task. In particular, the same dataset was compressed into a smaller number of polynomial representations for the shared syllables in the SSE $(N=26)$ versus the set of entire call types $(N=31)$ or distinct syllables $(N=62)$. Because each unit was characterized by 6 parameters, the SSE required considerably fewer features (i.e., 156) than the other two experiments (186 for WCE and 372 for USE), reducing the computation time considerably. These shared syllables still classified calls to type as effectively as the WCE and USE characterizations, suggesting that this loss of information may have been offset by a simpler and more efficient alternative system of representation. Computer scientists have moved to a similar approach for human speech because improved recognition can be achieved using phonemes instead of words. (Of course, there remains the downstream need to derive words from these assembled phonemes.)

As described in the Introduction, chunking is the process by which information is segmented to relax the cognitive demands associated with retention and recall. The WCE and SSE proposed that a killer whale could construct its entire stereotyped vocal repertoire 
either by storing a large number of whole call types or by rearranging a smaller set of archived shared syllables, respectively. (The nearly tripled size of the syllable count when each was considered distinct instead of shared rendered the USE-based representation less likely from a chunking perspective. In particular, chunking facilitates recall by decomposing information into a set of constituent parts. With fewer parts, less memory must be devoted to store the pieces required to represent and understand the whole repertoire. The SSE demands only a third of the information used by the USE, permitting a more condensed and efficient representation.) The SSE approach supports the viewpoint that killer whale calls can be chunked into fewer and simpler phonological vocal units, which can generate the same repertoire defined by the larger and more complex set of whole call type contours used in the WCE. As mentioned above, the reduction in memory load afforded by the smaller shared syllable count may be offset by the need to retain the rules used to reconstitute the repertoire from these syllables.

Second, the 11 shared LFC and HFC syllables identified here were shared and reordered to generate a variety of different call types (Figure 5.11). The duration, Legendre polynomial coefficients and RMS frequency modulation score all determined which segments sorted together and merited collapse into a single category. Most call types were built from at least one of the shared syllables, supporting the notion that many of the calls were constructed from a set of common syllabic units. Indeed, 18 of the 31 call types were comprised entirely of shared syllables and another 5 contained at least one shared syllable (together constituting $53 \%$ of the calls, Table 5.4). Certain patterns of syllable usage emerged. Some syllables were used much more frequently than others, including LFC syllable 4 and HFC syllables 1 and 2. Distinct call types resulted when the same 
arrangements of HFC syllables were paired with different combinations of LFC syllables (and vice versa). When combined in tandem, 3 shared LFC and 2 shared HFC syllables were produced at the same position within a sequence, consistently beginning or ending multiple call types. For example, when paired, HFC syllable 2 always preceded HFC syllable 1. Syllables could be ordered more flexibly as well: 2 shared LFC and 1 shared HFC syllables were produced at different positions within a sequence. These observations may form the outlines of a phonological syntax-based rule system in which syllables are arranged in certain orders and combinations only, but further work is required to test this hypothesis.

The two examples given in the results (i.e., N72, N72.2, N72.3 and N16.1, N91, N91.2, N91.3) suggest a system in which new call types can be generated by concatenating additional syllables and interspersing them with periods of silence (the former) or stringing them together as a continuous vocalization (the latter). This study does not offer proof that the killer whales were actually creating their calls in this manner. (Training captive killer whales to synthesize calls by serially producing components heard from a loudspeaker and/or to decompose playback calls by producing the set of constituent segments would certainly offer important supporting evidence.) Such a system could, however, flexibly yield the size and kind of repertoire produced by these animals. In addition, new call types could be fashioned from existing call types simply by adding, deleting or reordering syllables. One of the basic patterns witnessed here indicated that the LFCs could be formed by linking successively longer strings of syllables together whereas the dominant HFC syllable combination was conserved across multiple call types.

Eight call types were constructed entirely from a set of unique syllables that were not shared with any other type. Because the shared syllables were formed from distinct 
segments that confused with one another when they were sorted, additional consolidation would have produced an even smaller number of unique units and consequently a greater number of call types formed from the set of shared units. A tradeoff emerged since further syllable collapse conflated previously distinct call types by characterizing them with identical internal syllabic orderings, reducing the total number of call types. This provided an effective lower bound on the total number of shared syllables, limiting the extent to which the segments should be collapsed.

Third, nearly half of the variable calls matched a stereotyped syllable with a score that rivaled at least $10 \%$ of repeated self-sorts of the actual syllable (Table 5.3, Figure 5.10). In other words, many of the variable calls, which generally have been investigated separately from stereotyped calls or dismissed altogether from analyses, sorted into the syllable categories generated from the stereotyped repertoire. This suggests that variable calls may differ less from stereotyped calls than previously thought and that many variable calls may represent different arrangements of the same phonological segments as are found in stereotyped calls.

The prospect that killer whales build their calls from smaller subunits is reinforced by the observation that compound calls can be constructed from whole stereotyped calls (Chapter 3; Strager, 1993; 1995). This suggests a nested system of vocal production in which similar rules of flexible sequencing assemble syllables into call types, which can then be assembled into compound calls. Certain syllables (e.g., 1 and 2) and call types (e.g., N66 and N67) participated more frequently in these assemblages than others. A large portion of the killer whale vocal repertoire can be defined by a system that flexibly generates new call types from a finite set of components but employs only a subset of the possible combination of 
these segments. This kind of vocal structure of smaller subunits building the repertoire is consistent with analyses conducted on Alaskan resident and transient killer whale stereotyped calls (Yurk, 2005) and on humpback whale song (Payne \& McVay, 1971; Payne et al., 1984; Suzuki et al., 2006), suggesting that it may characterize the vocal regimes of a broader array of marine mammal populations and species than previously anticipated.

Generally, a syllable included a set of traces that occupied a continuous time and frequency space. For example, syllable 8 was defined by segments gathered from three call types (N32, N64 and N89) that lasted $\sim 1 \mathrm{~s}$ and ranged between $500 \mathrm{~Hz}$ and $1 \mathrm{kHz}$. A few syllables were formed from call types whose contributions to the class segregated noticeably, causing larger amounts of temporal and/or spectral spread (Figure 5.11). This was due in part to the multivariate representation of each contour since a syllable category could have been formed from contours that shared a subset of the 6 temporal and spectral features used to determine collapse into a single class. Context may have influenced this variability as well. Most of the segments comprising syllable 4, for instance, were short and produced either separately or between periods of silence. Segments 16.12 and 91.02, however, were the longest contributors to syllable 4 and both were vocalized without interruption after an initial syllable. The immediate vocal context and position of a segment within a call type may have influenced its production and caused some of the variability observed in Figure 5.11 (see Pols, 1986 for a human speech analog in which vocal context and transition influence the acoustic structure of phonemes and how they are perceived). Finally, each segment was treated as a unified set that was subject to collapse with other segments. Both outliers and traces that approximated the mean of a syllable's distribution more closely were bundled together during collapse. Segment 72.33 in syllable 4, for example, was 
characterized by considerable scatter in terms of mean frequency (Figure 5.11), possibly arising from variability in production at the individual or group level.

A portion of the stereotyped calls from Pacific Northwest resident and transient killer whale matched successfully with the Norwegian syllables identified here (Table 5.3, Figure 5.10). This is especially striking considering the high performance standard needed to rank as well as the stereotyped Norwegian syllables. There are two alternative explanations for this result. The first concludes that because killer whales vocalize within a finite temporal and spectral range, it is to be expected that a certain proportion of signals will overlap between populations by chance. The second suggests that, similar to humans, each population of killer whales uses a portion of the common universal phonemic inventory to form its own subset of units to establish its vocal repertoire. The lower success rates of the stereotyped Pacific Northwest calls compared with the variable Norwegian calls (Table 5.3) do indicate important divergent properties between the populations that need to be considered.

These two hypotheses will be informed by further work on how similar syllable usage is among killer whale groups. The second explanation, for example, would receive additional support if the same syllable were to undergo the kind of cultural drift across multiple call types as has been observed at the level of the call type (Deecke et al., 2000). Similar to human speech and dialect patterns, previous research has indicated that killer whale vocalizations change over time as a result of cultural changes and copying errors (see Ford, 1991 for an early discussion of the issue and Deecke et al., 2000 for a demonstration; Miller \& Bain, 2000; Yurk, 2005). Cultural drift may also operate on the syllables if they are indeed the more basic units of vocal production. Just as different call types changed at 
different rates (Deecke et al., 2000), the temporal or spectral properties of different syllables may become altered depending on their pattern or frequency of usage. Supposing that these animals have production control over the individual syllables separately, further work should test the rates and kinds of syllable modification that occur over time. If shared syllables drift similarly across call types, this would support the view that calls are composed of discrete units subject to individual handling. This idea is analogous to the manner in human language in which the drift in production of certain vowels across words can lead to regional dialects and accents. Another experiment might involve training captive killer whales to respond to playbacks of a subset of calls by producing each call's constituent segments in a series separated by brief silence. Their vocal response to a new subset of call playbacks could then be investigated. If the animals produced the component segments of these new calls, the experiment would demonstrate that the animals were capable of parsing the vocalizations into syllables in a manner consistent with their training.

This study introduced a variety of new techniques based on the field of human speech recognition to analyze killer whale vocal repertoires. The pitch tracking algorithm used here has been productively applied elsewhere (Nousek et al., 2006; Miller et al., 2007), demonstrating its effectiveness in a variety of experimental contexts. The Legendre polynomials offered a simple representation of the contours that permitted robust classification to the call type categories previously defined by humans. The capacity to represent each contour with only 6 data points marked a considerable improvement in the amount of computation time and memory required to execute classification tasks. The $4^{\text {th }}-$ order Legendre polynomial fit offered a compromise between reducing the number of data points required while still achieving a fairly accurate fit. It is likely that reducing the order of 
the polynomial further (and therefore the number of data points associated with each contour) would have contributed to a compromised ability to discriminate between call types in the experiments presented here. Increasing the order, however, would have required longer computation time. Although a higher order would have fit the discontinuous traces slightly better, it would have introduced a set of unnecessary additional coefficients for the simpler continuous traces that may have interfered with categorization. Future work could consider this issue more closely by evaluating classification performance across a range of orders of the polynomial fit.

The 3 experiments could only be compared when each was afforded the same information to conduct the classification. All of the results were improved therefore by constraining the possible training set matches according to the number of segments and components in the test contour. If these constraints were relaxed in the context of a different study, similar classification performance results might be achieved by using a higher order Legendre polynomial fit. In addition, the Gaussian models provided a straightforward way to summarize the contributions of numerous contours through a single mean vector and covariance matrix. The speed and accuracy of these methods would facilitate real-time call type classification and analyses requiring more elaborate computations.

In the future, it would be useful to explore forming a full inventory of phonemes derived from the variable calls alone and investigating the overlap between this inventory and the stereotyped and variable phoneme inventories of both Norwegian and other populations. The ultimate test for the legitimacy of the perspective that syllables are the building blocks of killer whale vocal activity must come from the animals themselves. Playback studies in captivity could test whether animals are capable of discerning syllables by 
evaluating their performance on syllable matching and discrimination tasks. Further support for syllable sharing would be offered by killer whales that, after being trained to classify stimulus pairs as the same or different, sorted syllables roughly into the categories established here. This study offers incentive to continue exploring syntax in killer whales analytically and experimentally to improve our understanding of how these animals perceive and produce their vocal repertoire.

\subsection{Acknowledgements}

Stephanie Seneff and Peter Tyack offered numerous brainstorms, advice and guidance on the theoretical development of this chapter. Andy Solow offered comments on the evaluation and rarefaction components of the analysis. Chao Wang generously allowed me to use her pitch tracking algorithm on the killer whale calls, supplying her time and technical support to optimize its performance. The analysis benefited enormously from the dedicated hours of correcting of contour traces and discerning of overlapping calls provided by Sara Kim, Gary Matthias, Rebecca McGowan, Levi Schmidt, Katie Smyth, Clara Stefanov-Wagner, Marlene Brito, Deirdre Connolly, Maitagorri Schade and Ivan Dimitrov. Diane Rak, Katie Stanchak and Jia Xin Wu assisted with call type classification. Dr. Robert Gross offered a detailed explanation and programming hints for fitting the Legendre polynomials to the contour traces. Ghinwa Choueiter and Scott Cyphers helped me gain access to and program the otherwise impenetrable CSAIL SLS computers. The undergraduate student help was supported by the Massachusetts Institute of Technology Undergraduate Research Opportunities Program office and the Ocean Life Institute at the Woods Hole Oceanographic Institution. 


\section{Chapter 6. Conclusions}

\subsection{Framing comments}

There has been increasing interest in cultural traits that are transmitted socially through observation and learning in animals. While social learning can most easily be demonstrated in experiments with captive animals, methods for gathering indirect evidence have been proposed for wild animals. One method suggests that if ecological and genetic explanations can be discounted for behavioral differences between social groups or populations in the wild, such differences are likely to have arisen via cultural transmission. For example, Whiten et al. (1999) examined whether 39 different behaviors were absent, occasionally present or frequently present in 6 populations of chimpanzees (Pan troglodytes) across Africa. These authors concluded that the resulting unique behavioral arrays arose from culturally-transmitted differences between the populations. Although it is not possible to exclude genetic or ecological explanations entirely (see Humle \& Matsuzawa, 2002), Whiten et al. (1999) certainly brought more attention to the discussion of animal culture. Two years later, a lengthy discourse on the subject of culture in marine mammals was published (Rendell \& Whitehead, 2001). Killer whales (Orcinus orca) featured prominently in this article and the ensuing commentary since they exhibit a combination of three features that make the cultural acquisition of behavior likely in this species (see Chapter 1). First, their population-distinctive foraging strategies resemble the differences in feeding behavior between the chimpanzee populations described above. Second, their social structure provides a set of stable relationships where repeated learning and cultural transmission of behavior could occur. Finally, killer whales produce group-distinctive vocal repertoires that parallel the song repertoires of certain bird species and that exceed in complexity and 
diversity the vocal behavior of non-human primates including chimpanzees.

Captive experiments allow a controlled demonstration of social transmission and observational learning (e.g., Helfman \& Schultz, 1984; Warner, 1988; Galef, 1992; White et al., 2007). Such work, however, tends to be disconnected from the functional contexts of behavior in the wild, which can be difficult to track continuously. A hybrid approach is likely to be the most beneficial in which social learning studied in captivity is complemented by explorations of behavior in the wild, an ecologically- and evolutionarily-valid setting. From a functional perspective, it is important to understand the details of group- or population-specific behavioral differences as a starting point to search for evidence that killer whales engage in cultural transmission and social learning. The aim of my dissertation was to focus on this functional approach and use digital archival tags (DTAGs, Johnson \& Tyack, 2003) to examine the feeding and vocal behaviors of Norwegian killer whales more closely. The killer whales that have been studied in this population display carousel feeding behavior (Christensen, 1978; Similä \& Ugarte, 1993) and pod-specific stereotyped pulsed call repertoires (Strager, 1993; 1995). I explored the contributions of individual animals to carousel feeding groups, the relationships between individual movements and group vocal activity and the syntax of pulsed calls. My approach characterizes a constellation of behaviors that can be used in a manner similar to the behavioral array employed by Whiten et al. (1999). In particular, a set of companion studies should be designed in the future to examine the detailed movement, feeding and vocal behaviors between killer whale populations. These inquiries will provide a foundation for describing both the shared and divergent patterns that might be inherited or learned.

Once the details of these behaviors are mapped for at least a handful of populations, 
it is important to examine their acquisition by exploring their transmission. To prove social learning, it is first necessary to document the frequency and type of opportunities in which naïve individuals are exposed to more experienced individual(s) performing the behavior in question. The crucial set of experiments would then involve tracing the transmission and execution of either the very behaviors that distinguish different populations of killer whales or some arbitrary and novel behavior used by a particular group. The kind of longitudinal effort conducted on single individuals from birth until they can perform the behaviors in question is certainly served well by work done in captivity. This would allow a constant monitoring of the social and physical environment to ascertain whether and which external phenomena contribute to learning a behavior. Genetic explanations might still linger if these behaviors are transmitted primarily among related individuals. Alternatively, the wild provides a more natural experimental context but is much less controlled. It would be possible to introduce a novel behavior or vocalization (see Richards et al., 1984) to a freeranging population of animals and examine whether and how easily it is incorporated by a naïve individual into its repertoire. Captive studies would also permit a seeding of different behaviors in different individuals (e.g., see Horner et al., 2006; Hopper et al., 2007 for comparable chimpanzee studies), which would allow subsequent examination of which of these behaviors a new calf eventually adopts.

Such work would be complemented by studies in the wild of behavioral acquisition but it may be hard to observe an individual over the months or years necessary, especially given the restrictions on tagging very young animals. However, a combined tracking of social association with the dissemination of a novel behavior or behavioral modification in the wild would allow an assessment of whether social learning and cultural transmission 
occur naturally. Such a process was documented for the spreading of lobtail feeding among humpback whales (Megaptera novaeangliae) in New England waters (Weinrich et al., 1992). Therefore, an improved understanding of the movement and vocal phenomena as I have presented them in this dissertation, coupled with future work on their ontogeny and transmission, will help characterize the function of culture and social learning with respect to certain behaviors in killer whales.

\subsection{Chapter synopses}

\subsubsection{Chapter 2}

Killer whales display a wide diversity of foraging strategies and feeding preferences (see Chapter 1), which are influenced by their socially gregarious nature and the ecology and prey availability of their habitat. In particular, Norwegian killer whales feed in groups on herring (Clupea harengus) via carousel feeding, a set of behaviors that involves herding the fish from depth, corralling them into a ball that is trapped against the surface, individually tail slapping the edge of the ball and consuming the fish one by one (Christensen, 1978; Similä \& Ugarte, 1993; Domenici et al., 2000; Nøttestad et al., 2002; Simon et al., 2005). Previous research has restricted its exploration of carousel feeding to the group level because of the general difficulties associated with tracking individual marine mammals.

As outlined earlier, one goal of my dissertation was to probe how individual killer whales participated in carousel feeding groups. The central aim of Chapter 2 therefore was to utilize the tag data from individual animals to reconstruct as complete a spatial and temporal portrait as possible of carousel feeding activity. Using the acoustic signature of the percussive tail slap, the dataset was divided into two primary behavioral states: tail slapping 
(TS) and not tail slapping (NTS) periods. Tail slaps produced by the tagged animal were identified by a sudden change in the pitch signal as the animal moved from pointing downwards to upwards. The diving and movement details preceding and following a focal tail slap appeared to be controlled primarily by the position and shape of the fish ball. Group tail slapping rates were higher than previously characterized and individual tail slapping rates during carousel feeding varied considerably, suggesting either inter-individual or inter-carousel differences. TS episodes were characterized by a higher proportion of time spent at shallow depths (between 5 and 25m) and included or were immediately preceded by the deepest dives recorded by the tags. NTS periods, however, contained longer inter-dive intervals and slower excursions to depth. The variability in the measurements of the vertical velocity, fluking intensity, change in pointing angle and change in roll were elevated during TS compared to NTS episodes. These results were sensible since carousel feeding required active and consistent maneuvering about the herring at shallow depths once the fish had been brought to the surface.

I sought to differentiate the tight circling behavior associated with carousel feeding behavior in the literature from other periods of behavior. A linearity index (LI) was computed to measure the relative directedness or circuitousness of travel. TS episodes tended to be characterized by low LI values that resulted from the active circling of the animals to maintain proximity to or perhaps control the position of the fish ball. In contrast, NTS episodes were defined by higher LI values and thus more directional travel. One of two categories of behavioral sequence preceded the TS periods. The first resembled the pattern described previously in the literature in which broad horizontal looping and occasional deep dives by the animals gave way to the tighter looping and focal and non-focal 
tail slapping activity that typified carousel feeding. These behaviors implied a behavioral flow from putative corralling to tail slapping and eating. In the second type of sequence, the killer whales transitioned abruptly from direct straight line travel to carousel feeding, suggesting that they converged upon a group of fish that had already coalesced at the surface likely as the result of the corralling efforts of another group. Together, these results inform the dynamic and opportunistic nature of carousel feeding, a habitat-specific and likely culturally evolved foraging tradition.

\subsubsection{Chapter 3}

An index of association is commonly used in animal behavior to describe the strength of a social relationship. Such indexes for marine mammals have traditionally been limited to sightings of animals that surface at the same time and/or in the same location. The extent to which individuals overlap in space and time varies based on the behavioral context of the animals and the definitions and protocols established by different research studies (e.g., Würsig, 1978; Ballance, 1990; Bigg et al., 1990; Smolker et al., 1992). In addition, surface behavior is obviously limited to a set of intermittently-sampled 2D observations acquired from animals that maneuver and interact in 3D.

Chapter 3 explored a more detailed analysis of the association patterns of two pairs of simultaneously tagged killer whales. The work was intended to challenge the frequent assumption in the marine mammal literature that proximity or synchrony at the surface automatically translates to similar behaviors at depth. More specifically, the depth sensors on the tags afforded an opportunity to examine vertical association throughout the water column. A juvenile and adult female that were traveling within a larger group were tagged 
simultaneously and they traced very similar vertical profiles as they surfaced and dove in tight synchrony. These animals were never one another's nearest neighbor, however, suggesting that the entire social group was ascending and descending in unison through the water column. The vertical excursions of a second pair of simultaneously tagged animals, an adult female and male killer whale within a carousel feeding group, were unlinked, however. They occasionally overlapped but primarily dove out of phase with each other.

An important conclusion drawn from this chapter was that the presence or absence of vertical association in particular (and 3D association more generally) may have been managed more by the behavioral context of the individuals than by their social relationship. The group of animals in which the first killer whale pair was observed was silent as they swam, implying that they may have been relying on visual or passive acoustic cues to maintain their formation. During carousel feeding, by contrast, synchronous occupation of a depth layer by a group of animals could lead to the evacuation and escape of the corralled herring ball. Coordination of this foraging sequence requires a decoupling of the vertical profiles to keep the herring trapped, allowing individual animals to break rank one or two at a time to tail slap the ball and feed. Ultimately, when designing metrics of association, it is most important to consider the movements, relationships and behaviors that are functionally and socially relevant to the study animals.

\subsubsection{Chapter 4}

When investigating the function(s) of the vocal activity of killer whales, most research has matched overall changes in calling rate of a group with different behavioral states. Pulsed calls may relate to arousal level (a possible explanation for the elevated calling 
rates of transient killer whales after a kill, Deecke et al., 2005), function as an acoustic badge for group membership (Ford, 1989), provide an orientation cue to conspecifics (Miller, 2002) or allow animals to maintain contact with one another through matched counter-calling (Miller et al., 2004c). In only one study was a single call type, N21, paired predominantly with a particular behavior (Van Opzeeland et al., 2005). In general, however, the null hypothesis that killer whales produce and use their call types interchangeably has remained unchallenged.

This claim was explicitly tested in Chapter 4 by searching for coincident call type usage and movement behavior patterns. During NTS periods, calling bouts were consistently shallower and characterized by less variable vertical velocity than bouts lacking vocal activity. I investigated whether the vocal behavior associated with either putative corralling or putative travel preceding tail slapping (described earlier in Chapter 2) could be differentiated. Such a result might inform the function of and justify the distinction between these two sequences. The first category of broad looping consistent with putative corralling behavior was accompanied by high rates of vocal activity in all 4 instances, which may indicate a social communication function. However, two of the three instances of the second category of directional travel contained no calls. Visual observations were available for one of these cases, which documented the tagged (silent) group converging on another group of whales that was already engaged in feeding. This suggests that killer whales may eavesdrop to locate neighboring conspecifics that are acoustically active (producing pulsed calls and tail slaps) as they feed on herring that have already been corralled to the surface. We do not know the exact costs and benefits of attracting other whales to a carousel but the foraging animals do produce loud and percussive tail slaps, which would disclose their 
location regardless.

I split the call types into two broad categories according to whether they were characterized by a low frequency component alone (LFC) or low and high frequency components together $(\mathrm{L} / \mathrm{HFC})$. I predicted that TS periods would contain higher counts of L/HFC calls since these call types were more likely to offer orientation cues to conspecifics (Miller, 2002) than LFC calls. Group members may have used this information to help maintain the coherence of the herring ball for feeding. The results confirmed this prediction since 5 of the 7 recordings contained more L/HFC (and less LFC) calls than expected during TS periods and more LFC (and less L/HFC) calls than expected during NTS periods. Significant differences in rate of call type production were observed for all 7 animals showing both TS and NTS episodes. For six of the whales, the three call types contributing most to this significant difference followed the trend just observed in which LFC call types were more common during NTS periods and L/HFC call types during TS periods. These observations were consistent with the notion that certain call types were used preferentially during particular behaviors, providing evidence against the claim that call types were produced interchangeably. No relationship between call type and the raw movement measures was discovered, suggesting that either such an association does not actually exist or that the movement data did not capture the relevant behavioral or environmental data that triggered particular vocal activity.

\subsubsection{Chapter 5}

An elucidation of the basic units of vocal production yields insight into how the brain archives and builds acoustic communication signals in animals. In general, such 
vocalizations have been considered large and independent units. For example, most studies have focused on the pulsed call as the fundamental unit of killer whale vocal production (Ford, 1987; Strager, 1993; Filatova et al., 2004). An alternative approach might search for a set of shared vocal subunits that comprise these stereotyped and variable calls (see Yurk, 2005 for an analysis involving syllable divisions). Indeed, advances in digital signal processing and a conceptual move from using whole words to phonemes in human speech (Lee et al., 1989) have afforded improved performance. These results suggest that animal vocal repertoires might benefit from similar analytical consideration. Such an approach provides an important step towards exploring whether animal communication signals could be generated by recombining and rearranging a small and finite set of subunits. In Chapter 5, I used human speech processing techniques to explore whether a set of shorter segments shared across Norwegian killer whale call types operated as efficiently as whole calls to classify vocalizations to call type.

A pitch tracking algorithm developed for human telephone speech (Wang \& Seneff, 2000; Wang, 2001) was successfully applied to trace the fundamental frequencies of the killer whale pulsed calls. Calls were segmented based on brief gaps of silence or abrupt spectral shifts. Three experiments involving sets of 6 temporal and spectral summary measurements were designed to test call type classification efficiency. The whole call experiment (WCE) operated under the traditional view that considered the entire contour as the entity for sorting. The contours were then divided into segments that were either completely callspecific (unshared segment experiment, USE) or allowed to share between call types (shared segment experiment, SSE). The categorization results were statistically equivalent across these three experiments. The SSE approach required the least amount of information $(N=$ 
26 segments versus $N=31$ whole call types and $N=62$ distinct syllables) to perform at the same level of accuracy as the other experiments. The result that SSE required less information to classify calls just as successfully suggests that it is a more parsimonious method of representing the vocal behavior of these animals (see Lee et al., 1989). Nearly $75 \%$ of the call types contained at least one of the shared syllables, suggesting that many of the vocalizations were drawn from a common inventory of sounds. The LFCs were often built by concatenating different strings of syllables whereas the predominant HFC syllable sequence was shared across many call types.

Variable pulsed calls have generally been viewed as a miscellaneous vocal category that lacks a relationship with the stereotyped calls. In addition, stereotyped calls between populations are considered unrelated. These assumptions were not supported by my observation that Norwegian variable pulsed calls and resident and transient stereotyped calls from the Pacific Northwest all overlapped to differing degrees with the inventory of segments derived from the Norwegian stereotyped calls. The Norwegian variable calls demonstrated the closest overlap with the stereotyped calls, implying that variable calls may constitute unique combinations of the phonological segments derived from the stereotyped utterances. The small number of matches between the stereotyped Norwegian calls and the Pacific Northwest calls arose either because killer whales from different populations vocalize within the same temporal and spectral space and call types will naturally overlap by chance or because these different populations actually generate their vocalizations from a common syllabic inventory. Given the earlier discussion of cultural transmission, it is possible that killer whales do not learn the phonemes they produce but rather have a larger set of possible syllables at birth that are subsequently pruned with experience to allow the generation of 
their particular repertoire, a phenomenon called selection-based learning (Nelson \& Marler, 1994). However, the actual sequences and patterns of syllables may still be socially learned and culturally acquired.

\subsection{General conclusions}

I executed two new approaches to studying free-ranging killer whales in my dissertation. First, the digital tagging technology afforded a novel opportunity to track the movements of individual killer whales as they engaged in group feeding and vocal behaviors. The 3D orientation and position information yielded by the movement sensors allowed both gross comparisons of measurement means and variability during different behavioral states and fine examinations of pitch changes during focal tail slaps. These data demonstrated a set of phenomena across the whales including a highly circuitous path, more variable movements and a tail slap-induced abrupt pitch change during carousel feeding. Visual observations at the surface could not have provided information on individual tail slapping rates or continuously tracked an animal once it left the surface. The movement analysis uncovered an important dichotomy in the behavioral sequences anticipating carousel feeding. One scenario upheld the earlier description of looped corralling at depth giving way to the carousel while the other suggested a more direct convergence on a school of herring that had already been corralled.

The addition of group vocal behavior data reinforced this distinction since heightened vocal behavior accompanied the corralling in the first scenario but generally not the directed travel in the second. Vocal activity certainly accompanies the foraging behavior of numerous killer whale populations (e.g., Ford, 1989; Deecke et al., 2005; Van Opzeeland 
et al., 2005) and may function to help coordinate social feeding behavior. During corralling and carouseling, Norwegian killer whales may use stereotyped L/HFC calling to convey additional information about the location and orientation of conspecifics. A carousel feeding individual could keep track continuously of the positions of the other group members by monitoring their incessant vocal activity. In contrast, the silence of the directed travel in 2 of the 3 instances could be explained in a variety of ways (e.g., the animals were not interested in foraging, they were eavesdropping on conspecifics, they were foraging silently, they remained silent to prevent their prey from detecting them, etc.). Both the abrupt changes in direction that were sometimes observed and the actual convergence on at least one occasion of the traveling group with another carousel feeding group reinforce the eavesdropping hypothesis. During these periods, the silent group was likely coordinating their movements and behaviors visually.

Second, I conducted a set of experiments that profited from human speech processing algorithms and approaches. The field of speech recognition productively implements phonemic instead of whole word representations of human language (see Lee et al., 1989), and a similar approach was employed here. The results were consistent with the notion that killer whales may assemble their stereotyped and variable calls from a shared set of segments. If this finding can be confirmed, it would help guide our understanding of how these animals produce, process and store in memory their pulsed calls. For example, instead of archiving entire call types, these animals may store individual syllables and their various orderings to generate call repertoires. They might produce variable calls simply by rearranging these syllables into non-typical sequences. 


\subsection{Future directions}

There are several directions for future work. One important advance involves exploring whether cooperation and role playing or turn taking is occurring during carousel feeding or other behaviors. More than two simultaneous tagouts will provide important data about how these animals position and orient themselves relative to one another as they carousel feed and whether they role play and/or take turns as they corral and tail slap the fish. Role specialization is one way in which animals trying to solve a problem can coordinate their efforts. Some animals display highly specialized roles (e.g., lions (Panthera leo), Packer et al., 1990) while others engage in turn-taking behaviors in which animals cycle through behaviors to complement one another (e.g., Dusky dolphins (Lagenorhynchus obscurus), Würsig \& Würsig, 1980; Würsig, 1986). Norwegian killer whales might take turns during carousel feeding, for example, if different animals contained herring to prevent losing the fish while others tail slapped to consume the fish. It is therefore important to characterize the component behaviors produced by killer whales engaged in particular activity sequences and to identify when different individuals display each of the behaviors using multiple simultaneous tag records.

In addition, experimental tests of observational learning are important for establishing the possibility of social transmission of behavior among killer whales. Captive studies might involve seeding a different problem-solving approach with two different animals and observing whether and how these behaviors propagate through the social group. This kind of study would ground the work in the wild in the context of an experimental demonstration of the presence or absence of observational learning and cultural transmission. 
It is important to understand the predator-prey dynamics and interactions of killer whales and herring. Further work should focus on how killer whales and herring respond to the movements of one another spatially and temporally. This kind of integrated study could explore whether a particular conformation of herring is required before tail slapping is initiated. This could be achieved by combining further tagging work with a simultaneous monitoring of the herring position and geometry via active sonar or video. A comparative study involving other populations and species of marine mammals that feed on herring would provide a useful perspective on convergent or distinct behavioral strategies for feeding on a mobile coastal fish species. It could also explore whether any observed differences are genetic, ecological or culturally learned.

Icelandic orcas engage in carousel feeding as well, for example, but produce a low frequency I36 call that is thought to corral the herring (Simon et al., 2005; 2006; 2007). To link the I36 definitively with a corralling function, simultaneous acoustic recording and herring monitoring will be required in a preliminary study before conducting a playback experiment. Beyond killer whales, humpback whales in southeastern Alaska bubble net feed on herring in groups and a particular call reliably and immediately precedes their lunging ascent (D’Vincent et al., 1985). Simultaneous tagging and array recordings could help determine whether the call is produced consistently by the same animal or by the animal that occupies a certain position within the group's 3D spatial conformation. Using this integration of data recording technologies to study the feeding behavior of free-ranging marine mammals will offer important comparative insights into how marine animals forage in groups and whether they cooperate to do so.

Another productive direction for future research for this work will require assigning 
call production to individual animals. This will inform the nature of vocal interactions between individuals and help determine whether certain call types act as triggers for behavioral responses in particular group members. The use of a hydrophone array (Miller \& Tyack, 1998) in the Pacific Northwest was a start in this research direction as it yielded data to support the hypothesis that killer whales engage in matched counter-calling by responding to stereotyped calls with calls of the same type (Miller et al., 2004c). The results that I present in my dissertation are based on the movements of individual killer whales and the vocalizations of their groups. The use of an array accompanied by more digital tags that are simultaneously deployed or tags with more specialized sound localization possibilities would bring us a little closer towards identifying the vocalizing individuals. The major difficulty will be distinguishing individual animals that vocalize in very close proximity to one another. Until technical advances are able to compensate, sound localization may be restricted to behaviors in which vocalizing groups or individuals are sufficiently separated in space from each other. Any progress in this domain will offer insights into the social and behavioral function of killer whale vocal activity.

The syntax chapter motivates further inquiry into how animals acquire their stereotyped vocal repertoire. Human infants go through a babbling stage where they produce numerous phonemes but only some of these are subsequently retained for speech once they become verbal. To characterize the vocal development of killer whales, it would be useful to explore whether they acquire their repertoires by proceeding through a similar babbling stage. If syllables are produced in isolation or in unusual orders early in life, this might suggest that phonemes are largely inherited while the group-specific sequences are learned. If, however, the stable syllables do not emerge until later in vocal development, 
these might be vocally learned through imitation. This work would be conducted most productively in captivity where the vocal ontogeny and acoustic environment of a newborn calf could be tracked closely. If killer whales do babble, I would expect that early in development their vocal behavior would be characterized by more variable calls. As they learn their repertoire over time, they would produce increasing amounts of stereotyped calling activity until reaching a certain threshold, which may vary depending on behavioral or social context. The captive setting would also afford an opportunity to explore whether killer whales can learn new syllables and/or new orders of already established syllables. Such a demonstration would provide strong evidence for vocal learning and phonological syntax in these animals. 


\section{Chapter 7. REFERENCES}

Aburdene, M. F. \& Dorband, J. E. 1996. Parallel computation of discrete Legendre transforms. Proceedings of the International Conference on Acoustics, Speech, and Signal Processing: 6, 3225-3228.

Bain, D. E. 1986. Acoustic behavior of Orcinus: sequences, periodicity, behavioral correlates and an automated method for call classification. In: Behavioral Biology of Killer Whales (Ed. by Kirkevold, B. C. \& Lockard, J. S.), pp. 335-371. New York: A. R. Liss.

Baird, R. W., Abrams, P. A. \& Dill, L. M. 1992. Possible indirect interactions between transient and resident killer whales: Implications for the evolution of foraging specializations in the genus Orcinus. Oecologia: 89, 125-132.

Baird, R. W. \& Dill, L. M. 1995. Occurrence and behaviour of transient killer whales: seasonal and podspecific variability, foraging behaviour, and prey handling. Canadian Journal of Zoology: 73, 1300-1311.

Baird, R. W. \& Dill, L. M. 1996. Ecological and social determinants of group size in transient killer whales. Behavioral Ecology: 7, 408-416.

Baird, R. W. \& Whitehead, H. 2000. Social organization of mammal-eating killer whales: Group stability and dispersal patterns. Canadian Journal of Zoology: 78, 2096-2105.

Ballance, L. T. 1990. Residence patterns, group organization, and surfacing associations of bottlenose dolphins in Kino Bay, Gulf of California, Mexico. In: The Bottlenose Dolphin (Ed. by Leatherwood, S. \& Reeves, R. R.), pp. 267-283. San Diego, CA: Academic Press.

Beecher, M. D., Stoddard, P. K., Campbell, S. E. \& Horning, C. L. 1996. Repertoire matching between neighbouring song sparrows. Animal Behaviour: 51, 917-923.

Benoit-Bird, K. J. \& Au, W. W. L. 2003. Prey dynamics affect foraging by a pelagic predator (Stenella longirostris) over a range of spatial and temporal scales. Behavioral Ecology and Sociobiology: 53, 364-373.

Bertram, B. C. R. 1980. Vigilance and group size in ostriches. Animal Behaviour: 28.

Bigg, M. A., Olesiuk, P. F., Ellis, G. M., Ford, J. K. B. \& Balcomb, I., K. C. 1990. Social organization and genealogy of resident killer whales (Orcinus orca) in the coastal waters of British Columbia and Washington State. Report of the International Whaling Commission: 12, 383-405.

Boesch, C. 1994. Cooperative hunting in wild chimpanzees. Animal Behaviour: 48, 653-667.

Boesch, C. 2003. Is culture a golden barrier between human and chimpanzee? Evolutionary Anthropology: 12, 82 91.

Boesch, C., Marchesi, P., Marchesi, N., Fruth, B. \& Joulian, F. 1994. Is nut cracking in wild chimpanzees a cultural behavior? Journal of Human Evolution: 26, 325-338.

Boesch, C. \& Tomasello, M. 1998. Chimpanzee and human cultures. Current Anthropology: 39, $591-614$.

Böhner, J. \& Todt, D. 1996. Influence of auditory stimulation on the development of syntactical and temporal features in European starling song. Auk: 113, 450-456.

Bonafonte, A., Nogueiras, A. \& Rodriguez-Garrido, A. 1996. Explicit segmentation of speech using Gaussian models. In: Proceedings of the International Conference on Spoken Language Processing, pp. 1269-1272.

Bowles, A. E., Young, W. G. \& Asper, E. D. 1988. Ontogeny of stereotyped calling of a killer whale calf, Orcinus orca, during her first year. Rit Fiskideildar: 11, 251-275.

Bräger, S., Würsig, B., Acevedo, A. \& Henningsen, T. 1994. Association patterns of bottlenose dolphins (Tursiops truncatus) in Galveston Bay, Texas. Journal of Mammalogy: 75, 431-437.

Brown, C. R., Brown, M. B. \& Shaffer, M. L. 1991. Food-sharing signals among socially foraging cliff swallows. Animal Behaviour: 42, 551-564.

Brown, M. B. 1974. Identification of the sources of significance in two-way contingency tables. Applied Statistics: 23, 405-413.

Bshary, R., Wickler, W. \& Fricke, H. 2002. Fish cognition: A primate's eye view. Animal Cognition: 5, 1-13.

Byrne, R. W., Barnard, P. J., Davidson, L., Janik, V. M., McGrew, W. C., Miklosi, A. \& Wiessner, P. 2004. Understanding culture across species. Trends in Cognitive Sciences: 8, 341-346.

Byrne, R. W. \& Whiten, A. 1988. Machiavellian Intelligence: Social Expertise and the Evolution of Intellect in Monkeys, Apes, and Humans. Oxford: Oxford University Press.

Cairns, S. J. \& Schwager, S. J. 1987. A comparison of association indices. Animal Behaviour: 35, 1454-1469.

Caro, T. M. \& Hauser, M. D. 1992. Is there teaching in nonhuman animals? Quarterly Review of Biology: 67, 151-174. 
Cavalli-Sforza, L. L. \& Feldman, M. W. 1981. Cultural transmission and evolution: A quantitative approach. Princeton, NJ: Princeton University Press.

Cerchio, S. \& Dahlheim, M. 2001. Variation in feeding vocalizations of humpback whales Megaptera novaeangliae from southeast Alaska. Bioacoustics: 11, 277-295.

Chapman, L. J. \& Chapman, C. A. 1994. Observations on synchronous air-breathing in Clarias liocephalus. Copeia, 246-249.

Chen, S.-H. \& Wang, Y.-R. 1990. Vector quantization of pitch information in Mandarin speech. IEEE Transactions on Communications: 38, 1317-1320.

Christensen, I. 1978. The killer whale (Orcinus orca) in the northeast Atlantic. Fisken og Havet: 1, 23-31.

Clark, C. W. 1986. The evolutionary advantages of group foraging. Theoretical Population Biology: 30, 45-75.

Cocroft, R. B. 2005. Vibrational communication facilitates cooperative foraging in a phloem-feeding insect. Proceedings of the Royal Society B: Biological Sciences: 272, 1023-1029.

Connor, R. C. \& Norris, K. S. 1982. Are dolphins reciprocal altruists? The American Naturalist: 119, 358-374.

Connor, R. C., Smolker, R. \& Bejder, L. 2006. Synchrony, social behaviour and alliance affiliation in Indian Ocean bottlenose dolphins, Tursiops aduncus. Animal Behaviour: 72, 1371-1378.

Coussi-Korbel, S. \& Fragaszy, D. M. 1995. On the relation between social dynamics and social learning. Animal Behaviour. 50, 1441-1453.

Creel, S. \& Creel, N. M. 1995. Communal hunting and pack size in African wild dogs, Lycaon pictus. Animal Behaviour: 50, 1325-1339.

Cynx, J. 1990. Experimental determination of a unit of song production in the zebra finch (Taeniopygia guttata). Journal of Comparative Psychology: 104, 3-10.

D’Vincent, C. G., Nilson, R. M. \& Hanna, R. E. 1985. Vocalization and coordinated feeding behavior of the humpback whale in southeastern Alaska. Scientific Reports of the Whales Research Institute: 36, 41-47.

de Waal, F. B. M. 1999. Cultural primatology comes of age. Nature: 399, 635-636.

Deecke, V. B., Ford, J. K. B. \& Slater, P. J. B. 2005. The vocal behaviour of mammal-eating killer whales: communicating with costly calls. Animal Behaviour: 69, 395-405.

Deecke, V. B., Ford, J. K. B. \& Spong, P. 1999. Quantifying complex patterns of bioacoustic variation: Use of a neural network to compare killer whale (Orcinus orca) dialects. Journal of the Acoustical Society of America: 105, 2499-2507.

Deecke, V. B., Ford, J. K. B. \& Spong, P. 2000. Dialect change in resident killer whales: implications for vocal learning and cultural transmission. Animal Behaviour. 60, 629-638.

Deecke, V. B. \& Janik, V. M. 2006. Automated categorization of bioacoustic signals: Avoiding perceptual pitfalls. Journal of the Acoustical Society of America: 119, 645-653.

Deecke, V. B., Slater, P. J. B. \& Ford, J. K. B. 2002. Selective habituation shapes acoustics predator recognition in harbour seals. Nature: 420, 171-173.

Dittus, W. P. J. 1984. Toque macaque food calls: Semantic communication concerning food distribution in the environment. Animal Behaviour: 32, 470-477.

Dobson, C. W. \& Lemon, R. E. 1979. Markov sequences in songs of American thrushes. Behaviour: 68, 86105.

Domenici, P. 2001. The scaling of locomotor performance in predator-prey encounters: from fish to killer whales. Comparative Biochemistry and Physiology A: 131, 169-182.

Domenici, P., Batty, R. S., Similä, T. \& Ogam, E. 2000. Killer whales (Orcinus orca) feeding on schooling herring (Clupea harengus) using underwater tail-slaps: Kinematic analyses of field observations. The Journal of Experimental Biology: 203, 283-294.

Eales, L. A. 1985. Song learning in zebra finches: Some effects of song model availability on what is learnt and when. Animal Behaviour: 33, 1293-1300.

Eens, M., Pinxten, R. \& Verheyen, R. F. 1989. Temporal and sequential organisation of song bouts in the starling. Ardea: 77, 75-86.

Elgar, M. A. 1986. House sparrows establish foraging flocks by giving chirrup calls if the resources are divisible. Animal Behaviour: 34, 169-174.

Elisseeff, A. \& Pontil, M. 2002. Leave-one-out error and stability of learning algorithms with applications. In: Advances in Learning Theory: Methods, Models and Applications (Ed. by Suykens, J. A. K.). Amsterdam: IOS Press. 
Farabaugh, S. M., Linzenbold, A. \& Dooling, R. J. 1994. Vocal plasticity in budgerigars (Melopsittacus undulatus): Evidence for social factors in the learning of contact calls. Journal of Comparative Psychology: 108, 81-92.

Fertl, D., Acevedo-Gutierrez, A. \& Darby, F. L. 1996. A report of killer whales (Orcinus orca) feeding on a carcharhinid shark in Costa Rica. Marine Mammal Science: 12, 606-611.

Filatova, O. A., Burdin, A. M., Hoyt, E. \& Sato, H. 2004. A catalogue of discrete calls of resident killer whales (Orcinus orca) from the Avacha Gulf of Kamchatka Peninsula. Zoologicheskyi Journal: 83, 11691180.

Foote, A. D., Griffin, R. M., Howitt, D., Larsson, L., Miller, P. J. O. \& Hoelzel, A. R. 2006. Killer whales are capable of vocal learning. Biology Letters: 2, 509-512.

Ford, J. K. B. 1987. A catalogue of underwater calls produced by killer whales (Orcinus orca) in British Columbia. Canadian Data Report of Fisheries and Aquatic Sciences: 633, 1-165.

Ford, J. K. B. 1989. Acoustic behavior of resident killer whales (Orcinus orca) off Vancouver Island, British Columbia. Canadian Journal of Zoology: 67, 727-745.

Ford, J. K. B. 1991. Vocal traditions among resident killer whales (Orcinus orca) in coastal waters of British Columbia. Canadian Journal of Zoology: 69, 1454-1483.

Ford, J. K. B. \& Ellis, G. M. 2006. Selective foraging by fish-eating killer whales Orcinus orca in British Columbia. Marine Ecology Progress Series: 316, 185-199.

Ford, J. K. B., Ellis, G. M., Barrett-Lennard, L. G., Morton, A. B., Palm, R. S. \& Balcomb, K. C. 1998. Dietary specialization in two sympatric populations of killer whales (Orcinus orca) in coastal British Columbia and adjacent waters. Canadian Journal of Zoology: 76, 1456-1471.

Fripp, D., Owen, C., Quintana-Rizzo, E., Shapiro, A., Buckstaff, K., Jankowski, K., Wells, R. \& Tyack, P. 2005. Bottlenose dolphin (Tursiops truncatus) calves appear to model their signature whistles on the signature whistles of community members. Animal Cognition: 8, 17-26.

Furui, S. 1986. On the role of spectral transition for speech perception. Journal of the Acoustical Society of America: 80, 1016-1025.

Galef, B. G. 1992. The question of animal culture. Human Nature: 3, 157-178.

Galef, B. G. \& Allen, C. 1995. A new model system for studying behavioral traditions in animals. Animal Behaviour: 50, 705-717.

Gazda, S. K., Connor, R. C., Edgar, R. K. \& Cox, F. 2005. A division of labour with role specialization in group-hunting bottlenose dolphins (Tursiops truncatus) off Cedar Key, Florida. Proceedings of the Royal Society B: Biological Sciences: 272, 135-140.

Götmark, F., Winkler, D. W. \& Andersson, M. 1986. Flock-feeding on fish schools increases individual success in gulls. Nature: 319, 589-591.

Greenwood, M. 1946. The statistical study of infectious disease. Journal of the Royal Statistical Society, A: 109, 85110.

Guinet, C. \& Bouvier, J. 1995. Development of intentional stranding hunting techniques in killer whale (Orcinus orca) calves at Crozet Archipelago. Canadian Journal of Zoology: 73, 27-33.

Hailman, J. P. \& Ficken, M. S. 1986. Combinatorial animal communication with computable syntax: Chicka-dee calling qualifies as language by structural linguistics. Animal Behaviour: 34, 1899-1901.

Hauser, M. D., Chomsky, N. \& Fitch, W. T. 2002. The faculty of language: What is it, who has it, and how did it evolve? Science: 298, 1569-1579.

Helfman, G. S. \& Schultz, E. T. 1984. Social transmission of behavioural traditions in a coral reef fish. Animal Behaviour: 32, 379-384.

Herzing, D. L. 1996. Vocalizations and associated underwater behavior of free-ranging Atlantic spotted dolphins, Stenella frontalis and bottlenose dolphins, Tursiops truncatus. Aquatic Mammals: 22, 61-79.

Heuber, T., Chollet, G., Denby, B., Dreyfus, G. \& Stone, M. 2007. Continuous-speech phone recognition from ultrasound and optical images of the tongue and lips. In: Interspeech. Antwerp, Belgium.

Hile, A. G., Plummer, T. K. \& Striedter, G. F. 2000. Male vocal imitation produces call convergence during pair bonding in budgerigars, Melopsittacus undulatus. Animal Behaviour. 59, 1209-1218.

Hinde, R. A. 1976. Interactions, relationships and social structure. Man, New Series: 11, 1-17.

Hinde, R. A. 1979. Towards understanding relationships. London: Academic Press.

Hoelzel, A. R. 1991. Killer whale predation on marine mammals at Punta Norte, Argentina; food sharing, provisioning and foraging strategy. Behavioral Ecology and Sociobiology: 29, 197-204. 
Hoelzel, A. R., Natoli, A., Dahlheim, M. E., Olavarria, C., Baird, R. W. \& Black, N. A. 2002. Low worldwide genetic diversity in the killer whale (Orcinus orca): implications for demographic history. Proceedings of the Royal Society of London B: 269, 1467-1473.

Hoelzel, A. R. \& Osborne, R. W. 1986. Killer whale call characteristics: Implications for cooperative foraging strategies. In: Behavioral Biology of Killer Whales (Ed. by Kirkevold, B. C. \& Lockard, J. S.), pp. 373-403. New York: Alan R. Liss, Inc.

Hooker, S. K., Miller, P. J. O., Johnson, M. P., Cox, O. P. \& Boyd, I. L. 2005. Ascent exhalations of Antarctic fur seals: a behavioural adaptation for breath-hold diving? Proceedings of the Royal Society B: Biological Sciences: 272, 355-363.

Hopper, L. M., Spiteri, A., Lambeth, S. P., Schapiro, S. J., Horner, V. \& Whiten, A. 2007. Experimental studies of traditions and underlying transmission processes in chimpanzees. Animal Behaviour: 73, 1021-1032.

Horner, V., Whiten, A., Flynn, E. \& de Waal, F. B. M. 2006. Faithful replication of foraging techniques along cultural transmission chains by chimpanzees and children. Proceedings of the National Academy of Sciences of the United States of America: 103, 13878-13883.

Hosmer, D. W. \& Lemeshow, S. 2000. Applied Logistic Regression. New York: Wiley.

Huberty, C. J. \& Olejnik, S. 2006. Applied MANOVA and Discriminant Analysis: 2nd Edition. Hoboken, New Jersey: John Wiley \& Sons, Inc.

Hultsch, H. \& Todt, D. 1989. Memorization and reproduction of songs in nightingales (Luscinia megarbynchos): Evidence for package formation. Journal of Comparative Physiology A: 165, 197-203.

Humle, T. \& Matsuzawa, T. 2002. Ant-dipping among the chimpanzees of Bossou, Guinea, and some comparisons with other sites. American Journal of Primatology: 58, 133-148.

Imanishi, K. 1957. Identification: A process of enculturation in the subhuman society of Macaca fuscata. Primates: 1, 1-29.

Janik, V. M. 2000. Source levels and the estimated active space of bottlenose dolphin (Tursiops truncatus) whistles in the Moray Firth, Scotland. Journal of Comparative Physiology A: 186, 673-680.

Janik, V. M. \& Slater, P. J. B. 1997. Vocal learning in mammals. Advances in the Study of Behavior: 26, 59-99.

Janik, V. M. \& Slater, P. J. B. 2000. The different roles of social learning in vocal communication. Animal Behaviour: 60, 1-11.

Jefferson, T. A., Stacey, P. J. \& Baird, R. W. 1991. A review of killer whale interactions with other marine mammals: Predation to co-existence. Mammal Review: 21, 151-180.

Johnson, M. P. \& Tyack, P. L. 2003. A digital acoustic recording tag for measuring the response of wild marine mammals to sound. IEEE Journal of Oceanic Engineering: 28, 3-12. doi:10.1109/JOE.2002.808212.

Kramer, D. L. \& Graham, J. B. 1976. Synchronous air breathing, a social component of respiration in fishes. Copeia, 689-697.

Krebs, J. R. \& Kroodsma, D. E. 1980. Repertoires and geographical variation in bird song. In: Advances in the Study of Behaviour (Ed. by Rosenblatt, J. S., Hinde, R. A., Beer, C. \& Busnell, M.-C.). New York: Academic Press.

Kummer, H. 1971. Primate Societies: Group Techniques of Ecological Adaptation. New York: Aldine Atherton, Inc.

Laiolo, P. \& Jovani, R. 2006. The emergence of animal culture conservation. Trends in Ecology \& Evolution: 22, 5.

Laland, K. N. \& Hoppitt, W. 2003. Do animals have culture? Evolutionary Anthropology: 12, 150-159.

Lee, C.-H., Juang, B.-H., Soong, F. K. \& Rabiner, L. R. 1989. Word recognition using whole word and subword models. Proceedings of the International Conference on Acoustics, Speech, and Signal Processing: 1, 683686.

Lee, M. S., Kozhevnikov, A. A. \& Hahnloser, R. H. R. 2004. Neural mechanisms of vocal sequence generation in the songbird. Annals of the New York. Academy of Sciences: 1016, 153-170.

Lemon, R. E. \& Chatfield, C. 1971. Organization of song in cardinals. Animal Behaviour: 19, 1-17.

Macdonald, D. W. 1983. The ecology of carnivore social behavior. Nature: 301, 379-384.

Mann, J. 2000. Unraveling the dynamics of social life: Long-term studies and observational methods. In: Cetacean Societies: Field Studies of Dolphins and Whales (Ed. by Mann, J., Connor, R. C., Tyack, P. L. \& Whitehead, H.), pp. 45-64. Chicago: The University of Chicago Press. 
Marler, P. 1977. The structure of animal communication sounds. In: Recognition of Complex Acoustic Signals (Ed. by Bullock, T. H.), pp. 17-35. Berlin: Springer.

Martinez, D. R. \& Klinghammer, E. 1970. The behavior of the whale Orcinus orca: A review of the literature. Zeitschrift für Tierpsychologie: 27, 828-839.

Matkin, C. O. \& Saulitis, E. L. 1994. Killer whale (Orcinus orca) biology and management in Alaska. Contract No. T75135023, Marine Mammal Commission. Washington, DC.

McGregor, P. K. 1991. The singer and the song: On the receiving end of bird song. Biological Reviews: 66, 5781.

Mennill, D. J. \& Ratcliffe, L. M. 2004. Overlapping and matching in the song contests of black-capped chickadees. Animal Behaviour: 67, 441-450.

Miller, P. J. \& Tyack, P. L. 1998. A small towed beamforming array to identify vocalizing resident killer whales (Orcinus orca) concurrent with focal behavioral observations. Deep Sea Research II: 45, 1389-1405.

Miller, P. J. O. 2002. Mixed-directionality of killer whale stereotyped calls: a direction of movement cue? Behavioral Ecology and Sociobiology: 52, 262-270.

Miller, P. J. O. 2006. Diversity in sound pressure levels and estimated active space of resident killer whale vocalizations. Journal of Comparative Physiology A: 192, 449-459.

Miller, P. J. O. \& Bain, D. E. 2000. Within-pod variation in the sound production of a pod of killer whales, Orcinus orca. Animal Behaviour: 60, 617-628.

Miller, P. J. O., Johnson, M. P. \& Tyack, P. L. 2004a. Sperm whale behaviour indicates the use of echolocation click buzzes 'creaks' in prey capture. Proceedings of the Royal Society of London B: 271, 22392247. doi:10.1098/rspb.2004.2863.

Miller, P. J. O., Johnson, M. P., Tyack, P. L. \& Terray, E. A. 2004b. Swimming gaits, passive drag and buoyancy of diving sperm whales Physeter macrocephalus. The Journal of Experimental Biology: 207, 19531967.

Miller, P. J. O., Samarra, F. I. P. \& Perthuison, A. D. 2007. Caller sex and orientation influence spectral characteristics of "two-voice" stereotyped calls produced by free-ranging killer whales. Journal of the Acoustical Society of America: 121, 3932-3937.

Miller, P. J. O., Shapiro, A. D., Tyack, P. L. \& Solow, A. R. 2004c. Call-type matching in vocal exchanges of free-ranging resident killer whales, Orcinus orca. Animal Behaviour. 67, 1099-1107.

Mitani, J. C. \& Gros-Louis, J. 1998. Chorusing and call convergence in chimpanzees: Tests of three hypotheses. Behaviour: 135, 1041-1064.

Morton, A. B., Gale, J. C. \& Prince, R. C. 1986. Sound and behavioral correlations in captive Orcinus orca. In: Behavioral Biology of Killer Whales (Ed. by Kirkevold, B. C. \& Lockard, J. S.), pp. 303-333. New York: Alan R. Liss, Inc.

Nelson, D. A. \& Marler, P. 1994. Selection-based learning in bird song development. Proceedings of the National Academy of Sciences of the United States of America: 91, 10498-10501.

Noad, M. J., Cato, D. H., Bryden, M. M., Jenner, M. N. \& Jenner, K. C. S. 2000. Cultural revolution in whale songs. Nature: 408, 537-537.

Nøttestad, L. \& Axelsen, B. E. 1999. Herring schooling manoeuvres in response to killer whale attacks. Canadian Journal of Zoology: 77, 1540-1546.

Nøttestad, L., Fernö, A. \& Axelsen, B. E. 2002. Digging in the deep: killer whales' advanced hunting tactic. Polar Biology: 25, 939-941.

Nøttestad, L. \& Similä, T. 2001. Killer whales attacking schooling fish: Why force herring from deep water to the surface? Marine Mammal Science: 17, 343-352.

Nousek, A. E., Slater, P. J. B., Wang, C. \& Miller, P. J. O. 2006. The influence of social affiliation on individual vocal signatures of northern resident killer whales (Orcinus orca). Biology Letters: 2, 481-484.

Nowak, M. A., Plotkin, J. B. \& Jansen, V. A. A. 2000. The evolution of syntactic communication. Nature: 404, 495-498.

Nudds, T. D. 1978. Convergence of group size strategies by mammalian social carnivores. The American Naturalist: 112, 957-960.

Oleson, E. M., Calambokidis, J., Burgess, W. C., McDonald, M. A., LeDuc, C. A. \& Hildebrand, J. A. 2007a. Behavioral context of call production by eastern North Pacific blue whales. Marine Ecology Progress Series: 330, 269-284. 
Oleson, E. M., Wiggins, S. M. \& Hildebrand, J. A. 2007b. Temporal separation of blue whale call types on a southern California feeding ground. Animal Behaviour: 74, 881-894.

Packer, C. \& Ruttan, L. 1988. The evolution of cooperative hunting. The American Naturalist. 132, 159-198.

Packer, C., Scheel, D. \& Pusey, A. E. 1990. Why lions form groups: Food is not enough. American Naturalist. 136, 1-19.

Parrish, J. K. \& Edelstein-Keshet, L. 1999. Complexity, pattern, and evolutionary trade-offs in animal aggregation. Science: 284, 99-101.

Payne, K., Tyack, P. \& Payne, R. 1984. Progressive changes in the songs of humpback whales (Megaptera novaeangliae): A detailed analysis of two seasons in Hawaii. In: Communication and Behavior of Whales (Ed. by Payne, R.), pp. 9-57. Boulder, Colorado: Westview Press, Inc.

Payne, R. S. \& McVay, S. 1971. Songs of humpback whales. Science: 173, 585-597.

Pols, L. C. W. 1986. Variation and interaction in speech. In: Invariance and V ariability in Speech Processes (Ed. by Perkell, J. S. \& Klatt, D. H.). Hillsdale, NJ: Lawrence Erlbaum Associates, Publishers.

Pulliam, H. R. 1973. Advantages of flocking. Journal of Theoretical Biology: 38, 419-422.

Rendell, L. \& Whitehead, H. 2001. Culture in whales and dolphins. Behavioral and Brain Sciences: 24, 309-382.

Rendell, L. E. \& Whitehead, H. 2003. Vocal clans in sperm whales (Physeter macrocephalus). Proceedings of the Royal Society of London Series B: Biological Sciences: 270, 225-231.

Richards, D. G., Woltz, J. P. \& Herman, L. M. 1984. Vocal mimicry of computer-generated sounds and vocal labeling of objects by a bottlenosed dolphin (Tursiops truncatus). Journal of Comparative Psychology: 98, 10-28.

Riesch, R., Ford, J. K. B. \& Thomsen, F. 2006. Stability and group specificity of stereotyped whistles in resident killer whales, Orcinus orca, off British Columbia. Animal Behaviour: 71, 79-91.

Sapolsky, R. M. \& Share, L. J. 2004. A pacific culture among wild baboons: Its emergence and transmission. PLoS Biology: 2, 534-541.

Sato, K., Watanuki, Y., Takahashi, A., Miller, P. J. O., Tanaka, H., Kawabe, R., Ponganis, P. J., Handrich, Y., Akamatsu, T., Watanabe, Y., Mitani, Y., Costa, D. P., Bost, C. A., Aoki, K., Amano, M., Trathan, P., Shapiro, A. \& Naito, Y. 2007. Stroke frequency, but not swimming speed, is related to body size in free-ranging seabirds, pinnipeds and cetaceans. Proceedings of the Royal Society B: Biological Sciences: 274, 471-477.

Saulitis, E., Matkin, C., Barrett-Lennard, L., Heise, K. \& Ellis, G. 2000. Foraging strategies of sympatric killer whale (Orcinus orca) populations in Prince William Sound, Alaska. Marine Mammal Science: 16, 94109.

Schultz, T. \& Waibel, A. 2001. Language independent and language adaptive large vocabulary speech recognition. Proceedings of the International Conference on Spoken Language Processing.

Sharpe, F. A. \& Dill, L. M. 1997. The behavior of Pacific herring schools in response to artificial humpback whale bubbles. Canadian Journal of Zoology: 75, 725-730.

Similä, T. 1997. Sonar observations of killer whales (Orcinus orca) feeding on herring schools. Aquatic Mammals: 23, 119-126.

Similä, T., Holst, J. C. \& Christensen, I. 1996. Occurrence and diet of killer whales in northern Norway: seasonal patterns relative to the distribution and abundance of Norwegian spring-spawning herring. Canadian Journal of Fisheries and Aquatic Sciences: 53, 769-779.

Similä, T. \& Ugarte, F. 1993. Surface and underwater observations of cooperatively feeding killer whales in northern Norway. Canadian Journal of Zoology: 71, 1494-1499.

Simon, H. A. 1974. How big is a chunk? Science: 183, 482-488.

Simon, M., McGregor, P. K. \& Ugarte, F. 2007. The relationship between the acoustic behaviour and surface activity of killer whales (Orcinus orca) that feed on herring (Clupea harengus). Acta Ethologica: 10, 47-53.

Simon, M., Ugarte, F., Wahlberg, M. \& Miller, L. A. 2006. Icelandic killer whales Orcinus orca use a pulsed call suitable for manipulating the schooling behaviour of herring Clupea harengus. Bioacoustics: 16, 57-74.

Simon, M., Wahlberg, M., Ugarte, F. \& Miller, L. A. 2005. Acoustic characteristics of underwater tail slaps used by Norwegian and Icelandic killer whales (Orcinus orca) to debilitate herring (Clupea harengus). Journal of Experimental Biology: 208, 2459-2466.

Slater, P. J. B. 1973. Describing sequences of behavior. In: Perspectives in Ethology (Ed. by Bateson, P. P. G. \& Klopfer, P. H.), pp. 131-153. New York: Plenum Press. 
Slater, P. J. B. 1983. Sequences of song in chaffinches. Animal Behaviour: 31, 272-281.

Slater, P. J. B. 1986. The cultural transmission of bird song. Trends in Ecology and Evolution: 1, 94-97.

Smith, T. G., Siniff, D. B., Reichle, R. \& Stone, S. 1981. Coordinated behavior of killer whales, Orcinus orca, hunting a crabeater seal, Lobodon carcinophagus. Canadian Journal of Zoology: 59, 1185-1189.

Smolker, R. \& Pepper, J. W. 1999. Whistle convergence among allied male bottlenose dolphins (Delphinidae, Tursiops sp.). Ethology: 105, 595-617.

Smolker, R. A., Mann, J. \& Smuts, B. B. 1993. Use of signature whistles during separations and reunions by wild bottlenose dolphin (Tursiops sp.) mothers and infants. Behavioral Ecology and Sociobiology: 33, 393402.

Smolker, R. A., Richards, A. F., Connor, R. C. \& Pepper, J. W. 1992. Sex differences in patterns of association among Indian Ocean bottle-nosed dolphins. Behaviour: 123, 38-69.

Snowdon, C. T. 1982. Linguistic and psycholinguistic approaches to primate communication. In: Primate Communication (Ed. by Snowdon, C. T., Brown, C. H. \& Petersen, M. R.), pp. 212-238. Cambridge: Cambridge University Press.

Soha, J. A. \& Marler, P. 2001. Vocal syntax development in the white-crowned sparrow (Zonotrichia leucophrys). Journal of Comparative Psychology: 115, 172-180.

Solow, A. R. \& Smith, W. 1991. Detecting cluster in a heterogeneous community sampled by quadrats. Biometrics: 47, 311-317.

Stander, P. E. 1992. Cooperative hunting in lions: the role of the individual. Behavioral Ecology and Sociobiology: 29, 445-454.

Stephens, M. A. 1986. Tests for the Exponential Distribution. In: Goodness-of-Fit Techniques (Ed. by D'Agostino, R. B. \& Stephens, M. A.), pp. 421-459. New York: Marcel Dekker, Inc.

Strager, H. 1993. Catalogue of underwater calls from killer whales (Orcinus orca) in northern Norway. M.Sc. thesis, University of Århus.

Strager, H. 1995. Pod-specific call repertoires and compound calls of killer whales, Orcinus orca Linnaeus, 1758, in the waters of northern Norway. Canadian Journal of Zoology: 73, 1037-1047.

Suzuki, R., Buck, J. R. \& Tyack, P. L. 2006. Information entropy of humpback whale songs. Journal of the Acoustical Society of America: 119, 1849-1866.

Terrace, H. S. 1987. Chunking by a pigeon in a serial learning task. Nature: 325, 149-151.

Tyack, P. L. 1999. Communication and cognition. In: Biology of Marine Mammals (Ed. by Reynolds III, J. E. \& Rommel, S. A.), pp. 287-323. London: Smithsonian Institution Press.

Tyack, P. L. \& Sayigh, L. S. 1997. Vocal learning in cetaceans. In: Social Influences on Vocal Development (Ed. by Snowdon, C. \& Hausberger, M.), pp. 208-233. Cambridge: Cambridge University Press.

Van Opzeeland, I. C., Corkeron, P. J., Leyssen, T., Similä, T. \& Van Parijs, S. M. 2005. Acoustic behaviour of Norwegian killer whales, Orcinus orca, during carousel and seiner foraging on springspawning herring. Aquatic Mammals: 31, 110-119.

van Schaik, C. P., Ancrenaz, M., Borgen, G., Galdikas, B., Knott, C. D., Singleton, I., Suzuki, A., Utami, S. S. \& Merrill, M. 2003. Orangutan cultures and the evolution of material culture. Science: 299, 102-105.

van Schaik, C. P., Deaner, R. O. \& Merrill, M. Y. 1999. The conditions for tool use in primates: implications for the evolution of material culture. Journal of Human Evolution: 36, 719-741.

Wang, C. 2001. Prosodic Modeling for Improved Speech Recognition and Understanding. Ph.D. thesis, MIT.

Wang, C. \& Seneff, S. 1998. A study of tones and tempo in continuous Mandarin digit strings and their application in telephone quality speech recognition. Proceedings of the International Conference on Spoken Language Processing, 635-638.

Wang, C. \& Seneff, S. 2000. Robust pitch tracking for prosodic modeling in telephone speech. Proceedings of the International Conference on Acoustics, Speech, and Signal Processing.

Wang, C. \& Shapiro, A. D. In prep. A robust and versatile pitch tracking algorithm: From human speech to killer whale vocalizations.

Warner, R. R. 1988. Traditionality of mating-site preferences in a coral reef fish. Nature: 335, 719-721.

Weinrich, M. T., Schilling, M. R. \& Belt, C. R. 1992. Evidence for acquisition of a novel feeding behaviour: Lobtail feeding in humpback whales, Megaptera novaeangliae. Animal Behaviour: 44, 1059-1072.

White, D. J., Gros-Louis, J., King, A. P., Papakhian, M. A. \& West, M. J. 2007. Constructing culture in cowbirds (Molothrus ater). Journal of Comparative Psychology: 121, 113-122. 
Whitehead, H. 1996. Babysitting, dive synchrony, and indications of alloparental care in sperm whales. Behavioral Ecology and Sociobiology: 38, 237-244.

Whitehead, H. 2004. The group strikes back: Follow protocols for behavioral research on cetaceans. Marine Mammal Science: 20, 664-670.

Whitehead, H. \& Weilgart, L. 1991. Patterns of visually observable behavior and vocalizations in groups of female sperm whales. Behaviour: 118, 275-296.

Whiten, A. 2000. Primate culture and social learning. Cognitive Science: 24, 477-508.

Whiten, A. 2005. The second inheritance system of chimpanzees and humans. Nature: 437, 52-55.

Whiten, A. \& Boesch, C. 2001. The cultures of chimpanzees. Scientific American: 284, 60-67.

Whiten, A. \& Byrne, R. W. 1988. Taking (Machiavellian) intelligence apart: Editorial. In: Machiavellian Intelligence: Social Expertise and the Evolution of Intellect in Monkeys, Apes, and Humans (Ed. by Byrne, R. W. \& Whiten, A.), pp. 50-65. Oxford: Clarendon Press.

Whiten, A. \& Byrne, R. W. 1997. Machiavellian Intelligence II. Cambridge: Cambridge University Press.

Whiten, A., Goodall, J., McGrew, W. C., Nishida, T., Reynolds, V., Sugiyama, Y., Tutin, C. E. G., Wrangham, R. W. \& Boesch, C. 1999. Cultures in chimpanzees. Nature: 399, 682-685.

Whiten, A., Horner, V. \& de Waal, F. B. M. 2005. Conformity to cultural norms of tool use in chimpanzees. Nature: 437, 737-740.

Wilkinson, G. S. 1984. Reciprocal food sharing in the vampire bat. Nature: 308, 181-184.

Wilkinson, G. S. 1992. Information transfer at evening bat colonies. Animal Behaviour: 44, 501-518.

Williams, H. \& Staples, K. 1992. Syllable chunking in zebra finch (Taeniopygia guttata) song. Journal of Comparative Psychology: 106, 278-286.

Wilson, R. P., Liebsch, N., Davies, I. M., Quintana, F., Weimerskirch, H., Storch, S., Lucke, K., Siebert, U., Zankl, S., Müller, G., Zimmer, I., Scolaro, A., Campagna, C., Plötz, J., Bornemann, H., Teilmann, J. \& McMahon, C. R. 2007. All at sea with animal tracks; methodological and analytical solutions for the resolution of movement. Deep Sea Research II: 54, 193210.

Würsig, B. 1978. Occurrence and group organization of Atlantic bottlenose porpoises (Tursiops truncatus) in an Argentine bay. Biological Bulletin: 154, 348-359.

Würsig, B. 1986. Delphinid foraging strategies. In: Dolphin cognition and behavior: A comparative approach (Ed. by Schusterman, R. J., Thomas, J. A. \& Wood, F. G.), pp. 347-359. Hillsdale, NJ: Lawrence Erlbaum Associates.

Würsig, B. \& Würsig, M. 1977. The photographic determination of group size, composition and stability of coastal porpoises (Tursiops truncatus). Science, 755-756.

Würsig, B. \& Würsig, M. 1980. Behavior and ecology of the dusky dolphin, Lagenorbynchus obscurus., in the south Atlantic. Fishery Bulletin U.S.: 77, 871-890.

Yurk, H. 2005. Vocal culture and social stability in resident killer whales (Orcinus orca). Ph.D. thesis, University of British Columbia.

Yurk, H., Barrett-Lennard, L., Ford, J. K. B. \& Matkin, C. O. 2002. Cultural transmission within maternal lineages: vocal clans in resident killer whales in southern Alaska. Animal Behaviour: 63, 1103-1119.

Zentall, T. R. 2006. Imitation: Definitions, evidence, and mechanisms. Animal Cognition: 9, 335-353.

Zhu, D., Adda-Decker, M. \& Antoine, F. 2005. Different size multilingual phone inventories and contextdependent acoustic models for language identification. In: Interspeech. Lisbon, Portugal.

Zimmer, W. M. X., Tyack, P. L., Johnson, M. P. \& Madsen, P. T. 2005. Three-dimensional beam pattern of regular sperm whale clicks confirms bent-horn hypothesis. Journal of the Acoustical Society of America: 117, 1473-1485. doi:10.1121/1.1828501. 


\section{Appendix 1. AdDitional Movement Plots}

\section{A1.1 Pitch, depth and Az variation profiles centered on focal tail slaps}

Pitch (left), depth (center) and Az variation (right) profiles centered on focal tail slaps (gray dotted line). Each line color corresponds to a unique focal tail slap and is consistent across each horizontal triptych of plots.
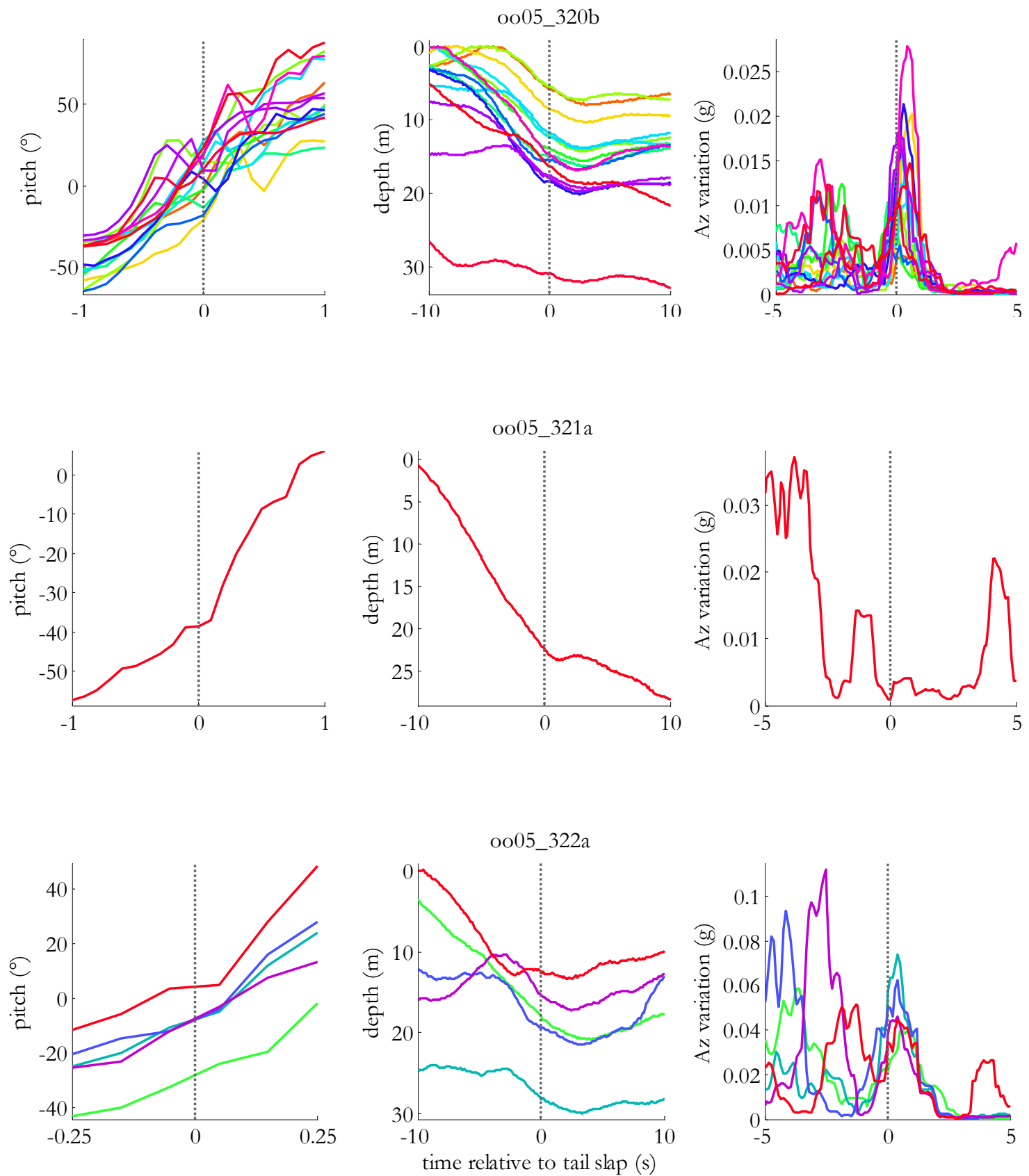

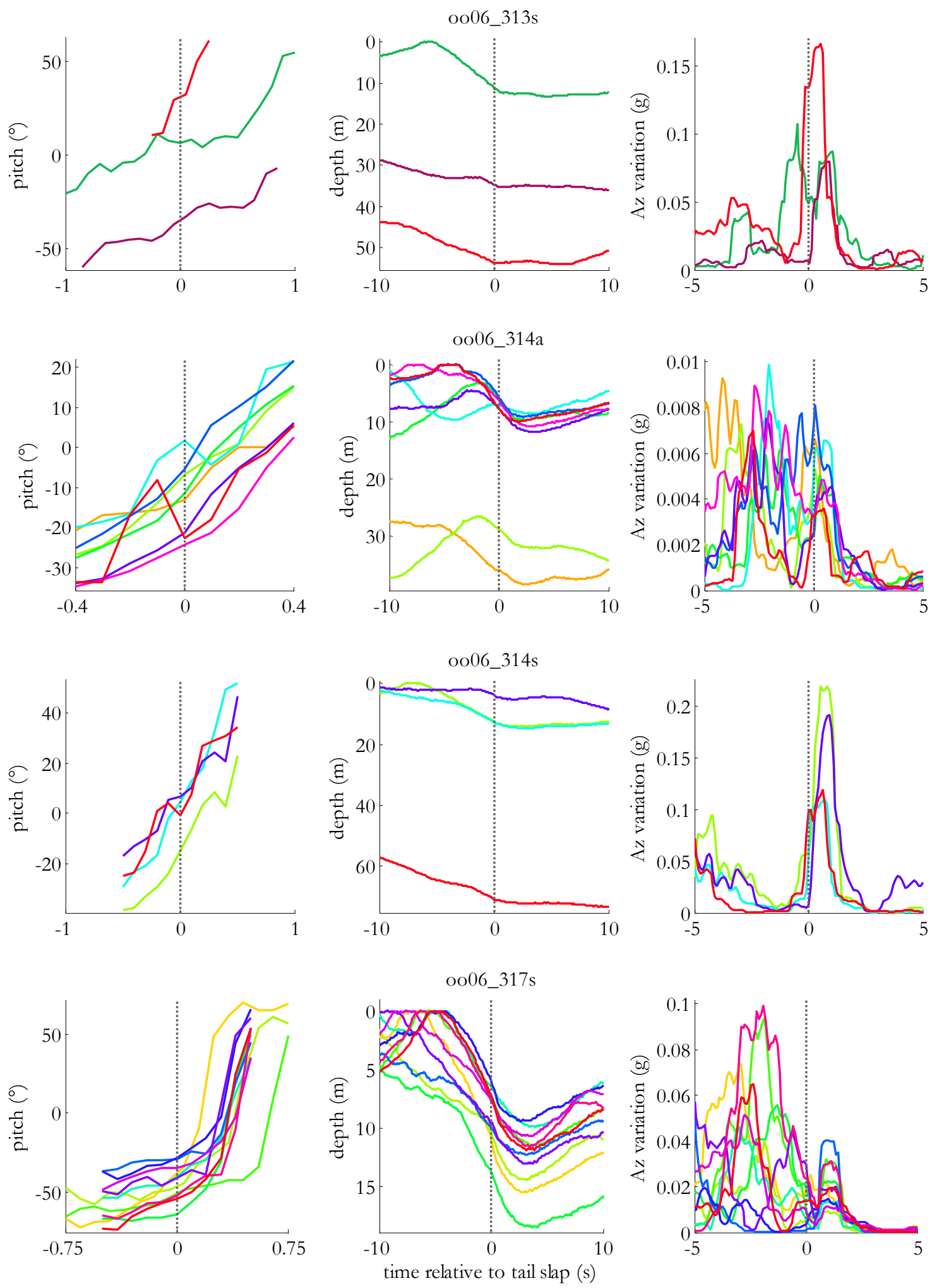


\section{A1.2 Pseudo-tracks}

The pseudo-track is a non-geo-referenced inertial path. Horizontal movement is plotted as relative distance and the beginning and ending of this track are shown ( $\square$ and $\odot$, respectively). In the left panel, depth is colorized, the filled gray circles represent tail slaps produced by a non-tagged individual, and the red circles indicate tail slaps produced by the tagged, focal animal. In the right panel, the red sections of the track correspond to periods of low linearity (thresholded using the focal tail slap data, see Chapter 2).
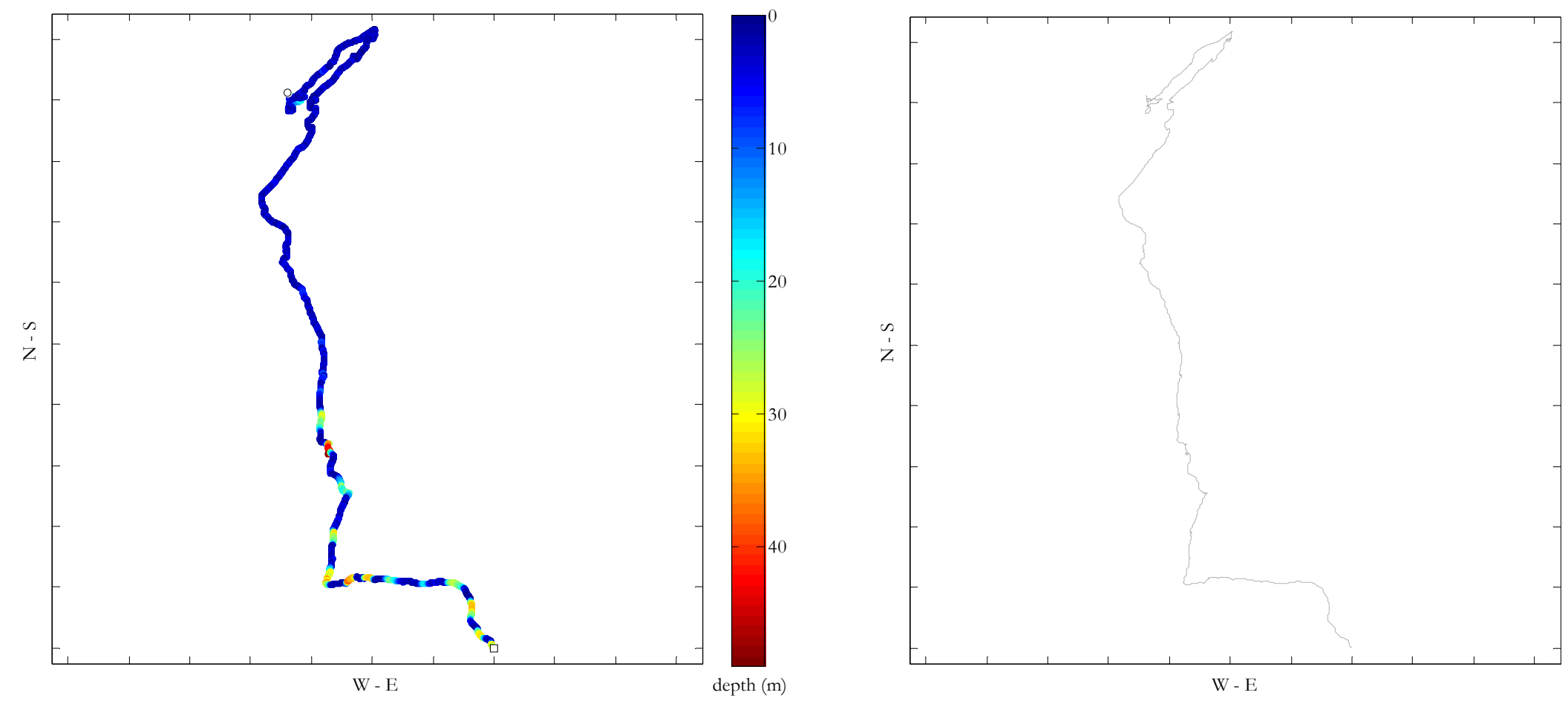

oo05 316a 


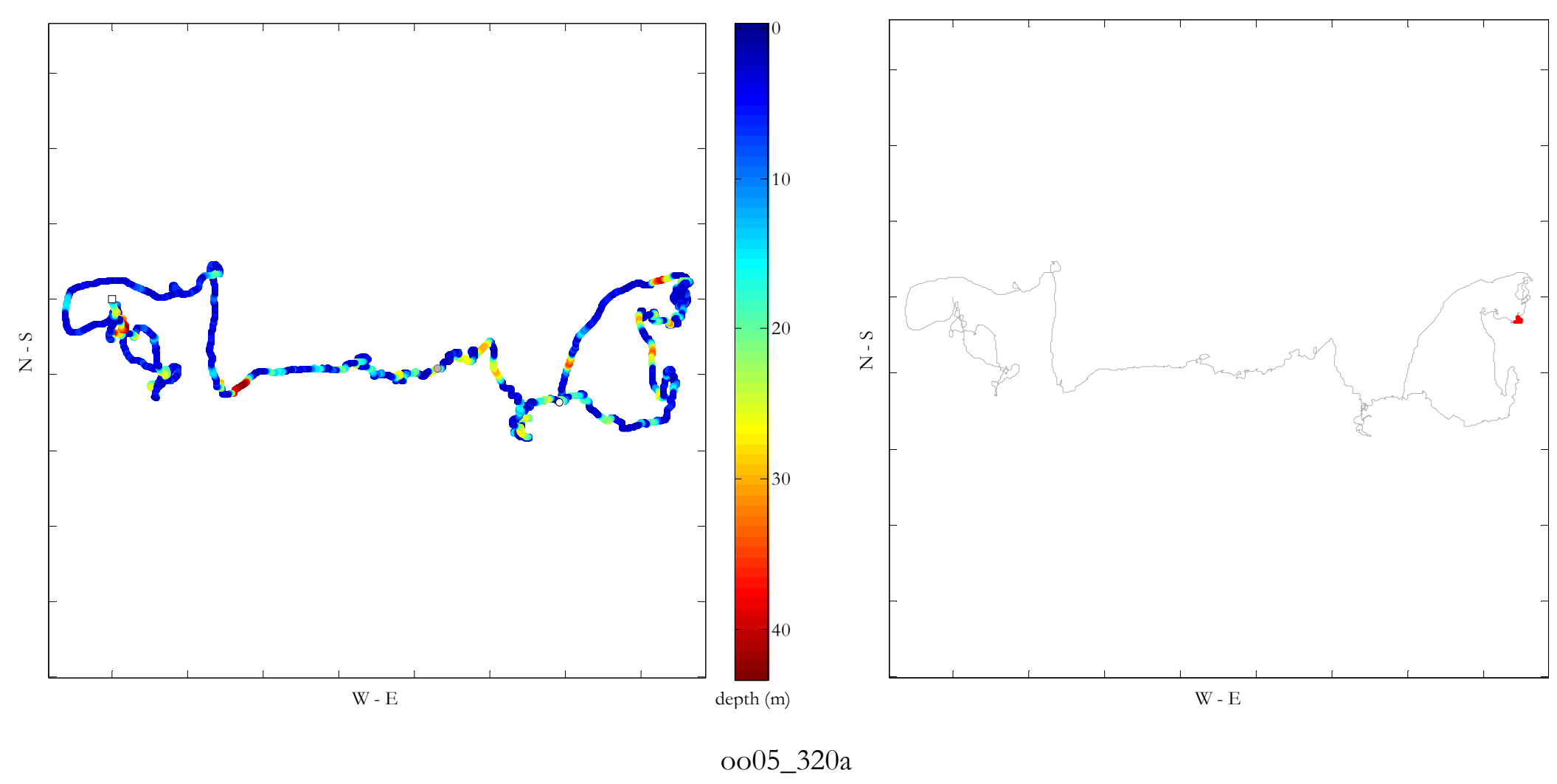




$$
w
$$




$$
M
$$




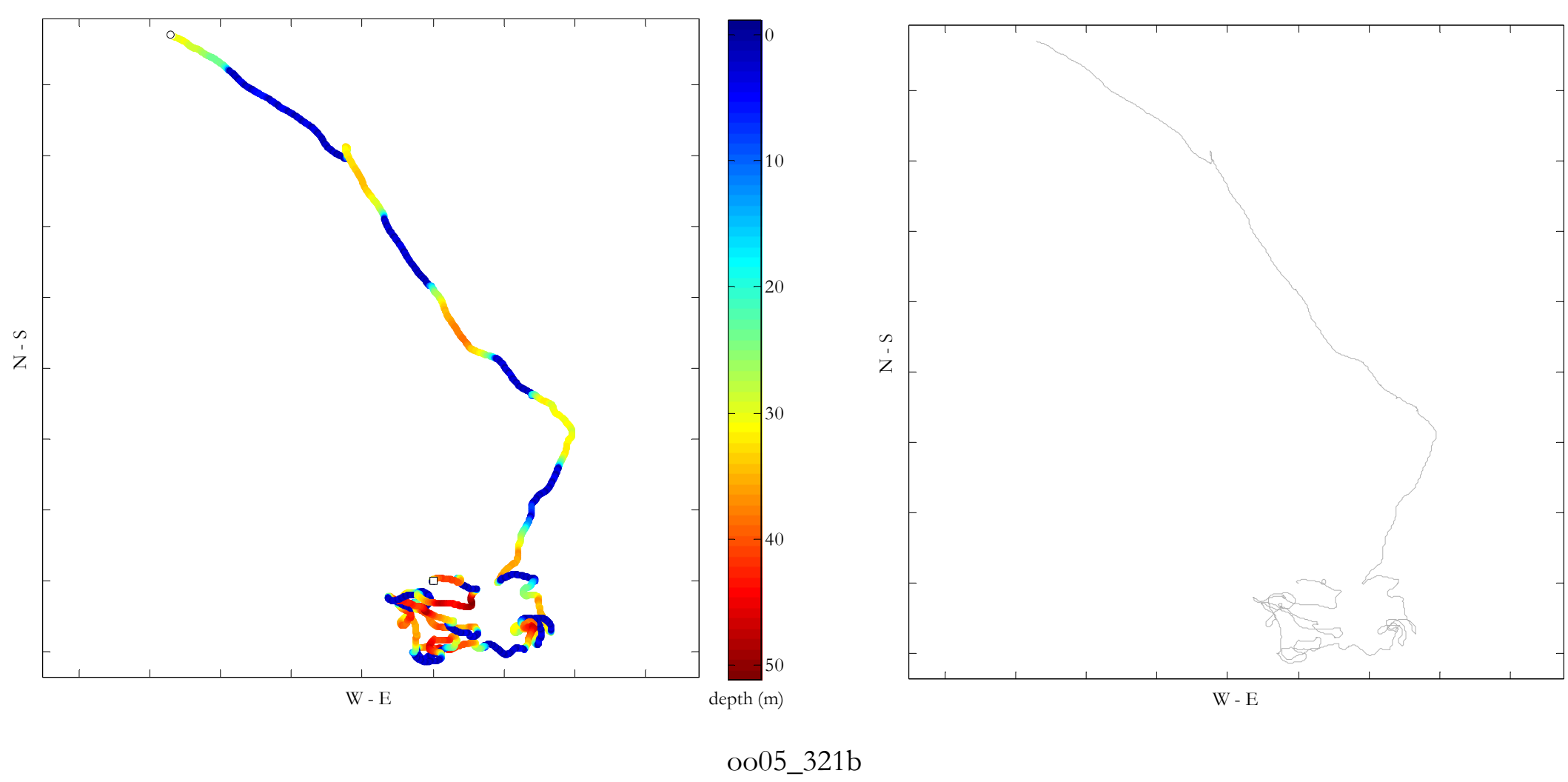



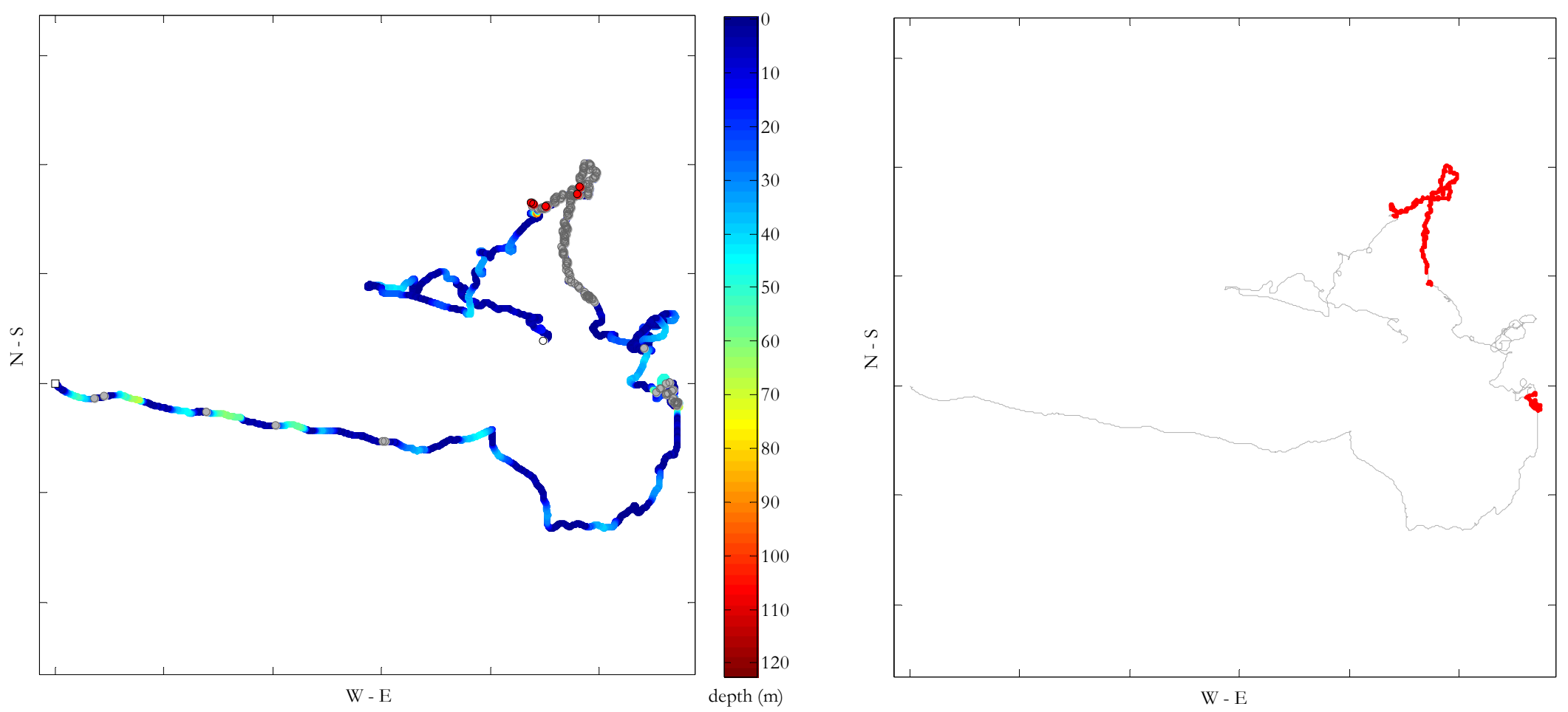

oo05_322a 

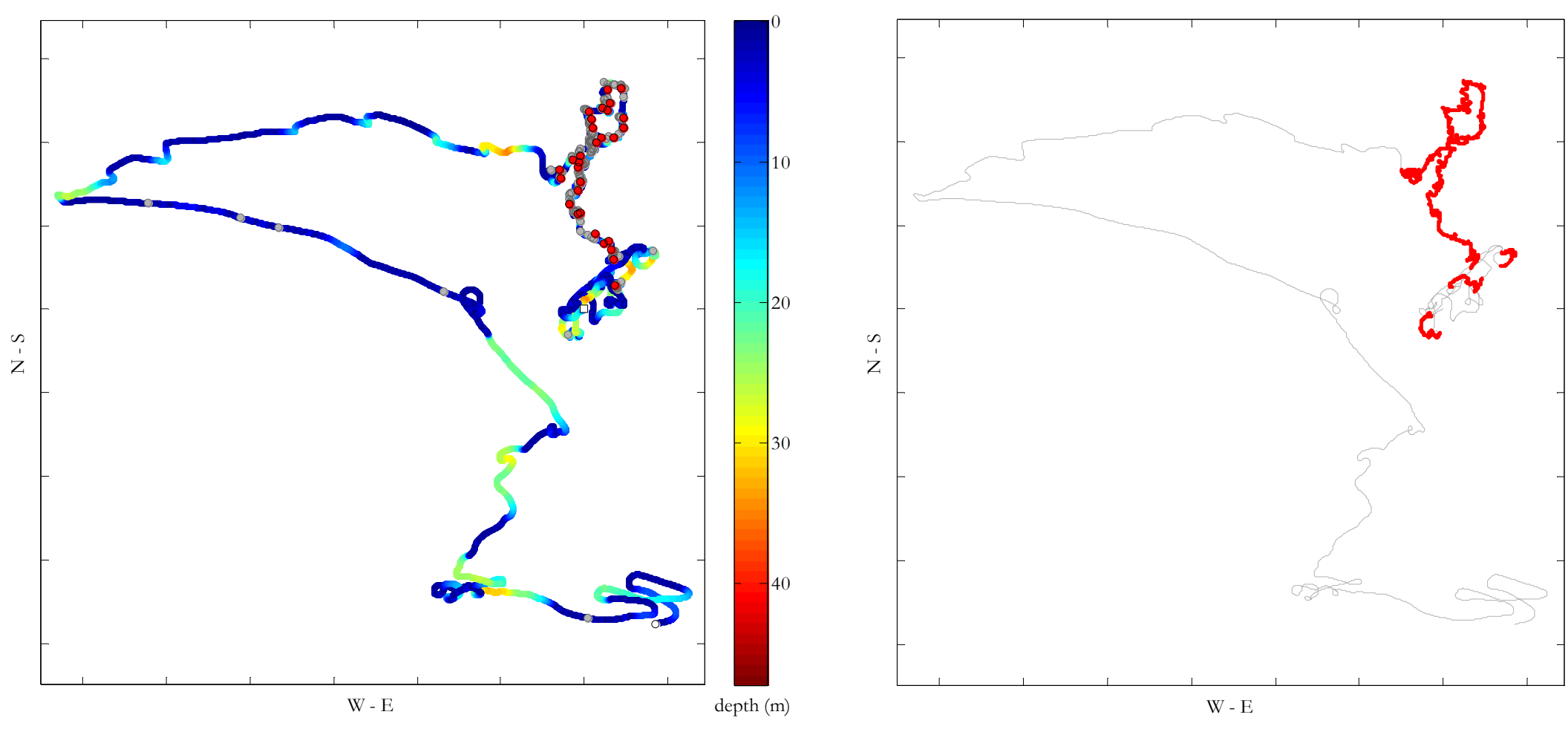

oo05_322b 


$$
4
$$



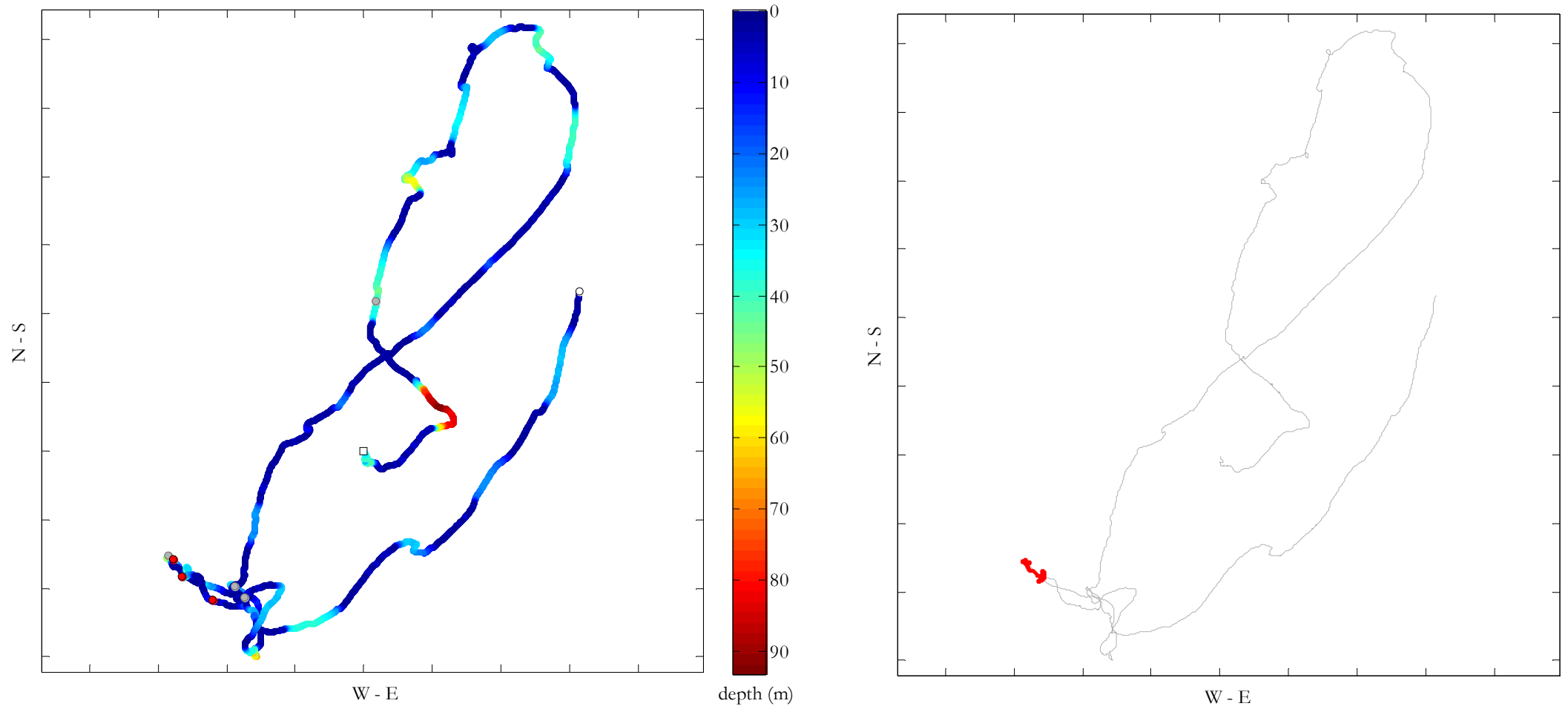

oo06_313s 


$$
V
$$



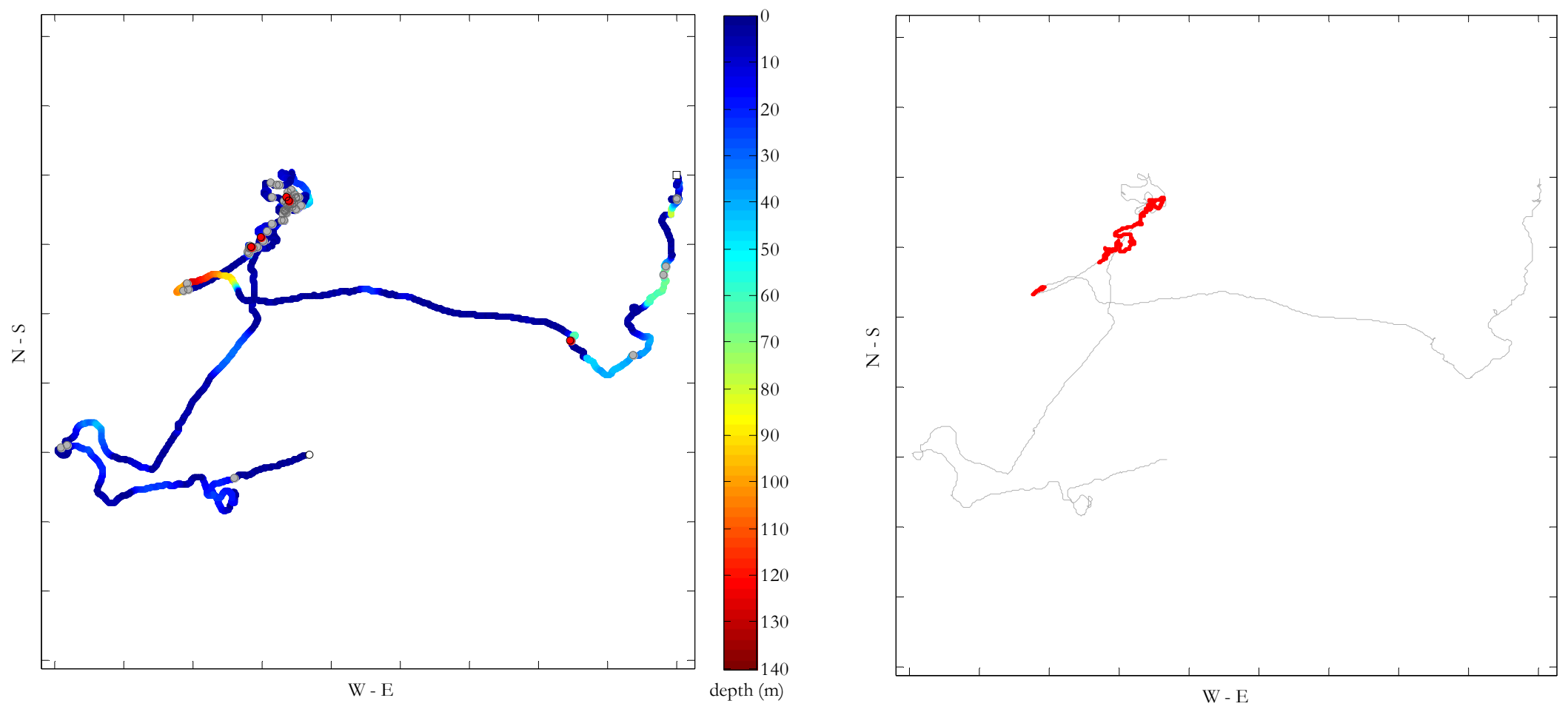

oo06_314s 
<smiles>[Y]C1(C)CC1(C)C</smiles> 


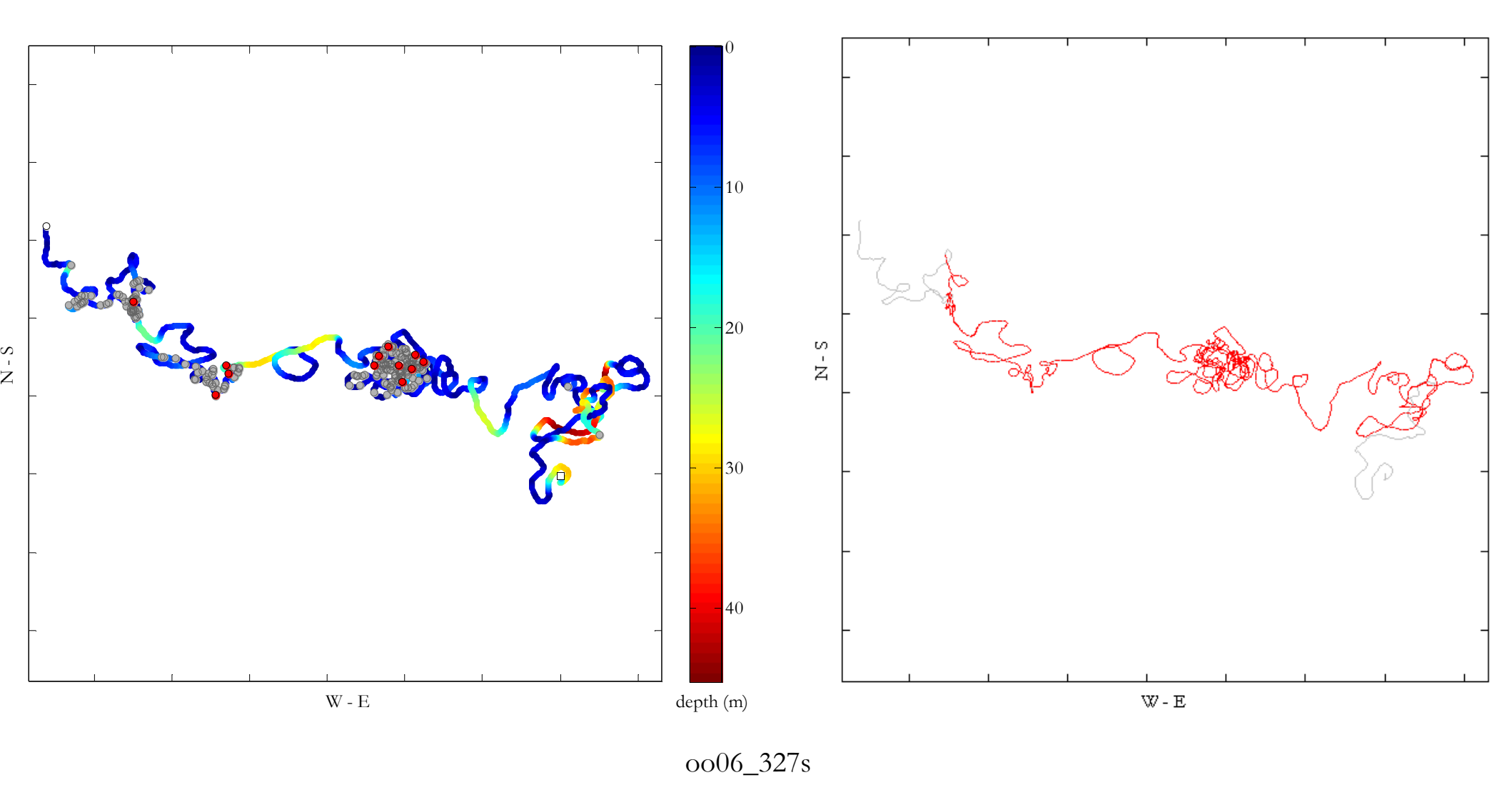





\section{A1.3 Movement data summary plots}

The linearity index (LI) is plotted as a thin continuous black line in the two uppermost panels and ranges from 0 (no displacement) to 1 (straight path). The LI plots two rough states: a low linearity (high circuitous) state when the animals were tail slapping and a high linearity (low circuitous) state when few tail slaps were observed (see Figure 2.9). Dive duration and maximum dive depth are indicated with the magenta squares and red circles in the left and right panels, respectively. The small gray squares and open black circles on the LI curve indicate non-focal and focal tail slaps, respectively. The two tail slapping (TS) periods are indicated by the black horizontal bars at the top of the plot. The low LI episodes are shown by the blue horizontal bars. Beginning in the second row and reading left to right, the remaining panels plot the change in pitch (degrees), depth $(\mathrm{m})$, change in roll (degrees), vertical velocity $(\mathrm{m} / \mathrm{s})$, residual heading (degrees) and variation in the $\mathrm{z}$-axis of the accelerometer (a proxy for fluking energy, relative units). Time is reported locally and runs identically along the $\mathrm{x}$-axis of each panel.

\section{O005_316a}
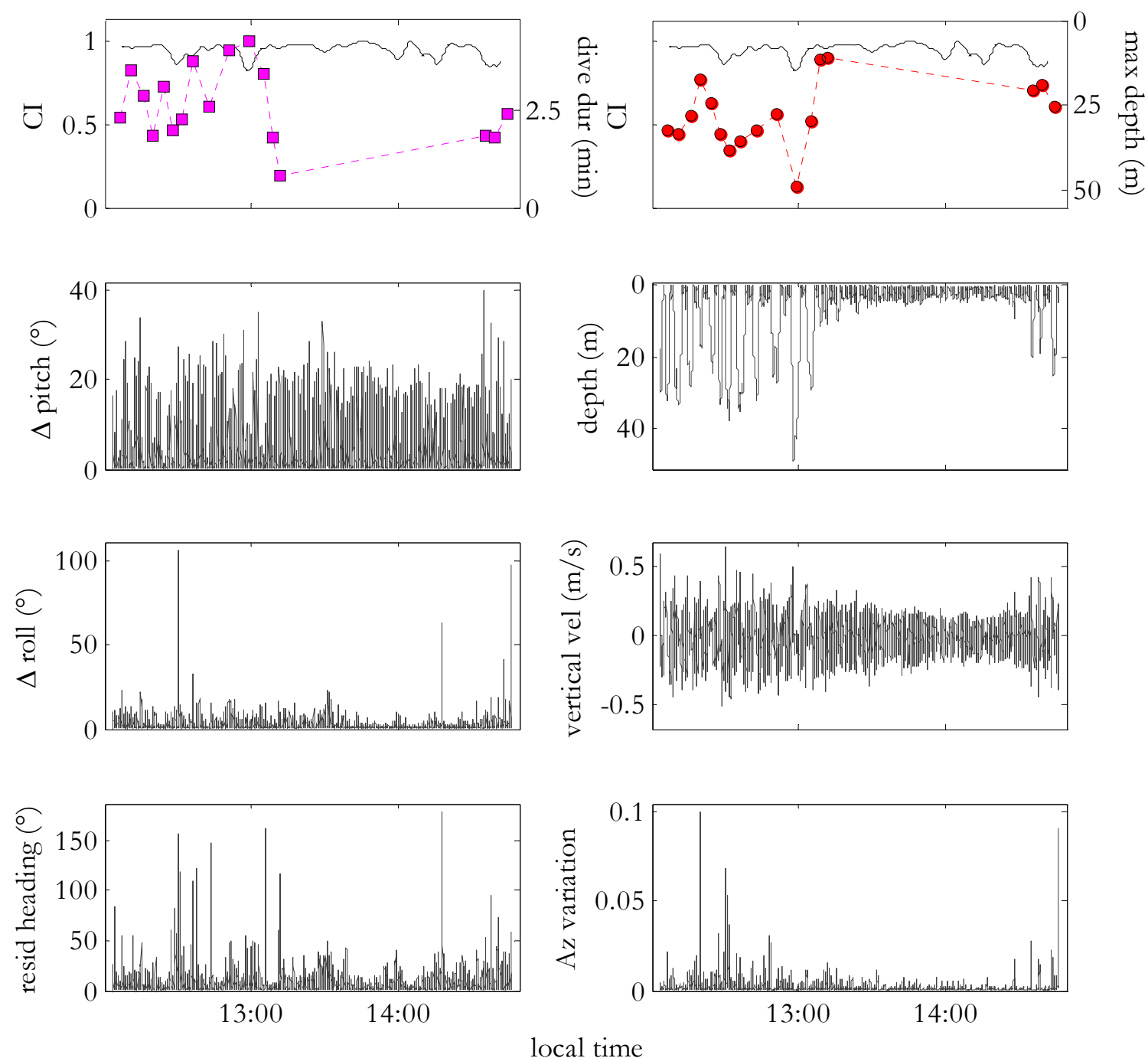

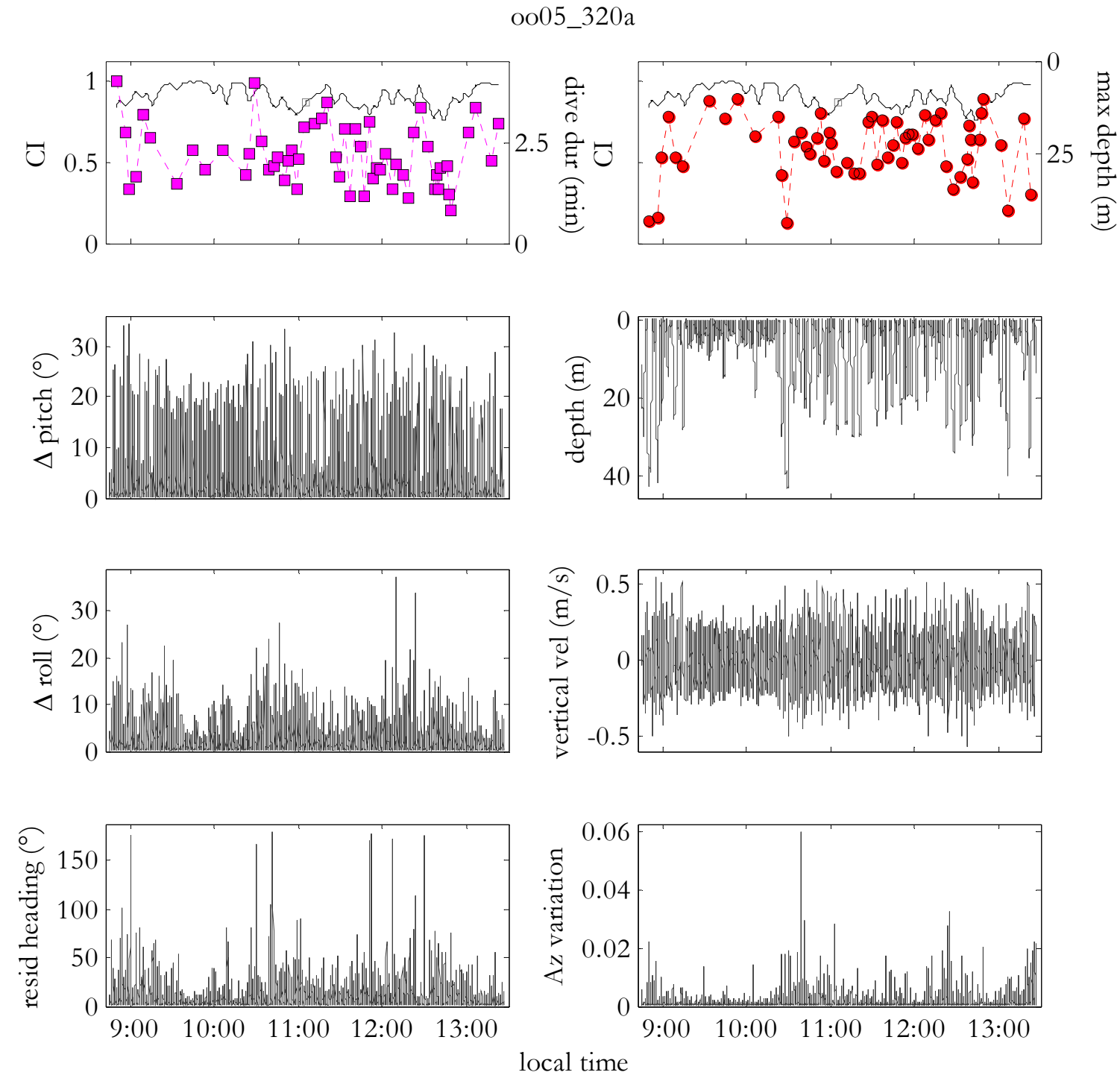

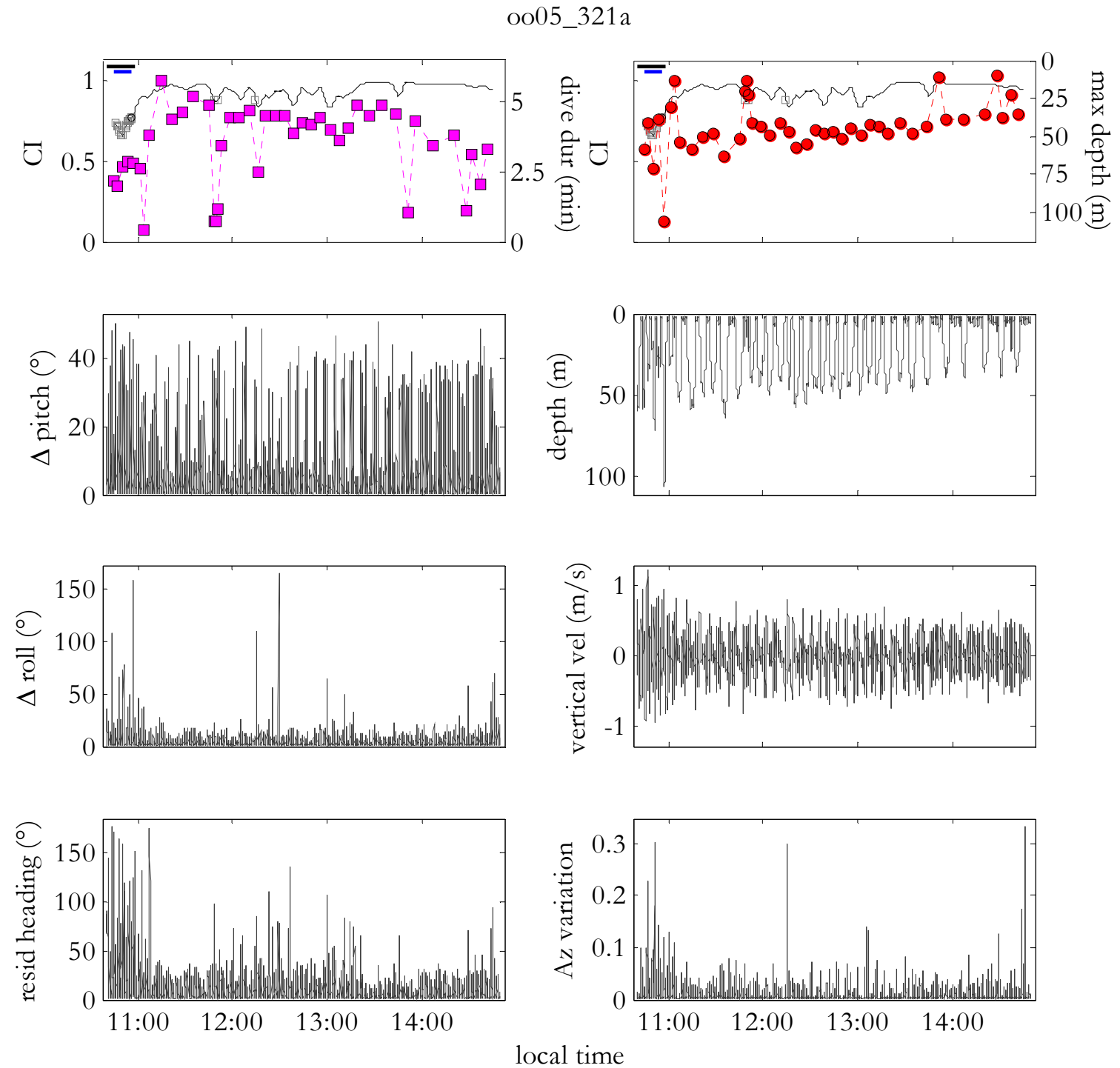

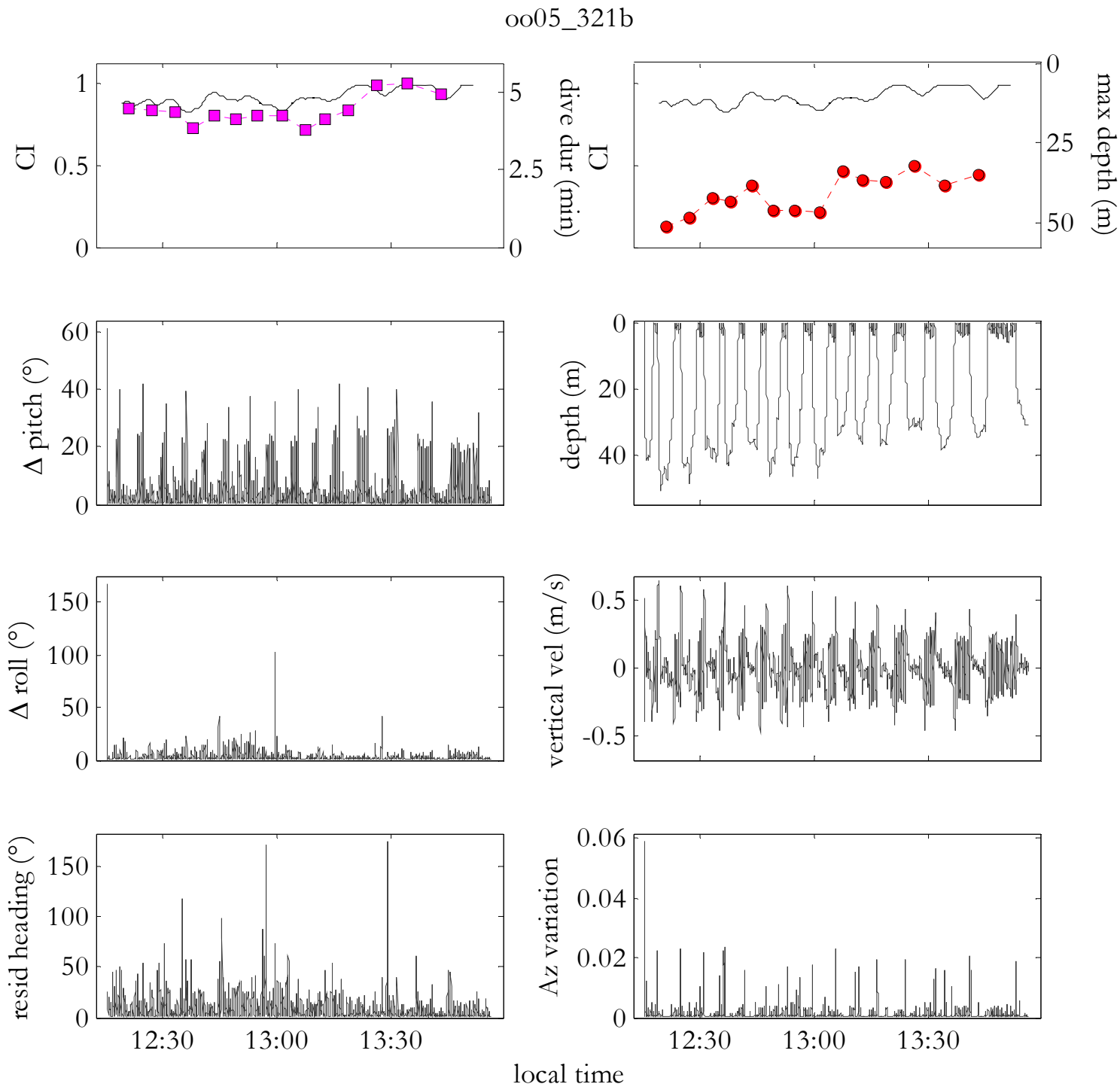
oo05_322b
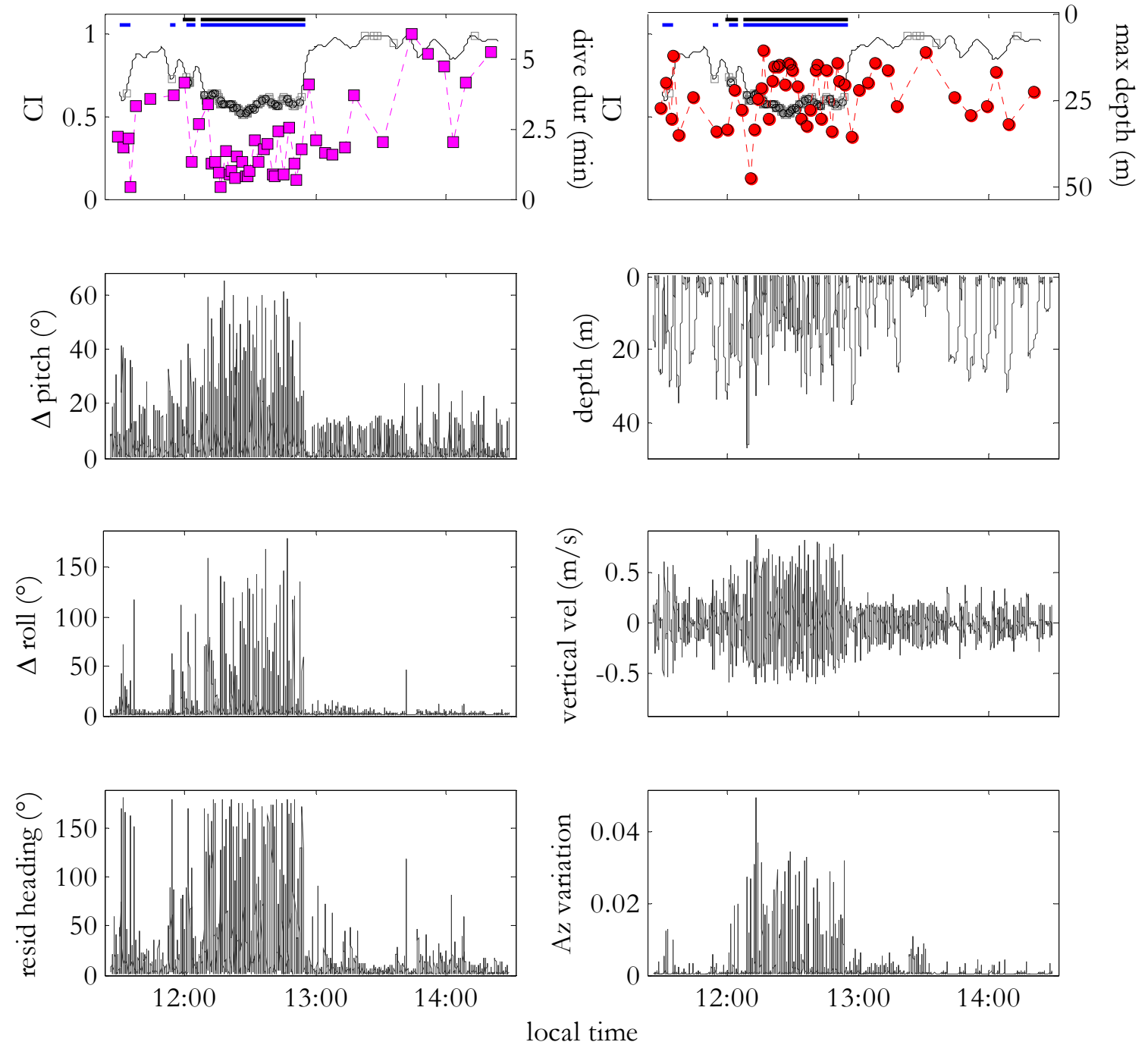

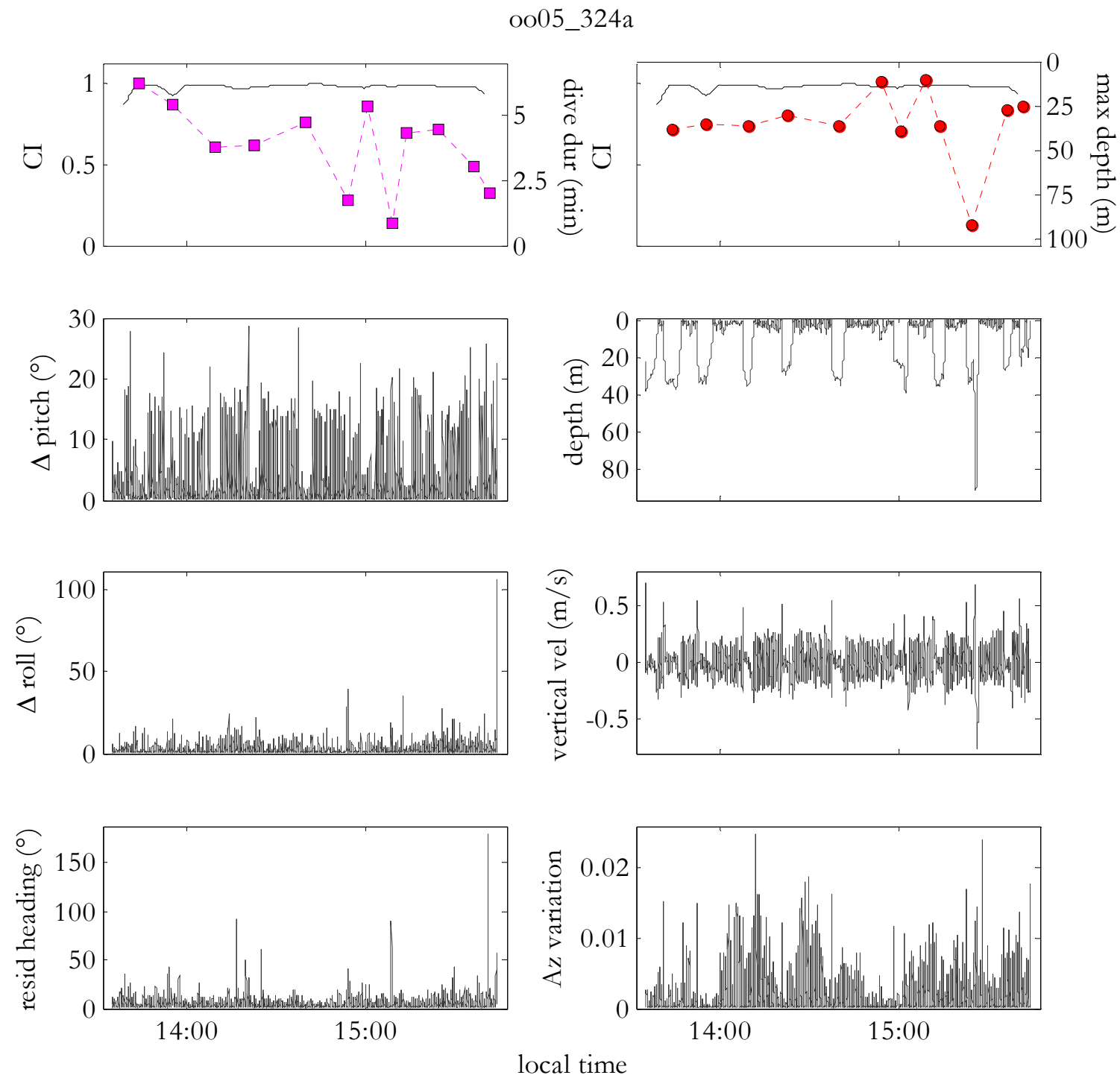

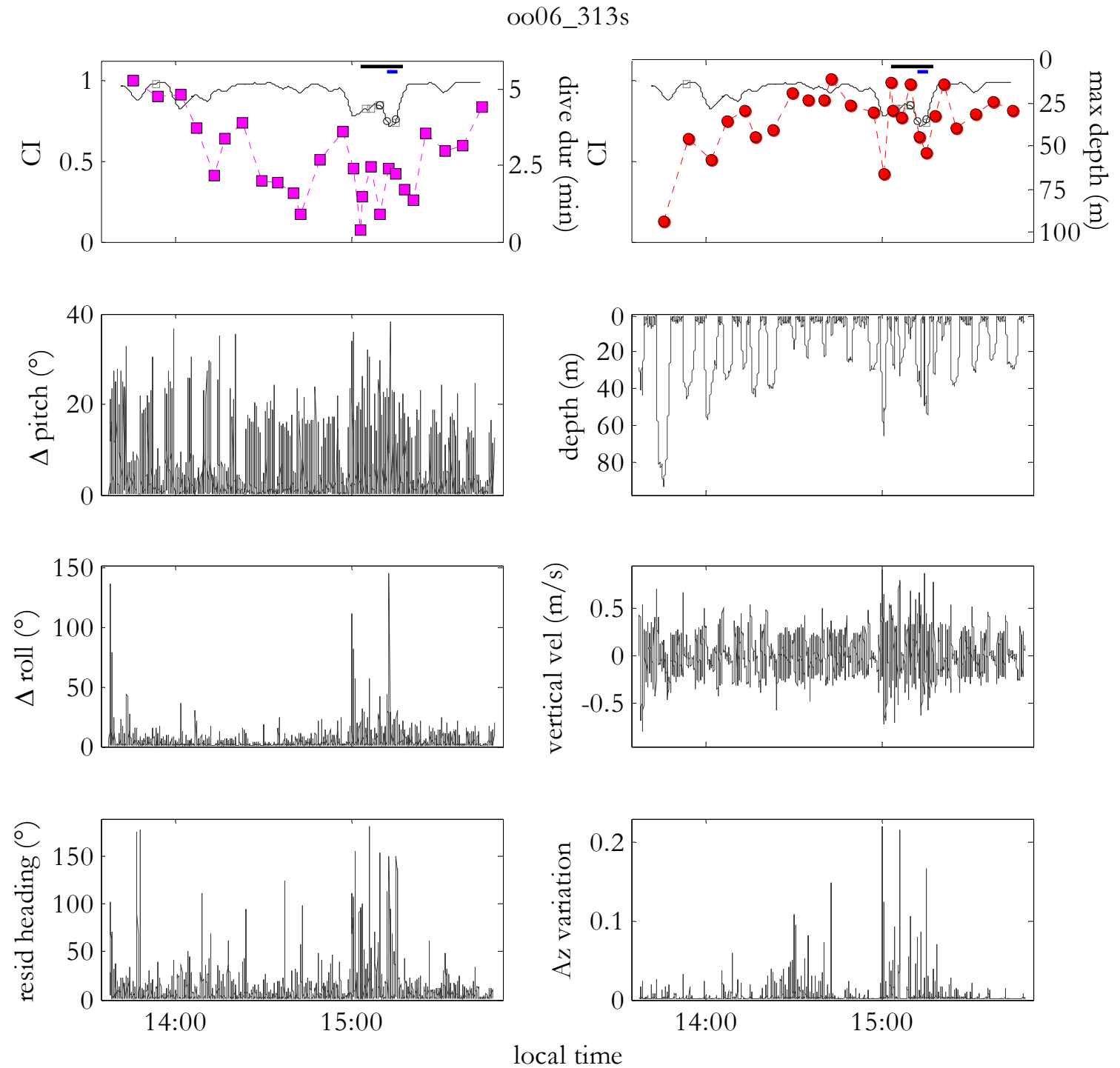

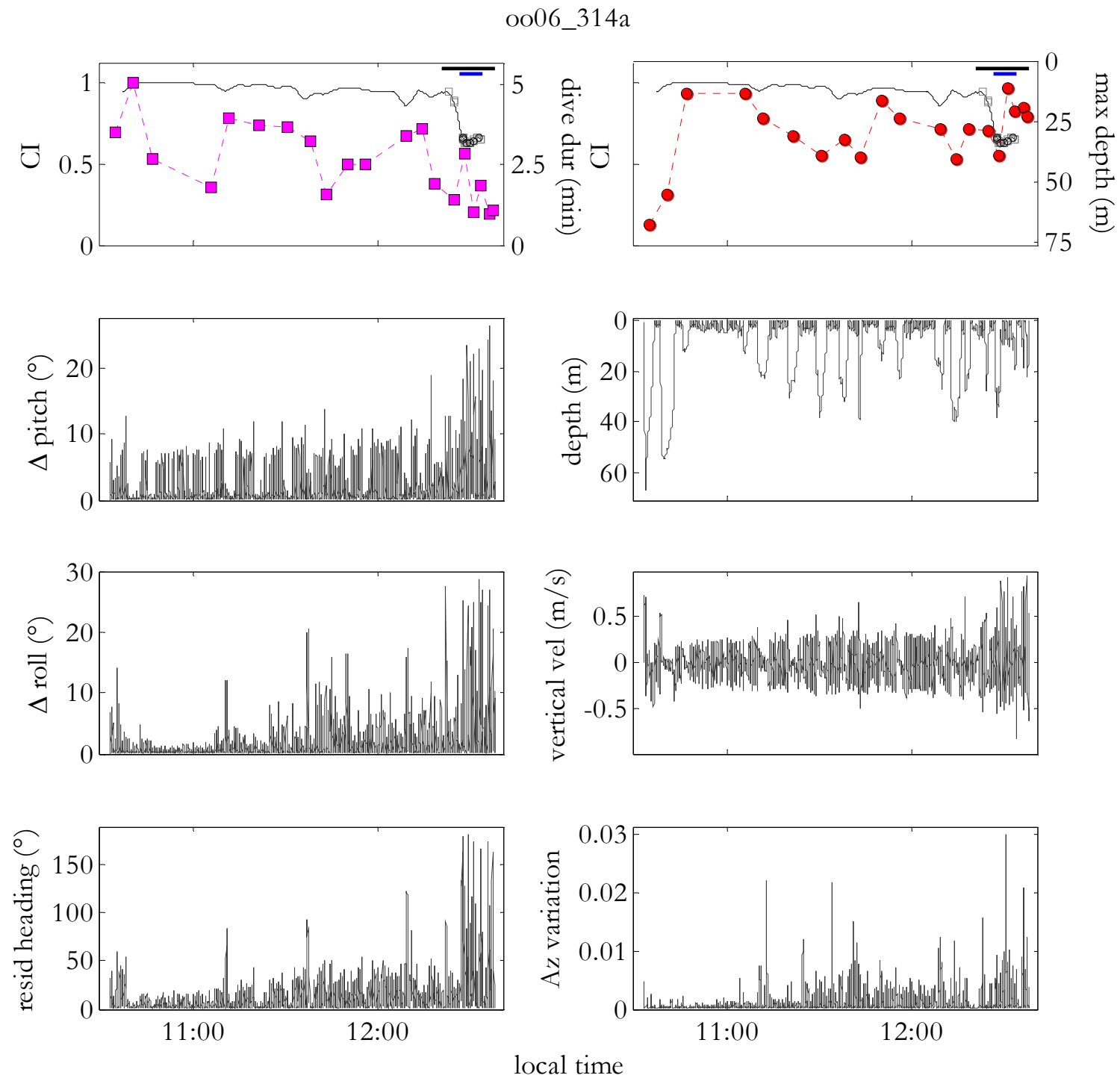

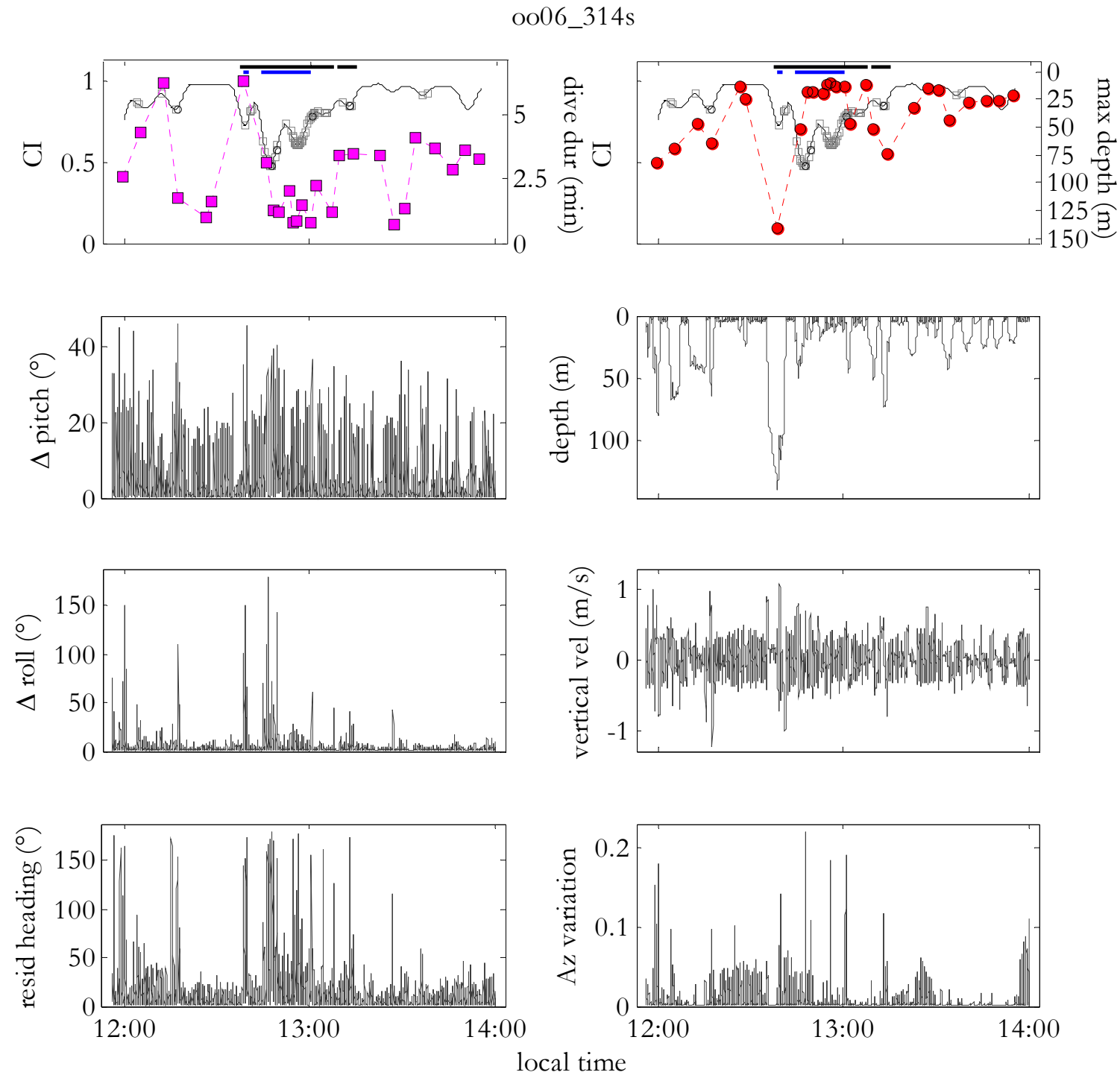

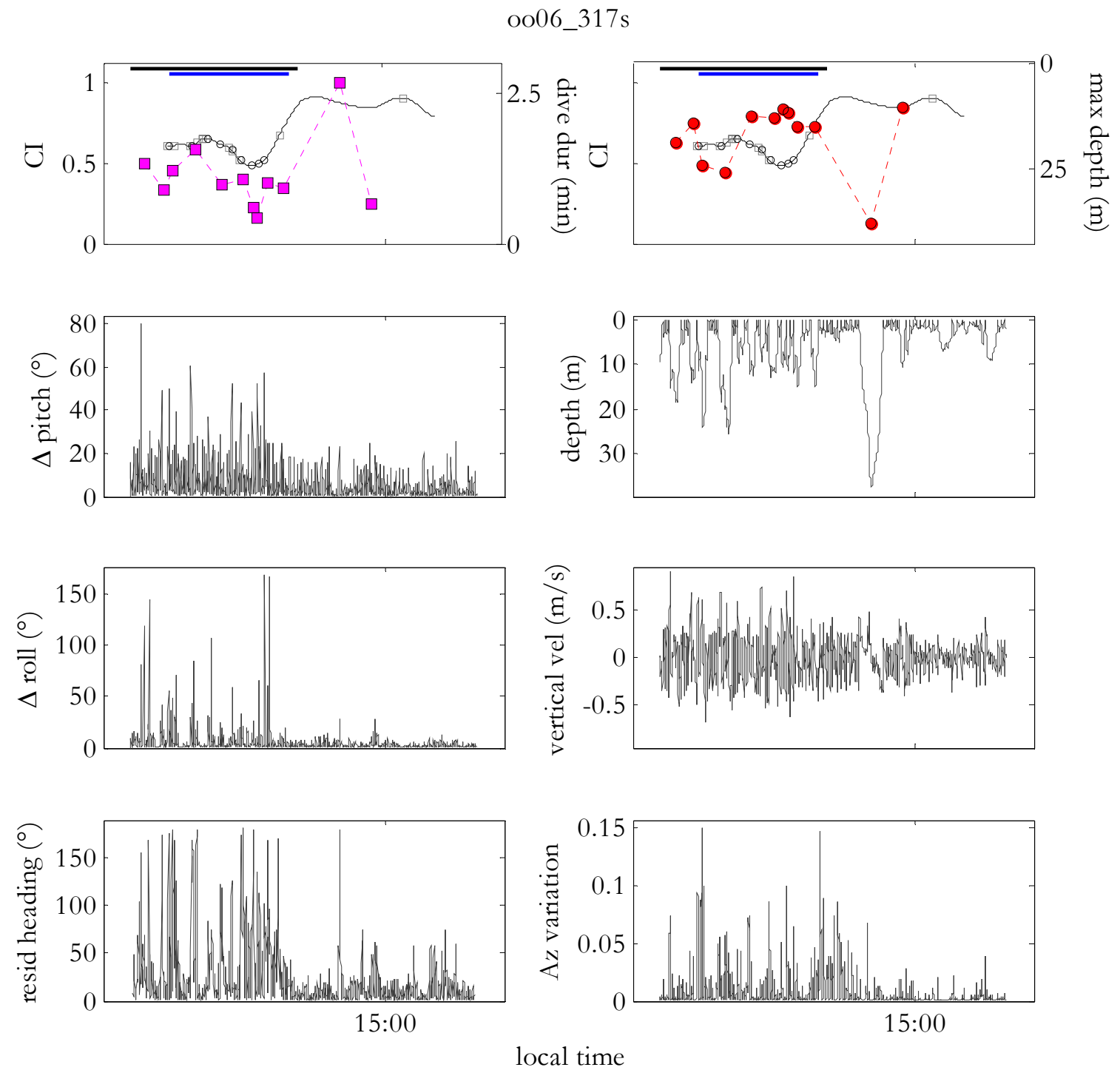

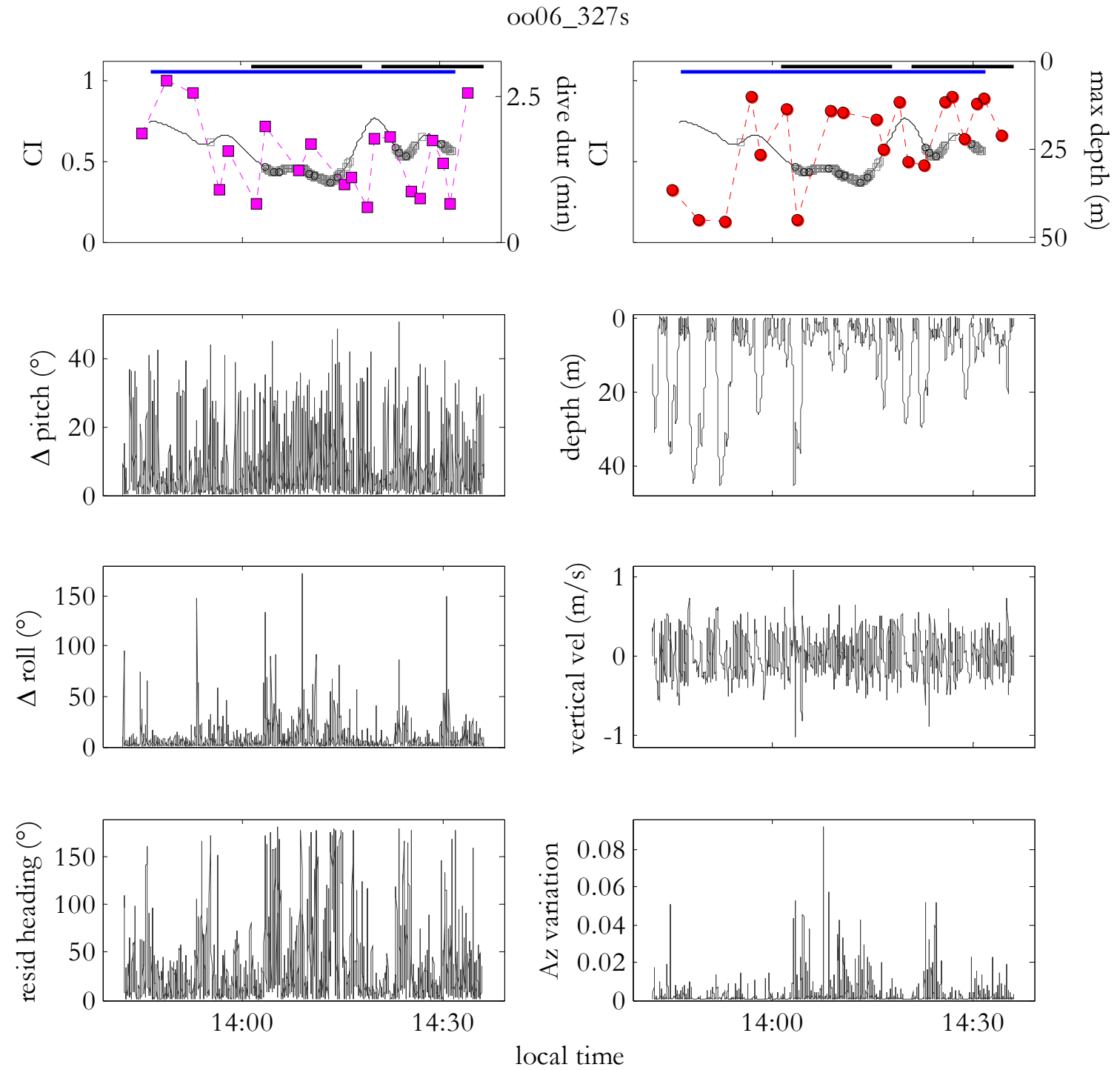


\section{APpendiX 2. NORWEgIAN CALl TyPES}

Spectrograms of Norwegian call types recorded during the tagouts listed in square brackets. Call types from N1 to N34 were described by Strager (1993), call types from N35 to N63 were defined by Van Opzeeland et al. (2005) and call types N64 to N103 were newly added here.
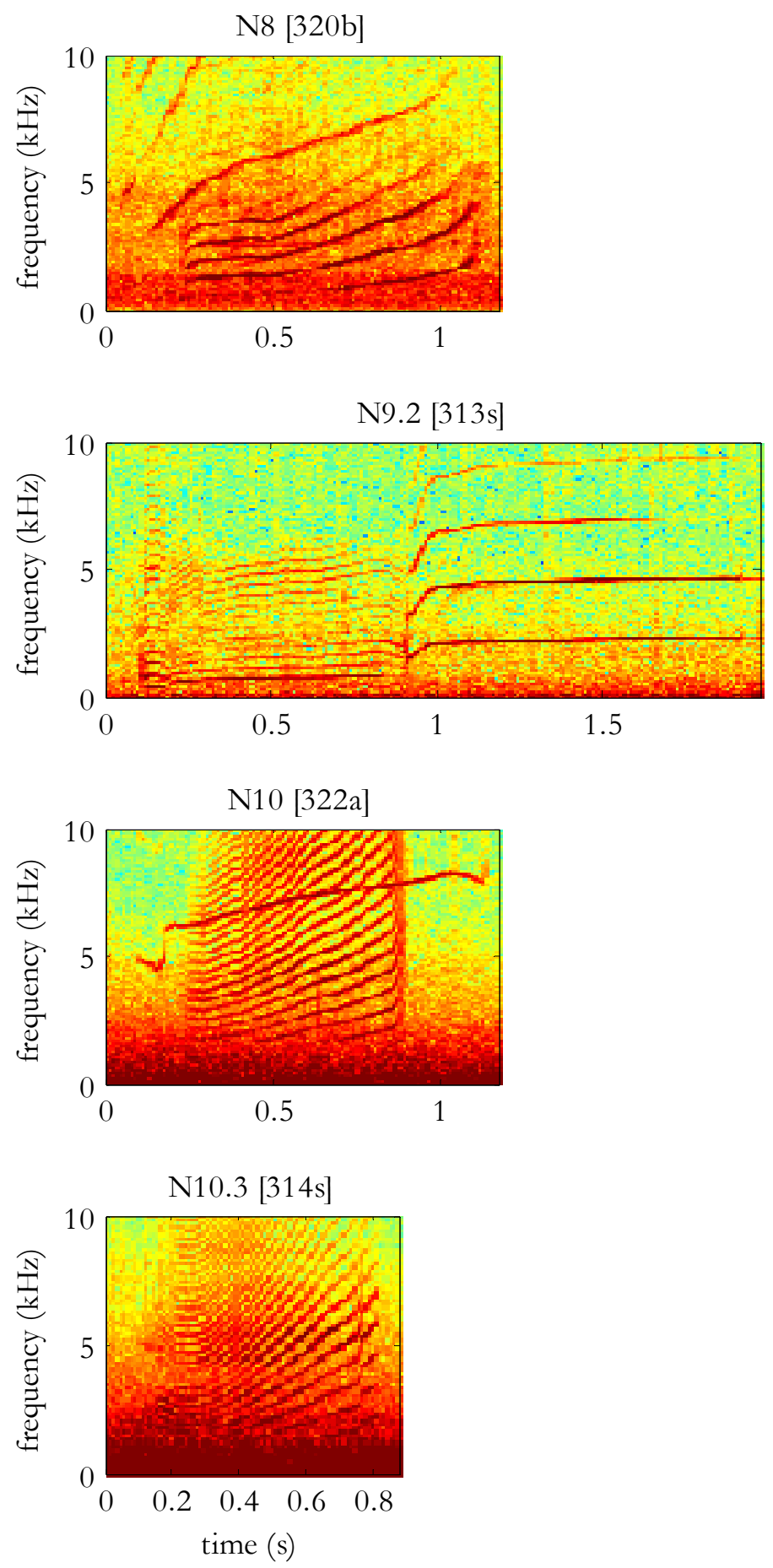

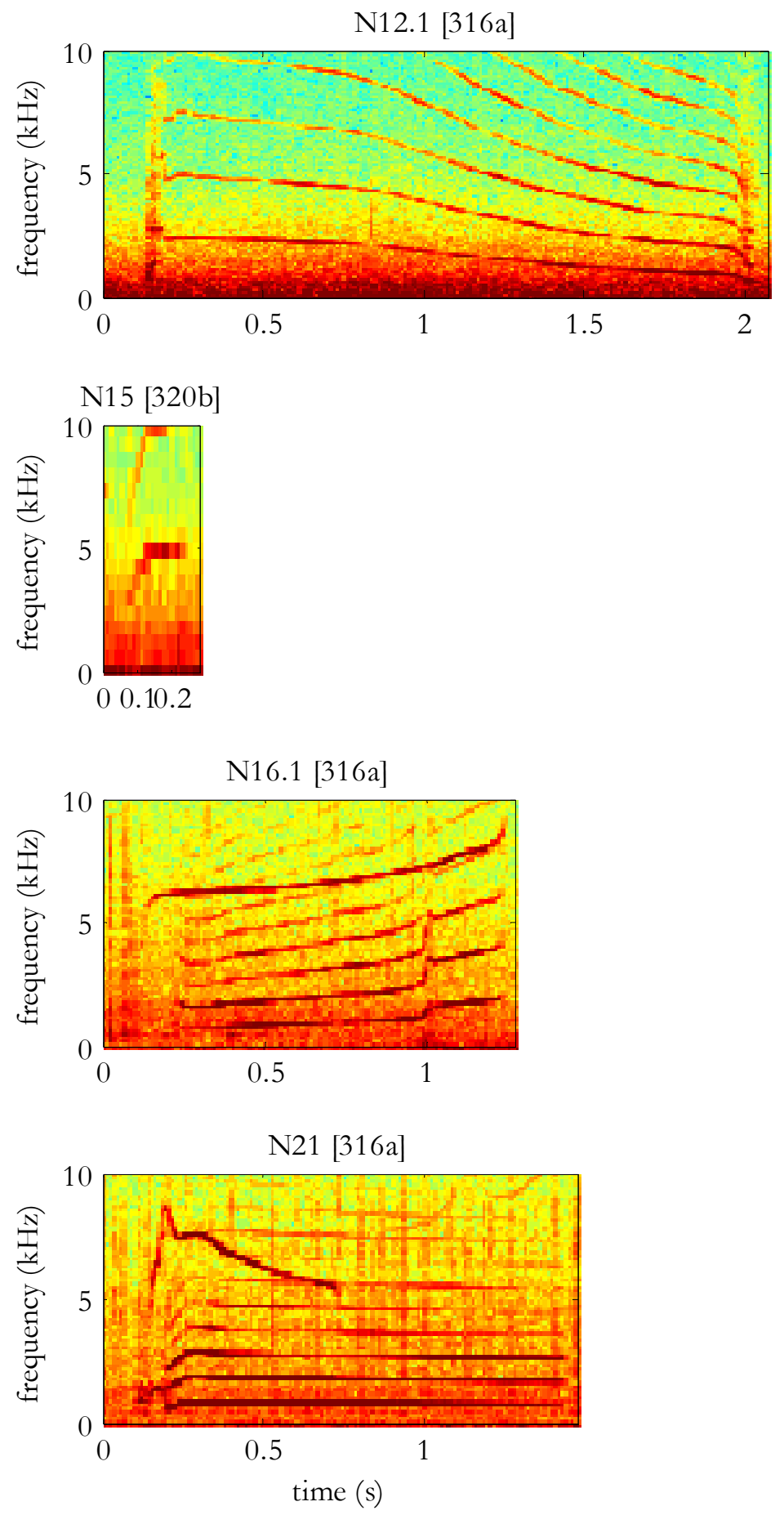

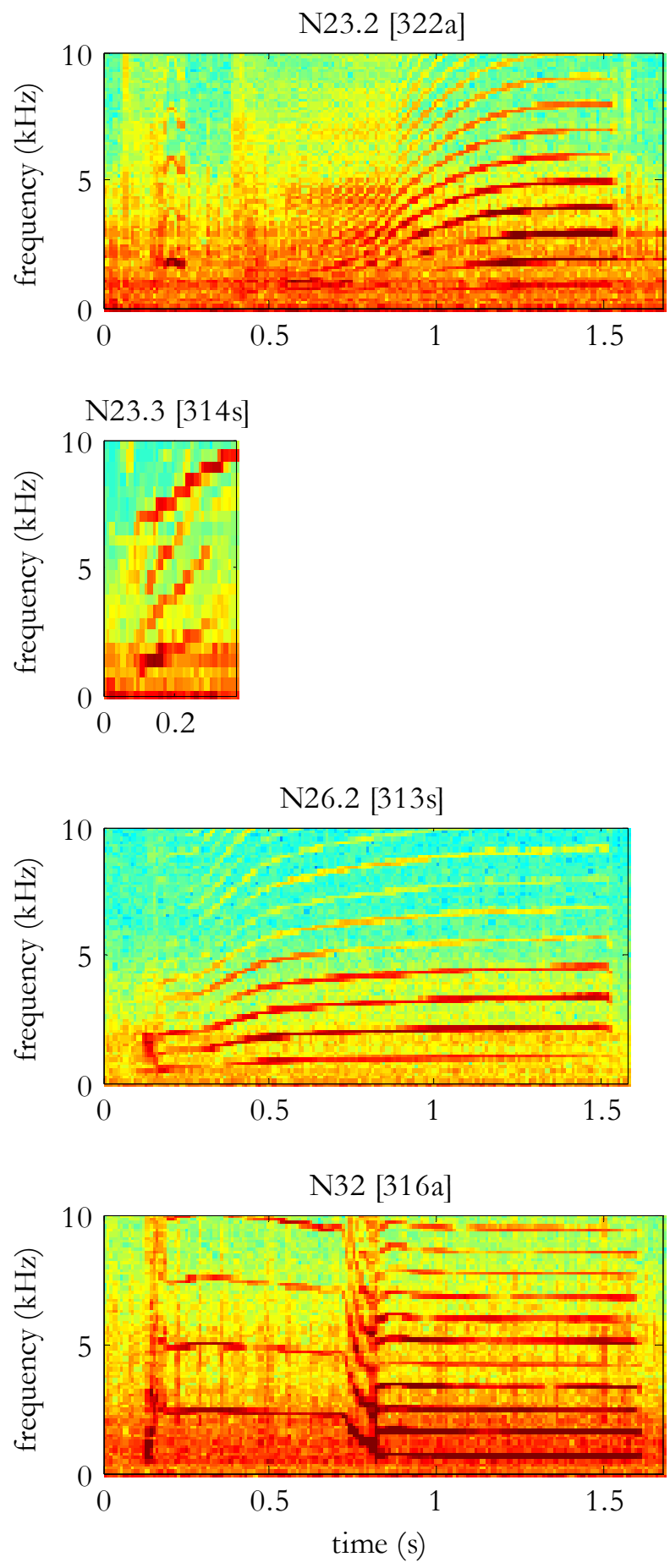

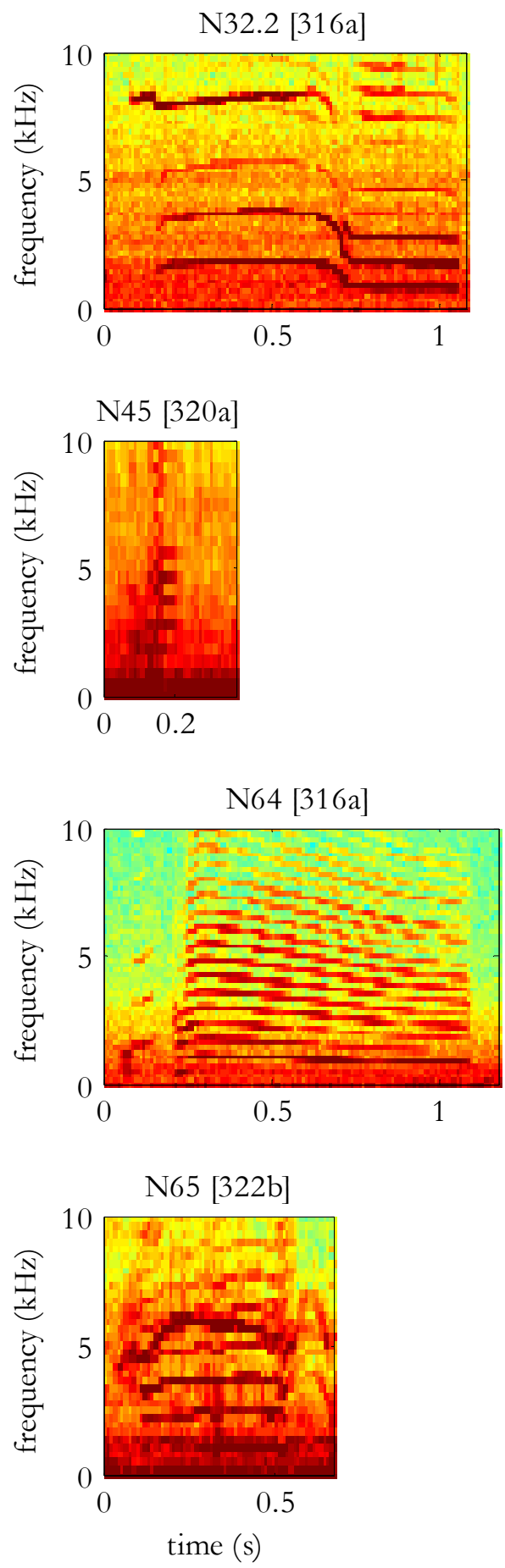

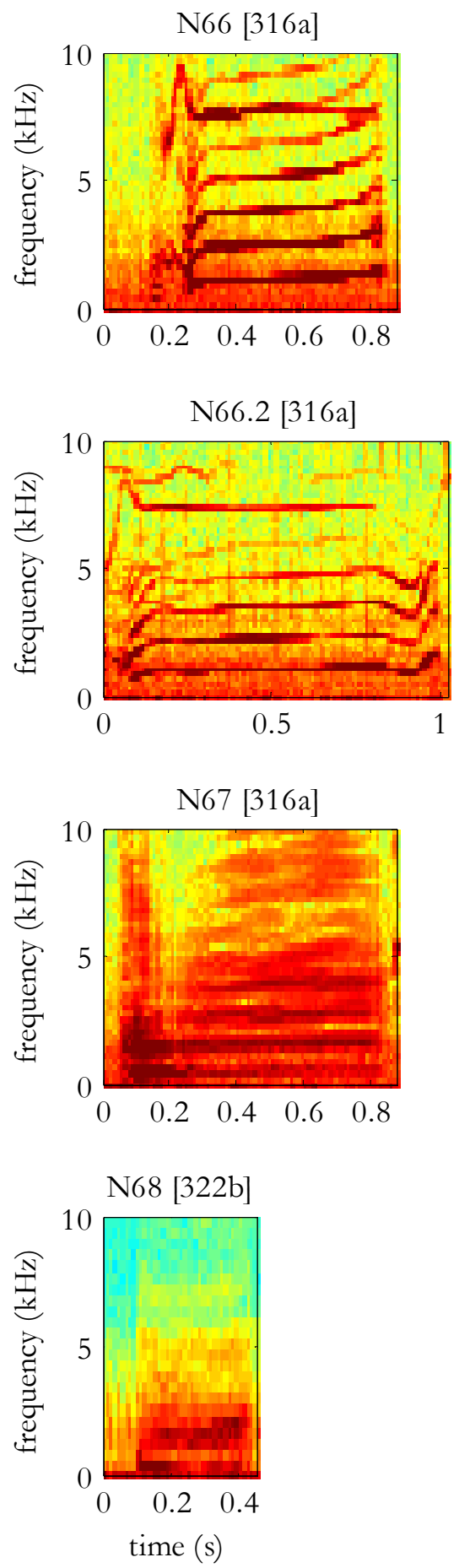

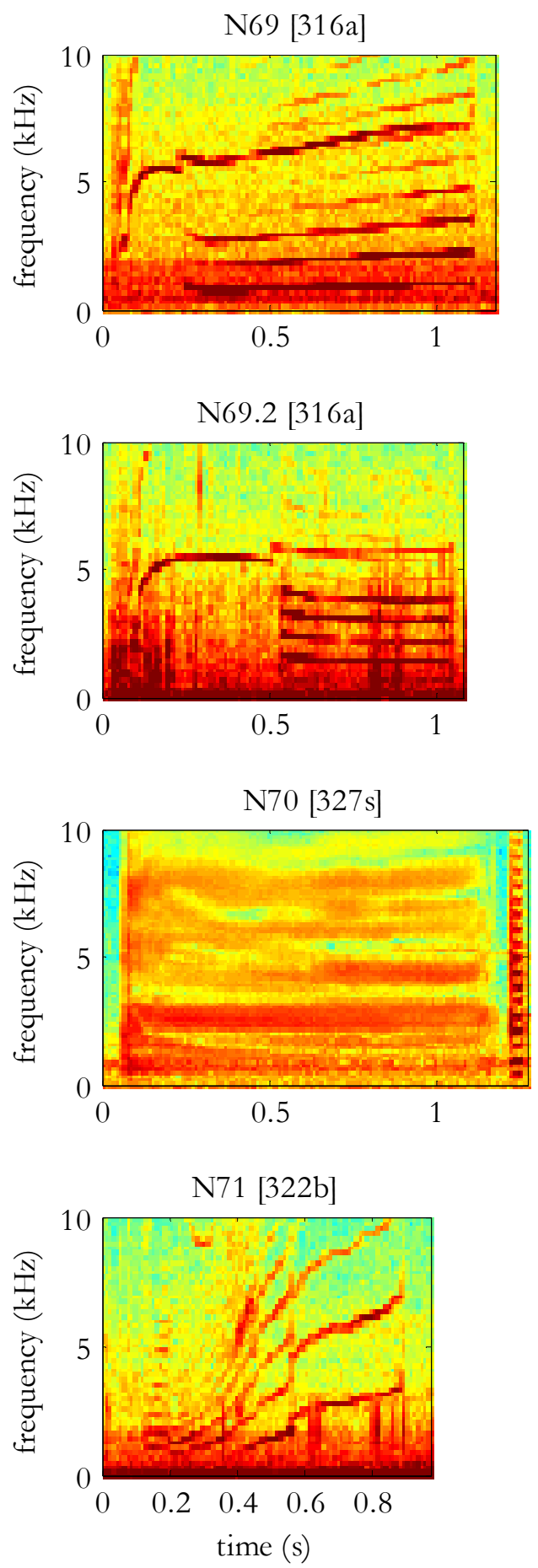

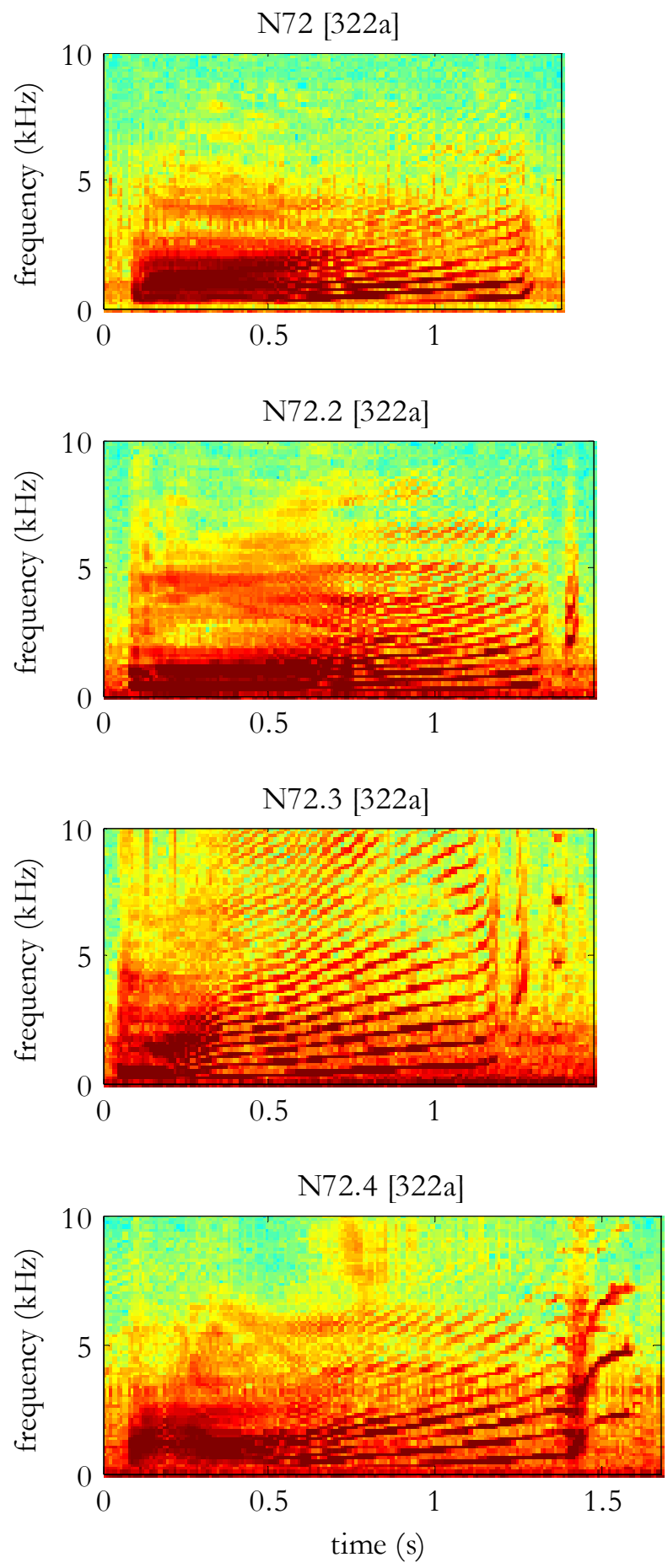

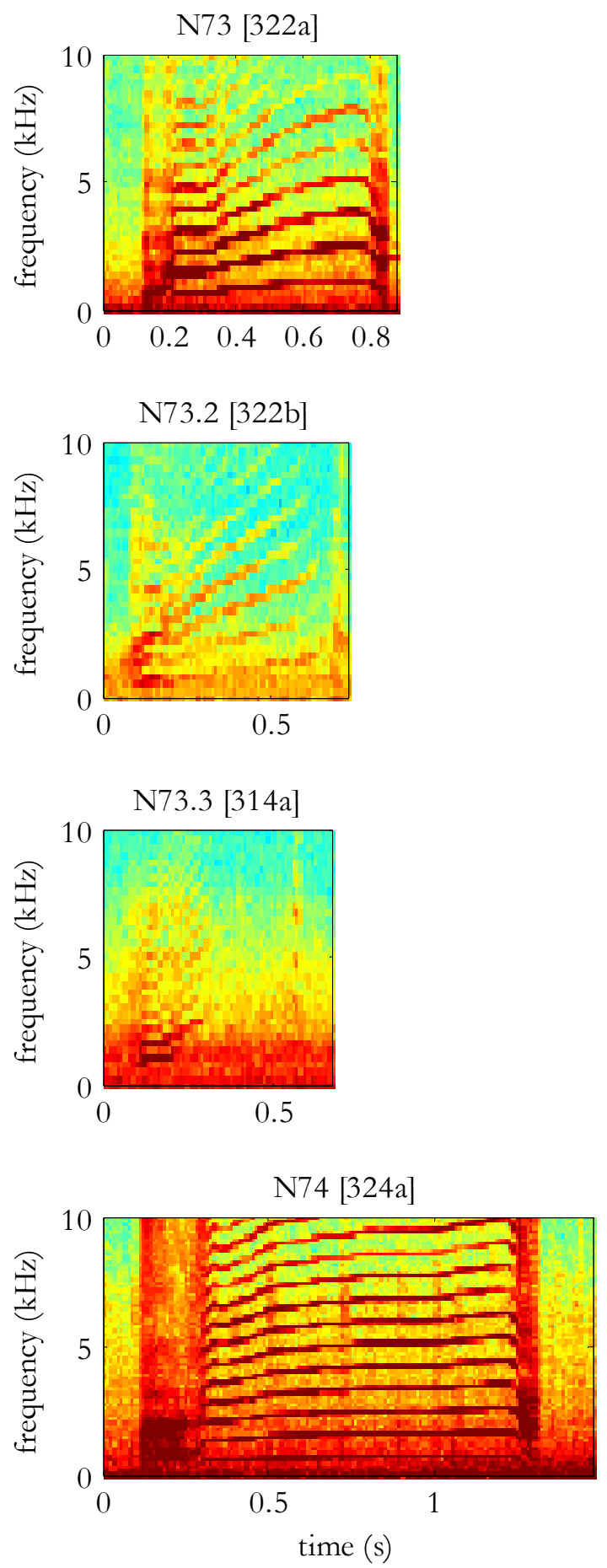

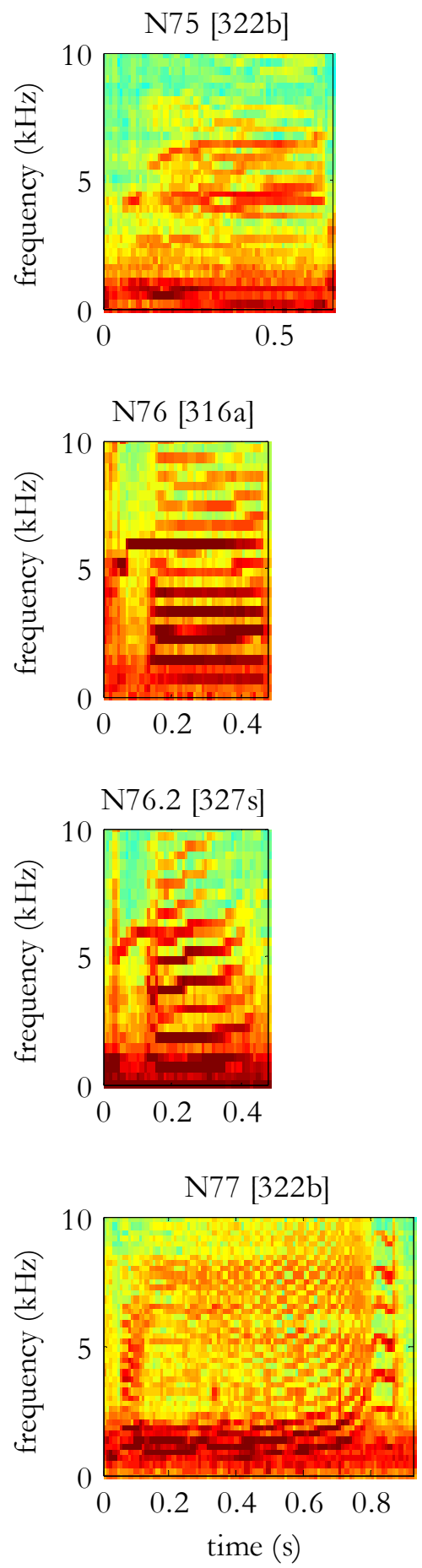

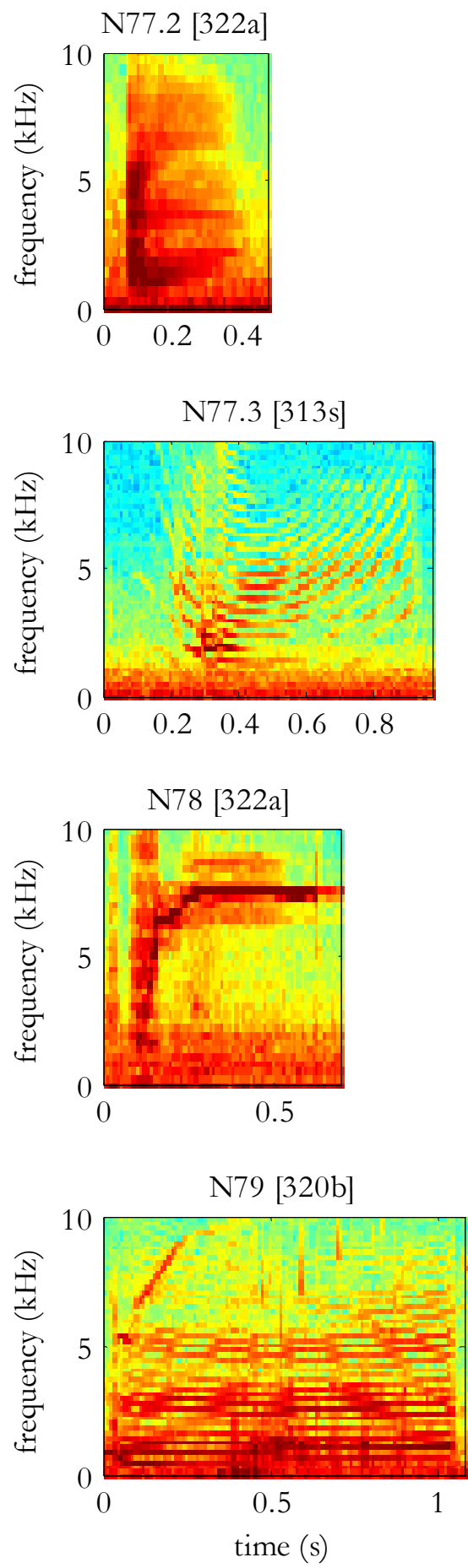

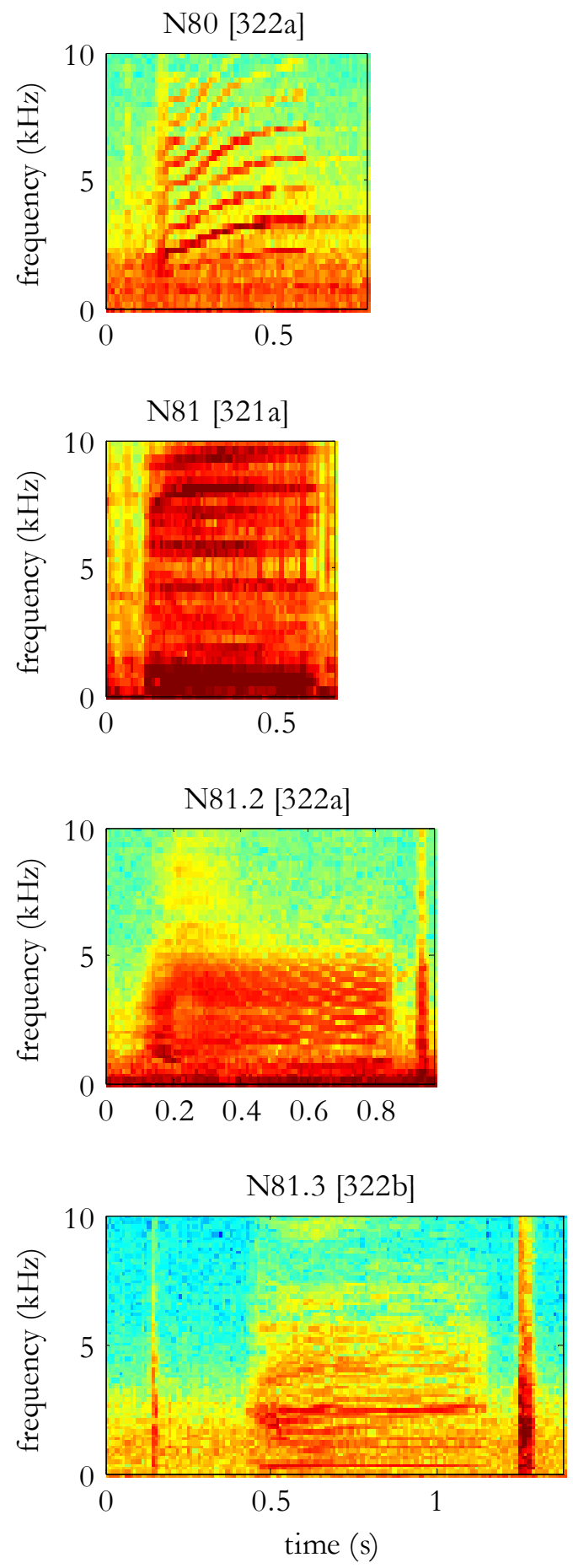

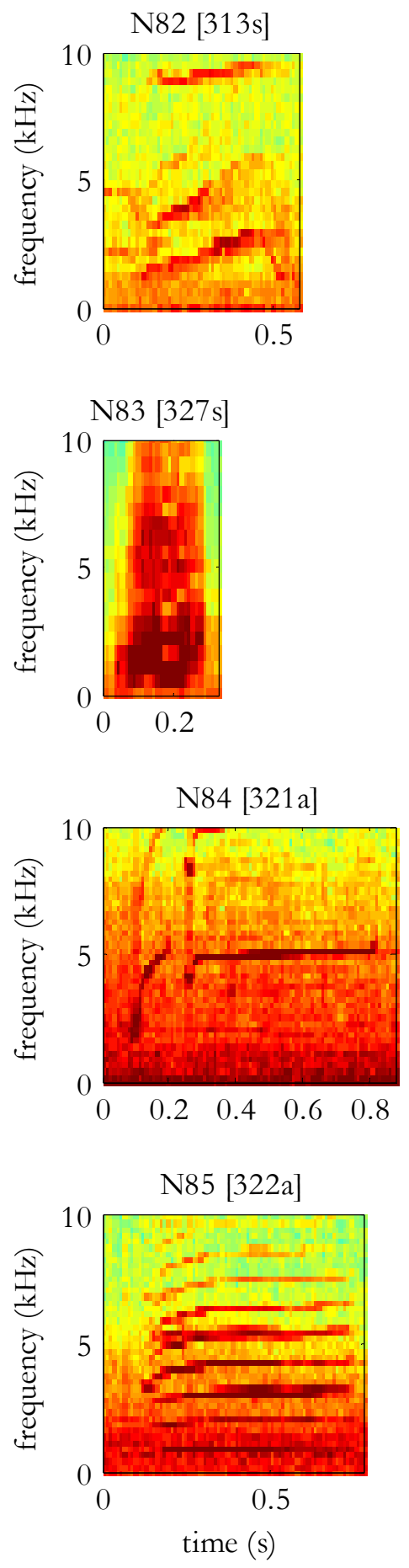

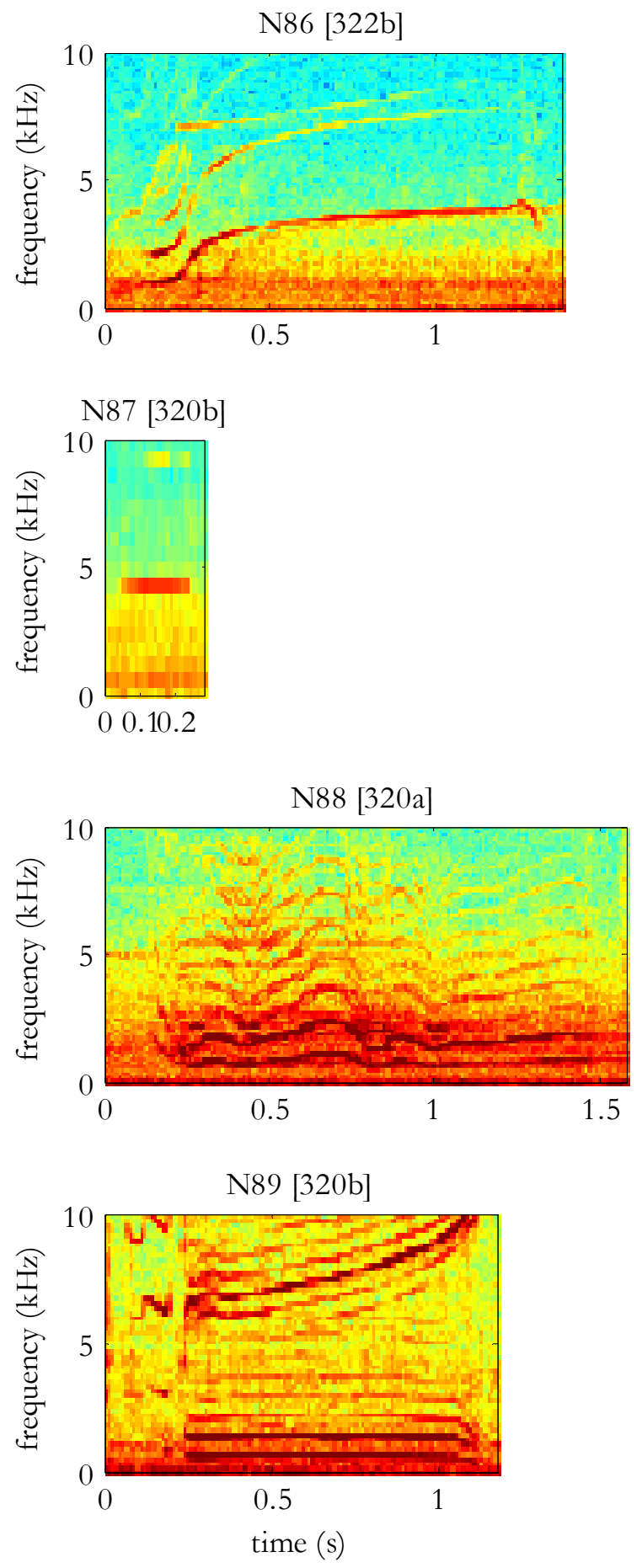

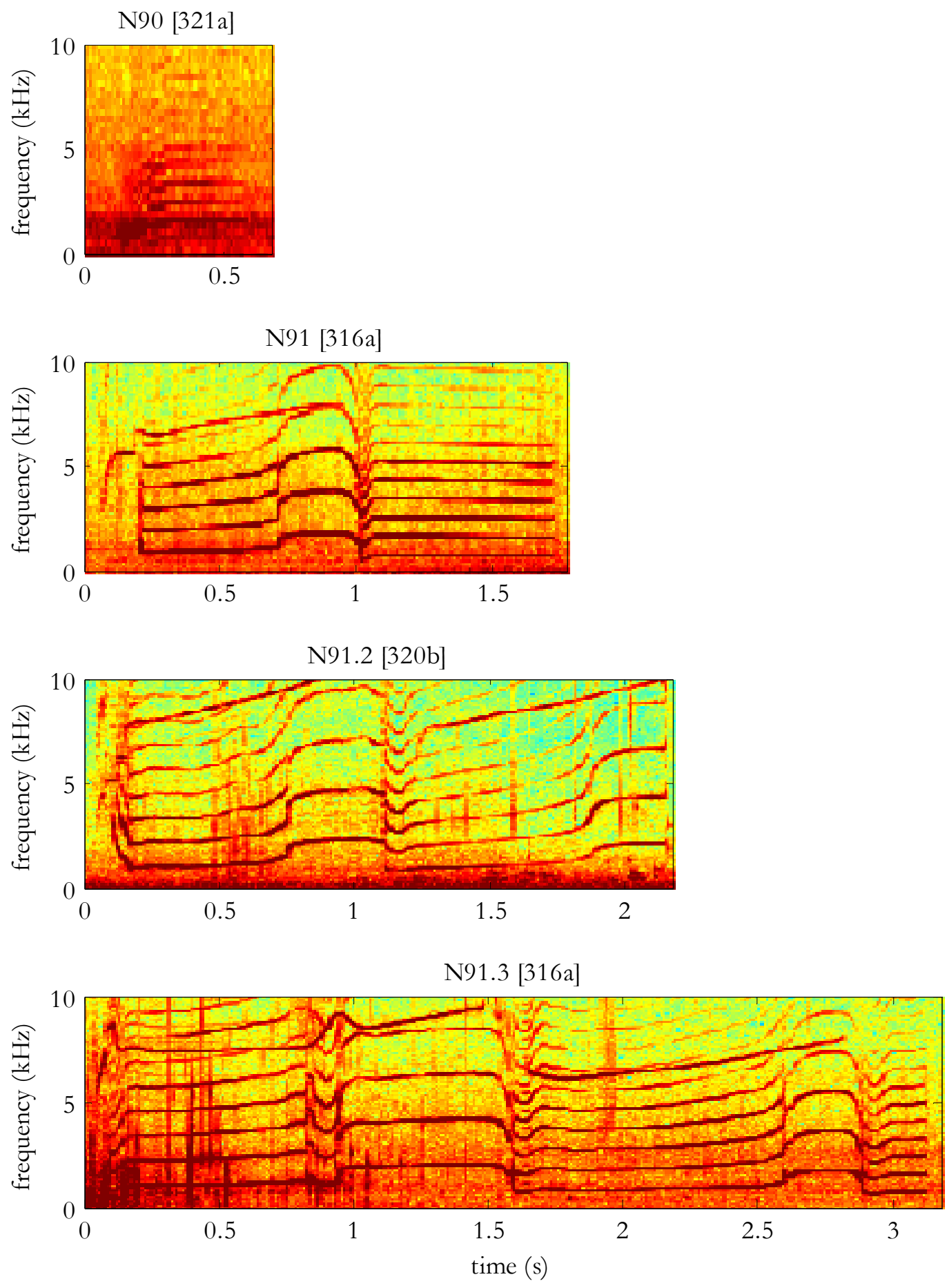

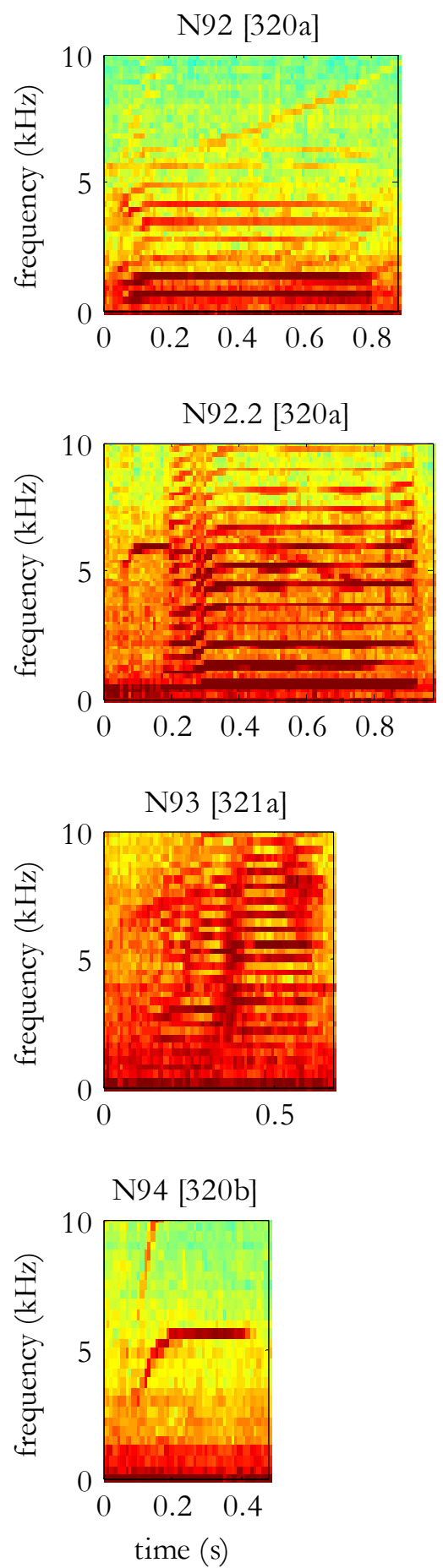

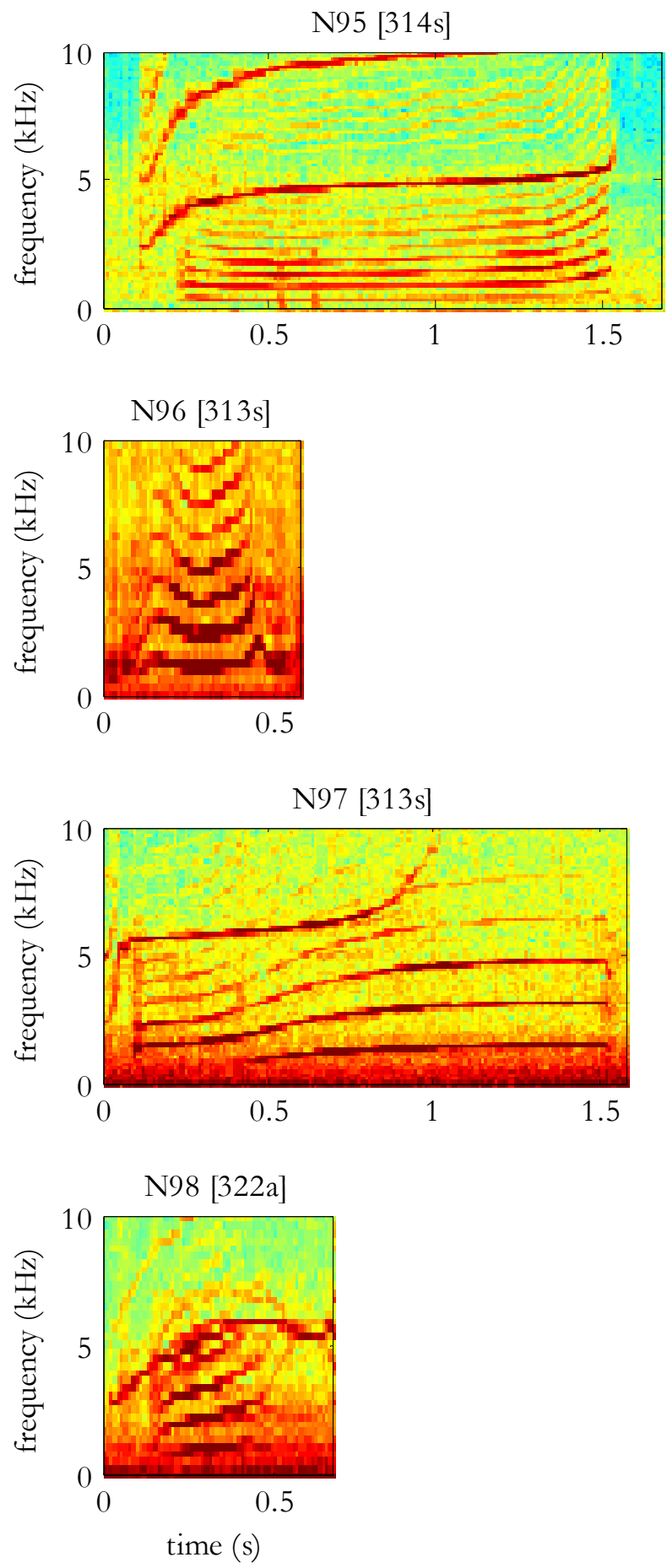

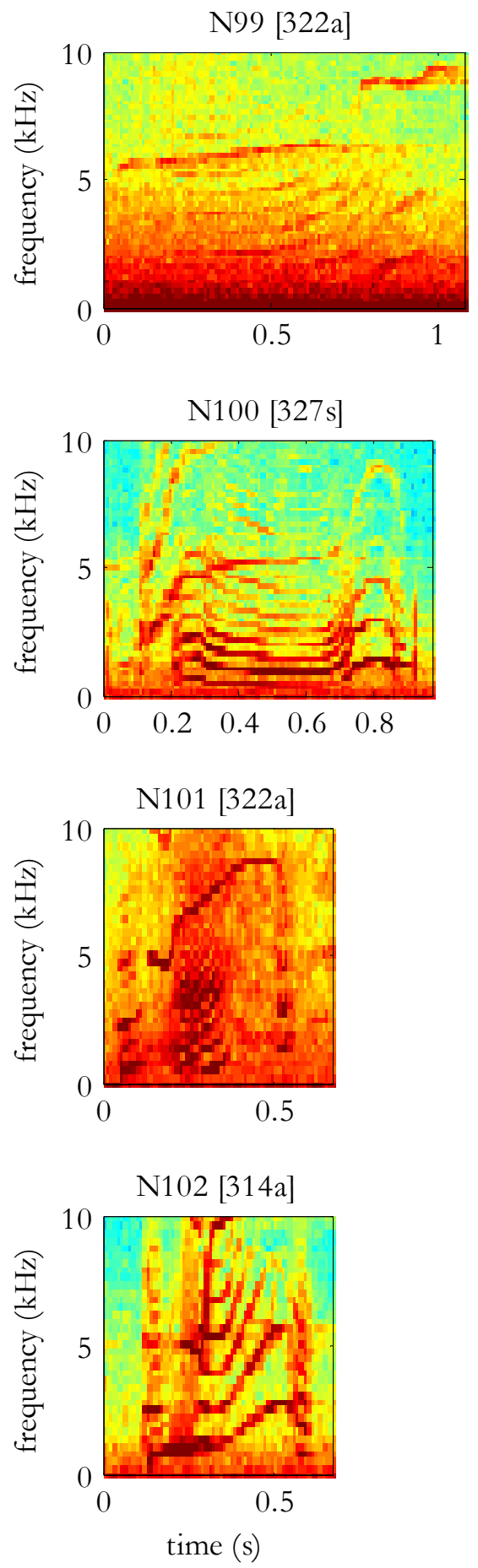


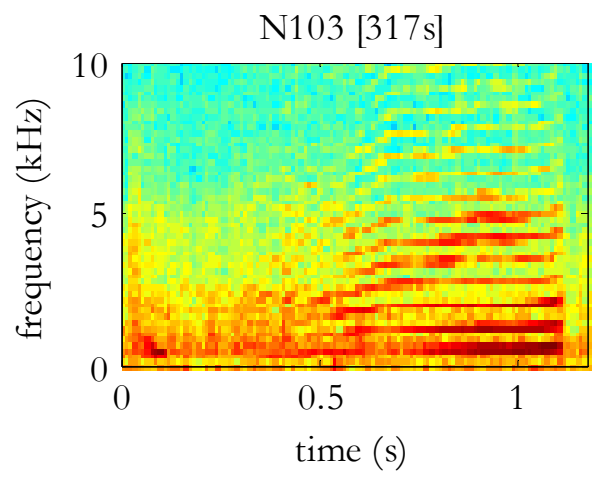




\section{APPENDIX 3. NORWEgIAN STEREOTYPED CALl CONTOURS}

All contour traces for each call type (see Chapter 5). LFCs are plotted in black and HFCs in gray.
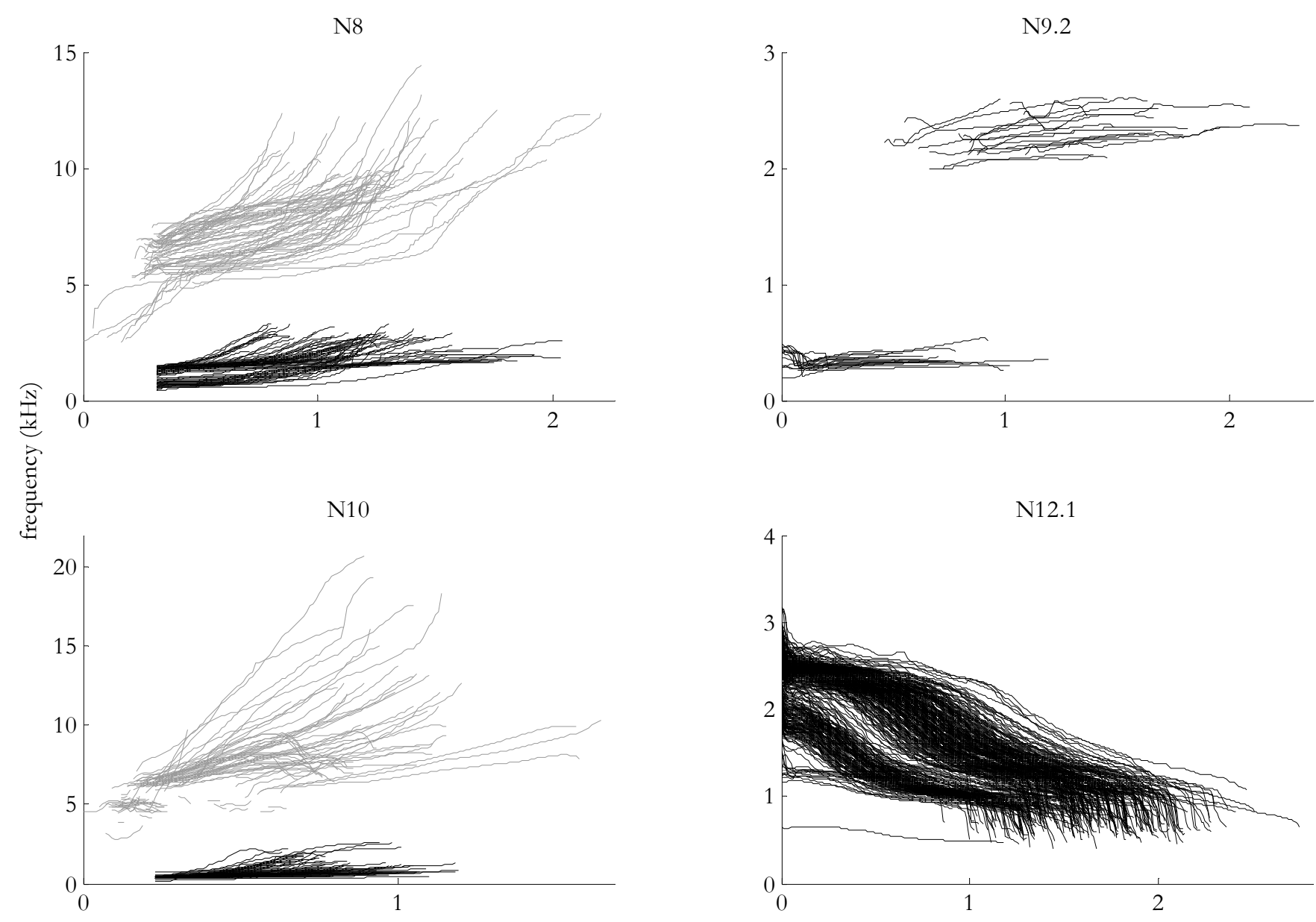

time (s) 
N16.1

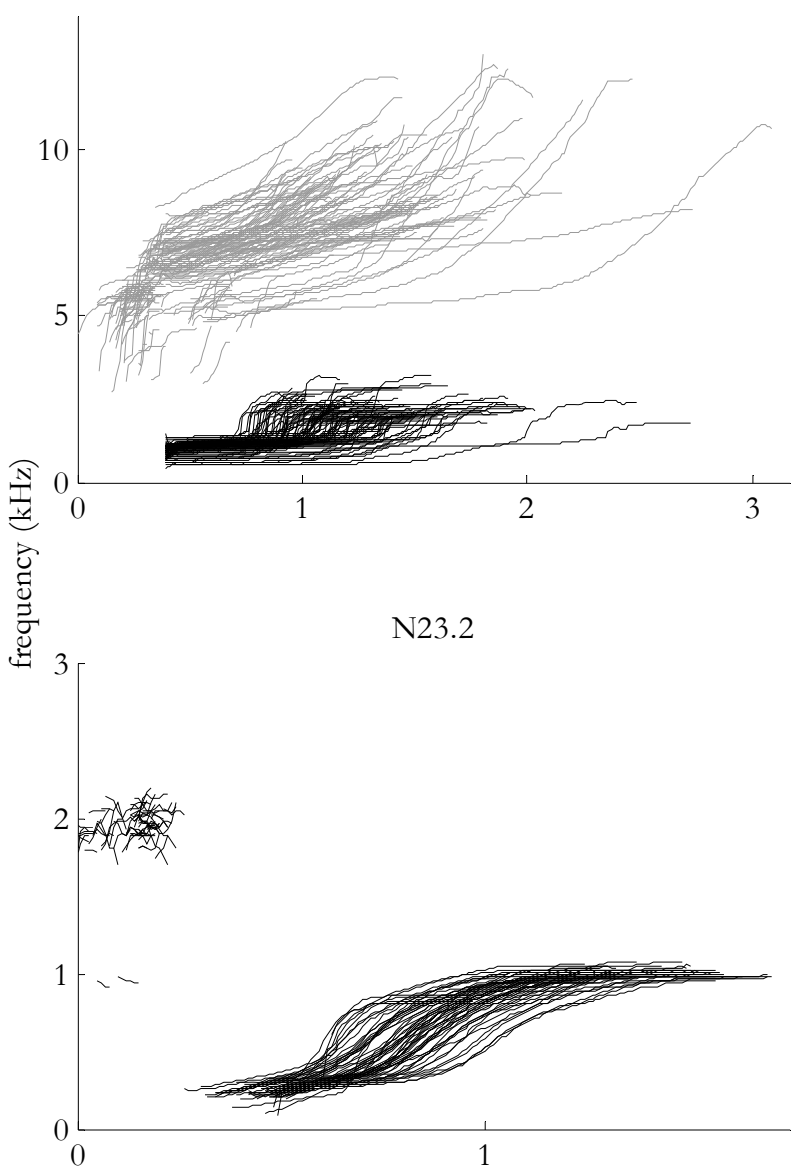

N21

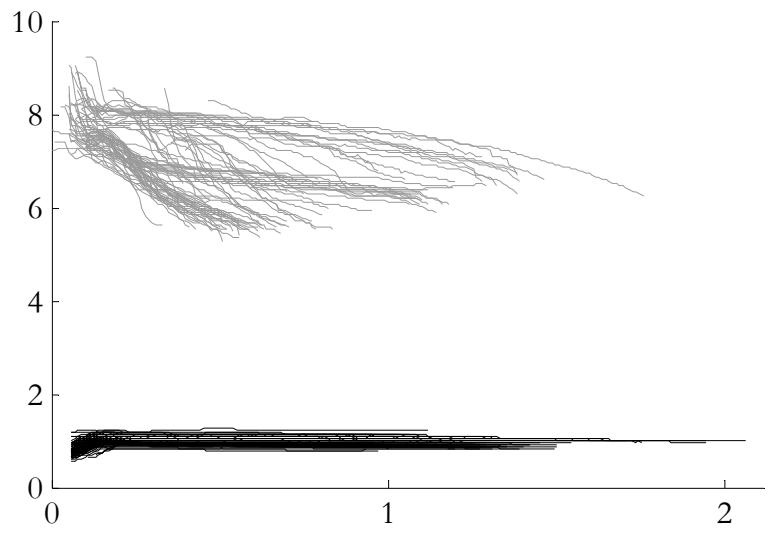

N23.3

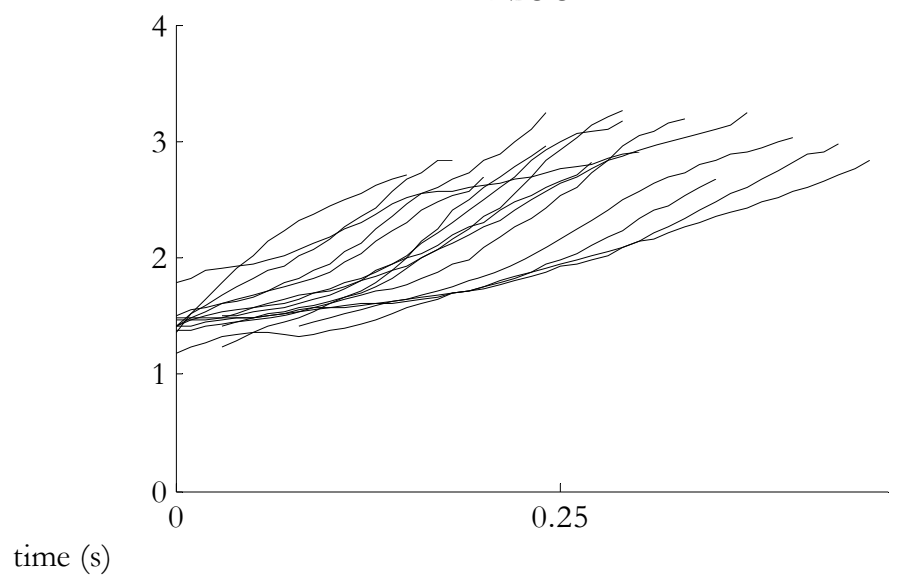


N26.2

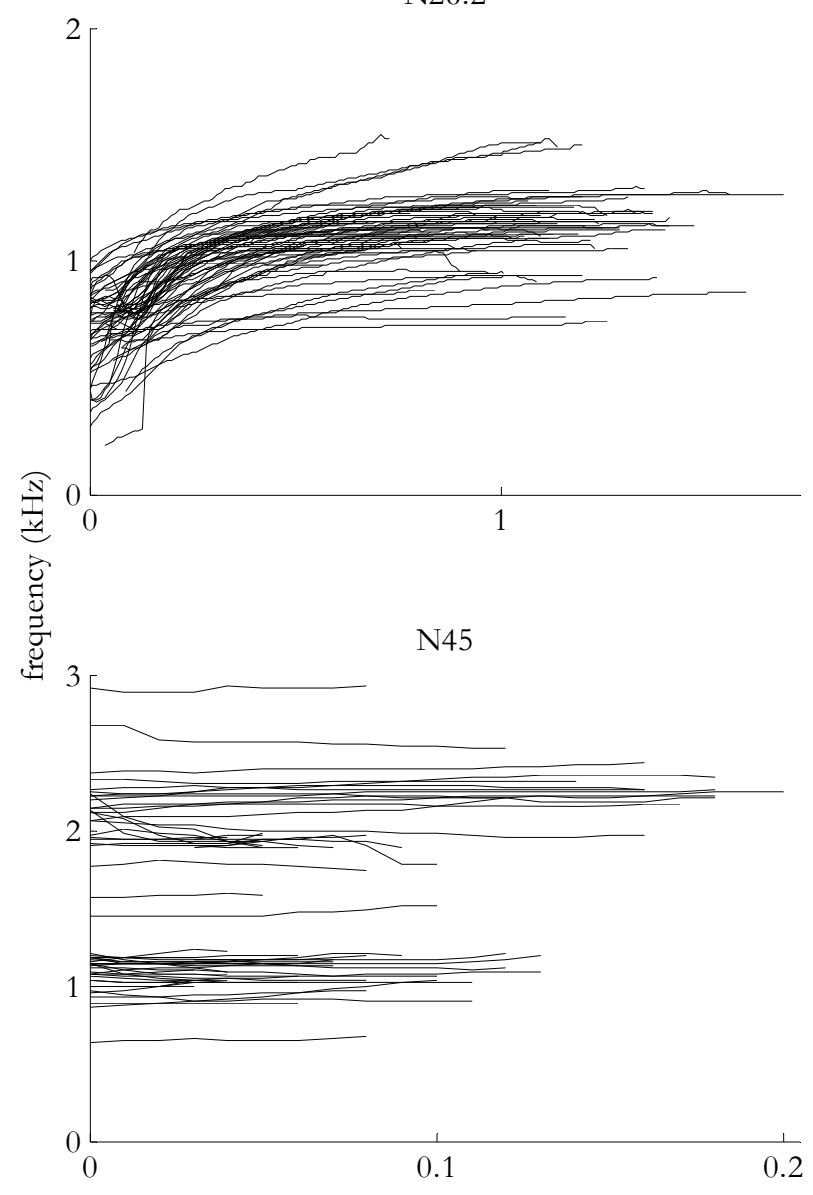

N32

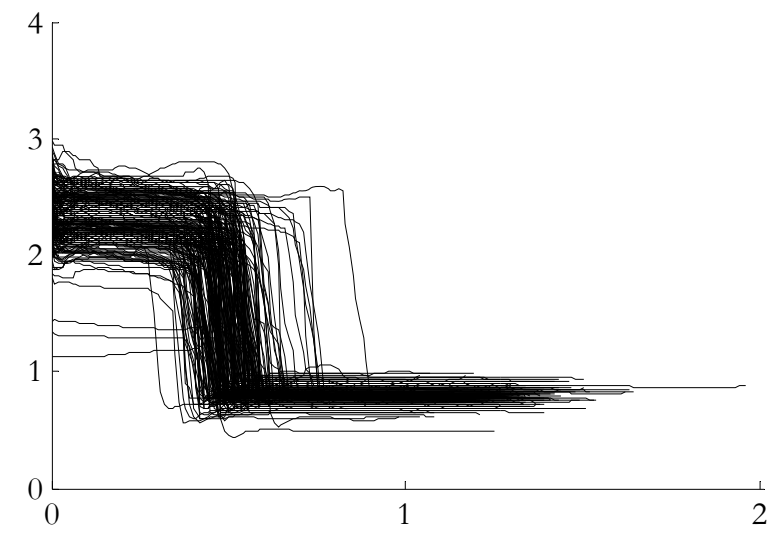

N64

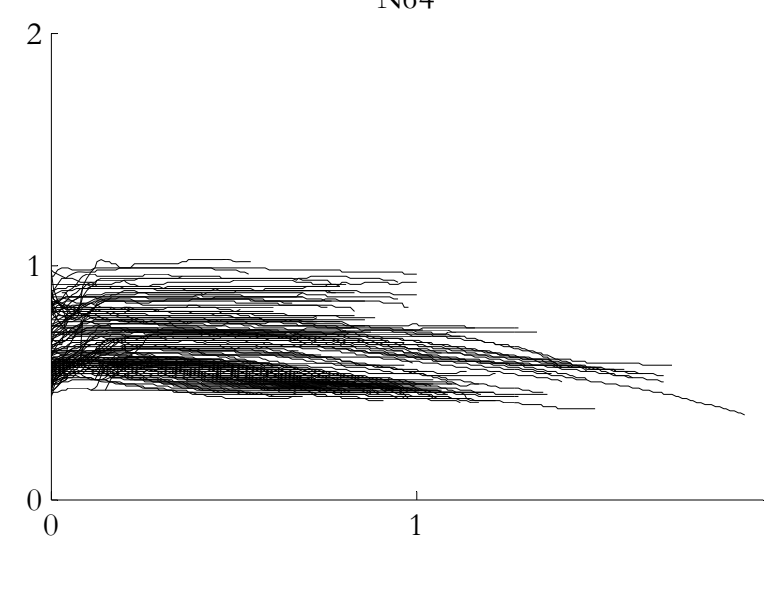



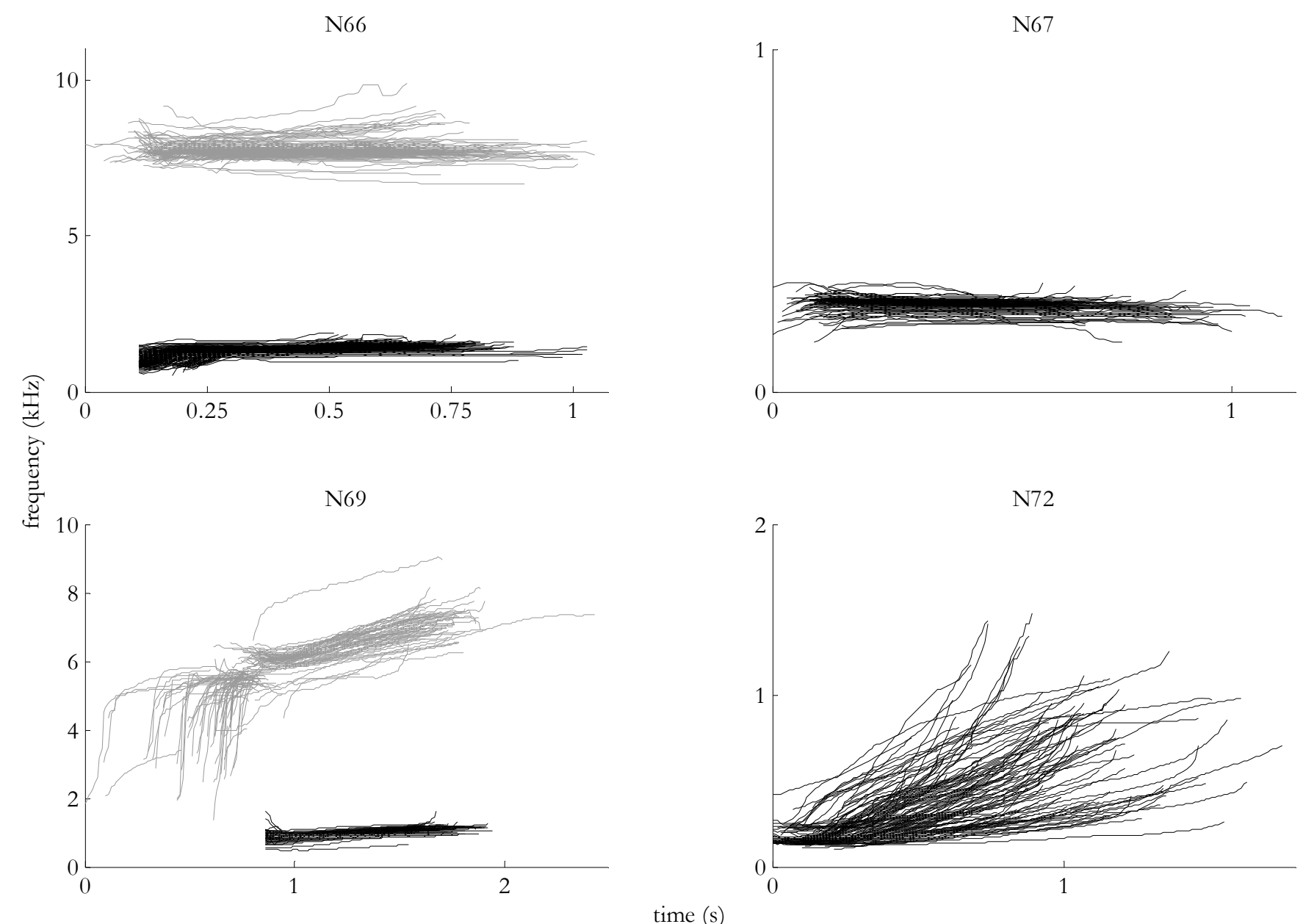
N72.2

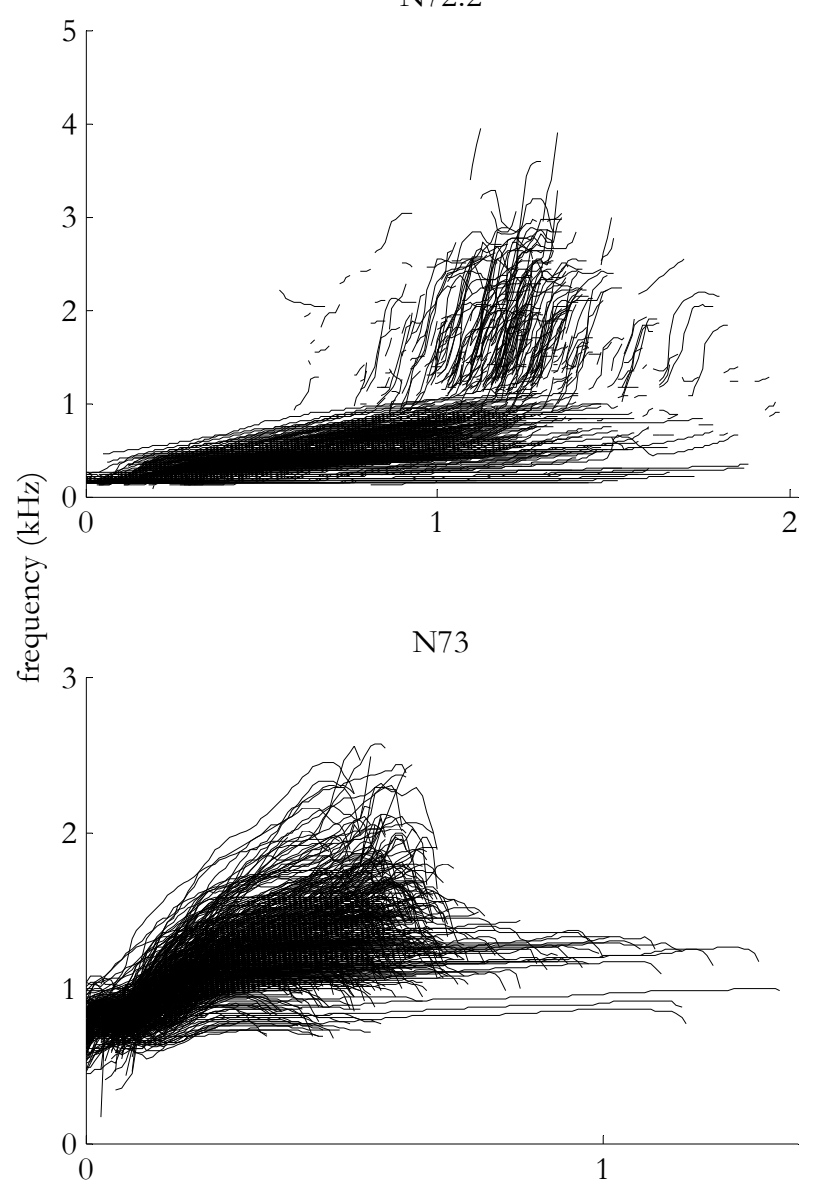

N72.3

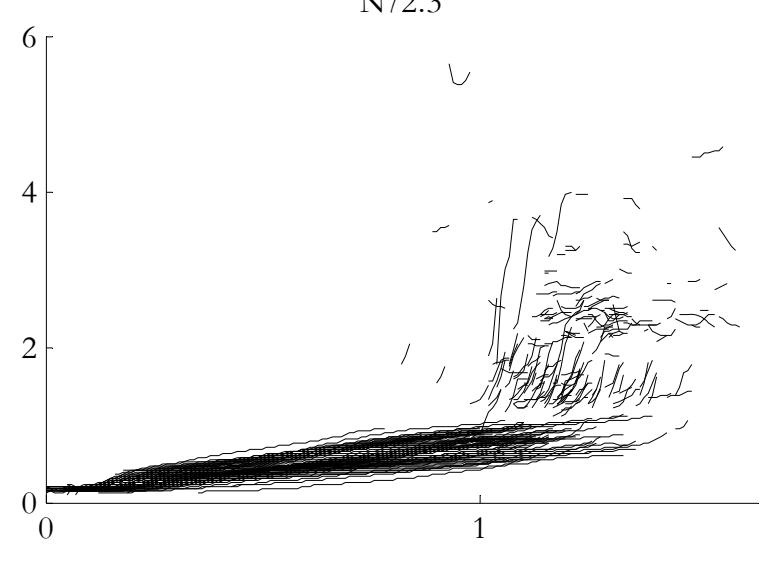

N76

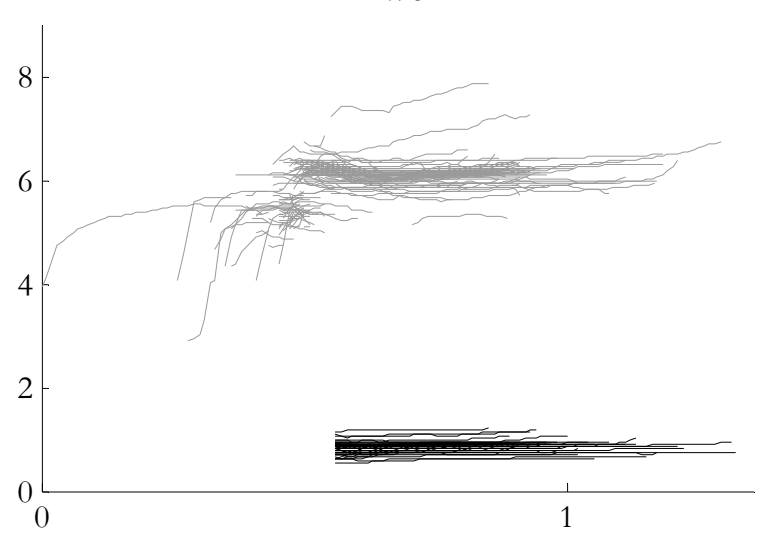

time (s) 
N76.2

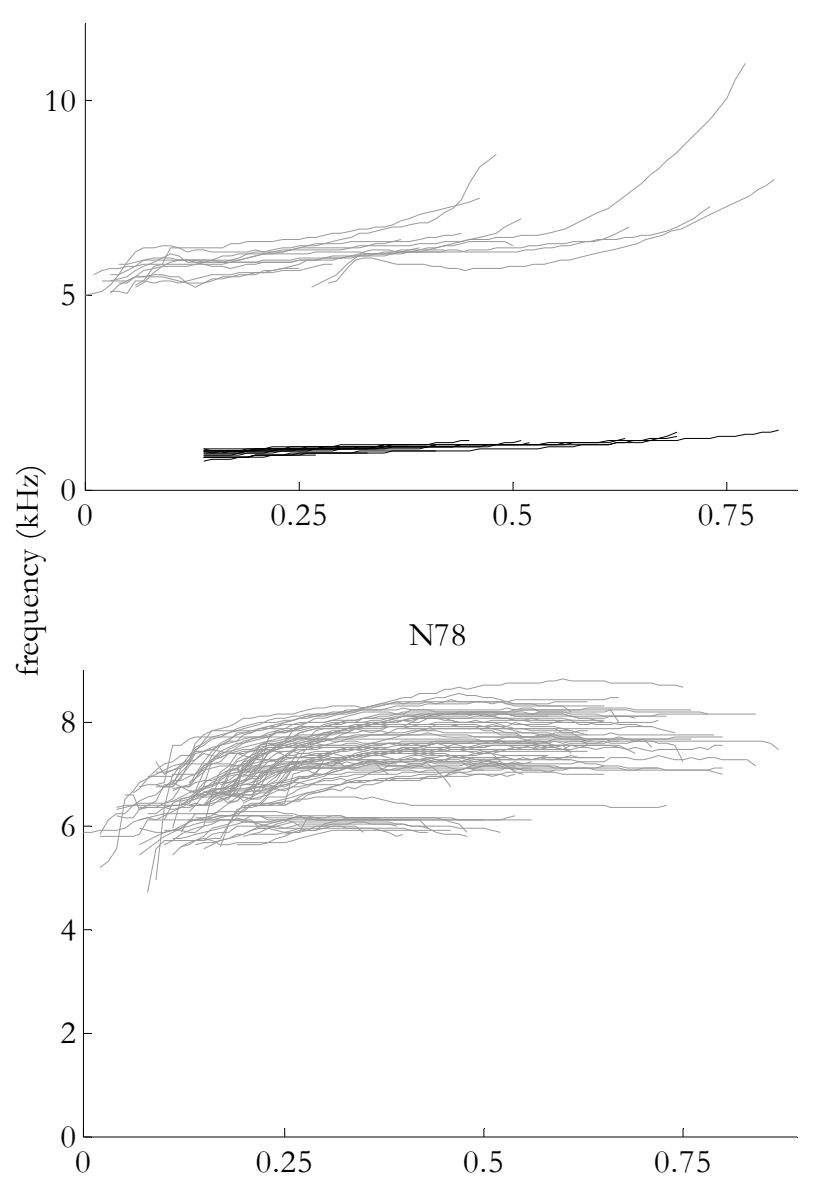

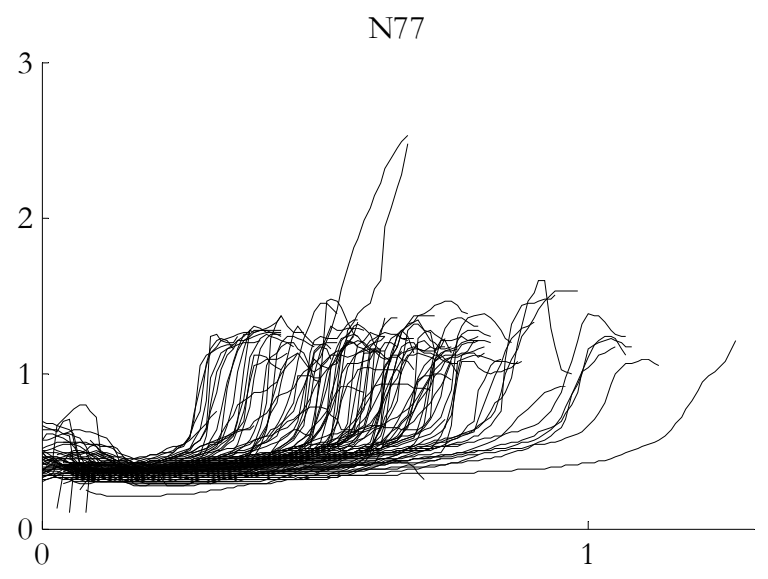

N79

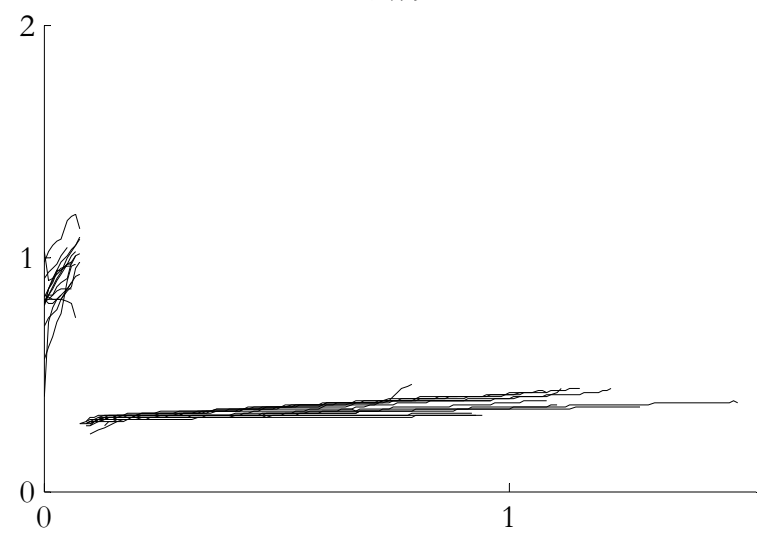

time (s) 

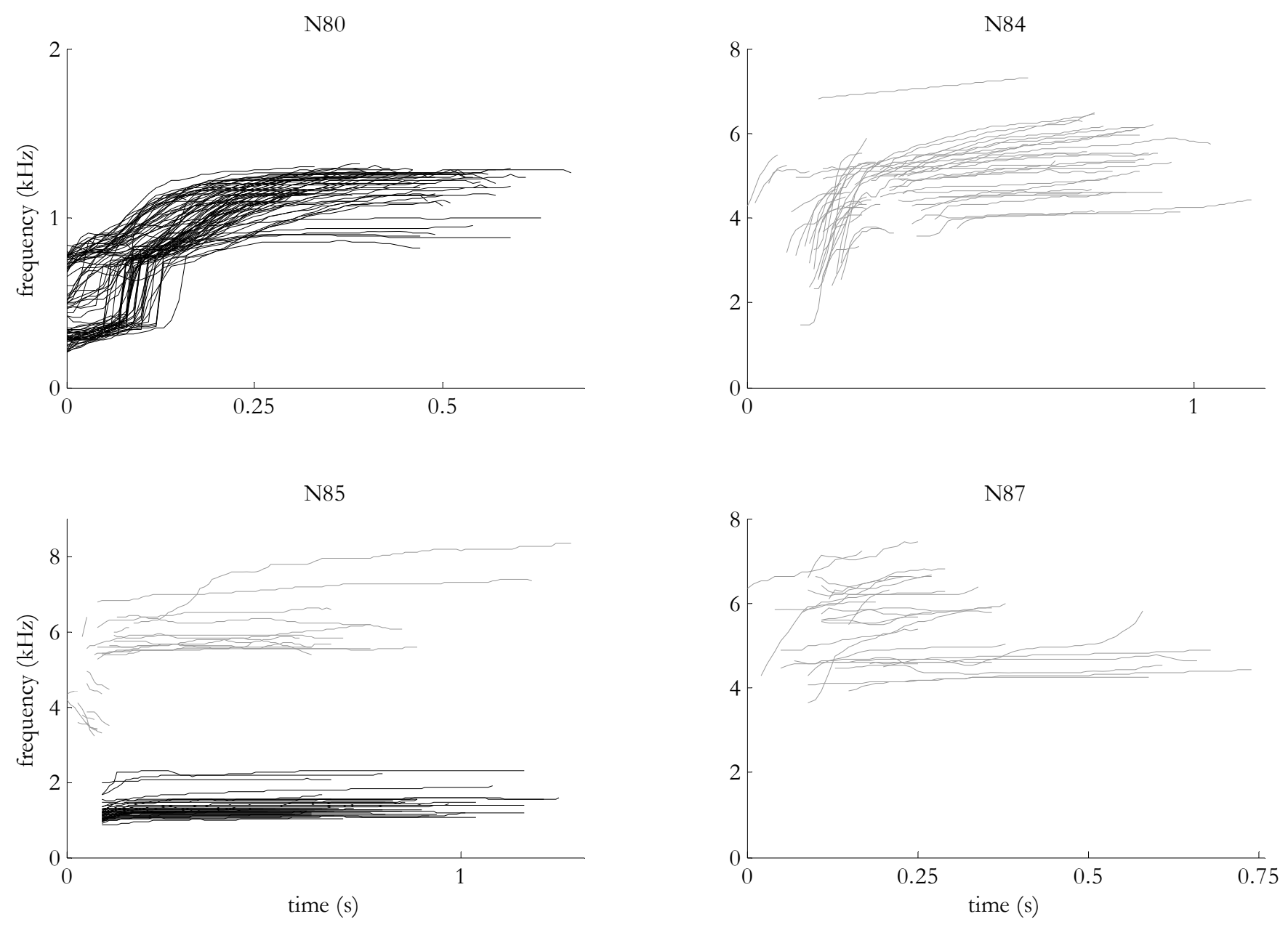
N89

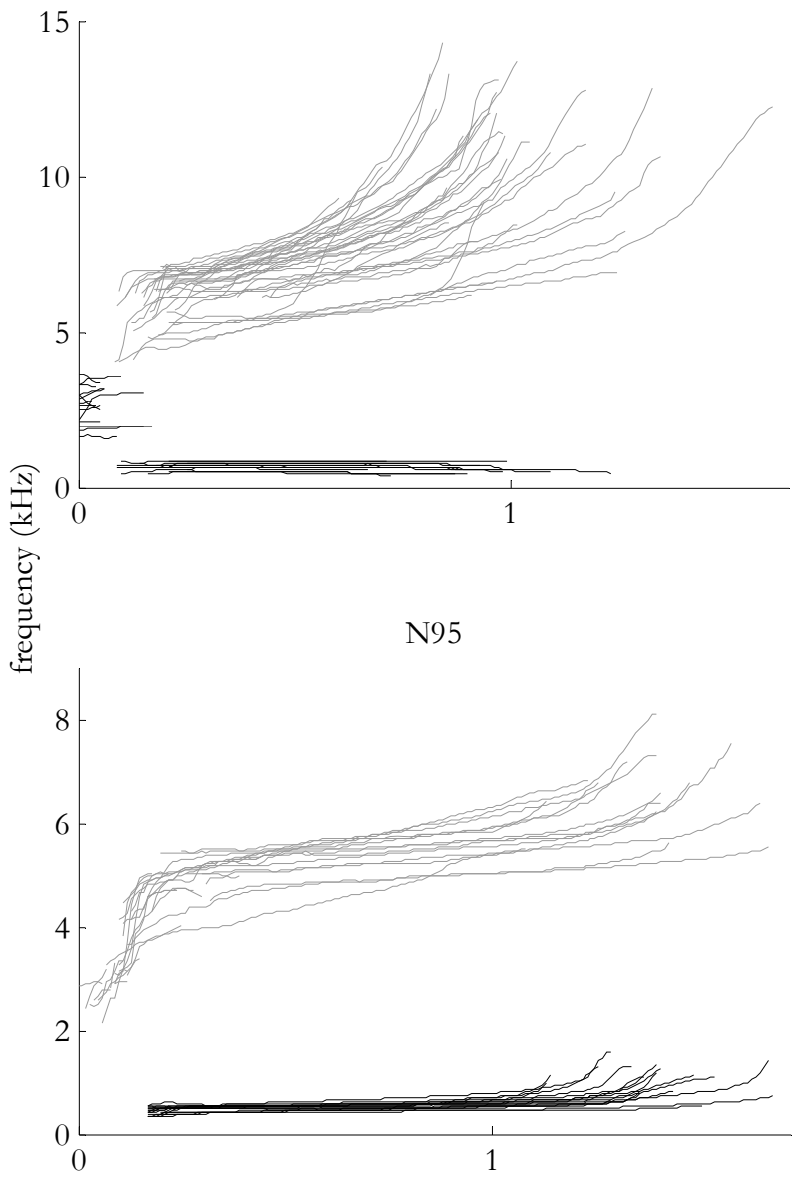

N91

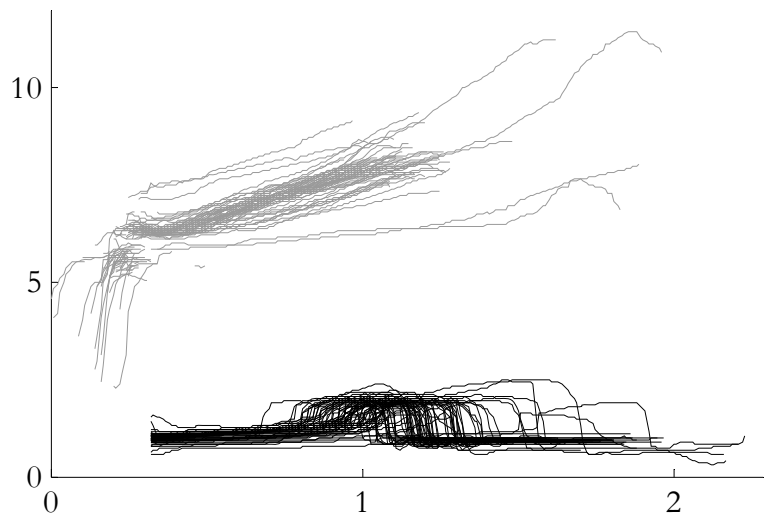

time (s) 


\title{
APPENDIX 4.
}

\section{Preliminary evidence for signature vocalizations among free-ranging narwhals (Monodon monoceros) ${ }^{\text {a) }}$}

\author{
Ari D. Shapiro ${ }^{\text {b) }}$ \\ Biology Department, Woods Hole Oceanographic Institution, MS \#50, Woods Hole, Massachusetts 02543
}

(Received 14 March 2006; revised 9 June 2006; accepted 21 June 2006)

Animal signature vocalizations that are distinctive at the individual or group level can facilitate recognition between conspecifics and re-establish contact with an animal that has become separated from its associates. In this study, the vocal behavior of two free-ranging adult male narwhals (Monodon monoceros) in Admiralty Inlet, Baffin Island was recorded using digital archival tags. These recording instruments were deployed when the animals were caught and held onshore to attach satellite tags, a protocol that separated them from their groups. The signature content of two vocal categories was considered: (1) combined tonal/pulsed signals, which contained synchronous pulsatile and tonal content; (2) whistles, or frequency modulated tonal signals with harmonic energy. Nonparametric comparisons of the temporal and spectral features of each vocal class revealed significant differences between the two individuals. A separate, cross-correlation measure conducted on the whistles that accounted for overall contour shape and absolute frequency content confirmed greater interindividual compared to intraindividual differences. These data are consistent with the hypothesis that narwhals produce signature vocalizations that may facilitate their reunion with group members once they become separated, but additional data are required to demonstrate this claim more rigorously. (1) 2006 Acoustical Society of America. [DOI: 10.1121/1.2226586]

PACS number(s): 43.80.Ka, 43.80.Ev [WAA]

Pages: $1695-1705$

\section{INTRODUCTION}

Signature vocalizations of animals acoustically encode individual or group identity and are characterized by unique sets of spectral and/or temporal attributes. The specific acoustic features required to distinguish between individuals according to their vocalizations have been measured in a host of taxa [e.g., birds: macaroni penguins (Eudyptes chrysolophus), Searby et al., 2004, chiropterans: evening bats ( $N y c-$ ticeius humeralis), Scherrer and Wilkinson, 1993, canids: timber wolves (Canis lupus), Goldman et al., 1995, primates: common marmosets (Callithrix jacchus), Jones et al., 1993, pinnipeds: subantarctic fur seals (Arctocephalus tropicalis), Charrier et al., 2001, 2003, cetaceans: bottlenose dolphins (Tursiops truncatus), Janik, 1999, Watwood et al., 2005]. Playback experiments have demonstrated that animals can recognize signature signals and have illustrated the diversity of contexts in which signature vocalizations are used, including facilitating recognition between an infant and one or both of its parents [cliff swallows (Hirundo pyrrhonota): Stoddard and Beecher, 1983, tree swallows (Tachycineta bicolor): Leonard et al., 1997, Mexican free-tailed bats (Tadarida brasiliensis mexicana): Balcombe, 1990, fur seals (Arctocephalus tropicalis): Charrier et al., 2001, 2003, bottlenose dolphins: Sayigh et al., 1998, Janik et al., 2006], mate-pair recognition [king penguins (Aptenodytes patagonicus): Lengagne et al., 2000], and group affiliation associated with territorial de-

\footnotetext{
"Portions of this work were presented in "Vocal behavior of free-ranging Arctic narwhals (Monodon monoceros)," Proceedings of the 16th Biennial Conference on the Biology of Marine Mammals, San Diego, CA, December 2005.

b) Author to whom correspondence should be addressed. Electronic mail: ashapiro@ whoi.edu
}

fense (North American bullfrog (Rana catesbeiana): Bee and Gerhardt, 2002, Arctic foxes (Alopex lagopus): Frommolt et al., 2003). There are selective benefits for the signals produced in these contexts. Recognition is very important when one or both parents must allocate a finite amount of resources to their offspring, a scenario in which confusion is associated with high fitness costs. Mates or groups of individuals that consistently defend one another, their young, or their territory can benefit from individual or group recognition because it provides a system for remembering with whom they have shared mutual investments.

The proximate methods for achieving signature recognition can include imprinting, habituation, associative learning, and vocal learning. Vocal learning occurs when the respiratory, phonatory, and/or filter systems are employed to render signals more or less similar to acoustic models that are encountered through experience with other individuals (Janik and Slater, 1997, 2000). Contact calls, generally used by animals when they become separated from their social partner(s) or group to first locate one another and then mediate reunion, contain signature content in certain species and appear to be vocally learned. When placed in social groupings of unfamiliar individuals, the contact calls of male budgerigars (Melopsittacus undulatus), for example, initially converged and subsequently underwent continuous and synchronous changes (Farabaugh et al., 1994). Evidence is accumulating for some species of nonhuman primates to possess vocal plasticity during adulthood despite its apparent absence during development (see review by Egnor and Hauser, 2004). Male chimpanzees (Pan troglodytes) produce pant hoots, long-distance vocalizations that seem to function in maintaining contact with and attracting allied individuals

* Reprinted with permission from Shapiro, A.D. Journal of the Acoustical Society of America, Vol. 120, Issue 3, Pages 1695-1705, 2006. (C) 2006, Acoustical Society of America. 
(Mitani and Nishida, 1993). Pant hoot convergence was observed among both chorusing dyadic pairs (Mitani and GrosLouis, 1998) and larger groups containing 3-11 adult males (Marshall et al., 1999). Similarly, several spectral and temporal parameters of the contact calls among pygmy marmosets (Cebuella pygmaea), referred to as trills, underwent parallel or convergent shifts between new adult mate pairs (Snowdon et al., 1997; Snowdon and Elowson, 1999). Comparable observations were made when two naïve pygmy marmoset social groups of mixed-age composition were introduced (Elowson and Snowdon, 1994).

In principle, it is possible to conclude that signals contain signature content by demonstrating more interindividual than intraindividual variability. To offer sufficient proof, this result must be shown for at least several exemplars of the signature signals of each of several individuals. One of the most striking examples of signature vocalizations is found among bottlenose dolphins whose signature whistles, first identified by Caldwell and Caldwell (1965), appear to function as vocally-learned contact calls. Among bottlenose dolphins, whistle imitation appears to be an important agent in the ontogeny of this signature vocalization (Sayigh et al., 1990, Miksis et al., 2002) and in social communication both in captivity (Tyack, 1986) and in the wild (Janik, 2000, Fripp et al., 2005). Bottlenose dolphin mother-calf pairs were more likely to orient towards one another's signature whistles when separated from each other and temporarily restrained than those of other individuals of the same corresponding age cohort (Sayigh et al., 1998, Janik et al., 2006). Captive animals produced their signature whistles most often when they separated themselves voluntarily and spontaneously from their mixed-age group by swimming into an adjacent tank compared to when they were swimming together (Janik and Slater, 1998). The remaining dolphins were also more likely to produce their signature whistles when an individual left the main tank. Finally, adult males that had strong social bonds with another male were most likely to use signature whistles when they were separated either due to temporary, artificial restraint or voluntarily when they were free-ranging, presumably to facilitate an eventual reunion (Watwood et al., 2005). Collectively, these studies reveal the importance of signature whistles in maintaining contact between bottlenose dolphin individuals in artificial and natural settings alike and in both involuntary and voluntary contexts.

Despite some debate (see McCowan and Reiss, 1995, 2001; Janik, 1999 for discussion; Smolker and Pepper, 1999), the studies just described have helped to solidify the case for signature whistles in bottlenose dolphins and suggest the possibility of signature vocalizations among other long-lived, social odontocetes in an underwater environment where acoustic signaling is the most reliable and efficient form of communication. In addition, signature information can also be encoded at the group level, which can form the basis for acoustic badges of membership (e.g., Boughman, 1997). For example, five of six shared call types among killer whales (Orcinus orca) revealed distinctive structural differences between matrilineal units (Miller and Bain, 2000) and it is possible that other signature details allow differentiation between individual animals as well (Nousek et al., in press). Cultural divergence of discrete call types appears to account for some of the subtle differences in the temporal and spectral features across these matrilineal units and even within pods (Deecke et al., 2000).

The principal challenge for studying the signature signals of marine mammals involves the difficulty of assigning vocalizations in the wild unambiguously to the individual animal that produced them. In this manuscript, the possibility of signature signals among free-ranging narwhals (Monodon monoceros) was examined by recording the acoustic activity of two individuals with digital archival tags. These gregarious, long-lived Arctic odontocetes migrate distances of thousands of kilometers in large numbers with subpopulations moving in a coordinated fashion (Hay and Mansfield, 1989; Dietz and Heide-Jørgensen, 1995; Laidre et al., 2004). They travel in groups that are often sex segregated and range in size from a few animals to dozens of individuals, although the stability or fluidity and interconnectedness of these assemblages remain unknown (reviewed in Hay and Mansfield, 1989).

Narwhals produce echolocation clicks with repetition rates between 2 and $>500 / \mathrm{s}$ (Ford and Fisher, 1978, Møhl et al., 1990), maximum frequencies reaching at least $160 \mathrm{kHz}$ (Miller et al., 1995) and maximal source levels reaching $218 \mathrm{~dB}$ re $1 \mu \mathrm{Pa}$ (Møhl et al., 1990). Miller et al.(1995) arbitrarily divided clicking into the two categories of train clicks produced at $\leqslant 30$ clicks/s and burst clicks produced at $\geqslant 40$ clicks/s. Pulsatile sounds featuring a repetition rate high enough to possess a tonal character with harmonically related sidebands (see Watkins, 1967) were called longer click series by Watkins et al. (1971) and pulsed tones by Ford and Fisher (1978). Characterized as narrow-band, these signals had durations between 0.56 and several seconds and spectral energy ranging from $500 \mathrm{~Hz}$ to $24 \mathrm{kHz}$. The repetition rate was generally constant although Watkins et al. (1971) reported a tendency for the repetition rate of these vocalizations to increase at the very beginning and slow down towards the end. In this manuscript, these signals will be referred to as combined tonal/pulsed signals. Finally, narrow-band, frequency modulated (FM) whistles have been described that generally last $<1.0 \mathrm{~s}$ (range: $0.1-6.0 \mathrm{~s}$ ) and have a frequency range between $300 \mathrm{~Hz}$ and $18 \mathrm{kHz}$ (Ford and Fisher, 1978; Møhl et al., 1990).

Although Ford and Fisher (1978) did not find any evidence for signature content among whistles, they speculated that the different pulsed tones in their recordings were produced by separate individuals as signature calls in a social context. They recorded series of the same tone growing louder and then softer, concluding that this resulted from one individual producing each series as it approached and then swam past a stationary hydrophone. This possibility was not conclusive since groups of animals were swimming by the recorder and multiple individuals could have been producing each tone. In addition, no data on differences in acoustic parameters were available to quantify the distinctiveness of the calls. In this study, we examined the possibility of signature vocalizations among free-ranging narwhals more closely. The results support this hypothesis for both combined tonal/pulsed signals and whistles, suggesting a social 


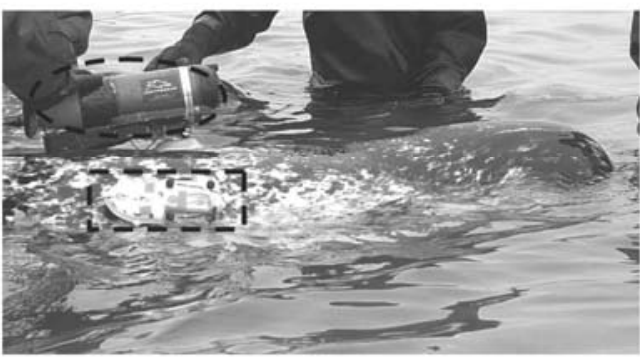

FIG. 1. Narwhal shown with Crittercam (contained within the dashed ellipse) and DTAG (contained within the dashed rectangle) attached immediately before release. Photograph courtesy of Rune Dietz.

function for vocal production that is distinctive either at the individual or group level. Further work is recommended to confirm signature vocal production among additional animals and to ascertain the natural function of these vocalizations in the wild.

\section{METHODS}

\section{A. Study area}

Field work was conducted from 8-23 August 2004 at Kakiak Point, Admiralty Inlet on Baffin Island in Nunavut, Canada $\left(73^{\circ} 40^{\prime} \mathrm{N}, 86^{\circ} 40^{\prime} \mathrm{W}\right)$. The inlet has a maximum depth of $720 \mathrm{~m}$. Groups of narwhals ranging from approximately 5-30 individuals (pers. obs.) traveled into the inlet at this time of year once the ice had mostly melted. The field camp occupied a position about $500 \mathrm{~m}$ from a site used intermittently by the Inuit to hunt narwhals.

\section{B. Equipment}

This experiment employed a digital archival tag (DTAG) developed by Johnson and Tyack (2003) featuring a single hydrophone, pressure and temperature sensors, and a triaxial accelerometer and magnetometer, which recorded to flash memory. The sampling rate of the hydrophone was set to $96 \mathrm{kHz}$ while the other sensors sampled at $50 \mathrm{~Hz}$. A 16 bit $\mathrm{ADC}$ was used. Sigma delta conversion provided an effective antialiasing filter, dispensing with aliasing caused by energy exceeding the Nyquist frequency of $48 \mathrm{kHz}$. The tag attached noninvasively to individual animals via suction cups and its release was coupled to the release mechanism of the National Geographic Crittercam (see Marshall, 1998) that was deployed simultaneously. A VHF transmitter signaled the location of an attached tag intermittently as the animal surfaced and then regularly once the tag was released and floated to the water's surface.

\section{Capturing and tagging protocol}

The DTAG was deployed in collaboration with a satellite tagging project that required working with the animals onshore. As described by Dietz et al. (2001), a $50 \mathrm{~m}$ long and $10 \mathrm{~m}$ deep black net with $20 \times 20 \mathrm{~cm}$ mesh was oriented perpendicular to the shore and kept afloat with 7-8 white buoys. The net was secured to the shore and in the water.

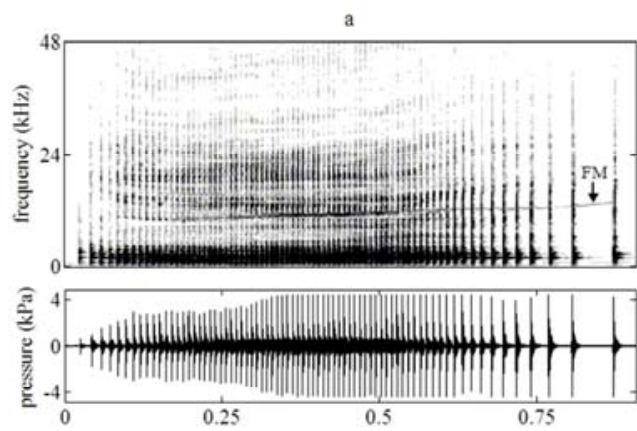

b
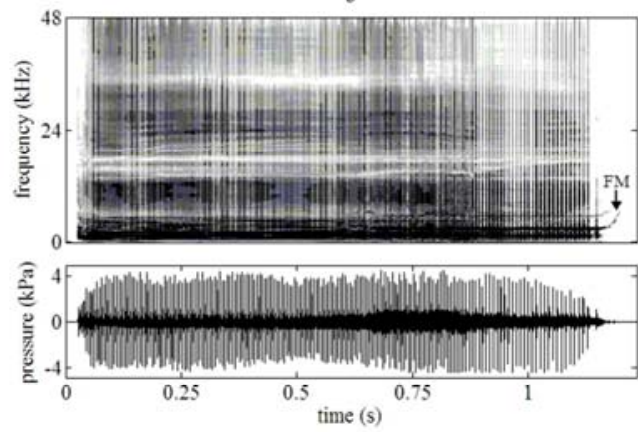

FIG, 2. Sample spectrograms (larger, top plots) and waveforms (smaller, bottom plots) of a combined tonal/pulsed signal produced by individual (a) $\mathrm{mm} 224$ and (b) $\mathrm{mm} 226$ with a FFT size and frame length of 512 points, $50 \%$ window overlap, and a maximum frequency displayed of $48 \mathrm{kHz}$. The low frequency energy associated with most of the pulses is likely due to the resonance of the air sacs involved in sound production or transmission. The solid arrows in both spectrograms indicate the synchronous FM tonal component produced by the tagged animal.

When weather conditions permitted, the net was deployed and monitored constantly for caught animals, signaled by the submergence of at least one of the buoys. As soon as a whale became entangled, two boats were dispatched immediately to bring the animal to the surface to breathe and tow it to shore with the assistance of a land-based crew hauling on the net. Once an animal was caught, the remainder of its group moved out of visual range, presumably continuing their migration deeper into the inlet. A fluke belt was used to keep the animal ashore and oriented with its head submerged and pointed into the water while its blowhole had access to the air at all times. Three males and five females were captured in all. During satellite tag attachment, blood samples were collected to assess overall health and stress levels.

Just before two of the adult males and one of the adult females were guided back to deeper water, a DTAG was attached to their dorsal sides $\sim 0.5 \mathrm{~m}$ caudal to the blowhole (Fig. 1). These animals were not followed visually once they were released from shore so it was not possible to determine whether they eventually reunited with their group members. The VHF signal was monitored from the field camp on shore using two handheld yagi antennae. Once a regular VHF sig- 

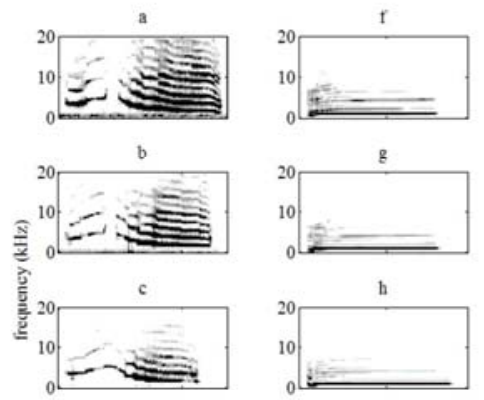

d
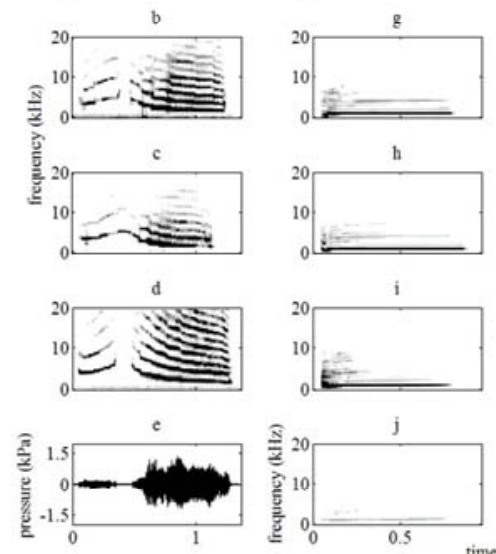

b
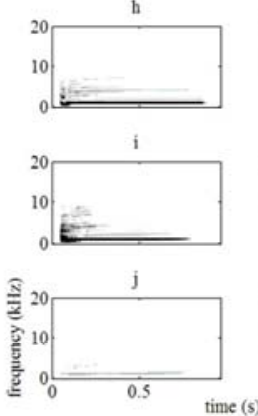
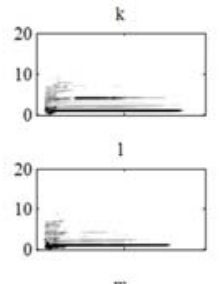

$\mathrm{m}$
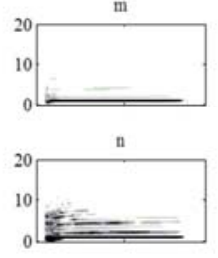

$\circ$

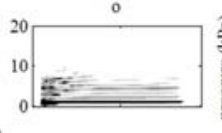

nal was detected from a tag that had released from the animal and the weather permitted, a boat was dispatched for recovery. If the VHF signal grew too faint to detect from shore, tracking was conducted from a higher altitude on the nearby cliffs for improved range. The first tag recorded for $2.54 \mathrm{~h}$ (male mm224), the second tag for $12.14 \mathrm{~h}$ (male mm226), and the third tag was not recovered. These two tagged males entered the inlet two days apart, strongly suggesting that they belonged to different social groups. The data were offloaded and burned to $\mathrm{CD}$ in duplicate in the field.

\section{Vocalization extraction}

The $14.68 \mathrm{~h}$ of recordings were audited by listening to and visually examining the spectrograms in $15 \mathrm{~s}$ segments. Focal (tagged animal) vocalizations were marked according to their starting time and vocal category. It was assumed that vocalizations with a relatively high signal to noise ratio (SNR) belonged to the focal animal and not a neighboring nonfocal animal. Although this assumption could not be verified visually because the tagged narwhals were not followed, it was true for at least the first few dives since no group members were observed in the immediate vicinity. Much softer sounds were often heard on the recordings, presumably from more distant, vocalizing nonfocal animals. The SNR of these focal vocalizations was computed by comparing the root of the mean of the squared pressure (RMS) along the window containing $90 \%$ of the signal energy to a segment of noise of the same duration immediately preceding the signal (Madsen, 2005). The analysis presented here only excluded echolocation clicks, or broadband pulses of energy with interclick intervals usually greater than $100 \mathrm{~ms}$. Nearly all remaining vocalizations were considered that could be divided into the two discrete categories of (1) combined tonal/pulsed signals (Fig. 2), defined as uninterrupted pulsatile vocalizations with a synchronously produced FM tonal

J. Acoust. Soc. Am., Vol. 120, No. 3, September 2006

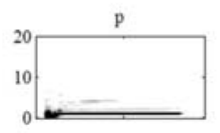

q
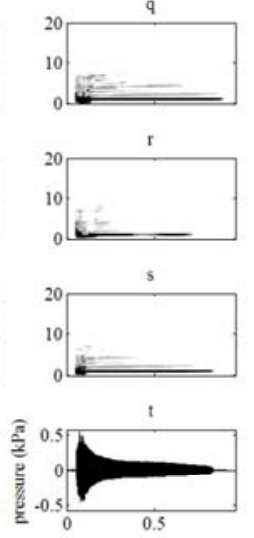

FIG. 3. Spectrogram composite of all four whistles of $\mathrm{mm} 224$ (a-d) and 14 of the 17 whistles of $\mathrm{mm} 226$ (f-s) with a FFT size and frame length of 512 points, $50 \%$ window overlap, and a maximum frequency displayed of $20 \mathrm{kHz}$. The remaining 3 whistles of mm226 resembled those displayed here but were excluded for grapla here but were excluded for graphical convenience. The waveforms displayed in subplots $\mathrm{e}$ and $\mathrm{t}$ are of the same whistles used to generate subplots $\mathrm{d}$ and $\mathrm{s}$, respectively. component, a low mean interpulse interval (IPI $<13 \mathrm{~ms}$ ), and high pulse number $(>49)$, and (2) whistles (Fig. 3), which were characterized by FM, tonal energy with several harmonics. See the discussion in this manuscript for a comparison of these designations to earlier classification schemata. All vocalizations were saved as separate wav files. Individual $\mathrm{mm} 224$ produced 42 combined tonal/pulsed signals and 4 whistles and mm226 produced 31 combined tonal/ pulsed signals and 17 whistles.

\section{E. Analysis of combined tonal/pulsed signals}

Pulses were located automatically using customized Matlab 7.1 (The MathWorks, Inc.) software that, given a user-provided threshold value, triggered on and marked abrupt peaks in the pressure waveform. Subsequent inspections of all waveforms were made to select undetected and remove erroneously marked pulses. A nonparametric Wilcoxon rank sum test with a Bonferroni correction was used to examine whether the four parameters of average IPI, duration, number of pulses, and pulse repetition rate were significantly distinguishable between the two individuals. The pulse repetition rate and the normalized pulse number were also plotted as functions of the normalized duration to provide a visual means of comparing these sounds.

\section{F. Whistle extraction and analysis}

The fundamental frequency contour of each whistle spectrogram (FFT size and frame length of 2048 points with $50 \%$ window overlap) was traced by hand with customized Matlab software (Fig. 4). One hundred equally spaced points were extracted from these contours and normalized to a time axis between 0 and 1 (see Watwood et al., 2004, 2005). Two tests of similarity were conducted on these whistle contours:

Ari Shapiro: Preliminary evidence for narwhal signature vocalizations 


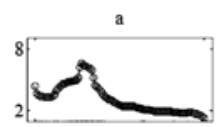

b
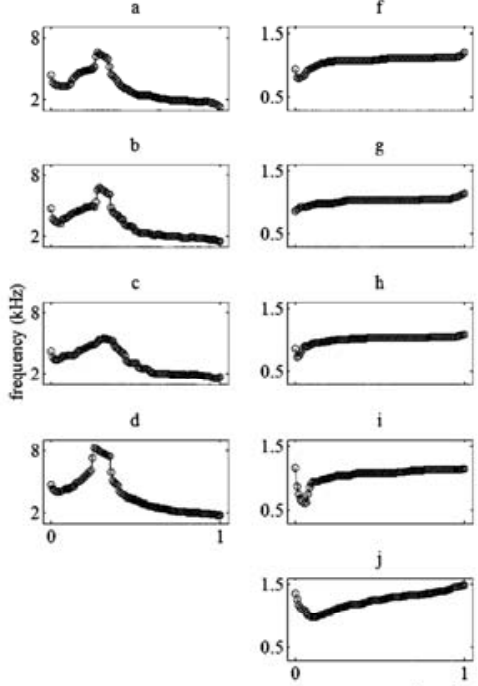

time (normalized units)
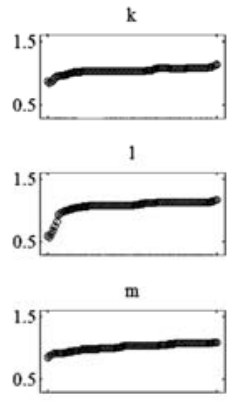

n
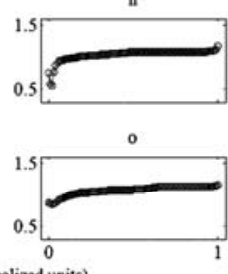
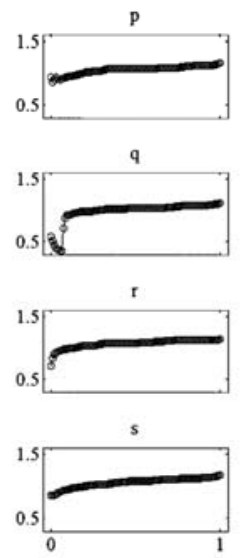

FIG. 4. Digitized traces of the fundamental frequency contours of the whistles displayed in Fig. 3. Each trace is shown with 100 equally spaced points that have been normalived on a horizontal time axis from 0

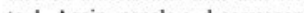
to 1 . Again, panels a-d correspond to the whistles produced by $\mathrm{mm} 224$ and $\mathrm{f}-\mathrm{s}$ to those by $\mathrm{mm} 226$. Note the difference in the frequency ranges for the two individuals.

\section{Nonparametric comparison}

One temporal (original duration before normalization) and five spectral (minimum, maximum, mean, initial, and ending frequencies) features were determined for every whistle (Fig. 5). These parameters were selected because they summarized the timing and coarse frequency content of the whistles. The differences between the finer aspects of the

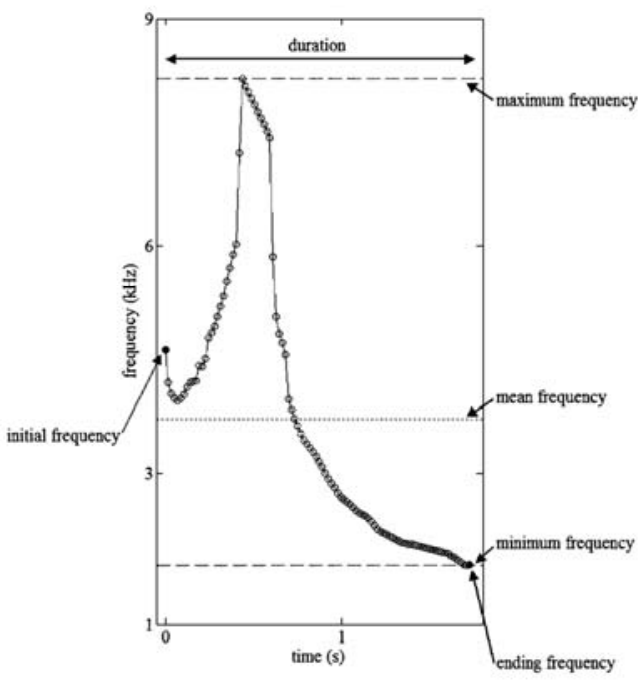

FIG. 5. Illustration of temporal and spectral features extracted from a traced whistle produced by mm224 (subplot d in Figs. 3 and 4). The initial and ending frequencies are indicated by filled circles $(\bullet)$ while the other spectral components are marked with horizontal dashed lines. Note that the mean frequency is closer to the minimum frequency because $\sim 60 \%$ of the whistle's frequency content lies below $3700 \mathrm{~Hz}$

J. Acoust. Soc. Am., Vol. 120, No. 3, September 2006

Ari Shapiro: Preliminary evidence for narwhal signature vocalizations frequency contours were reserved for the cross-correlation comparison. Again, a nonparametric Wilcoxon rank sum test with a Bonferroni correction was used to compare these features between individuals.

\section{Cross-correlation comparison}

Cross-correlation is often used in signal processing as a tool for determining the similarity between two signals. Because the frequency ranges of the whistles from the two individuals were distinct (e.g., the average maximum frequency of $\mathrm{mm} 226$ was less than the average minimum frequency of mm224), the cross-correlation measurement used here was designed to account for overall contour shape and absolute frequency content. In the equation

$$
\sum_{i=1}^{100} \frac{\left|\left(f_{A}(i)-f_{B}(i)\right)\left(f_{A}(i)-f_{B_{m}}(i)\right)\right|}{\overline{f_{A}(i)+f_{B}(i)}},
$$

$i$ is the sample number that ranges between 1 and 100, $f_{A}(i)$ and $f_{B}(i)$ correspond to the $i$ th frequency value of contours $A$ and $B$, respectively, and $f_{B_{m}}(i)$ is the $i$ th frequency value of contour $B$ after it has been slid along the frequency axis to minimize the frequency differences between contours $A$ and $B$. These terms are illustrated in Fig. 6. Larger values of this cross-correlation measure indicated greater differences between contours than smaller values. A value of 0 would reveal no difference at all in contour shape. A constrained, nonlinear minimization routine was used to determine $f_{B_{0}}(i)$. The first term in the product of the numerator of (1) is the difference between points along the actual contours normalized in time [Fig. 6(a)]. The second term, however, returns a smaller number if the frequency modulation pattern is similar between the whistles regardless of the absolute frequency offset of the two [Fig. 6(b)]. Whistles therefore could have achieved a higher similarity 


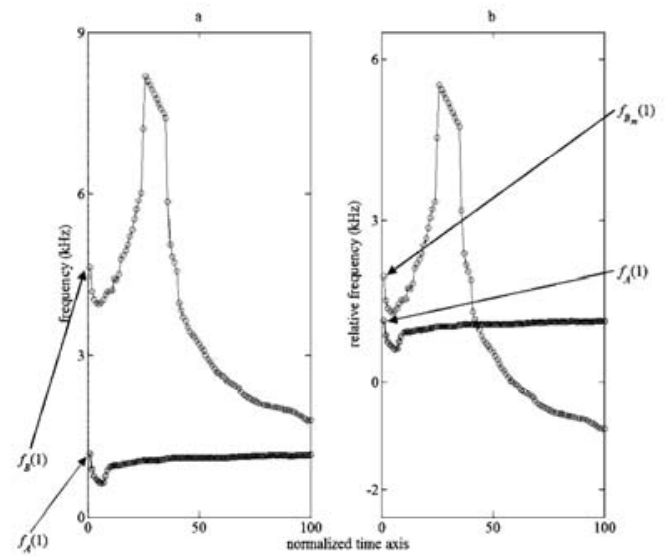

FIG. 6. Illustration of points used for cross-correlation comparison of whistles (see text for the equation). In (a), contour $A$ (darker, from mm226: subplot $\mathrm{i}$ in Figs. 3 and 4 ) and $B$ (lighter, from mm224: subplot d in Figs. 3 and 4) are depicted normalized in time with their original frequency content. In (b), contour $B$ has been shifted along the frequency axis to minimize the frequency difference between the two contours. All 100 points along the contours were used to compute Eq. (1).

ranking [a smaller value of (1)] by overlapping in absolute frequency, possessing similar overall contour shapes or both.

\section{RESULTS}

Tables I and II list the summary measurements of the combined tonal/pulsed signals and whistles. With the exception of a single whistle assigned to mm226 with a SNR of $13.9 \mathrm{~dB}$, the remaining vocalizations produced by both animals were characterized by a SNR of at least $28.2 \mathrm{~dB}$. Combined tonal/pulsed signals were produced throughout the water column but tended to concentrate at particular depths (roughly $70 \mathrm{~m}$ for $\mathrm{mm} 224$ and $20 \mathrm{~m}$ for mm226, Fig. 7). Whistle production occurred between 20 and $100 \mathrm{~m}$ for $\mathrm{mm} 224$ but was confined to the upper $30 \mathrm{~m}$ for mm226 (Fig. 7). Both vocal categories were recorded throughout the diving sequence, indicating that the behavioral or environmental contexts in which these vocalizations occurred were not gen-

TABLE I. Summary statistics of the acoustic features of combined tonal/ pulsed signals.

\begin{tabular}{crrrr}
\hline \hline & Mean & Std. & Min. & Max. \\
\hline mm224, & $n=42$ & & & \\
Duration (s) & 1.6 & 0.7 & 0.6 & 2.7 \\
Average IPI (ms) & 12.9 & 4.3 & 9.0 & 36.1 \\
Number of pulses & 128.1 & 45.4 & 49.0 & 202.0 \\
mm226, & $n=31$ & & & \\
Pulse repetition rate (pulses/s) & 82.3 & 14.2 & 28.1 & 112.8 \\
Duration (s) & 1.2 & 0.1 & 1.0 & 1.2 \\
Average IPI (ms) & 6.3 & 0.3 & 5.6 & 6.8 \\
Number of pulses & 186.2 & 9.6 & 156.0 & 201.0 \\
Pulse repetition rate (pulses/s) & 160.8 & 7.4 & 147.5 & 180.5 \\
\hline
\end{tabular}

J. Acoust. Soc. Am., Vol. 120, No. 3, September 2006
TABLE II. Summary statistics of the acoustic features of whistles.

\begin{tabular}{rrrrr}
\hline \hline & Mean & Std. & Min. & Max. \\
\hline & $\mathrm{mm} 224, n=4$ & & & \\
Duration (s) & 1.19 & 0.08 & 1.09 & 1.26 \\
Minimum frequency $(\mathrm{Hz})$ & 1549 & 201 & 1292 & 1775 \\
Maximum frequency $(\mathrm{Hz})$ & 7181 & 1386 & 5460 & 8844 \\
Mean frequency $(\mathrm{Hz})$ & 3638 & 370 & 3405 & 4190 \\
Initial frequency $(\mathrm{Hz})$ & 4773 & 277 & 4496 & 5145 \\
Ending frequency $(\mathrm{Hz})$ & 1572 & 216 & 1292 & 1788 \\
& & & & \\
Duration $(\mathrm{s})$ & 0.78 & 0.04 & 0.68 & 0.85 \\
Minimum frequency $(\mathrm{Hz})$ & 718 & 156 & 360 & 980 \\
Maximum frequency $(\mathrm{Hz})$ & 1177 & 111 & 1095 & 1501 \\
Mean frequency $(\mathrm{Hz})$ & 1012 & 71 & 895 & 1240 \\
Initial frequency $(\mathrm{Hz})$ & 939 & 236 & 641 & 1501 \\
Ending frequency $(\mathrm{Hz})$ & 1160 & 90 & 1095 & 1486 \\
\hline \hline
\end{tabular}

erally restricted to a very narrow depth or time. The two animals responded differently immediately after handling. Many combined pulsed/tonal signals (17 of 42) and one whistle were produced by individual $\mathrm{mm} 224$ just after release on his first dive lasting only $10.8 \mathrm{~min}$. Individual $\mathrm{mm} 226$ was vocally active, however, between hours 4 and 10 of the deployment where he reached a maximum depth of about $125 \mathrm{~m}$ (data not shown). He did not produce any combined tonal/pulsed signals or whistles for the first 24 dives that exceeded roughly $10 \mathrm{~m}$ following his release, a response more closely resembling the silent reaction observed and discussed by Finley et al. (1990) of narwhals exposed to envi-

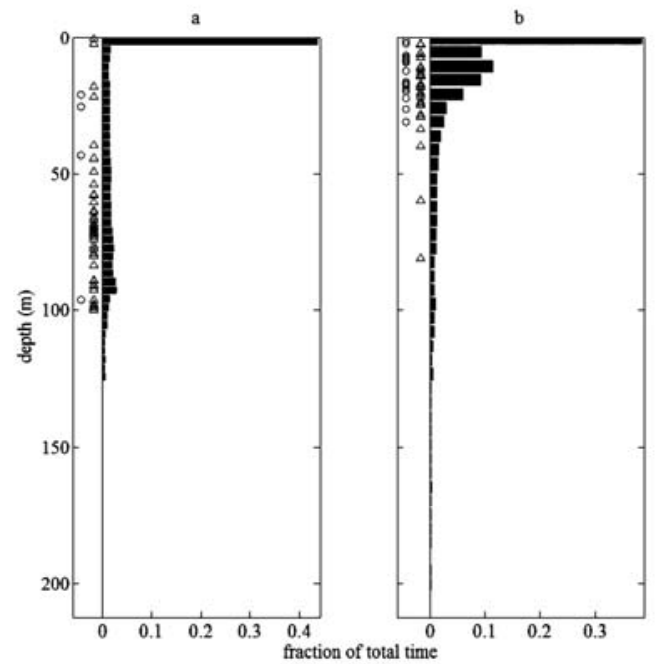

FIG. 7. Approximate depths where combined tonal/pulsed signals (triangles, $\Delta$ ) and whistles (circles, $O$ ) were produced adjacent to a frequency histogram of depth bins (bars) for $\mathrm{mm} 224$ (a) and $\mathrm{mm} 226$ (b). The frequency plotted on the abscissa is expressed as a fraction of the total amount of time spent at all depths. The maximum depths achieved for mm224 and mm226 during the DTAG deployments were roughly 125 and $210 \mathrm{~m}$, respectively.

Ari Shapiro: Preliminary evidence for narwhal signature vocalizations 

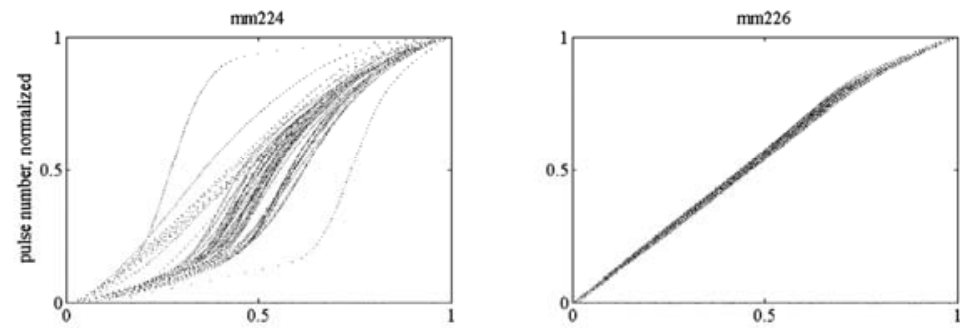

FIG. 8. Visual representations of temporal features of combined tonal/ pulsed signals. Normalized pulse number (top two panels) and pulse repetition rate (pulses per second, bot-
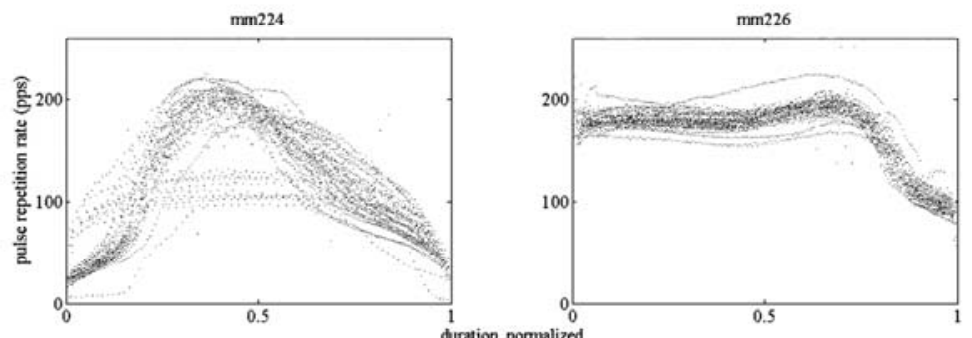
tom two panels) as a function of nortom malized duration. Note the clear differences in general morphology of these plots between the two individuals.

ronmental disturbances. Whistles were less common than combined tonal/pulsed signals, as reported in earlier studies (Ford and Fisher, 1978; Miller et al., 1995).

The combined tonal/pulsed signals lasted between 0.55 and 2.68 s and contained between 49 and 202 pulses. Spectrograms revealed the synchronous production of both pulsatile energy in the form of repeated broadband impulses and a tonal, FM component by the tagged animals (Fig. 2). The FM component was not an analytical artifact of the pulsatile energy (see Watkins, 1967) because the fundamental frequency of the tonal feature was inconsistent with the repetition rate of the pulses. It is likely that at least two sound generating apparatuses are required to produce these combined tonal/pulsed signals to achieve pulsatile and FM energy content simultaneously. Combined tonal/pulsed signals were characterized by pulse rates between 28 and 113 pulses/s for mm224 and between 148 and 180 pulses/s for $\mathrm{mm} 226$. Figure 2 reveals additional low frequency energy associated with each pulse, which is likely the consequence of resonance of the air sacs of the tagged animal and not reverberations from or echoes off of elements along the inlet bottom. Indeed, no echoes consistent with target localization or monitoring position in the water column were detected in the audio record. All whistles were between 0.68 and $1.26 \mathrm{~s}$ with frequencies ranging between 360 and $8844 \mathrm{~Hz}$. Three of the four whistles produced by mm224 were characterized by a brief $(0.18-0.24 \mathrm{~s})$ upsweep, followed by a pause and longer $(0.62-0.66 \mathrm{~s})$ downsweep [Figs. 3(a), 3(b), and 3(d)]. The fourth whistle was continuous but still showed an upsweep preceding the downsweep [Fig. 3(c)]. All seventeen whistles produced by $\mathrm{mm} 226 \mathrm{con}-$ tained a brief $(0.026-0.091 \mathrm{~s})$ broadband segment with energy that peaked between about 500 and $700 \mathrm{~Hz}$ and then decayed steadily until disappearing above $8-10 \mathrm{kHz}$ followed by a flat, constant frequency tone that lasted for the remainder of the whistle [Figs. 3(f)-3(s), 3 whistles are not shown]. The whistles were all of about the same intensity except for a quieter one recorded on the tag attached to mm226 [Fig. 3(j)], which may have been softer, may have been produced by a nonfocal animal located further from the tag, or may have had a transmission path that was partially obscured by the animal or tag components before reaching the hydrophone.

The uniqueness of each of the two sets of combined tonal/pulsed signals and whistles was apparent from simple visual inspection. Among the combined tonal/pulsed signals the patterns of how the relative timing and repetition rate of the pulses varied as a function of normalized duration differed between the two individuals (Fig. 8). All measured features for both the combined tonal/pulsed signals and whistles differed significantly between the two animals (Wilcoxon rank sum test with a Bonferroni correction, $P=0.002$ for combined tonal/pulsed signals duration, $P<0.001$ for remaining combined tonal/pulsed signals measurements, $P$ $=0.011$ for whistle ending frequency and $P=0.008$ for remaining whistle measurements). The whistles produced by $\mathrm{mm} 224$ were longer and higher in every measurement compared to those belonging to $\mathrm{mm} 226$, which were shorter and lower. Indeed, the minimum frequency of mm224 was $1549 \pm 201 \mathrm{~Hz}(\bar{x} \pm \mathrm{sd})$ and the maximum frequency of $\mathrm{mm} 226$ was $1177 \pm 111 \mathrm{~Hz}(\bar{x} \pm \mathrm{sd})$, values that did not overlap even a single standard deviation away (Table II). The cross-correlation test on the whistles revealed dramatic differences for the interindividual comparisons (between $\mathrm{mm} 224$ and $\mathrm{mm} 226$ ) and only slight differences among the intraindividual comparisons (Table III). The interindividual results were more different than the intraindividual results by 1-2 orders of magnitude. 
TABLE III. Cross-correlation comparison of whistles between the same and different individuals. These data were computed in arbitrary units with higher values indicating a greater difference between the contours being compared. The intraindividual comparisons are italicized.

\begin{tabular}{rrr}
\hline \hline & $\mathrm{mm} 226$ & $\mathrm{~mm} 224$ \\
\hline $\mathrm{mm} 224$ & 127250 & 5348 \\
$\mathrm{~mm} 226$ & 716 & \\
\hline
\end{tabular}

\section{DISCUSSION AND CONCLUSIONS}

Two free-ranging narwhals each produced an acoustically distinctive set of combined tonal/pulsed signals and whistles. Visual and aural inspection and nonparametric and cross-correlation analyses all demonstrated striking interindividual differences among these vocalizations and intraindividual temporal and spectral fidelity. These results support the claim of Ford and Fisher (1978) that narwhals produce individually distinctive signature vocalizations. The recordings analyzed here also contained numerous faint combined tonal/pulsed signals and whistles produced by nonfocal animals. These observations are consistent with the conclusion that these vocal categories are regularly produced by freeranging narwhals in this area.

The function of these vocalizations remains uncertain, but they do not appear to facilitate foraging. When feeding, some odontocetes produce a sequence of regularly spaced echolocation clicks that precede a buzz, or a series of clicks characterized by a dramatically elevated repetition rate [e.g., sperm whales (Physeter macrocephalus): Miller et al., 2004, Blainville's beaked whales (Mesoplodon densirostris): Madsen et al., 2005]. It seems unlikely that the narwhal vocalizations quantified here were used for foraging purposes since no echolocation clicks were detected immediately before the combined tonal/pulsed signals or the whistles. Indeed, the kind of clicking behavior characterized by changes in repetition rate and amplitude that is associated with foraging has been recorded from narwhals in previous studies (see Møhl et al., 1990; Miller et al., 1995), but was not observed here.

Combined tonal/pulsed signals and whistles may play a role in social communication based on their stereotypy (Ford and Fisher, 1978) and the signature content shown by the limited dataset presented here. These distinctive vocalizations might serve as contact calls to facilitate reunions of individuals with their group members in a manner similar to that observed in captive and free-ranging bottlenose dolphins (Janik and Slater, 1998; Watwood et al., 2005). Unlike the studies conducted with bottlenose dolphins to identify pair bonds or alliances among males (Connor et al., 1992, 2001; reviewed in Wells, 2003), little work has been completed to describe the social structure and group relationships among individual narwhals. Based on personal observations, the narwhals entering Admiralty Inlet traveled in groups ranging in size from roughly 5 to 30 animals. The group members traveling with the tagged animals vacated the area while their companions were detained on shore. The vocalizations of more distant animals that were recorded in this study were usually faint, suggesting that for the tagged animals, the

J. Acoust. Soc. Am., Vol. 120, No. 3, September 2006 dives occurring after their capture were likely solitary events. In addition, no other animals appeared in accompanying video footage recorded from a Crittercam (with a visual range extending between 3 and $20 \mathrm{~m}$ depending on the light level) attached to $\mathrm{mm} 226$ during the first hour following its release. These observations are consistent with the hypothesis that the combined tonal/pulsed signals and/or whistles were used by these two narwhals as contact calls in an effort to regain contact with their groups. However, actual reunions with other animals were not obvious from the acoustic record.

Because these two animals were likely members of different groups traveling into Admiralty Inlet, an alternative explanation consistent with the results presented here is that these vocalization classes may have been distinctive at the level of the social group (see Terhune et al., 2001; Weiß et al., 2006). Another possibility is that combined tonal/pulsed signals and whistles are actually used as signature vocalizations to cue conspecifics about individual identity. The whistle of mm226 was characterized by nearly constant frequency except for the brief noisy segment at the very beginning. From an information theory perspective, a flat whistle encodes less information compared to a frequency modulated whistle. It is possible, however, that even flat whistles of consistently distinctive durations or pitches could be used to distinguish between individuals. The contours of the whistles of $\mathrm{mm} 226$ appeared very similar to the FM component of the combined tonal/pulsed signals of this animal. The whistles produced by $\mathrm{mm} 224$, however, contained the frequency modulation expected of signature vocalizations and did not resemble this animal's combined tonal/pulsed signals FM component.

The combined tonal/pulsed signals described here most closely resembled the longer click series and the pulsed sounds described by Watkins et al. (1971) and Ford and Fisher (1978), respectively. All of these vocalizations were characterized by a combination of pulses and a tonal signal. Watkins et al. (1971) described the repetition rate of their longer click series tending to increase before becoming constant and eventually slowing down, somewhat similar to the trend observed in the combined tonal/pulsed signals described here (Fig. 8). In both the combined tonal/pulsed signals recorded in this study and their equivalents described in Watkins et al. (1971) and Ford and Fisher (1978), the synchronously-produced FM component creating the tonal quality in these sounds was not due exclusively to harmonically-related sidebands of the repetition rate (see Watkins, 1967). Note in Fig. 2 that the pitch of the FM component does not always correspond to the repetition rate of the pulses. For the combined tonal/pulsed signals produced by $\mathrm{mm} 226$, for example, the FM component begins over halfway through the signal without any observable change in repetition rate. Also, the tonal energy persists even as the pulses slow down at the end of the combined tonal/ pulsed signals attributed to individual mm224 [Fig. 2(a)] and continues beyond the conclusion of the pulses in the signal assigned to $\mathrm{mm} 226$ [Fig. 2(b)]. The pulsatile component of

Ari Shapiro: Preliminary evidence for narwhal signature vocalizations 
the combined tonal/pulsed signals quantified here was characterized by a higher upper frequency limit (up to $48 \mathrm{kHz}$ ) compared to earlier recordings.

Previous studies (Watkins et al., 1971; Ford and Fisher, 1978) reported observing tonal signals with properties that were both similar to and different from the whistles analyzed here. The frequency ranges overlapped but the whistles that were recorded here had higher harmonics, extending the upper bound of these tonal vocalizations to at least $48 \mathrm{kHz}$. Earlier published tones were either constant in frequency or swept upwards or downwards, again consistent with the whistles presented here (Fig. 3). Both earlier works, however, described whistles as narrow-band signals lacking additional detectable harmonic content. All of the whistles recorded for this study were typified by a fundamental FM component and harmonic energy, a difference which may have resulted from the higher sampling rate of the recording equipment and/or the elevated signal to noise ratio due to the close proximity of the hydrophone to the whale.

The cross-correlation test used in this analysis was modified slightly from those described in other studies of signature vocalizations (e.g., Buck and Tyack, 1993; McCowan, 1995; Janik, 1999; Watwood et al., 2005). In general, similarity between whistle contours can result either coarsely from a general overlap in frequency range and/or more finely from comparable frequency modulation (e.g., loop number, overall shape). The time-invariant crosscorrelation test used here incorporated both of these components into its final measurement. A continuum was possible ranging from dissimilar (minimal frequency overlap and contour resemblance) to very similar (maximal frequency overlap and contour resemblance). Because the first term of the product ranked similarity according to both overlap and contour and the second term according to contour only, intermediate scores of similarity were also possible. This was particularly important since the frequency ranges of the whistles from the two narwhals were mostly nonoverlapping. The possibility of contour shape resemblance was excluded by the unambiguous results of the cross-correlation test (Table III)

These findings suggest possible directions for future work. Tagging and recording the combined tonal/pulsed signals and whistles of multiple narwhals from other groups would provide data that could support or reject the conclusions made here. If these signals do possess signature content, further study could ascertain whether they are distinctive at the individual or group level. Critical to determining the function of these vocalizations will be an assessment of the behavioral context in which these vocalizations are produced and the stability of group composition and size over short and long time scales. If narwhals are capable of differentiating between individuals acoustically, quantifying these aspects of group dynamics would provide starting estimates for the number of animals with which a single individual is interacting and therefore between which it should be able to distinguish. Playback experiments would be useful for identifying the temporal and spectral features of the combined tonal/pulsed signals and whistles that the animals may be using to facilitate differentiation. An understanding of the ontogeny of these sounds to determine if vocal learning plays any role in their acquisition or development requires acoustic data from the same animals and their groups collected longitudinally over many years.

Signature whistles appear to be used by bottlenose dolphins as contact calls in a variety of contexts (Janik and Slater, 1998; Sayigh et al., 1998; Watwood et al., 2005). If narwhals, another gregarious odontocete, similarly use their combined tonal/pulsed signals and whistles as contact calls when separated from conspecifics, the procedure described here affords an opportunity to make recordings in this context while the animals are detained ashore. Under this hypothesis, an involuntary separation of the sort imposed here would cause the animals and/or their group members to vocalize in an effort to regain contact.

Despite the small sample size, the data presented in this manuscript provide supportive evidence for at least two classes of signature vocalizations among free-ranging narwhals at the individual or group level. Future work focused on the ontogeny, function, and acoustic characteristics of the combined tonal/pulsed signals and whistles produced by narwhals is required to develop an improved understanding of the vocal and social behavior of this elusive Arctic animal.

\section{ACKNOWLEDGMENTS}

This manuscript benefited from the insights, thorough revisions, and helpful suggestions provided by Peter Tyack. Special thanks to Mark Johnson, Kira Barton, Alex Bocconcelli, Amanda Hansen, Tom Hurst, and Alex Shorter for DTAG and field support and to Rune Dietz, Jack Orr, Mehdi Bakhtiari, Sila Akikuluk, Sandie Black, Moe Keenainak, Martin Nweeia, Hans-Christian Schmidt, Seemee Tunraq, and Keith Yip for their generous assistance in the field. I am grateful to Amanda Searby and Stephanie Watwood for comments on the contour comparisons and to two anonymous reviewers whose suggestions helped to improve this manuscript. I thank the WHOI Academic Programs office, the National Science Foundation Research Fellowship, and the National Defense Science and Engineering Graduate Fellowship for financial support. This field operation was funded by the Greenland Institute of Natural Resources, the National Environmental Research Institute, Department of Fisheries and Oceans, Nunavut Wildlife Management Board and the Danish Cooperation for the Environment in the Arctic (DANCEA). Additional logistical support was provided by the Polar Continental Shelf Project. This work was conducted under permit No. SLE-04/05-9 issued by the Canadian Department of Fisheries and Oceans and animal use protocol FWI2004-2005-10.

Balcombe, J. P. (1990). "Vocal recognition of pups by mother Mexican free-tailed bats, Tadarida brasiliensis mexicana," Anim. Behav. 39 960-966.

Bee, M. A., and Gerhardt, H.C. (2002). "Individual voice recognition in : territorial frog (Rana catesbeiana)," Proc. R. Soc. London, Ser. B 269. 1443-1448.

Boughman, J. W. (1997). "Greater spear-nosed bats give group-distinctive calls," Behav. Ecol. Sociobiol. 40, 61-70.

Buck, J. R., and Tyack, P.L. (1993). "A quantitative measure of similarity fo Tursiops truncatus signature whistles," J. Acoust. Soc. Am. 94. $2497-2506$ 
Caldwell, M. C., and Caldwell, D.K. (1965). "Individualized whistle contours in bottlenose dolphins (Tursiops truncatus)," Nature (London) 207. 434-435.

Charrier, I., Mathevon, N., and Jouventin, P. (2001). "Mother's voice recognition by seal pups," Nature (London) 412, 873.

Charrier, I., Mathevon, N., and Jouventin, P. (2003). "Vocal signature recognition of mothers by fur seal pups," Anim. Behav, 65, 543-550.

Connor, R. C., Heithaus, M. R., and Barre, L. M. (2001). "Complex social structure, alliance stability and mating access in a bottlenose dolphin 'super-alliance'," Proc, R. Soc, London, Ser. B 268, 263-267.

Connor, R. C., Smolker, R. A., and Richards, A. F. (1992). "Two levels of alliance formation among male bottlenose dolphins (Tursiops sp.)," Proc. Natl. Acad. Sci. U.S.A. 89, 987-990.

Deecke, V. B., Ford, J. K. B., and Spong, P. (2000), "Dialect change in resident killer whales: implications for vocal learning and cultural transmission," Anim. Behav, 60, 629-638.

Dietz, R., and Heide-Jørgensen, M. P. (1995). "Movements and swimming speed of narwhals Monodon monoceros, equipped with satellite transmitters in Melville Bay, northwest Greenland," Can. J. Zool. 73, 2106-2119.

Dietz, R., Heide-Jørgensen, M. P., Richard, P. R., and Acquarone, M. (2001). "Summer and fall movements of narwhals (Monodon monoceros) from northeastern Baffin Island towards northern Davis Strait," Arctic 54. 244-261.

Egnor, S. E. R., andHauser, M. D. (2004). "A paradox in the evolution of primate vocal learning," Trends Neurosci. 27, 649-654.

Elowson, A. M., andSnowdon, C. T. (1994). "Pygmy marmosets, Cebuella pygmaea, modify vocal structure in response to changed social environment," Anim. Behav. 47, 1267-1277.

Farabaugh, S. M., Linzenbold, A., and Dooling, R. J. (1994). "Vocal plasticity in budgerigars (Melopsittacus undulatus) - Evidence for social fac tors in the learning of contact calls," J. Comput. Phys. 108, 81-92.

Finley, K. J., Miller, G. W., Davis, R. A., and Greene, C. R. (1990), "Reactions of belugas, Delphinapterus leucas, and narwhals, Monodon monoceros, to ice-breaking ships in the Canadian high Arctic," Can. Bull. Fish. Aquat. Sci. 224, 97-117

Ford, J. K. B., and Fisher, H. D. (1978). "Underwater acoustic signals of the narwhal (Monodon monoceros)," Can. J. Zool. 56, 552-560.

Fripp, D., Owen, C., Quintana-Rizzo, E., Shapiro, A., Buckstaff, K., Jankowski, K., Wells, R., and Tyack, P. (2005). "Bottlenose dolphin (Tursiops truncatus) calves appear to model their signature whistles on the signature whistles of community members," Anim. Cogn. 8, 17-26.

Frommolt, K. H., Goltsman, M. E., and MacDonald, D. W. (2003). "Barking foxes, Alopex lagopus: Field experiments in individual recognition in a territorial mammal," Anim. Behav, 65, 509-518.

Goldman, J. A. Phillips, D. P., and Fentress, J. C. (1995). "An acoustic basis for maternal recognition in timber wolves (Canis lupus)," J. Acoust. Soc. Am. 97, 1970-1973.

Hay, K. A., and Mansfield, A. W. (1989). "Narwhal: Monodon monoceros Linnaeus, 1758," in Handbook of Marine Mammals: Volume 4, River Dol. phins and the Larger Toothed Whales, edited by S. H. Ridgway and R. Harrison (Academic, London), pp. 145-176.

Janik, V. M. (1999). "Pitfalls in the categorization of behaviour: a comparison of dolphin whistle classification methods," Anim. Behav. 57, 133143.

Janik, V. M. (2000). "Whistle matching in wild bottlenose dolphins (Tursiops truncatus)," Science 289, 1355-1357.

Janik. V. M.. Sayigh, L. S., and Wells, R. S., (2006). "Signature whistle shape conveys identity information to bottlenose dolphins," Proc. Natl, Acad. Sci. U.S.A. 103, 8293-8297.

Janik, V. M., and Slater, P. J. B. (1997). "Vocal learning in mammals," Adv. Study Behav. 26, 59-99.

Janik, V. M., and Slater, P. J. B. (1998). "Context-specific use suggests that bottlenose dolphin signature whistles are cohesion calls," Anim. Behav. 56, 829-838.

Janik, V. M., and Slater, P. J. B. (2000). "The different roles of social learning in vocal communication," Anim. Behav, 60, 1-11.

Johnson, M. P. and Tyack. P. L. (2003). "A digital acoustic recording tag for measuring the response of wild marine mammals to sound," IEEE J. Ocean. Eng. 28, 3-12.

Jones, B. S., Harris, D. H. R., and Catchpole, C. K. (1993). "The stability of the vocal signature in phee calls of the common marmoset, Callithrix jacchus," Am. J. Primatol 31, 67-75.

Laidre, K. L., Heide-Jørgensen, M. P., Logdson, M. L., Hobbs, R. C., Heagerty, P., Dietz, R., Jørgensen, O. A., and Treble, M. A. (2004). "Seasonal

J. Acoust. Soc. Am., Vol. 120, No. 3, September 2006 narwhal habitat associations in the high Arctic," Mar. Biol. (Berlin) 145 $821-831$

Lengagne, T., Aubin, T., Jouventin, P., and Lauga, J. (2000). "Perceptual salience of individually distinctive features in the calls of adult king penguins," J. Acoust. Soc. Am. 107, 508-516.

Leonard, M. L., Horn, A. G., Brown, C, R., and Fernandez, N. J. (1997),

"Parent-offspring recognition in tree swallows, Tachycineta bicolor," Anim. Behav. 54, 1107-1116.

Madsen, P. T. (2005). "Marine mammals and noise: Problems with root mean square sound pressure levels for transients," J. Acoust. Soc. Am. 117, 3952-3957.

Madsen, P. T., Johnson, M., de Soto, N. A.. Zimmer, W. M. X., and Tyack, P. (2005). "Biosonar performance of foraging beaked whales (Mesoplodon densirostris)," J. Evol. Biol. 208, 181-194.

Marshall, A. J., Wrangham, R. W., and Arcadi, A. C. (1999). "Does learning affect the structure of vocalizations in chimpanzees?," Anim. Behav, $\mathbf{5 8}$ $825-830$.

Marshall, G. J. (1998). "Crittercam: An animal-borne imaging and data logging system," Mar. Technol. Soc. J. 32, 11-17.

McCowan, B. (1995). "A new quantitative technique for categorizing whistles using simulated signals and whistles from captive bottlenose dolphins (Delphinidae, Tursiops truncatus)," Ethology 100, 177-193.

McCowan, B. and Reiss, D. (1995). "Quantitative comparison of whistle repertoires from captive adult bottlenose dolphins (Delphinidae, Tursiops truncatus)-A re-evaluation of the signature whistle hypothesis," Ethology 100, 194-209.

McCowan, B., and Reiss, D. (2001). "The fallacy of 'signature whistles' in bottlenose dolphins: A comparative perspective of 'signature information' in animal vocalizations," Anim. Behav, 62, 1151-1162

Miksis, J. L., Tyack, P. L., and Buck, J. R. (2002), "Captive dolphins, Tur siops truncatus, develop signature whistles that match acoustic features of human-made model sounds," J. Acoust. Soc. Am. 112, 728-739,

Miller, L. A., Pristed, J., Møhl, B., and Surlykke, A. (1995). "The clicksounds of narwhals (Monodon monoceros) in Inglefield Bay, Northwest Greenland," Marine Mammal Sci. 11, 491-502.

Miller, P. J. O., and Bain, D. E. (2000). "Within-pod variation in the sound production of a pod of killer whales, Orcimus orca," Anim. Behav. 60 . 617-628.

Miller, P. J. O., Johnson, M. P., and Tyack, P. L. (2004). "Sperm whale behaviour indicates the use of echolocation click buzzes 'creaks' in prey capture," Proc. R. Soc. London, Ser. B 271, 2239-2247.

Mitani, J. C., and Gros-Louis, J. (1998). "Chorusing and call convergence in chimpanzees: Tests of three hypotheses," Behaviour 135, 1041-1064.

Mitani, J. C, and Nishida, T. (1993), "Contexts and social correlates of lon distance calling by male chimpanzees," Anim. Behav, 45, 735-746.

Møhl, B., Surlykke, A., and Miller, L. A. (1990). "High intensity narwhal clicks," in Sensory Abilities of Cetaceans, edited by J. Thomas and R. Kastelein (Plenum, New York), pp. 295-303.

Nousek, A. E., Slater, P. J. B., and Miller, P. J. O. (in press). "The influence of social affiliation on individual vocal signatures of northern resident killer whales (Orcinus orca)," Proc, R. Soc. London, Ser B.

Sayigh, L. S., Tyack, P. L., Wells, R. S., and Scott, M. D. (1990). "Signature whistles of free-ranging bottenose dolphins, Tursiops truncatus: Motheroffspring comparisons," Behav. Ecol. Sociobiol. 26, 247-260.

Sayigh, L. S., Tyack, P. L., Wells, R. S., Solow, A. R., Scott, M. D., and Irvine, A. B. (1998). "Individual recognition in wild bottlenose dolphins: a field test using playback experiments," Anim. Behav, 57, 41-50.

Scherrer, J. A., and Wilkinson, G. S. (1993). "Evening bat isolation calls provide evidence for heritable signatures," Anim. Behav. 46, 847-860. Searby, A., Jouventin, P., and Aubin, T. (2004). "Acoustic recognition in macaroni penguins: An original signature system," Anim. Behav. 67. 615-625.

Smolker, R., and Pepper, J. W. (1999). "Whistle convergence among allied male bottlenose dolphins (Delphinidae, Tursiops sp.)," Ethology 105 595-617

Snowdon, C. T., and Elowson, A. M. (1999). "Pygmy marmosets modify call structure when paired," Ethology 105, 893-908,

Snowdon, C. T., Elowson, A. M., and Roush, R. S. (1997) "Social influences on yocal development in New World primates," in Social Influences on Vocal Development, edited by C. T. Snowdon and M. Hausberger (Cambridge University Press, Cambridge), pp. 234-248.

Stoddard, P. K., and Beecher, M. D. (1983). "Parental recognition of offspring in the cliff swallow," Auk 100, 795-799.

Terhune, J. M., Healey, S. R., and Burton, H. R. (2001). "Easily measured 
call attributes can detect vocal differences between Weddell seals from two areas," Bioacoustics 11, 211-222.

yack, P. (1986). "Whistle repertoires of two bottlenosed dolphins, Tursiops truncatus: Mimicry of signature whistles?" Behav. Ecol. Sociobiol. 18, 251-257.

Watkins, W. A. (1967). "The harmonic interval: fact or artifact in spectral analysis of pulse trains," in Marine Bioacoustics, edited by W. N. Tavolga (Pergamon, New York), pp. 15-43

Watkins, W. A., Schevill, W. E., and Ray, C. (1971). "Underwater sounds of Monodon (Narwhal)," J. Acoust. Soc. Am. 49, 595-599.

Watwood, S. L., Owen, E. C. G., Tyack, P. L., and Wells, R. S. (2005).

"Signature whistle use by temporarily restrained and free-swimming bottlenose dolphins, Tursiops truncatus," Anim. Behav. 69, 1373-1386. Watwood, S. L.. Tyack, P. L., and Wells, R. S. (2004). "Whistle sharing in paired male bottlenose dolphins, Tursiops truncatus," Behav. Ecol. Sociobiol. 55, 531-543.

WeiB, B. M., Ladich, F., Spong, P., and Symonds, H. (2006). "Vocal behavior of resident killer whale matrilines with newborn calves: The role of family signatures," J. Acoust. Soc. Am. 119, 627-635.

Wells, R. S. (2003). "Dolphin Social Complexity: Lessons from Long-Term Study and Life History," in Animal Social Complexity: Intelligence, Culture, and Individualized Societies, edited by F. B. M. de Waal and P. L. Tyack (Harvard University Press, Cambridge, Massachusetts), pp. 32-56. 


\section{APPENDIX 5. TESTING FOR ORIENTATION RESPONSES OF INDIVIDUAL SPERM WHALES TO A VARYING SONAR EXPOSURE LEVEL ${ }^{*}$}

\section{A5.1 Abstract}

Research examining responses of marine mammals to sound stimuli is often limited by small sample sizes or the reporting of a single exposure level for each subject, which has restricted analysis of dose-response functions. Here we report on a statistical technique for titrating a continuous behavioral response parameter against a range of sound exposure levels for each subject. We analyzed the angular orientation responses of three tagged sperm whales with respect to a sonar source operating between $2-15 \mathrm{kHz}$ as a function of a varying received sound exposure level. During each experiment, the source level was gradually increased, or ramped up, and then maintained. A method accounting for serial correlation and based on circular regression was used to test the null hypothesis of no effect of received level on angular orientation for two whales. Our analysis did not find a significant effect for approach towards or avoidance of the source as a function of received level, $90 \%$ of which ranged from $106-137 \mathrm{~dB}$ re $1 \mu \mathrm{Pa}^{2}$ s. This statistical technique proved robust to test for avoidance responses more generally, underscoring its utility for empirical evaluation of the assumption that animals will avoid harmful exposures during ramp up or a sound source approach.

\section{A5.2 Introduction}

The extent to which marine mammals are influenced by anthropogenic sound

\footnotetext{
* This manuscript has been submitted to Marine Mammal Science for publication with Peter L. Tyack and Andrew R. Solow as co-authors.
} 
sources, including sonars used to detect and locate objects underwater, is a contentious issue. Marine mammals have evolved mechanisms to use sound to communicate and orient in the sea, whose physical properties favor sound for rapid long-distance communication. Over the past century, humans have had global impacts on the ocean acoustic environment. Ships have elevated the deep ocean ambient noise by 10-100 fold and naval sonar exercises have been found to cause some whales to strand and die (Cox et al., 2006). The largest problem for managing the risks of sound involves our ignorance of its effects on marine mammals.

Part of the difficulty in measuring these effects analytically lies in defining the qualitative and quantitative nature of possible responses and in determining their spatial and temporal extent. Individual and species variability and a wide diversity of stimulus categories, exposure levels, and patterns of presentation contribute to the complexity of these analyses. Controlled exposure experiments (CEEs), however, have proven useful in addressing conservation concerns by describing dose-response relationships for the behavioral responses of animals to acoustic exposure (Tyack et al., 2003). In a CEE, the behavioral response of one or more focal individuals is monitored over time during exposure to a stimulus with acoustic characteristics that are varied in a controlled fashion. Most earlier CEEs have associated one received level with a behavioral response but given the limited number of subjects typically available, this has hindered the development of dose-response functions. There is a need to develop CEE protocols and associated analyses that allow a rigorous testing of how behavioral response parameters vary over a range of acoustic exposure for each subject.

One might expect animals to avoid an aversive stimulus by moving away from the source. Many acoustic mitigation protocols gradually increase, or ramp up, an anthropogenic 
sound stimulus from the lowest level practicable to its full operating intensity. The most common goal of this procedure is to allow an exposed animal the opportunity to detect the sound at safe exposure levels and move away from the zone of potential injury near the source, as demonstrated for certain species of baleen whales (Malme et al., 1984; McCauley et al., 2000; Richardson et al., 1990). However, it is also possible that ramp up may make it more likely for the animal to habituate to the sound and remain in the area. Another goal of ramp up could be to habituate animals to particular signals at levels below which a potentially risky response may be evoked. Our analysis, which was designed to examine whether subjects showed avoidance responses as a function of their acoustic exposure, is ideal for testing whether animals will remain in or vacate an area when introduced to sounds of steadily increasing level, and to define the received sound exposure level at which this response occurs.

This paper presents a new analysis method aimed at identifying a response in the angular orientation of individual marine mammals to a sound source. The method was applied to data collected from mid-frequency sonar tests on tagged sperm whales (Physeter macrocephalus). Previous opportunistic observations of sperm whale behavior in the presence of sonars or pingers (Watkins \& Schevill, 1975; Watkins, 1977; Watkins et al., 1985; 1993) suggested that avoidance responses may occur, but did not involve rigorous analyses. The cruises described here have been used for several studies: to test whether mid-frequency sonar could detect sperm whales; to define the 3D beam pattern of sperm whale clicks (Zimmer et al., 2003; 2005); and to determine whether the behavior of the whales was affected by the received sound exposure level (RL) of the sonar (this paper). Given the low sample sizes that often accompany studies on the effects of anthropogenic noise on whales, 
the analysis presented here benefits from evaluating behavioral responses of each individual subject against a range of acoustic RLs, using each presentation of the sonar stimulus as a data point. The method addresses serial dependence in the dataset with an approach that considers the continuous orientation response of each sperm whale subject to varying RLs of mid-frequency sonar when the source was on and of the background noise level when the source was off.

Previous experiments of this type have often taken the individual subject as the unit of analysis and utilized stationary sources (Malme et al., 1984) or a relatively straight line approach of the source (Miller et al., 2000; Nowacek et al., 2004). These analyses extracted a single measurement for each animal (e.g., the closest point of approach) as a function of the maximum RL at the whale (Richardson et al., 1990). The method presented here, however, examined the possibility of a relationship between orientation response and RL for an individual animal whose relative position with respect to the vessel was changing throughout the exposure. The sonar source was moved in a variable, circling path around the animal as it swam, covering the full $360^{\circ}$ several times over during each experiment. Our analysis took advantage of exposing a whale that could change its orientation with respect to the vessel much more rapidly than the ship was moving.

Any method that uses a data series from the same subject must consider possible serial correlation between consecutive measurements. Animal movements can be serially dependent (especially for a large whale over the short intervals of $15 \mathrm{~s}$ between sonar pings) because the direction of an individual at time $t$ can often be predicted fairly well by its orientation and position at time $t-1$. The sequence of RLs was also characterized by serial dependence. The rotation test used here to assess significance controlled for serial 
dependence in both data streams by examining the dependence of the sequence of angular orientation data on the rotated sequences of RL data, preserving any serial dependence across each rotation. More specifically, only the relative timing - and not the internal order of the orientation and RL data series was altered between rotations, maintaining the serial dependence of each data stream.

\section{A5.3 Materials and methods}

\section{A5.3.1 Experimental design}

\section{A5.3.1.1 Field protocol}

Three exposures of mid-frequency sonar were conducted with adult male sperm whales from 2001-2003 in the Ligurian Sea, Italy using a quiet research vessel (R/V Alliance). A cantilevered pole mounted on a rigid hull inflatable boat was used to attach a digital archival tag (DTAG) to a focal sperm whale (Moore et al., 2001; Johnson \& Tyack, 2003). The DTAG contained a hydrophone, depth sensor, and a tri-axial accelerometer and magnetometer, which all recorded to flash memory (Johnson \& Tyack, 2003, see Table A5.1 for sampling rates). After tagging, the vessel circled the focal animal for the remainder of the experiment, maintaining a distance between $150 \mathrm{~m}$ and $4.5 \mathrm{~km}$. Visual observers recorded the bearing and range to the focal animal during surfacings using reticule estimates from Fujinon Big Eye 25x150 binoculars. Reticule numbers were converted to range with Gratran 1.0.0 (C) J.C. Gordon) using published calibration factors (Kinzey \& Gerrodette, 2001) and equations (Gordon, 1990; Lerczak \& Hobbs, 1998).

After the whale was tagged, a pre-exposure control period preceded the initiation of exposure to sonar sounds broadcast from an omnidirectional source (Table A5.1). The 


\begin{tabular}{|c|c|c|c|}
\hline year & 2001 & 2002 & 2003 \\
\hline calendar day & 2 October & 10 July & 8 September \\
\hline experiment duration $(\mathrm{h})$ & 6.93 & 5.12 & 7.61 \\
\hline exposure start (h into experiment) & 1.01 & 2.15 & 2.09 \\
\hline exposure end (h into experiment) & 3.14 & 4.88 & 5.66 \\
\hline duration of exposure $(\mathrm{h})$ & 2.13 & 2.73 & 3.58 \\
\hline number of pauses $(>4 \mathrm{~min})$ & 2 & 3 & 1 \\
\hline total length of pauses $(\mathrm{h})$ & 0.66 & 0.93 & 0.08 \\
\hline sampling rate: hydrophone $(\mathrm{kHz})$ & 32 & 32 & 96 \\
\hline sampling rate: movement sensors $(\mathrm{Hz})$ & 5.88 & 5.88 & 5 \\
\hline sonar ping mean frequencies $(\mathrm{kHz})$ & $2.6,3.8,8.0,15.0$ & $2.0,3.0$ & $2.0,3.0$ \\
\hline interval between pings (s) & 15 & 15 & 15 \\
\hline input sonar ping duration (s) & 0.1 & 0.1 & 0.4 \\
\hline number of complete dives & 8 & 5 & 8 \\
\hline maximum dive depth $(\mathrm{m})$ & 899.6 & 1171.0 & 903.9 \\
\hline
\end{tabular}

Table A5.1. Descriptive measurements made for each experiment. The italicized sonar ping frequency in 2001 was near the Nyquist frequency of $16 \mathrm{kHz}$. Because it was not possible to measure the RLs of these pings reliably, they were excluded from the analysis.

source level (SL) was increased gradually from $150 \mathrm{~dB}$ re $1 \mu \mathrm{Pa}$ RMS and was operated to maintain a received sound level (RL) at the whale below $160 \mathrm{~dB}$ re $1 \mu \mathrm{Pa}$ RMS, following the conditions of the research permit and selected to be well below that considered to pose a risk of injury. RL was approximated by modeling transmission loss for the estimated range to the whale. Sperm whales usually begin producing regular echolocation clicks soon after they dive but cease during ascent and while at the surface (Madsen et al., 2002; Miller et al., 2004; Watwood et al., 2006). A hydrophone array was used to track the tagged animal as it was clicking (Zimmer et al. 2005). The sonar was only active when the location of the animal was known sufficiently to ensure the exposure did not exceed the permitted limit. In 2001 and 2002, the sonar was turned off when the animal stopped clicking as it started its ascent, leading to pauses in the exposure exceeding 4 minutes. In 2001, the 4 frequencies were repeated in a fixed sequence while in 2002 and 2003, the 2 and $3 \mathrm{kHz}$ pings were alternated. The interval between pings was $15 \mathrm{~s}$ for all exposures. After exposure, the vessel continued to 
circle the tagged whale. The DTAG was programmed to release from the sperm whale and a VHF tracking beacon was used to recover the tag for data offloading.

\section{A5.3.1.2 Received sound exposure level (RL) calculations}

The RL of each sonar ping was calculated from the DTAG recordings, corresponding to the approximate level encountered by the tagged animal. Echolocation clicks and buzzes (Miller et al., 2004) of the focal or neighboring non-focal animals coincided with some of the pings. Because these clicks often had sound pressure levels that were substantially higher than the sonar pings, they were removed using Adobe Audition (Adobe Systems, Inc.) to prevent them from interfering with the computation of RL. When extracting the clicks, small segments of the sonar pings were also removed, but the short duration of the clicks compared to the overall length of the pings rendered the effect on the RL calculations negligible. Ping echoes from surface and bottom bounces were excluded from the RL determinations because their amplitudes were generally at least $20 \mathrm{~dB}$ lower than the direct arrivals. All programming for the remainder of the analysis was conducted in Matlab (The MathWorks, Inc.).

The duration and bandwidth of the sonar pings changed between years (Tables A5.1 \& A5.2). A 2-pole Butterworth band-pass filter was designed to exclude extraneous, nonping energy and was centered at the mean frequency of each ping. The $-3 \mathrm{~dB}$ endpoints serving as the filter cut-off frequencies were chosen as twice the bandwidth flanking the upper and lower frequency bounds of the ping to avoid filtering away any ping energy. The RL of each ping was calculated as the sound exposure level, or energy flux density, given by the sum of the squared pressure over the duration of the ping in $\mathrm{dB}$ re $1 \mu \mathrm{Pa}^{2} \mathrm{~s}$ : 


\begin{tabular}{|c|c|c|c|c|c|}
\hline & & \multicolumn{4}{|c|}{ output $(90 \%$ range $)$} \\
\hline $\begin{array}{l}\text { ping: year, } \\
\text { frequency }\end{array}$ & $\begin{array}{c}\text { bandwidth } \\
(\mathrm{Hz})\end{array}$ & duration (s) & $\begin{array}{c}\text { SEL (dB re } \\
\left.1 \mu \mathrm{Pa}^{2} \mathrm{~s}\right)\end{array}$ & $\begin{array}{c}\text { SPL } L_{\mathrm{RMS}}(\mathrm{dB} \text { re } \\
\left.1 \mu \mathrm{Pa}^{2} \mathrm{~s}\right)\end{array}$ & $\begin{array}{c}\mathrm{SPL}_{\mathrm{p}-\mathrm{p}}(\mathrm{dB} \text { re } \\
\left.1 \mu \mathrm{Pa}^{2} \mathrm{~s}\right)\end{array}$ \\
\hline 2001, all frequencies & 200 & $0.07,0.12$ & 83,109 & 93,119 & 109,133 \\
\hline $\begin{array}{l}\text { 2002, both } \\
\text { frequencies }\end{array}$ & 100 & $0.07,0.13$ & 102,125 & 113,135 & 125,148 \\
\hline $2003,2.0 \mathrm{kHz}$ & 200 & \multirow{2}{*}{$0.30,1.76$} & \multirow{2}{*}{112,138} & \multirow{2}{*}{112,143} & \multirow{2}{*}{131,156} \\
\hline $2003,3.0 \mathrm{kHz}$ & 400 & & & & \\
\hline
\end{tabular}

Table A5.2. Bandwidth, duration and level (energy flux density or sound exposure level (SEL), sound pressure level RMS (SPLRMs), and sound pressure level peak-peak (SPLp-p)) of sonar frequencies used in all years. The frequency of each ping increased from $f_{0}-1 / 2 b$ to $f_{0}+1 / 2 b$ where $f_{0}$ corresponds to the mean frequency given in Table A5.1 and $b$ to the bandwidth listed here. The ranges listed here defined the middle $90 \%$ of the data values.

$$
\mathrm{RL}=10 \log \int_{0}^{T} p^{2}(t) d t=10 \log \left(\frac{1}{T} \int_{0}^{T} p^{2}(t) d t\right)+10 \log (T)
$$

where $p(t)$ is the instantaneous pressure as a function of time $t$, the duration $T$ was defined to contain 90\% of the energy in a window surrounding the ping (Blackwell et al., 2004; Madsen, 2005), and the term $\frac{1}{T} \int_{0}^{T} p^{2}(t) d t$ corresponds to the squared pressure (RMS) of the signal.

Energy flux density provided a realistic measurement of the total amount of acoustic energy from the sonar ping impinging on the tagged animal. Because it was not possible to calculate the RL of the pings centered near the Nyquist frequency of the tag recording (i.e., the 15 $\mathrm{kHz}$ pings of 2001 sampled at $32 \mathrm{kHz}$ ), they were dropped from the analysis.

During the intervals when the sonar source was off, the background noise RL was calculated. These measurements were made by selecting noise clips once every $15 \mathrm{~s}$ that excluded echolocation clicks and were of the same duration as the pings, filtering them as described above by cycling through the $2-4$ unique $f_{0}$ 's as though sonar pings were present and computing the energy flux density. Background noise calculations during creaks and 
surfacing periods were made by averaging the preceding and succeeding noise RL values. For the remainder of this paper, "sonar RL" refers to the levels of the sonar pings whereas "RL" also includes the periods without sonar where background noise RL was quantified instead.

\section{A5.3.1.3 Animal and vessel paths}

A 3D path of the tagged animal was generated from the DTAG movement and depth data (Johnson \& Tyack, 2003). This path was geo-referenced by range estimations of the tagged individual at the surface recorded by visual observers using binocular reticules. Incorporating a \pm 0.1 reticule reporting inaccuracy, error ovals were calculated around the coordinate pairs of each sighting. The positions of the animal were located within their respective error ovals to minimize the scaling of the track segments between surfacings. GPS latitude and longitude data of the vessel's course were converted to horizontal 2D coordinates positioned at the water surface. Both animal and vessel tracks were re-sampled to $1 \mathrm{~Hz}$. Figures A5.1 and A5.2 illustrate the corrected whale tracks in 2D and 3D, respectively.

\section{A5.3.2 Data preparation and statistical methods}

\section{A5.3.2.1 The directional time-series}

A directional time-series containing the focal animal's orientation relative to the playback vessel was expressed as a single angle in radians once each second. Values of $0, \pi / 2$ and $\pi$ corresponded to the animal facing directly away from the vessel, broadside to the vessel, and towards the vessel, respectively. The directional time-series was produced by. 


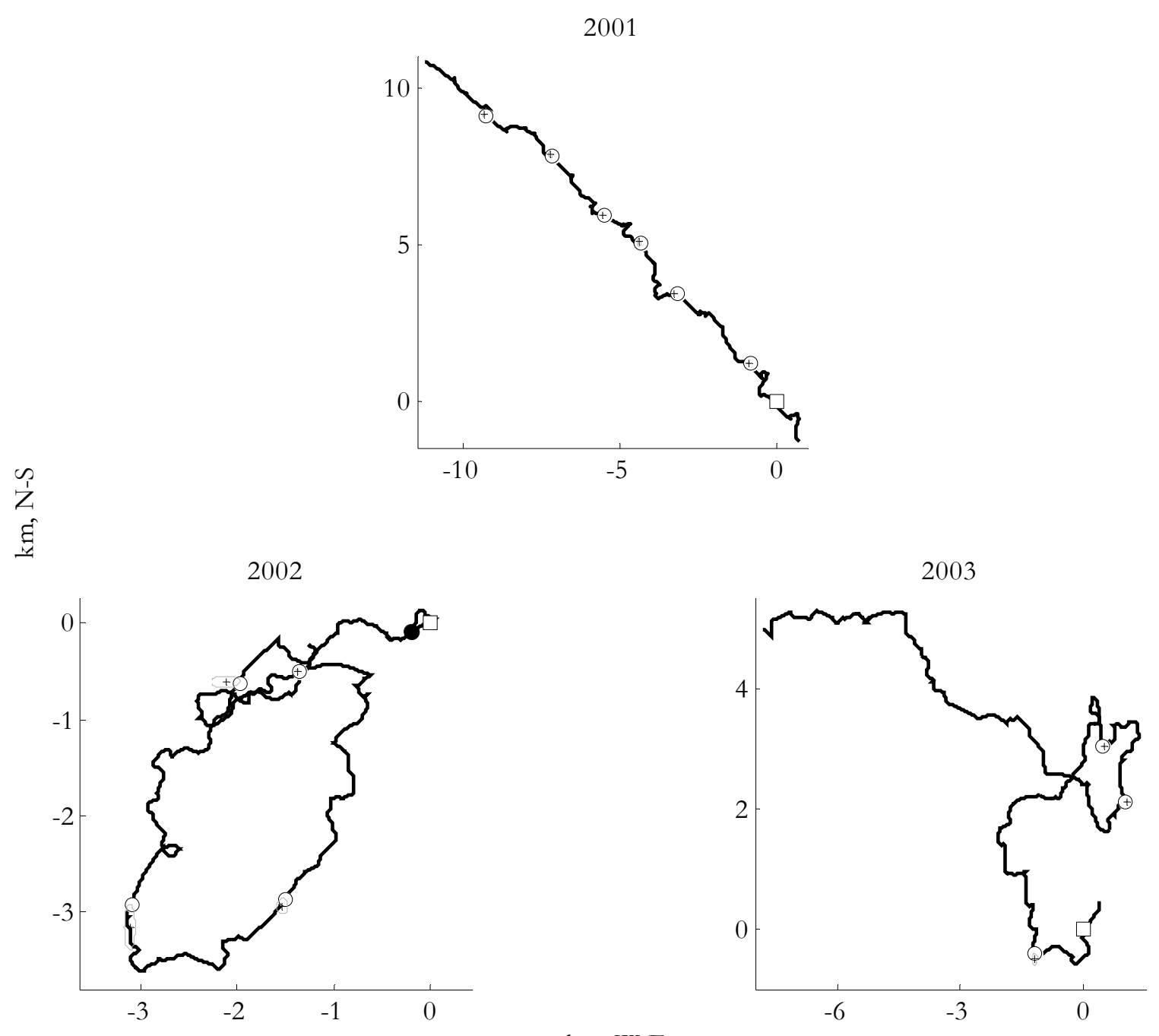

$\mathrm{km}, \mathrm{W}-\mathrm{E}$

Figure A5.1. The adjusted animal paths are displayed as the heavy, dark black lines. Observer-sighted locations (black + ) with \pm 0.1 reticule error ovals (gray) are indicated. All sightings (O) were placed within (or on) their associated error ellipses to minimize the manipulation required to adjust the remaining positions. (Some of the error ellipses are too small to be visible relative to the sighting circles.) The beginning of the whale track is indicated $(\bullet)$ and the first sighting position are marked $(\square)$.

calculating the arccosine of the dot product of the vector pointing from the vessel to the animal and the heading vector of the whale (Figure A5.3). Because we were concerned with the animal pointing towards versus away, but not left versus right, we used the absolute value of the pointing angle. 


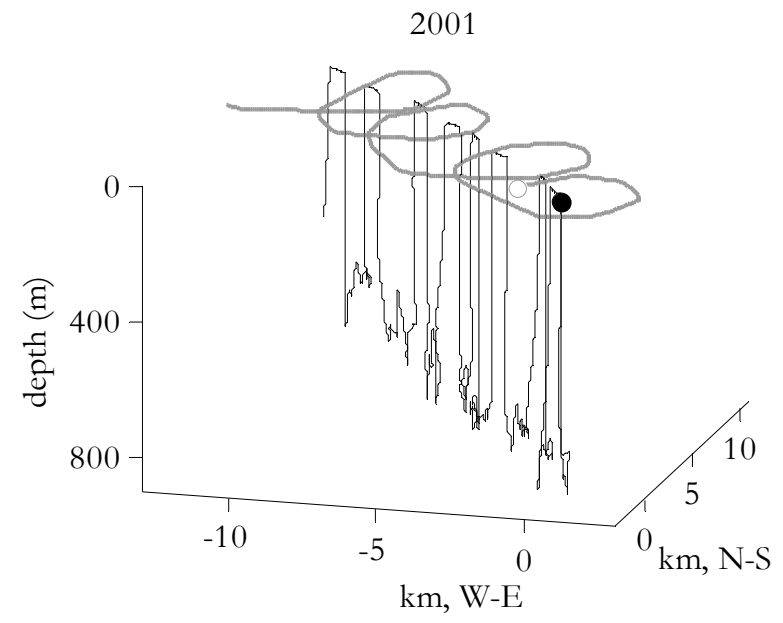

2002

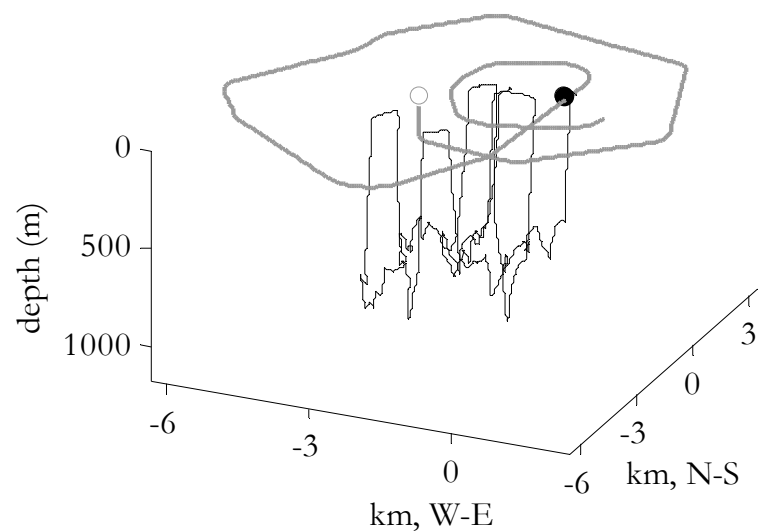

2003

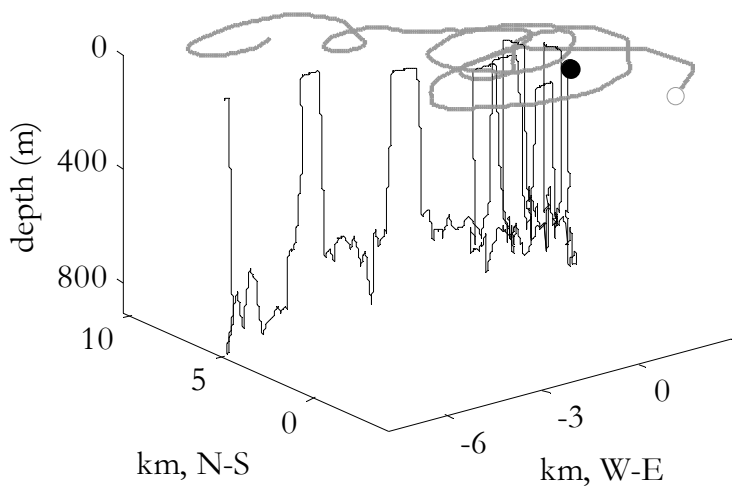

Figure A5.2. The 3D track of each sperm whale (thin black line) is displayed with the surface track of the vessel (thick gray line). The starting points of the whale and vessel paths are indicated ( $\bullet$ and $\odot$, respectively).

\section{A5.3.2.2 Assumptions}

The time-series data were determined by the angular orientation of the whale and position of the whale and the vessel. The regression model developed below assumed that any dependence of the time-series orientation data on sonar RL was not controlled by vessel movement but instead was driven by the whale altering its orientation relative to the vessel as a function of RL. This assumption was generally valid under this experimental design since the vessel was slowly steered to continue circling the whale (see Introduction) 


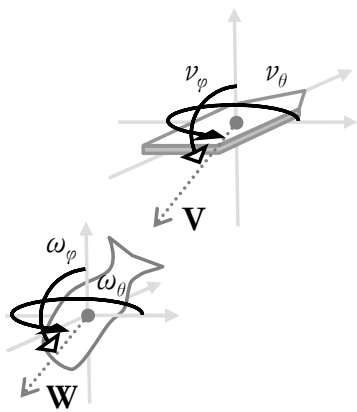

Scenario 1

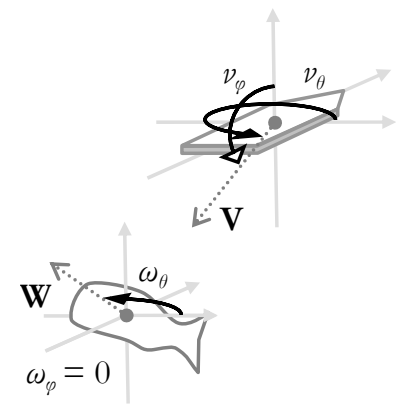

Scenario 2

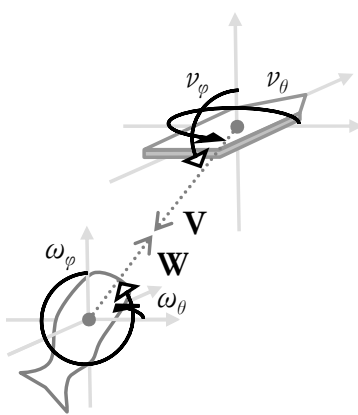

Scenario 3

Figure A5.3. Scenarios illustrating the computation of the directional time-series. Scenario 1, Animal facing away from the vessel: The vector directed from the vessel to the animal $(\mathbf{V})$ is parallel to the vector of the animal's heading $(\mathbf{W})$. In other words, $\left|v_{\theta}-\omega_{\theta}\right|=0$ and $v_{\varphi}=\omega_{\varphi}$, which means $\cos ^{-1}(\mathbf{V} \cdot \mathbf{W})=0$. Scenario 2 , Animal broadside to the vessel: Oriented broadside, the value of $\omega_{\varphi}$ is always zero. In addition, because $\mid v_{\theta}-$ $\omega_{\theta} \mid=\pi / 2, \cos ^{-1}(\mathbf{V} \cdot \mathbf{W})=\pi / 2$. Scenario 3 , Animal facing towards the vessel: $\mathbf{V}$ and $\mathbf{W}$ are anti-parallel. Now, $\mid v_{\theta}$ $-\omega_{\theta} \mid=\pi$ and $v_{\varphi}=-\omega_{\varphi}$, $\operatorname{soc} \cos ^{-1}(\mathbf{V} \cdot \mathbf{W})=\pi$.

independent of changes in RL, while the whale could change orientation in a matter of seconds. Other patterns in the movement of the vessel may have unintentionally influenced RL, however, violating this critical assumption. For example, the vessel might have steered in front of an animal that was swimming with a fairly constant heading. As the vessel pulled in front of the animal's path and the distance between the vessel and animal closed, the RL would consequently increase (given a constant SL). Because we assumed that an angular orientation changing as a function of RL was due to the whale, it would have appeared that the animal was orienting more towards the vessel with closing range and a corresponding increase in RL. Data characterized by this phenomenon were excluded from the final analysis.

\section{A5.3.2.3 Circular regression on residual deviations from path trend}

To determine whether RL affected the movement behavior of the focal animal, a regression of the directional time-series on RL was conducted. Because the angular response 
was a circular variable, ordinary regression analysis was not appropriate. We used $|\theta|$, ranging between 0 and $\pi$, as our orientation variable because we were not interested in whether whales turned left or right but only towards or away. Let $\theta$ be the true orientation of the focal animal relative to the vessel, which ranged between $\pm \pi$. The basic assumption underlying the analysis was that the probability density function (pdf) of $\theta$ has the form:

$$
f(\theta)=\frac{1}{2} \operatorname{VM}(\theta ; \mu(\mathrm{RL}), \varkappa)+\frac{1}{2} \mathrm{VM}(\theta ;-\mu(\mathrm{RL}), \varkappa)
$$

where

$$
\operatorname{VM}(\theta ; \mu, x)=\left[2 \pi I_{0}(x)\right]^{-1} \exp [x \cos (\theta-\mu)]
$$

is the von Mises pdf with mean $\mu$, concentration parameter $x$ and the zero-order Bessel function $I_{0}(\varkappa)$. The von Mises distribution is the standard distribution used in modeling circular data (see Batschelet, 1981 for a review). Under the model in (A5.1), $\theta$ followed a mixture of two von Mises distributions with opposite means, equal scales and equal mixing weights. This mixture model reflected the physical and behavioral equivalence of an animal orienting to the left or to the right of the vessel by the same angle. The mean function $\mu(\mathrm{RL})$ was assumed to have the form:

$$
\mu(\mathrm{RL})=\varrho+g(\beta \cdot \mathrm{RL})
$$

where $\varrho$ is a constant and $g(u)=2 \tan ^{-1}(u)$ (Fisher \& Lee, 1992; Fisher, 1993). It is straightforward to show that the corresponding pdf of $|\theta|$ is given by:

$$
h(|\theta|)=\left[2 \pi I_{0}(\varkappa)\right]^{-1}(\exp [\varkappa \cos (|\theta|-\mu(\mathrm{RL}))]+\exp [\varkappa \cos (\theta+\mu(\mathrm{RL}))]) .
$$

The parameter $\beta$ in this model (see (A5.3)) governed the dependence of $|\theta|$ on RL. The null hypothesis, $H_{0}: \beta=0$, that there was no such dependence could be tested against the general alternative hypothesis, $H_{1}: \beta \neq 0$, using the likelihood ratio statistic defined as: 


$$
\Lambda=2\left(\log \left(L_{1}\right)-\log \left(L_{0}\right)\right)
$$

where $\log \left(L_{1}\right)$ and $\log \left(L_{0}\right)$ are the maximized values of the log likelihood under $H_{1}$ and $H_{0}$, respectively. The log likelihood for this model is:

$$
\begin{aligned}
& L(\varrho, \beta, \varkappa)=-n \log I_{0}(\varkappa)+ \\
& \quad \sum_{j=1}^{n} \log \left(\exp \left[\varkappa \cos \left(\left|\theta_{j}\right|-\mu\left(\mathrm{RL}_{j}\right)\right)\right]+\exp \left[\varkappa \cos \left(\left|\theta_{j}\right|+\mu\left(\mathrm{RL}_{j}\right)\right)\right]\right)
\end{aligned}
$$

where the observations consist of pairs $\left(\left|\theta_{j}\right|, \mathrm{RL}_{j}\right), j=1,2, \ldots, n$. The maximum likelihood (ML) estimates of the parameters $\varrho, \beta$ and $x$ under $H_{1}$ were found by maximizing (A5.6) using a constrained nonlinear optimization routine. The procedure was identical under $H_{0}$ except that the optimization was subject to the restriction that $\beta=0$. In both cases, maximization was conducted numerically under the identifiability constraint $\mu(\mathrm{RL})>0$. In undertaking this analysis, we considered the possibility of a delayed response to sonar RL, which would have indicated that the animal was responding to the stimulus after a constant time delay. We lagged the values of sonar RL used in fitting the unrestricted model and compared alternative lags via the maximized log likelihood but because there was no

evidence of a delayed response, we proceeded by using synchronous observations of $|\theta|$ and RL.

\section{A5.3.2.4 Testing significance}

A randomization test was conducted to determine whether the null hypothesis could be rejected in favor of the alternative. To account for the serial dependence of both the RL and $|\theta|$ data, we preserved the order of each data stream in the randomization test. Under the null hypothesis that the animal's angular response was independent of RL, the $|\theta|$ data 
were rotated (with their order maintained) by a random amount relative to the RLs (also with their order maintained) and a new maximum log likelihood and set of maximum likelihood estimates of the parameters were calculated for the null and alternative models. The rotation test was conducted 100 times and the likelihood ratio test was used as described above to compare the fit of the rotated data under both models. This test assessed whether the introduction of the parameter $\beta$ into the model, indicating some dependence of angular response on RL, yielded a significantly better fit of the actual data than under the null compared to the rotated datasets. The value of $\Lambda$ from the original, synchronous data was compared to the distribution of $\Lambda$ computed from the rotated datasets to determine the significance level. This test assumed no dependence of the angular response on RL when calculating the distribution of $\Lambda$.

\section{A5.4 Results}

Descriptive measurements and plots of the ping-by-ping sound exposure levels (RL) received at each whale are presented for each controlled exposure in Table A5.1 and Figure A5.4. In all three years, the source level (SL) of the sonar began at $150 \mathrm{~dB}$ RMS re $1 \mu \mathrm{Pa}$ at $1 \mathrm{~m}$, yielding an RL at the whale below the noise floor. As the sonar SL was gradually ramped up, the target RL grew each year with the mean RL increasing from 100 in 2001 to 118 in 2002 to $129 \mathrm{~dB}$ re $1 \mu \mathrm{Pa}^{2}$ s in 2003 (for 90\% ranges and other RL units, see Table A5.2). In 2001, there were 3 exposure periods separated by pauses exceeding 4 min, and the average sonar RL for each exposure period was 91,106 , and $101 \mathrm{~dB}$ re $1 \mu \mathrm{Pa}^{2} \mathrm{~s}$. There were 4 such periods in 2002 with average RLs of 92, 116, 119, and $122 \mathrm{~dB}$ re $1 \mu \mathrm{Pa}^{2} \mathrm{~s}$. The two intervals in 2003 had average RLs of 129 and $136 \mathrm{~dB}$ re $1 \mu \mathrm{Pa}^{2}$ s. All experiments exposed the animals to 

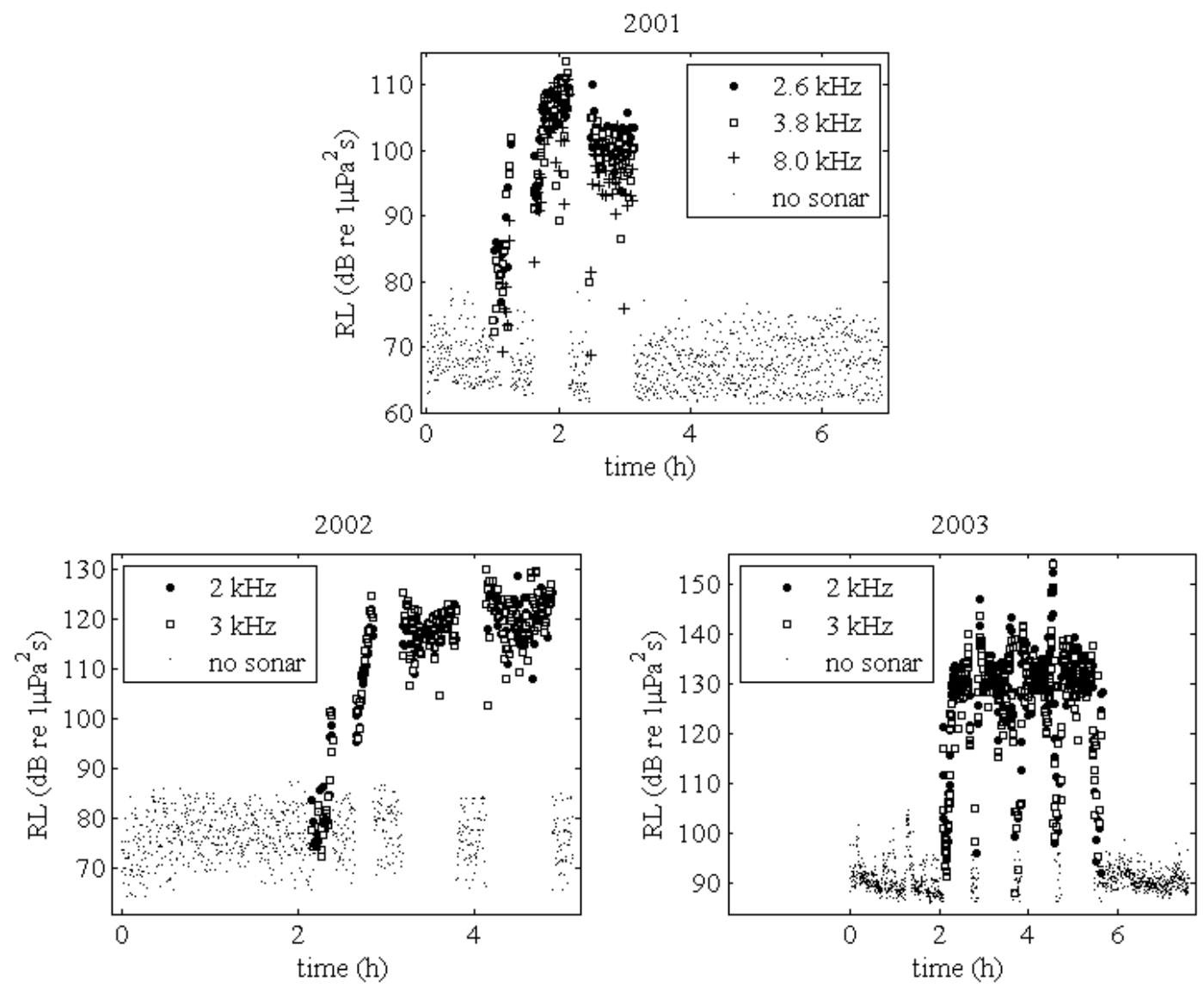

Figure A5.4. Received sound exposure level data plotted as energy flux density (dB re $1 \mu \mathrm{Pa}^{2} \mathrm{~s}$ ) as a function of time for 2001-2003.

increasing average sonar RL with each successive exposure period except 2001. In 2001 and 2003, lower frequency pings averaged about $1 \mathrm{~dB}$ higher than higher frequency pings; in 2002, the average difference between frequencies was less than $1 \mathrm{~dB}$.

The path morphologies of the animals differed substantially between the years (Figures A5.1 \& A5.2). In 2001, the sperm whale traveled mainly towards the northwest while the ship circled the whale at a range that varied between 1.2 and $5.2 \mathrm{~km}$, a pattern confirmed using a passive sonar system for acoustic localization of the animal (Zimmer et al., 2005). The tagged animal followed a curved path in 2002 with a small loop towards the 
end while in 2003 the sperm whale traced a more circuitous path initially, eventually settling on a westward travel direction. The number of complete dives and the maximum depth achieved by each animal are listed in Table A5.1. The resulting directional time-series data reveal low frequency trends from the vessel's movement about the animal superimposed on high frequency fluctuations in the animal's heading (Figure A5.5).

We excluded the 2001 dataset from subsequent analysis because the vessel passed in front of the whale, which was continuing on a steady course. This violated the assumption described in the Methods that the motion of the vessel did not cause an interaction between RL and orientation of the whale with respect to the vessel. The whale tracks in 2002 and 2003 were more variable and we did not observe the same kind of interaction as seen in 2001. We concluded that retaining the 2002 and 2003 datasets for the remainder of the analysis was appropriate.

The analysis was designed to test whether there was a relationship between RL and each whale's orientation with respect to the sound source. This allowed testing for effects such as increased avoidance with increased exposure compared to a null hypothesis of no relationship between the whale's orientation and RL. In both 2002 and 2003, we were unable to reject the null hypothesis in favor of the alternative because $\Lambda$ was not significant (2002: $P$ $=0.88,2003: P=0.73)$. No visible relationship was evident between the angular variable $|\theta|$ and RL (Figure A5.6). In particular, substantial amounts of spread characterized the $|\theta|$ data both when the source was both on and off. Similar amounts and ranges of spread among the angular orientation data were also found when comparing the ramp-up periods and the span immediately preceding them, reinforcing the conclusion of no effect. A nonuniform distribution of $|\theta|$ is present in 2002 with data mostly absent from the "towards" 
2001

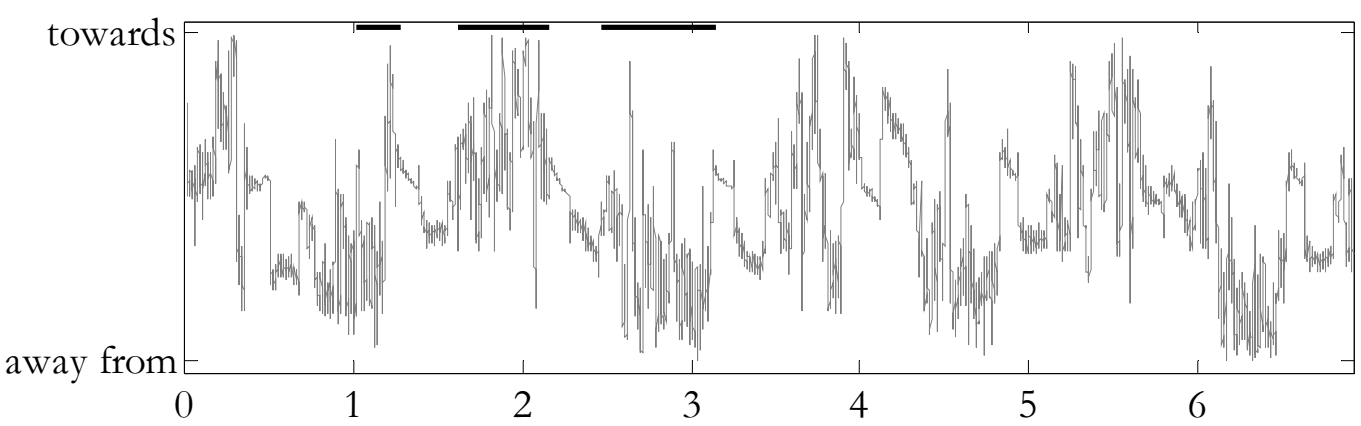

2002

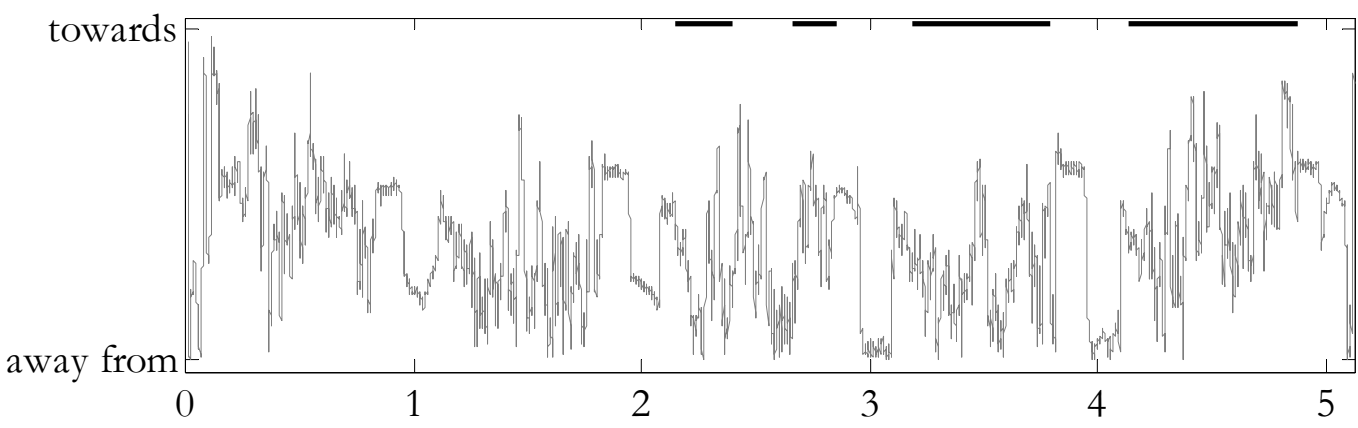

2003

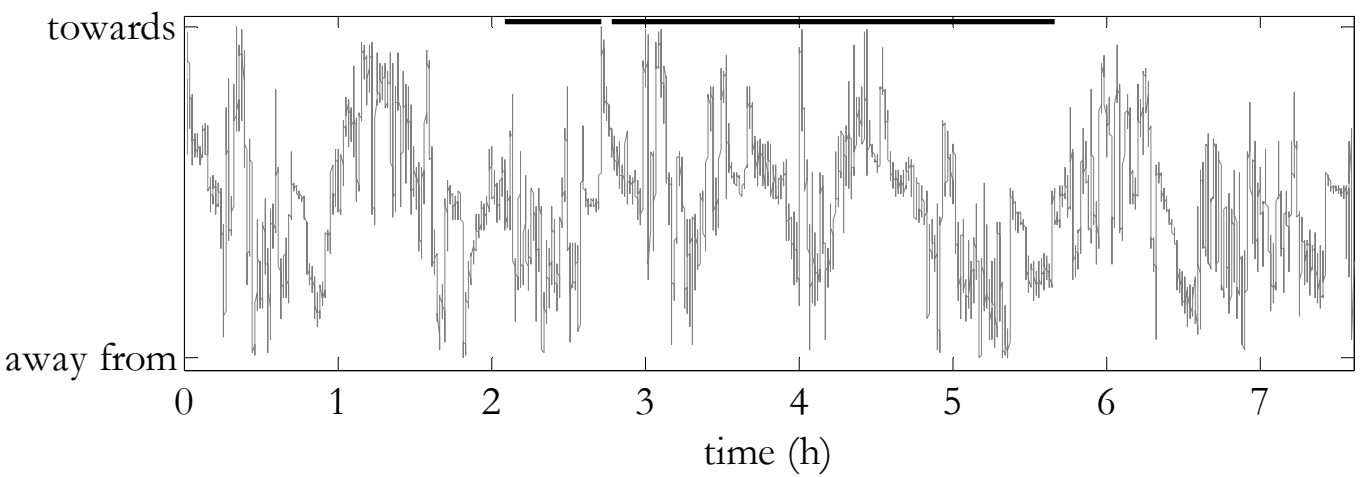

Figure A5.5. Directional time-series data plots. The ordinate ranges from 0 (away from) to $\pi$ (towards) as illustrated in Figure A5.3. Black horizontal lines at the top of the plots correspond to the intervals during which the sonar source was active. The low frequency trend of the vessel's path and the high frequency jitter of the animal's movements are simultaneously visible in these plots.

condition at all RLs (Figure A5.6). While it is possible that the whale was responding to the quiet ship when the sonar was off, we believe that the vessel's slow turning rate as it traced one larger loop followed by a smaller, shorter loop allowed the whale to remain slightly 

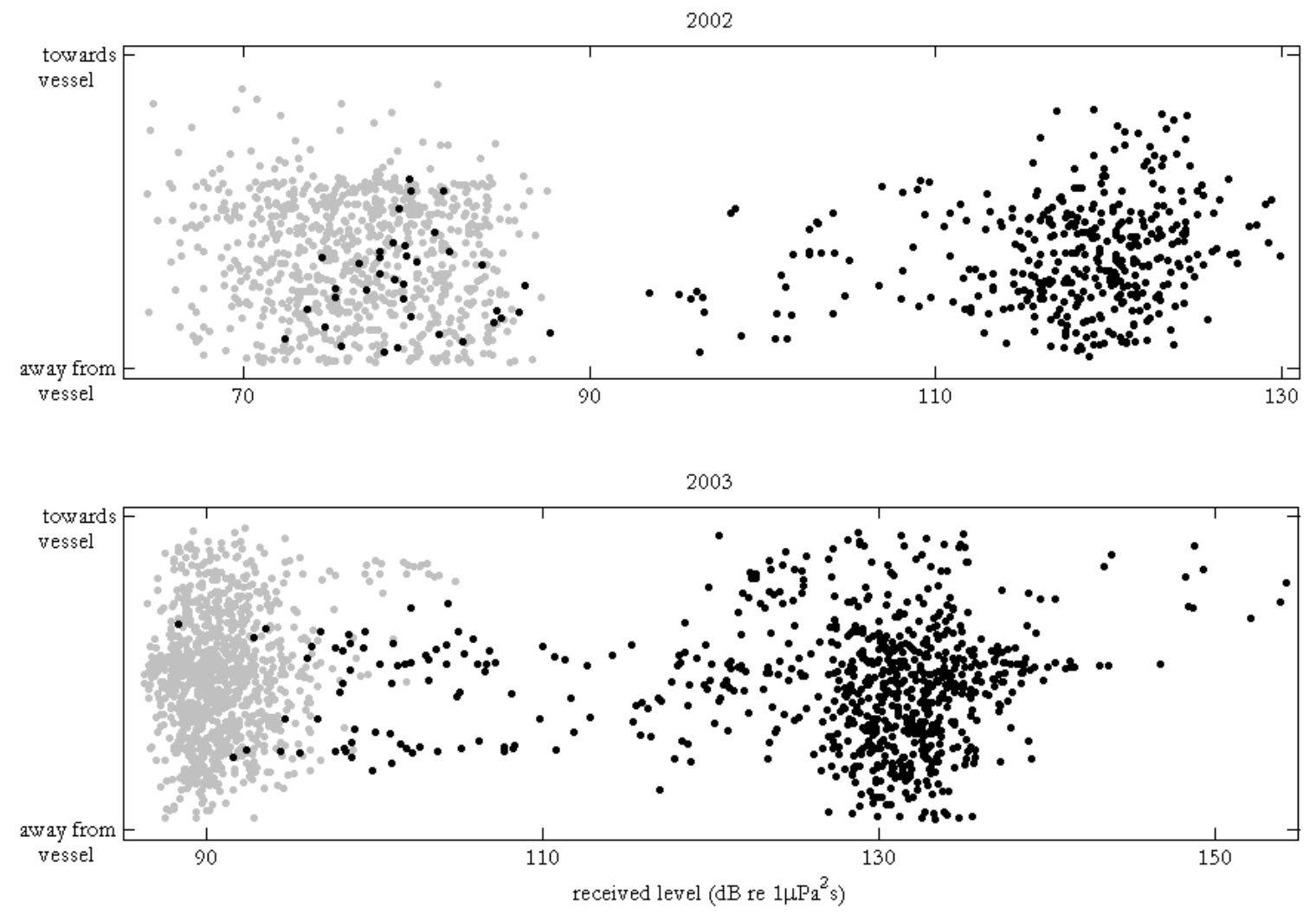

Figure A5.6. Orientation of whale with respect to the source vessel plotted as $|\theta|$ vs. sonar RL $(\bullet)$ and noise RL ( ). The ordinate range is the same as in Figure A5.4.

ahead of the vessel for the duration of the experiment. By monitoring the orientation of the whale as a function of RL in both the presence and absence of sonar, we can conclude that this non-uniform distribution was a consequence of the geometry of the ship circling the whale and did not result from the sound exposure.

\section{A5.5 Discussion}

A directional time-series of angular orientations was calculated from the 3D path of tagged sperm whales relative to a moving sonar source vessel in three separate controlled exposure experiments (CEEs). The experiment from 2001 was discarded from the full analysis because the angular response variable could have been affected by the movements 
of the vessel as well as by the animal. We were unable to reject the null hypothesis of no dependence of angular orientation on received sound exposure level (RL) in 2002 and 2003, suggesting that increasing RL over the range studied did not elicit a systematic angular response of the animals towards or away from the sonar source. The two whales behaved similarly in response to rather different exposure ranges: the minimum and maximum RLs in 2003 were roughly 16 and $24 \mathrm{~dB}$ higher than in 2002, respectively.

A substantial benefit of the analytical approach developed here is the ability to derive meaningful results from each whale in a small number of total experiments, each involving many serially dependent exposures. Earlier studies that associated each exposed individual with a single RL (see Introduction) required dozens of experiments to develop an adequate sample size for testing how responses varied with RL, a difficult undertaking given field costs, logistical challenges, and regulatory limitations. Our analysis, however, was able to evaluate a behavioral response parameter against a large range of exposure levels for each individual subject, controlling for serial dependence in the dataset. Ideally, only a handful of experiments would be required to gather enough information to relate behavioral responses to a range of RLs. However, extrapolating results from individuals to the population level would require conducting these tests on at least a few representatives of each relevant age and sex class and within each meaningful seasonal and behavioral context. The more variable the dose-response curves between individuals, the larger the sample size of subjects that would be required to draw meaningful conclusions and make informed recommendations with respect to the entire population.

The start of the exposure protocol used here involved steadily increasing the RL (Figure A5.4) in a manner consistent with the operation of anthropogenic acoustic signals 
that slowly approach while transmitting, with mitigation measures that ramp up the level of a source, and with an animal slowly approaching a stationary sound source. Ramp up procedures have commonly relied on the premise that animals will move away from a sound source at levels well below the threshold that causes injury. By the time the sound stimulus nears its full source level (SL), the exposed animals will ideally have maneuvered to a safer range where the risk of injury from the stimulus has been reduced. But it has also been suggested that ramp up may make animals more likely to habituate to lower levels of anthropogenic sounds, which may be beneficial if it prevents animals from panicking at higher (but non-injurious) levels. Alternatively, habituation could be detrimental if it led to lack of avoidance of harmful stimuli. While ramp up has become an established mitigation protocol, there have been few studies on whether the assumed avoidance behavior or habituation actually takes place (but see Malme et al., 1984; McCauley et al., 2000; Richardson et al., 1990). The predictions that ramp up will stimulate avoidance or cause habituation and potentially reduce responsiveness seem contradictory, which highlights the need for empirical studies on its effectiveness in meeting management goals. The method presented here can be used to quantify how any behavioral response relates to acoustic dosage. Using orientation as the response measure, our analysis can test the common assumption that animals will demonstrate avoidance behavior during ramp up.

When designing experiments to test the effects of ramp up versus an approach with constant SL on avoidance behavior, it is important to both titrate the levels at which avoidance might happen during a realistic exposure sequence and to balance with appropriate controls. The RLs, for example, should be spread more uniformly between the minimum and maximum exposure levels to avoid concentrating the data over the narrow 
exposure ranges analyzed here. Although this could be achieved by randomizing RL on a ping-by-ping basis between a lower and upper bound, this proposal is hardly realistic because it eliminates both the very structure of the ramp up and the animal's ability to anticipate and respond to a monotonically changing trend in exposure level. Another possibility involves the source circling the animal to maintain a constant range and balancing ramp up with ramp down exposures to cover the full RL space by changing the SL. This scenario is unrealistic for most sonars and seismic surveys, however, because vessels do not circle the animals that they encounter. Most ships towing a hydrophone, for example, must travel in a straight line during data collection, which causes a ship to pass by animals at varying ranges.

Instead, we recommend introducing each individual subject to multiple pass bys, each involving either a ramp up or a transmission using a constant SL. The order of presentation would be semi-randomized so that the first two exposure regimes would be different (i.e., ramp up and then constant or constant and then ramp up), allowing an exploration, for example, of whether ramp up can facilitate habituation. To test whether the vessel's position with respect to the whale may impact response, the design would involve balanced linear pass bys involving passing the vessel in front of and behind the animal (summarized in Figure A5.7). This setup effectively titrates the dose-response curve by allowing full coverage of the RL and bearing (from the vessel to the animal after folding from $\theta$ to $|\theta|$ ) spaces, allowing the animal to select its orientation as the vessel moves and controlling for possible position effects to avoid the problem introduced by the 2001 dataset. Multiple exposures within a single experiment allow comparisons of the direction and magnitude of responses across trials to test for habituation or sensitization across pass bys as well as within a single pass by. This experimental design therefore explores whether 


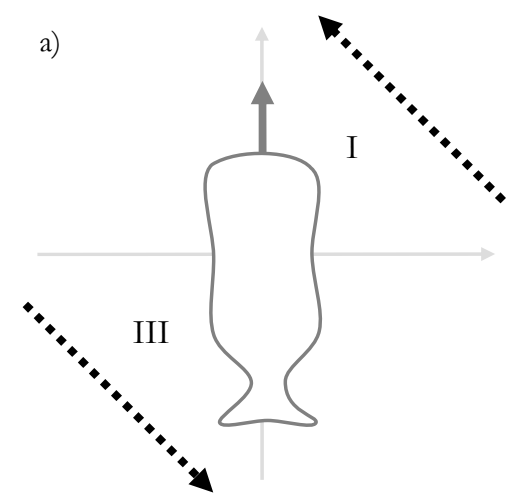

b)

Quadrant: III, I, I, III, I, I, III, ..

Ramp: $\uparrow-\uparrow \uparrow-\uparrow-\ldots$

Figure A5.7. (a) Proposed experimental design for controlled exposure experiments involving ramp up. Once the general heading trend of the study animal (thick, dark gray line) can be determined, the vessel can either close its distance with the whale by passing in front from broadside in quadrant I or move behind from broadside in quadrant III as the whale swims away from the path of the vessel (thick, dotted black lines). The source can either be ramped up $(\uparrow)$ or maintained at a constant level $\left(^{-}\right)$. Each exposure period therefore allows four possible combinations of vessel movement and source level trend. (b) A sequence was determined using a semi-randomized order; one sample realization is listed here. This design covers the full RL space and all bearing angles from the vessel to the animal (after folding from $\theta$ to $|\theta|$ ) and balances vessel approaches with retreats and increasing with constant levels. See text for further discussion.

behavioral responses are influenced by exposure level, range to the source and the approach or retreat of the vessel. Analyses from such a protocol could also explore how animals respond as a function of time and repeated exposure.

The analytical approach presented here garners the full value of each exposure by evaluating a continuous behavioral measure in relation to a full range of exposure levels. We also propose an experimental design and analysis to test whether whales do avoid sound sources at levels below those that pose a risk of injury, similar to the kinds of studies conducted with baleen whales (Malme et al., 1984; McCauley et al., 2000; Richardson et al., 1990). This is a critical assumption used to justify many mitigation measures, but has not been well tested, especially for odontocetes. As an applied research approach used to develop and implement effective and safe mitigation standards, CEEs are a useful method to provide realistic assessments of how anthropogenic sounds actually impact behaviorally 
complex marine mammals in a variety of specific settings, and to test whether common mitigation measures actually achieve their conservation goals.

\section{A5.6 Acknowledgements}

The NATO Undersea Research Centre planned and executed the sonar exposure experiments described here. The research was supported by ONR, SERDP, and the US Navy's environmental compliance division. The authors thank the R/V Alliance crew and scientific team for gathering the data analyzed here. We are grateful to J. Gordon for his advice on range calculations, P. Madsen for his guidance on computing RL and P. Miller for general assistance with the development of this manuscript. W. Zimmer and J. Haun offered helpful comments on later drafts of this manuscript. Tamara and Kristie Shapiro provided aesthetic insight into the design of the figures. A. D. S. was supported by a National Defense Science \& Engineering Graduate Fellowship and a National Science Foundation Research

Fellowship. This research adhered to all applicable legal and regulatory requirements and all institutional guidelines. The CEEs were conducted under the NMFS research permits 9811578 and 981-1707. 


\section{A5.7 References}

Batschelet, E. 1981. Circular Statistics in Biology. Toronto: Academic Press.

Blackwell, S. B., Lawson, J. \& Williams, M. T. 2004. Tolerance by ringed seals (Phoca bispida) to impact pipe-driving and construction sounds at an oil production island. Journal of the Acoustical Society of America: 115, 2346-2357. doi:10.1121/1.1701899.

Cox, T. M., Ragen, T. J., Read, A. J., Vos, E., Baird, R. W., Balcomb, K., Barlow, J., Caldwell, J., Cranford, T., Crum, L., D'Amico, A., D'Spain, G., Fernandez, A., Finneran, J., Gentry, R., Gerth, W., Gulland, F., Hildebrand, J., Houser, D., Hullar, T., Jepson, P. D., Ketten, D., MacLeod, C. D., Miller, P., Moore, S., Mountain, D. C., Palka, D., Rommel, S., Rowles, T., Taylor, B., Tyack, P., Wartzok, D., Gisiner, R., Mead, J. \& Benner, L. 2006. Why beaked whales? Report of workshop to understand the impacts of anthropogenic sound. Journal of Cetacean Research and Management: 7, 177-187.

Fisher, N. I. 1993. Statistical analysis of circular data. New York: Cambridge University Press.

Fisher, N. I. \& Lee, A. J. 1992. Regression models for an angular response. Biometrics: 48, 665-677.

Gordon, J. C. 1990. A simple photographic technique for measuring the length of whales from boats at sea. Reports of the International Whaling Commission: 40, 581-588.

Johnson, M. P. \& Tyack, P. L. 2003. A digital acoustic recording tag for measuring the response of wild marine mammals to sound. IEEE Journal of Oceanic Engineering: 28, 3-12. doi:10.1109/JOE.2002.808212.

Kinzey, D. \& Gerrodette, T. 2001. Conversion factors for binocular reticles. Marine Mammal Science: 17, 353 361.

Lerczak, J. A. \& Hobbs, R. C. 1998. Calculating sighting distances from angular readings during shipboard, aerial, and shore-based marine mammal surveys. Marine Mammal Science: 14, 590-599.

Madsen, P. T. 2005. Marine mammals and noise: Problems with root mean square sound pressure levels for transients. Journal of the Acoustical Society of America: 117, 3952-3957. doi:10.1121/1.1921508.

Madsen, P. T., Payne, R., Kristiansen, N. U., Wahlberg, M., Kerr, I. \& Mohl, B. 2002. Sperm whale sound production studied with ultrasound time/depth-recording tags. Journal of Experimental Biology: 205, 1899-1906.

Malme, C. I., Miles, P. R., Clark, C. W., Tyack, P. \& Bird, J. E. 1984. Investigations of the potential effects of underwater noise from petroleum industry activities on migrating gray whale behavior - Phase 2: January 1984 migration. Cambridge, MA: Bolt, Beranek and Newman Inc.

McCauley, R. D., Fewtrell, J., Duncan, A. J., Jenner, C., Jenner, M.-N., Penrose, J. D., Prince, R. I. T., Adhitya, A., Murdoch, J. \& McCabe, K. 2000. Marine seismic surveys -- A study of environmental implications. APPE A Journal, 692-708.

Miller, P. J. O., Biassoni, N., Samuels, A. \& Tyack, P. L. 2000. Whale songs lengthen in response to sonar. Nature: 405, 903.

Miller, P. J. O., Johnson, M. P. \& Tyack, P. L. 2004. Sperm whale behaviour indicates the use of echolocation click buzzes 'creaks' in prey capture. Proceedings of the Royal Society of London B: 271, 22392247. doi:10.1098/rspb.2004.2863.

Moore, M. J., Miller, C. A., Morss, M. S., Arthur, R., Lange, W. A., Prada, K. G., Marx, M. X. \& Frey, E. A. 2001. Ultrasonic measurement of blubber thickness in right whales. Journal of Cetacean Research and Management: 2, 301-309.

Nowacek, D. P., Johnson, M. P. \& Tyack, P. L. 2004. North Atlantic right whales (Eubalaena glacialis) ignore ships but respond to alerting stimuli. Proceedings of the Royal Society of London B: 271, 227-231. doi:10.1098/rspb.2003.2570.

Richardson, W. J., Wursig, B. \& Greene Jr., C. R. 1990. Reactions of bowhead whales, Balaena mysticetus, to seismic exploration in the Canadian Beaufort sea. Journal of the Acoustical Society of America: 79, 11171128.

Tyack, P., Gordon, J. \& Thompson, D. 2003. Controlled exposure experiments to determine the effects of noise on marine mammals. Marine Technology Society Journal: 37, 41-53.

Watkins, W. A. 1977. Acoustic behavior of sperm whales. Oceanus: 20, 50-58.

Watkins, W. A., Daher, M. A., Fristrup, K. M. \& Howald, T. J. 1993. Sperm whales tagged with transponders and tracked underwater by sonar. Marine Mammal Science: 9, 55-67. 
Watkins, W. A., Moore, K. E. \& Tyack, P. 1985. Sperm whale acoustic behaviors in the southeast Caribbean. Cetology: 49, 1-15.

Watkins, W. A. \& Schevill, W. E. 1975. Sperm whales (Physeter catodon) react to pingers. Deep-Sea Research: 33, 123-129.

Watwood, S. L., Miller, P. J. O., Johnson, M., Madsen, P. T. \& Tyack, P. L. 2006. Deep-diving foraging behaviour of sperm whales (Physeter macrocephalus). Journal of Animal Ecology: 75, 814-825. doi:10.1111/j.1365-2656.2006.01101.x.

Zimmer, W. M. X., Johnson, M. P., D'Amico, A. \& Tyack, P. L. 2003. Combining data from a multisensor tag and passive sonar to determine the diving behavior of a sperm whale (Physeter macrocephalus). IEEE Journal of Oceanic Engineering: 28, 13-28. doi:10.1109/JOE.2002.808209.

Zimmer, W. M. X., Tyack, P. L., Johnson, M. P. \& Madsen, P. T. 2005. Three-dimensional beam pattern of regular sperm whale clicks confirms bent-horn hypothesis. Journal of the Acoustical Society of America: 117, 1473-1485. doi:10.1121/1.1828501. 


\section{Appendix 6. Transmission loss Patterns from Acoustic Harassment and Deterrent Devices Do Not Always Follow Geometrical SpREading Predictions*}

\section{A6.1 Abstract}

Acoustic harassment and deterrent devices have become increasingly popular mitigation tools for negotiating the impacts of marine mammals on fisheries. The rationale for their variable effectiveness remains unexplained but high variability in the surrounding acoustic field may be relevant. In the present study, the sound fields of one acoustic harassment device and three acoustic deterrent devices were measured at three study sites along the Scandinavian coast. Superimposed onto an overall trend of decreasing sound exposure levels with increasing range were large local variations in sound level for all sources in each of the environments. This variability was likely caused by source directionality, interping source energy level variation and multi-path interference. Rapid and unpredictable variations in the sound level as a function of range deviated from expectations derived from spherical and cylindrical spreading models and conflicted with the classic concept of concentric zones of increasing disturbance with decreasing range. Under such conditions, animals may encounter difficulties when trying to determine the direction to and location of a sound source, which may complicate or jeopardize avoidance responses.

\section{A6.2 Introduction}

Marine mammals interact with aquaculture and fisheries in a variety of ways. They

\footnotetext{
* This manuscript has been submitted to Marine Mammal Science for publication with Jakob Tougaard, Poul Boel Jørgensen, Line A. Kyhn, Jeppe Dalgaard Balle, Cristina Bernardez, Arne Fjälling, Junita Karlsen and Magnus Wahlberg as co-authors.
} 
can consume stocks or catch directly, inflict harm upon the catch and the fishing gear, introduce fecal coliform bacteria or parasites, and become severely or fatally caught in the gear (reviewed in Hammond \& Fedak, 1994; Dawson et al., 1998; Nash et al., 2000). These interactions should be limited both to protect the animals and to reduce the economic losses incurred by the fisheries. Acoustic approaches have been developed to alert the animals to the presence of gear or to encourage them to vacate an area (see Jefferson \& Curry, 1996 for a review). Repeated usage of an offensive stimulus, however, can lead to habituation, sensitization, attraction (once the sound has been associated with the presence of food) or, if loud enough, hearing damage. The use of gunshots, explosives, firecrackers and biological sounds have been largely ineffective in deterring marine mammals from fisheries, possibly for the reasons mentioned above (Shaughnessy \& Semmelink, 1981; Jefferson \& Curry, 1996).

The playback of artificial sounds intended to mitigate conflicts between marine mammals and fisheries have met with mixed results. Such playback devices can be separated into two categories. Low level acoustic deterrent devices (ADDs, commonly referred to as "pingers") are designed to displace animals temporarily from a region. On the other hand, high level acoustic harassment devices (AHDs, or "seal scarers") are loud enough to cause pain and discourage predation (e.g., Milewski, 2001). ADDs and AHDs differ in their output source energy levels (SLs) and frequency bands. ADDs typically operate in the 10-100 kHz band and emit SLs below $150 \mathrm{~dB}$ re $1 \mu \mathrm{Pa}^{2} \mathrm{~s} @ 1$ m, whereas AHDs operate mainly between 5 and $30 \mathrm{kHz}$ at levels often exceeding $170 \mathrm{~dB}$ re $1 \mu \mathrm{Pa}^{2} \mathrm{~s} @ 1$ m (Northridge et al., 2006). (See Madsen, 2005 for an explanation of level measurements and units.)

ADDs and AHDs are currently used to mediate many marine mammal-fishery 
interactions worldwide. After introducing ADDs, several studies have documented actual changes in the behavior of harbor porpoises (Phocoena phocoena), one of the species most at risk of by-catch, leading to a reduction in entanglement (e.g., Kraus et al., 1997; Trippel et al., 1999) and in local abundance (Johnston, 2002; Olesiuk et al., 2002). More than half of the New Zealand Hector's dolphins (Cephalorhynchus hectori) observed in one study avoided “white pinger" ADDs (manufactured by Dukane ${ }^{\circledR}, f_{0}=9.6 \mathrm{kHz}$, pulse length $=400 \mathrm{~ms}$ ) attached to gillnets (Stone et al., 2000). In a trial involving AHDs in the Baltic Sea, depredation losses of salmon in traps due to gray seals (Halichoerus grypus) were halved, doubling the landed catch (Fjälling et al., 2006). Also, killer whales (Orcinus orca) were strongly displaced by AHDs in a study conducted in British Columbia (Morton \& Symonds, 2002). As a result of these kinds of findings, ADDs and AHDs have become increasingly popular for abating marine mammal interactions with fisheries (Johnston \& Woodley, 1998). Indeed, pingers are now mandatory in several types of gill-net fisheries around the world and have been suggested as a possible mitigation solution to by-catch associated with commercial trawling (de Haan et al., 1997; Reeves et al., 2001).

Not all experiments, however, have encountered this level of success. Cox et al. (2001) reported habituation of free-ranging harbor porpoises to one Dukane NetMark 100 pinger $(10 \mathrm{kHz}, 132 \mathrm{~dB}$ re $1 \mu \mathrm{Pa} @ 1 \mathrm{~m})$. These animals partially habituated to both Airmar $\left(10 \mathrm{kHz}, 132 \mathrm{~dB}\right.$ re $\left.1 \mu \mathrm{Pa}_{\mathrm{RMS}} @ 1 \mathrm{~m}\right)$ and SaveWave Black Save pingers $(30-160 \mathrm{kHz}, 155 \mathrm{~dB}$ re $1 \mu \mathrm{Pa}_{\mathrm{RMS}} @ 1 \mathrm{~m}$ ) over a 48 -day course involving repeated activation and deactivation of these devices (Jørgensen, 2006). Quick et al. (2004) reported survey results indicating that despite the elevated usage of AHDs, damage to Scottish marine salmon farms by harbor (Phoca vitulina) and gray seals increased between 1987 and 2001. Similarly, sea lions (Otaria 
flavescens) damaged catches in gillnets containing active pingers more often than those without pingers (Bordino et al., 2002). The by-catch levels of Franciscana dolphins (Pontoporia blainvillę), however, did fall in this same study when the pingers were active. The mechanisms leading cetaceans and pinnipeds to avoid or become attracted to fishing operations with functional ADDs and AHDs remain uncertain (Kraus, 1999; Quick et al., 2004; but see Akamatsu et al., 1996; Kastak et al., 2005; Kastelein et al., 2006 for explorations of tolerance and habituation thresholds in seals and sea lions). This calls for research that examines how ADDs and AHDs actually function and transmit signals into the water. Quantifying the sound exposure level (SEL) of these devices will yield an improved understanding of the acoustic field to which animals are exposed when approaching a pinger underwater. Simple spherical and cylindrical spreading models and their associated zones of increasing impact with decreasing range (Richardson et al., 1995) may not be applicable for sound transmission in every instance (e.g., DeRuiter et al., 2006; Madsen et al., 2006). Although Terhune et al. (2002), for example, depicted that received levels varied greatly as a function of range for AHDs in the Bay of Fundy, Canada, the sound field of an ADD in the same area displayed less variability with range (e.g., Cox et al., 2001).

The nature of the sound field may be highly dependent on several factors including geographic location, habitat morphology, the time-frequency characteristics of the emitted signals, and the depth of source and receiver. Shallow water can lead to multi-path propagation in which sound reflected off of both the water's surface (including associated wave action) and the ocean bottom interferes constructively and destructively to create a complicated pattern of signal intensity as a function of range. This phenomenon may make it quite difficult to move away from a sound source by swimming down an intensity gradient 
in order to minimize exposure. A detailed characterization of the sound fields of these devices is needed to understand their possible influence on marine mammal behavior.

In this study, we test whether typical ADD and AHD signals propagate according to the spherical or cylindrical spreading that is generally assumed when discussing zones of increasing impact (Richardson et al., 1995). We also explore the issue of variable SELs at close and distant ranges to several types of pingers and a single AHD in three shallow water environments in Sweden and Denmark.

\section{A6.3 Materials and Methods}

\section{A6.3.1 Field sites}

Three study sites were selected for the sound transmission experiments (Figure A6.1). The first was situated in a bay south of the island of Saltö, Sweden (referred to here as the "Saltö" field site, $\left.58^{\circ} 51.7^{\prime} \mathrm{N}, 11^{\circ} 08.6^{\prime} \mathrm{E}\right)$. The bottom of the bay was relatively smooth, 13-20 m deep and was comprised of a mixture of mud and sand patches. Saltö was utilized on 5 June (SSs for Saltö, Sweden, summer) and 23, 24 and 29 September 2005 (SSf for Saltö, Sweden, fall). The second field site, used on 23, 24, and 29 September 2005, was located in another bay on the eastern side of the island of Sydkoster (referred to here as the

“Kosterhamn” or KSf field site, $\left.58^{\circ} 52.7^{\prime} \mathrm{N}, 11^{\circ} 05.4^{\prime} \mathrm{E}\right)$. The sandy seafloor graded smoothly from a depth of $12 \mathrm{~m}$ where the experiment was conducted to more than $20 \mathrm{~m}$ at the entrance of the deep fjord. The final site employed on 9 September 2005 was located in the shallow, sloping waters (5-15 m) of Jammerland Bay, Storebælt, Denmark (called “Jammerland" or JDf here, $55^{\circ} 36.0^{\prime} \mathrm{N}, 11^{\circ} 05.1$ 'E) and was characterized by a hard, sandy bottom. These sites were representative of locations with respect to depth, topography, and 


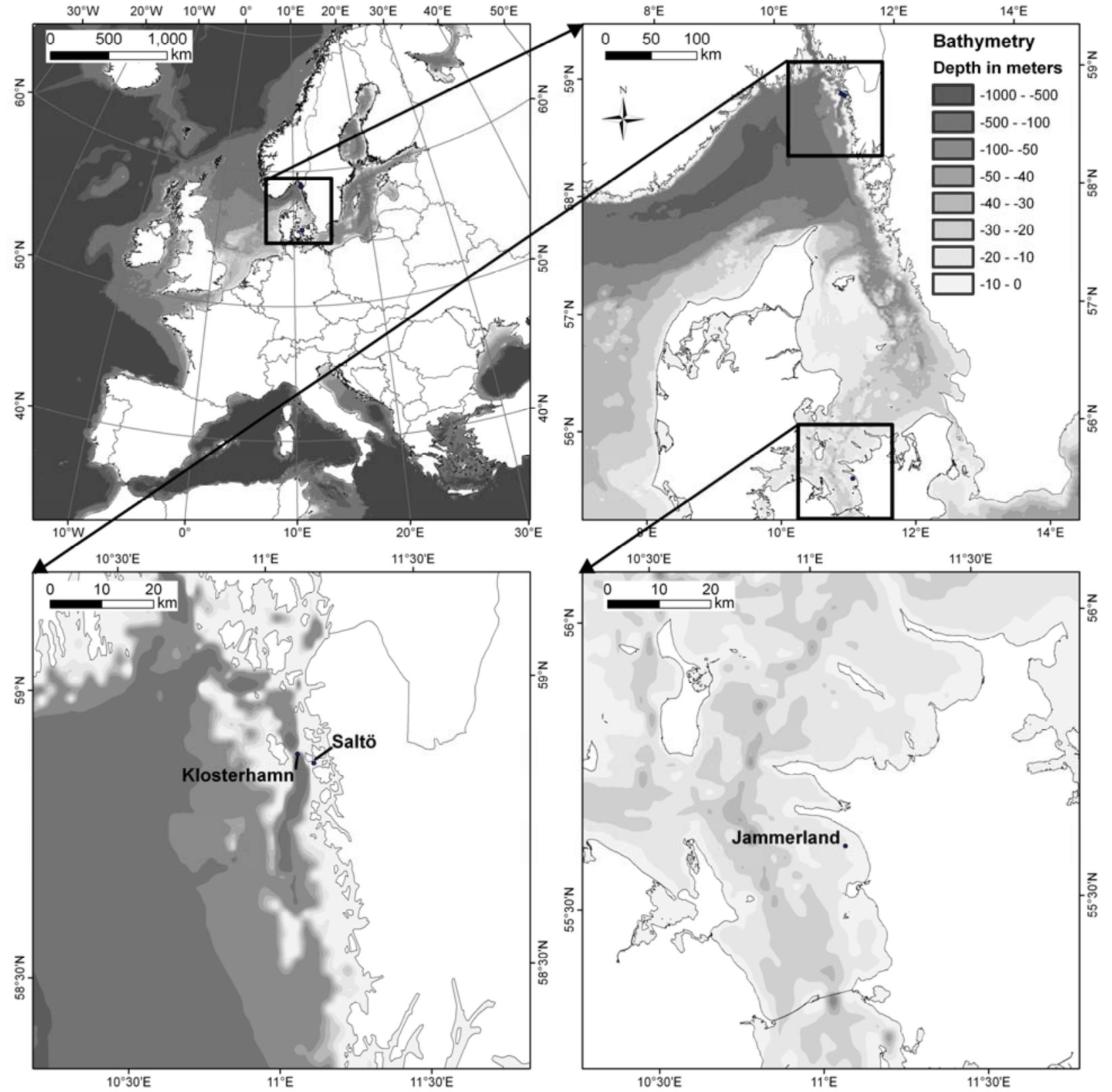

Figure A6.1. Maps of study locations.

bottom structure where pingers have been deployed by the fisheries. For all sites, sea state varied between 0 and 2 during recordings. 


\begin{tabular}{|c|c|c|c|c|c|c|}
\hline Sound source & Manufacturer & Field site $^{\mathrm{a}}$ & $\begin{array}{c}\text { Approximate } \\
\text { source level } \\
\text { (dB re } 1 \mu \mathrm{Pa} \\
\text { RMS@1 } 1 \mathrm{~m} \text { ) }\end{array}$ & $\begin{array}{c}\text { Frequency } \\
(\mathrm{kHz})\end{array}$ & Signal type ${ }^{b}$ & $\begin{array}{c}\text { Average } \\
\text { duration }(\mathrm{ms})\end{array}$ \\
\hline ADD & Airmar & "SSf, KSf & 132 & 9.8 & C & 300 \\
\hline ADD & Airmar & JDf & 132 & 10 & C & 300 \\
\hline ADD & Aquamark & SSf, KSf & 145 & $20-160$ & $\mathrm{C}, \mathrm{S}$ & 300 \\
\hline ADD & SaveWave & JDf & 155 & $30-120$ & $\mathrm{~S}^{\mathrm{c}}$ & $200-425$ \\
\hline AHD & Lofitech & SSs, KSf & 193 & 15.6 & $\mathrm{C}$ & 200 \\
\hline
\end{tabular}

a SSs: Saltö, Sweden, spring; KSf: Kosterhamn, Sweden, fall; SSf: Saltö, Sweden, fall; JDf: Jammerland, Denmark, fall

b C: constant frequency; S: frequency sweep

c The SaveWave pinger produced a series of upward-modulated frequency sweeps, which were of variable duration and rich in harmonics. The SLs of these signals were similar. Sweeps were repeated up to 4 times per signal. Signals were repeated with a variable interval of up to several tens of seconds. All parameters changed randomly from one signal to the next.

Table A6.1. Specifications of sound sources described in this study.

\begin{tabular}{c|c|c|c} 
Field site & Hydrophone & Recording unit & Sound source \\
\hline \hline SSs & BK 8101 & DAT & AHD \\
\multirow{2}{*}{ SSf } & Reson TC 4032 & & Airmar \\
& Reson TC 4034 & DAB & Aquamark \\
\hline \multirow{2}{*}{ KSf } & Reson TC 4032 & & Airmar \\
\cline { 2 - 2 } & Reson TC 4034 & & AHD, Aquamark \\
\hline \multirow{2}{*}{ JDf } & Reson TC 4032 & DAB & SaveWave, Airmar
\end{tabular}

Table A6.2. Equipment used at each field site with corresponding amplification and filtering details. Abbreviations: B\&K = Brüel and Kjær (Danish hydrophone company), DAT = Digital Audio Tape Recorder, $\mathrm{HP}=$ high pass filter; LP = low pass filter, DAB=Data Acquisition Board. SSs: Saltö, Sweden, spring, KSf: Kosterhamn, Sweden, fall, SSf: Saltö, Sweden, fall, JDf: Jammerland, Denmark, fall. All hydrophones were calibrated in the laboratory before fieldwork.

\section{A6.3.2 Sound sources}

Table A6.1 lists the specifications for the sound sources and Figure A6.2 provides the waveforms, spectra and spectrograms of the acoustic output of each device.

\section{A6.3.3 Experimental protocol}

There were a few differences in how the data were gathered and the setup of the recording chain between the field sites. Details of the equipment variability are listed in 

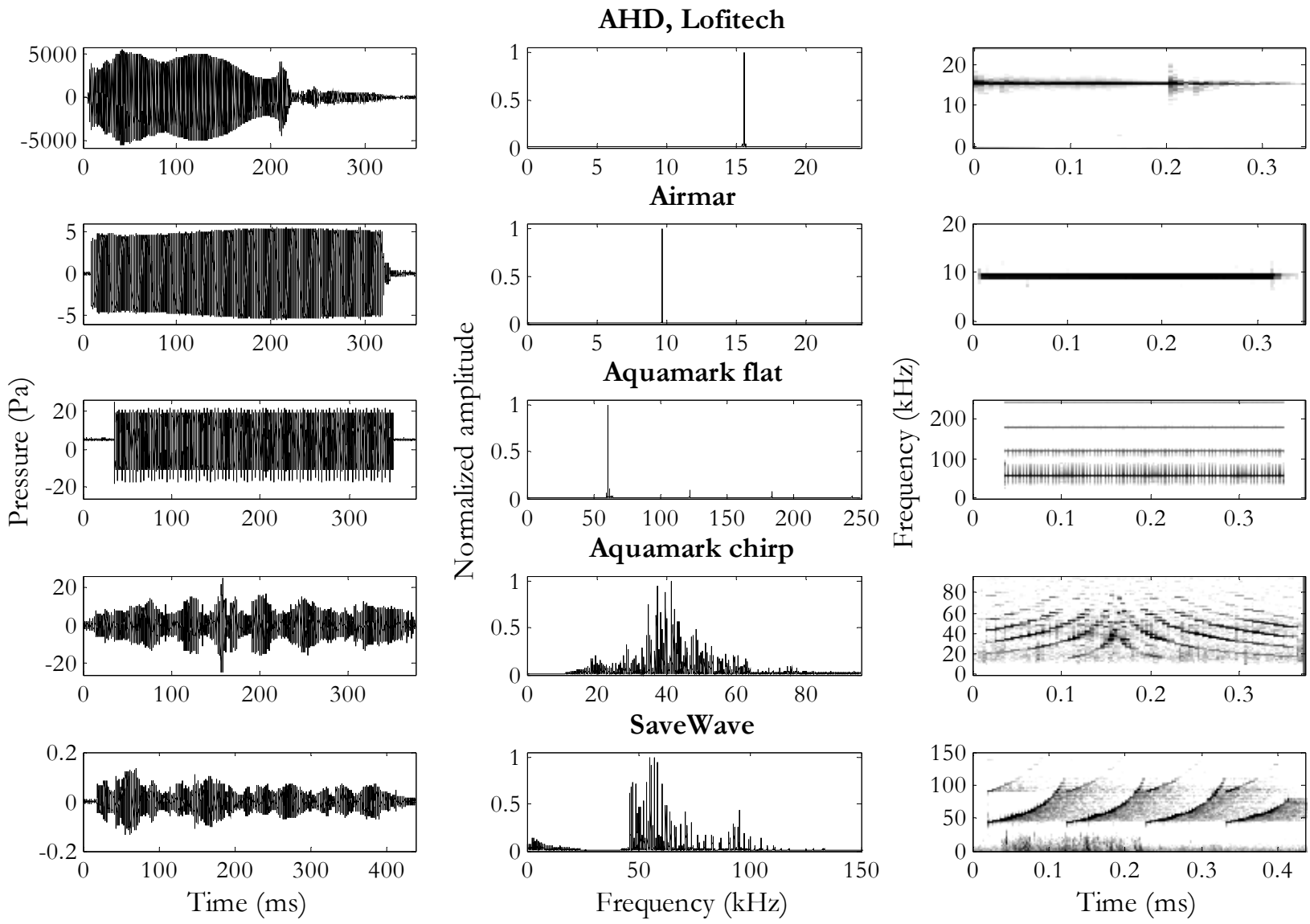

Figure A6.2. Waveforms (left), spectra (center) and spectrograms (right) for each of the sound sources. The SaveWave signal was an example taken from the larger repertoire of signals (see Table A6.1). Sweep duration, start and end frequencies and number of repetitions changed randomly from signal to signal.

Table A6.2. The sound sources were deployed singly at a fixed depth either by suspending them from a buoy or the edge of a boat at the two Swedish sites. Measurements at Jammerland took place as part of a separate study on habituation of porpoises to pingers and employed a 5 x 3 array of 15 SaveWave pingers spaced $200 \mathrm{~m}$ apart and a $5 \times 11$ array of 55 Airmar pingers spaced $100 \mathrm{~m}$ apart. All pingers were attached approximately $0.5 \mathrm{~m}$ below the surface at the end of buoys measuring $2 \mathrm{~m}$ in length (fashioned from bamboo sticks lashed to a lead weight and a Styrofoam float). The two arrays were separated by about 5 


\begin{tabular}{c|c|c|c} 
Sound source & Field site & Recording duration (min) & Number of signals measured \\
\hline \hline \multirow{2}{*}{ Lofitech AHD } & KSf & 54 & 388 \\
\cline { 2 - 4 } & SSs & 93 & 538 \\
\multirow{3}{*}{ Airmar ADD } & SSf & 41 & 423 \\
& KSf & 62 & 211 \\
\cline { 2 - 4 } & JDf & 12 & 35 \\
\hline \multirow{2}{*}{ Aquamark ADD } & SSf & 41 & 58 \\
\hline SaveWave ADD & KSf & 62 & 50 \\
\hline
\end{tabular}

Table A6.3. Recording duration and number of signals analyzed for each sound source and field site. See Table A6.1 for abbreviations.

$\mathrm{km}$.

Recordings at all sites were made by towing a previously calibrated hydrophone from a small boat that drifted or was rowed very slowly past the sound source to cover both distant and close ranges. The Reson TC 4032 and BK 8101 hydrophones had cylindrical elements and became directional receivers at frequencies above $20 \mathrm{kHz}$. The Reson TC 4034 had a spherical element and was thus omni-directional at all frequencies. All hydrophones were calibrated in the laboratory before experiments commenced to ensure that sensitivities were in agreement with the standards given by the producers. For one set of experiments (SSs, JDf), the depth of the hydrophone was held constant at 2, 3 or $5 \mathrm{~m}$. For the other experiments (SSf, KSf), a Star-Oddi CTD tag was attached $10 \mathrm{~cm}$ above the hydrophone element. This tag logged depth, salinity and temperature once every second and the data were downloaded at the end of each experiment. The sampling rates for all experiments ranged between 48 and $500 \mathrm{kHz}$ depending on the recording system and the pinger that was being characterized. All data from the recording unit were stored on a laptop computer. Table A6.3 lists the recording duration and number of signals analyzed for each experiment. A handheld GPS was used at the Jammerland field site to provide the 
location of the sound sources. At the two other sites, a frequency shift keying (FSK)-

modulated representation of GPS location was synchronously recorded to allow subsequent pairing of all received signals with their absolute locations (see Møhl et al., 2001).

The SL and directionality of the AHD were measured in a harbor near the field site prior to the field experiment. No boat activity was present at the time of this test. For the Airmar and Aquamark pingers, the measurements were made in an echo-free tank. The hydrophone was fixed one meter from the transmitting element of the ADD or AHD and the entire setup was lowered to depth. To evaluate the directionality of the ADD or AHD, SL was calculated from several pings emitted at each of several orientations of the ADD or AHD relative to the hydrophone.

\section{A6.3.4 Ping detection}

Using customized Matlab (Mathworks, Inc.) software, ping detection was partially automated by locating ping events in the recording that exceeded a user-defined amplitude threshold. To qualify for analysis, a ping needed to fulfill 3 criteria. It had to 1) be at least $10 \mathrm{~dB}$ louder than an interval of silence of the same duration immediately preceding the ping, 2) correspond to the durations listed in Tables A6.1 and A6.3) be confirmed by the user. Signals from Jammerland were identified manually in the recordings because they were characterized by a poorer signal-to-noise ratio (SNR) resulting from the greater distances separating the pingers from the hydrophone. 


\title{
A6.3.5 Calculations
}

\author{
A6.3.5.1 Range
}

The latitude, longitude and depth of each source and receiver were all converted into 3D meter space. At the Jammerland field site, the Cartesian distance between the receiver and the closest pinger source was computed as the range. For the two other sites, the Cartesian distance was simply calculated between the receiver and the single source.

\section{A6.3.5.2 Sound Exposure Level (SEL)}

All pings of constant frequency (see Table A6.1) were band-pass filtered around their central frequency using a two-pole Butterworth filter to exclude extraneous, non-ping energy. For frequency sweep signals, a two-pole Butterworth band pass filter was applied above and below the lowest and highest frequencies contained within the signal. The received acoustic energy of every ping was computed as the energy flux density, or SEL (for sound exposure level), defined as the logarithm of the sum of the squared pressure over the ping duration in $\mathrm{dB}$ re $1 \mu \mathrm{Pa}^{2} \mathrm{~s}$ :

$$
\mathrm{SEL}=10 \log \int_{0}^{T} p^{2}(t) d t=10 \log \left(\frac{1}{T} \int_{0}^{T} p^{2}(t) d t\right)+10 \log (T)+120
$$

where $p(t)$ is the instantaneous pressure at time $t$ and the duration $T$ of the signal contains 90\% of the energy (Blackwell et al., 2004; Madsen, 2005). A calibration signal of known sound level was routed through the entire recording chain and used as a reference for the computations.

The SaveWave signals contained energy beyond the range of the flat frequency response of the hydrophone. To compensate for this reduced sensitivity, these signals were 
Airmar

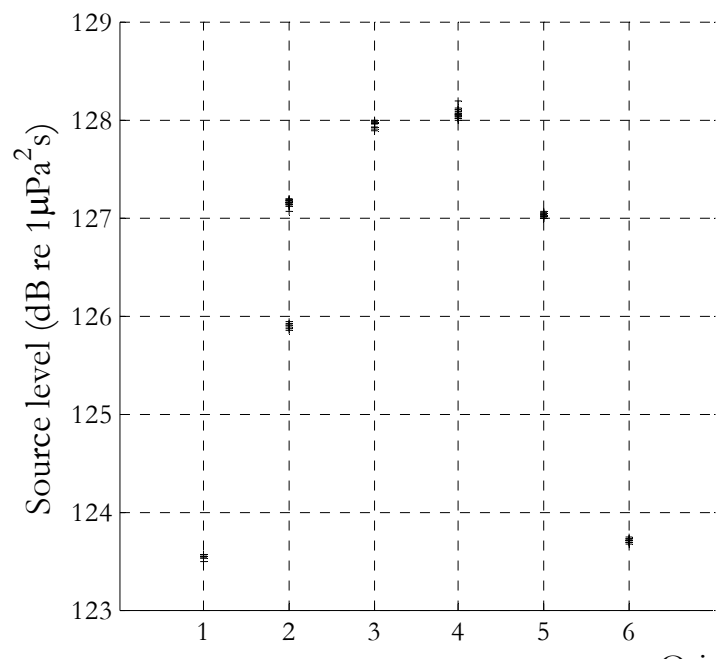

Aquamark 100

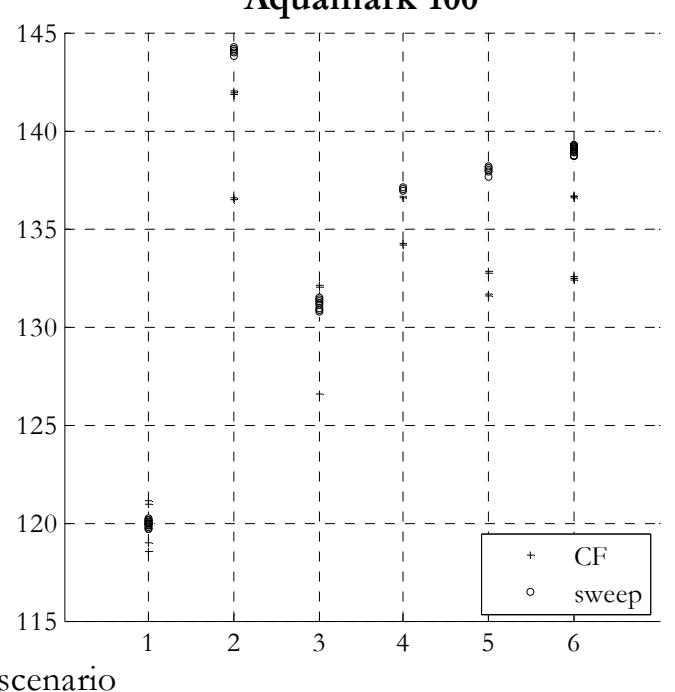

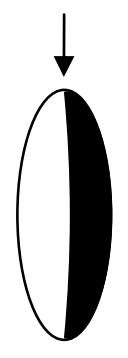

1

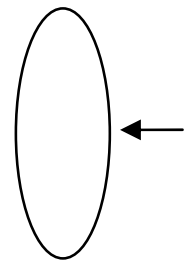

2

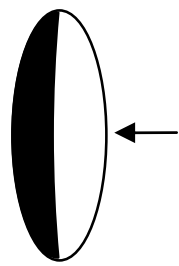

3

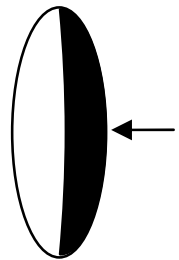

4

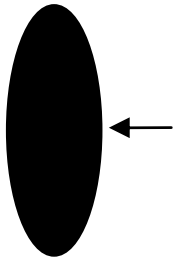

5

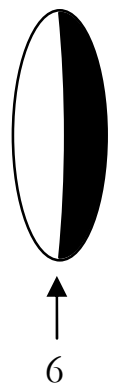

Figure A6.3. Source energy level (at one meter distance) of the Airmar and Aquamark pingers recorded in various directions. The levels of the $\mathrm{CF}$ (constant frequency) and sweep ping are denoted uniquely ( + and $\circ$, respectively). The orientation scenarios 1-6 of the pingers and receivers are illustrated graphically beneath the plots. The pinger (black and white oval) was recorded from the direction indicated by the origin of the arrow. The first pinger was recorded from its north pole, the middle four from the equator at four different pinger orientations and the final image from the south pole.

adjusted by amplifying the high frequencies in this range. At the greatest distances where the SNR was poor, the SELs from the SaveWave were calculated once the energy of the background noise immediately preceding the signal was subtracted. Airmar recordings from Jammerland were similarly characterized by a poor SNR at large distances. These ping levels were therefore determined by the peak of the average power spectrum calculated over the complete signal duration. 


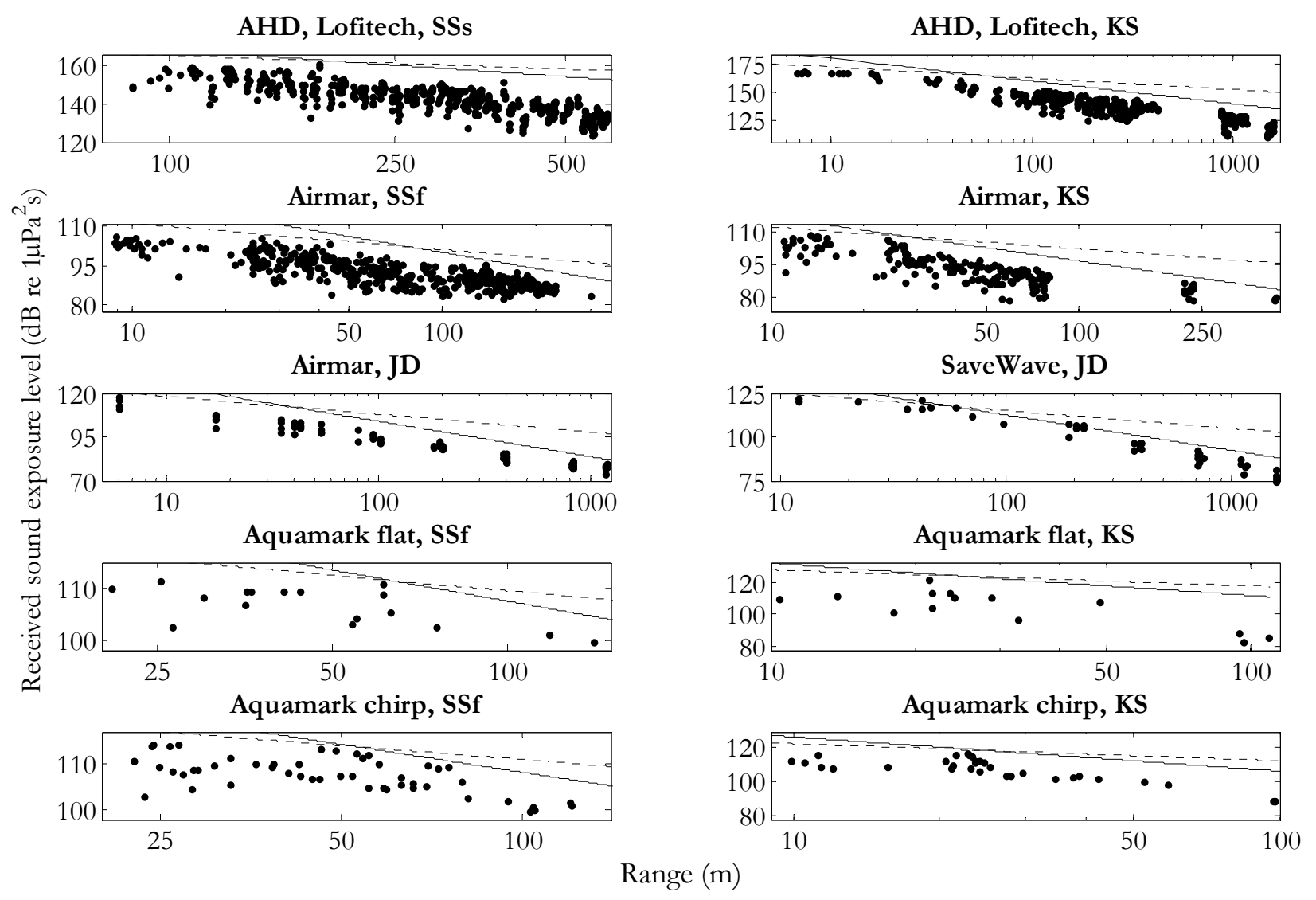

Figure A6.4. Received sound exposure level as a function of range. Slopes obeying cylindrical and spherical spreading laws and absorption are shown by the dotted and solid lines, respectively.

\section{A6.4 Results}

Figure A6.3 displays the SL measurements of the Airmar and Aquamark in different directions, revealing anomalies of up to 4.7 and $25.7 \mathrm{~dB}$, respectively. Figure A6.4 plots SEL as a function of range for all sound sources in each environment. The lines indicating spherical and cylindrical spreading are not intended to compare the expected and actual SELs but rather to show patterns of the slope predicted by these basic models. Figure A6.4 illustrates that despite an overall trend for SEL to decrease with increasing distance, a tremendous amount of dynamic range in the SEL existed over a given range. This 


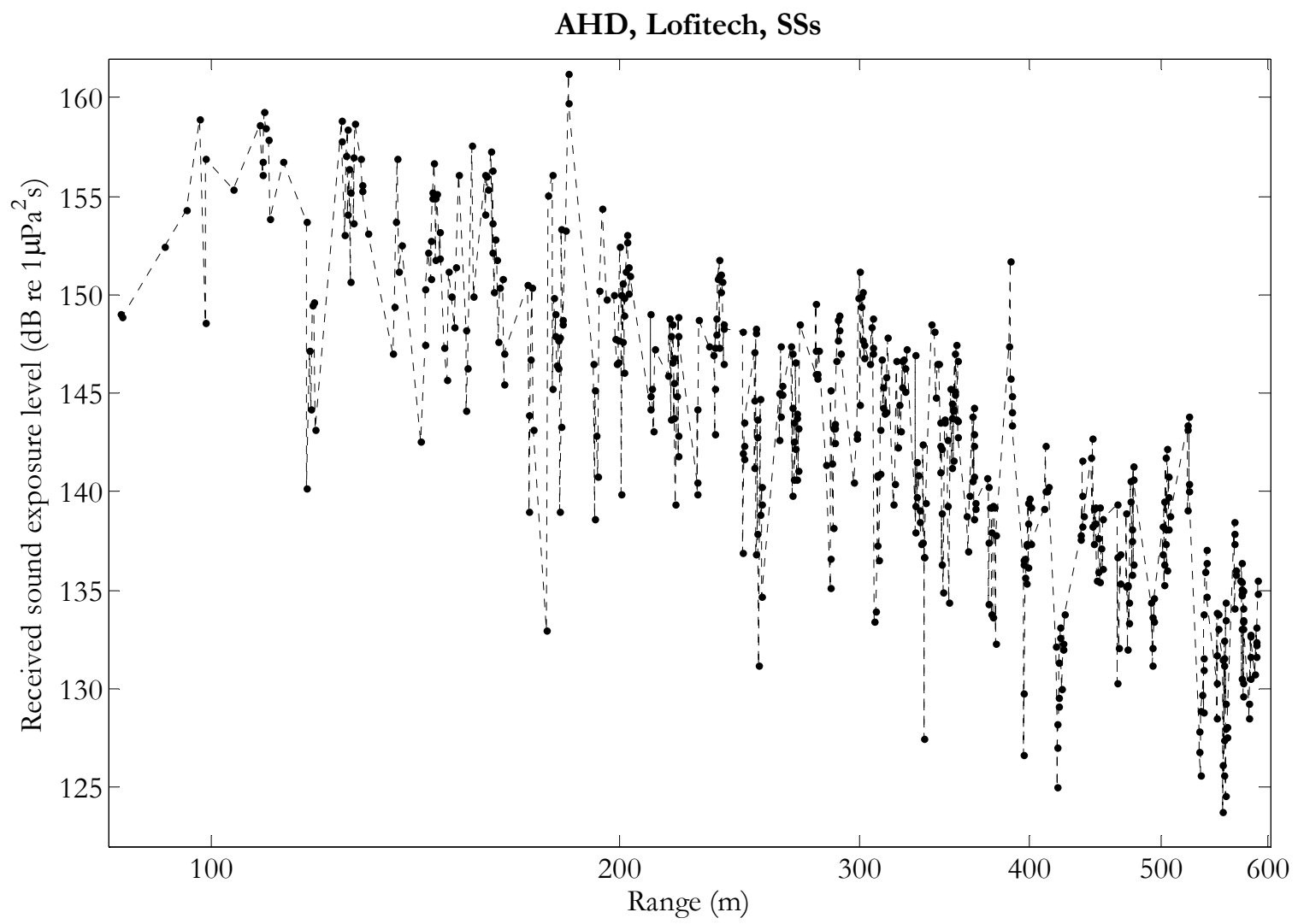

Figure A6.5. Received sound exposure level from a Lofitech AHD source as a function of range for a recording using a hydrophone that continuously approached a stationary pinger. Imagining an animal moving along a trackline similar to the one here, a steadily reliable decrease with increasing range would not occur since the levels fluctuate dramatically. See text for further elaboration.

phenomenon appeared consistently in the plots for all of the sound sources and environments.

The upper left subpanel of Figure A6.4 is enlarged in Figure A6.5 to show that fluctuations in SEL at a particular range were often much greater than those between two rather different ranges. Figure A6.5 can also be viewed as the series of SELs that an animal would encounter if it were traveling directly towards or away from the AHD Lofitech source. An animal traveling away from the AHD would experience a constantly fluctuating SEL, generally trending downwards, but with successive pings in the sequence increasing 
and decreasing unpredictably.

\section{A6.5 Discussion}

There was a pronounced variability in SELs of up to $19 \mathrm{~dB}$ at constant ranges out to beyond $1 \mathrm{~km}$ from the AHD (Lofitech). For the ADDs (i.e., the Airmar, Aquamark and SaveWave pingers), the variability was less pronounced at long ranges. At a range of $100 \mathrm{~m}$, there was up to $10 \mathrm{~dB}$ of variation for the Airmar pinger and up to $6 \mathrm{~dB}$ for the Aquamark 100 (Figure A6.4). The overall trend of decreasing SEL with increasing range from the ADD or AHD (Figures A6.4 \& A6.5) was disrupted by interference patterns. Such variability and deviation from spherical or cylindrical spreading expectations, even at large distances from the source, conflicts with the classic description of concentric zones of increasing disturbance with decreasing range (Richardson et al., 1995). This also poses a difficulty for an animal attempting to predict level on a fine scale and orient with respect to this variable intensity gradient. The spatial extent of these zones is clearly difficult to predict, especially given the plasticity of an animal's thresholds of detection, injury and avoidance resulting from its motivation, behavior and physiological state.

One of the motivating concerns for launching this study was the possibility that constructive interference could generate unpredictable pinger SEL hotspots of sufficiently high intensity that might lead to unexpected hearing damage in marine mammals. Although the recorded levels fell below the intensities that caused temporary threshold shifts and temporary losses of hearing sensitivity (i.e., $195 \mathrm{~dB}$ re $1 \mu \mathrm{Pa} 2 \mathrm{~s}$, Finneran et al., 2005), Figures A6.4-A6.5 reveal that moving away from the source did not necessarily guarantee that SEL would decrease. This alters the way in which we should understand an animal's perception 
of an AHD- or pinger-emitted sound field. While swimming away from a sound source, the animal could be exposed to dramatic sound level variations over very small spatial scales. Theoretically, the sound level may shift by several orders of magnitude within a fraction of a meter (Wahlberg, 2006). If the animal integrates time of arrival and phase shift differences between its ears with a series of level cues and these two sets of sensory cues oppose one another, it may be difficult to determine the direction to and location of the sound source. Natural orientation cues may also be obscured by artificial signals through masking and from temporary threshold shifts reported to occur at levels below those measured here (Schlundt et al., 2000). This possibility conflicts with the hypothesis that animals learn to avoid an area due to an acoustic deterrent. The rapid and unpredictable variations in the sound intensity as a function of range to the pinger may seriously confuse the animal and make avoidance responses more complicated than intended. If the animal uses subsequent pings to improve its ability to assess directionality of a signal (as indicated by Kastelein et al., 2007), this problem becomes more serious.

We still need to test whether large spatial variations in SELs prevent animals from reacting appropriately to ADD and AHD signals. Besides the actual problem of detection and determination of the direction to the sound source, the behavior of the animals may be influenced by a learning component that needs to be addressed. Grey seals lifted their heads out of the water in response to AHD signals (Bordino et al., 2002; Fjälling et al., 2006) and physiological (Clark, 1991), behavioral (Olesiuk et al., 2002) and masking (Southall et al., 2000) effects have been observed. Further studies between acoustic deterrents and marine mammal responses are required to examine how animals behave around and react to fishing nets with and without pingers. These issues could be addressed by comparing the acoustic 
measurements of the pinger signals reported here with the behavior of animals swimming through the sound field.

The variability in the SEL may be an important factor to consider when evaluating the implementation of acoustic mitigation devices in fishery regimes. The dynamic characteristics of a trawl, for example, could influence the source directionality and multipath interference, potentially contributing to even larger SEL fluctuations than observed under static conditions. Some newly developed acoustic mitigation devices (i.e., DDD02F) operate with SLs higher than $160 \mathrm{~dB}$ re $1 \mu \mathrm{Pa}^{2} \mathrm{~s}$, further contributing to concerns surrounding their implementation (Dalgaard Balle \& Larsen, unpublished data).

The variability in SELs observed in this study could have been caused by a combination of inter-ping SL variations, bathymetry, wave action influencing the surface reflections, multi-path interference, and source directionality. Salinity and temperature effects were unlikely to have played a strong role because neither a pronounced halocline nor thermocline was observed (measured at SSf and KSf with the Star-Oddi CTD tag) and because computer modeling has demonstrated that such an influence would be rather small for the ranges of interest here (Westerberg \& Spiesberger, 2002). Source directionality and multi-path propagation will now be explored more explicitly. The pingers were mounted vertically to record signals from the broadside axis and thereby minimize directionality effects. The Airmar pinger showed sub-dB variations in its inter-ping SL when recorded in a fixed direction, whereas the Aquamark 100 showed a larger variation, possibly because of slight variations in SL for the various sound types emitted (Figure A6.3). The broadside SL of the Airmar pinger varied less than $2 \mathrm{~dB}$ when rotating the pinger about its axis (Figure A6.3). Therefore, because the Airmar pingers were recorded at small angles relative to their 
axis of symmetry, most of the variability in their SELs as a function of range was attributed to multi-path propagation. Multi-path modeling demonstrates that variability of the magnitude observed here can result from the interference of direct, surface-reflected and bottom-reflected rays (Wahlberg, 2006).

For the Aquamark pinger, the transmission beam pattern was more complicated and variable and depended on which of the two types of signals was being emitted (Figure A6.3). The SL was not only variable between the pinger's axis of symmetry and broadside, but it also varied by $13 \mathrm{~dB}$ on the broadside when rotated about its axis of symmetry. It was not clear to what extent the source directionality and multi-path variation each contributed to the SEL variation for the Aquamark pinger. The signals produced by the SaveWave pingers were variable in duration and frequency spectrum, causing the transmitted energy to vary from one signal to the next, which may at least partially explain the observed SEL variability.

The soft and hard bottom locations did not produce clear differences in the SEL variability. This is surprising since a softer bottom should have rendered fewer multi-paths, leading to a less complicated SEL pattern as a function of range. The soft bottom may have reflected sound better than expected, diminishing the differences in acoustic propagation between the experimental sites. In addition, the soft bottom site was shallower than the hard bottom site, which may have confounded the possible effects of bottom properties on multi-path propagation.

The efficiency of pingers, quantified both in terms of their power demands and the quantity of sound that they are able to discharge, may be improved by decreasing the duration of the emitted signal, which would lead to a reduction in the interference patterns measured here. This suggestion must be balanced, however, with the important issue that to 
obtain a maximum effect, the signal loudness should exceed some critical threshold for an animal's particular integration time that will produce the desired avoidance or disturbance response. More work is required to explore the behavior of seals and porpoises in relation to ADD and AHD sound sources with realistic SLs and their interaction with fishing gear in light of more complex, non-geometrical spreading models. The interplay between conservation and marine mammal and fishery interactions must continue to be engaged by consistent research efforts that explore the ways in which these ADDs and AHDs actually operate and influence the animals that they are intended to target.

In conclusion, we found that signals from ADDs and AHDs did not propagate in a coastal environment according to the simple models of spherical or cylindrical spreading that posit zones of increasing impact with decreasing range (Richardson et al., 1995). The acoustic field to which animals are exposed when approaching a pinger underwater is thus complicated and not easily described by these concentric zones of responsiveness, masking and discomfort relative to the range from the ADD/AHD. Instead, the SEL varied severalfold within very short distances, likely as a result of the interference of direct, surfacereflected and bottom-reflected rays (Wahlberg, 2006). The behavior of seals and cetaceans in relation to the sound field of ADDs and AHDs should be prioritized in future research.

\section{A6.6 Acknowledgements}

S.G. Lunneryd helped with logistics and field work. N.I. Bech, V. Teloni, A. Ungfors and S. Viitasalo assisted during field work. Equipment was kindly loaned by B. Møhl and P.T. Madsen (Århus University, Denmark) and S.G. Lunneryd (Swedish Board of Fisheries). Pingers were provided by Airmar Technology Corporation and with support 
from SaveWave. The project was funded by the Swedish Fishermen Association, the Swedish Board of Fisheries, Aage V. Jensen Foundations, Danish Forest and Nature Agency, The Nordic Research Council and the Carlsberg Foundation. Additional logistical support was furnished by the Oticon Foundation and Reson A/S. The authors wish to thank S.G. Lunneryd, J. Hagberg and S. Königsson (Swedish Board of Fisheries), F. Larsen (Danish Institute for Fisheries Research), P.T. Madsen and two anonymous reviewers for comments and ideas that significantly improved earlier drafts of this manuscript. A.D. Shapiro received financial support from the National Defense Science and Engineering Graduate Fellowship and the WHOI Academic Programs Office. 


\section{A6.7 References}

Akamatsu, T., Nakamura, K., Nitto, H. \& Watabe, M. 1996. Effects of underwater sounds on escape behavior of Steller sea lions. Fisheries Science: 62, 503-510.

Blackwell, S. B., Lawson, J. \& Williams, M. T. 2004. Tolerance by ringed seals (Phoca hispida) to impact pipe-driving and construction sounds at an oil production island. Journal of the Acoustical Society of America: 115, 2346-2357. doi:10.1121/1.1701899.

Bordino, P., Kraus, S., Albareda, D., Fazio, A., Palmerio, A., Mendez, M. \& Botta, S. 2002. Reducing incidental mortality of Franciscana dolphin Pontoporia blainvillei with acoustic warning devices attached to fishing nets. Marine Mammal Science: 18, 833-842.

Clark, W. W. 1991. Recent studies of temporary threshold shift (TTS) and permanent threshold shift (PTS) in animals. Journal of the Acoustical Society of America: 90, 155-163.

Cox, T. M., Read, A. J., Solow, A. \& Tregenza, N. 2001. Will harbour porpoises (Phocoena phocoena) habituate to pingers? Journal of Cetacean Research and Management: 3, 81-86.

Dawson, S. M., Read, A. \& Slooten, E. 1998. Pingers, porpoises and power: Uncertainties with using pingers to reduce bycatch of small cetaceans. Biological Conservation: 84, 141-146.

de Haan, D., Dremiere, P. Y., Woodward, B., Kastelein, R. A., Amundin, M. \& Hansen, K. 1997. Prevention of by-catch of small cetaceans in pelagic trawl by technical means (project CETASEL). ICES.

DeRuiter, S. L., Tyack, P. L., Lin, Y.-T., Newhall, A. E., Lynch, J. F. \& Miller, P. J. O. 2006. Modeling acoustic propagation of airgun array pulses recorded on tagged sperm whales (Physeter macrocephalus). Journal of the Acoustical Society of America: 120, 4100-4114. doi:10.1121/1.235970.

Finneran, J. J., Carder, D. A., Schlundt, C. E. \& Ridgway, S. H. 2005. Temporary threshold shift in bottlenose dolphins (Tursiops truncatus) exposed to mid-frequency tones. Journal of the Acoustical Society of America: 118, 2696-2705.

Fjälling, A., Wahlberg, M. \& Westerberg, H. 2006. Acoustic harassment devices reduce seal interaction in the Baltic salmon-trap, net fishery. ICES Journal of Marine Science: 63, 1751-1758. doi:10.1016/j.icesjms.2006.06.015.

Hammond, P. S. \& Fedak, M. A. 1994. Grey seals in the North Sea and their interactions with fisheries. pp. 1-157. Cambridge: Ministry of Agriculture, Fisheries and Food.

Jefferson, T. A. \& Curry, B. E. 1996. Acoustic methods of reducing or eliminating marine mammal-fishery interactions: do they work? Ocean \& Coastal Management. 31, 41-70.

Johnston, D. W. 2002. The effect of acoustic harassment devices on harbour porpoises (Phocoena phocoena) in the Bay of Fundy, Canada. Biological Conservation: 108, 113-118.

Johnston, D. W. \& Woodley, T. H. 1998. A survey of acoustic harassment device (AHD) use in the Bay of Fundy, NB, Canada. Aquatic Mammals: 24.1, 51-61.

Jørgensen, P. B. 2006. Habituation and habitat exclusion of wild harbour porpoises (Phocoena phocoena) in response to pingers. thesis, University of Copenhagen and University of Århus, Denmark.

Kastak, D., Southall, B. L., Schusterman, R. J. \& Kastak, C. R. 2005. Underwater temporary threshold shift in pinnipeds: Effects of noise level and duration. Journal of the Acoustical Society of America: 118, 3154-3163. doi:10.1121/1.204712.

Kastelein, R. A., de Haan, D. \& Verboom, W. C. 2007. The influence of signal parameters on the sound source localization ability of a harbor porpoise (Phocoena phocoena). Journal of the Acoustical Society of America: 122, 1238-1248.

Kastelein, R. A., van der Heul, S., Terhune, J. M., Verboom, W. C. \& Triesscheijn, R. J. V. 2006. Deterring effects of $8-45 \mathrm{kHz}$ tone pulses on harbour seals (Phoca vitulina) in a large pool. Marine Environmental Research: 62, 356-373. doi:10.1016/j.marenvres.2006.05.004.

Kraus, S. D. 1999. The once and future ping: Challenges for the use of acoustic deterrents in fisheries. Marine Technology Society Journal: 33, 90-93.

Kraus, S. D., Read, A. J., Solow, A., Baldwin, K., Spradlin, T., Anderson, E. \& Williamson, J. 1997. Acoustic alarms reduce porpoise mortality. Nature: 388, 525.

Madsen, P. T. 2005. Marine mammals and noise: Problems with root mean square sound pressure levels for transients. Journal of the Acoustical Society of America: 117, 3952-3957. doi:10.1121/1.1921508. 
Madsen, P. T., Johnson, M., Miller, P. J. O., Aguilar Soto, N., Lynch, J. \& Tyack, P. 2006. Quantitative measures of air-gun pulses recorded on sperm whales (Physeter macrocephalus) using acoustic tags during controlled exposure experiments. Journal of the Acoustical Society of America: 120, 2366-2379. doi:10.1121/1.2229287.

Milewski, I. 2001. Impacts of salmon aquaculture on the coastal environment: A review. In: Marine Aquaculture and the Environment: A Meeting for Stakeholders in the Northeast (Ed. by Tlusty, M. F., Bengston, D. A., Halvorson, H. O., Oktay, S. D., Pearce, J. B. \& Rheault Jr., R. B.), pp. Pages 166-197. Falmouth, MA: Cape Cod Press.

Møhl, B., Wahlberg, M. \& Heerfordt, A. 2001. A large-aperture array of nonlinked receivers for acoustic positioning of biological sound sources. Journal of the Acoustical Society of America: 109, 434-437. doi:10.1121/1.1323462.

Morton, A. B. \& Symonds, H. K. 2002. Displacement of Orcinus orca (L.) by high amplitude sound in British Columbia, Canada. ICES Journal of Marine Science: 59, 71-80. doi:10.1006/jmsc.2001.1136.

Nash, C. E., Iwamoto, R. N. \& Mahnken, C. V. W. 2000. Aquaculture risk management and marine mammal interactions in the Pacific Northwest. Aquaculture: 183, 307-323.

Northridge, S., Fortuna, C. \& Read, A. 2006. Guidelines for technical measures to minimise cetaceanfishery conflicts in the Mediterranean and Black Seas. ACCOBAMS.

Olesiuk, P. F., Nichol, L. M., Sowden, M. J. \& Ford, J. K. B. 2002. Effect of the sound generated by an acoustic harassment device on the relative abundance and distribution of harbor porpoises (Phocoena phocoena) in Retreat Passage, British Columbia. Marine Mammal Science: 18, 843-862.

Quick, N. J., Middlemas, S. J. \& Armstrong, J. D. 2004. A survey of antipredator controls at marine salmon farms in Scotland. Aquaculture: 230, 169-180. doi:10.1016/S0044-8486(03)00428-9.

Reeves, R. R., Read, A. J. \& Notarbartolo-di-Sciara, G. 2001. Report of the workshop on interactions between dolphins and fisheries in the Mediterranean: Evaluation of mitigation alternatives. Roma: ICRAM.

Richardson, W. J., Greene, C. R., Jr., Malme, C. I. \& Thompson, D. H. 1995. Marine mammals and noise. San Diego, CA: Academic Press.

Schlundt, C. E., Finneran, J. J., Carder, D. A. \& Ridgway, S. H. 2000. Temporary shift in masked hearing thresholds of bottlenose dolphins, Tursiops truncatus, and white whales, Delphinapterus leucas, after exposure to intense tones. Journal of the Acoustical Society of America: 107, 3496-3508.

Shaughnessy, P. D. \& Semmelink, A. 1981. Attempts to develop acoustic methods of keeping Cape fur seals Arctocephalus pusillus from fishing nets. Biological Conservation: 21, 141-158.

Southall, B. L., Schusterman, R. J. \& Kastak, D. 2000. Masking in three pinnipeds: Underwater, lowfrequency critical ratios. Journal of the Acoustical Society of America: 108, 1322-1326.

Stone, G. S., Cavagnaro, L., Hutt, A., Kraus, S., Baldwin, K. \& Brown, J. 2000. Reactions of Hector's dolphins to acoustic gillnet pingers. Wellington: Department of Conservation.

Terhune, J. M., Hoover, C. L. \& Jacobs, S. R. 2002. Potential detection and deterrence ranges by harbor seals of underwater acoustic harassment devices (AHD) in the Bay of Fundy, Canada. Journal of the World Aquaculture Society: 33, 176-183.

Trippel, E. A., Strong, M. B., Terhune, J. M. \& Conway, J. D. 1999. Mitigation of harbour porpoise (Phocoena phocoena) by-catch in the gillnet fishery in the lower Bay of Fundy. Canadian Journal of Fisheries and Aquatic Sciences: 56, 113-123.

Wahlberg, M. 2006. Sound propagation of signals from two pingers and an acoustic harassment device in shallow waters. Report for Naturens Stemme and Aarhus University.

Westerberg, H. \& Spiesberger, J. L. 2002. The range of acoustic pingers in the Baltic and the North sea. In: ASCOB ANS 10th AC meeting. Bonn, Germany. 